MORPHOLOGICALLY GOVERNED ACCENT IN OPTIMALITY THEORY

A Dissertation Presented

by

JOHN D. ALDERETE

\author{
Submitted to the Graduate School of the \\ University of Massachusetts Amherst in partial fulfillment \\ of the requirements for the degree of \\ DOCTOR OF PHILOSOPHY
}

May 1999

Department of Linguistics 
(C) Copyright by John D. Alderete 1999

All Rights Reserved 


\section{NEAR KLAMATH}

We stand around the burning oil drum and we warm ourselves, our hands and faces, in its pure lapping heat.

We raise steaming cups of coffee to our lips and we drink it with both hands. But we are salmon

fisherman. And now we stamp our feet on the snow and rocks and move upstream, slowly, full of love, toward the still pools.

-Raymond Carver, Fires 


\section{ACKNOWLEDGEMENTS}

This was the first time that I worked on a project of this size and scope and I am indebted to the members of my committee, John McCarthy, Lisa Selkirk, John Kingston, and Bob Rothstein, for their guidance and support throughout the endeavor. I am also grateful to Alan Prince, who, while not a formal member of the committee, went beyond his role as a consultant and provided many important comments on all aspects of this thesis. I found it tremendously encouraging to sit across from these linguists and find that they had put aside the time to read and respond cogently to the bulky materials stuffed in their boxes. Thank you for allowing me the space to develop my ideas, slowing me down when I was rushing, and sweating the details when I no longer could.

I would also like to express my gratitude to all those who have contributed over the years to my professional and intellectual development. There is a long road between putting together a good homework solution and writing a dissertation, and the support I received from my committee and the larger faculty at UMass was essential to my arriving at where I am today. Many others aside from the committee have helped me come to understand myself as a linguist, but those at the forefront of my mind include Emmon Bach, Roger Higgins, Angelika Kratzer, and Ellen Woolford, and also my teachers and advisors at UC Santa Cruz, Junko Itô, Sandra Chung, and Bill Ladusaw.

The idea that served as the point of departure for this thesis, namely that the accentual properties of roots play a major role in governing word accent, was first hit upon in the summer of 1995 in the weekly brown bag meetings of John McCarthy's NSF grant. I am grateful to John for providing that environment, and for the creative input and expert advice of the participants of those meetings, including Jill Beckman, Laura Benua, Amalia Gnanadesikan, Suzanne Urbanczyk, and in later years, Toni Borowsky, Patrik Bye, Katy Carlson, Paul De Lacy, Mark Harvey, Anna Lubowicz, Caroline Jones, Jennifer Smith, and Rachel Walker. Other opportunities for presenting my research contributed greatly to the preparation of this thesis, including a series of informal meetings at Rutgers and MIT, and invited talks at Cornell University, SUNY Stoney Brook, University of British Columbia, University of Texas at Austin, and University of Victoria. Thanks to the audiences of these gatherings for their helpful comments and questions.

Like most dissertations in linguistics, the argumentation developed in this one revolves around the presentation of evidence, and I would like to thank the language experts and consultants who helped in the collection, organization, and interpretation of this evidence. I am especially indebted to Jane Hill for carefully answering my questions about Cupeño and for making available to me her unpublished fieldnotes and a tape of Cupeño speech, which enabled me to test the predictions of my analysis in a rigorous way. I also owe a great deal to Bob 
Rothstein for explaining many aspects of Russian morphology and phonology to me. Thanks also to Keren Rice and Ed Cook for their advice on linguistic structures in Tahltan, and for helping me clarify a set of problems for after the dissertation. I am also glad to have had the opportunity to converse with Ben Hermans about all aspects of phonology, but especially to get his input on Limburg Dutch, which greatly improved the case study of this language. Thanks is also due to David Payne who helped me gain a better understanding of accent in Aguaruna, in addition to several other aspects of the language. A special commendation is in order for my Japanese consultants, Kiyomi Kusumoto, Junko Shimoyama, Mariko Sugahara, and Hisao Tokizaki, both for helping me find examples and providing a background for classifying and analyzing them, and to Coletta Omamo, who served as a consultant for a field methods class in which data from Luo was collected.

I have also learned a great deal through conversations and correspondence with a number of other linguists. Thanks to the following for their input and critical perspective during the development of the ideas in this thesis: Brett Baker, Eric Baković, Lee Bickmore, Abdelaziz Boudlal, Ellen Broselow, Ioana Chitoran, Abigail Cohn, Megan Crowhurst, Ewa CzaykowskaHiggins, Diamandis Gafos, Dale Hartkemeyer, Bruce Hayes, Paul Hopkins, José Ignacio Hualde, Abdelkrim Jebbour, René Kager, Yoonjung Kang, Motoko Katayama, Ed Keer, Michael Kenstowicz, Armin Mester, Scott Myers, Marc van Oostendorp, Jaye Padgett, Steve Parker, Joseph Pater, Douglas Pulleyblank, Anthi Revithiadou, Patricia Shaw, Ayako Tsuchida, Hubert Truckenbrodt, Charles Ulrich, Draga Zec, and Cheryl Zoll.

I am also grateful to my friends and colleagues at UBC, including Guy Carden, Douglas Pulleyblank, Michael Rochemont, and Patricia Shaw, for helping me settle into a new routine and giving me useful professional advice concerning the completion of this thesis. In addition, they were instrumental in making possible a seminar that enabled me and its participants, Susan Blake, Tanya Bob, Suzanne Gessner, Ikuyo Kaneko, Eunsook Kim, and Eric Rosen, to probe further into the ideas of my thesis. A special thanks is due to Leora Bar-el and Brent Whitted for carefully proof reading the final manuscript and giving helpful stylistic advice.

Finally, I must mention those who, through their friendship and kind support, made grad school a little more doable and a lot more fun. A special thanks is due to Kathy Adamczyk, Lynne Ballard, and Terri Braun for their chit-chat, diligent administrative assistance, and patience when I made unreasonable requests. Thanks also to my classmates and fellow graduate students, Bart Hollebrandse, Andre Isaak, Kiyomi Kusumoto, Winnie Lechner, Deanna Moore, Maribel Romero, and Junko Shimoyama, who helped make these past years stimulating and enjoyable and reminded me on a regular basis of the importance of kicking up my heels at the end of the week. Lastly, I would like to express my deepest gratitude to the following people for their generous support of a non-academic nature during the course of my career, Kuldhir Bhati, Ulrike Demske-Neumann, Joe Dove, Tim Dunn, Carolyn Eastman, Joe Eskenazi, Mike Green, Ian Harper, Dañiel Hernandez, Abdelkirm Jebbour, Kyle Johnson, Sandra Lai, Winnie Lechner, Yanti Mirdayanti, Scott Mobley, Charito Torrefranca, Jing Wang, and Than Than Win. I could not have completed this thesis without these people.

With love and gratitude, this dissertation is dedicated to my parents. 


\author{
ABSTRACT \\ Morphologically Governed Accent in Optimality Theory \\ John D. Alderete \\ Directed by: Professor John J. McCarthy
}

This dissertation examines the influence of morphological factors on stress and pitch accent. Two basic types are recognized. In root-controlled accent, inherent accent in a root overrides inherent affix accent; as a result, affixal accent is only realized in words with unaccented roots. In affix-controlled accent, the presence of a particular affix triggers one of several accentual mutations in the stem: deletion of accent (or a "dominance effect"), insertion of an accent (often known as preor post-accentuation), and accent shift or "flop".

I argue that these two types of accentual behavior, despite important differences, are united under the rubric of faithfulness constraints in Optimality Theory. Root-controlled accent is a consequence of the privileged faithfulness status of roots over affixes, as has been shown in other empirical domains such as vowel harmony. Affix-controlled accent is due to a novel type of constraint, anti-faithfulness, which evaluates a pair of morphologically related words and requires an alternation in the shared stem.

The principal case of root-controlled accent studied in this dissertation is the Uto-Aztecan language Cupeño. In addition, I show how the accentual systems of Japanese and Russian fall within the scope of root faithfulness constraints. The study of these cases leads to a substantive restriction on the range of edge effects in accent systems, and clarifies a role for root accentedness in blocking morpho-accentual processes.

A number of properties of affix-controlled accentual processes are identified and shown to follow from the anti-faithfulness thesis. Affix-controlled accent is (I) morphologically triggered, (II) stem-mutating, and (III) grammar dependent. (I-II) follow from the assumption that anti-faithfulness operates on related words: forcing an alternation in a pair of words ensures that affix-controlled accent is morphological because it contrasts two word classes. Furthermore, as a relation between words, anti-faithfulness only affects the interval of a word which occurs throughout a paradigm, namely the stem (II). Finally, anti-faithfulness does not fully specify how a pair of words should differ accentually; its specific effects therefore depend on the larger grammar in which it is embedded (III).

Affix-controlled accent in Russian, Japanese, Cupeño, Limburg Dutch, and Aguaruna (Jivaroan) is investigated in a series of case studies. I argue that anti-faithfulness constitutes an integrated theory of the diverse morpho-accentual phenomena found in these languages, explains the important differences between the accentual properties of roots and affixes, and establishes parallels with non-accentual affix-controlled phenomena. 


\section{TABLE OF CONTENTS}

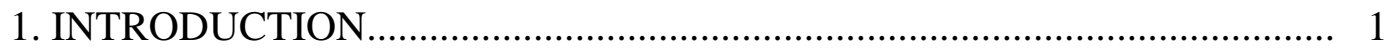

1.1 Morphologically Governed Accent in Optimality Theory ....................... 1

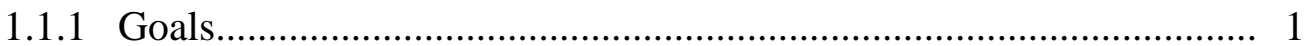

1.1.2 Synopsis of Theoretical Arguments .......................................... 4

1.1.3 Overview of the Dissertation .................................................... 11

1.2 Lexical Accent and Prosodic Faithfulness ............................................. 12

1.2.1 Remarks on the Notion 'Accent' ................................................ 12

1.2.1.1 Observations ........................................................... 12

1.2.1.2 The Representation Question .......................................... 15

1.2.2 A Theory of Prosodic Faithfulness ............................................ 17

1.2.2.1 The Constraints ............................................................. 17

1.2.2.2 Application of the Constraints ........................................ 19

1.2.2.3 Consequences for Culminative Accent ........................... 22

2. ROOT-CONTROLLED ACCENT IN CUPEÑ O _...................................... 28

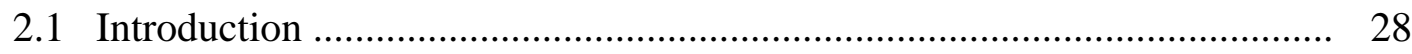

2.2 Theoretical Background: Root and Affix Faithfulness ........................... 30

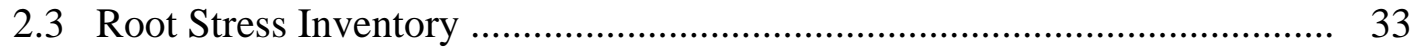

2.3.1 The Data ............................................................................... 34

2.3.2 The Analysis ..................................................................... 35

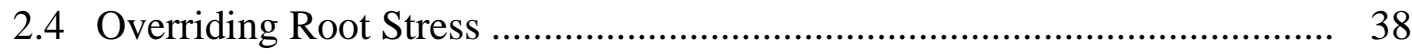

2.4.1 Data and Observations ............................................................ 38

2.4.2 The Analysis ......................................................................... 42

2.4.3 Extending the Analysis ....................................................... 47

2.5 Discussion of Alternatives ................................................................. 54

2.5.1 A Level-Ordering Account ..................................................... 54

2.5.2 A Cyclic Analysis ....................................................................... 56

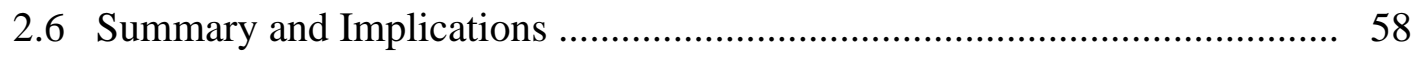




\section{RESTRICTED EDGE EFFECTS IN ROOT-CONTROLLED ACCENT}

SYSTEMS

3.1 Restricted Edge Orientation .................................................................. 60

3.1.1 Factorial Typology …………………………........................... 60

3.1.2 Empirical Issues ................................................................... 64

3.2 Extended Case Study: Modern Russian …………………………….......... 66

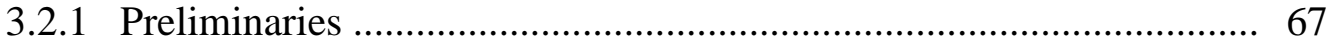

3.2.2 Noun Stress: The Basic Patterns ..................................................... 68

3.2.3 Extending the Analysis: Verb Stress and Prefixed Words ................ 74

3.3 Extended Case Study: Tokyo Japanese ……………............................... 80

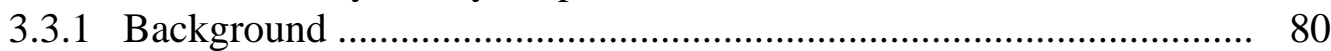

3.3.2 Analysis of Japanese Word Accent .................................................. 83

3.3.3 Analysis of Noun-Noun Compounds ............................................... 85

3.3.4 Influences of Prefixation on Word Accent ....................................... 96

3.4 Summary and Conclusion ..................................................................... 109

4. TRANSDERIVATIONAL FAITHFULNESS AND ANTI-FAITHFULNESS 112

4.1 Morphemic Sources of Accentual Regularity ………................................ 113

4.2 Transderivational Correspondence Theory …………………………....... 116

4.2.1 Introductory Remarks ................................................................. 116

4.2.2 Transderivational Faithfulness in Stress Neutral Affixation .............. 119

4.2.2.1 Theoretical Assumptions ................................................... 119

4.2.2.2 Application to Stress Neutral Affixation ............................ 121

4.3 Transderivational Anti-Faithfulness ..................................................... 128

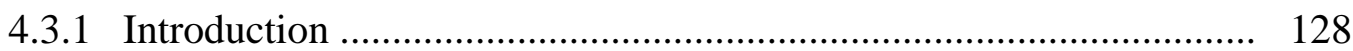

4.3.2 Transderivational Anti-Faithfulness ............................................... 131

4.3.2.1 Theoretical Assumptions .................................................... 131

4.3.2.2 Application to Exchange Processes ..................................... 135

4.3.3 Implications of Transderivational Anti-Faithfulness ........................ 141

\section{THE ROLE OF TRANSDERIVATIONAL ANTI-FAITHFULNESS IN} MORPHO-ACCENTUAL PHENOMENA ............................................... 147

5.1 Towards an Integrated Theory of Affix-Controlled Accent ……................. 147

5.1.1 Properties of Affix-Controlled Accent ............................................. 147

5.1.2 Affix-Controlled Accent as Prosodic Anti-Faithfulness ................... 150 
5.2 Dominance Effects as Transderivational Anti-Faithfulness

5.2.1 The Problem .............................................................................. 153

5.2.2 The Proposal: Dominance Effects as the Negation of MAX-PrOM ... 156

5.2.3 Case Study: Dominance Effects in Russian ..................................... 163

5.24 Implications ........................................................................... 173

5.2.5 Discussion of Alternatives ............................................................ 182

5.3 Pre/Post-Accentuation as Transderivational Anti-Faithfulness ................... 190

5.3.1 The Problem ......................................................................... 190

5.3.2 The Proposal: Pre- and Post-Accentuation as Negation of

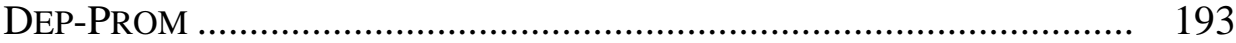

5.3.3 Implications ............................................................................ 200

5.3.3.1 Strict Base Mutation in Pre- and Post-Accentuation ........... 200

5.3.3.2 Dominant/Recessive Pre-Accentuation in Tokyo Japanese 204

5.3.3.3 Factorial Typology ........................................................... 207

5.3.4 Discussion of Alternatives .......................................................... 210

5.4 Accent Shifts as Transderivational Anti-Faithfulness ……......................... 216

5.4.1 The Problem ............................................................................ 216

5.4.2 Dragging Tone Mutation in Limburg Dutch: Evidence for

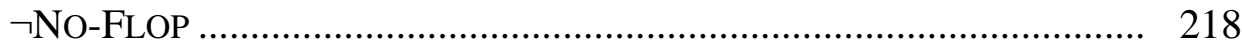

5.4.2.1 Data and Observations ..................................................... 218

5.4.2.2 Tone in Monomorphemic Words ........................................ 220

5.4.2.3 Tonal Mutation in Derived Words as $\neg$ No-Flop-ToNE ...... 223

5.4.3 Case Study: Accent Shift in Tokyo Japanese ................................... 227

5.4.4 Extended Case Study: Accent in Aguaruna ……………………..... 234

5.4.4.1 Distribution of Accent in Underived Words ......................... 235

5.4.4.2 Vowel Deletion and Accent Shift ........................................ 237

5.4.4.3 Accent Shift in Derived Words ........................................... 245

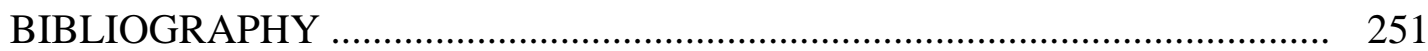




\section{Chapter 1. Introduction}

\subsection{Morphologically Governed Accent in Optimality Theory}

This dissertation is about accent systems in which word structure has an important role in the characterization of accentual processes. These processes affect phonological categories like stress and tone, but importantly, they cannot be described with reference to sound structure alone. Morphological factors like the accentual properties of roots and certain diacritically marked affixes must also be recognized. As many accent systems studied here have a strict limitation of one accent per word, one major accentual process examined in this thesis is Accent Resolution, the deletion of accent in words with more than one inherently accented morpheme. This pattern of deletion often shows a preference for retention of accent in the root, which underscores one important function of morphological structure. Other morpho-accentual phenomena examined here include morphologically triggered de-accentuation (or "dominance effects"), accent insertion (often know as preand post-accentuation), and certain accentual shifts. The occurrence of these processes is, in many cases, directly tied to affixation, and so they too are inherently morphological. The focus here is almost exclusively on word accent, as the accentual processes under examination are mostly word-level phenomena, but some parallels with other levels of structure are made throughout this thesis.

\subsubsection{Goals}

This thesis has two basic goals. The first goal is more or less a descriptive one and involves arguing for the existence of two distinct types of morpho-accentual processes (1). The first type is an analogue to a well-known type of vowel harmony system where the features of root vowels have the effect of 'overriding' the featural specifications in prefixes and suffixes. In many accent systems, the presence of an accent in the root likewise overrides accent in affixes, hence the term 'root-controlled accent'. The second type is fundamentally different from the first. Generally linked to affixation, 'affix-controlled' accentual processes require a change in the accentuation of the base, which is usually the root or the stem.

(1) a. Root-Controlled Accent (RCA): inherent accent in the root precludes the realization of accent elsewhere in the word.

b. Affix-Controlled Accent (ACA): the attachment of an affix correlates with a mutation of the accent in the base of affixation.

The second goal, related to the first, is to argue for a specific theoretical model which accounts for these two types of morpho-accentual processes in an explanatory way.

To my knowledge, the first characterization of an accent system in terms of rootcontrol is due to Hill \& Hill 1968, who describe stress-accent in the Uto-Aztecan language Cupeño. In this language, both roots and affixes have an accented/unaccented contrast, but when an inherently accented root combines with an accented affix, the root accent 'wins', as exemplified below with some characteristic examples (the roots are underlined). 
(2) Root-Controlled Accent in Cupeño (Hill \& Hill 1968)
a. /pé + yax/
$\rightarrow$ pé-yax
/pé + yax + qál/
$\rightarrow \quad$ pe-ya-qál
'He says'
/pé + yax + qál/
b. /pé + ?áyu + qál/
$\rightarrow \quad$ pe-?áyu-qal
/pé + pulín + qál/
$\rightarrow$ pe-pulín-qal
'S/he was wanting'
'She gave birth'
'He was saying'

Part of the descriptive aim of this thesis is to extend this idea to other, better-known accent systems like Russian and Japanese. In the analyses of these systems, root-controlled accent has a very similar role, causing the deletion of an accent outside of the root. The parallels between these systems run deeper than this, however, which can be seen by examining a second pattern of RCA.

RCA has an equally important role in blocking the application of other accentual processes. For example, Cupeño has a set of suffixes which place an accent on the rootfinal syllable (3a), but this process is blocked in words with accented roots because such an insertion would preclude the realization of a root accent $(3 b)$.

(3) Blocking Effect of RCA in Cupeño
a. /wena + 'nuk/
$\begin{array}{ll}\rightarrow & \text { wená-nuk } \\ \rightarrow & \text { ne-má-č i }\end{array}$
'having put in'
/né + ma + ‘̌ il
'with my hand(s)'
b. /méme + 'yke/ $\rightarrow$ méme-yke
/tívii?e $+{ }^{\prime m a a}+$ le/ $\rightarrow \quad$ tívi?-me-l
'to the ocean'
'small round basket'

Likewise, in Russian and Tokyo Japanese, certain patterns of pre-accentuation are only found in words with unaccented roots, an observation which extends to other morphoaccentual processes. In sum, RCA has the effect of privileging roots in the realization of inherent accent, both in the concatenation of more than one inherently accented morpheme and in the application of morpho-accentual processes.

In the second type of morpho-accentual process, affix-controlled accent, affixes are in charge accentually. However, this process is not simply the symmetric counterpart to RCA; it shows a different kind of behavior altogether. The main characteristic of ACA which sets it apart from RCA is that ACA demands a change in the accentuation of the base to which the affix is attached. Thus, affix-controlled processes run counter to the underlying force behind RCA because they demand a change in base prosody, which typically contains the root. Three affix-controlled processes examined in detail here are illustrated below with some examples from Tokyo Japanese. 
(4) Affix-Controlled Accent in Tokyo Japanese (Poser 1984, McCawley 1968)

a. Dominance effects require a deletion of base prosody

$$
\begin{array}{llll}
\text { /edo }+\mathrm{kko} / & \rightarrow & \text { edo-kko } & \text { 'native of Tokyo' } \\
/ \text { kóobe }+\mathrm{kko} / & \rightarrow & \text { koobe-kko } & \text { 'native of Kobe' }
\end{array}
$$

b. Pre-accentuation requires an insertion of accent into the base

$$
\begin{array}{llll}
\text { /yosida }+\mathrm{ke} / & \rightarrow & \text { yosidá-ke } & \text { 'the Yoshida family' } \\
\text { /nisímura }+\mathrm{ke} / & \rightarrow & \text { nisimurá-ke } & \text { 'the Nishimura family' }
\end{array}
$$

c. Accent shifts require a shift of base prosody

$$
\begin{array}{llll}
/ \text { kúzu }+ \text { ya/ } & \rightarrow & \text { kuzú-ya } & \text { 'junkman' } \\
\text { /toma }+ \text { ya/ } & \rightarrow & \text { toma-ya } & \text { 'mat seller' }
\end{array}
$$

A common type of affix-controlled process, exemplified in (4a) with the suffix $-k k o$, is deletion of the prosody of the base to which the affix is attached. It is clear why this type of deletion, sometimes called a 'dominance effect', is antagonistic to RCA: RCA strives to preserve the accent of the root, while -kko demands deletion of the root accent. Another type of affix-controlled process is pre-accentuation (or post-accentuation for prefixes), which causes insertion of an accent somewhere in the base, as shown in (4b). Preaccentuation may also run counter to the imperative to realize root accent in this system because, as is typical, there is one accent per word in Japanese; the insertion of an accent thus entails the deletion of the base accent, as in nisimurá-ke. A final type of ACA involves accent shift or "flop", exemplified in (4c) with the suffix -ya. While not in direct conflict with RCA, accent shifts of this kind resemble the other types of ACA in that they mutate the accent of the base. The accentual change demanded by -ya is a shift of the prosody of the base, as observed in words with accented stems like kuzú-ya. To summarize, affixcontrolled accent runs counter to RCA in requiring a change of the accentuation in the base of affixation.

The identification of these two morpho-accentual processes raises the following two questions for the theory of morphologically governed accent.

1. How are the differences between RCA and ACA to be described and explained?

2. How is the conflict between RCA and ACA to be resolved?

The observation that RCA and ACA are fundamentally different poses the formal question of how to distinguish them as separate classes of morpho-accentual phenomena. Furthermore, the inherent difference between RCA and ACA leads to situations of conflict where RCA demands preservation of a root accent, while ACA requires a change of the root accent, even the deletion of it. Moreover, the negotiation of this conflict is a subtle matter. In some contexts RCA wins, as found in pre-accentuation in Cupeño. In the case of Japanese, however, ACA wins, as the pre-accenting suffix -ke induces an insertion of accent across the board, even in words with accented roots. How then is the conflict between these morpho-accentual phenomena to be modelled in a way that accounts for the observed cross-linguistic differences?

There is also a larger theoretical issue to be addressed in the analysis of these morpho-accentual processes. Both RCA and ACA have clear parallels in other morpho- 
phonological alternations and these parallels require explanation. As alluded to above, RCA is like root-controlled vowel harmony in that the phonological patterns observed in words are determined by the roots contained in these words. Affix-controlled accentual processes also have well-established parallels with non-accentual processes. Preaccentuation, for example, can be compared to the length alternations induced by certain affixes, like pre-lengthening suffixes found in many languages (e.g., Yidiñ, Dixon 1977): both pre-accenting and pre-lengthening suffixes trigger a change of the phonological makeup of their bases. These considerations lead to a third important question, which has both descriptive and theoretical implications.

3. What accounts for the parallels to RCA and ACA found in other areas of phonology?

If the parallels pointed to above are valid, then it would be a significant liability if the ultimate theory of RCA and ACA did not make these connections. A major goal of this thesis, therefore, is to account for the properties of ACA and RCA with principles that are generally available in linguistic theory. The correct theory, by this desideratum, should account for the range of accentual processes with theoretical constructs which are not specific to a theory of accent, but rather, sufficiently general to extend to other morphologically governed phonological patterns.

\subsubsection{Synopsis of Theoretical Arguments}

A fundamental goal of linguistic theory is to make sense of language particular facts with universal principles. Optimality Theory (OT) takes a particular approach to achieving this goal (Prince \& Smolensky 1991, 1993, McCarthy \& Prince 1993b). ${ }^{1}$ Universal constraints are posited, often having a basis in theories of language production, perception, and processing. This assumption entails that there is universal set of constraints, dubbed $C O N$, and that these constraints are present in the grammar of every language. To account for cross-linguistic differences, these well-formedness constraints are ranked, or prioritized, on a language particular basis. In this ranking, violation of a constraint is tolerated if it leads to the satisfaction of another, higher-ranking, constraint.

(5) Some Core Assumptions in Optimality Theory

a. Universality of Constraints: linguistic behavior is modelled as the interaction of universal constraints.

b. Constraint Ranking and Violability: constraints are ranked on a language particular basis; violation of a given constraint is tolerated if it leads to the satisfaction of a higher-ranking constraint.

In this context, it is important to account for language particular patterns in tandem with the typological regularities which cross-cut languages. Optimality Theory, because of these inherent assumptions, is responsible for both types of observations. The core tenets of OT bring new insight into both language particular and cross-linguistic patterns found in morphologically governed accent systems, as I show below in a synopsis of the theoretical arguments developed in subsequent chapters.

In most OT work in phonology, two types of constraints are recognized (6). There are the so-called Markedness constraints, which characterize various forms of surface well-

\footnotetext{
${ }^{1}$ For an introduction to the technical aspects of Optimality Theory, e.g., the generation and evaluation of candidate forms, see the references cited above, in addition to McCarthy \& Prince 1994a, 1995, Beckman 1997 [1998], and Benua 1997 [1998].
} 
formedness and are often motivated in terms of ease of articulation or perception. These constraints assess outputs and determine the markedness of a given form as a function of its constraint violations. For the present purposes, however, the more important constraint type is Faithfulness. Generally speaking, Faithfulness constraints require a lexical form to remain unchanged at the surface. Thus, if lexical-to-surface Faithfulness is fully satisfied, then the output duplicates the input in every way.

(6) Constraint Types in Optimality Theory

a. Markedness: applies to output forms and characterizes surface well-formedness.

b. Faithfulness: applies to a pair of related forms and requires them to match for different aspects of linguistic structure.

There is a fundamental tension between Markedness and Faithfulness constraints. On the one hand, Markedness pushes a form towards simplification and loss of marked structure. Faithfulness, on the other hand, pushes back, striving for preservation of lexical distinctions, even at the expense of marked or complex structure at the surface. While Markedness constraints have an important role in accent systems, especially in regulating phonologically predictable accentual patterns, Faithfulness to lexical accent is fundamental as it is at the heart of the explanation for the morphological factors examined in this thesis.

One central role of the Faithfulness constraints is to provide the basis for a theory of phonemic accent. The presence of a phonemic contrast in a language entails a ranking in which Faithfulness for the contrastive feature outranks the relevant Markedness constraints that neutralize this contrast. This reasoning applies with equal force when prosodic features such as stress and tone give rise to surface contrast. If Faithfulness to lexical prosody outranks the set of constraints which bring about a regular pattern for this prosody, the result is a system with contrastive accent. The reverse ranking, on the other hand, produces a grammar without this contrast. In sum, phonemic versus predictable accent is determined through constraint ranking in this theory.

The approach to accentual contrast as satisfaction of Faithfulness to lexical prosody leads to a principled analysis of the first type of morpho-accentual process, root-controlled accent. Converging sources of evidence have surfaced recently which lead to the conclusion that there are distinct Faithfulness constraints for roots and affixes. A direct source of evidence derives from psycho-linguistic studies showing the privileged role of roots in lexical access and storage (see Beckman 1997 [1998] for a review). In addition, the distinction between Root and Affix Faithfulness is motivated on purely linguistic grounds (as argued in McCarthy \& Prince 1995, Selkirk 1995a,b, Urbanczyk 1996, and Beckman 1997 [1998]). The privileged status of roots over affixes leads to the following intrinsic ranking among the Faithfulness constraints.

(7) Morphologically-Dispersed Faithfulness (McCarthy \& Prince 1995)

\section{Root Faith > Affix Faith}

This inherent ranking accounts for two characteristic observations. First, roots generally license a wider range of contrasts than affixes. This observation is ensured by the ordering in (7) because any constraint ranked above Root Faith must also outrank Affix Faith. Therefore, any restriction that holds on the distribution of a feature of the root must also hold of affixes, which effectively precludes a contrast in affixes which is not present in roots. In addition to deriving restrictions in inventories, the above ordering predicts that roots will have a privileged status in phonological alternations. In familiar root-controlled 
vowel harmony systems, for example, the demands of the system require that there be a single featural specification in a given harmonic domain; in situations where a root and affix have conflicting featural specifications, morphologically-dispersed Faithfulness guarantees faithful treatment of the features of the root, yielding the observed root-control pattern. In sum, the ranking Root Faith $\gg$ Affix Faith assigns a privileged Faithfulness status to roots, which is found in both phonological inventories and alternations. Furthermore, these results foreground the importance of constraint ranking because the satisfaction of a high-ranking constraint is decisive.

With these assumptions in hand, root-controlled accent is explained with the same basic tools used in the analysis of other root-controlled phenomena. Typically, words may only have a single accent; thus, in words with more than one inherently accented morpheme, all but one is deleted. If the word contains an accented root and one or more accented affixes, satisfaction of Root Faithfulness has the effect of precluding the realization of affix accent, as shown below.

(8) Root-Controlled Accent in Cupeño as Root Faithfulness

\begin{tabular}{|l|c|c|}
\hline /pé + ?áyu + qál/ & FAITH(Accent) Root & FAITH(Accent)Affix \\
\hline \hline a. $\quad$ pé-?ayu-qal & $* !$ & $*$ \\
\hline b. $\rightarrow$ pe-?áyu-qal & & $* *$ \\
\hline
\end{tabular}

Preservation of affix accent in (8a) entails a violation of FAITH(Accent) Root, which is fatal for the first candidate given the rank of this constraint in the system. The winning form is thus the one which preserves the root accent at the expense of the loss of affix accent (8b). This analysis therefore explains the pattern of root retention in terms of the same constraint ranking used in the analysis of a non-accentual phenomena like vowel harmony, establishing a parallel between these two systems in a formal way. In addition to this result, there are two important consequences that this approach to RCA has for accent systems in general.

The first consequence is that the role of directionality in accent systems is significantly reduced. The inherent ranking Root Faith $\gg$ Affix Faith predicts that, in languages with an accentual contrast in both roots and affixes, root accent generally takes precedence over affix accent. This prediction is indeed interesting because it attributes a role for the morphological structure in Accent Resolution (AR) that has been formerly treated in terms of phonological directionality. For example, the retention of stem accent in many Indo-European languages is argued in Kiparsky \& Halle 1977 to be due to a principle which assigns a word level accent to the first inherently accented morpheme of a word. Likewise, Poser 1984 employs a principle of directional AR which favors deletion of all but the first lexical accent in Japanese minor phrases. These accounts, however, lack the crucial evidence from prefix + root sequences which shows that AR must be governed by directionality. The absence of a class of prefixes which 'win out' over a following stem accent invites a re-analysis of these phonological accounts in terms of root-controlled accent. In the RCA analysis, the absence of a class of prefixes which take precedence over a root accent is a predicted consequence of the privileged Faithfulness status of roots. It appears, therefore, that two additional accent systems, which have been previously analyzed in different way, also fall within the scope of Root Faithfulness constraints.

The theory of RCA developed here does not deny a role for directionality in accent systems altogether. Rather, morphologically-segregated Faithfulness predicts that rootcontrolled AR is primary, and when Faithfulness is indecisive, other constraints may take 
effect. Faithfulness is not decisive when it is crucially dominated. Thus, when a constraint requiring accent to appear at a designated edge dominates Faithfulness to lexical prosody, a directional pattern of AR may emerge. Also, Faithfulness is not decisive in word types where inherently accented morphemes are of equal status. For example, in Cupeño, words with an unaccented root and more than one accented affix show a preference for realizing the rightmost affix accent. In such a scenario, rightmost accent surfaces when the grammar does not require faithful treatment of a root accent. To summarize, overriding root accent is primary, applying to all cases where Root and Affix Faithfulness to accent is decisive.

A second important consequence of the proposed theory is that it clarifies a distinct type of morpho-accentual process, affix-controlled accent. In contrast to root-controlled accent, which assigns enhanced Faithfulness to roots, affix-controlled accent actively mutates the base of affixation, as depicted below for dominant affixes in Japanese.

$$
\text { RCA in Cupeño }
$$

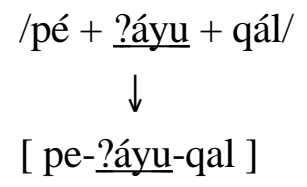

ACA in Japanese

$$
\begin{aligned}
& \frac{\text { kóobe }}{\ddagger}+\mathrm{kko} / \\
& \text { [ koobe-kko ] }
\end{aligned}
$$

INPUT

OUTPUT

The analysis of RCA follows from the privileged Faithfulness properties of roots. I argue that the analysis of ACA, by contrast, is due to a new type of constraint, Anti-Faithfulness. Roughly speaking, Anti-Faithfulness is the negation of Faithfulness. While Faithfulness resists an alternation, Anti-Faithfulness specifically requires one, as observed with the deletion of accent in words with -kko. The distinction between RCA and ACA can thus be characterized as two different lines of development in the formalization of Faithfulness. RCA is due to Root Faithfulness; ACA is the result of affix-induced Faithfulness reversals which bring about a change in the accentuation of the base of affixation.

While Anti-Faithfulness forms the basis of an explanatory theory of ACA, it is also motivated in non-accentual phonology. In particular, this constraint type is indispensable in the analysis of exchange processes, i.e., morpho-phonological alternations which involve a full rotation of two classes of phonological elements. A well-known example of this type is the voicing exchange found in the Nilotic language Luo, where the [voice] specification for the stem-final obstruent in the singular is reversed in the corresponding plural.

(10) Voicing Exchange in Luo (Gregerson 1972, Okoth-Okombo 1982)

$\begin{array}{lll}\text { a. } \begin{array}{l}\text { Singular } \\ \text { reč }\end{array} & \begin{array}{l}\text { Plural } \\ \text { bed-e } \\ \text { rej-e }\end{array} & \begin{array}{l}\text { 'arm' } \\ \text { 'fish' }\end{array} \\ \text { b. } \begin{array}{c}\text { č ogo } \\ \text { luedo }\end{array} & \begin{array}{l}\text { ć ok-e } \\ \text { luet-e }\end{array} & \text { 'bone' } \\ \text { 'hand' }\end{array}$

Cases such as these pose an interesting challenge to OT because no ranking of Markedness and Faithfulness constraints yields the observed exchange (a point demonstrated in Moreton 1996). Succinctly, any ranking of these two classes of constraints which maps one type of segment onto another is invariably one-way, i.e., /A/ $\rightarrow[\mathrm{B}]$, but /B/ $\rightarrow[\mathrm{B}]$, 
not /B/ to [A] as observed in Luo. In order to produce an unfaithful mapping, as in /A/ $\rightarrow[\mathrm{B}]$, Faithfulness for the relevant feature must be ranked below the constraint banning segments of type $A$; if this ranking holds, then the grammar will not change $/ B /$ to $[A]$ as well because this mapping violates the constraint banning [A]. The up-shot is that exchange processes are formally intractable in OT if grammars are characterized as a ranking of just Markedness and Faithfulness constraints.

The introduction of Anti-Faithfulness constraints solves the problem posed by exchange rules. The Faithfulness constraint for [voice] has, by hypothesis, a corresponding Anti-Faithfulness constraint, $\neg$ FAITH(voice), which requires a change of the [voice] specification of the stem-final obstruent. The ranking of this constraint above its related Faithfulness constraint therefore describes the full rotation of this feature observed in Luo.

(11) Voicing Exchange in Luo as Anti-Faithfulness

\begin{tabular}{|cc|c|c|}
\hline Input & Output & $\neg$ FAITH(voice) & FAITH(voice) \\
\hline \hline a. $/$ bat $\rightarrow$ & be $[\mathrm{d}]-\mathrm{e}$ & $*$ & $*$ \\
\cline { 2 - 4 } & $*$ be $[\mathrm{t}]-\mathrm{e}$ & $* !$ & \\
\hline \hline b. $/$ č ogo/ $\rightarrow$ & č o[k]-e & & $*$ \\
\cline { 2 - 5 } & *c o[g]-e & $* !$ & \\
\hline
\end{tabular}

Both parts of the exchange are predicted by $\neg$ FAITH(Voice) because this constraint specifically requires a change of the [voice] specification in the base. This solution therefore motivates Anti-Faithfulness as an important theoretical construct and shows that it is operative in non-accentual morpho-phonological alternations.

A host of properties of affix-controlled accent are identified in this thesis and shown to follow from Anti-Faithfulness when this constraint type is applied to morphologically related words, i.e., when it is 'transderivational' in the sense of Benua 1997 [1998]. First, ACA is morphological in the sense that it induces an opposition between a base and a derived form. As a relation between words, Anti-Faithfulness forces an alternation in the paradigm which has the effect of marking and re-enforcing salient morphological distinctions. Second, affix-controlled accent is always base-mutating, meaning that it always affects the base of affixation. This observation also follows from the transderivational implementation: Anti-Faithfulness only affects the subconstituent of the word which occurs throughout a paradigm, namely the base. Third, affix-controlled accentual processes are 'grammar dependent', which means that their output is constrained by the independently motivated grammar of accent. Anti-Faithfulness explains grammar dependence because it does not fully specify the ways in which two words must differ accentually; it asserts that they must be different, and the rest of the grammar predicts how this difference is realized (a point returned to below).

In a sense, the approach to ACA as Transderivational Anti-Faithfulness (TAF) is an explicit formal statement of the insights that underlie many traditional analyses of diacritic properties of affixes (see especially Garde 1968 et seq. on Slavic languages, Fudge 1984 on English, and Carlson 1976, 1989 on Interior Salish). These analyses differentiate morpheme classes with various accentual diacritics, e.g., 'pre-stressing' or 'accentdeleting', etc.; furthermore, language particular prioritizations for these diacritic properties determine the outcome in words which are marked for more than one diacritic (see Carlson 1976 et seq. for a particularly elaborate hierarchical ordering). The formalization proposed 
here, however, establishes the basis of a highly restrictive theory of accentual processes because of the nature of Transderivational Anti-Faithfulness and the inherent properties of Optimality Theory. First, TAF theory establishes substantive limitations on the range of affix-controlled processes. Since ACA is derived as Faithfulness reversals, the range of affix-controlled accentual processes must be described in terms of violations of existing Faithfulness constraints. Thus, the theory of Accentual Faithfulness defines a set of constraints which govern the realization and distribution of accent (12a). These Accentual Faithfulness constraints have corresponding Anti-Faithfulness constraints (12b), which effectively predict the range of possible affix-controlled accentual processes.

\section{(12) Faithfulness and Anti-Faithfulness for Accent}
a. MAX-ACCENT: no accent deletion
b. ᄀMAX-ACCENT: obligatory accent deletion
DEP-ACCENT: no accent insertion
$\neg$ DEP-ACCENT: obligatory accent insertion
NO-FLOP-ACCENT: no accent shift
$\neg$ NO-FLOP-ACCENT: obligatory accent shift

The fundamental notion of Faithfulness in OT thus has a role in the analysis of ACA too, through the negation of the independently needed Accentual Faithfulness constraints. The constraints which are essential to the analysis of phonemic accent have corresponding AntiFaithfulness constraints, which in turn characterize a restrictive typology of affix-controlled morpho-accentual processes.

The TAF theory of affix-controlled accent is also constrained by the larger grammar in which Anti-Faithfulness is employed. Succinctly, a TAF constraint requires a change in the base of affixation, but the realization of this change is predicted by the independently motivated grammar of accent. Applying this reasoning to dominance effects in Japanese, the suffix - $k k o$ requires a deletion of base prosody by activation of the TAF constraint $\neg$ MAX-ACCENT. It is the rest of the grammar, however, which determines the result of this deletion process. Since unaccented words are left unaccented by default in Japanese, as shown by miyako 'city', so too are forms which result from de-accentuation.

(13) Grammar Dependent ACA: Dominance Effects in Japanese

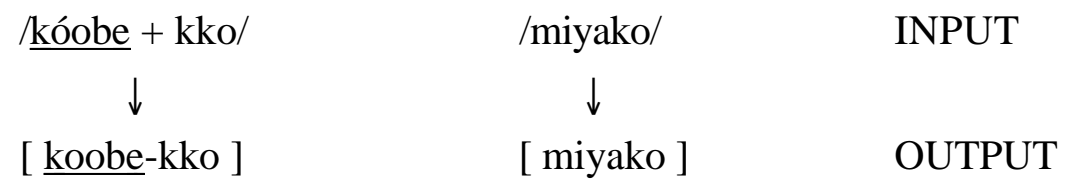

As obligatory violations of Faithfulness, TAF makes the prediction that the output of affixcontrolled accentual processes is intimately tied to the default accentual structures found elsewhere in the system. The finding that all of these processes exhibit some form of grammar dependence is therefore strong support for the proposed theory.

Returning to the observed conflict between RCA and ACA, OT provides the right tools for modelling this antagonism as well. Recall that certain suffixes in Cupeño trigger the insertion of an accent in the base. This observation is accounted for in (14a) as an effect of the Anti-Faithfulness constraint, $\neg$ DEP-ACCENT, which calls for the observed insertion of an accent. The loser fails to insert an accent, and as a result incurs a fatal violation of $\neg$ DEP-ACCENT. However, accented roots block pre-accentuation, which is derived by ranking the Root Faithfulness constraint demanding the realization of accent, namely MAX-ACCENT Root, above the Anti-Faithfulness constraint, as shown in (14b). 
(14) Pre-Accentuation with Blocking Effects in Cupeño

\begin{tabular}{|c|c|c|c|c|}
\hline Input & & Output & MAX-ACCENT Root $_{\text {RAX }}$ & $\neg$ DEP-ACCENT \\
\hline \multirow[t]{2}{*}{ a. / $\underline{\text { wena }}+$ nuk $_{\text {pre }} /$} & $\rightarrow$ & wená-nuk & & \\
\hline & & *wena-nuk & & $* !$ \\
\hline \multirow[t]{2}{*}{ b. / $\underline{\text { méme }}+\mathrm{yke}_{\mathrm{pre}} /$} & $\rightarrow$ & méme-yke & & $*$ \\
\hline & & *memé-yke & $* !$ & \\
\hline
\end{tabular}

The conflict between these two competing forces is described with one of the fundamental assumptions of OT, namely that the constraints are ordered with respect to each other in a constraint hierarchy. Furthermore, this ranking is established on a language particular basis; in one language Root Faithfulness may be ranked above the Anti-Faithfulness constraint $\neg$ DEP-ACCENT, which as illustrated above for Cupeño, accounts for blocking effects. However, another language may have the reverse ranking, yielding preaccentuation across the board, as exemplified above for - $k e$ in Japanese. In sum, this variation is treated as a language-internal prioritization of universal constraints.

To summarize the main ideas, two lines of development in the characterization of Faithfulness in Optimality Theory define a theory of morphologically governed accent. A set of Accentual Faithfulness constraints are employed in the analysis of phonemic accent. A relatively straightforward modification of these constraints, namely their division into the morphological categories root and affix, leads to a principled explanation of root-controlled accent. With morphologically-dispersed Faithfulness, overriding root accent is a consequence of the privileged Faithfulness properties of roots, as has been shown in other empirical domains such as vowel harmony. Affix-controlled accent, on the other hand, is due to a new constraint type, Anti-Faithfulness, which models this type of morphoaccentual process as a Faithfulness reversal. Applied between a base and derivative, AntiFaithfulness explains the fact that affix-controlled accentual processes are inherently morphological and base-mutating. Furthermore, the Anti-Faithfulness thesis leads to a restrictive typology of morpho-accentual processes; these processes are limited to operations that can be derived by obligatory violations of Faithfulness constraints and that result in language particular default patterns for accent.

Concerning the larger theoretical implications, the theory of morphologically governed accent proposed in this dissertation employs principles which have very general applications in phonology. The notion of Faithfulness crucial in the characterization of phonemic accent is no less crucial in the analysis of other types of phonemic contrast. Furthermore, the privileged Faithfulness status for roots found in accent systems is also characteristic of non-accentual phonological systems, and thus the ordering of Root and Affix Faithfulness extends to both types of systems. Lastly, the approach to affixcontrolled accent as obligatory violations of Faithfulness constraints also has some currency outside of accent systems; it is critical to the analysis of exchange processes and it forms the base of a general theory of morpho-phonological alternations. These descriptive and analytical assumptions therefore lead to the conclusion that root-controlled accent is a special case of a cross-linguistic trend favoring retention of information in the root, and likewise, that affix-controlled accent is just a special type of phonological alternation instantiating a morphological contrast. 


\subsubsection{Overview of the Dissertation}

The next section of this chapter provides an introduction to the properties of the accent systems examined in this thesis and the theoretical background in autosegmental and metrical theory relevant for these systems. A theory of Prosodic Faithfulness is then proposed, and the constraints responsible for phonemic accent are defined and applied to a concrete example. The rest of the thesis is summarized below as an overview of what is to come in later chapters.

§2. Root-Controlled Accent in Cupeño. This chapter is a detailed case study of stressaccent in Cupeño. After a close look at stress in isolated roots, the interaction between root and affix stress is examined and the morphologically-dispersed Faithfulness constraints are employed in the analysis of the basic fact that root accent overrides affix accent. The root-controlled analysis of Cupeño stress-accent is contrasted with some plausible alternatives and a host of predictions of the analysis are summarized.

§3. Restricted Edge Effects in Root-Controlled Accent Systems. This chapter studies the implications of the analysis of root-controlled accent in Cupeño for other languages. The consequences of the universal ordering Root Faith >> Affix Faith are studied and a prediction is outlined which posits a significant restriction on the scope of directionality in accent systems. This prediction is then examined in detail in two languages, Russian and Japanese. In developing close formal analyses of the regular and productive accentual patterns found in these languages, it is shown that these languages do in fact conform to the restrictive theory of edge effects stemming from morphologically-segregated Faithfulness.

§4. Transderivational Faithfulness and Anti-Faithfulness. This chapter provides the necessary background for analyzing affix-controlled accent in a general theory of morpho-phonological alternations. As the theory of Anti-Faithfulness is developed in Transderivational Correspondence Theory (Benua 1997 [1998]), a review of the basic concepts of this theory is given in the context of a discussion of stress-neutral affixation in English. Next, the theory of Anti-Faithfulness is motivated and applied to the case of voicing exchange in Luo. The implications of this theory are also studied and a set of predictions for affix-controlled accentual processes are clarified.

§5. The Role of Transderivational Anti-Faithfulness in Morpho-Accentual Phenomena. This chapter applies the theory of Transderivational Anti-Faithfulness to affixcontrolled accent, arguing that this theory establishes the observed parallels between morpho-accentual processes and morpho-phonological alternations in general. It begins by discussing the properties which distinguish affix-controlled accent from rootcontrolled accent and the underlying functions of these distinct accentual processes in the larger architecture of the grammar. Subsequently, each affix-controlled process is examined in the context of a series of case studies. It is shown that the TAF theory of affix-controlled processes provides an explanatory account of the properties which characterize ACA and distinguish this theory from several previous approaches. The resulting theory is an integrated whole, accounting for a heterogeneous body of accentual patterns as forced violations of existing Faithfulness constraints. 


\subsection{Lexical Accent and Prosodic Faithfulness}

The proposal to study accent systems is in some ways problematic because the term 'accent' has been used in so many different ways and applied to a wide range of phonological phenomena. For some, the term accent is often equated with 'pitch accent' in systems like Japanese, and as accent is realized tonally in this system, accent would appear to be restricted to just those systems with linguistic uses of f0 (fundamental frequency). In practice, however, lexical stress systems like Russian are dubbed pitch accent systems too, either because the system under analysis developed from a true pitch accent system, as with Russian, or simply to emphasize the phonological similarities between stress-accent in one language and non-stress accent languages like Japanese. The latter case is exemplified by the stress-accent language Cupeño (Uto-Aztecan), where the ancestor language was clearly a stress language (Munro 1977, see Kiparsky \& Halle 1977 on a comparison between Cupeño and other Indo-European languages like Russian). ${ }^{2}$

Accent systems are also sometimes called 'restricted tone systems' to clarify the differences between accent and tone. While differentiating accent and tone is not always a straightforward matter, accent systems typically differ from tonal systems in the nature of the accentual contrast and the types of accentual processes. These properties will be examined in detail below, but an important point is that, in some theories, accent simply involves a type of tone system where tonal contrasts are more restricted and processes involving tone can operate over long distances. Any characterization of the notion accent will have to account for these differences, and furthermore, it should formalize the intuition shared by many that there is a notion of accent that is independent of the phonetic realization of accent. That is, an adequate characterization should account for the striking similarities between languages like Russian and Japanese (an observation first made by in Jakobson 1963, 1965 I believe), despite the obvious fact that accent in these two systems have different phonetic correlates.

In the next subsection, I list of properties which characterize the accent systems studied throughout this thesis. I also identify some theoretical approaches to accent, including various autosegmental and metrical theories, and I justify choosing a metrical theory of accent. Then, in $\S 1.2 .2$, I propose a formal theory of lexical accent in terms of Faithfulness to underlying prosody, discuss its advantages over previous approaches to accent systems, and clarify how it is used in the individual case studies which follow.

\subsubsection{Remarks on the Notion 'Accent'}

\subsubsection{Observations}

To start with a relatively theory-neutral characterization, accent is often a cover term for systems with suprasegmental features like stress and tone, which have the following phonological properties.

\footnotetext{
${ }^{2}$ Distinctive tone in a related Uto-Aztecan language, Northern Tepehuan, is probably an innovation, brought about by the loss of certain laryngeals (see Bascom 1965 for details).
} 


\section{(15) General Properties of Accent Systems ${ }^{3}$}

a. Contrastiveness: accent is unpredictable and therefore may bring about contrast in otherwise identical words.

b. Edge Effects: accent is often assigned or attracted to a designated edge of a word or phrase (cf. 'delimitative accent' from Trubetzkoy 1939).

c. Culminativity and Accent Resolution: there can be at most one accent per word; in words with more than one inherently accented morpheme, all but one is deleted.

d. Accentual Processes: accentual processes are limited to deletion, insertion, and shift of accent; these processes may take place over long distances.

The distribution of suprasegmentals is, in part, unpredictable in accent systems, and they may therefore lead to a surface contrast in otherwise identical words. For example, stress-accent in Russian is unpredictable and brings about a contrast in words such as bágrit' 'to spear fish' and bagrit' 'to paint crimson'. Accent in Japanese, though realized as a tonal event, is likewise contrastive and also yields a surface contrast, e.g., hási 'chopsticks', hasí 'bridge', and hasi 'edge'. Accentual contrasts such as these differ from those found in so-called 'free tone' systems, such as Yoruba, in that the contrast is not paradigmatic; that is, it is not due to an opposition among an inventory of accentual or tonal units, e.g., a high versus low tone contrast in a single tone-bearing unit. Rather, the contrast is only in the location of accent in the surface form or the presence or absence of accent, as in the case of Tokyo Japanese. Concretely, the total number of contrasts possible in an accent system is $n+1$, where $n$ stands for the number of sponsors for accent in a given word and an additional contrast (the ' +1 ' part) allows for the absence of accent in a form. On the other hand, a system with a paradigmatic contrast may admit as many contrasts per sponsor multiplied by the number of accentual or tonal types in the language, which is clearly less restricted. In sum, languages such as Russian and Japanese have in common that accent is unpredictable and therefore has a contrastive function, ${ }^{4}$ but this accentual contrast is more restricted than the range of contrasts observed in tonal systems.

Another unifying property of accent is that accent is often attracted to the edge of a prosodic or morphological unit. Thus, various contexts may require accent to appear at a designated edge, or alternatively, may privilege realization of an accent which is closer to a given edge constituent. These two types of edge effects may be distinguished as EDGE TROPISM versus EDGE ORIENTATION, respectively. The former accounts for the Trubezkovian notion of delimitative accent, where accent is co-extensive with an edge. Edge tropism is observed in Cupeño in words which are composed exclusively of unaccented morphemes. In such forms, accent is tropic to the first syllable of the word, as in /yax $+\mathrm{em} / \rightarrow$ yáx-em 'say! (second person plural)'. Cupeño also exemplifies the other type of edge effect, edge orientation. In words with more than one inherently accented morpheme, the rightmost accent is realized in the surface form, e.g., /pé + yax + qál/ $\rightarrow$

${ }^{3}$ This set of properties is based on the characterizations of accent given in Hyman 1977, 1978, McCawley 1978, Clements \& Goldsmith 1984, Beckman 1986, van der Hulst \& Smith 1988, Odden 1995 and references therein.

${ }^{4}$ Beckman 1986 observes that accent typically does not carry the same distinctive load as other segmental features, a point supported by psycholinguistic research leading to the conclusion that lexical stress is not directly used in lexical access strategies (see Culter \& Clifton 1984 and Culter 1986). Whatever the reason for this observation concerning the function of accent, the distribution of accent is nonetheless phonologically unpredictable, and this requires a linguistic analysis on a par with segmental features which are also unpredictable. 
pe-ya-qál 'he was saying', cf. /pé + yax/ $\rightarrow$ pé-yax 'he says', which shows a bias towards realizing inherent accent as close as possible to the right edge of the word, though non-final accent is still found in the system. Edge effects such as these are not unique to accent systems as autosegmental tone may also show the same patterns of edge tropism and orientation. But this property of accent systems distinguishes accent from other phonological features which are not mobile in the same sense as accent and will be important in the characterization of Faithfulness to underlying accent in §1.2.2.

A third important property observed in accent systems is that there may be an overarching constraint in the system which has the effect of requiring a single accent in a given domain, often the lexical word. When an accent system is subject to this constraint, accent is said to be CULMINATIVE. ${ }^{5}$ In Russian, for example, there is a single rise and fall in intensity per word. Likewise in Japanese, minor phrases may only have a single fall in pitch over the accented syllable. Culminativity in these systems leads to a characteristic type of morpho-accentual process, Accent Resolution. When a word is composed of more than one inherently accented morpheme, only one may realize its accent because accent is culminative. Therefore, in multiply accented lexical forms, the mapping from input to output may be viewed as a type of competition for a unique surface accent. In this way, accent systems may be contrasted from other types of tonal systems and stress systems where there is no 'uniqueness requirement' on accent. Thus, tone languages like Yoruba do not limit the number of tones in a form to exactly one, and consequently, there is no loss of all but one lexical tone.

Finally, accent systems may be characterized by the types of processes operating on accent. Accent systems often have only three types of processes: deletion, insertion, and shift of accent. Deletion of accent has already be mentioned as a culminativity effect: in words with more than one accented morpheme, only one accent can be realized, which in turn results in the deletion of all other lexical accents. Insertion of accent may also be modelled as an effect of culminativity: if the underlying representation of a form does not have a lexically specified accent, an accent is inserted to satisfy an existence requirement for accent embodied in culminativity. While deletion and insertion of accent may be due to other factors, both phonological and morphological, culminativity plays a major role in deriving these two processes in the cases studies presented here. Finally, an accent may also shift from its lexical sponsor in the mapping from lexical to surface forms. For example, if the vowel which is associated with an accent is deleted, the lexical accent may shift to another position in the surface form, as with syncopated vowels in the Jivaroan language Aguaruna (\$5.4.4). One common way of contrasting these accentual processes with those found in tone systems is that processes of tone assimilation and dissimilation are typically subject to stricter locality conditions, essentially requiring that the target and trigger be in adjacent syllables or moras (see Odden 1995 and references therein). The accentual processes outlined above, however, may take place over long distances and are typically not subject to the same locality conditions. For example, Accent Resolution in Russian and Japanese does not require accent on two adjacent syllables in order to trigger the deletion of accent; this accentual process simply deletes all lexical accents but one, whether they are local or not. To summarize, accent systems may have three types of

\footnotetext{
5The term 'culminative' has been used differently in different contexts: Trubetzkoy 1939 defines culminative features as features which make an appearance exactly one time in a given domain, while Liberman \& Prince 1977 and Hyman 1977 use the term differently for stress systems to mean essentially that there is always a stress peak which is more prominent than all others in the word. For the purposes of the case studies presented here, both uses of the term are appropriate, though in the next subsection, some remarks are made as far as how to distinguish these different observations in terms of the bracketed grid structures commonly assumed in metrical stress theory.
} 
processes, accent deletion, accent insertion, and accent shift, and these processes may operate over long distances.

While accent systems share a host of phonological properties, it is important to note that the phonetic correlates to accent may be significantly different. That is, accent systems have similar phonological properties, but the phonetic attributes of accent may differ from language to language. Thus, stressed syllables in Russian have an intensity peak and are perceived as louder than unstressed syllables (Jones \& Ward 1969). In Japanese, on the other hand, the primary cue for accent is the fall in f0 directly following the accented syllable (see Beckman 1986 and references therein). While some early work on stress and accent has suggested that f0 is a major factor in cueing stress as well as tone (Hyman 1977, 1978, based on Fry 1955, 1958), it seems clear that there is no universal phonetic correlate to accent as I have characterized it. Beckman 1986 provides experimental evidence supporting a distinction between stress-accent and non-stress accent, the latter being represented in Tokyo Japanese where f0 is the primary correlate to accent. In stress-accent systems, as exemplified by English in Beckman's study, phonetic cues other than pitch are used, including intensity, length, and possibly other phonetic properties, such as phonation type and vowel quality. Jones \& Ward's 1969 characterization of stress in Russian shows a clear role for amplitude and duration of stressed syllables, even in post-focus environments, which effectively classifies Russian as a stress-accent language. Thus, while accent in Russian and Japanese behaves similarly in the phonology, the phonetics of accent is quite different in these languages.

\subsubsection{The Representation Question}

These observations concerning the phonetics and phonology of accent lead to some interesting questions regarding the representation of accent. The phonology of accent in Russian and Japanese supports a direct comparison, suggesting that they should be represented with the same phonological structures. On the other hand, the phonetic correlates of accent are vastly different; if the phonological representation of accent requires a connection with the phonetic implementation of accent, entails different phonological representations for accent. It would appear therefore that there are two basic approaches to the 'representation question'.

(16) Two Basic Approaches to Representation of Accent

a. Different Phonologies Approach: phonological representations for accent are determined on a language-particular basis and have universal phonetic interpretations.

b. Different Phonetics Approach: accent is given a consistent phonological representation which has a language-particular phonetic interpretation.

Fleshing out the first approach, accent systems like Russian and Japanese require a lexical representation for accent. After all, accent is contrastive in these languages, and the domain of idiosyncratic distinctions such as these is the lexicon. On the assumption that Russian and Japanese have different phonologies, one clear avenue of analysis is that accent in Russian is encoded with the features in the representation of metrical stress, e.g., grid marks or diacritics marking the heads of stress feet. As accent in Japanese is realized with an f0 contour, the obvious choice here is to posit an autosegmental feature, presumably a tonal melody, or simply a linked high tone, over the lexically accented syllable. In the Different Phonologies theory, therefore, lexical accent is encoded with the phonological features which best describe the phonetic properties associated with accent in 
these languages. An approach of this kind is taken in the autosegmental literature where languages with non-stress accent are represented with linked tones, see for example Pulleyblank 1986, Poser 1984, Archangeli \& Pulleyblank 1984, Blevins 1993. The Different Phonologies approach leaves stress-accent languages like Russian and Cupeño with a different analysis, presumably lexical markings for stress feet or a set of minor rules which characterize the lexically idiosyncratic stress patterns.

The alternative approach presented in (16b) is that there is a single phonological feature for lexical accent in Russian and Japanese and the phonetic consequences of this feature is dealt with on a language-particular basis. The Different Phonetics approach is often taken in the metrical literature, with the underlying assumption that lexical accent is lexically specified stress and that stressed syllables may be assigned other phonological features by rule, or that stress is realized differently from one language to the next. See for example Zubizarreta 1982, Hayes 1980, Bennett 1981, Abe 1981, Prince 1983, HV, Sietsema 1989, Melvold 1990, Prince 1990, Zec 1994, Hayes 1995, McCarthy 1995, Pater 1995, van der Hulst 1995, Féry 1996, Revithiadou 1997; Idsardi 1992, Kubozono 1995, Katayama 1995, 1998. The Different Phonetics approach is of course not wedded to a metrical representation of accent, and other researchers have posited non-metrical accentual features. For example, Clements \& Goldsmith 1984 and Goldsmith 1984 employ an accentual diacritic which guides the association of a pitch accent (=tonal melody) to the lexical sponsor. Also, employing the feature system of Vanderslice \& Ladefoged 1972, Beckman \& Edwards 1994 encode lexical stress in English with a diacritic feature for accent, which is again not directly tied to metrical structures. These specific implementations of the Different Phonetics theory of accent have in common that the phonological representation of accent does not have a unitary phonetic realization, which, as we will see, makes possible certain generalizations in the phonology of a wider range of accent systems.

While the matter of the lexical representation of accent is not directly relevant to the core issues of this thesis, I assume a metrical theory of accent, following the leading ideas in Prince 1983, HV, and Idsardi 1992. Before stating my formal assumptions, I will briefly summarize my reasons for choosing this specific theory. One important reason for formalizing accent as a prominence on the metrical grid is that it explains the phonological similarities among accent systems. Thus, the basic observation that accent systems have at most one accent receives a natural account if, by hypothesis, accent is encoded as a prominence which embodies a strong position in a bracketed grid structure, i.e., either a grid mark or an '*'. Metrical representations are inherently culminative because of their hierarchical structure (see Hayes 1995), and so culminative accent, in a sense, comes for free. If, on the other hand, accent is represented as stress-accent in one system, and for example, as a linked tone in another, then culminativity effects do not follow in both systems, and therefore a direct parallel cannot be made. Furthermore, the non-local character of accentual processes is explained in the metrical interpretation of the Different Phonetics approach, but it does not necessarily follow in other theories. Concretely, the loss of non-adjacent lexical prominences is expected in the metrical theory because a lexical accent is deleted if it does not form the head of a stress foot. On the other hand, the deletion of tone due to certain cooccurrence constraints on tone is subject to more stringent locality requirements (Goldsmith 1976, Odden 1995, Myers 1987a). These points will be made more explicit below after a formal theory of lexical accent is proposed.

A second argument in favor of the metrical theory just described is that it leads to a more restricted theory of morpho-accentual processes, an argument originally due to Bennett 1981, cf. Poser 1984, but in a different form. As outlined above, accent systems may be characterized by a set of accentual processes, deletion, insertion and shift. These processes may be phonological or morphological, i.e., triggered by special affixes or only 
in certain word classes. In other words, there is a restricted set of accentual processes which have counterparts in the morphology, a fact that is clearly relevant for the representation of accent. While all of the pieces are not in place to make this argument succinctly, the theory of morphological accent developed in this dissertation assumes that accentual processes with a morphological basis are derived as reversals of Faithfulness constraints governing the relation between lexical and surface accent. If the Faithfulness constraints operate on the prominence structures employed in metrical theories, then certain phonological operations can be systematically ruled out. For example, the fact that a prominence on the grid is never associated with more than one subordinate element supports the conclusion that grids never 'spread'; thus, it follows that there is no Faithfulness constraint against this phonological operation. Because there is no Faithfulness constraint against spreading of accent, there will never be a morphological process calling for the reversal of an anti-spreading constraint, i.e., a morphological process expressing an imperative to spread. Thus, an advantage of the metrical theory of accent is that it restricts the range of possible morpho-accentual processes.

To summarize this background discussion, following many previous approaches, I employ an accentual feature that does not have a direct phonetic interpretation. The assumption that this accentual feature is a prominence on the grid establishes a clear parallel in the phonology between stress-accent systems like Russian and Cupeño and non-stress accent languages like Japanese. Though these assumptions are motivated in their own right, if it turns out that the lexical representation of accent in these languages must be different, then this finding will not directly affect the basic arguments to be made here. Thus, if Russian has lexical stress but Japanese has linked tone structure, then the explanations for root-controlled and affix-controlled accent does not substantively change; these explanations lie in principles applying to Faithfulness constraints generally, and not just to a specific type of Faithfulness. Indeed, all that matters in the construction of the theory of accent here is that these languages have an object in the underlying representation to be faithful to. If this is true, then the exact nature of the Faithfulness constraints is quite irrelevant to the analysis of RCA and ACA, as the analysis is one that is defined in terms of Faithfulness generally.

\subsubsection{A Theory of Prosodic Faithfulness}

\subsubsection{The Constraints}

We require a set of constraints to govern the relation between lexical and surface accent, which I will refer to collectively as 'Prosodic Faithfulness'. Prosodic Faithfulness must therefore make reference to the assumed prominence structure, which requires the notion of Correspondence developed in McCarthy \& Prince 1995.

\section{(17) Correspondence}

Given two strings $\mathrm{S}_{1}$ and $\mathrm{S}_{2}$, correspondence is a relation $R$ from the elements of $\mathrm{S}_{1}$ to those of $S_{2}$. Elements $\alpha \in S_{1}$ and $\beta \in S_{2}$ are referred to as correspondents of one another if $\alpha R \beta$.

As McCarthy \& Prince make clear, the set of correspondent elements that can be referred to by the Faithfulness constraints is not limited to segments; these elements may include autosegmental features like moras, tone, and importantly, prominence structure. As discussed above, accent is encoded as a lexical prominence, i.e., a grid mark over an accented vowel in the underlying representation. The Prosodic Faithfulness constraints given below make reference to lexical and surface prominence and require related strings to 'match' in this prominence structure. 
(18) Prosodic Faithfulness (PROS-FAITH)

MAX-PROM: $\quad$ For $x$ a prominence, $\forall \mathrm{x} \exists \mathrm{x}^{\prime}\left[\mathrm{x} \in \mathrm{S}_{1} \rightarrow \mathrm{x}^{\prime} \in \mathrm{S}_{2} \& \mathrm{x} R \mathrm{x}^{\prime}\right]$

'Every prominence in $S_{1}$ must have a correspondent in $S_{2}$.'

DEP-PROM: $\quad$ For $x$ a prominence, $\forall \mathrm{x} \exists \mathrm{x}^{\prime}\left[\mathrm{x} \in \mathrm{S}_{2} \rightarrow \mathrm{x}^{\prime} \in \mathrm{S}_{1} \& \mathrm{x} R \mathrm{x}^{\prime}\right]$

'Every prominence in $\mathrm{S}_{2}$ must have a correspondent in $\mathrm{S}_{1}$.'

\section{NO-FLOP-PROM}

For $x$ a prominence, $y$ a sponsor, and $z$ an autosegmental link, $\forall \mathrm{x} \forall \mathrm{y} \forall \mathrm{z}\left[\mathrm{x}\right.$ and $\mathrm{y}$ are associated via $\mathrm{z}$ in $\mathrm{S}_{1} \rightarrow$

$\exists \mathrm{x}^{\prime} \exists \mathrm{y}^{\prime} \exists \mathrm{z}$ such that $(\mathrm{x}, \mathrm{y}, \mathrm{z}) R\left(\mathrm{x}^{\prime}, \mathrm{y}^{\prime}, \mathrm{z}^{\prime}\right)$ and $\mathrm{x}^{\prime}$ and $\mathrm{y}^{\prime}$ are associated via $\mathrm{z}^{\prime}$ in $\mathrm{S}_{2}$. 'Corresponding prominences must have corresponding sponsors and links.'

The above constraints distinguish between two forms of Faithfulness to underlying prosody, which in turn, are responsible for the range of accentual contrasts commonly found in accent systems. The first two constraints, MAX-PROM and DEP-PROM, govern Faithfulness to the presence or absence of prominence in related forms. When properly ranked, these constraints yield a contrast between accented and unaccented morphemes. This type of contrast can take two forms. In a system in which every word has an accent, like Russian and Cupeño, words with accented morphemes are faithful to their lexical prominence, which contrasts with words that have no underlying accent and, as a result, receive a default accentual pattern. Alternatively, the accented/unaccented contrast may directly manifest in surface words, as in Japanese where words with no underlying prominence are distinguished from accented forms by the absence of accent in the output. In both systems, MAX-PROM plays a crucial role in enforcing Faithfulness to a lexical prominence. The difference between these two results from the ranking of DEP-PROM: in languages like Russian, DEP-PROM is relatively low-ranking, as unaccented forms receive an inserted accent; in languages like Japanese, by contrast, DEP-PROM is relatively highranking, ensuring that unaccented forms in the input will not receive a non-lexical accent in the output.

A different form of Faithfulness concerns the position of prominence in related forms. Unless otherwise motivated, the position of prominence does not change in the mapping from one structure to another, and Faithfulness to the position of accent is governed by NO-FLOP-PROM. Thus, if NO-FLOP-PROM is high-ranking in the grammar, specifically ranked above constraints which assert a fixed position for prominence structures, then a word with $n$-numbered syllables or moras will have $n$ number of accentual contrasts because the lexical position for accent must be maintained. Importantly, this contrast in the position of accent is only observed if MAX-PROM is also suitably highranked; NO-FLOP-PROM is only relevant for a prominence which has a counterpart in the input, which is of course governed by MAX-PROM. Since MAX-PROM (and DEP-PROM) bring about an additional contrast, i.e., the presence or absence of an accent, these constraints together yield the set of contrasts characteristic of accent systems discussed above, namely $n+1$, where $n$ is equal to the number of sponsors for accent in a given form.

In the characterization of Prosodic Faithfulness above, accent is understood as an autosegmental unit, namely a grid mark, instead of a property of a segment itself or the result of underlying foot structure, as sometimes assumed (Inkelas 1994, Kenstowicz 1995a, McCarthy 1995, 1997, Benua 1997 [1998], Itô, Kitagawa, \& Mester 1996). That is, the constraints which mitigate against the deletion (MAX), insertion (DEP), and migration (NO-FLOP) of accent, are more like the Faithfulness constraints employed in the 
treatment of other autosegmental objects like moras (McCarthy 1997) or tone (Bickmore 1996, Zoll 1996b, Yip 1996, Myers 1997a). The reason for this assumption is not part of a plan to rule out metrical constituency in accent systems altogether - there are many good reasons for wanting bracketed grids or stress feet in surface representation of accent. The rationale here that the principal reasons for positing foot structure in underlying representations, e.g., affix-controlled processes like pre- and post-accentuation, will receive a different treatment in the latter half of the thesis. Since the theory of these processes no longer requires foot structure in the underlying representation, Faithfulness to the hierarchical relations embodied in foot structure is not necessary in the input. Furthermore, as will be illustrated in detail in chapter 5, the Prosodic Faithfulness constraints given here have 'echoes' in the morphology: the attachment of an affix triggers a violation of one of these constraints. In morphologically triggered accentual processes, then, it is imperative to separate the different types of Faithfulness to underlying prosody precisely as it is done here in order to account for the differences among affixes which lead to deletion, insertion, or shift of an accent.

\subsubsection{Application of the Constraints}

Let us apply these constraints to a concrete example as a means of illustrating the basic assumptions of the theory of Prosodic Faithfulness. Two closely related Cupan languages (Uto-Aztecan), Cupeño and Cahuilla, differ in the behavior of stress in roots. Cupeño has a contrast between initial and pen-initial stress, while Cahuilla has uniform initial stress (Munro 1977, 1990).

$\begin{array}{llll}\text { Cupeño } & \text { Cahuilla } & \\ \text { ?. } & \text { ?ámúl } & \text { 'agave' } \\ \text { qaṣ ály } & \text { qásily } & \text { 'sagebrush' } \\ \text { qewál } & \text { kíyul } & \text { 'fish' } \\ \text { kaxál } & \text { qáxal } & \text { 'quail' } \\ & & \\ \text { b. } & \text { tévet } & \text { tévat } & \text { 'conifer' } \\ & \text { wáxeč ily } & \text { wáxač ily } & \text { 'frog' } \\ \text { wí?et } & \text { wí?at } & \text { 'oak' } \\ \text { sú?iš } & \text { sú?išs } & \text { 'jackrabbit' }\end{array}$

Stress is therefore phonemic in Cupeño, but predictable in Cahuilla. In OT, the presence of a phonemic contrast is an indication of high-ranking Faithfulness, as Faithfulness requires a contrast present in the lexical form to surface in the output. This assumption applies with equal force when stress, or other suprasegmental features, introduce a contrast, which is illustrated by the following schematic rankings.

(20) Phonemic versus Predictable Accent through Constraint Ranking

a. Phonemic Accent: PROS-FAITH >> PHONO

b. Predictable Accent: PHONO >> PROS-FAITH

In this theory, the distinction between phonemic and predictable accent is determined by the ranking of the Prosodic Faithfulness constraints relative to other 
phonological constraints on the distribution of accent. In Cupeño, the observed contrast in the position of accent is described by ranking two PROS-FAITH constraints above the constraint responsible for deriving regular initial stress, INITIAL-PROM. In a grammar characterized by this ranking, an input with a lexical prominence on the second syllable will be paired with an output which also has a prominence over the second syllable, as illustrated below. Where needed, indices are marked on prominence structure to indicate corresponding prominences; in the forms below, two grid marks bearing the same index indicates that they stand in correspondence according to the definition given in (17) above.

(21) Phonemic Accent in Cupeño

\begin{tabular}{|c|c|c|c|}
\hline $\begin{array}{rr} & \mathrm{X}_{1} \\
\text { Input: } & \text { ?amúl }\end{array}$ & MAX-PROM & NO-FLOP-PROM & INITIAL-PROM \\
\hline 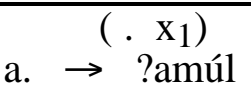 & & & $*$ \\
\hline $\begin{array}{ll} & \left(\mathrm{x}_{1} .\right) \\
\text { b. } & \text { ?ámul }\end{array}$ & & $* !$ & \\
\hline $\begin{array}{ll} & \left(\mathrm{x}_{2} \cdot\right) \\
\text { c. } & \text { ?ámul }\end{array}$ & $* !$ & & \\
\hline
\end{tabular}

Given the OT principles of constraint ranking and violability, the predicted output may violate a given constraint if such a violation will lead to the satisfaction of high-ranking constraints. Thus, when comparing the first and last candidates above, candidate (21c) is ruled out because it violates MAX-PROM as a means of satisfying lower-ranking INITIALPROM. Concretely, the failed candidate has deleted the lexical accent because the lexical prominence $\mathrm{x}_{1}$ does not have a correspondent, or a 'counterpart', in the surface form. The failed candidate in (21b) is also unfaithful to the input, but in a different way: the lexical prominence has not been deleted but shifted from its lexical position in the input, leading to a crucial violation of NO-FLOP-PROM. The winning candidate is therefore the one which is fully faithful to both the presence of an accent and its position in the input (21a), despite its poor edge-alignment by INITIAL-PROM.

The result achieved by this grammar is the desired opposition between forms like ?amúl and forms which are not marked for second syllable stress, e.g., tévet. If such forms are either marked lexically for initial stress, or completely unmarked, they will receive initial stress by the ranking of constraints given above. ${ }^{6}$ This result is ensured by the ranking of two important PROS-FAITH constraints, namely MAX-PROM and NO-FLOPPROM, above INITIAL-PROM, which favors the realization of the prosodic properties of the input over perfect edge-alignment.

In contrast to this ranking of constraints, the reverse ranking in Cahuilla accounts for regular initial stress in roots, as illustrated below. Regardless of the accentual properties of lexical roots in Cahuilla, inputs are mapped onto outputs which have uniform initial stress, even when such a mapping leads to a violation of PROS-FAITH, as in the input-output pairs shown in (22b).

\footnotetext{
${ }^{6}$ The empirical issues of restricting the stress contrast to initial and pen-initial syllables, and the ranking of INITIAL-PROM relative to the anti-insertion constraint, is taken up in $\$ 2.3$, where a more thorough analysis of Cupeño root stress is given.
} 
(22) Predictable Accent in Cahuilla

\begin{tabular}{|c|c|c|c|c|c|}
\hline & Inputs & & Outputs & INITIAL-PROM & PROS-FAITH \\
\hline \multirow[t]{2}{*}{ a. } & /tévet/ & $\rightarrow$ & tévet & & \\
\hline & & & * tevét & $* !$ & * \\
\hline & /CVCV́ / & $\rightarrow$ & $\mathrm{CV} \mathrm{CV}$ & & $*$ \\
\hline & & & * CVCV́ & $* !$ & \\
\hline
\end{tabular}

In this way, the presence or absence of a phonemic contrast in accent is derived through constraint ranking. To summarize the above results, phonemic accent in Cupeño involves the ranking of a set of PROS-FAITH constraints above a phonological constraint which requires a systematic pattern (23a). If the reverse ranking holds, on the other hand, the result is a predictable pattern of accent, as observed in the case of Cahuilla (23b). Furthermore, the effects of these two schematic rankings can be combined to account for what might be dubbed 'hybrid accent' systems, i.e., systems where accent is contrastive in some contexts, but certain over-arching constraints limit the distribution of the accentual contrast. For example, Spanish nouns have a contrast in the position of stress in that stress may fall on any of the last three syllables of the word; but antepenultimate stress is systematically avoided if the penultimate syllable is bimoraic (Harris 1983). By interleaving the PROS-FAITH constraints between two purely phonological constraints, this type of limited contrast can be achieved in a direct way (see Pater 1995, Alderete 1996, Revithiadou 1998, and Baerman 1998 for analyses of such systems along these lines).

(23) Summary of Results

\begin{tabular}{|l|l|l|}
\hline Classification & Schematic Ranking & Examples \\
\hline \hline a. Phonemic Accent & PROS-FAITH >> PHONO & Cupeño \\
\hline b. Predictable Accent & PHONO > PROS-FAITH & Cahuilla \\
\hline c. 'Hybrid' Accent & PHONO $_{1}>$ PROS-FAITH > PHONO 2 & Spanish Nouns \\
\hline
\end{tabular}

Of course restricted phonemic contrasts can be approached differently in terms of constraints on underlying representations themselves; these are the Morpheme Structure Constraints (MSC) which are commonly used in rule-based accounts of such restrictions, however they are conceived (see Hammond 1989b and Franks 1991; Inkelas 1994, Pater 1994, Katayama 1995 for different formal approaches to restricted accentual contrasts). One important argument for the treatment of such systems as constraint domination is that it solves a classic problem in Generative Phonology concerning the treatment of constraints on the inventory, namely the Duplication Problem (see Kenstowicz \& Kisseberth 1977 and references therein). In many accent systems, the constraints on the distribution of contrast also play a role in governing the output of certain accentual processes. For example, unaccented words in Russian receive a stress on the first vowel of the inflectional ending, e.g., /stol $+\mathrm{u} / \rightarrow$ stol-ú 'table (dative plural)'; furthermore, special accent-deleting suffixes in Russian, e.g., $-a \check{c}$, trigger a deletion of the stem accent which results in ending stress as well: /púz + ač $+\mathrm{u} / \rightarrow$ puz-ač $-u$ 'man with paunch'. Clearly, the constraint requiring ending stress is operative in both cases, showing that a single constraint has a role in the analysis of restricted suffix stress and the stress pattern resulting from deaccentuation. 
In the theory proposed here, this constraint is the same constraint, whose application is restricted exclusively to output forms (see $\$ 3.2$ and $\S 5.2 .3$ for the details of the analysis). Consistent with the OT principle of the RICHNESS OF THE BASE, there are no languages particular restrictions on the input. Therefore, the restriction governing the range of possible stress patterns in unaccented words may not be stipulated of lexical forms because it is a restriction specific to Russian. This reasoning entails that the constraint yielding ending stress is a surface-oriented constraint, which may of course extend to the analysis of ending stress in de-accented structures. In a rule-based theory with MSCs, on the other hand, a constraint on possible inputs applies to the lexical inventory, yielding ending stress in unaccented words. This constraint is in additional to a constraint yielding ending stress in forms like puz-ǎ́-ú. The argument is thus that the approach to restricted phonemic contrast with surface-oriented constraints is superior to the theory with MSCs because the latter requires constraints operative in different components of the grammar which achieve essentially the same result. ${ }^{7}$

\subsubsection{Consequences for Culminative Accent}

Recall that accent systems always have a single most prominent accent, entailing the resolution of accent in words with more than one lexical accent. In addition, in words with no lexical accent, an accent is often supplied to the surface representation. These two observations characterize two different meanings for the notion 'culminativity', both the classical sense of Trubetzkoy 1939 and the sense it which it is commonly used in studies of metrical stress (see Liberman \& Prince 1977, Hyman 1977, Hayes 1995).

(24) Culminativity of Accent (relative to a domain $D$ )

a. Existence requirement: every $D$ has an accent.

b. Uniqueness requirement: every $D$ has exactly one accent that is greater than all others.

To say that some constituent $D$ has culminative accent entails that every instance of $D$ has an accent. Furthermore, culminativity of accent entails that there be a single accent that stands out among all others in $D$, which can be satisfied simply by the existence of a single accent per $D$.

The role of prosody is largely undisputed in the analysis of culminativity; the assumption that lexical prominences are mapped directly onto bracketed grid structure derives culminative accent in a simple and direct way. A fundamental assumption in metrical stress theory is that prosodic structures are hierarchically structured (Liberman \& Prince 1977, Halle \& Vergnaud 1978, Selkirk 1980, among many others), as in the hierarchy of prosodic categories arranged below.

\footnotetext{
${ }^{7}$ See Prince \& Smolensky 1993, Pater 1995, 1996, Myers 1997a, Tesar \& Smolensky 1997, and Beckman 1997 for further discussion of the solution to the Duplication Problem and conspiracies in general in Optimality Theory.
} 
Within the present OT framework, the hypothesis that prosodic categories are hierarchically organized breaks down into a set of well-formedness constraints, given in (26). Thus, each of these constraints may be ranked on a language particular basis, accounting for the common finding that, while these principles of prosodic organization dictate a target for optimal prosodic structure, they may, in fact, be violated in specific contexts. For example, Itô \& Mester 1992 argue convincingly that certain unpaired syllables in Japanese word clippings are directly associated with the prosodic word, rather than being footed by a non-binary foot.

(26) Constraints on Prosodic Domination (Selkirk 1995 [1996], cf. Itô \& Mester 1992)

LAYEREDNESS: No $\mathrm{C}^{\mathrm{i}}$ dominates a $\mathrm{Cj}, \mathrm{j}>\mathrm{i}$, e.g., 'No $\sigma$ dominates F.'

HEADEDNESS: Any $\mathrm{C}^{\mathrm{i}}$ must dominate a $\mathrm{C}^{\mathrm{i}-1}$ (except if $\mathrm{C}^{\mathrm{i}}=\sigma$ [or consistent with (4), except if $\left.\mathrm{C}^{\mathrm{i}}=\mu\right]$ ), e.g., 'A PrWd must dominate a F.'

EXHAUSTIVITY: No $\mathrm{C}^{\mathrm{i}}$ immediately dominates a constituent $\mathrm{Cj}, \mathrm{j}<\mathrm{i}-1$, e.g., 'No PrWd immediately dominates a $\sigma . '$

NONRECURSIVITY: No $\mathrm{C}^{\mathrm{i}}$ dominates $\mathrm{C}$, $\mathrm{j}=\mathrm{i}$, e.g., 'No $\mathrm{F}$ dominates a $\mathrm{F}$.'

The culminativity requirements follow from these assumptions inherent to the organization of prosodic structure, plus a basic assumption about the nature of headed constituents discussed below. First, the existential requirement follows from the principle of HEADEDNESS, and the standard assumption that accent is a property of the head of a prosodic foot. The principles of prosodic organization require that every PrWd must have a foot, and since accent is a property of the head of a prosodic foot, the existence requirement follows from these basic assumptions. The same type of result can be modelled for phonological phrases because phrases contain PrWds, and are thus subject to the restrictions on PrWds, including the requirement that they have an accent. Furthermore, the head of a given PCat is more than just an obligatory element at the immediately subordinate level in the hierarchy; the head specifies a structural relationship between itself and other members at the same level (see e.g., Liberman \& Prince 1977, Hayes 1980, Selkirk 1980). Thus, in purely arboreal theories of prosodic structure characteristic of early metrical theories, as well as the bracketed grid theories of Hammond 1984, HV, and Hayes 1995, the head specifies a relation between a strong element, i.e., the head, and a weak element, i.e., the non-head. Since every PrWd has a single head foot, and every foot has a single head syllable, it follows that if accent is assigned to a head syllable in the head foot, there can only be a single 'main accent', or a single accent greater in prominence than all others.

To illustrate these results in Cupeño, if an input has more than one lexical prominence, only one of them can be faithfully retained in the related output because there is only a single head foot in the output (27a). Furthermore, given a form with no 
underlying accent, as in (27b), the related surface form will be supplied with a prominence as a consequence of the assumption that all words must have a prosodic foot, and hence must have a prominence in the head syllable of that foot.

(27) Culminative Accent in Cupeño 8

\section{Underlying Representation}
$\stackrel{x}{x} \underset{x}{x}$ /pé $+\underline{\text { ?áyu }}+$ qál/
b. /yax $+\mathrm{em} /$

$\begin{array}{ll}\rightarrow \quad & (\mathrm{x} .) \\ \text { pe-?áyu-qal } \\ & (\mathrm{x} .) \\ \rightarrow \quad \text { yáx-em }\end{array}$

Surface Form

'S/he was wanting'

'Say' (plural imperative)

Importantly, 'culminativity effects' such as these may be derived over long distances, which, as mentioned above, is characteristic of accent systems in general. It makes no difference in the analysis of culminativity above whether the lexical prominences are on adjacent or non-adjacent syllables; what matters is that only one can be realized as the head of the main stress foot.

One question that arises in this theory is whether or not a lexical prominence can give rise to contrastive accent that is not the primary accent in a surface form. After all, accent is equated with prominence on the grid in this theory, and this prominence structure is also the stuff that makes up the heads of non-primary feet. So, in languages which allow non-head feet, can Faithfulness to a lexical prominence likewise give rise to a contrastive secondary prominence? Without further stipulation, clearly the theory of Prosodic Faithfulness proposed here does allow for this possibility, which leads to an interesting empirical question: do languages have contrastive secondary stresses (potentially realized as tone or other suprasegmentals)? While it is sometimes asserted that only primary stress is contrastive in lexical accent systems (van der Hulst 1994), the number of reported cases with unpredictable secondary prominence casts some doubt on this claim. In Modern Hebrew, for example, the assignment of secondary stress to the first or second syllable of the word is unpredictable and must therefore be specified for individual words (Bolozky 1980: 277). Secondary (=non-final) stress in Tübatulabal is likewise assigned on a wordby-word basis and is not predictable from the phonological make-up of the form (Voegelin 1935, see also Kager 1989, Crowhurst 1991). A third case concerns the directionality of secondary stress in the Peruvian language Huariapano: secondary stress is assigned on alternating syllables, but whether the stress trains run from left-to-right or right-to-left is again a lexical property of individual words (Parker 1998, 1994). All of these cases have in common the fact that a pattern of non-primary prominence is not phonologically predictable, which is exactly the type of lexical idiosyncrasy predicted by the theory of Prosodic Faithfulness assumed here. ${ }^{9}$

\footnotetext{
${ }^{8}$ This result for Cupeño relies on the assumption that lexical prominences cannot be 'realized', i.e., faithfully retained in a bracketed grid, in a non-head foot. There are two possible means of ensuring this result: either there are no non-head feet in outputs, consistent with the absence of secondary stresses, or the lexical prominences are on a grid which marks the most prominent foot, and so realization of a foot-level prominence is not sufficient. The latter state of affairs seems to be the correct account of Cupeño, as is made clear in chapter 2 .

${ }^{9}$ The difficulty in hearing secondary stress may perhaps explain why impressionistic studies do not always report irregularities in secondary stress. Pressing further, if secondary stress is hard to hear, its apparent markedness may be due to a failure to hear such deviations in stress in language learning. Concretely, deviations from a regular pattern of stress is more audible, and hence more reliably retained, for primary stresses than secondary ones, which would appear to explain the preference for lexical primary stress over non-primary stress implicit in van der Hulst's claim.
} 
The theory of phonemic accent through constraint interaction also has an important implication for an often noted property of accent in underlying representations. In many accent systems, the general trend of 'one accent per word' is also carried over into the lexical inventory, with the effect that individual morphemes also have at most one accentual prominence. Clearly, the same constraints at work in restricting the surface forms extend into the lexicon somehow, raising the question of how underlying forms themselves are subject to said culminativity requirements. In the literature on lexical stress systems, this fact is taken as highly significant and leads to the conclusion that the same universal principles at work in assigning predictable and systematic stress are also at work in assigning lexical stress. Thus, Tsay 1991 approaches lexical stress as different instantiations of a universal set of parameter settings for stress. Building on this idea, Idsardi 1992 proposes a theory of Lexical Edge Marking (LEM), which draws on a restricted set of rule types and posits a metrical bracketing in lexical entries as the representation of lexical stress. In other words, stress in the lexicon is assigned 'by rule', and as a consequence of this assumption, the properties of these stress rules are carried over to lexical stress. As pointed out explicitly by Idsardi, since the rules of regular and systematic stress only assign a single stress, it follows that this feature of stress rules is inherited by the principles of lexical stress markings, thereby explaining the fact that there is only a single lexical stress per morpheme.

This basic result also follows from the theory of lexical accent proposed here, once the principles of learning inputs is properly understood, except the current theory does not have the descriptive short-comings of LEM theory. Intuitively, the parallel between these two theories works as follows. Given that there may only be a single prominence in the output, it is of absolutely no use to posit underlying forms with more than one prominence. If the learner goes to the trouble to do such a thing, the lexical form will invariably lead to unfaithful mappings, i.e., ones in which one of the prominences is deleted, and so morphemes with more than one prominence are never learned as a part of the lexical inventory. The principle implicit in this informal statement is not one of grammar construction, but rather, a principle of learning the inputs of a language. As shown in Prince \& Smolensky 1993, Tesar \& Smolensky 1993, 1998, the principles of harmonic evaluation may be employed in both the learning of a grammar and the lexical forms of a particular language. The learning of inputs is governed

by Lexicon Optimization (LO), which is given below.

(28) Lexicon Optimization (Tesar \& Smolensky 1998, cf. Prince \& Smolensky 1993) Suppose given an overt structure $\rho$ and a grammar. Consider all structural descriptions (of all inputs) with overt part equal to $\rho$; let the one with maximal Harmony be $p$, a parse of some input $I$. Then $I$ is assigned as the underlying form of $\rho$.

Given a grammar in which a set of constraints ensuring culminative accent (see discussion above) outranks the PROS-FAITH constraints, culminative accent is guaranteed in lexical forms by LO. As illustrated below, a lexical form with more than one lexical prominence will never realize all of them, because of the role of CULMINATIVITY in the system, and so such a mapping will always have a breach of Faithfulness (29a). When compared with a different input-output mapping with a single lexical prominence which produces same overt structure, the latter will always be chosen by LO; the IO-map in (29b) is more harmonic than the one in (29a), and therefore it is chosen as the lexical form. ${ }^{10}$

\footnotetext{
${ }^{10}$ This result is also obtained if the learner has not yet arrived at the correct ranking of constraints; see Tesar 1998 and Tesar \& Smolensky 1998 for discussion of the application of these principles in the acquisition of both inputs and constraint rankings in tandem.
} 
(29) Culminativity in Lexical Inventory through Lexicon Optimization

\begin{tabular}{|c|c|c|c|}
\hline Input & Output & CULMINATIVITY & MAX-PROM \\
\hline \multirow[t]{2}{*}{ a. $\quad / \sigma \sigma \sigma o ́ \sigma l ~ \rightarrow$} & $\dot{\sigma} \sigma \sigma$ & & $*$ \\
\hline & ó ớ & $* !$ & \\
\hline \multirow[t]{2}{*}{ b. $\rightarrow / \delta \sigma \sigma / \rightarrow$} & ó $\sigma \sigma$ & & \\
\hline & $\sigma \sigma^{\prime} \sigma$ & & $* !$ \\
\hline
\end{tabular}

In this way, the grammatical constraints requiring culminative accent in outputs is 'cycled back' into inputs in the learning of lexical forms. An important property of this account which distinguishes it from various alternatives is that culminativity of inputs is derived by a language particular ranking of universal constraints. That is, there is an intimate relationship between the grammar of outputs and the principles of learning lexical forms: in both cases, it is harmony relative to the language particular constraint hierarchy that matters. Thus, if a language allowed for multiple prominence in outputs, it will likewise allow for multiple prominence in inputs. The theory of accent proposed here will have no trouble with cases reported to have lexical accent in more than one position in the word (see Idsardi 1992 for discussion of the relevant cases). The presence of more than one prominence entails that the culminativity requirements are subjugated to MAX-PROM, which in turn allows for more than one lexical prominence in a given form. In contrast, if lexical accent is assigned by rule, as in LEM theory, then an additional provision needs to be stipulated in order to accommodate non-culminative lexical accent.

A final difference between the current theory and theories of lexical accent assignment by rule is that the latter approach makes substantive restrictions on the set of possible lexically accented positions. In essence, lexical accent must fall on a position which is attested as a position of primary stress in some language, e.g., final, initial, or penultimate position, etc. In languages which have a wider range of lexically accented positions, like Russian and Japanese, forms with accent in marked positions must be treated as exceptions on a par with loan words or structures with a complex morphological analysis (see for example Idsardi 1992: 52 for such lexical edge markings in Russian). In the theory of lexical accent as Prosodic Faithfulness, the lexical positions for accent are also restricted, but only by the constraints operative in the language under analysis. Thus, Russian allows stress on any syllable in the stem, so there is nothing precluding the acquisition of an input with, for example, accent on the third syllable from the beginning of a word that has six syllables. Again, the constraints on the distribution of surface accent play an important role in dictating accent in lexical forms, as has just be illustrated for culminativity requirements. Thus, in languages with looser restrictions on surface accent, like Russian, the theory provides a descriptively adequate treatment of lexical accent, which, in other theories, leads to additional stipulation in to accommodate marked positions for accent.

To summarize, I have proposed a theory of Prosodic Faithfulness in which accent is encoded lexically as prominence on the grid, and Faithfulness to this prominence entails realization of the lexical prominence in the head of a prosodic foot, represented as a bracketed grid. Culminative accent in this theory is explained in terms of requirements on this bracketed structure, i.e., the requirement that every grouping must have a head, and that there is only one head foot in a given accentual domain. Furthermore, restrictions on the surface distribution of accent were stated in the grammar as well-formedness constraints which may dominate the Prosodic Faithfulness constraints. These surface-oriented 
constraints also have an indirect effect on lexical forms because the principle of Lexicon Optimization systematically excludes lexical forms which would otherwise lead to gratuitous constraint violation. The proposed theory therefore has the descriptive power to extend to the reported cases of contrastive secondary prominence, systems with emergent lexical accent in more than one position per word, and languages like Russian and Japanese where accent is in principle possible in any position in the word. In general, the restrictions on both lexical and surface accent come from the language particular rankings of universal constraints. 


\section{Chapter 2. Root-Controlled Accent in Cupeño}

\subsection{Introduction}

A fundamental observation in the accent system of Cupeño ${ }^{11}$ (Uto-Aztecan) is that inherent stress in roots overrides inherent stress in affixes (Hill \& Hill 1968). That is, the system recognizes a distinction between accented and unaccented roots, and inherently accented roots cause the deletion of stress in inherently accented prefixes and suffixes. This is illustrated with the following forms.

(1) Accented Roots with Accented Affixes
a. pe-mí?aw-lu
/pé + mí?aw + lu/
'He came'
$3 \mathrm{sg}+\mathrm{COME}+\mathrm{MOTION}$
b. ?áyu-qa
/?áyu + qá/
'He wants'
WANT+PRES.SING

(2) Unaccented Root $\sqrt{ } y a x$ with Accented Affixes
a. né-yax
'I said'
/né + yax/
$1 \mathrm{sg}+\mathrm{SAY}$
b. ne?en ya-qá?
'I say'
/ne?en yax + qál
$1 \mathrm{sg}+$ SAY+PRES.SING

The accented roots in (1) win out over the person marker, pé-, and the singular present suffix, $-q a$, because affix stress is overridden by root stress. Inherent stress in affixes only emerges in words containing unaccented roots, as shown in (2). In sum, there is a rank order in the system, with an imperative to realize inherent stress in roots over inherent stress in affixes.

Cross-linguistically, roots are special in another way, which can be seen by examining languages with phonemic stress. In Sanskrit, for example, the position of accent is contrastive in roots, but accent in suffixes is limited to the first vowel of the suffix (Kiparsky 1973). Likewise, in the Athapaskan language Tahltan, stress is contrastive in roots but not in affixes, as the position of affix stress is predictable from the root stress (Cook 1972, Nater 1989). The basic observation in both cases is therefore that roots are privileged in the phonemic inventory, sponsoring a richer set of accentual contrasts than other morphological domains.

\footnotetext{
${ }^{11}$ Cupeño, now extinct, was a Takic language spoken in Southern California. The data examined in this chapter are drawn from Hill 1967 (H), Hill \& Hill 1968 (H\&H), Hill \& Nolasquez 1973 (given with page.sentence number), Crowhurst 1994 (C), and a set of unpublished fieldnotes provided for me by Jane Hill ( $\mathrm{JH})$. A note on transcription: /e/ is the symbol used here for schwa, /?/ for glottal stop, and $/ \eta /$ for velar nasal.
} 
In this chapter, the connection between overriding root stress and the privileged status of roots in inventories is explained as the interaction of Faithfulness constraints in Optimality Theory (Prince \& Smolensky 1991, 1993). In particular, the cross-linguistic observation that roots have a wider range of accentual contrasts than affixes motivates the introduction of distinct Root and Affix Faithfulness constraints, with Root Faith ranked above Affix Faith (McCarthy \& Prince 1995). With this inherent ranking, overriding root stress in Cupeño is explained as a straightforward case of constraint conflict: root stress overrides affix stress because the constraint responsible for realizing stress in roots is topranked. In sum, the observation that root stress overrides affix stress in Cupeño is treated as a special case of the cross-linguistic tendency for roots to license a wider range of contrasts than affixes.

One important goal of this chapter, therefore, is to provide further evidence for the segregation of Faithfulness constraints into the morphological domains Root and Affix, thereby supporting the findings of McCarthy \& Prince 1995, Selkirk 1995a, Urbanczyk 1996, and Beckman 1997 [1998], among others. Morphologically-dispersed Faithfulness is shown to be essential in the explanation of the diverse aspects of Cupeño accent, extending to the analysis of complicated morpho-accentual phenomena. A second goal is to motivate the Faithfulness-based analysis by contrasting it with plausible alternatives. Both of the alternatives examined here employ phonological levels, or strata, in some crucial way, and the assumptions inherent to these approaches are shown to have descriptive and theoretical problems. Finally, I present a complete analysis of accent in Cupeño, bringing a wide range of observations in this complex accentual system to bear on contemporary issues in theoretical phonology.

The rest of the chapter is structured as follows. The next section (\$2.2) lays out the theoretical background necessary for the analysis of Cupeño accent. $\$ 2.3$ then examines stress in isolated roots and gives the constraint ranking necessary for the root stress inventory. In $\$ 2.4$, these rankings are incorporated in the larger analysis of stress in fully formed words. Two alternatives to the Faithfulness-based account are subsequently considered in $\$ 2.5$, namely the level-ordered account given in Crowhurst 1994 and a multistratal account along the lines of Halle \& Vergnaud 1987a. The last section (\$2.6) summarizes the main results of the chapter and discusses some of the implications of the core ideas. 


\subsection{Theoretical Background: Root and Affix Faithfulness}

Recent work in Optimality Theory has argued for a set of Faithfulness constraints for roots which is distinct from the Faithfulness constraints for affixes. The evidence for this distinction comes in various forms, which are summarized below.

(3) Evidence for Privileged Status of Roots

a. Evidence from inventories (McCarthy \& Prince 1995, Beckman 1997 [1998], Urbanczyk 1996, Parker 1997, Adisasmito-Smith 1998): Roots tend to admit a wider range of contrasts than affixes, allowing for marked structure which is absent in affixes.

b. Evidence from phonological alternations (McCarthy \& Prince 1995, Selkirk 1995a,b, Pater 1996, Blake 1998): Phonological alternations may be 'rootcontrolled'; that is, there is a premium set for realization of phonological features of the root over features in an affix.

c. Psycholinguistic evidence (see Beckman 1997 [1998] for a survey): Word recognition studies provide support that lexical storage and access is root-based and not affix-based.

The first form of evidence comes from phonological inventories. In many inventories, roots license a wider range of contrasts than affixes, but the reverse state of affairs never obtains. With distinct Root and Affix Faithfulness, this asymmetry in the distribution of contrast may be accounted for in terms of familiar types of constraint interaction (discussed directly below).

A second form of evidence is that roots tend to have privileged Faithfulness properties in alternations, favoring retention of information in roots over information in affixes. For example, consider a well-known case of root-controlled vowel harmony in Akan. In this language, $[ \pm \mathrm{ATR}]$ is contrastive in root vowels, but it is predictable in affixes. As illustrated below, $[ \pm \mathrm{ATR}]$ specifications in prefixes and suffixes are determined by the root to which they attach.

(4) Root-Controlled Vowel Harmony in Akan (Clements 1981)
a. e-bu-o 'nest'
$\varepsilon-\underline{b U}-\mathrm{O} \quad$ 'stone'
b. o-be-tu-i 'he came and dug (it)'
O-be-tU-I 'he came and threw (it)'

Another example illustrating a root-controlled alternation is [labial] dissimilation in Tashlhiyt Berber. In this case, certain derivational prefixes lose their [labial] specification when they combine with a root bearing a primary [labial] specification, as illustrated by the contrast between (5a) and (5b) below.

(5) Root-Controlled Labial Dissimilation in Tashlhiyt Berber (Selkirk 1993, 1995b)
a. m-xazar $\sqrt{x z r}$ 'scowl'
b. n-fara
$\sqrt{\text { fra }}$ 'disentagle'

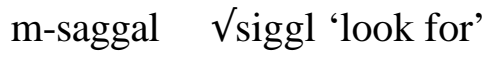
$n-\hbar$ ašš am $\sqrt{h}$ šš m 'be shy'

Again, retention of information in the root, in this case Place features, is more important than retention of affix information. 
These two patterns of root privilege are interpreted by McCarthy \& Prince 1995 as evidence for a universal ordering among the morphologically dispersed Faithfulness constraints (see also the references listed in (3) above for developments and further discussion).

(6) Meta-Constraint on Constraint Rankings (McCarthy \& Prince 1995) 12

$$
\text { Root Faith >> Affix Faith }
$$

This inherent ranking explains root-controlled phenomena in terms of the same constraint interaction required in the analysis of root-affix asymmetries in inventories. Given the ordering of Faithfulness constraints above, any restriction that holds on the distribution of a property of a root must also hold of affixes, effectively precluding a contrast in affixes which is not sponsored in roots.

This same ranking also extends to root-controlled alternations, since it asserts that retention of a property in a root is always better than retention of the same property in an affix. To illustrate this result for Akan, consider the following ranking of constraints for [ \pm ATR] Faithfulness for roots and affixes.

(7) Morphologically-Dispersed Faithfulness in Akan (after McCarthy \& Prince 1995)

$$
\text { IDENT(ATR) Root, PHONO > IDENT(ATR) })_{\text {Affix }}
$$

A grammar with this ranking will allow a $[ \pm \mathrm{ATR}]$ contrast in roots because IDENT(ATR) Root is not dominated by any crucial phonological constraints. Predictable $[ \pm \mathrm{ATR}]$ specification in affixes, on the other hand, derives from the domination of IDENT(ATR)Affix. Thus, if one or more affixes have a $[ \pm A T R]$ specification which conflicts with that of the root, it will be overridden by the root's [ \pm ATR] specification, as depicted below.

\begin{tabular}{|c|c|c|c|c|}
\hline Input: & $\begin{array}{r}\varepsilon+\underline{b u}+O \\
-A+A-A\end{array}$ & IDENT(ATR) $_{\text {Root }}$ & HARMONY & IDENT(ATR) Affix \\
\hline a. & $\begin{array}{c}\varepsilon-\underline{b u}-O \\
-A+A-A\end{array}$ & & $* !$ & \\
\hline b. & $\begin{array}{l}\varepsilon-\underline{b U}-O \\
-A\end{array}$ & $* !$ & & $*$ \\
\hline c. $\rightarrow$ & $e-\frac{b u}{+A}-o$ & & & $* *$ \\
\hline
\end{tabular}

(8) Root-Controlled ATR Spread in Akan

\footnotetext{
${ }^{12}$ Root privilege is interpreted here as an ordering between distinct Root and Affix Faithfulness constraints; but an equally coherent analysis would be to posit Root Faithfulness distinct from context-free Faithfulness, i.e., a theory in which there is no Affix Faithfulness. Since both approaches establish a parallel between root-controlled accent and other phonological phenomena, I do not distinguish them here. These distinct theories do, however, make testable predictions for different types of edge effects in rootcontrolled accent systems, which are discussed in detail in chapter 3.
} 
An over-arching constraint, HARMONY, requires a single [ \pm ATR] specification per harmony domain. With Root Faithfulness top-ranked in the constraint hierarchy, the $[ \pm$ ATR] specification of the root will always override the affix's $[ \pm$ ATR] specification. Importantly, the Faithfulness constraints make direct reference to the input specification of distinct morphological categories; such morphological and lexical information is crucial in distinguishing (8b) and (8c).

The ordering of Root and Affix Faithfulness, motivated here on purely linguistic grounds, also appears to have a functional basis in some word recognition studies. In a number of recognition experiments, a priming effect is found in words that share the same root; further, no such effect is found in words that share the same affix, leading to the conclusion that lexical access and storage are root-based (see Beckman 1997 [1998] for a review). In light of these findings, a grammar that assigns special Faithfulness properties to roots will aid considerably in the on-line processing of words. Root Faithfulness in effect precludes the destruction of information in the root, leaving intact properties of the input which are critical in lexical look-up strategies.

In many cases, the morphological root is co-extensive with an underived stem, and so there is no means of distinguishing these two domains in terms of their retention of lexical information. In some contexts, however, stem-forming affixes show privileged Faithfulness properties which are uncharacteristic of affixes generally and therefore call for a notion of Stem Faithfulness which is distinct from Root Faithfulness (see Revithiadou 1997 for an alternative). While not directly attested in Cupeño, certain derivational suffixes in Russian exhibit these Faithfulness properties; in \$5.2.3 the intermediate rank of these morphemes is treated as an effect of ranking Stem Faithfulness between Root and Affix Faithfulness. For the moment, however, I will ignore this formal distinction and treat simplex stems as roots, which will therefore be governed by the Root Faithfulness constraints.

The ordering of Faithfulness constraints given in (5) above is not specific to [ \pm ATR] or segmental features more generally. It is a proposal which extends to all aspects of Faithfulness, including the Prosodic Faithfulness constraints given in \$1.2.2.1. Thus, applying the inherent ordering in (6) to the PROS-FAITH constraints yields the following ranking of constraints.

\section{(9) Root-Controlled Accent Systems}

PROS-FAITHRoot $_{\text {R PROS-FAITH }}$ Affix

Before delving into the facts of Cupeño, let us briefly consider the role of this ranking in accentual inventories cross-linguistically. As mentioned above, it is often the case that roots sponsor a wider range of contrasts than affixes; this trend is also observed when accent is responsible for the surface contrast. For example, in Sanskrit, the location of accent is unpredictable in roots, giving rise to surface contrasts in the presence or absence of accent and its surface position in the root. Polysyllabic affixes, on the other hand, when they receive an accent, always have initial accent (Kiparsky 1973). Likewise, in Tahltan (Northern Athapaskan), the presence or absence of accent is contrastive within roots. However, accent is predictable in affixes, basically falling on every other syllable counting from the root stress (Cook 1972, Nater 1989). In both cases, therefore, accent is more restricted in affixes than in roots. 
With the distinction between Root and Affix Faithfulness, restricted affix stress becomes a simple matter of ranking the relevant prosodic well-formedness constraint, as shown in (10). The limitations on affix prosody derive from the ordering of various constraints relative to Root and Affix Faithfulness.

(10) Restricted Affix Inventories

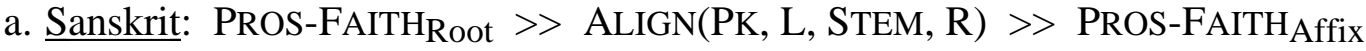
b. Tahltan: PROS-FAITHRoot $>$ RHYTHM $>$ PROS-FAITHAffix

The ranking for Sanskrit yields the observed accentual contrasts in roots because of the high-ranked status of PROS-FAITH Root. But accent in affixes is predictable because the Alignment constraint ensures that if accent is on the affix it will always fall on the first syllable, or equivalently, the syllable directly following the stem, which is derived here through a subcategorization-type Alignment constraint (see McCarthy \& Prince 1993a). The intrinsic ordering of Root and Affix Faithfulness also extends to the skewed accentual inventory in Tahltan: roots support a contrast in accent, but accent in affixes must be on alternating syllables because of the force of RHYTHM (Hung 1994), which dominates PROS-FAITH Affix. In sum, the distinction between Root and Affix Faithfulness applied to the PROS-FAITH constraints provides the correct tools for describing restricted accentual inventories in affixes.

The ordering of the PROS-FAITH constraints in (9) above also has a role in the resolution of accent in words with more than one inherently accented morpheme. Substituting MAX-PROM in this ranking will set a premium on preserving inherent root accent over inherent affix accent. As this pattern of root privilege will support a major theme in the case studies that follow, I state the effects of this inherent ordering in prose.

\section{(11) Root-Controlled Accent Hypothesis (derived from (9))}

In lexical-to-surface mappings of a word with more than one inherent accent, if accent is deleted, accent in the root is realized over accent elsewhere in the word.

As with root-controlled vowel harmony, root-controlled accent follows from the same principles responsible for restricted affix inventories. Concretely, the ordering of Root and Affix Faithfulness employed in the analysis of restricted affix accent in Sanskrit and Tahltan extends to accentual alternations, explaining root-controlled accent as a special case of the cross-linguistic trend for root privilege. In the next two sections the Root-Controlled Accent Hypothesis will guide the study of the interaction between root and affix stress in Cupeño, and subsequently in chapter 3, it will be applied to accent in Russian and Japanese.

\subsection{Root Stress Inventory}

In this section, the observations characterizing the root stress inventory of Cupeño are presented (\$2.3.1) and are then analyzed in OT terms (\$2.3.2). The goal of the analysis is to demonstrate how emergent lexical prosody and restrictions on surface stress are characterized in terms of a language particular ranking of the Prosodic Faithfulness constraints. 


\subsubsection{The Data}

While earlier work on Cupeño assumed that stress in roots was unpredictable, more recent research has shown that the observed root stress patterns are not completely irregular (Munro 1990, Crowhurst 1994). Stress is contrastive in certain contexts to be described below, but if a root has a long vowel, that vowel is stressed. The examples below are typical, showing long vowel stress in bare roots (12) and conjugated verbs (13). Most of the roots in these examples are no longer than two syllables, which apparently reflects the canonical pattern. ${ }^{13}$
a. máasive-t
xéene
péexwen
náač i
híima?ay
b. tevxáa-qa ?iyúune muháan

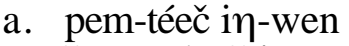 č em-náaxč in

b. pe?-ič áay-wen taváan-pe-qal

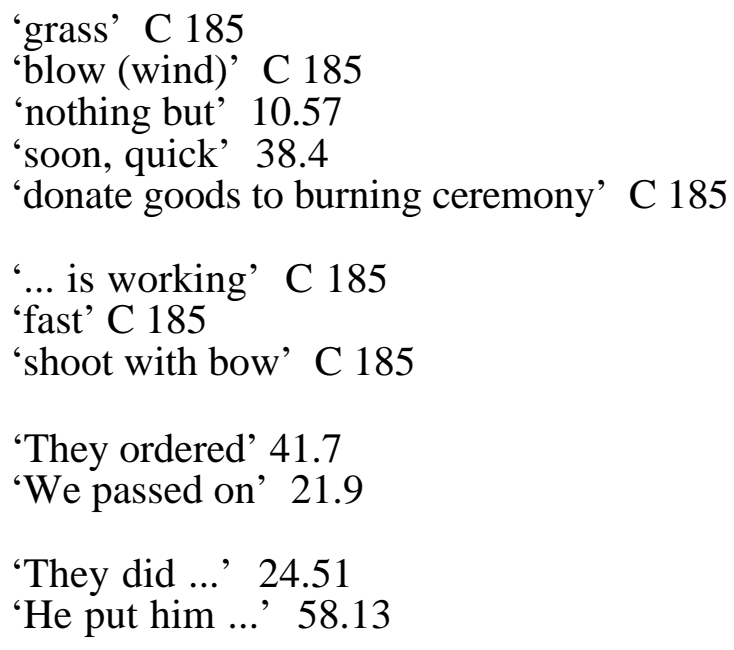

Long vowel stress also has the effect of precluding stress on a short vowel in the same word. That is, there are no roots with long vowels where stress falls on a syllable with a short vowel. The historical developments leading up to Cupeño stress, as described in Munro 1990, supports this observation. Pre-Cupeño stressed the root-initial vowel, or the second vowel if it was long; otherwise default stress fell on the initial syllable.

Subsequently, contrastive vowel length was lost in unstressed syllables. Thus, the fact that vowel length was only preserved in stressed syllables effectively rules out the possibility of short-vowel stress in forms with long vowels. Summarizing the above discussion in synchronic terms, one key observation governing the distribution of accent in roots is that long vowels attract stress. ${ }^{14}$

In contrast to this predictable part of the stress system, stress is contrastive in roots which do not contain long vowels: stress may fall on either the first or second syllable, as shown by the nouns in (14) and the conjugated verbs in (15).

\footnotetext{
${ }^{13}$ It is rare to find roots composed of three or four syllables with post-peninitial stress; this observation has prompted Crowhurst 1994 to invoke an initial two syllable window for stress in roots. The observations on canonical morpheme shape in Hill 1967: 184 ff., however, suggest that such a constraint may in fact be unnecessary because of the rarity of simplex roots greater than two syllables. Also, a cursory inspection of the lexical resources uncovers some exceptions to the two syllable window: iš mivíy 'things', tukumáy 'tomorrow', and piš ?emáy 'just then'.

${ }^{14}$ Stress in Spanish loans, e.g., váaka-?am 'cattle' and kaváayu-?um 'horses', also conforms to this pattern of long vowel stress. But stressed vowels in both Spanish and English loans tend to be long in Cupeño, suggesting that vowel length in these forms is phonological. Considering the role of duration in signaling stress in these languages, however, the most sensible approach to this problem seems to be that stressed vowels in the source languages are perceived as long, and hence represented as such lexically.
} 
(14)
a. sú?i-šs ‘jackrabbits' 10.63
púki-yka 'by (to) the door' 9.25
máxi?č -am 'greens' 9.4
kúpa-nax 'from Cupa' 29.1
kwíni-ly 'acorns' 29.1

(15)
a. pe-mí?awlu 'He came' 9.1
č em-yáyax
pem-híwen
pem-náyxi
'We try to ...' 9.7
'They stopped' 21.9
'They fought' 1.15
b. temá-1 'ground' 29.4
atáx?-am 'the people' 29.1
savá-1 'grass' 29.4
kawí-š 'rock' 29.4
sevé-1 'wind' 9.16
si?áyi-šs ‘cracked acorns’ 29.7

b. pe-pulín-qal '... gives birth' 43.5

č em-tewáš 'We lost' 125

While there may be a historical account of these patterns, the initial-peninitial stress contrast is synchronically unpredictable. This fact has led Hill \& Hill 1968 and Munro 1977 to classify Cupeño as a 'lexical stress' language, i.e., a language in which stress alone may introduce contrast among roots.

To summarize, the inventory of stress patterns observed in roots (excluding monosyllables) is given in (16).

(16) Root Stress Inventory

$\begin{array}{llll}\text { a. Predictable Long Vowel Stress } & \text { b. Contrastive Stress Elsewhere } \\ \text { CV́ VCV } & \text { CVCCV́ V } & \text { CV́ CVC } & \text { CVCV́ } \\ & & \text { C } \\ \text { xéene } & \text { tevxáa } & \text { sú?išs } & \text { temál }\end{array}$

Any analysis of the root stress inventory must account for the fact that long vowels are always stressed, and at the same time, it must allow for lexically determined initial or peninitial stress in forms with no long vowels.

\subsubsection{The Analysis}

I assume essentially the same foot structures proposed in Crowhurst 1994 to account for certain correspondences between the accent system and the prosodic morphology of the habilitative construction (see also Hill 1970, McCarthy 1979a, 1997, McCarthy \& Prince 1986, 1990). In particular, roots are consistently parsed into rightheaded feet in the output, even if this results in a monomoraic foot. ${ }^{15}$ This is illustrated below.

\footnotetext{
${ }^{15}$ The evidence for iambic feet in the analysis of stress is indirect, but strong. There is a relationship between the overall shape of the habilitative and the surface stress of its base form: the stressed vowel is always followed by two syllables in the habilitative, e.g., č ál—čá?a?al 'husk' and páč ik—páč i?ik 'leach acorns'. Assuming that feet are uniformly right-headed, it is possible to describe the prosodic morphology of the habilitative as a bipodal unit. Thus, finally-accented stems receive two epenthetic syllables, i.e., [( $\check{\mathrm{c}}$ á)(?a?al)], while disyllabic stems with initial stress only get one inserted syllable, as in [(pá)(̌̌ i?ik)], because one of the stem syllables can be recruited in the final foot.
} 
(17) Uniform Right-Headed Feet

$\begin{array}{llll}(\mathrm{x}) & (\cdot \mathrm{x}) & (\mathrm{x}) & (. \mathrm{x}) \\ \text { xéene } & \text { tevxáa } & \text { sú?iš } & \text { temál }\end{array}$

In constraint-based terms, uniform iambs entail a constraint ranking in which RHTYPE $=$ IAMB dominates RHTYPE $=$ TROCHEE (Prince \& Smolensky 1993). In addition, Foot Binarity must be ranked below the Prosodic Faithfulness constraints (PROS-FAITH) because the iambic requirement may have the effect of creating non-binary feet in cases like [(sú)?iš ], as depicted in the following tableau.

(18) Emergence of Lexical Initial Stress

\begin{tabular}{|c|c|c|}
\hline $\begin{array}{c}\mathrm{X} \\
\text { /sú?ǐs / }\end{array}$ & PROS-FAITH & FTBIN \\
\hline $\begin{array}{ll} & (. \mathrm{x}) \\
\text { a. } & \text { su? } \tilde{\mathrm{s}}\end{array}$ & $* !$ & \\
\hline $\begin{array}{l}(\mathrm{x}) \\
\mathrm{b} . \rightarrow \text { sú? }\end{array}$ & & * \\
\hline
\end{tabular}

The losing candidate is the unfaithful one, because the first vowel in the input has a prominence, but the related vowel in the output has no corresponding prominence, hence violating PROS-FAITH. The constraint violations in the first candidate can be due either to deletion of an accent, i.e., a MAX-PROM violation, or, if the grid mark in the input stands in correspondence with the surface prominence, then a violation of NO-FLOP-PROM is incurred. Either scenario is sufficient to motivate the domination of FTBIN. The winner, therefore, is the candidate which matches the input prosody exactly, at the expense of a FTBIN violation.

In this way, the constraint system accounts for the lexically determined stress contrast: variation in the position of stress is licensed by high-ranking Faithfulness. By the same reasoning, the surface restrictions on the stress inventory are achieved through the domination of Prosodic Faithfulness. In particular, predictable long vowel stress is derived by ranking the Weight-to-Stress Principle (Prince 1990) above PROS-FAITH. Assuming that only CVV syllables (and not CVC) are heavy (Crowhurst 1994), this correctly yields the observed pattern of long vowel stress.

It is useful, given the Richness of the Base (see discussion in §1.2), to show this result with a form that has an underlying prominence on a short vowel. ${ }^{16}$ For example, if a root such as Vtevxaa has an inherent accent on the first vowel, an unfaithful mapping results because the WSP dominates PROS-FAITH.

\footnotetext{
${ }^{16}$ To clarify, there is no overt evidence for a prominence on a short vowel in this form, but it is necessary to derive the restrictions on the inventory from any possible input, as the Richness of the Base entails that there are no language particular restrictions on the input. Therefore, prominence is freely distributed in the input, and the assumed ranking of constraints ensures the winning output forms will have stress on a heavy syllable.
} 
(19) Predictable Long Vowel Stress

\begin{tabular}{|l|c|c|}
\hline & X \\
/tevxaa/ & WSP & PROS-FAITH \\
\hline \hline a. $\quad \begin{array}{c}(\mathrm{x} .) \\
\text { tévxaa }\end{array}$ & $* !$ & \\
\hline b. $\rightarrow \quad \begin{array}{c}(. \times \mathrm{x}) \\
\text { tevxáa }\end{array}$ & & $*$ \\
\hline
\end{tabular}

The loser above has stress on a closed syllable, leaving the subsequent heavy syllable unstressed. Since CVC syllables are not heavy, this candidate incurs a fatal violation of the WSP, leaving the candidate which has undergone an accent shift as the optimal form.

Next recall that Cupeño lost contrastive vowel length in unstressed syllables. While not attested in synchronic alternations, this fact is an integral part of the root stress inventory and must therefore come within the scope of the analysis. It turns out that this systematic gap receives a direct explanation by considering further the role of the WSP in the system. Any unstressed long vowel constitutes a violation of the WSP; hence, vowel shortening can be induced by ranking the WSP above the Faithfulness constraint governing the realization of vowel length, WT-IDENT (McCarthy 1995, Urbanczyk 1996).

(20) Vowel Shortening by the WSP

\begin{tabular}{|l|c|c|}
\hline /CVVCV́ V/ & WSP & WT-IDENT \\
\hline \hline a. CVVĆ́ V & $* !$ & \\
\hline b. $\rightarrow$ CVCŕ V & & $*$ \\
\hline
\end{tabular}

The losing candidate suffers from a fatal WSP violation because it has an unstressed long vowel, so the form with shortening is the optimal output. In general, the WSP, in its topranked position, results in shortening of any unstressed long vowel. ${ }^{17}$

\footnotetext{
${ }^{17}$ Though they are shortened on the surface, long vowels actually fail to delete in contexts where short vowels would drop by regular rules of syncope. Thus, shortening and syncope may be understood here as a kind of 'chain-shifting' phenomenon, as found, for example, in Woleaian (Micronesian), where final vowels are deleted but long final vowels are merely shortened. While formal treatment of this fact would lead us too far afield, the greater degree of Faithfulness for long vowels can be handled straightforwardly in terms of locally-conjoined Faithfulness constraints (see Kirchner 1996 for an approach to chain-shifting rules in these terms).
} 
To summarize, the constraint rankings argued for thus far are given below.

(21) Summary Ranking

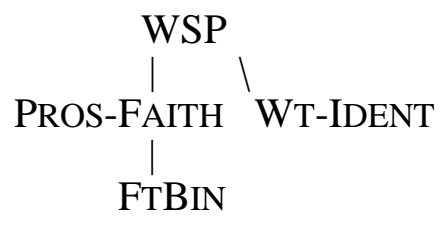

The ranking in which PROS-FAITH dominates FTBIN accounts for distinctive stress in the inventory of accentual patterns observed in roots. With the assumed iambic requirement undominated, this ranking means that initially-accented roots will surface with a non-binary foot. The domination of PROS-FAITH by the WSP, on the other hand, accounts for the predictable part of the stress system, namely the observation that long vowels are always stressed. Finally, the ranking of the WSP above WT-IDENT accounts for the absence of unstressed long vowels.

\subsection{Overriding Root Stress}

In this section, the influence of inherent root stress is examined and analyzed in larger words. The section begins with a detailed study of the interaction between root and affix stress $(\S 2.4 .1)$, followed by the proposed analysis $(\$ 2.4 .2)$. The analysis is then extended in $\$ 2.4 .3$ to account for pre-accenting suffixes and the special phonology of the nominalizer.

\subsubsection{Data and Observations}

Inherent accent in roots overrides accent in affixes (Hill \& Hill 1968). This is shown by the behavior of accented affixes when they combine with different classes of roots. When an accented prefix or suffix is attached to an unaccented root, inherent accent in the affix surfaces. However, when these same affixes attach to an accented root, the root accent prevails. The behavior of the two classes of roots is illustrated below, starting with unaccented roots.

The accented affixes of which I am able to find good examples are listed below. The accented prefixes are the person prefixes listed in (22), which are used to mark the person and number of subjects and possessors. The accented suffixes are listed in (23) (not including pre-accenting suffixes, which are treated in $\$ 2.4 .3$ ).

(22) Accented Prefixes

$\begin{array}{lll}1 & \text { né- } & \text { č ém- } \\ 2 & \text { ?é- } & \text { ?ém- } \\ 3 & \text { pé- } & \text { pém- } \\ & \text { Singular } & \text { Plural }\end{array}$


(23) Accented Suffixes

$\begin{array}{ll}\text {-qál } & \text { 'past durative marker' (PAST.DUR) } \\ \text {-qá } & \text { 'present singular marker' (PRES.SING) } \\ \text {-1́ } & \text { 'object marker' (OBJECT) } \\ \text {-í } & \text { 'nominalizer' (NOM) }\end{array}$

When one of these accented prefixes or suffixes combines with an unaccented root, inherent accent in the affix surfaces, as shown below for three roots classified by Hill \& Hill 1968 as unaccented.

a. Accented Prefix Wins

$\begin{array}{llll}\text { /né + yax/ } & \rightarrow & \text { né-yax } & \text { 'I say' JH } \\ \text { /pé + yax/ } & \rightarrow & \text { pé-yax } & \text { 'He says' 1.15 } \\ \text { /ć ém + yax/ } & \rightarrow & \text { ć ém-yax } & \text { 'We say' 21.6 } \\ \text { /pém + yax + wen/ } & \rightarrow & \text { pém-yax-wen } & \text { 'They said' 42.28 }\end{array}$

b. Accented Suffix Wins /ne?ep né + yax + qál/ /ne?en yax + qál

/pé + yax + qál/

/mi + yax + qál

$\rightarrow \quad$ né?ep ne-ya-qál

$\rightarrow \quad$ ne?en ya-qá?

$\rightarrow \quad$ pe-ya-qál

$\rightarrow \quad$ mi-ya-qá?

'I was saying' JH

'I say' JH

'He was saying' 1.9

He tells them 38.49

a. Iné $+\max +? \mathrm{e} / \quad \rightarrow$ né-max-?e $\quad$ (I) to give ...' JH

$\pi \tilde{\mathrm{c}} \mathrm{em}+\max +? \mathrm{e} / \quad \rightarrow \quad$ č é?-max-?e

$/ ? \mathrm{i}+$ pém + max $\quad \rightarrow \quad$ ?i-pé?-max

'(We) to give ...' JH

'They gave you ...' JH

b. $/ \max +$ qál $\quad \rightarrow \quad$ max-qá? $\quad$ '... giving ...'JH

$/ ? \mathrm{i}+$ né $+\max +$ qáll $\quad \rightarrow \quad$ ?i-ne-max-qál $\quad$ 'I was giving you' JH

ᄃ́ im + pé $+\max +$ qál/ $\rightarrow \quad$ č im-pe-max-qál 'He was giving us' JH
a. /né + wen/
$\rightarrow \quad$ né-wen
ć ém + wen/
$\rightarrow \quad$ č́m-wen
/né + wen + pi/
$\rightarrow \quad$ né-wene-pi
/ć ém + wen + pi/
$\rightarrow \quad$ č ém-wene-pi
'I put' JH
'We put' JH
'(I) to put it in' JH
'(We) to put it in' JH
b. /né + wen + qál/
/wen + qál
$\rightarrow \quad$ ne-wen-qál
$\rightarrow \quad$ wen-qál
'I was putting' JH
'... put (it) ...' JH

As is evident from the examples above, when a word has more than one accented affix, it is the rightmost one in the word which surfaces with stress, e.g., /né + wen + qál/ $\rightarrow$ [newen-qál]. This pattern also holds when the competition is between two accented suffixes, as shown by the following examples which both contain the past durative and objective case marker.

(27) Rightmost Accented Suffix Wins

$$
\begin{array}{llll}
\text { /yax }+ \text { qál + 1́/ } & \rightarrow & \text { yex-qel-í } & \text { 'While ... was saying' H\&H } 236 \\
\text { /?é }+ \text { yax }+ \text { qál + í/ } & \rightarrow & \text { ?e-ya-qal-1́ } & \text { '... what you said' JH }
\end{array}
$$

When an unaccented root combines with an unaccented affix, however, the word is assigned default initial stress, as exemplified below. 
(28) Default Initial Stress
a. /yax $+\mathrm{em} / \quad \rightarrow$ yáx-em '(You Pl) say!' JH
/̌ em $+\check{c}$ eme yax + wel $\rightarrow$ č em-č eme yáx-we 'We say' JH
/ne?qwen ya + ?a/ $\quad \rightarrow \quad$ ne?qwen yá-?a $\quad$ 'I can say' JH
b. $/ \max +\mathrm{em} / \quad \rightarrow$ máx-em $\quad \rightarrow$ Give! (Pl)' C 186
$/ \max +\mathrm{an} / \quad \rightarrow \quad$ máx-an $\quad$ 'Give it to me' JH
$/ \mathrm{max}+\mathrm{a}$ ?ěs $/ \quad$ máx-a?ěs $\quad \rightarrow \quad$ Give it to us' JH
c. /wen + em/ $\rightarrow$ wén-em 'Put it in (Pl subj)' JH
/wen + al $\quad \rightarrow \quad$ wén-a $\quad$ 'Put it in (Sg)' JH

While many of the examples above with emergent prefix stress also have initial stress, as noted in Hill \& Hill 1968: 235, stressed prefixes may surface with non-initial stress. In the examples below, an object marker prefix separates the stressed prefix from the beginning of the word, yielding prefix stress on the second syllable of the word. ${ }^{18}$

(29) Non-Initial Prefix Stress
a. mi-né-tew
mi-pé-tew
mi-č ém-tew
'I saw them'
'He saw them'
'We saw them'
b. pi-pú-kuṣ
'He ... took it'
/pi + pé + kus /
$3 \mathrm{sg}+3 \mathrm{sg}+\mathrm{TOOK}$
c. pi-pé-wen
/pi + pé + wen/
'He put it'
$3 \mathrm{sg}+3 \mathrm{sg}+\mathrm{PUT}$
d.

\begin{tabular}{|c|c|}
\hline $\begin{array}{l}\text { ?i-pé?-max } \\
\text { 'They gave you ...' }\end{array}$ & $\begin{array}{l}\text { /?i+ pém+ max/ } \\
2 \mathrm{sg}+3 \mathrm{pl}+\mathrm{GIVE}\end{array}$ \\
\hline $\begin{array}{l}\text { mi-né-maxe-n-pi } \\
\text { '(I) to give them ...' }\end{array}$ & $\begin{array}{l}/ \mathrm{mi}+\text { né }+\max +\mathrm{n}+\mathrm{pi} / \\
3 \mathrm{pl}+1 \mathrm{sg}+\mathrm{GIVE}+\mathrm{X}+\mathrm{FUT}\end{array}$ \\
\hline $\begin{array}{l}\text { mi-pé?-max-wen } \\
\text { 'They were giving ...' }\end{array}$ & $\begin{array}{l}\text { /mi }+ \text { pém }+\max +\text { wen/ } \\
\text { 3pl+3pl+GIVE+PRES.IMPER }\end{array}$ \\
\hline
\end{tabular}

In contrast to the forms above containing unaccented roots, when an inherently accented affix combines with an accented root, root accent always prevails. This is shown below for each affix type individually (30), and with accented roots which combine with both accented prefixes and suffixes (31).

\footnotetext{
${ }^{18}$ The object markers preceding the stressed prefixes here cannot be clitics because they do not meet the requirements for stand-alone pronouns stated in Hill \& Nolasquez 1973: $122 \mathrm{ff}$.
} 
(30) Root Accent Overrides Affix Accent

a. Root-Controlled De-Accenting in Prefixes

\begin{tabular}{|c|c|c|c|}
\hline é + ๆíy + pi/ & $\rightarrow$ & pe-ๆíy-pi & 'He would go away' 1.15 \\
\hline e + yax/ & $\rightarrow$ & pe-ๆéye-yax & 'It shakes' 1.17 \\
\hline$v+1 u /$ & & & ‘He came’ 9.1 \\
\hline & $\longrightarrow$ & & ssed on' 21.9 \\
\hline ven/ & $\rightarrow$ & & went out' 29.2 \\
\hline & $\rightarrow$ & & t angry' 1.15 \\
\hline & & pem-č í?-lyu-wen & 'They went gathering' 2 \\
\hline
\end{tabular}

b. Root-Controlled De-Accenting in Suffixes

$\begin{array}{llll}\text { /píq + pe + qál/ } & \rightarrow & \text { píq-pe-qal } & \text { ‘... touched him’ 43.31 } \\ \text { /mi + k'áw + pe + qál/ } & \rightarrow & \text { mi-k'áw-pe-qal } & \text { 'He was calling them’ 44.1 } \\ \text { /nánva + ya + qál } & \rightarrow & \text { nánva-ya-qa } & \text { '... is done' 44.9 } \\ \text { /?áyu + qál } & \rightarrow & \text { ?áyu-qa } & \text { ‘... (He) wants' 23.31 }\end{array}$

(31) Root-Controlled De-Accenting

\begin{tabular}{|c|c|c|c|}
\hline é + ?áyu + qál/ & & pe-?áyu-qal & 'He was wanting' 1.14 \\
\hline & & pe-túl-qa & 'He finished' 42.22 \\
\hline ál/ & & pe-háw-pe-qal & 'He sang' 42.22 \\
\hline & & pe-pulín-qal & 'She gave birth' 43.5 \\
\hline $1+$ pe/ & & ne-níy-qal-i-pe & 'When I go away' 1.1 \\
\hline
\end{tabular}

To summarize, the interaction between root and affix accent may be described as follows (roots are underlined).

(32) Summary of Cupeño Accent

-If the root contains an inherently accented vowel, that vowel receives the unique word stress:

$$
\begin{array}{ll}
/ \ldots \text { róot... } / & \rightarrow \\
/ \text { pé }+\underline{\text { túl }}+\text { qál } \rightarrow & {[\ldots \text { róot } \ldots]}
\end{array}
$$

-In words without an accented root, the rightmost accented vowel in an affix bears word stress:

$$
\begin{array}{lll}
/ \ldots \text { áf }+\ldots+\text { áf } / & \rightarrow & {[\ldots \text { af }+\ldots+\text { áf }]} \\
/ \text { pé }+ \text { yax }+ \text { qál } / & \rightarrow & {[\text { pe-ya-qál }]} \\
\text { /yax }+ \text { qál }+1 / / & \rightarrow & {[\text { yex-qel-í }]}
\end{array}
$$

-If the word does not contain an inherently accented morpheme, the first vowel receives the word stress:

$$
\left.\begin{array}{lll}
/ \sigma \sigma \ldots / & \rightarrow & {\left[\begin{array}{ll}
0 & \sigma
\end{array}\right]} \\
/ \mathrm{yax}+\mathrm{em} / & \rightarrow & {[\text { yáx-em }}
\end{array}\right]
$$




\subsubsection{The Analysis}

There is always a single phonetic stress per word in Cupeño, ${ }^{19}$ and so when more than one accent is present in the underlying representation, only one can be realized as stress. That is, stress is culminative in Cupeño, and so only one inherently accented morpheme can faithfully project its lexical prominence. Therefore, when two or more morphemes combine, each endowed with a lexical word-level prominence, all but one of the prominences must be deleted (see $\S 1.2 .2 .3$ for discussion of the prosodic analysis of culminativity). The analysis proposed below is that this competition for a unique word accent is negotiated through the interaction of a set of ranked well-formedness constraints, i.e., the interaction of Faithfulness constraints, segregated by morphological domains, and Alignment constraints, which favor accent that is closest to a designated edge.

As mentioned in $\$ 2.2$, I follow McCarthy \& Prince 1995 in assuming that Faithfulness constraints are segregated into the morphological domains Root and Affix, and that Root Faith always outranks Affix Faith. This ordering applies to the set of Prosodic Faithfulness constraints, yielding MAX-PROM Root $\gg$ MAX-PROMAffix. This natural division in the PROS-FAITH constraints is necessary cross-linguistically to describe the restrictions observed in the accentual inventories of affixes. What is more, this ordering explains the interaction between root and affix stress in Cupeño.

The following tableaux illustrate the basic result. As shown in (33), when the competition for the unique word accent is between an accented prefix and an accented root, the accented root wins. Likewise, when the competition is between an accented root and an accented suffix, the root will again prevail with the word stress, as shown in (34).

(33) Root-Controlled De-Accenting in Prefixes: / áf + róot ... / $\rightarrow$ [ af-róot ... ]

\begin{tabular}{|c|c|c|}
\hline $\begin{array}{c}\mathrm{x}_{1} \mathrm{x}_{2} \\
\text { /pé + mí?aw + lu/ }\end{array}$ & MAX-PROMRoot & MAX-PROM $_{\text {Affix }}$ \\
\hline $\begin{array}{ll} & \left(\mathrm{x}_{1}\right) \\
\text { a. } & \text { pé-mi?aw-lu }\end{array}$ & $* !$ & \\
\hline $\begin{array}{l}\left(. \mathrm{x}_{2}\right) \\
\text { b. } \rightarrow \quad \text { pe-mí?aw-lu }\end{array}$ & & $*$ \\
\hline
\end{tabular}

\footnotetext{
${ }^{19}$ Bright \& Hill 1967: 355-56 mention in passing a pattern of alternating stress counting from the primary stress, but unfortunately do not give any examples. This statement appears to run counter to most work on Cupeño which explicitly deny the existence of secondary stresses (e.g. Hill \& Hill 1968: 236), which is consistent with my own listening of a tape of Cupeño speech provided for me by Jane Hill. If it turns out that Cupeño does in fact have secondary stresses, this finding does not affect the result derived here. The distinction between primary and secondary stress implicit here is consistent with culminative stress, which can be analyzed in terms of the prosodic analysis of culminative accent generally given in §1.2.2.3.
} 
(34) Root-Controlled De-Accenting in Suffixes: / ... róot + áf / $\rightarrow$ [ ... róot-af ]

\begin{tabular}{|c|c|c|}
\hline $\begin{array}{c}\mathrm{x}_{1} \quad \mathrm{x}_{2} \\
\text { /?áyu + qál }\end{array}$ & MAX-PROMRoot & MAX-PROMAffix \\
\hline $\begin{array}{ll} & \left(. \quad x_{2}\right) \\
\text { a. } & \text { ?ayu-qá }\end{array}$ & $* !$ & \\
\hline b. $\rightarrow$ ? ?áyu-qa & & $*$ \\
\hline
\end{tabular}

The same result obtains when an accented root combines with both an accented prefix and an accented suffix, as depicted in (35). Here again, the outcome is overriding root stress because of the universal ordering between Root and Affix Faithfulness.

(35) Root-Controlled De-Accenting: / áf + róot + áf / $\rightarrow$ [ af-róot-af ]

\begin{tabular}{|c|c|c|}
\hline $\begin{array}{c}\mathrm{x}_{1} \mathrm{x}_{2} \mathrm{x}_{3} \\
\text { /pé + túl + qál }\end{array}$ & MAX-PROMRoot & MAX-PROM $_{\text {Affix }}$ \\
\hline a. $\quad\left(\begin{array}{c}\left.\mathrm{x}_{3}\right) \\
\text { a.tul-qá }\end{array}\right.$ & $* !$ & $*$ \\
\hline $\begin{array}{ll} & \left(\mathrm{x}_{1}\right) \\
\text { b. } & \text { pé-tul-qa }\end{array}$ & $* !$ & $*$ \\
\hline $\begin{array}{c}\left(. \mathrm{x}_{2}\right) \\
\text { c. } \rightarrow \text { pe-túl-qa }\end{array}$ & & $* *$ \\
\hline
\end{tabular}

When the competition is instead between two accented affixes, the Faithfulness constraints cannot be decisive, as the MAX-PROM violations are equal in such a case. The decision therefore falls to some other constraint, which in this case, is the lower ranked Alignment constraint, ALIGN-R(PK, PrWd), which favors right-aligned stress peaks. This constraint thus picks the candidate with the rightmost affix stress, as shown below for a word with an accented prefix and suffix.

(36) Rightmost Affix Stress: / áf + root + áf ... / $\rightarrow$ [ af-root-áf ... ]

\begin{tabular}{|c|c|c|}
\hline $\begin{array}{c}\mathrm{x}_{1} \quad \mathrm{x}_{2} \\
\text { /pé-yax-qál/ }\end{array}$ & MAX-PROM & ALffix \\
\hline \hline $\begin{array}{c}\left(\mathrm{x}_{1}\right) \\
\text { pé-yax-qal }\end{array}$ & $*$ & yax-qal! \\
\hline $\begin{array}{c}\left(. \mathrm{x}_{2}\right) \\
\text { b. } \rightarrow \text { pe-yax-qál }\end{array}$ & $*$ & \\
\hline
\end{tabular}

This result obtains in words with two stressed suffixes, e.g. /yax + qál $+1 /$ / $\rightarrow$ [yex-qel-1́]. The correct outcome here is more harmonic than a form which stresses the penultimate suffix, e.g. [yex-qál-i], because the former better satisfies ALIGN-R(PK, PrWd). 
The fact that inherent accent can be realized non-finally in Cupeño shows that MAX-

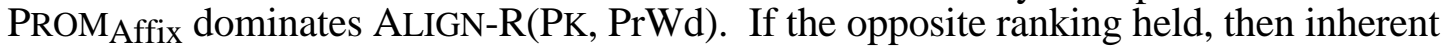
accent could only surface word-finally, which is not true for Cupeño. In the tableau below, lexical accent emerges in a non-final vowel, despite the resulting violation of ALIGN-R(PK, PrWd).

(37) Non-Final Prefix Stress: / áf ... / $\rightarrow$ [áf ... ]

\begin{tabular}{|l|c|c|}
\hline & $\begin{array}{c}\mathrm{x}_{1} \\
\text { /pé }+ \text { yax/ }\end{array}$ & MAX-PROM Affix \\
\hline \hline $\begin{array}{c}\left(. \mathrm{x}_{2}\right) \\
\text { pe-yáx }\end{array}$ & $* !$ & \\
\hline b. $\rightarrow \begin{array}{c}\left(\mathrm{x}_{1}\right) \\
\text { pé-yax }\end{array}$ & & $\mathbf{y a x}$ \\
\hline
\end{tabular}

To give an interim summary of the results, the inherent ordering between Root and Affix Faithfulness explains the each pattern of root retention depicted above. That is, regardless of affix type, if an inherently accented root combines with an inherently accented affix, the accent of the root prevails because of the inherent ranking of MAX-PROMRoot above MAX-PROM Affix. Affixal accent only emerges in words with unaccented roots because in such cases there is no root accent to realize. Finally, in words with more than one accented affix, the rightmost affix accent wins because of the role of ALIGN-R(PK, PrWd) in the system.

Recall from \$2.4.1 that words which do not have a lexical accent have initial stress. Alongside the rightward orientation of affix accent, this is a pattern of conflicting directionality which Crowhurst 1994 compares to default-to-opposite edge orientation in unbounded stress systems. Naturally, it is desirable to account for this pattern of 'rightmost lexical accent/otherwise default initial stress' with the same basic toolbox employed in the analysis of default-to-opposite stress, and this is the spirit in which the following analysis is proposed.

At the heart of most recent approaches to default-to-opposite stress is a ranking of conflicting Alignment constraints. Thus, Zoll 1997 and Walker 1996 employ opposing Alignment constraints formulated to contrast different syllable types, i.e., heavy versus light; Baković 1998 approaches the problem in terms of constraint conflict between Alignment constraints defined at different levels of metrical structure (cf. Prince 1983); and similar constraint rankings are used in Kenstowicz 1995b, Hewitt \& Crowhurst 1996, and Crowhurst \& Hewitt 1997. For concreteness, I follow Baković 1998 in distinguishing among levels of metrical structure, which I refer to here as stress prominence and stress peaks, and model the conflicting edge effects as a similar type of constraint interaction. My proposal is thus that lexical accents are encoded as stress peaks, i.e., a level 3 grid mark shown below, and stress peaks are oriented to the right edge of the word. Stress prominences (level 2 grids) on the other hand are left-edge oriented, and so the leftmost syllable receives a superordinate grid mark in words with no inherent accent, as shown below. 
(38) Default-to-Opposite Stress in Cupeño

a. Rightmost Accented Syllable

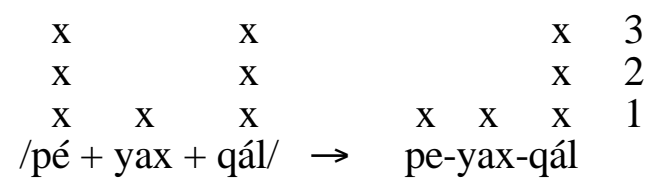

b. Leftmost Prominent Syllable

$$
\begin{array}{ccr} 
& & 3 \\
x \quad x & x \quad x & 2 \\
\text { /yax }+ \text { em/ } \rightarrow \text { yáx-em } & 1
\end{array}
$$

While it has no phonetic consequences for stress, the distinction between different grid levels is crucial in the analysis: the rightmost level 3 grid mark (=lexical accent) wins in a multiply accented structure (38a), but when the underlying representation has no inherent accent, the level two grid mark (=prominent syllable) is leftmost (38b). ${ }^{20}$ This result is very much on par with the treatment of default-to-opposite edge stress in the aforementioned analyses, except the default pattern of initial stress does not have a level 3 prominence. The generalization governing this non-uniform set of structures above is thus that a level 3 grid is not inserted if it is not needed to distinguish a most prominent syllable from all others. The constraint interaction giving this result therefore involves ranking DEP-PROM above the constraint requiring a level 3 grid prominence, namely

HEADEDNESS(PrWd) (see $§ 1.2 .2 .3$ for the definition of this constraint and its interaction with the PROS-FAITH constraints). With this ranking, stress peaks will only be present in the output if they are also present in the input, correctly predicting the input-output mappings given above in (38).

The Alignment constraints which are responsible for the conflicting directionality effects are given below.

INIT-PROM $\equiv \operatorname{ALIGN}((\mathrm{x})-2, \mathrm{~L}$, PrWd, L): the left edge of every level $2 x$ must coincide with the left edge of some PrWd.

Align-R(PK, PrWd $) \equiv \operatorname{Align}((\mathrm{x})-3, \mathrm{R}, \operatorname{PrWd}, \mathrm{R})$ : the right edge of every level $3 x$ must coincide with the right edge of some PrWd.

INIT-PROM refers to stress prominences and characterizes the imperative for prominence on the initial syllable, as illustrated in the tableau below.

(40) Default Initial Stress: $/ \sigma \sigma \ldots / \rightarrow\left[\begin{array}{ll}\sigma & \sigma\end{array} \ldots\right]$

\begin{tabular}{|l|l|c|}
\hline \multicolumn{1}{|c|}{$/$ yax + em/ } & ALIGN-R(PK, PrWd) & INIT-PROM \\
\hline \hline a. $\quad \begin{array}{l}\text { yax-ém })-2 \\
\text { b. } \rightarrow \text { yáx-em }\end{array}$ & & yax! \\
\hline
\end{tabular}

A stress peak cannot be inserted in this system, so words with no inherent accent receive default initial stress because the left edge is the preferred edge for level 2 grid marks. On the other hand, level 3 prominence is oriented to the right edge of the word; therefore, in

\footnotetext{
${ }^{20}$ See Antilla \& Bodomo 1996 for a parallel result in Dagaare where lexical accent is likewise argued to be more prominent than non-lexical accent.
} 
words with more than one inherently accented affix, the rightmost inherent accent wins as an effect of ALIGN-R(PK, PrWd). As illustrated below, this result requires ranking ALIGN$\mathrm{R}(\mathrm{PK}, \mathrm{PrWd})$ above INIT-PROM, because the presence of a level 3 grid mark entails a subordinate level 2 grid mark, which by INIT-PROM, is properly aligned at the left edge of the word.

(41) Rightmost Affix Stress: / áf + root + áf ... / $\rightarrow$ [af-root-áf ... $]^{21}$

\begin{tabular}{|c|c|c|}
\hline $\begin{array}{c}\mathrm{x}_{\mathrm{a}} \mathrm{x}_{\mathrm{b}} \\
/ \mathrm{pé}+\mathrm{yax}+\mathrm{qál} /\end{array}$ & ALIGN-R(PK, PrWd) & INIT-PROM \\
\hline $\begin{array}{ll}\text { a. } & \left(x_{a}\right)-3 \\
& (x)-2 \\
& \text { pé-yax-qal }\end{array}$ & yax-qal! & \\
\hline b. $\begin{array}{ll}\quad\left(\cdot x_{b}\right)-3 \\
\rightarrow \quad \text { pe-yax-qál }\end{array}$ & & pe-yax \\
\hline
\end{tabular}

In sum, the conflicting patterns of directionality are handled as conflicting Alignment constraints which refer to different levels of prominence structure; where these constraints conflict, rightmost inherent accent prevails because of the ranking of ALIGN-R(PK, PrWd) above INIT-PROM.

One question this analysis raises concerns the behavior of level 2 grid marks: are lexical stress prominences faithfully preserved in output forms, and if so, how are they influenced by the conflicting Alignment constraints? As there is no need to distinguish between level 2 and 3 grid marks with the Prosodic Faithfulness constraints, it is clear that a lexical level 2 prominence would be preserved, and hence will pattern with stress prominences elsewhere in the system if present. However, it turns out that the lexical inventory does not have this type of prominence structure, given the principles of learning discussed in $\$ 1.2 .2 .3$. The overt structure needed to posit a lexical accent in the first place is surface stress. Given a choice between positing a lone level 2 grid mark over the stressed syllable or a column of grid marks reaching to level 3, it is clear that the latter option will be chosen because the input-output mapping resulting from such a decision is more harmonic than if a level 3 grid is not assumed in the underlying representation. Positing lexical accent as a level 3 prominence therefore avoids a violation of HEADEDNESS(PrWd), given the ranking assumptions laid out above.

To summarize the results of this section so far, the observations concerning root and affix stress in Cupeño are captured by the following constraint rankings.

\footnotetext{
${ }^{21}$ The change to alphabetic indices here in marking the correspondence relations between prominences is simply to avoid confusion with the numbering for grid levels and is of no theoretical significance.
} 
(42) Summary Ranking

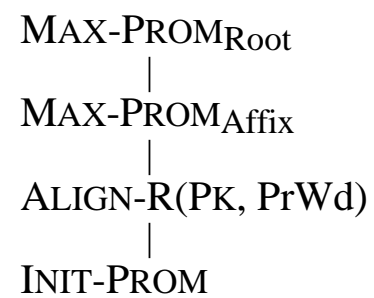

Because of the intrinsic ranking between Root and Affix Faithfulness, MAX-PROMRoot dominates MAX-PROM ${ }_{\text {Affix }}$; this ranking explains the fundamental observation that root stress overrides affix stress. This ranking is also shown to be crucial in the analysis of two additional morpho-accentual phenomena explored in the next subsection. The Alignment constraint for word-level prominence, ALIGN-R(PK, PrWd), is ranked below MAXPROM $_{\text {Affix }}$, accounting for the fact that lexical prominences may be non-final. ALIGN$\mathrm{R}(\mathrm{PK}, \mathrm{PrWd})$ is responsible for the observed rightward edge orientation of lexical accent which is found in words with more than one accented affix. Finally, the lowest ranking constraint, INIT-PROM, describes the pattern of initial stress by default in words with no inherently accented morphemes and is dominated by ALIGN-R(PK, PrWd) because of the structural assumptions of stress peaks versus stress prominences. ${ }^{22}$

\subsubsection{Extending the Analysis}

The discussion so far has focused squarely on the interaction between root and affix stress where the affixes are themselves stressed. A large number of affixes in Cupeño, however, may contribute an accent, but do not themselves surface with stress. These are the pre-accenting suffixes, which typically cause accent to fall on the root-final vowel. Any analysis of Cupeño accent must account for these cases, and as will be shown directly below, the ideas developed so far provide a clear line of analysis for pre-accentuation. The analysis will also be extended to account for the unique phonology of the nominalizer suffix $-i$.

\footnotetext{
${ }^{22}$ René Kager (personal communication) points out an interesting prediction of this analysis, given the position of the WSP in the system (see \$2.3.2): a long vowel in an affix could attract stress, even when attached to an inherently accented root. Interestingly, there appear to be no affixes with long vowels, save one suffix, -kwáani 'for the sake of' (from Hill \& Nolasquez's 1973 list of affixes), which is probably derived historically from the bare noun stem -kwaan 'worth, value'. The absence of long vowels in affixes therefore suggests an additional role for Root and Affix Faithfulness, where the constraint banning long vowels is interleaved between Root and Affix Faithfulness for vowel length.
} 
A list of the pre-accenting suffixes in Cupeño is given below. ${ }^{23}$

(43) Pre-Accenting Suffixes (see Hill 1967, Hill \& Hill 1968, and Hill \& Nolasquez 1973)

\begin{tabular}{|c|c|}
\hline -?aaw & 'at' \\
\hline$-\check{c} \mathrm{i}$ & 'with, by means of' (WITH) \\
\hline -maa & 'diminutive' (DIM) \\
\hline -nuukV & 'punctual subordinator' (PUNCT) \\
\hline$-\eta а$ & 'in' \\
\hline -пa?aw & 'on' \\
\hline$-\eta a x$ & 'from' \\
\hline -pe & 'place of' \\
\hline -wi & 'augmentative' (AUG) \\
\hline$-\mathrm{i}$ & 'objective case' (OBJECT) \\
\hline -yka & 'to' \\
\hline
\end{tabular}

The examples below illustrate the behavior of the pre-accenting suffixes. One consistent fact in these examples is that the accent contributed by the pre-accenting suffix always surfaces on the root-final syllable, as shown in (44) with some monosyllabic and disyllabic stems.

(44) Unaccented Root + Pre-Accenting Suffix
a. /wena + nuk $_{\text {pre }} / \quad \rightarrow \quad$ wená-nuk
'having put it' H: 192
b. $/ \mathrm{ki}+\eta \mathrm{a}_{\text {pre }} /$
/ki $+\eta \mathrm{xax}_{\text {pre }} /$

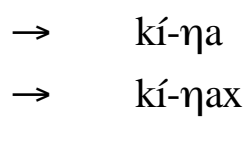
'at the house' 2.63
'in the house'
10.42
c. $/ \mathrm{ku}+\mathrm{yka}$ prel
kú-yka
'into the fire'
3.136

The following three sets of data show the behavior of the pre-accenting suffixes when they follow a sequence of accented prefix + unaccented root. As illustrated in all three sets, the accent contributed by the pre-accenting suffix always wins out over an accented prefix. ${ }^{24}$ Also, the forms in (48) show the same pattern as those in (45-47), except the roots are disyllabic, which shows that the size of the root does not affect the dominance relations between the affixes involved.

\footnotetext{
${ }^{23}$ A distinction is made in Hill \& Hill 1968 between the suffixes given in (40) and ones which are claimed to yield root-initial stress in unaccented forms, e.g. -we 'present imperfect (plural subject)', -weene 'past imperfect'. The evidence given for this two-way distinction is largely based on theory-internal assumptions with regard to syncope, and for this reason I will only discuss the root-final accenting suffixes.

${ }^{24}$ Unfortunately it is impossible at this time to determine if the accent contributed by a pre-accenting suffix also takes precedence over an inherent suffix accent. The indeterminacy of the data, however, will not have crucial implications for the analysis presented here.
} 
(45) Accented Prefix + Unaccented Root $\sqrt{m a}$ 'hand' + Pre-Accenting Suffix
$\begin{array}{lll}/ \text { né }+ \text { ma }+\check{c} \mathrm{i}_{\text {pre }} / & \rightarrow & \text { ne-má-č } \mathrm{i} \\ / \mathrm{pé}+\mathrm{ma}+\check{c} \mathrm{i}_{\text {pre }} / & \rightarrow & \text { pe-má-č } \mathrm{i}\end{array}$
'with my hands'
$\mathrm{H}: 192$
'by the hand'
10.52
/pé + ma $+i_{\text {prel }} \quad \rightarrow \quad$ pe-má-y
'his paws (obj.)'
8.126
cf. /pém + ma/
$\rightarrow \quad$ pé?-ma
'their hands'
8.130

(46) Accent Prefix + Unaccented Root $\sqrt{ } y u$ 'head' + Pre-Accenting Suffix
/né + yu $+\mathrm{i}_{\text {pre }}$
$\rightarrow \quad$ ne-yú-y
'my hair (obj.)'
23.71
/pém + yu $+\mathrm{i}_{\text {pre }} / \quad \rightarrow \quad$ pum-yú-y
'their heads (obj.)'
14.206
/pém + yu + ๆa pre/

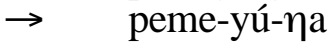
'on their heads'
H: 192

(47) Accented Prefix + Unaccented Root $\sqrt{k i}$ 'house' + Pre-Accenting Suffix, cf. (42)
/pé $+\mathrm{ki}+$ ykaprel $\rightarrow$ pe-kí-yka
/pé $+\mathrm{ki}+\mathrm{i}_{\text {pre }} \quad \rightarrow \quad$ pe-kí-y
/pé $+\mathrm{ki}+$ ?aw prel $\rightarrow$ pe-kí-?aw
$\check{\mathrm{c}} \mathrm{em}+\mathrm{ki}+$ ?awprel $\rightarrow$ č em-kí-?aw
'to his house'
10.49
$/$ pém $+\mathrm{ki}+\mathrm{i}_{\text {pre }} /{ }^{\mathrm{p}} \quad \rightarrow \quad$ pem-kí-y
lém $+\mathrm{ki}+\mathrm{i}_{\text {prel }}$
$\rightarrow \quad$ em-kí-y
'his house (obj.)'
12.148
'at (his) house' 12.154
'in our homes' 21.1
'their homes (obj.)' 22.18
'your homes (obj.)' 23.20

(48) Accented Prefix + Unaccented Root (Disyllabic) + Pre-Accenting Suffix
/pé + tama + naprel $\rightarrow$
pe-tamá-na
'in his mouth'
1.37
/pé + qewi + ?aw pre $/ \rightarrow$ pe-qewí-?aw
$/$ pé + qillyi + ?awprel $\rightarrow$ pe-qilý́-?aw
'at (on) its forehead'
'on nape of his neck'
11.108
15.240

A significant and important fact of pre-accentuation in Cupeño is that this process is blocked in words that have inherently accented roots (Hill \& Hill 1968). That is, when a pre-accenting suffix attaches to an accented root, no accent is inserted by the suffix and the root accent is realized, as illustrated below.

(49) Accented Prefix + Accented Root + Pre-Accenting Suffix (Hill \& Hill: 236)
/?ísi + lye $+\mathrm{i}_{\text {pre }}$
$\rightarrow \quad$ ?ísi-ly-i
/méme + yke prel $\rightarrow$ méme-yke
'coyote (obj.)'
tívi? + maapre + le/
$\rightarrow \quad$ tívi?-ma-l
'to the ocean'
'small round basket'

To summarize the main features of pre-accentuation, pre-accenting morphemes cause root-final stress. Also, pre-accenting morphemes win out over accent in a prefix, but lose to a root accent. The analysis given below builds on the ideas developed in $\$ 2.4 .2$ in accounting for these facts. ${ }^{25}$

In order to study the interaction between pre-accentuation and inherent accent in roots and affixes, it is necessary to have a concrete analysis of pre-accentuation. While at present there is no 'standard' theory of pre-accentuation, I will commit for the moment to a specific analysis as a means of developing my argument for the root-controlled analysis of Cupeño accent. The analysis of pre-accentuation in Cupeño in particular, and pre- and post-accentuation in general, will be significantly modified in chapter 5, section 3 , where this phenomenon is studied in detail. However, as is made clear in the analysis of Cupeño

\footnotetext{
${ }^{25}$ Hill \& Hill 1968: 236 suggest that there is a locality condition on the distance between the pre-accenting suffix and the root, essentially that there cannot be more than one intervening suffix. I have not be able to find an ample body of examples supporting this observation, so this condition is not analyzed here.

However, in §5.3, where pre-accentuation in Cupeño is revisited, I sketch an analysis, assuming that the locality condition is in fact significant, as this type of condition is an important theoretical implication following from the theory of pre-accentuation developed there.
} 
presented there, the changes in the theory of pre-accentuation do not affect the argument presented here for root-controlled accent, as the same principles apply in both analyses.

Observationally, pre-accenting suffixes have two basic requirements: they must appear to the right of a root or stem, and they must directly follow a stressed syllable. Following Kager 1996, this combination of alignment properties can be formalized within Generalized Alignment Theory (McCarthy \& Prince 1993a), as in the complex constraint below. ${ }^{26}$

(50) PRE-ACCENT $\equiv$ Align(Affix pre $_{\text {, L }}$, Root, R) $\&_{\text {or }}$ Align(Affix $_{\text {pre }}$, L, PROM, R) The left edge or pre-accented suffixes (a lexically marked affix class) must coincide with both the right edge of the root and the right edge of a prominent syllable.

According to the above constraint, pre-accenting affixes are formal suffixes: they have the subcategorization requirements typical of suffixes, namely coincidence at the left edge of the affix with the right edge of the root. In addition, pre-accenting suffixes must be leftaligned to the right edge of a prominent syllable, whether this prominence is an emergent lexical accent or an inserted prominence. PRE-ACCENT must therefore be ranked above INIT-PROM, as words with an unaccented root and a pre-accenting suffix receive non-initial stress, in contrast with words which have no inherently accented morphemes (see \$2.4.1 and §2.4.2. for data and analysis). Thus, PRE-ACCENT has a dual role: it ensures that the pre-accenting morpheme is a suffix and that it is properly aligned with a stressed syllable, which may be a non-initial syllable, as illustrated below.

(51) Root-Final Stress in Pre-Accentuation: $/ \ldots$ root $+\mathrm{af}_{\text {pre }} / \rightarrow\left[\ldots\right.$ róot-af $\left.{ }_{\text {pre }}\right]$

\begin{tabular}{|c|c|c|}
\hline /wena $\mid$ nukpre/ & PRE-ACCENT & INIT-PROM \\
\hline $\begin{array}{ll} & (\mathrm{x}) \\
\text { a. } & \text { wéna } \mid \text { nuk }_{\text {pre }}\end{array}$ & $* !$ & \\
\hline b. $\rightarrow$ wená $\mid$ nuk pre & & $*$ \\
\hline
\end{tabular}

The two candidates above differ in their obedience to the prominence subcategorization requirement. The losing candidate has initial stress, and while this pattern leads to satisfaction of INIT-PROM, it is fatal because the pre-accenting suffix is not properly aligned with the stressed syllable. The winner is thus the form with root-final stress because such a pattern satisfies PRE-ACCENT.

Concerning the interaction between roots and pre-accenting suffixes, root accent overrides pre-accentuation, as it does with accented suffixes. The explanation of this fact is very much on par with the explanation of overriding root stress given above. The competition for word stress is again a result of top-ranked Root Faithfulness.

\footnotetext{
${ }^{26}$ The conjunction of the two Alignment constraints here is derived through the disjunctive mode defined in Hewitt \& Crowhurst 1996, according to which the complex constraint is violated if either of the conjuncts are violated (cf. Smolensky 1993 et seq. where violations of conjoined constraints involves violation in both conjuncts).
} 
(52) Blocking Effect due to Root Accentedness: / ... róot $+\mathrm{af}_{\text {pre }} / \rightarrow[\ldots$ róot-af pre $]$

\begin{tabular}{|l|c|c|}
\hline $\begin{array}{c}\mathrm{x}_{1} \\
\text { /tívi?e } \mid \mathrm{maa}_{\text {pre }}+\mathrm{le} /\end{array}$ & MAX-PROMRoot & PRE-ACCENT \\
\hline \hline a. $\quad\left(. \mathrm{x}_{2}\right)$ \\
tivié $\mid \mathrm{maa}_{\text {pre-le }}$ & $* !$ & \\
\hline b. $\rightarrow$ tívi?e $\mid$ maa $_{\text {pre-le }}$ & & $*$ \\
\hline
\end{tabular}

This result truly shows the importance of Prosodic Faithfulness in the analysis. Here, the competition is between two morphemes, both of which yield stress on the root. Therefore, it is only by considering the lexical sources of accent, and its morphological affiliation, that the correct outcome is arrived at. In particular, the inherent root stress here wins not because stress surfaces within the root; the accent contributed by the pre-accenting suffix surfaces as well. Rather, it is the affiliation with the root which leads to satisfaction of topranked MAX-PROMRoot.

Turning next to words with an accented prefix and a pre-accenting suffix, for example, /pé $+\mathrm{ma}+\mathrm{i}_{\text {prel }} \rightarrow$ pe-má-y 'his paws (objective)', the lack of evidence concerning the interaction between accented suffixes and pre-accenting suffixes makes it difficult to be sure about the constraint rankings involved here. However, the two possible scenarios can be handled straightforwardly as follows. If a pre-accenting suffix generally wins out over affixes, that is, pre-accenting suffixes cause root-final accent in words which either have an accented prefix or suffix, then this observation can be accounted for with the

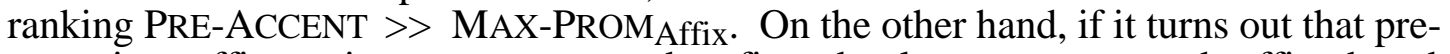
accenting suffixes win out over accented prefixes, but lose to an accented suffix, then these patterns are consistent with the general pattern of rightward edge orientation. After all, the pre-accenting suffix brings an accent closer to the right edge of the word than a prefix accent can, which fares better on ALIGN-R(PK, PrWd). Thus, while the data does not

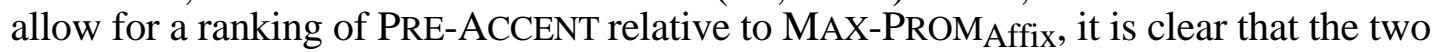
logically possible states of affairs concerning the interaction between the accented affixes and the pre-accenting suffixes can be countenanced within the set of assumptions laid out here.

As a final puzzle, let us examine the special phonology of the nominalizer $-i$, which is characterized by Hill \& Hill 1968: 236 as being intermediate between a root accent and an affix accent. The nominalizer is weaker than a root accent because it is not stressed when it combines with an accented root, as shown in (53). But it is stronger than a suffix accent, as shown by the fact that it can cause deletion of a subsequent accent (54), going against the pattern of retention of the rightmost affix accent. 
(53) Intermediate Behavior of the Nominalizer $-i$

$\begin{array}{ll}\text { wíw-i-š } & \text { /wíwe }+\mathrm{i}+\text { č e/ } \\ \text { 'acorn mush' } & \text { ROOT+NOM+ABSO } \\ \text { páč ik-i-̌̌s } & \text { /páč iike+ i + č e/ } \\ \text { 'leached acorn meal' } & \text { ROOT+NOM+ABSO }\end{array}$

(54) Intermediate Behavior of the Nominalizer $-i$
a. yex-í-qe-t
'one who is going to say'
/yax $+\mathrm{i}+\mathrm{qá}+$ te/
$\mathrm{SAY}+\mathrm{NOM}+\mathrm{PRES}+\mathrm{ABSO}$
b. $\mathrm{k}^{\mathrm{w}} \mathrm{a}$ ?-1́-qa-t
'I'm gonna eat ...'
$/ \mathrm{k}^{\mathrm{w}} \mathrm{a}+\mathrm{i}+\mathrm{qá}+$ te/
$\mathrm{k}^{\mathrm{w}} \mathrm{a}-$ ?1́-q-te-m
'we're gonna eat ...'
$\mathrm{EAT}+\mathrm{NOM}+\mathrm{PRES}+\mathrm{ABSO}$
c. max-í-qa-t
$/ \mathrm{k}^{\mathrm{w}} \mathrm{a}+\mathrm{i}+\mathrm{qa}+$ te $+\mathrm{m} /$
EAT+NOM+PRES+ABSO+PLUR
ne-max-1́-ve-nax
$/ \mathrm{max}+\hat{i}+\mathrm{qa}+\mathrm{te} /$
GIVE+NOM+PRES+ABSO
'(the way) I always do'
/né + max + í + ve + ๆax prel
$1 \mathrm{sg}+\mathrm{GIVE}+\mathrm{NOM}+\mathrm{SUBORD}+\mathrm{FROM}$

When compared to other morpho-accentual phenomena examined in subsequent chapters, the behavior of the nominalizer suggests several possible analyses. For example, as a stem-forming suffix, -i may be ascribed the special Faithfulness properties characteristic of derivational affixes to account for the retention of its accent over an affix accent (see especially Revithiadou 1997). Alternatively, the nominalizer could be approached as another well-known accentual class, namely dominant morphemes, which trigger a deletion of a neighboring accent (see chapter $\$ 5.2$ for detailed discussion), and in turn allows the suffix to realize its own inherent accent. Third, as a noun-forming affix, the intermediate status of -i may also be attributed to a Faithfulness effect due to its membership in the class of nouns, providing further support for the notion of Noun Faithfulness proposed in Smith 1996, 1997. For the matters at hand, however, the specific details of the analysis are not directly relevant. The importance of the nominalizer is that it has an intermediate status among the accentual types of morphemes in Cupeño, e.g., roots versus affixes, and thus, it must be distinguished from these types in a formal way.

Consistent with the line of analysis pursued in this thesis, morphemes of different accentual types are distinguished through constraint ranking. Together with the ranking assumptions laid out above, this approach to the nominalizer provides an additional argument for the distinction between Root and Affix Faithfulness, as I will now illustrate.

Let us assume that there is a rankable constraint, STRESS-TO-í, which specifically requires stress on the nominalizer. This constraint must be ranked above MAX-PROMAffix to account for the fact that $-i$ is stressed over an inherently accented affix, as shown below. 
(55) Special Behavior of Nominalizer -í: / ...i áf / $\rightarrow$ [.. i-af $]$

\begin{tabular}{|l|c|c|}
\hline & \multicolumn{1}{|c|}{$\mathrm{x}_{1}$} \\
\hline \hline a. $\quad \begin{array}{c}\left(\cdot \mathrm{x}_{1}\right) \\
\text { yax-i-qát }\end{array}$ & STRESS-TO-i & MAX-PROMAffix \\
\hline b. $\rightarrow$ yax-í-qat & $* !$ & \\
\hline
\end{tabular}

The nominalizer loses to a root accent, however, which shows that STRESS-TO- $i$ is dominated by MAX-PROMRoot. The result of this ranking decision is illustrated below.

(56) Root-Controlled De-Accenting in the Nominalizer: / ... róot $+i / \rightarrow$ [ ... róot- $i]$

\begin{tabular}{|c|c|c|c|}
\hline $\begin{array}{c}\mathrm{x}_{1} \\
\text { /páč ike }+\mathrm{i}+\check{c} \text { e/ }\end{array}$ & MAX-PROMRoot & STRESS-TO-í & MAX-PROMAffix \\
\hline a. $\quad \stackrel{\left(. \mathrm{x}_{2}\right)}{\text { pač } \mathrm{ike-1́-č} \mathrm{e}}$ & $* !$ & & \\
\hline $\begin{array}{ll} & \left(\mathrm{x}_{1} \cdot\right) \\
\text { b. } \rightarrow & \text { páč ike-i-č e }\end{array}$ & & $*$ & $*$ \\
\hline
\end{tabular}

The up-shot is thus that the special phonology of the nominalizer further substantiates the distinction between Root and Affix Faithfulness in the analysis. The intermediate status of the nominalizer is directly characterized by ranking STRESS-TO- $i$ between Root and Affix Faithfulness.

To summarize the results of this section, the rankings shown below build on the constraint system of $\$ 2.4 .2$ to account for pre-accentuation and the behavior of the nominalizer $-i$.

(57) Summary Ranking

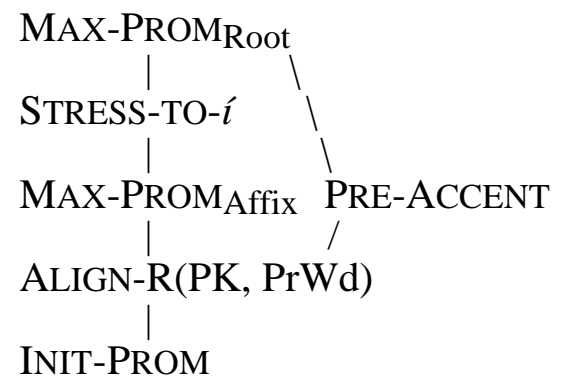

Distinguished lexically from the accented suffixes, the pre-accenting suffixes attach to a root which ends in a stressed syllable. This subcategorization requirement, embodied in PRE-ACCENT, is ranked above the Alignment constraints because it may bring non-final and non-initial stress. However, PRE-ACCENT is ranked below MAX-PROM Root because the presence of a root accent precludes pre-accentuation. Finally, the intrinsic ordering

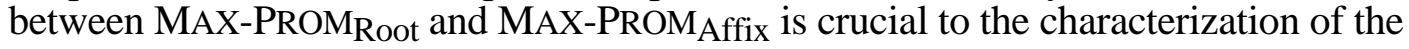


intermediate strength of the nominalizer, providing the right slot for the ranking of STRESSTO- $i$ in the system.

To complete the analysis, the constraint rankings from $\S 2.3$ must be incorporated into the system in (57) above. Specifically, the WSP is ranked above the Prosodic Faithfulness constraints MAX-PROMRoot and NOFLOP-PROM, which in turn both dominate FTBIN. The former ranking accounts for predictable long vowel stress, and the latter accounts for the lexical contrast between initial and peninitial stress.

In conclusion, the distinction between Root and Affix Faithfulness has two important functions in the analysis. First, it explains overriding root stress, even in subtle cases involving pre-accentuation. Second, it is instrumental in the analysis of the nominalizer. Thus, these two independently established ranking arguments converge on the same result, providing strong evidence for the distinction between Root and Affix Faithfulness.

\subsection{Discussion of Alternatives}

In this section, two alternatives to the Faithfulness-based analysis of overriding root stress are considered: the level-ordering analysis proposed in Crowhurst 1994 (\$2.5.1), and a cyclic account along the lines of Halle \& Vergnaud 1987a (\$2.5.2).

\subsubsection{A Level-Ordering Account}

Crowhurst 1994 gives a level-ordering account of overriding root stress. The crux of the analysis centers on a lexical distinction between accented and unaccented roots: accented roots have a lexical foot, and unaccented roots do not. Furthermore, on the root cycle prior to affixation (the Level 1 phonology), a word tree is built only over accented roots because of an additional assumption that feet may not be inserted at this stage. Accented roots hence leave the Level 1 phonology with word-level prosodic analysis, while unaccented roots exit with no prosodic structure above the syllable. At the next level, a different stress rule is proposed for affix stress, accounting for the difference between accented and unaccented roots with a two level grammar.

This analysis is depicted graphically in (58) below. The inputs to the Level 1 phonology differ in the presence of a lexical foot, and they are likewise distinguished in the output by the presence of a word-level category. The root syllable in (58b) cannot by parsed directly by the prosodic word because this option violates the principle of Strict Layering (Selkirk 1984), and furthermore, this form cannot be supplied with an epenthetic foot because this strategy is not available. 
(58) Level 1 Phonology

INPUT

a.

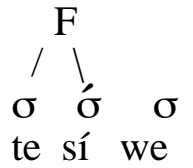

OUTPUT

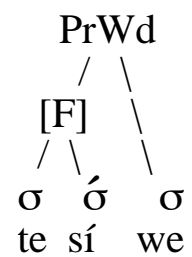

b.

$\begin{array}{rr}\sigma & \sigma \\ \operatorname{yax} & \text { yax }\end{array}$

When these outputs are then subjected to the Level 2 phonology, the difference between accented and unaccented roots is exploited in the following way. Words with accented roots already have word-level structure, which in turn determines the position of the main stress foot (59a). On the other hand, words with unaccented roots will be devoid of such structure, and can therefore be assigned rightmost affix stress with a different set of stress principles (59b).

(59) Level 2 Phonology

INPUT

a.

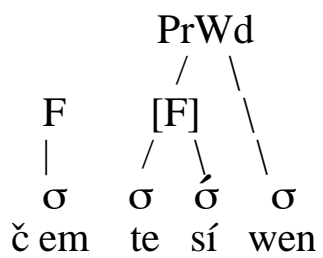

OUTPUT

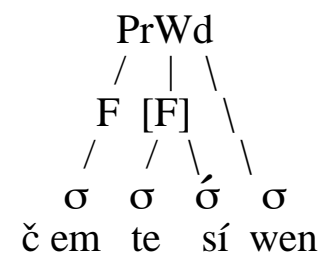

b.
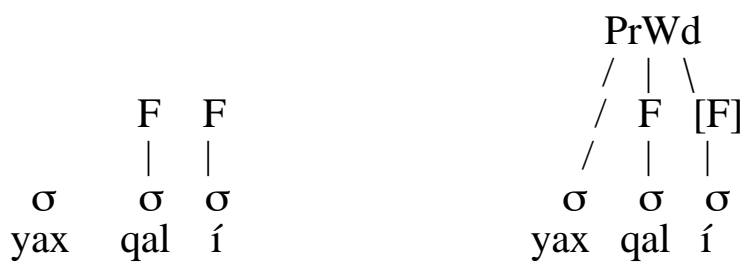

In summary, the level-ordering analysis accounts for overriding root stress by defining a root cycle prior to affixation in which certain principles of prosodic organization apply, effectively distinguishing accented and unaccented roots in the relevant way. Crucial to this analysis, therefore, is the assumption that the grammar cycles on bound roots. This claim, however, has been argued against extensively in the literature (see e.g. Kiparsky 1982b and Inkelas 1989 and references therein). The empirical finding in these works is that bare bound roots do not form domains for cyclic rules. It would seem, therefore, that the level-ordering account bases its analysis on an assumption for which there is little cross-linguistic support.

There is an additional empirical problem with this analysis, stemming from the distinction made between accented and unaccented roots. The Level 1 phonology 
distinguishes between accented roots and unaccented roots by the presence of word-level prosodic structure. In effect, unaccented roots are clitics when they leave the Level 1 phonology. As it happens, Cupeño's Level 2 phonology supplies a word tree, so unaccented roots do not retain their clitic-like status. However, there is nothing inherent to the level-ordering analysis that ensures that this necessary step would take place. So the typological prediction is made that there should be some language where unaccented roots behave like clitics post-lexically. To my knowledge, however, no such language exists. For example, in Tokyo Japanese, unaccented roots have no specific prosodic properties other than their lack of tone structure. It seems, therefore, that the core idea of the levelordering analysis has little empirical support outside of Cupeño.

\subsubsection{A Cyclic Analysis}

A different approach to overriding root stress can be modelled in the multi-stratal framework given in Halle \& Vergnaud 1987a (HV). In this work, dominant morphemes are distinguished from recessive ones through cyclicity. In particular, dominant affixes are cyclic morphemes which are represented on a metrical plane that is distinct from that of other morphemes. Thus, in the examples from Vedic Sanskrit below, the accent of the dominant suffix -in is represented on a different autosegmental plane than the one for the roots and noncyclic suffixes.

$$
\begin{array}{lll}
\text { /ráth+ịn +e/ } & \text { rath-ín -e } & \text { 'charioteer' (dative singular) } \\
\text { /mitr+ịn +e/ } & \text { mitr-ịn -e } & \text { 'befriended' (dative singular) }
\end{array}
$$

Furthermore, cyclic affixation triggers a copying process from one metrical plane to the plane of the cyclic affix. This copying is governed by the Stress Erasure Convention (SEC), which essentially states that information about stress generated on previous cycles is carried over only if the affixed constituent is not a domain for the cyclic stress rules. Thus, as depicted below, the accented/unaccented contrast in roots is lost when they combine with dominant (cyclic) suffixes.

(61) Dominant Affixes in Vedic Sanskrit (HV)

\begin{tabular}{|l|l|l|l|}
\hline Cyclic Stratum & (Accented) & (Unaccented) & \\
\hline Cycle 1 & $\begin{array}{l}* \\
\text { ráth }\end{array}$ & mitr & \\
\hline Cycle 2 & $\begin{array}{c}* \\
\text { ráth-ín } \\
*\end{array}$ & $\begin{array}{c}\text { mitr-ịn } \\
*\end{array}$ & \\
\hline OUTPUT & $\begin{array}{c}* \text { rath-ín } \\
*\end{array}$ & $\begin{array}{c}\text { mitr-ịn } \\
*\end{array}$ & Root accent deleted by the SEC. \\
\hline
\end{tabular}

In this illustration, accented and unaccented roots are distinguished by the presence of stress above the root. This information is represented on a metrical plane apart from the one marking stress on cyclic affixes, which is placed directly below the form. Hence, when root stress is copied at Cycle 2, this information is lost because the larger constituent 
forms a domain for the cyclic stress rules, in effect neutralizing the accentual contrast in roots.

Consider next the application of the basic proposal to overriding root stress in Cupeño. Suppose that the direction of copying can be parameterized on a languageparticular basis. That is, suppose that instead of copying from the root stress plane to the cyclic plane, as in Vedic, stress information for affixes is copied to the root stress plane. Assuming that the affixed constituents form cyclic domains effectively accounts for overriding root stress with the SEC. This result is illustrated in the chart below.

(62) Overriding Root Stress in Cupeño

\begin{tabular}{|l|l|l|l|}
\hline Cyclic Stratum & (Accented) & (Unaccented) & \\
\hline Cycle 1 & $\begin{array}{c}* \\
\text { tesíwe }\end{array}$ & yax & \\
\hline Cycle 2 & $\begin{array}{c}* \\
\text { č em-tesíwe } \\
*\end{array}$ & $\begin{array}{c}\text { yax-qál-í } \\
* *\end{array}$ & \\
\hline OUTPUT & č em-tesíwe & $\begin{array}{c}* \\
\text { yáx-qal-i }\end{array}$ & Affix stress deleted by SEC. \\
\hline
\end{tabular}

With accented roots, it is clear how the SEC applies to give the correct result: when copying from the affix plane to the root plane, information specified for affixes is lost because the larger constituent forms a cyclic domain. This same principle, however, gives an incorrect result for words with unaccented roots. On a par with the accented roots, affix stress is lost with cyclic affixation, yielding a metrical plane with no stress information whatsoever, and which therefore receives a default initial stress. In sum, just as dominant affixes neutralize the accentual contrast in the roots they attach to, roots in Cupeño would neutralize the accentual contrast in affixes, leading to the incorrect outcome above with *yáx-qal-i.

The only way around this descriptive problem is to posit a feature [+/- cyclic], which governs the possibility of copying from the affix plane when applied to roots. That is, accented roots must be marked [+cyclic] in order to require copying, which results in deletion of affix stress, while unaccented roots must be marked [-cyclic] to preclude this copying. Going beyond the lack of explanatory insight, this approach leads to a more serious empirical problem. By introducing cyclicity as a marker of dominance which is independent of the accentedness contrast, the cyclic approach essentially claims that these two features will cross-classify roots in some language. However, a recent paper (Inkelas 1996) which surveyed effects such as these in a variety of languages, found that the dominant/recessive distinction is not used in any language to classify roots (see $\$ 5.2 .4$ for further discussion). The application of such a feature to account for overriding root stress in Cupeño, therefore, seems to make an empirical prediction for which there is no crosslinguistic support. 


\subsection{Summary and Implications}

In this chapter, I have developed a comprehensive analysis of accent in Cupeño. At every stage in the analysis, the notion of Prosodic Faithfulness has played an important role in describing the diverse aspects of the system. First, Prosodic Faithfulness provided the formal means of characterizing phonemic stress. This set of constraints was segregated into Root and Affix Faithfulness constraints to account for the cross-linguistic observation that roots license a wider range of accentual contrasts than affixes. This division was in turn employed in the explanation of dominant root stress, a pattern which pervades the accent system of Cupeño. Finally, distinct Root and Affix Faithfulness proved essential in extending the analysis to the far corners of the system, including the analysis of preaccentuation and the intermediate strength of the nominalizer.

In developing the Faithfulness-based analysis, I have made connections between these observations in an accent system and diverse phonological phenomena. By characterizing Cupeño accent as root-controlled, one can see parallels to well-known vowel harmony systems (McCarthy \& Prince 1995, Selkirk 1995a, Beckman 1997 [1998]) and other segmental processes like dissimilation (Holten 1995, Selkirk 1995b, Alderete 1997b, Blake 1998). The domain-sensitive constraints employed in the analysis of Cupeño are in no way specific to this language and have been applied to a wide range of phenomena. The analysis proposed here therefore accomplishes one of the central goals in linguistic theory, namely the description of language particular patterns with universal principles.

In addition, I have argued for this analysis by contrasting it with the plausible alternatives. It was shown that, in contrast to the Faithfulness-based analysis, the alternatives employing phonological levels lead to descriptive problems and loss of generalization. First, the level-ordering account was shown to rely on the assumption that bare bound roots form cyclic domains, and this assumption was challenged on empirical grounds. Second, the cyclic alternative was shown to have a descriptive problem with dominant root stress, and the fix-up to this problem led to an ad hoc feature system which was also challenged. In summary, the available alternatives to the Faithfulness-based analysis are inferior on empirical and theoretical grounds.

I would like to conclude with a brief discussion of some further issues which are raised by the main ideas developed here. The first issue involves the examination of a set of languages which also require root stress, but where this requirement is apparently not a function of phonemic stress. For example, roots are always stressed in the Nicobarese language Nancowry, but the distribution of stress is predictable, falling on the last vowel of the root (Radhakrishnan 1981). Likewise, Chukchee has predictable root stress, typically falling on the rightmost vowel of the root (Krause 1979). A third case is Nisgha, where root-based stress exhibits an interesting interaction between edge alignment and quantity sensitivity (see Shaw 1992 and references therein). In these languages, there is a constraint requiring root stress, but this constraint cannot be an input-output Faithfulness constraint because it applies to all the roots in the language. This observation, therefore, raises the question of whether there are two sets of constraints in Universal Grammar, both of which encourage root stress. That is, the analysis of Cupeño proposed here involves a Faithfulness constraint which encourages realization of inherent root stress over affix stress, but a cross-linguistic perspective reveals a need for a constraint which also encourages root stress, but only evaluates outputs.

Evidence from the Athapaskan language Tahltan appears to resolve this issue because in this language both constraints function independently in the same system. A fundamental component of the Tahltan stress system is that every root must have a stress (Cook 1972), which classifies this language with Nancowry and Chukchee. Furthermore, 
stress is also assigned to every odd syllable counting from the root stress, resulting in fixed

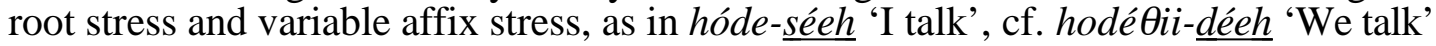
(roots are underlined). Stress in polysyllabic forms is not fully predictable, however, as Nater 1989 shows that stress introduces phonemic contrast in longer words. Thus, stress is contrastive in roots, leading to variation in stress in longer words; furthermore, every root must have a stress. In this system, therefore, both a Faithfulness constraint for root stress and an over-arching constraint requiring every root to bear stress are needed (see Hewitt 1992, Kennedy 1994, and Fitzgerald 1997 for some leading ideas).

A second issue concerns the retention of accent in words with more than one inherently accented morpheme. According to the Root-Controlled Accent (RCA) hypothesis, accent in the root is retained over accent elsewhere in the word. An interesting feature of this hypothesis is that it assigns a role to the morphology in the description of a pattern that has formerly been treated as a directionality effect. Thus, in a very influential paper, Kiparsky \& Halle 1977 describe accent retention in a variety of Indo-European languages in terms of a principle of edge orientation, according to which accent is lost in all but the first inherently accented morpheme in a word. Poser 1984 assumes a similar principle in the analysis of non-stress accent in Japanese. These precedents raise the issue of whether both the assumptions embodied in the RCA hypothesis and this principle of edge orientation are indeed necessary in the analysis of the retention of accent crosslinguistically.

It is helpful to return to the analogy of vowel harmony systems developed in $\$ 2.2$ as a way of addressing this theoretical and empirical issue. The well-known cases of back (and round) harmony in Finnish and Turkish show alternations in suffixes which are amenable to a left-to-right spreading rule, an analysis which also involves a principle of directionality (see Bach 1968 and Lightner 1972). Considered alongside root-controlled [ \pm ATR] harmony in Akan (Clements 1981), however, an equally coherent analysis of Finnish and Turkish is that the specification for vowel features in the suffix is rootcontrolled. In this analysis, the suffixing preference in these languages only gives the appearance of a directionality effect, but it is the vowel specifications in the root that have the formal role in the analysis.

In a similar way, the analysis of root-controlled accent in Cupeño presented here sheds new light on the two accent systems mentioned above. ${ }^{27}$ In particular, Japanese, as well as many Indo-European languages, shows a strong preference for suffixing morphology. Thus, in stem + suffix sequences where both morphemes have a lexical accent, the deletion of accent in the suffix may be analyzed in terms of the same principles of root-control employed in Cupeño. In the absence of prefixed structures which can decide between the two approaches, the RCA analysis is thus quite attractive because it explains retention of root accent in terms of a universal ordering between Root and Affix Faithfulness. Furthermore, root accentedness has the same effect of blocking morphoaccentual processes like pre- and post-accentuation in these languages, which is also explained in terms of Root Faithfulness. I therefore propose to investigate the interaction between root and affix accent in Russian and Japanese, and much of the next chapter is dedicated to developing this argument in more detail.

\footnotetext{
${ }^{27} \mathrm{An}$ important difference between vowel harmony systems and accent systems is that only the former exhibits blocking effects from opaque vowels, for example low vowels in Akan. The analysis of this difference stems from the theory of autosegmental spreading: because root-control in vowel harmony systems is achieved through linking of a feature substantive constraints on these linkages can prohibit spreading across or through an opaque vowel; since grid marks cannot spread, such blocking effects are not possible in accentual systems described in terms of prominence on the grid.
} 


\section{Chapter 3.}

\section{Restricted Edge Effects in Root-Controlled Accent Systems}

\subsection{Restricted Edge Orientation}

\subsubsection{Factorial Typology}

Much of the discussion of the previous chapter focuses on the consequences of culminative accent in Cupeño. The over-arching requirement that words have a single stress-accent in this language creates a competition in words with more than one inherent accent. Furthermore, the resolution of accent in multiply accented words, which I will refer to as Accent Resolution (AR), leads to the identification of a set of factors which determine which lexical accent is retained. The novel aspect of the analysis of Cupeño is that a role for word structure is recognized in AR. Thus, the finding that root accent overrides affix accent is argued to derive from a principle of root-control, according to which retention of a root accent is favored over retention of accent in an affix. Rootcontrolled AR is apparently not typical, however, as many have argued for a phonological principle at work in AR. For example, in a very influential paper, Kiparsky \& Halle 1977 argue that AR in many Indo-European languages is governed by directionality: a word level prominence is assigned to the lexical accent that is closest to the beginning of the word (1a). Likewise, Poser's 1984 rule of Accent Resolution in Tokyo Japanese is characterized in terms of directionality, favoring retention of a leftmost lexical accent.

\section{(1) Accent Resolution with Directionality}

a. Basic Accentuation Principle (Kiparsky \& Halle 1977): If a word has more than one accented vowel, the first of these gets the word accent. [If a word has no accented vowel, the first vowel gets the word accent.]

b. Accent Resolution (Poser 1984): Delete all but the leftmost accent within a minor phrase.

The existence of root-controlled AR in Cupeño, alongside directional AR, raises the question of whether Universal Grammar countenances both morphological and phonological principles in the characterization of this accentual phenomenon. Furthermore, if this question is answered affirmatively, are there principles which can predict when the morphological principle of root-control applies, as opposed to the phonological principle of directionality? ${ }^{1}$

The way to approach these typological questions in Optimality Theory is to consider the implications of the only mechanism for language variation in OT, constraint permutation, for the constraints at work in each type of AR. Starting first with directional AR, this type of pattern implies a constraint, EDGEMOST, which sets a premium for accent that appears at a designated edge (after Prince \& Smolensky 1993; see also McCarthy \&

\footnotetext{
${ }^{1}$ See Beckman 1995, 1997 [1998] for discussion of a related issue concerning various factors at work in vowel harmony.
} 
Prince 1993a). In §1.2.1.1, two distinct types of edge effects are identified: categorical edge tropism and gradient edge orientation. The type of edge effect predicted by the EDGEMOST constraint is in large part due to the rank of EDGEMOST in the system (though the way its violations are interpreted is also important — see Zoll 1996a and Gafos 1996b). Thus, if EDGEMOST is undominated, the result is edge-tropic accent that is co-extensive with the favored edge. The rules in (1), by contrast, are characterized by the second type; they favor accent which is as close as possible to the left edge, yet allow for non-initial accent. EDGEMOST has a role in yielding this type of edge effect too, but in these contexts, it is dominated by other constraints in the system.

The EDGEMOST constraint is also ranked on a language particular basis in relation to the constraints at work in root-controlled AR, i.e., the MAX-PROM constraints from $\S 1.2 .2 .1$. Furthermore, the fact that root accent takes precedence over affix accent entails that there is a natural ordering between Root and Affix Faithfulness. The explanation of overriding root accent given in $\$ 2.4$ rests on this universal ordering because it provides a basis for understanding root-controlled AR in Cupeño as a special case of a more general pattern of root privilege. Therefore, three constraints are at work in the analysis of directional and root-controlled AR: EDGEMOST, MAX-PROMRoot, and MAX-PROMAffix; also, MAX-PROM Root, by hypothesis, always ranks above MAX-PROM Affix $_{\text {because of the }}$ universal ordering Root Faith $\gg$ Affix Faith.

These background assumptions, together with the assumption that the EDGEMOST constraint may be freely re-ranked relative to the two MAX-PROM constraints, yields the following factorial typology. The resulting typology is characterized in (3) with a brief description for each grammar.

(2) Factorial Typology with Decisive EDGEMOST Constraint

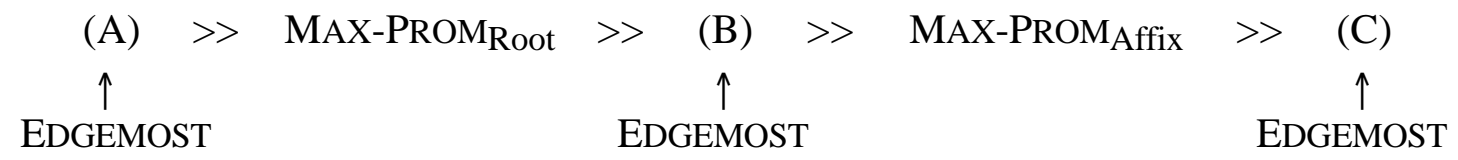

(3) Grammars Resulting from Factorial Typology

Grammar A: accent is delimitative (always coincides with a designated edge) in all contexts; accent is not contrastive.

Grammar B: accent is contrastive in roots, but delimitative in words with unaccented roots. In words with more than one accented root, the edgemost (i.e., closest to a designated edge) accented root wins.

Grammar C: accent is contrastive in roots and affixes, but a root accent takes precedence over an affix accent. In words with more than one accented morpheme of the same morphological class, the edgemost accent wins. In words with no accented morphemes, accent is delimitative.

If accent is not contrastive in a languages described by these grammars, it is edge-tropic, or locked into an edgemost position, because of the unmitigated force of EDGEMOST, as in grammar A. The gradual demotion of EDGEMOST, however, brings about a loosening of the edge requirements on accent, and consequently, the emergence of an accentual contrast in roots and affixes (B and C). In the grammars with contrastive accent, a pattern of edge orientation is found. If accent is sponsored by a morpheme of the same morphological class (that is, all are roots or affixes), then the accent which is closest to the favored edge is 
retained. Interestingly, this pattern of edge orientation is only permitted under very special circumstances in this factorial typology, which stems from the intrinsic ordering of MAX-

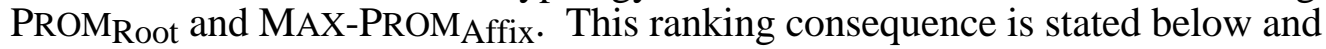
subsequently tested against the three grammars shown above.

\section{(4) Restricted Edge Orientation (REO)}

Edge orientation for accent is only observed in contexts where PROS-FAITH is indecisive.

PROS-FAITH is decisive if it predicts which, among possibly many, inherently accented morpheme realizes its lexical accent. In words with accented morphemes of the same morphological class, however, the ordering of MAX-PROMRoot and MAX-PROMAffix is indecisive, and so other factors, like phonological directionality, can take effect. REO therefore predicts that root-control is primary because all grammars in which PROS-FAITH is not crucially dominated favor realizing a root accent over an affix accent; directional AR only comes in word types that cannot be resolved on the basis of morphological factors. To show the primacy of root-controlled AR, however, it is necessary to study the characteristics of each grammar more carefully and to see that they are all in fact consistent with REO.

In the illustrations which follow, EDGEMOST is set for the left edge of the word, hence LEFTMOST, to compare these results with a common type of system. This decision is entirely arbitrary, however, and so it is clear that the same points hold of other systems which exhibit different types of edge effects. Starting first with a familiar system, grammar $\mathrm{C}$ in many ways resembles accent in Cupeño. Words with an accent on the root in the input realize this lexical accent at the surface, even if the root accent is not leftmost in the word (5a), or if retention of the root accent entails the loss of an affix accent (5b). Importantly, the input-output mapping in (5b) is consistent with REO because the Prosodic Faithfulness constraints are crucial for predicting the outcome, as shown by the conflicting constraint violations for the MAX-PROM constraints. The result depicted in $(5 \mathrm{c})$, by contrast, is pure directional AR: the leftmost affix wins. It turns out that this input-output pair is also compatible with REO because in a sequence of accented affixes, Prosodic Faithfulness is not decisive, and so the lower-ranking constraint LEFTMOST takes effect and gives the observed directionality effect.

(5) Grammar C: Root-Controlled Accent with Restricted Edge Orientation

\begin{tabular}{|c|c|c|c|c|c|}
\hline Input & & Output & MAX-PROMRoot & MAX-PROMAffix & LEFTMOST \\
\hline \multirow[t]{2}{*}{ a. /af + róot/ } & \multirow[t]{2}{*}{$\rightarrow$} & af-róot & & & $*$ \\
\hline & & *áf-root & $* !$ & & \\
\hline \multirow[t]{2}{*}{ b. /áf + róot/ } & \multirow[t]{2}{*}{$\rightarrow$} & af-róot & & $*$ & $*$ \\
\hline & & *áf-root & $* !$ & & \\
\hline \multirow[t]{2}{*}{ c. /áf + áf + root/ } & \multirow[t]{2}{*}{$\rightarrow$} & áf-af-root & & $*$ & $* !$ \\
\hline & & *af-áf-root & & $*$ & \\
\hline \multirow[t]{2}{*}{ d. /af + áf + root/ } & \multirow[t]{2}{*}{$\rightarrow$} & af-áf-root & & & * \\
\hline & & *áf-af-root & & $* !$ & \\
\hline
\end{tabular}

This hypothetical example therefore illustrates the primacy of morphological factors in AR: edge orientation is only found in contexts where the ordering of Root and Affix Faithfulness is not enough to predict the winner. 
REO is also consistent with a grammar in which LEFTMOST is promoted in the constraint hierarchy to a position above MAX-PROMAffix. Like grammar C, such a constraint system also favors retention of a root accent over leftmost accent, as illustrated in (6a) and (6b). The principle difference between this system and that described by grammar $\mathrm{C}$ is that only the latter has an accentual contrast in affixes. In grammar $\mathrm{B}$, affix accent is predictable, and so accent on a non-initial affix is lost (6d), which contrasts with the outcome above in $(5 \mathrm{~d})$. What these two grammars have in common, however, is that edge orientation only occurs when Prosodic Faithfulness is not relevant in deciding the winner. Thus, the role of the MAX-PROM constraints in negotiating between two accented morphemes is only apparent in $(6 \mathrm{~b})$ where the favored pattern of edge orientation is not observed; in (6c), by contrast, neither MAX-PROM constraint is active, and so the pattern of leftward edge orientation emerges.

(6) Grammar B: Contrastive Accent in Roots and Predictable Accent in Affixes

\begin{tabular}{|c|c|c|c|c|c|}
\hline Input & & Output & MAX-PROMRoot & LEFTMOST & MAX-PROMAffix \\
\hline \multirow[t]{2}{*}{ a. /af + róot/ } & \multirow[t]{2}{*}{$\rightarrow$} & af-róot & & $*$ & \\
\hline & & *áf-root & $* !$ & & \\
\hline \multirow[t]{2}{*}{ b. /áf + róot/ } & \multirow[t]{2}{*}{$\rightarrow$} & af-róot & & $*$ & $*$ \\
\hline & & *áf-root & $* !$ & & \\
\hline \multirow[t]{2}{*}{ c. /áf + áf + root/ } & \multirow[t]{2}{*}{$\rightarrow$} & áf-af-root & & & $*$ \\
\hline & & *af-áf-root & & $* !$ & $*$ \\
\hline \multirow[t]{2}{*}{ d. /af + áf + root/ } & \multirow[t]{2}{*}{$\rightarrow$} & áf-af-root & & & $*$ \\
\hline & & af-áf-róot & & $* !$ & \\
\hline
\end{tabular}

The last case, grammar A, also accords with REO because the MAX-PROM constraints are crucially dominated by LEFTMOST. In this system, accent is delimitative, and so regardless of the lexical properties of roots and affixes, accent always marks the left edge of the word.

(7) Grammar A: Delimitative Leftmost Accent

\begin{tabular}{|c|c|c|c|c|c|}
\hline Input & & Output & LEFTMOST & MAX-PROMRoot & MAX-PROM $_{\text {Affix }}$ \\
\hline \multirow[t]{2}{*}{ a. /af + róot/ } & $\rightarrow$ & áf-root & & * & \\
\hline & & *af-róot & $* !$ & & \\
\hline \multirow[t]{2}{*}{ b. /áf + róot/ } & $\rightarrow$ & áf-root & & * & \\
\hline & & *af-róot & $* !$ & & $*$ \\
\hline \multirow[t]{2}{*}{ c. /áf + áf + root/ } & $\rightarrow$ & áf-af-root & & & $*$ \\
\hline & & *af-áf-root & $* !$ & & $*$ \\
\hline \multirow[t]{2}{*}{ d. /af + áf + root/ } & $\rightarrow$ & áf-af-root & & & * \\
\hline & & af-áf-root & $* !$ & & \\
\hline
\end{tabular}

In this scenario too, then, the patterns of edge orientation observed in cases like (7b-c) arise when Prosodic Faithfulness is not a predictor of which lexical accent is retained in the surface form. 
The finding here is thus that no ranking of MAX-PROMRoot, MAX-PROMAffix, and LEFTMOST gives a purely directional pattern of AR. That is, the ranking of LEFTMOST relative to the intrinsically ordered Prosodic Faithfulness constraints never produces a system with contrastive accent and where edge orientation completely ignores the internal structure of words. If this theory is correct, then in systems with contrastive accent, inherent accent in roots should always take precedence over inherent accent in an affix; edge orientation is thus only found among morphemes of equal status with respect to the morphologically dispersed Faithfulness constraints. The rest of this chapter studies the implications of this restriction on the scope of edge orientation, both in the context of close formal analyses of accent in two languages, Russian and Japanese, and from a broader perspective on Accent Resolution in a wider range of languages developed directly below.

\subsubsection{Empirical Issues}

The predictions of Restricted Edge Orientation appear, at first glance, to be a rather strong, as there are several examples which have been described precisely in terms of directional AR. As mentioned above in (1), directionality is integral to the analyses given in Kiparsky \& Halle $1977(\mathrm{~K} \& \mathrm{H})$ of AR in the Indo-European languages Russian, Lithuanian, and Sanskrit. The principle argued to be at work in these systems, the Basic Accentuation Principle (BAP), is a straightforward pattern of leftward edge orientation. Furthermore, BAP has been a very influential tool in describing AR in non-Indo-European languages. A very similar idea is employed in Poser 1984 for Japanese minor phrases, in Idsardi 1992 for stress placement in Shuswap, Spokane, and Moses-Columbia Salish, cf. Czaykowska-Higgins 1993, in Hualde \& Bilbao 1993 for Getxo Basque, and in Payne 1990 for the Jivaroan language Aguaruna (p. 181). The loss of all but the first high tone in many Bantu languages, rather like Poser's Accent Resolution, is also often described in terms of leftward edge orientation. Finally, while not described in precisely these terms, the tonomechanics of Northern Tepehuan (Uto-Aztecan) given in Bascom 1959 show a pattern of AR similar to that found in Japanese and Bantu.

(8) Accent Resolution with Straight Leftward Edge Orientation
a. Russian
Halle 1973, 1996, K\&H, HV, Melvold 1990, Idsardi 1992
b. Lithuanian
K\&H, HV, Blevins 1993, Halle 1996
c. Sanskrit
K\&H, Kiparsky 1982c, 1984b, HV, Halle 1996
d. Japanese
Poser 1984
e. Interior Salish
Carlson 1976, 1989, Czaykowska-Higgins 1993, Black 1996
f. Getxo Basque
Hualde \& Bilbao 1992, 1993, Hualde 1991
g. Aguaruna
Payne 1978, 1990, Larson 1956
h. Northern Tepehuan
Bascom 1959, 1965, Woo 1970, Kim 1996
i. Misc. Bantu
See Myers 1997 for a survey

If these systems present valid cases of purely directional AR, which is oblivious to word structure, then the theory of root privilege developed here will have to be modified to accommodate them. However, upon closer inspection, there are reasons to doubt the analysis of these systems in terms of directionality, and so these cases, in fact, may not refute the restricted theory of edge effects embodied in REO. In the interest of pursuing this more restrictive theory, therefore, it is worthwhile to probe further into the characterization of edge effects in these systems. 
The first problem is that most accounts of these languages do not give the crucial evidence required to show a pattern of directional AR. As mentioned above, most of the languages are said to follow a BAP-type principle with leftward edge orientation.

Considering a sample of the sequences of accented morphemes consistent with this pattern, only one runs counter to the patterns predicted by Restricted Edge Orientation.

(9) Some Affixed Structures in Directional Accent Resolution
a. /róot + áf/
$\rightarrow \quad$ róot-af
b. /áf + róot/
áf-root
$\neq$ REO
c. /root + áf + áf/
root-áf-af

If the root accent is retained over a suffix accent, as in (9a), this pattern is of course compatible with both directional and root-controlled AR, and so it is inconclusive. Moreover, the retention of accent in the first of a sequence of two accented suffixes (9c), as found for example in Getxo Basque (Hualde \& Bilbao 1993) and Russian (Melvold 1990), is also inconclusive. This pattern of AR is consistent with REO because in these affixed structures, the morphemes are of equal status, and so Prosodic Faithfulness is indecisive. However, the pattern shown in the prefixed structure in (9b), if found across the board, is diagnostic of directional AR because PROS-FAITH is relevant in such a context and prefix retention is contrary to the expected pattern. How then do these prefixed structures behave in the languages in (8) above?

Many of these systems only have a few prefixes, or lack prefixation altogether, which precludes using these affixed structures as a reliable test for directional AR (though this is clearly not the case for Bantu languages). For example, Basque (King 1994) and Aguaruna are exclusively suffixing ${ }^{2}$ and so they are consistent with REO. In other languages, like Russian and Japanese, prefixes are productive or mildly productive in certain word classes, but fully unproductive in others, providing little help in determining the scope of edge orientation. In these systems, a common strategy is to mark the prefixes as outside the domain of accentual rules. Thus, Melvold 1990 assumes that prefixes in Russian verbs are non-cyclic, and hence do not trigger a second pass of stress assignment, or do so only under special circumstances. Also, Carlson 1989: 204 notes that prefixes are never stressed in Spokane, which requires a similar set of assumptions to those needed for Russian. Finally, while Northern Tepehuan appears to have directional AR, as noted in Woo 1970: 19, prefixes play no role in tone assignment, so they too are outside the scope of tonal resolution in this system. To summarize, it appears that the evidence from prefix + root sequences in these languages does not give strong support for an analysis in terms of directional AR, and hence, they do not directly contradict REO.

There is another fact about these systems which is curious in a purely phonological analysis of AR. Most, if not all, of the accent systems listed in (8) exhibit a specific pattern of directionality, namely leftward edge orientation. It is the leftmost inherently accented morpheme which surfaces with stress in Russian and a pitch fall in Japanese, and this pattern is duplicated in each of the accent systems listed above. ${ }^{3}$ Unlike other types of

\footnotetext{
${ }^{2}$ Though David Payne (personal communication) notes that Aguaruna has a semi-productive prefix which forms causatives. As a derivational affix, however, it may not be helpful in diagnosing directionality in this language.

${ }^{3}$ Some Salishan languages show a pattern of rightward orientation for stress in a sequence of so-called 'strong' suffixes, which adds an additional complication in these cases. However, in Moses Columbia Salish, this pattern is in conflict with the leftward pattern for stress found elsewhere in the language, which
} 
directionality in phonology, as in the assignment of prosodic structure, directional AR in these cases seems to always be set for the left edge. If AR is a matter of directionality, then the expectation is that there should also be patterns of AR showing rightward edge orientation. ${ }^{4}$

A brief look at a set of cases previously treated in terms of directional AR therefore turns up two interesting findings. First, the analyses of these systems often lack the evidence from prefix + root sequences which is needed to show that they must be governed by directionality. Second, a directional asymmetry is found in these cases, showing a strong preference for leftward edge orientation. The conclusion that I draw from these findings is that these systems need to be examined in more detail before they can be taken as counterexamples to REO, and the next two sections give special attention to affixed structures in Russian and Japanese with this issue in mind. A second, perhaps more speculative, inference that can be drawn is that these cases are amenable to an analysis very much in line with the one given for Cupeño in chapter 2. Indeed, the lack of a class of prefixes which override accent in a following root is exactly the predicted pattern if these systems have root-controlled AR. Furthermore, the morphological analysis of AR can also make sense of the observed directional asymmetry. Languages often show a preference for suffixes over prefixes (see Greenberg 1966, Hawkins \& Gilligan 1988), a trend sometimes expressed in terms of implicational statements like, 'if a language has prefixes, it also has suffixes'. The absence of a robust set of prefixes in a root-controlled system may thus give the appearance of a directionality effect in AR because the root is always word-initial. If the cases mentioned above are truly root-controlled, then the preference for suffixing morphology found in these systems (except Bantu) would give the illusion of leftward edge orientation. Of course, this pattern is just an illusion because if the Root-Controlled Accent hypothesis applies to these cases as well, then they are fully symmetric: they all favor retention of a root accent over an affix accent.

From these considerations, the notion of root-control may indeed have some currency beyond the accent system of Cupeño. First, the absence of a class of prefixes whose inherent accent takes precedence over the root accent is expected. Second, the apparent directional asymmetry may be accounted for in terms of a general preference for suffixing morphology. Moreover, the analysis of AR in these systems explains the pattern of root retention in terms of a general pattern of root privilege, accounting for AR with the same basic assumptions at work in root-controlled vowel harmony and dissimilatory phenomena. I therefore propose to examine two of the accent systems listed above in more detail and consider the hypothesis that these systems too have root-controlled accent.

\subsection{Extended Case Study: Modern Russian}

The section has two goals. The first goal is to present an analysis of the stress patterns in Modern Russian which is consistent with one of the restrictions on edge effects derived in $\$ 3.1$, namely that edge orientation effects are only observed in contexts where Faithfulness is not decisive. As discussed above, Russian has formerly been approached as a case of unrestricted edge orientation, i.e., in terms of an analysis in which 'the leftmost inherently accented morpheme wins'. I argue below, however, that an alternative to this analysis is also viable, which is consistent with the restrictive theory of edge effects; thus, this alternative is superior to the previous approach on theoretical grounds. The second

shows that the behavior of these special suffixes requires a different treatment (see Czaykowska-Higgins 1993 and Idsardi 1992, and §4.1).

${ }^{4}$ Myers 1997b suggests that this directional asymmetry is due to a perceptual difficulty with non-initial high tones, though this approach is not extended to stress languages. 
objective here is to construct an analysis of the basic stress system which can be called upon in $\$ 5.2 .3$ in the analysis of de-accenting suffixes. The theory of accent-deleting affixes proposed in chapter 5 makes the prediction that the accentual pattern resulting from de-accentuation is a default structure in the language. It is therefore necessary to establish a language particular default for Russian stress in order to test this prediction. The accentual defaults will be deduced from the constraint rankings given below for the analysis of stress in inflected words, and their role will then be extended to a wider range of data in chapter 5 , including certain minor accentual patterns involving a shift of stress in inflectional paradigms and derived nouns and verbs.

The rest of this section is organized as follows. The first subsection provides the necessary linguistic background on Russian, essentially stating my assumptions about Russian morphology and the phonetics of accent. The next subsection, §3.2.2, presents an analysis of the major stress patterns in underived nouns, which is a necessary point of departure because these patterns form the core of any description of Russian stress. Finally, §3.2.3 broadens the empirical scope further, examining the implications of the analysis for stress in verbs and prefixed words. It will be shown here that the analysis of Russian stress in terms of root-control is consistent with the findings in these areas, and therefore this analysis is consistent with the more restrictive theory of edge effects proposed here.

\subsubsection{Preliminaries}

Let us begin the study of Russian nominal stress with some preliminaries of Russian word structure from Townsend 1975: IC. First, roots in Russian typically have the shape CVC, where C stands for one or more consonants and V stands for one vowel. Roots of Church Slavonic origin always end in consonants, but a few are vowel-initial. Some roots have the shape CVRVC, with the medial $\mathrm{R}$ representing a resonant consonant. In most cases, however, there is a related Church Slavonic root with a single syllable. Other polysyllabic roots are often derivatives from roots with one syllable or borrowings.

Nouns are formed by attaching to the stem a set of inflections for gender, case, and number, as shown in the sketch below. The term 'stem' thus refers to the word minus inflections, which in this section simply involves a bare root.

(10) Morphological Frame for Underived Nouns

$$
[\text { Root }]_{\text {Stem }}+\text { Infl }
$$

Suffixation plays a very important role in word-formation, and as I will show in §5.2.3, noun-forming suffixes interact in interesting ways with the root accent. It is necessary, however, to determine the basic accentual principles in underived nouns before examining derived nouns. In contrast to suffixation, prefixation is much less important in nouns. While there are many prefixes, they are typically unproductive or have a low degree of productivity (Townsend 1975), which is probably why they are rarely taken into consideration in discussions of noun accentuation. Prefixed nouns and prefixed verbs have some important similarities, and so prefixed nouns will be addressed in §3.2.4., where I examine verb stress as well as the accentual properties of prefixes generally.

With respect to the phonetics of stress, Russian words have a single stress prominence per word, and this prominence is realized as a peak in intensity and greater duration than the neighboring syllables (see Jones \& Ward 1969 for more details). Russian also has a phonological pattern of vowel reduction in which non-high vowels are reduced 
in unstressed syllables. These patterns suggest that vowel quality may also have a role in cueing stress.

\subsubsection{Noun Stress: The Basic Patterns ${ }^{5}$}

As illustrated below, a fundamental observation in many nominal paradigms is that stress is fixed on the stem. The words with disyllabic stems given below, while somewhat rare in Russian, illustrate that stress may either fall on the first or second syllable, e.g.

kómnat-a versus tetrád'.

\begin{tabular}{|c|c|c|c|c|}
\hline \multicolumn{5}{|c|}{ 1) Fixed Stem Stress (by } \\
\hline $\mathrm{SG}$ & I & II & III & IV \\
\hline Nominative & rák & kómnat-a & tetrád' & bl'úd-o \\
\hline Accusative & rák-a & kómnat-u & tetrád' & bl'úd-o \\
\hline Genitive & rák-a & kómnat-i & tetrád'-i & bl'úd-a \\
\hline Dative & rák-u & kómnat-e & tetrád'-i & bl'úd-u \\
\hline Instrumental & rák-om & kómnat-oj & tetrád'-ju & bl'úd-om \\
\hline Locative & rák-e & kómnat-e & tetrád' -i & bl'úd-e \\
\hline \multicolumn{5}{|c|}{ RUminat-c } \\
\hline Nominative & rák-i & kómnat-i & tetrád' -i & blúd-a \\
\hline Accusative & rák-ov & kómnat-i & tetrád' -i & blúd-a \\
\hline Genitive & rák-ov & kómnat & tetrád'-ej & blúd \\
\hline Dative & rák-am & kómnat-am & tetrád'-am & blúd-am \\
\hline Instrumental & rák-am’i & kómnat-am'i & tetrád'-am'i & blúd-am’i \\
\hline \multirow[t]{2}{*}{ Locative } & rák-ax & kómnat-ax & tetrád' -ax & blúd-ax \\
\hline & 'crayfish' & 'room' & 'exercise book' & 'dish' \\
\hline
\end{tabular}

This pattern of fixed stress accounts for roughly $92 \%$ of the nominal paradigms in all declension classes. For a significant majority of nouns, therefore, stress is fixed on a stem vowel.

A second important stress pattern in nouns is fixed stress on the inflectional ending. Since most of the inflections are monosyllabic, this stress pattern gives stress on the first vowel following the stem, as shown below.

$\begin{array}{ccll}\text { Fixed Inflection } & \text { Stress } & & \\ \text { stól } & \text { č 'ert-á } & \text { vešč 'estv-ó } & \text { Nominative Singular } \\ \text { stol-ú } & \text { č 'ert-é } & \text { vešč 'estv-ú } & \text { Dative Singular } \\ \text { stol-ám } & \text { č'ert-ám } & \text { vešč 'estv-ám } & \text { Dative Plural } \\ \text { 'table' } & \text { 'characteristic' } & \text { 'substance' } & \end{array}$

There is a small percentage of nouns, mostly from declension class II, with fixed inflection stress, but with initial stress in the nominative plural, e.g., skovorod-a ' 'frying pan (nom sg)', cf. skóvorod-i (nom pl), or in both the nominative plural and the accusative singular, e.g., borod-á 'beard (nom sg), cf. bórod-u (acc sg), bórod-i (nom pl). Since these forms constitute less than a tenth of one percent of the total number of nouns, I will simply treat them as exceptions which are lexically marked for initial stress in the appropriate grammatical cases.

\footnotetext{
${ }^{5}$ Most of the data presented in this section are from Brown et al. 1996, which differs from many generative descriptions of noun stress in classifying the nominal paradigms by declension class, rather than by gender markings (as in, e.g., Melvold 1990). I am convinced by the arguments presented in Brown et al. (and references cited therein), and I follow this work in classifying the data in this way. However, the argumentation presented here does not hinge crucially on this choice of data organization.
} 
The frequencies for these two patterns given below support the following generalization (based on the generalizations formulated in Brown et al. 1996): stress is either fixed on a stem vowel or on the first vowel of the inflectional ending.

(13) Frequencies for Predominant Stress Patterns ( $n=43,996$; from Zaliznjak 1977)

Fixed Stem Stress $\quad 92 \%$

Fixed Inflection Stress $\quad 6 \%$

This generalization accounts for roughly $98 \%$ of the nouns, and when considering the size of Zaliznjak's corpus, it is sensible to assume that these two patterns of fixed stress constitute the core set of stress patterns. Furthermore, the remaining nominal paradigms conform to a basic pattern which distinguishes them from the major patterns examined here. Thus, in contrast to the fixed stress patterns here, the residual nominal paradigms exhibit two patterns of mobile stress, both of which have an opposition between the singular and plural case forms, as shown below with some partial paradigms.

(14) Mobile Stress Patterns

$\begin{array}{cl}\text { a. } & \text { Stem-Initial/Inflection Stress } \\ \text { tél-o } & \text { kólokol } \\ \text { tél-u } & \text { kólokol-u } \\ & \\ \text { tel-á } & \text { kolokol-á } \\ \text { tel-ám } & \begin{array}{l}\text { kolokol-ám } \\ \text { 'body' }\end{array}\end{array}$
b. Inflection/Stem-Final Stress dir-á kolbas-á Nominative Singular dir-é kolbas-é Dative Singular

dír-i kolbás-i Nominative Plural
dír-am kolbás-am Dative Plural

The pattern of mobile stress in (14a) has initial stress in singular forms, but ending stress in the plural. Likewise, the mobile stress patterns in (14b) show a related pattern of mobile stress: singular forms have ending stress while the plural forms have stem-final stress. While a handful of nouns stray from these two patterns in having anomalies within the singular or plural sub-paradigms, the basic pattern here is that the singular inflected forms have a fixed stress pattern, as do the plural inflected forms, and that these two fixed patterns are different (see Stankiewicz 1962 and references therein for discussion of this opposition). It appears therefore that the mobile stress patterns, constituting roughly $2 \%$ of the data, can be safely set aside and analyzed in a different way. I will return to the analysis of mobile stress in chapter 5 when the theoretical background for understanding these patterns has been sufficiently established.

The general strategy for analyzing Russian noun stress is the same as with the analysis of Cupeño: stress is 'root-controlled' in the sense that it is governed by the accentual properties of the obligatory constituent of the stem. With the Prosodic Faithfulness constraints for roots top-ranked, as shown below, root stress will override affix stress. Thus, in a word with an accented root, inherent accent in the root will be preserved throughout the paradigm, giving the observed pattern of fixed stress.

Furthermore, because the Root Faithfulness constraints dominate other prosodic wellformedness constraints, the position of stress may contrast in polysyllabic roots. 
(15) Root-Controlled Stress in Russian
a. MAX-PROMRoot $>$ PHONO, MAX-PROMAffix
b. NO-FLOP-PROMRoot $>$ PHONO

The Root Faithfulness constraints given above have no say in words with unaccented roots, and so in such contexts the low-ranking constraints play a decisive role. In particular, a purely phonological constraint, which I will now motivate, becomes active and requires stress to appear on the ending.

In characterizing the constraint responsible for ending stress, it is helpful to briefly compare this pattern in Russian with a related pattern in Sanskrit. In Sanskrit thematic nouns (nouns which have a theme vowel), there is also a basic distinction between stems which have a fixed accent on the stem and stems which have an accent on a post-stem vowel, namely the theme vowel. For the latter class of stems, the so-called 'oxytone stems', Kiparsky 1973 argues for a post-stem accent rule which specifically posits an accent on the vowel directly following the stem. The evidence for the restriction to the directly following vowel is that it limits accent to the first syllable of polysyllabic suffixes, which, while not significant for the case endings, is correctly borne out for verbal suffixes (Kiparsky 1973: 810). It would seem, then, that a parallel constraint is at work in Russian, which is the spirit in which I propose the following constraint (see also 'the Oxytone Rule' of Halle 1973).

(16) Post-STEM-PROM (PSP) $\equiv$ Align (PROM, L, Stem, R)

The left edge of the stress prominence must coincide with the right edge of some stem.

POST-STEM-PROM is formulated as a subcategorization type constraint in the constraint schemata provided in Generalized Alignment (McCarthy \& Prince 1993). The effect of stress on the first vowel of the inflectional ending is thus the same type of effect observed in suffixation generally: the left edge of a prominence (i.e., the left edge of the vowel dominated by a grid mark) must co-incide with the right edge of the stem, giving post-stem stress. Formulated as a gradient Alignment constraint, PSP requires a prominence on the first vowel of the inflectional ending, or as close as a prominence can get to this position, which will be crucial in the analysis presented below. ${ }^{6}$

The results of these constraint rankings will now be illustrated in a series of tableaux. First, words with fixed stem stress are assumed to have an inherently accented root, and so $\sqrt{ }$ rák shown below is endowed with a lexical prominence. Assuming that the plural ending is inherently accented as well (a possibility we must entertain, given Richness of the Base), these two morphemes compete for the unique word prominence. The inherent ranking between Root and Affix Faithfulness gives overriding root stress because, as with the parallel set of facts in Cupeño, failure to preserve the root accent leads to a fatal violation of MAX-PROMRoot.

\footnotetext{
6This constraint finds additional support in Pierrehumbert \& Beckman's 1988 analysis of 'initial lowering' effects in Tokyo Japanese, where the boundary tone of a preceding minor phrase appears on the initial mora of the following phrase; hence, the operative constraint appears to be ALIGN(L\%, L, Minor Phrase, R).
} 
(17) Overriding Root Stress in Russian

\begin{tabular}{|c|c|c|}
\hline $\begin{array}{c}\mathrm{x}_{1} \mathrm{X}_{2} \\
\text { /rák }+ \text { í/ }\end{array}$ & MAX-PROMRoot & MAX-PROM $_{\text {Affix }}$ \\
\hline \hline $\begin{array}{c}\mathrm{x}_{2} \\
\text { rak-1 }\end{array}$ & $* !$ & \\
\hline $\begin{array}{c}\mathrm{X}_{1} \\
\text { rák-i }\end{array}$ & & $*$ \\
\hline
\end{tabular}

If, on the other hand, the plural ending is unaccented, the outcome is the same. Since MAX-PROM Root dominates PSP, inherent root stress wins over default stress on the inflectional ending.

Root Faithfulness has another role in the analysis, namely that it ensures that the accent of the root does not shift forward to the favored post-stem position. As shown below, if the root accent flops to the inflection, this option leads to a violation of NO-FLOPPROMRoot, and since this constraint dominates PSP, the winning candidate is the form which does not undergo the shift.

(18) Lack of Accent Shift

\begin{tabular}{|c|c|c|}
\hline $\begin{array}{c}\mathrm{x}_{1} \mathrm{X}_{2} \\
\text { /rák }+1 /\end{array}$ & NO-FLOP-PROMRoot & POST-STEM-PROM \\
\hline \hline $\begin{array}{c}\mathrm{x}_{1} \\
\text { rak-1 }\end{array}$ & $* !$ & \\
\hline $\begin{array}{c}\mathrm{X}_{1} \\
\text { rák-i }\end{array}$ & & $*$ \\
\hline
\end{tabular}

Because of the rank of NO-FLOP-PROM Root, the root accent does not shift. This result leads to a further prediction which is borne out in Russian, namely that polysyllabic roots will show a contrast in accent position within the root. As shown with the inputoutput mappings below, disyllabic roots show such a contrast because failure to preserve the accent, and realize it in a position corresponding to its lexical position, leads to violation of either MAX-PROMRoot or NO-FLOP-PROMRoot.

(19) Positional Contrast in Disyllabic Roots

\begin{tabular}{|c|c|c|c|c|c|}
\hline Input & Output & MX-PMRt & NO-FLOP-PMRt & PSP & MX-PMAf \\
\hline \multirow[t]{3}{*}{ a. /kómnat + í/ $\rightarrow$} & kómnat-i & & & $*$ & $*$ \\
\hline & *komnát-i & & $* !$ & $*$ & $*$ \\
\hline & *komnat-1́ & $* !$ & & & \\
\hline \multirow[t]{3}{*}{ b. /tetrád' + í/ } & tetrád'-i & & & $*$ & $*$ \\
\hline & \multirow{2}{*}{$\begin{array}{l}\text { *tétrad'-i } \\
\text { *tetrad'-í }\end{array}$} & & $* !$ & $*$ & $*$ \\
\hline & & $* !$ & & & \\
\hline
\end{tabular}


Other stem-internal elements, such as derivational suffixes, also participate in this contrast, but since their Faithfulness properties are not governed by Root Faithfulness, they are treated in \$5.2.4., where the derivational suffixes are analyzed.

So far we have only been concerned with the ranking consequences of the grammar in (15) above for words with accented roots. In words with unaccented roots, however, the Root Faithfulness constraints such as MAX-PROMRoot are irrelevant because there is not an underlying accent to realize. The lower-ranking constraints therefore become active in these word types and give default ending stress. For example, a word with an unaccented root such as $\sqrt{s t o l}$, receives stress on the first vowel of the inflectional ending because this is the position prescribed by POST-STEM-PROM. As shown below, this result is obtained even if the inflectional ending is unaccented. Thus, the two plural endings $i$ and -am' $i$ get a stress on the first vowel because this structure properly aligns the surface prominence with the right edge of the stem

(20) Default Ending Stress with Unaccented Root

\begin{tabular}{|lr|c|c|}
\hline \multicolumn{1}{|c|}{ Input } & \multicolumn{1}{c|}{ Output } & MAX-PROMRoot & POST-STEM-PROM \\
\hline \hline a. /stol $+\mathrm{i} / \mathrm{stol-1}$ & & \\
\hline & *stól-i & & $* !$ \\
\hline \hline b. /stol + am'i/ $\rightarrow$ stol-ám'i & & $* !$ \\
\hline & *stol-am'í & & \\
\hline
\end{tabular}

The same result holds for most cases if the endings are inherently accented, which will be illustrated at the close of the discussion where the accentedness of the endings is clarified.

The constraint POST-STEM-PROM also has a role in words with unaccented stems and null inflections. As exemplified below, if a noun with fixed inflection stress has a null inflection, as with the nominative singular in first declension nouns, stress falls on the next closest vowel, i.e., the stem-final vowel.

(21) Fixed Inflection/Stem-Final Stress

$\begin{array}{lllll}\text { topór } & \text { slovár' } & \text { p'iróg } & \text { karás' } & \text { Nominative Singular } \\ \text { topor-ú } & \text { slovar'-ú } & \text { p'irog-ú } & \text { karas'-ú } & \text { Dative Singular } \\ \text { topor-ám } & \text { slovar'-ám } & \text { p'irog-ám } & \text { karas'-ám } & \text { Dative Plural } \\ \text { 'axe' } & \text { 'dictionary' } & \text { 'pie' } & \text { 'carp' } & \end{array}$

Because of the gradient nature of PSP, this observation receives a natural explanation in terms of the minimal violation of this constraint. As illustrated in the tableau below, the candidate with stem-final stress is the winner because it posits the accent closer to the right edge of the stem than the available alternative.

\footnotetext{
${ }^{7}$ This result also provides an argument against employing the constraint DEP-PROM Root in the analysis of fixed inflection stress, in effect banning the insertion of accent in roots in words with no inherent accent and leaving the inflection as the only viable option. This anti-insertion constraint is clearly irrelevant in the analysis of stem-final stress in words with null inflections, so the analysis with PSP covers more empirical ground.
} 
(22) Minimal Violation of POST-STEM-PROM

\begin{tabular}{|c|c|c|}
\hline /topor $+\varnothing /$ & PROS-FAITHRoot $_{\text {POST-STEM-PROM }}$ \\
\hline \hline tópor $\varnothing$ & & $* * !$ \\
\hline$\rightarrow$ topór- $\varnothing$ & & $*$ \\
\hline
\end{tabular}

Furthermore, as we will see in §5.3, PSP plays a crucial role in the analysis of certain patterns of mobile stress. In both of these patterns, ending stress is found throughout either the plural case forms or the singular case forms, providing further evidence for ending stress as a default. While it is impossible to establish this claim without an analysis in hand, it is clear that a constraint such as POST-STEM-PROM will have a role in the analysis of these patterns as well.

As discussed in $§ 1.2$, given Richness of the Base, it is necessary to derive the inventory of possible stress patterns without imposing a Russian-specific constraint on underlying representations. The analysis presented here meets this requirement, as the constraint rankings motivated above will generate all and only the observed stress patterns without language-particular restrictions on the input. Thus, the following illustration shows the predicted outcomes in all possible input-output mappings, factoring in both the accentedness of roots and endings. I assume, for ease of exposition, that accentual shifts are not possible here, which is a safe assumption, given the ranking of No-FLOPPROMRoot in the grammar.

(23) Inventory of Noun Stress Patterns

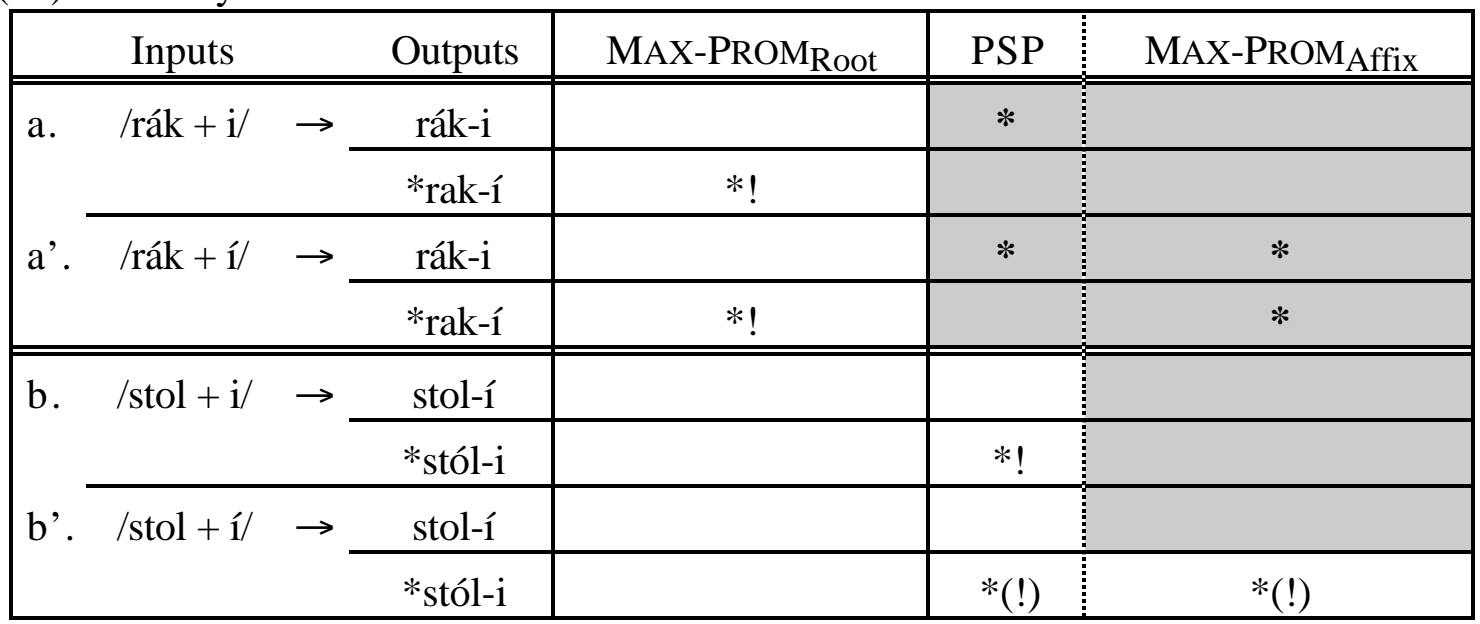

The point to be emphasized here is that the winning output form is predicted purely on the basis of the accentual properties of the root. Thus, if the input contains an accented root, the prediction is that the root will realize its accent in the output, regardless of the accentedness of the ending (23a-a'). Likewise, if the input is a word with an unaccented root, the resulting output has inflection stress, even if the ending is itself unaccented (23b).

We have not as yet determined the ranking of POST-STEM-PROM relative to MAXPROMAffix, or any of the Affix Faithfulness constraints, as this issue has not as yet been directly relevant for the empirical matters at hand. The evidence needed to settle this ranking issue is the presence or absence of an accentual contrast in the position of accent in affixes, and in this area, Russian morphology is not especially helpful. Most all of the endings are monosyllabic, except the instrumental plural -am' $i$ and perhaps a few others as 
well, if suffixes with yers are counted. This set is clearly not a large enough sample to decide whether Russian affixes have a contrast in the position of accent. Furthermore, there are only about 4 or 5 disyllabic derivational suffixes that are clearly not morphologically complex, which does not add much to the baseline here. It is certainly true, however, that when they are stressed, the polysyllabic suffixes have stress on the initial syllable (a point emphasized in Stankiewicz 1993: 185); thus, if POST-STEM-PROM

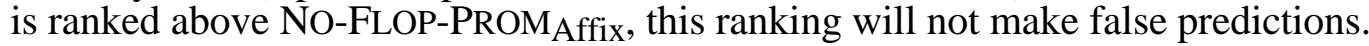
Moreover, as alluded to above, when polysyllabic suffixes attach to oxytone stems in Sanskrit, the result is always accent on the first vowel of the suffix. To be consistent with Sanskrit, therefore, I hypothesize that this pattern is also significant in Russian, which justifies the following ranking.

\section{(24) Root-Controlled Accent in Russian}

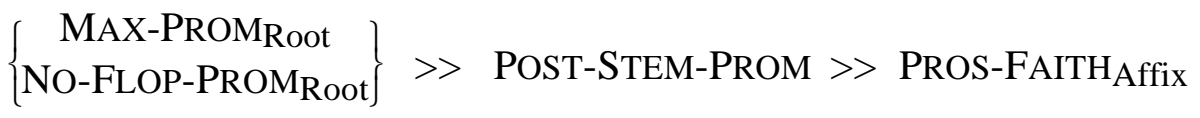

To summarize the results of this constraint system, accent is root-controlled in Russian nouns. That is, in words with accented (simplex) stems, inherent accent in the stem always prevails, as predicted by the ranking of the Root Faithfulness constraints MAX-PROMRoot and NO-FLOP-PROMRoot above all other constraints. In words with unaccented stems, these constraints are irrelevant, and the decision therefore falls to the next highest constraint in the hierarchy, namely POST-STEM-PROM, which ensures stress on the first vowel of the inflectional ending. The above ranking therefore accounts for the core set of stress patterns, namely fixed stem stress and fixed inflection stress, as a consequence of the accentual properties of the root.

The analysis presented above applies the theory of root-controlled accent to Russian nouns. The next question to be addressed therefore is, how does the analysis apply to the stress patterns found in other word classes? Specifically, does it extend to the accentuation of adjectives and verbs? Concerning adjectives, both Halle 1973a and Melvold 1990 emphasize that the principles of accentuation in adjectives are fundamentally the same as those found in nouns, and so it appears that investigating adjective stress will not turn up a new set of challenges. Stress in verbs, however, presents a new empirical problem, namely the accentuation of prefix + root sequences. Since these sequences may present crucial evidence for or against the root-controlled analysis, it is worthwhile studying prefixed verbs in some detail.

\subsubsection{Extending the Analysis: Verb Stress and Prefixed Words}

There is a basic distinction between thematic and athematic verbs in Russian. Thematic verbs are derived verbs which are essentially the product of attaching one of a set of theme vowels to a root. Athematic vowels are underived and therefore do not have a theme vowel. As thematic verbs involve certain complications which are irrelevant to the main issues at hand, I will focus exclusively on athematic verbs here, but see Halle 1973a and Melvold 1990 for comprehensive discussions. ${ }^{8}$

\footnotetext{
${ }^{8}$ Much of the data and descriptive generalizations below are drawn directly from these key works.
} 
The following morphological frame describes the composition of athematic verbs.

(25) Morphological Frame for Athematic Verbs

$$
\left[(\text { prefix })+\operatorname{root}+\left\{\begin{array}{l}
1 \\
e
\end{array}\right\}+\operatorname{suffix} \text { Agr }\right]
$$

Verbs in Russian have complex inflections consisting of a tense suffix and an agreement suffix. The inflections are shown in the forms below, which illustrate the range of possible stress patterns in verbs.

(26) Stress Patterns in Verbs

a. Present tense verbs

$\begin{array}{llll}\text { Pattern A } & \text { Pattern B } & \text { Pattern C } & \text { Pattern D } \\ \text { léz-u } & \text { pek-ú } & \text { ž iv-ú } & \text { strig-ú } \\ \text { léz-eš } & \text { peč -óš } & \text { ž iv'-óš } & \text { striž -óš } \\ \text { léz-et } & \text { peč -ót } & \text { ž iv'-ót } & \text { striž -ót } \\ & & & \\ \text { léz-em } & \text { peč -óm } & \text { ž iv'-óm } & \text { striž -óm } \\ \text { léz-ete } & \text { peč -óte } & \text { ž iv'-óte } & \text { striž -óte } \\ \text { léz-ut } & \text { pek-út } & \text { ž iv-út } & \text { strig-út } \\ \text { 'crawl' } & \text { 'bake' } & \text { 'live' } & \text { 'shear' }\end{array}$

b. Past tense verbs

$\begin{array}{lllll}\text { Masculine } & \text { léz } & \text { pék } & \text { ž í-l } & \text { stríg } \\ \text { Feminine } & \text { léz-la } & \text { pek-lá } & \text { ž i-lá } & \text { stríg-la } \\ \text { Neuter } & \text { léz-lo } & \text { pek-ló } & \text { ž í-lo } & \text { stríg-lo } \\ \text { Plural } & \text { léz-li } & \text { pek-lí } & \text { ž í-li } & \text { stríg-li }\end{array}$

Using some common alphabetic labels, the descriptions for the patterns exemplified above are given below (after Halle 1973a and Melvold 1990).

(27) Four Basic Patterns

a. Pattern A: fixed stem stress.

b. Pattern B: fixed stress on the inflection (when there is one)

c. Pattern C: stem-initial stress in the masculine, neuter, plural past tense forms, ending stress elsewhere.

d. Pattern D: ending stress in present tense forms, and stem-final stress in the past.

The point to be emphasized here is that the same basic stress patterns in nouns are also found in the verbs shown above. Thus, patterns A and B are exactly on a par with the core patterns observed in nouns: stress is either fixed on the stem or on the inflectional ending. Furthermore, verbs show the same two patterns of mobile stress; compare the verb stress patterns shown above with the nominal paradigms in (14). Pattern $\mathrm{C}$ has initial stress in a subset of the past tense forms, and ending stress elsewhere, which is mirrored in the pattern of singular-plural opposition found in stems like kólokol 'bell'. Also, pattern D stress is directly parallel to singular-plural opposition in words like kolbás-a 'sausage', 
ending stress in one grammatical class, and stem-final stress in another. In sum, the range of possible patterns are the same in both verbs and nouns. ${ }^{9}$

At this point, I can only give a partial story for verb stress, as the principles governing patterns $C$ and D stress have not yet been proposed. It is clear, however, that the same basic analysis for nouns may be extended to verb stress, which is satisfactory for the moment. This interim sketch will be sufficient to cover prefixed verbs and nouns, which is the focus of this section. The full range of stress patterns in verbs will be revisited and analyzed in chapter 5, where the principles governing mobile stress are introduced.

The patterns of fixed stress, i.e., patterns A and B, are covered by the constraint system developed in the previous section, as I will now demonstrate. First, fixed stem stress (pattern A) may be straightforwardly analyzed as the realization of inherent accent in roots. Hence, regardless of the accentuation of the inflections, if one assumes that the root $\sqrt{ } l e ́ z$ is inherently accented, then the grammar given in (24) for nouns will preserve the root accent, giving fixed stem stress (28a). Moreover, fixed inflection stress in verbs is treated in exactly the same way as nouns: if one assumes that in such words the root is unaccented, then it will receive default inflection stress, as shown in (28b).

(28) Fixed Stress in Verbs: Patterns A and B

\begin{tabular}{|c|c|c|c|c|}
\hline Input & & Output & MAX-PROMRoot & POST-STEM-PROM \\
\hline \multirow[t]{2}{*}{ a. /léz $+\mathrm{u} /$} & & léz-u & & $*$ \\
\hline & & *lez-ú & $* !$ & \\
\hline \multirow[t]{2}{*}{ b. /pek $+\mathrm{u} /$} & & pek-ú & & \\
\hline & & *pék-u & & $* !$ \\
\hline
\end{tabular}

Importantly, these results do not depend on the accentedness of the inflections: if the present tense inflection here was inherently accented, it would still go unstressed in the case of léz-u, and it would still be stressed in the case of pek-ú. This result follows from the rank of the Root Faithfulness constraints, as it was observed in root + inflection sequences in nouns in $\$ 3.2 .2$.

As for patterns $\mathrm{C}$ and $\mathrm{D}$ stress, these patterns of mobile stress resemble the corresponding nominal patterns in that they show oppositions between well-defined grammatical classes. An obvious comparison is seen in pattern D stress with verbs like $\sqrt{ }$ strig where present tense forms have fixed ending stress, which contrasts with the related past tense forms which have stem-final stress. The opposition in pattern $\mathrm{C}$ in verbal paradigms like $\sqrt{z}, v$ is more subtle, but equally coherent. Thus, there is a basic opposition again between present and past tense forms, except in this pattern the opposition goes in a different direction. Specifically, past tense forms generally have initial stress (excluding feminine past tense forms), while present tense forms have ending stress. As for the ending stress in the feminine forms, this pattern is the manifestation of a different morphological opposition, namely between masculine and feminine forms. There is abundant evidence in Russian for such an opposition, as illustrated with the following contrasts in derived nouns (see especially Halle 1973a: 340 ff.). In masculine-feminine

\footnotetext{
${ }^{9}$ According to Halle 1973a, fixed inflection stress is the predominate pattern in athematic verbs, which contrasts with noun stress where fixed stem stress is the most common. It is not clear if this difference is significant, given the vast differences in the baselines for each system (there are far fewer verbs than nouns in Halle's samples).
} 
noun pairs derived with the suffix - $O k$, for example, if the stem is unaccented the noun pairs differ in stress, e.g., pastuš kí versus pastúš ki '(dear little)

shepherds/shepherdesses'. This pattern is more akin to pattern D stress, as it is an opposition between ending stress (masculine) and stem-final stress (feminine), but the overall point still holds: the stress system is being used to mark an opposition between two grammatical classes. In sum, while pattern $\mathrm{C}$ stress appears to be somewhat complicated, the mobile stress patterns in verbs can be cogently described in terms of a contrast between pairs of grammatical classes, which is directly on a par with the singular-plural oppositions found in nominal mobile stress patterns. So, once these tools have been developed for noun stress, they may be straightforwardly extended to verbs, as is done in the case study on Russian derived nouns in chapter 5.

The comparison between noun and verb stress given above shows two things. First, it shows that the principle of root-control employed in the analysis of noun stress applies with equal force in verbs to describe the two patterns of fixed stress (patterns A and B). Second, it draws explicit parallels between the morphological oppositions found in nouns and verbs, which in turn suggests that these patterns of mobile stress (i.e., patterns $\mathrm{C}$ and $\mathrm{D}$ ) should be treated together. The productive patterns of prefixation in verbs present a further empirical domain in which to test the RCA hypothesis because such patterns yield prefix + root structures which are directly relevant for this analysis.

Remarkably, it turns out that the only pattern of verb stress that is affected by prefixation is pattern C (Melvold 1990). That is, fixed stem stress and fixed ending stress are generally not affected by prefixation (excluding one prefix to be discussed below). Furthermore, pattern D stress is generally not changed in prefixed verbs. This finding is highly significant because if the Russian stress is modelled in terms of root-control, prefixes should have no effect on fixed stem stress at all, as I have shown above with the inflectional suffixes. In what follows, I examine the effect of prefixation on pattern $\mathrm{C}$ stress and consider its implications for root-controlled accent in Russian. Then, the discussion is generalized to prefixes in nouns, which are far less productive, but show a related pattern.

Verbs which show pattern $\mathrm{C}$ stress show a related pattern when the verb root is combined with a prefix, as illustrated below with some past tense forms.

$\begin{array}{cccc}\text { (29) Past Tense forms with } \sqrt{z} \text { iv 'live'; pro-ž } i v & \text { 'live (a period of time)' } \\ \text { Masculine } & \text { ž í-1 } & \text { pró-ž i-l } & \text { pro-ž í-l } \\ \text { Feminine } & \text { ż i-lá } & \text { pro-ž i-lá } & \text { pro-ž i-lá } \\ \text { Neuter } & \text { ž í-lo } & \text { pró-ž i-lo } & \text { pro-ž í-lo } \\ \text { Plural } & \text { ž í-li } & \text { pró-ž i-li } & \text { pro-ž íli } \\ & & \text { Standard } & \text { Colloquial }\end{array}$

Recall from above that pattern $\mathrm{C}$ stress shows root-initial stress in all the past tense, save the feminine past form. In prefixed verbs, the same pattern of mobile stress may hold, which appears to correspond to a more prevalent, perhaps colloquial usage. Alternatively, initial stress in the non-feminine forms may be extended to word-initial stress, with stress falling on the first syllable of the prefix, which corresponds roughly to the standard pronunciation one might learn in school. ${ }^{10}$ It is clear, however, that these two patterns are simply different realizations of pattern $\mathrm{C}$ stress, with some variation in the delimitation of the domain to which initial stress is assigned.

\footnotetext{
10Melvold 1990: 299 mentions in passing that it appears that if a 'standard' pronunciation is possible, so too is the 'colloquial' stress pattern. Perhaps this tendency reflects a regularization of root-initial stress in the non-feminine forms.
} 
This pattern of variation, while it may yield stress on the prefix, is fully consistent with the assumption that accent is root-controlled. Succinctly, the roots in these contexts cannot be inherently accented, and so the prefix is not 'in competition' with an accented root. To flesh out the logic more concretely, the chief diagnostic for accented roots is fixed stem stress; pattern C stress does not have fixed stem stress, and so it follows that the verb roots with pattern $\mathrm{C}$ mobile stress are not inherently accented. Furthermore, since overriding root accent is only found in words with both an accented root and an accented affix, these data do not reveal anything about the hypothesis that accent is root-controlled in Russian. This reasoning is more or less consistent with most previous generative approaches to this pattern because mobile stress of this kind is only possible in words with unaccented roots (Halle 1973, 1996, Melvold 1990, Idsardi 1992). In §5.2.3, I will also develop an analysis with this assumption, which will establish this point more solidly with a specific analysis.

There is one prefix that is consistently accented in certain contexts, which may even have the effect of stealing the accent from an inherently accented root. This is the prefix $v i$ 'out-', which perfectivizes the stem it attaches to (a general property of verbal prefixes in Russian). As illustrated below, $v i$ - is always stressed in the perfective, but never in the imperfective. ${ }^{11}$

(30) Derived Verbs with $v i$ -

$\begin{array}{llll} & \text { Perfective } & \text { Imperfective } & \\ \text { p'isát' } & \text { ví-p'isat' } & \text { vi-p'ísivat' } & \text { 'write/write out' } \\ \text { skazát' } & \text { ví-skazat' } & \text { vi-skázivat' } & \text { 'say/express' } \\ \text { vest'í } & \text { ví-vest'i } & \text { vi-vod'it' } & \text { 'lead out }\end{array}$

This verbal prefix therefore appears to pose a challenge to the root-controlled analysis because it competes with an accent from a root and wins in perfective forms. However, the accentuation of derived imperfectives shows that the pattern is more complicated, involving an intricate interplay between the imperative to stress $v i$ - and the patterns of stem and ending stress required in the imperfectives. Given that $v i$ - is the sole prefix showing this pattern in the entire language (including some twenty productive verbal prefixes), rather than abandon the root-controlled analysis, it seems sensible to construct an analysis of $v i$ which accounts for its peculiar behavior. In chapter 5, I analyze this prefix on a par with other affixes which idiosyncratically delete the accent of the stem to which they attach (the so-called 'dominant affixes'). On this analysis, the behavior of the imperfectives falls out quite naturally from the fact that the perfective forms serve as the input to derived imperfectives, which also induce a specific morpho-accentual process, namely preaccentuation.

Finally, I will conclude the discussion with a brief look at prefixed nouns. Prefixation in nouns and adjectives is far less important than in verbs as most prefixes vary between having a low degree of productivity and being completely unproductive. According to Townsend 1975, there are two basic types of prefixed nouns, shown below.

\footnotetext{
${ }^{11}$ Browne 1978 notes the following exception: vígljadet' 'look like, appear' which is necessarily an imperfective given its inherent meaning.
} 
(31) Two Types of Prefixed Nouns (Townsend 1975)

a. Nouns with prefixes based on prepositions used in prepositional phrases

$\begin{array}{llll}\text { bez-rabót'ica } & \text { 'unemployment' } & \text { bez rabóti } & \text { 'without work' } \\ \text { za-kavkáz'o } & \text { 'Transcaucasia' } & \text { za Kavkázom } & \text { 'beyond the Caucasus' } \\ \text { pod-lésok } & \text { 'underbrush' } & \text { pod lésom } & \text { 'under the forest' }\end{array}$

b. Nouns with prefixes added to independent words

$\begin{array}{llll}\text { ne- } & \text { 'non-' } & \text { ne-znán'ie } & \text { 'ignorance' } \\ \text { pod- } & \text { 'sub-' } & \text { pod-grúppa } & \text { 'sub-group' } \\ \text { pra- } & \text { 'proto-' } & \text { pra-jazík } & \text { 'proto-language' } \\ \text { so- } & \text { 'co-' } & \text { so-ávtor } & \text { 'co-author' }\end{array}$

Given the paucity of examples, no clear patterns emerge concerning stress in prefixed nouns. A handful of prefixes in my sample, however, surface with stress, e.g., pr'i-gorod 'suburb' and prá-ded 'great grandfather', which of course requires some thought. One sound line of analysis for these cases is that they pattern with the prefixed verbs discussed above with pattern $\mathrm{C}$ stress. This tack is invariably the case with pr'i-gorod, as gorod is an independent word with mobile stress (and thus has an unaccented root). Moreover, the fact that one type of nominal prefix is derived from a preposition further supports this parallel, as most verbal prefixes are also derived historically from prepositions (Townsend 1975). A final piece of evidence bearing on this comparison between prefixed verbs and nouns is that prepositions were at one time included in the stress domain to which initial stress is assigned in pattern C mobile stress (see Halle 1973a: 318-19). Thus, while the stem gorod receives initial stress in the present day prepositional phrases shown below, the historically prior pattern had stress on the preposition.

(32) Stress in Prepositional Phrases

$$
\begin{array}{ll}
\text { za górod < zá gorod } & \text { 'out of town (to the countryside)' } \\
\text { za górodom < zá gorodom } & \text { 'outside of town (in the countryside)' }
\end{array}
$$

Thus, it is clear that prefixed nouns with stress on the prefix may be approached in terms of pattern $\mathrm{C}$ stress, which, as clarified above, is consistent with the root-controlled accent analysis.

To bring this case study to a close, I have examined the full range of stress patterns in underived nouns and verbs and constructed an analysis of most of these patterns which is consistent with the restricted edge effects derived in $\S 3.1$. Thus, while there are many previous analyses of Russian stress which describe certain patterns in terms of unrestricted edge orientation, an analysis in terms of root-controlled accent is also possible, and indeed, highly desired because it is consistent with a restrictive theory of edge effects.

Furthermore, I have examined a range of prefixed structures, with an eye towards evidence for or against the two basic analyses, and this investigation has only turned up one context which might pose a problem for the RCA hypothesis, namely verbs with the prefix vi-. As I suggested above, the peculiar behavior of this prefix may be analyzed in terms of a morpho-accentual process involving deletion, as will be shown in chapter 5, so this case does not provide compelling evidence one way or the other. I conclude, therefore, in favor of the RCA analysis of Russian, chiefly because it is consistent with Restricted Edge Orientation. Chapter 5 will present additional arguments in favor of this approach. 


\subsection{Extended Case Study: Tokyo Japanese}

The primary goal of this section is to consider the implications of the RootControlled Accent (RCA) hypothesis for the accent system of Tokyo Japanese. This goal is achieved by first proposing an analysis of the basic facts of Japanese word accent in terms of root-control and then studying the consequences for accent in affixed words. The analysis of the basic facts also satisfies a secondary goal of this section, which is to establish a consistent set of assumptions which will be necessary in the treatment of the various morpho-accentual processes in Japanese examined in chapter 5.

A first pass through the literature on Japanese accent ${ }^{12}$ would lead one to the conclusion that Japanese constitutes a counter-example to the RCA hypothesis as affixed structures have been described in terms of a principle of edge orientation, namely 'the leftmost inherent accent wins'. Thus, a BAP-like (Basic Accentuation Principle, Kiparsky \& Halle 1977) rule has been used to account for the fact that stem accent wins out over various inherently accented suffixes, such as the conditional suffix -tára, e.g., /yóm + tára/ $\rightarrow$ yón-dara 'if he reads', cf. /yob + tára / $\rightarrow$ yon-dára 'if he calls'. This stem and suffix interaction is of course consistent with a RCA-driven analysis, but the two analyses differ in their treatment of prefix accent. The analysis which employs the BAP predicts that inherent accent in a prefix will override accent in a root. In contrast, the RCA hypothesis predicts the absence of such a pattern and overriding root accent, as seen in Cupeño. The basic finding here is that there are no prefixes which override root accent, which provides empirical support for the analysis in terms of root-control. This analysis is also motivated on theoretically grounds because it is compatible with a restrictive theory of edge effects and explains resolution of accent in Japanese as a general pattern of root privilege.

This section is organized as follows. I begin with the necessary preliminaries in $\S 3.3 .1$, presenting the essential features of word accent in Tokyo Japanese and some basics of Japanese word structure. The next subsection (\$3.3.2) gives a constraint-based analysis of the basic accentual system and clarifies the predictions of the RCA hypothesis. Taking a slight detour, I examine the accentuation of noun-noun compounds in $\S 3.3 .3$, as compounds are directly relevant to prefix accent, and I present a new analysis of this construction. The set of assumptions motivated up to this point in the study will then be applied in $\$ 3.3 .4$ to the analysis of the various influences of prefixation on word accent. Finally, I close the section by showing that the various types of prefixes are in fact consistent with the predictions of the RCA hypothesis, which supports the overall argument that word accent in Japanese is root-controlled.

\subsubsection{Background}

A basic fact in Japanese is that accent is contrastive, both for the position of accent and its presence or absence in a word. Thus, accent alone may introduce contrast among otherwise identical words, as shown below with some examples familiar from McCawley 1968.

(33) Contrastive Accent

$$
\text { hási 'chopsticks' hasí 'bridge' hasi 'edge' }
$$

\footnotetext{
${ }^{12}$ The phonetics and phonology of Standard Tokyo Japanese and other Japanese dialects have been extensively investigated; see McCawley 1968, Chew 1973, Haraguchi 1977, 1991, Poser 1984, Beckman 1986, Beckman \& Pierrehumbert 1986, Pierrehumbert \& Beckman 1988, Kubozono 1988 [1993], and references therein.
} 
The convention I use to mark accent is to place an acute accent directly on the accented vowel, which differs from some work where the accented vowel directly after that vowel, e.g., ha'si 'chopsticks'. This accent marks a tonal event in which pitch drops directly after the accented vowel (illustrated below); thus, accent in Japanese differs from accent in Russian and Cupeño, where pitch is not a primary correlate to surface accent.

The position of accent is contrastive in Japanese, but not all positions in a word may bear accent. In syllables greater than a single mora, the pitch accent always falls on the first mora. This restriction on the inventory of accentual contrasts also has important consequences in various accentual rules. For example, foreign words typically receive accent on the syllable containing the third mora from the end of the word. Thus, the preantepenultimate mora is accented if it and the subsequent mora are tautosyllabic, e.g. hambáagaa 'hamburger'. Based on evidence such as this, Japanese is often referred to as a 'mora-counting', but 'syllable-accenting' accent language (see McCawley 1968: 133 ff. and Poser 1990a for discussion and additional evidence). ${ }^{13}$

A second important qualification concerning the distribution of surface accent is that contrastive accent is largely a property of nouns (see Smith 1997 for extensive discussion and analysis). Other word classes, such as verbs and adjectives, conform to certain regular patterns with accent typically falling on the syllable with the penultimate or antepenultimate mora. Furthermore, loans exhibit some accentual regularities, with accent mostly falling on the antepenultimate, pre-antepenultimate, or initial mora of the word. Following previous accounts (e.g., Katayama 1995, 1998, Kubozono 1995, Smith 1997; see also Zubizarreta 1982, Abe 1981, and Bennet 1981), I assume that these regularities are due to an effect of an Alignment constraint which requires an accentual prominence to appear on the head of a prosodic foot.

Concerning phonological phrasing, words are grouped into phonological constituents, called minor phrases, which are in turn organized into so-called major phrases ${ }^{14}$. Minor phrases are characterized by two important facts. First, a minor phrase may only have a single pitch accent, meaning that there can only be one rise and fall in f0 per minor phrase. Also, in phrases where the first mora is unaccented, there is a rise in pitch to the second mora, which then rises to a following accent, if there is one, and gradually falls to the final syllable if the phrase has no accent. This rise at the onset of a phrase is often referred to as 'initial lowering'. The basic features of minor phrases in Japanese are illustrated by the pitch contours given below.

\section{(34) F0 Contours of Minor Phrases}

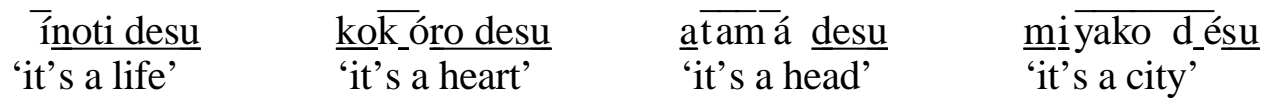

As shown by the above examples, each minor phrase begins at a low point for f0, rises to a high point over and beyond the second mora of the word. In words with accent on the initial syllable, as with the first example, there is a smaller rise from a higher, or 'weak' low, at the onset of the first mora, an effect which is also observed in phrases which begin with syllables containing two moras (see Pierrehumbert \& Beckman 1988 for further

\footnotetext{
${ }^{13}$ Another restriction on the distribution of accent is that there is an avoidance of devoicing accented vowels. The interaction between accent and vowel devoicing is very complex, involving both phonological and phonetic factors. It is not clear, therefore, to what extent vowel devoicing restricts the phonological inventory of accentual patterns (see Tsuchida 1997 for extensive discussion of this interaction).

${ }^{14}$ The terminology is due to McCawley 1968, cf. 'accentual phrase' and 'intonational phrase' of Pierrehumbert \& Beckman 1988.
} 
discussion). In addition, each example has a fall in pitch somewhere in the phrase, and as mentioned above, this fall directly follows the inherently accented vowel.

An important qualification which is needed before we can proceed is that I develop an analysis of accent in words, not minor phrases. While there are important similarities between words and minor phrases, there is also an important difference. As noted in Poser 1984, the generalization concerning edge orientation in Japanese accent is absolute in minor phrases, but this same generalization is subject to exceptions in words. For example, Japanese has a set of so-called 'dominant' suffixes which steal accent from the stem, counter-exemplifying the claim that the leftmost accent wins. For this reason, we focus here on word accent, leaving aside the problem of initial lowering and the observed edge orientation in phrasal accent (as this accent is certainly of the restricted type). The problem of immediate interest therefore is the characterization of the fact that inherent accent is contrastive in Japanese words.

Before moving to the analysis of contrastive accent, I will briefly review some basic features of Japanese morphology which will be relevant in the subsequent analysis. ${ }^{15}$ Concerning the concatenative morphology, affixation is mostly agglutinative. While affixes of both Sino-Japanese (SJ) and native origin are common, SJ affixes tend to be more productive (as they have most recently been introduced). Japanese has a rich inventory of suffixes, several of which will be examined in chapter 5, but a great many of these tend to behave like the second member of a compound (see Appendix I of McCawley 1968 for a long list). As for the prefixes, they are fewer in number, and attach mostly to nouns, but a few do attach to adjectives, e.g., $h i$ - 'slight', $o$ - 'honorific', ko- 'slight', and $o$ - may also attach to verbs, but the result is an adjective. Japanese also has several recently borrowed English prefixes, as in suupaa- 'super-', nyu- 'new', posuto- 'post', mini'mini', and these tend to pattern with the Aoyagi prefixes (meaning that words that contain them may have two rises and falls in pitch, see $\$ 3.3 .4)$.

Another very important word-forming process in Japanese is compounding. Concerning noun-noun compounds, there appears to be two basic types (see Poser 1984: $47 \mathrm{ff}$. and Otsu 1980). ${ }^{16}$ The first type are the so-called $d v a n d v a$ compounds, as in eda-ha 'branches and leaves' (eda + ha) and kúro-siro 'black and white' (kúro + síro). As illustrated with these examples, $d v a n d v a$ compounds involve semantic conjunction of two morphemes and are characterized by the lack of Rendaku voicing in the first obstruent of the second member. Most noun-noun compounds, however, are not of the dvandva type. Non- $d v a n d v a$ compounds, referred to as 'loose compounds' by Otsu, show sequential voicing and semantically involved modification of one member by another. For example, the initial obstruent is voiced in the following examples: /ori + kami/ $\rightarrow$ ori-gami 'folding paper' and /yama + tera/ $\rightarrow$ yama-dera 'mountain temple'. While the accentuation of dvandva compounds is straightforward, following the pattern of 'leftmost accent wins' observed in minor phrases, accent in loose compounds is far less straightforward. As the proper treatment of loose compounds bears directly on the analysis of the accentuation of prefixes, this classic problem will be studied in detail in $\S 3.3 .3$ where an analysis is proposed in terms of Word Binarity constraints and default-to-opposite edge orientation.

With this linguistic background, we may now proceed to the analysis of the basic accentual system of Japanese.

\footnotetext{
${ }^{15}$ This characterization of Japanese morphology draws heavily on Kageyama 1982, Abe 1985, Shibatani $1990 \S 10$ and Bloch 1946a,b.

${ }^{16}$ Otsu actually distinguishes among three types of compounds, including a less well defined class of 'strict compounds' which are semantically opaque and do not undergo Rendaku.
} 


\subsubsection{Analysis of Japanese Word Accent ${ }^{17}$}

Consistent with the theoretical assumptions laid out in the introduction, the lexicalto-surface mappings for the content words in (34) will be represented as the input-output mappings given below. That is, inherent accent is represented with a lexical prominence (marked here with an acute accent) which is mapped onto surface forms with the corresponding prominence structure. This surface prominence structure is aligned with a pitch accent, $\mathrm{H}^{*} \mathrm{~L}$, which in conjunction to other rules of phonetic implementation, accounts for the phonetic differences among these words.

(35) Contrastive Accent in Nouns

$\begin{array}{llll}\text { /ínoti/ } & \rightarrow & \text { ínoti } & \text { 'life' } \\ \text { /kokóro/ } & \rightarrow & \text { kokóro } & \text { 'heart' } \\ \text { /atamá/ } & \rightarrow & \text { atamá } & \text { 'head' } \\ \text { /miyako/ } & \rightarrow & \text { miyako } & \text { 'city' }\end{array}$

The lexical-to-surface correspondence in prominence structure is governed by the Prosodic Faithfulness constraints (see $\$ 1.2$ for the formal definitions). In the usual way, the accentual contrasts observed in Japanese are accounted for by ranking the PROS-FAITH constraints above a set of Markedness constraints: PROS-FAITH $>$ M. The dominated Markedness constraints in Japanese are given below.

(36) Dominated Markedness Constraints

Align-L(PM, PrWd): Prominence must be aligned to the left edge of prosodic word.

Align-R(PM, PrWd): Prominence must be aligned to the right edge of prosodic word.

NONFINALITY: The head (foot, syllable) of the prosodic word must not be final.

With these Markedness constraints dominated, the accentual contrasts allowed in the input will be mapped onto corresponding outputs, as depicted below.

(37) Prosodic Faithfulness derives Positional Contrast

\begin{tabular}{|c|c|c|c|c|c|c|}
\hline Input & & Output & PROS-FAITH & ALIGN-R & ALIGN-L & NONFINAL \\
\hline \multirow[t]{2}{*}{ a. /ínoti/ } & $\rightarrow$ & ínoti & & $*$ & & \\
\hline & & *inotí & $* !$ & & $*$ & $*$ \\
\hline \multirow[t]{2}{*}{ b. /kokóro/ } & $\rightarrow$ & kokóro & & $*$ & $*$ & \\
\hline & & *kókoro & $* !$ & $*$ & & \\
\hline \multirow[t]{2}{*}{ c. /atamá/ } & $\rightarrow$ & atamá & & & $*$ & $*$ \\
\hline & & *atáma & $* !$ & $*$ & $*$ & \\
\hline
\end{tabular}

These input-output pairs do not reveal all of the intricacies of a fully articulated constraint system, which would be too cumbersome here (but see analysis of compounds presented

${ }^{17}$ This analysis presented here has been inspired by the work of Katayama 1995, 1998, Smith 1997, and Kubozono 1995. 
below for more detail). However, they do show the important rankings which will be relevant for later analysis. First, the ensemble of PROS-FAITH constraints must outrank the two Alignment constraints. If this ranking did not hold, then non-final accents in the input would be paired with right-aligned accents in the output, as in the failed candidate in (37a); and conversely non-initial lexical accents would be left-aligned (37b). It is also necessary to assume also that PROS-FAITH dominates NONFINALITY because words with accent on the last mora do not shift accent to a non-final mora (37c).

The role of the two Prosodic Faithfulness constraints, MAX-PROM and NO-FLOPPROM, is clear from this brief illustration: lexical prominences are not deleted or not shifted in these input-output pairs. The role of DEP-PROM, on the other hand, is less transparent, but still crucial in the overall analysis. High-ranking DEP-PROM accounts for the fact that a default accent is not inserted in words like miyako, which do not have a surface pitch accent. Therefore, the ranking of DEP-PROM above a constraint calling for a prominence in every word, i.e., HEADEDNESS(PrWd) of Selkirk 1995 [1996] (see $\S 1.2 .2 .3$ ), accounts for the faithfulness to the absence of underlying accent. Together with MAX-PROM, DEP-PROM effectively accounts for the second accentual contrast between accented and unaccented words.

The positional and accented/unaccented contrasts shown above motivate the ranking of the Prosodic Faithfulness constraints above a set of Markedness constraints. The limitation of accent to the initial mora of a syllable shows that PROS-FAITH is also dominated. For concreteness, I follow Haraguchi 1991 in assuming that this restriction is due to a high-ranking constraint which requires the prominence to appear on the head mora of a syllable, which in a bimoraic syllable is the first mora in Haraguchi's analysis. Thus, I assume that the 'syllable-accenting' character of Tokyo Japanese is accounted for by ranking ACCENT-TO-HEAD $(\sigma)$ above PROS-FAITH constraints, though nothing crucial hinges on this assumption.

The various Prosodic Faithfulness constraints have not thus far been distinguished for their position in the word, and as entailed by the Root-Controlled Accent hypothesis, these constraints are sensitive to the distinction between roots and affixes. Thus, Japanese, like all languages, has the constraint ranking given below.

(38) Root-Controlled Accent in Japanese

$$
\text { PROS-FAITHRoot }_{\text {R PROS-FAITH }} \text { Affix }
$$

This ranking, which is an inherent ranking that does not need to be learned in Japanese, is responsible for the preference for realizing accent in stems over accent in suffixes. Thus, on a par with the same set of results in Cupeño and Russian, inherently accented suffixes lose their accent when they attach to accented stems, as in /yóm+tára/ $\rightarrow$ yón-dara 'if he reads', because PROS-FAITHRoot is ranked above PROS-FAITH Affix. The same inherent ranking predicts that inherent accent in stems will win out over inherent accent in prefixes, and this prediction is studied in detail in the final subsection of this case study.

The ranking given below summarizes the basic results established thus far in the analysis (the four lowest rankings constraints are in an unordered stratum). 
(39) Japanese Word Accent (Interim Ranking)

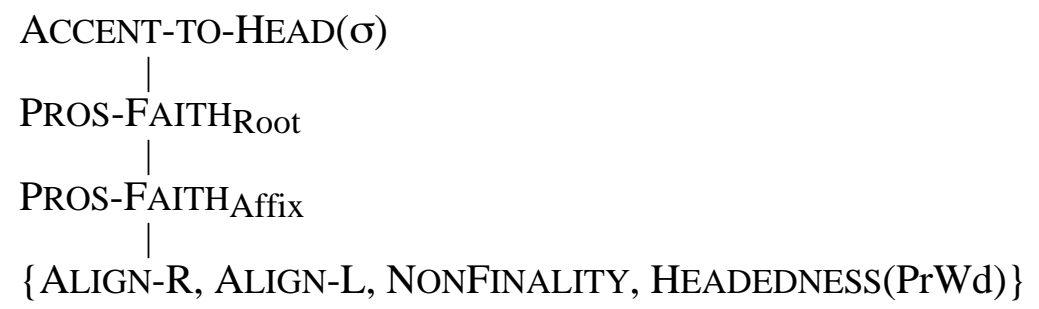

With ACCENT-TO-HEAD $(\sigma)$ ranked above the Prosodic Faithfulness constraints, lexical prominence will be limited to the first mora of a heavy syllable, the head mora of the syllable. The Prosodic Faithfulness constraints in turn dominate a set of Markedness constraints, which accounts for two kinds of accentual contrasts observed in the system. First, the contrast in the position of accent is derived by ranking PROS-FAITH above three constraints which require accent to appear at the left or right edge of the PrWd or in nonfinal positions. Second, the ranking of the Prosodic Faithfulness constraint DEP-PROM above HEADEDNESS(PrWd) accounts for the contrast between accented and unaccented words by banning the insertion of non-lexical prominence. An important empirical point is that this ranking pairs unaccented lexical words with unaccented surface forms, which will be crucial in the analysis of morphologically de-accented structures in chapter 5.

\subsubsection{Analysis of Noun-Noun Compounds}

An examination of noun-noun compounds in Japanese is essential for two reasons. First, it will enable us to establish some ranking relations that have not yet been established in the analysis of the basic facts given above. The analysis of compound accent, therefore, will clarify some important constraint rankings which will be needed in the characterization of default accentual patterns elsewhere in the system (investigated in detail in chapter 5). A second reason for studying compounds, which will become more apparent in the next subsection, is that many prefixes behave like the first member of a compound. Thus, a thorough account of prefix accent will first require an analysis of compound accent.

The accentuation of noun-noun compounds is a classical problem in Japanese phonology which has led to many contributions to the study of Japanese accent, including Martin 1952, Hirayama 1960, Chew 1964, McCawley 1968, Hiraguchi 1983, Poser 1984, 1990a, and Kubozono 1988 [1993], 1995, 1997. It is not possible to review all of this work here, nor is it desirable, as a thorough review would lead us too far afield. It is, however, necessary to be clear on some empirical matters, which involve comparing two recent analyses, namely Poser 1990a and Kubozono 1995.

Excluding dvandva compounds from the picture, the description of noun-noun compounds involves determining a 'default' position for accent; this accentual default is sensitive to the prosodic size of the second member. Thus, if the second member is 'long', i.e., trimoraic or greater, then the default position for accent is the first syllable of the second member, as shown by the behavior of compounds in which neither member has an inherent accent, e.g., /me + kusuri/ $\rightarrow$ me-gúsuri 'eye wash'. On the other hand, when the second member is 'short' (two moras or less), then the characterization of the default position depends on certain assumptions about how to treat exceptions. Following Chew 1964, Poser 1990a describes two distinct default patterns in compounds with short second position nouns: (i) word-final accent in the first member $\left(\mathrm{N}_{1}\right)$ when the second member $\left(\mathrm{N}_{2}\right)$ is unaccented (with some exceptions), as in /kábuto + musi/ $\rightarrow$ kabutó-musi 'beetle', and (ii) unaccented compounds when the second member is accented on the final syllable, 
e.g., /garasu + tamá/ $\rightarrow$ garasu-dama 'glass bead'. Kubozono 1995 reduces this two-fold pattern to a single accentual default, namely default final accent in $\mathrm{N}_{1}$, by assuming that compounds surfacing without an accent are exceptional and are treated on a par with other exceptions. I follow Kubozono's descriptive approach here because, as he shows quite convincingly, this approach enables a unification of certain generalizations about extrametrical elements. Let us first consider Kubozono's generalizations and see how this unification applies to particular examples. ${ }^{18}$

While Kubozono describes the default patterns in foot-based terms, it is possible to achieve the same descriptive insight without this construct, and this is the spirit in which the following generalization is formulated.

\section{(40) Noun-Noun Compounds in Tokyo Japanese (after Kubozono 1995)}

If the second member of a noun-noun compound has an inherent accent on a syllable other than the final syllable, keep it. Otherwise, assign a default accent.

The difference between noun-noun compounds and nouns generally is therefore that compounds introduce a kind of final syllable extrametricality; and in cases where the second noun is unaccented (either lexically or through extrametricality), a special accentual principle applies. Employing the long versus short distinction mentioned above, this principle assigns accent to a default position, which is characterized as follows.

(41) Default Accent with Long and Short Distinction (cf. Poser 1990)

a. Default with long $\mathrm{N}_{2}$ : if $\mathrm{N}_{2} \geq \mu \mu \mu$, accent the first syllable of $\mathrm{N}_{2}$.

b. Default with short $\mathrm{N}_{2}$ : if $\mathrm{N}_{2} \leq \mu \mu$, accent the last syllable of $\mathrm{N}_{1}$.

Moving now to some examples, this set of generalizations gives the correct results for compounds with a long second member, as illustrated below.

(42) Compounds with Long Second Member

$\begin{array}{llll}\text { a. /oo + kámakiri/ } & \rightarrow & \text { oo-kámakiri } & \text { 'big mantis' } \\ \text { /yámato + nadésiko/ } & \rightarrow & \text { yamato-nadésiko } \\ \text { /nise + karakása/ } & \rightarrow & \begin{array}{l}\text { nise-karakása } \\ \text { 'Japanese lady' } \\ \text { 'paper umbrella' }\end{array} \\ \text { b. /ne + syoogatú/ } & \rightarrow & \text { ne-syóogatu } & \text { 'lazy New Years holiday' } \\ \text { /áisu + koohíi/ } & \rightarrow & \text { aisu-kóohii } & \text { 'ice coffee' } \\ \text { /minami + amerika/ } & \rightarrow & \text { minami-ámerika } & \text { 'South America' }\end{array}$

Starting with the examples in (42a), the accent of $\mathrm{N}_{2}$ is non-final, and so it is preserved in the surface form. The examples in (42b), on the other hand, show the initial default pattern in words of this type. If $\mathrm{N}_{2}$ has accent on the final syllable, as in the first two examples, it cannot be preserved because of final syllable extrametricality, and so these forms receive

\footnotetext{
${ }^{18}$ While I follow Kubozono in admitting a set of exceptions to be accounted for in some other way, I will not go into them in detail here, as that would lead us too far from the focus of this section. But see Kubozono 1995 for some attractive proposals.
} 
default initial accent. Lastly, if the second member is unaccented, it also receives initial accent, as shown with minami-ámerika.

Concerning compounds with a short second member, the same basic accentual principles are at work, but we find a different default pattern. Hence, if a non-initial syllable is accented, then this accent is preserved in the output, as shown below in (43a). If, however, $\mathrm{N}_{2}$ has final syllable accent, or is unaccented, the resulting compound surfaces with final accent on the first member of the compound, a strikingly different pattern from the forms in (42) above.

(43) Compounds with Short Second Member

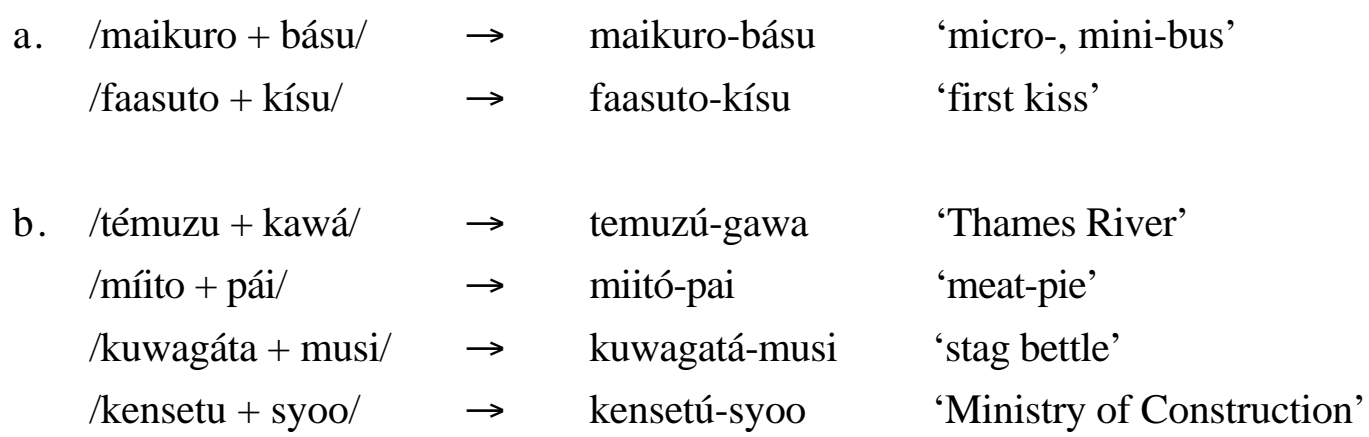

The importance of Kubozono's generalization is therefore that it employs the principle of final syllable extrametricality across the board, that is, in all noun-noun compounds (40). This approach differs, therefore, from Poser's 1990a approach in which compounds with long $\mathrm{N}_{2}$ 's are assumed to have an extrametrical foot, while short $\mathrm{N}_{2}$ 's are not subject to an extrametricality requirement. Kubozono has thus clarified a pattern that applies to compounds with both long and short second members and also broadened the empirical coverage of the analysis to cases like /nise + karakása/ $\rightarrow$ nise-karakása (where Poser's analysis predicts default initial accent). A final point is that the generalization given in (40) is formally different than Kubozono's characterization of default accent, which essentially states that default accent falls on the non-final foot derived with a foot-parsing algorithm. The system used here is descriptively identical to Kubozono's, however, because it gives the same default for compounds with short and long second members. It differs only in the theoretical assumption that the default is derived in terms of feet.

To focus the following discussion, consider the following observations.

(44) Salient Observations in Noun-Noun Compounds

a. Final Syllable Extrametricality: the final syllable of the whole compound is ineligible for accent.

b. De-Accentuation of $\mathrm{N}_{1}$ : all initial members are treated as unaccented.

c. Prosodic Minimum on Accented Noun: a preference is given to positing accent on nouns larger than two moras.

d. Default-to-Opposite Edge: if the accent occurs in $\mathrm{N}_{1}$, it is rightmost; if it occurs in $\mathrm{N}_{2}$ (and $\mathrm{N}_{2}$ is unaccented), it is leftmost.

The first two observations are rather straightforward, and it is clear how to proceed in analyzing them. Thus, following Kubozono 1995 (see also Poser 1990a), I will treat the 
extrametricality effects as the result of a NONFINALITY constraint (Prince \& Smolensky 1993), though some additional assumptions are necessary to make the analysis consistent with the constraint system given in $\$ 3.3 .2$ (see below). As for the observation in (44b), morphological processes often correlate with the neutralization of an accentual contrast; indeed, there is abundant evidence in Japanese for such a morpho-accentual process. Therefore, in completing the picture here, it seems sensible to approach this pattern as a dominance effect induced by the morphological process of compounding, an idea which is originally due to McCawley 1968 I believe. The remaining observations are somewhat more subtle, but as they too have clear precedents in the literature, the parallels found in other languages will suggest a clear line of analysis.

Starting first with (44c), the characterization of the default positions given above show a prosodic requirement on the accented noun: if it is suitably large, accent appears on the second member, but if $\mathrm{N}_{2}$ does not meet the three mora threshold, accent falls on the first member. Prosodic minima of this kind are very common cross-linguistically, and the standard treatment of this observation is in terms of a binarity requirement on the prosodic structure of the word (McCarthy \& Prince 1986 et seq., Hayes 1995, Itô \& Mester 1992). Thus, one clear line of analysis is to posit a binarity requirement of some kind on the noun which receives the accent of the larger compound. This approach will require some additional consideration of the internal structure of compounds, essentially positing the binarity requirement on the prosodic head of the compound.

The next question is, once the correct noun has been accented, where does the accent fall? The answer to this question also seems to have clear parallels in other accent systems, as the default patterns for the first and second members show a clear pattern of default-to-opposite edge accent. Thus, if the first member is accented, accent falls on the final syllable. This pattern is in contrast to the accentuation of the second member, which receives initial accent by default (in cases where lexical accent is not preserved). I analyze this pattern in the same way as in other languages, modelling this effect in terms of constraint conflict between different Alignment constraints. To sum up the new ideas, I propose to use a binarity requirement on the head of the prosodic compound to describe the prosodic minimum on the accented element; once this contextually determined head is identified, the constraint rankings for default-to-opposition edge tropism will take effect, explaining the conflicting edge effects.

Starting first with the effect of final syllable extrametricality, there are two important differences between the behavior of accent in noun-noun compounds and nouns generally that need to be addressed. First, bare nouns may have final syllable accent, but compound nouns may not. Furthermore, as shown in the previous subsection, the default accentual pattern for nouns is to be unaccented, but with compounds, the default pattern is either word-initial accent (with long $\mathrm{N}_{2}$ ) or word-final accent (with short $\mathrm{N}_{2}$ ). These disparities in the inventory of possible accent patterns and the characterization of accentual defaults call for some new theoretical developments which I will now introduce.

The presence of an accentual contrast of the final syllable in non-compound nouns shows that NONFINALITY is dominated by the Prosodic Faithfulness constraints. This ranking, however, cannot be the same ranking which is responsible for the distribution of accent in compound nouns because in this context final accent is not possible. To deal with this restriction, I propose to relativize NONFINALITY to a different prosodic category for compounds than the one already in use for bare nouns, namely PrWd. Thus, I will employ a higher level prosodic category which I will provisionally call P-Comp, for prosodic compound (see Peperkamp 1997 and references therein). The prosodic words which dominate the segments of the nouns are thus grouped together under one P-Comp, as shown below. 
(45) Proposed Structures for Compounds

a.<smiles>CCPCPC(C)=P</smiles>

b.<smiles>CCNC(=O)CPC</smiles>

As a prosodic category, P-Comp must have a head. While the best P-Comp is rightheaded, meaning the structurally subordinate PrWd head is on the right as in (45a), it may also be left-headed (45b) under duress, i.e., when the second member does not meet the prosodic size requirement for heads. The constraint interaction which derives these results will be returned to below.

Returning to the matters at hand, the proposed structures for noun-noun compounds solves the two problems outlined above in one stroke. Thus, extrametricality effects observed only in compounds may be explained by relativizing NONFINALITY to PComp and ranking this constraint as shown below. With the relativized NONFINALITYPComp constraint ranked above the Prosodic Faithfulness constraints (46a), the effect is that accent may not appear in the final syllable of compounds, but may in nouns otherwise. Furthermore, it is natural to posit a head PrWd of P-Comp. Since it is a fundamental characteristic of headed structures is to have an accent, the constraint responsible for this accent-to-head tropism may be ranked above DEP-PROM to give the required accent in compound nouns (46b).

(46) Final Syllable Extrametricality Effects

a. NONFINALITYP-Comp >> PROSFAITH: preserve the accent of $\mathrm{N}_{2}$, unless it appears in the final syllable of the compound.

b. ACCENT-TO-HEAD(P-Comp) > DEP-PROM: the head of the compound must have an accent.

The following two tableaux illustrate the results of these rankings. Starting with (46a), because NONFINALITYP-Comp dominates the Prosodic Faithfulness constraints, final syllable accent is not allowed in this construction, as illustrated below. Accent in the second member is preserved if the satisfaction of Faithfulness does not lead to a violation of NONFINALITYP-Comp, as in the input-output pairs in (47a) and (47b). On the other hand, if satisfaction of PROS-FAITH leads to violation of NONFINALITYP-Comp, a default pattern is assigned, as in (47c) and (47d). 
(47) Final Syllable Extrametricality Effects

\begin{tabular}{|c|c|c|c|}
\hline Input & Output & NONFINALP-Comp & PROS-FAITH \\
\hline \multicolumn{4}{|c|}{ a. /yámato + nadésiko/ $\rightarrow$ yamato-nadési $\langle$ ko $\rangle$} \\
\hline & *yamato-nádesi $\langle\mathrm{ko}\rangle$ & & $* !$ \\
\hline \multirow[t]{2}{*}{ b. /nise + karakása/ } & $\rightarrow$ nise-karaká $\langle\mathrm{sa}\rangle$ & & \\
\hline & *nise-káraka $\langle$ sa $\rangle$ & & $* !$ \\
\hline \multirow[t]{2}{*}{ c. /ne + syoogatú/ } & $\rightarrow$ ne-syóoga $\langle\mathrm{tu}\rangle$ & & $*$ \\
\hline & $*^{*}$ e-syooga $\langle$ tú $\rangle$ & $* !$ & \\
\hline \multirow[t]{2}{*}{ d. /áisu + koohíi/ } & $\rightarrow$ aisu-kóo $\langle$ hii $\rangle$ & & $*$ \\
\hline & *aisu-koo〈híi $\rangle$ & $* !$ & \\
\hline
\end{tabular}

The second ranking in (46) describes the fact that compounds are, as a rule, always accented. Thus, by ranking ACCENT-TO-HEAD(P-Comp) above DEP-PROM, the PrWd head of the P-Comp must have an accent, as shown below. (The head of the P-Comp is underlined.)

(48) Obligatory Accent Insertion in Compounds

\begin{tabular}{|c|c|c|}
\hline /minami + amerika/ & ACCENT-TO-HEAD(P-Comp) & DEP-PROM \\
\hline$\rightarrow$ minami-ámerika & & * \\
\hline minami-amerika & $* !$ & \\
\hline
\end{tabular}

To summarize, the typical pattern for compounds is for them to be accented, which differs from bare nouns, which are preferentially unaccented in some contexts because DEP-PROM dominates ACCENT-TO-HEAD(PrWd). Furthermore, noun-noun compounds differ from nouns in general because they are subject to final syllable extrametricality; this fact requires the domination of the PROS-FAITH constraints by the NONFINALITY constraint specific to compounds.

Moving next to the problem of the distinct default positions for accent, as alluded to above, I propose to treat this fact by assuming that long and short compounds are prosodized differently, and as a result, these different prosodic structures yield two distinct defaults. The structural differences stem from a contextually determined position for the head PrWd, which is derived by the following prosodic well-formedness constraint.

(49) WORD-BINHead (after Itô \& Mester 1992)

The head PrWd of the prosodic compound must be binary; in effect, the PrWd prosodic head must be at least trimoraic.

Like the prosodic foot, the prosodic word subordinate to P-Comp is subject to a binarity requirement, essentially requiring a branching structure at the level directly below the prosodic word. The effect of this constraint, when applied to the head PrWd, is that this prosodic head must either consist of a pair of feet, or a foot plus a syllable, giving the effect that the head can be no less than trimoraic (assuming that feet must be binary as well). The constraint WORD-BINARITYHead can therefore be employed in explaining the distinction between short and long $\mathrm{N}_{2}$ 's: the three mora minimum needed to satisfy this constraint is exactly on a par with the requirement for the second member to be 'long'. By ranking the 
Word Binarity constraint relative to another constraint, ALIGN-R-HEAD, the contextually determined headedness effect mentioned above can be straightforwardly derived. Thus, the force of ALIGN-R-HEAD is that the PrWd head of the compound would like to be the second member, which gives $\mathrm{N}_{2}$ as the head when $\mathrm{N}_{2}$ is big enough, i.e., three moras or more, as in (50a). When $\mathrm{N}_{2}$ is less than trimoraic, however, it cannot be the head PrWd

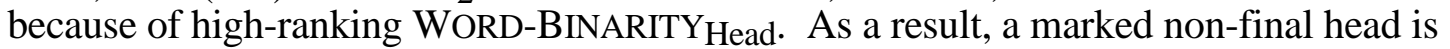
chosen, as illustrated in (50b). ${ }^{19}$

(50) Headedness in Noun-Noun Compounds
a. $\quad$ Long Second Member
b. Short Second Member
$\left[\ldots[\mu \mu \mu \ldots]_{\mathrm{Hd}}\right]$
$\left[[\ldots]_{H d} \mu(\mu)\right]$

This proposal is illustrated with the constraint interaction depicted in the following tableau.

(51) Long/Short Distinction via Word Binarity

\begin{tabular}{|c|c|c|c|}
\hline Input & Output & WORD-BIN Head & ALIGN-R-HEAD \\
\hline \multirow[t]{2}{*}{ a. /yámato + nadésiko/ } & $\rightarrow \quad$ [yamato-[nadesiko]] & & \\
\hline & [[yamato]-nadesiko] & & $* !$ \\
\hline \multirow[t]{2}{*}{ b. /míito + pái/ } & $\rightarrow$ [[miito $]-p a i]$ & & $*$ \\
\hline & [miito-[pai]] & $* !$ & \\
\hline
\end{tabular}

When both the first and second member of a compound are suitably binary, the grammar chooses in favor of a rightmost PrWd head, as shown by the input-output mappings in (51a). But when the preferred PrWd is not large enough, a marked position for the head is chosen, as in (51b).

With these assumptions in place, we are in a position to explain the two default positions for accent in compounds in terms of a phenomenon which has clear parallels in other languages, namely default-to-opposite edge tropism. The analysis unfolds as follows. Accent must be a property of the PrWd head by ACCENT-TO-HEAD(P-Comp), and so when $\mathrm{N}_{2}$ is long, $\mathrm{N}_{2}$ must be accented in order to satisfy this constraint. When $\mathrm{N}_{2}$ is short, on the other hand, $\mathrm{N}_{1}$ is the head, and so it must be accented. Thus, as illustrated below, by assuming that the default position for accent is at the right edge of the PrWd, in compounds with a short $\mathrm{N}_{2}$, the accent will be rightmost in the head PrWd by default (52a). In compounds with long $\mathrm{N}_{2}$ 's, however, $\mathrm{N}_{2}$ is the head, and so accent is assigned to the second member. But in just this context, accent cannot fall on the final syllable because the final syllable is extrametical. Accent cannot be rightmost, so it defaults to the left edge (52b).

\footnotetext{
${ }^{19}$ Concerning the prosodic analysis of compounds with sub-minimal nouns, there are at least two options: (i) they can be directly associated with the P-Comp, or (ii) they can be grouped into a separate PrWd which is in turn dominated by P-Comp. As I do not know of any empirical evidence which can decide between these two analyses, I leave both options open.
} 
(52) Divergent Accentual Defaults as Default-to-Opposite
a. Rightmost Accent with Short $\mathrm{N}_{2}$
b. Leftmost Accent with Long $\mathrm{N}_{2}$
$[[\underline{\sigma} \ldots \stackrel{\sigma}{\sigma}]+\mu(\mu)]$
$\left[\ldots+\left[\underline{\downarrow} \ldots\langle\underline{\sigma}\rangle_{-}\right]\right]$

Let us formalize these ideas with some constraint rankings. Following Baković 1998, I assume that default-to-opposite edge accent follows as a consequence of the domination of one Alignment constraint, which in turn activates a lower-ranking Alignment constraint (see also Samek-Lodovici 1998 and Nelson 1998 for related results and Kenstowicz 1995b and Zoll 1997 on the role of conflicting Alignment constraints in default-to-opposite stress). In particular, the rankings required for 'default rightmost accent, otherwise, leftmost accent', are given below.

(53) Default-to-Edge Orientation in Japanese Compound Accent

NONFINALITYP-Comp $\gg$ Align-R(PROM, PrWd) $\gg$ Align-L(PROM, PrWd)

Before demonstrating this result, however, it is necessary to account for the absence of an accentual contrast in the first member. That is, in contrast to $\mathrm{N}_{2}$, compounding generally suppresses the lexical accents of $\mathrm{N}_{1}$, in effect making all first members unaccented. Following McCawley 1968 and Kurata 1984, I analyze this as a dominance effect, i.e., a deletion of accent that is triggered by the application of the morphological process itself. As this kind of morpho-accentual process is the topic of a discussion in chapter 5, I will not go into the details of the analysis here, except to say that there is a rankable constraint, DE-ACCENT- $\mathrm{N}_{1}$, which causes de-accentuation of the first member of a compound because it is ranked above the Prosodic Faithfulness constraints.

(54) Dominance Effect in First Member

DE-ACCENT-N $1>$ PROS-FAITH

Now that all of the ranking arguments have been established, I will demonstrate the results of this system with a series of tableaux. Starting first with compounds that have short $\mathrm{N}_{2}$ 's, as shown in the tableau below, when the first member is unaccented, the expected pattern is the observed one, namely rightmost accent in the word (55b). Putting accent on the final syllable of the whole compound, a different rightmost accent, results in a violation of both ACCENT-TO-HEAD(P-Comp) and NONFINALITYP-Comp, and so this option is ruled out (55a). Furthermore, initial accent in $\mathrm{N}_{1}$ is also marked because it results in a violation of ALIGN-R, which is ranked above ALIGN-L, and so this candidate is eliminated as well (55c).

(55) Rightmost Default with Short Second Member ( $\mathrm{N}_{1}$ is Unaccented.)

\begin{tabular}{|c|c|c|c|c|}
\hline /kensetu + syoo/ & ACCENT-TO-HEAD & NONFINAL & ALIGN-R & ALIGN-L \\
\hline a. $\quad[[$ kensetu $]$-syóo $]$ & $* !$ & $*$ & & \\
\hline b. $\rightarrow$ [[kensetú]-syoo $]$ & & & & $*$ \\
\hline c. $\quad[$ kénsetu $]$-syoo $]$ & & & $* !$ & \\
\hline
\end{tabular}

Rightmost accent in $\mathrm{N}_{1}$ is also predicted if this noun is accented on a non-final syllable because it will be de-accented by the constraint ranking given in (54), but to compare the 
compound structures above with some related compounds with accented $\mathrm{N}_{2}$ 's, I return to this result below.

Moving next to compounds with long second members, when such an $\mathrm{N}_{2}$ has final syllable accent, rather than being faithful to this lexical accent, which would violate highranking NONFINALITYP-Comp (56a), an initial accent is posited, because this candidate fares better on the low-ranking constraint ALIGN-L (56c). Importantly, I assume that violations of the Alignment constraints here are interpreted categorically (see Zoll 1996a for motivation and discussion), and thus, a close-to-rightmost accent, as in candidate (56b), is not good enough. Finally, ALIGN-R cannot be satisfied here by positing an accent in the first member, as in (56d), because this option puts accent on a non-head, which violates ACCENT-TO-HEAD(P-Comp).

(56) Leftmost Default with Long Second Member ( $\mathrm{N}_{2}$ is Accented.)

\begin{tabular}{|c|c|c|c|c|}
\hline /ne + syoogatú/ & ACCENT-TO-HEAD & NONFINAL & ALIGN-R & ALIGN-L \\
\hline a. ne-syooga $\langle\underline{\text { tú }}\rangle$ & & $* !$ & & * \\
\hline b. ne-syoogá $\langle\underline{\text { tu}}\rangle$ & & & $*$ & $* !$ \\
\hline c. $\rightarrow$ ne-syóoga $\langle\underline{\mathrm{tu}}\rangle$ & & & $*$ & \\
\hline d. né-syooga $\langle\underline{t u}\rangle$ & $* !$ & & & \\
\hline
\end{tabular}

Thus, while the language on a whole prefers rightmost accent, in compounds with a final head, the extrameticality requirement triggers a default-to-opposite edge effect.

Significantly, this result holds regardless of whether the final syllable is inherently accented or not: compounds with unaccented second members also receive default initial accent, as the following tableau shows.

(57) Leftmost Default with Long Second Member ( $\mathrm{N}_{2}$ is Unaccented.)

\begin{tabular}{|l|c:c|c|c|}
\hline$/$ minami + amerika/ & ACCENT-TO-HEAD & NONFINAL & ALIGN-R & ALIGN-L \\
\hline \hline a. $\quad$ minami-ameriká & & $* !$ & & $*$ \\
\hline b. minami-ameríka & & & $*$ & $* * !$ \\
\hline c. $\rightarrow$ minami-ámerika & & & $*$ & \\
\hline d. $\quad$ minamí-amerika & $* !$ & & & \\
\hline
\end{tabular}

Consistent with the above results, accent is assigned to the head PrWd, hence ruling out (57d). And since ALIGN-R cannot be satisfied because of final syllable extrametricality, the winner is the form with initial accent on the head prosodic word because this candidate does best on ALIGN-L.

The same principles of default edge effects are predicted in structures which are deaccented, as with compounds that have accented $\mathrm{N}_{1}$ 's (and short $\mathrm{N}_{1}$ 's, which trigger the non-standard left-headed structure). Thus, when the non-final accent of $\mathrm{N}_{1}$ is deleted, ruling out fully faithful accentuation (58a), the resulting pattern is default right-aligned accent (58b), as observed above with lexically unaccented $\mathrm{N}_{1}$ 's. 
(58) Dominance Effect in First Member

\begin{tabular}{|l|c|c|c|c|}
\hline /kuwagáta + musi/ & DE-ACCENT-N & PROS-FAITH & ALIGN-R & ALIGN-L \\
\hline \hline a. $\quad$ kuwagáta-musi & $* !$ & & & \\
\hline b. $\quad$ kúwagata-musi & & $*$ & $* !$ & \\
\hline c. $\rightarrow$ kuwagatá-musi & & $*$ & & $*$ \\
\hline
\end{tabular}

This result is in fact consistent with a general prediction made in chapter 5 , namely that morphologically triggered de-accentuation of this kind always brings about a default pattern of accent. De-accentuation in compounds is thus consistent with this prediction because it causes default right-aligned accent.

A final puzzle to be dealt with is the Faithfulness effect in compounds like the following: /maikuro + básu/ $\rightarrow$ maikuro-bá $\langle s u\rangle$. Such cases have a short second member, and so the first noun is the head of the prosodic compound. Given the ranking of ACCENT-TO-HEAD(P-Comp) over Prosodic Faithfulness, the system currently predicts that accent should fall somewhere in the first member, since it is the head PrWd. This case can be straightforwardly accounted for, however, if we employ the distinction used throughout this thesis, namely the one between the anti-deletion constraint, MAX-PROM, and the antiinsertion constraint, DEP-PROM. Specifically, the desired result can be achieved by ranking these two constraints as shown below.

(59) Evidence for the Distinction between MAX-PROM and DEP-PROM

\begin{tabular}{|l|c|c|c|}
\hline /maikuro + básu/ & MAX-PROM & ACCENT-TO-HEAD & DEP-PROM \\
\hline \hline a. $\rightarrow$ maikuro-bá $\langle$ su $\rangle$ & & $*$ & \\
\hline b. maikuró-ba $\langle\mathrm{su}\rangle$ & $* !$ & & $*$ \\
\hline
\end{tabular}

Walking through the above candidate set, the loser is out because it fails to realize the inherent accent in básu and the constraint governing the realization of inherent accent, MAX-PROM, is ranked above ACCENT-TO-HEAD(P-Comp). The winner therefore is the form that has an accent outside of the head PrWd as a means of satisfying this high-ranking Faithfulness constraint. Crucially, this Faithfulness effect only holds with inherent accent in the second member of a compound, as lexical accents in the first member are wiped out by DE-ACCENT-N $\mathrm{N}_{1}$. Because of this last ranking, inherent accent in the first member is not realized when $\mathrm{N}_{2}$ is long, which accords with the observed facts.

To summarize the above constraint rankings, I have argued for the following partial ordering of constraints. 
(60) Summary Rankings for Noun-Noun Compounds

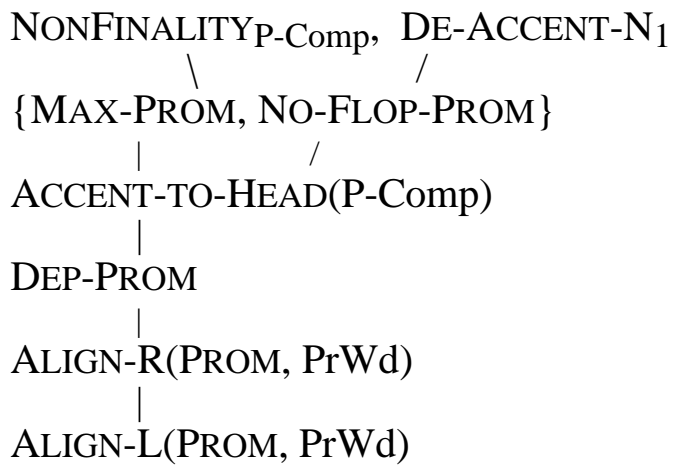

The second column, with the Word Binarity constraint top-ranked, accounts for the position of the head in the prosodic compound, which was shown to be sensitive to the size of the second member. This context-sensitive headedness effect in turn leads to the analysis of the divergent default patterns in terms of default-to-opposite edge orientation. Because ALIGN-R dominates ALIGN-L, as shown in the first column, the accentual default in compounds with initial heads is rightmost in the word. But when the head is final in the word, ALIGN-R cannot be fully satisfied, and so the lower-ranking Alignment constraint, ALIGN-L, takes effect, giving leftmost accent in the word. The remaining rankings dealt with some further peculiarities of the compound accent rule. For example, the highranking constraint DE-ACCENT- $\mathrm{N}_{1}$, accounts for the neutralization of accentual contrast in the first member, which appears to be a necessary stipulation. Finally, the ranking of ACCENT-TO-HEAD(P-Comp) between the two Faithfulness constraints is needed to account for the fact that accent insertion is a possible means of satisfying the imperative to put accent in the head PrWd, while deletion of inherent accent (in the second noun) is not.

Before moving on to examine prefixing structures, it is worth mentioning some of the advantages of the analysis proposed here. First, this analysis handles a complex set of phenomena with arguably universal constraints. Thus, many of the basic ideas used here, e.g., word binarity, default-to-opposite, and dominance effects, have clear parallels in other systems, and I relate the components of Japanese compound accent to these other phenomena by using the same, or formally related, well-formedness constraints. The analysis therefore differs from the one offered in Kubozono 1995 which treats the two default patterns in terms of a non-standard interpretation of Alignment constraints.

The second important advantage of the analysis proposed here is that it clarifies the constraint rankings which characterize the accentual defaults elsewhere in the language. Thus, in addition to the accentual default observed above in underived words, i.e., unaccented structures, there are two additional defaults which crop up in different corners of Japanese morpho-phonology. 
(61) Accentual Defaults Elsewhere in Japanese (cf. Poser 1984)

a. Default Structure 1: unaccented words, pattern resulting from dominant unaccented affixes, main pattern of exceptions in compounds

b. Default Structure 2: rightmost accent, as in first member of noun-noun compounds, the position to which accent is shifted in word with accent-shifting suffixes, many deverbal nouns, (ante-) penultimate accent in loans, verbs, adjectives

c. Default Structure 3: leftmost accent, as in second member of compounds, some deverbal nouns, and some loans

As we will see in chapter 5, the default rightmost/leftmost accent is also important in the outcome of certain accent shifts triggered by suffixation, which falls out from the constraint rankings given here because the suffixed structures are compound-like. Thus, when the accent falls on the first member, the accent is rightmost in the PrWd, but when it occurs on the second member, it is leftmost, exactly as we have seen above in noun-noun compounds. Deverbal nouns also support the right edge default pattern here, as this is the main pattern when an accented verb is changed to a noun, e.g., hiraki 'opening, closet', cf. hiráku 'open'. Deverbal nouns formed from accented verbs may also have initial accent, perhaps showing variation in the application of NONFINALITY $(\sigma)$ in these cases. Thus, if ALIGN-R dominates ALIGN-L, and the accentuation of the verb must change (a typical affixcontrolled process) then the prediction is that the accent of the verb base will gravitate to the right edge of the word in forming a noun. Or, if this is not possible because of $\operatorname{NONFINALITY}(\sigma)$, then accent defaults to the left edge of the word. Clearly these sketches do not cover all the morpho-accentual processes in Japanese, but the characterizations of the different defaults deriving from the analysis of compounds given here do seem helpful in accounting for other morpho-accentual processes, and are therefore worth pursuing further. ${ }^{20}$

\subsubsection{Influences of Prefixation on Word Accent ${ }^{21}$}

Now that an analysis of compound accent has been proposed, some further implications of the Root-Controlled Accent hypothesis can be examined in more detail. In particular, the accentual behavior of prefixes will be studied, as the interaction between prefixes and the bases to which they attach is directly relevant for the RCA hypothesis. The basic empirical finding here is that prefixes in Japanese may be sorted into three welldefined classes with respect to their accentual behavior: (i) prefixes which behave like the first member of a compound, (ii) dominant post-accenting prefixes which delete the accent of the base and bring about stem-initial accent, and (iii) so-called 'Aoyagi prefixes' which induce a prosodic organization of the word such that it has two minor phrases. I will show in this subsection that the behavior of these prefix classes is consistent with the RCA hypothesis, and thus, that this restrictive hypothesis may be maintained.

\footnotetext{
${ }^{20}$ One interesting problem which is not straightforwardly predicted here is the pattern of pre-antepenultimate accent in loans ending in either LLH or HLH ( $\mathrm{L}=$ light, $\mathrm{H}=$ heavy), a pattern which is uncovered and analyzed in Katayama 1995, 1998. The problem here is how to account for the variation between preantepenultimate, as in herikóputaa 'helicopter' and antepenultimate, as in bitámiN 'vitamin'. Katayama argues that this variation requires a gradient Alignment constraint for the ante-penultimate pattern, which is in different from the analysis of noun-noun compounds here, which relies on a categorical interpretation of Alignment violations.

${ }^{21}$ The examples presented in this section come from various Japanese dictionaries, namely Masuda 1974, NHK 1985, and Akinage 1981, and have been checked with at least two native speakers of Tokyo Japanese.
} 
At first blush, there seem to be a few examples that provide direct evidence against the RCA hypothesis. Thus, there are some examples that show that prefixation leads to the deletion of the accent of the stem, e.g., sin-getsu 'new moon' and kó-bari 'little needle', cf. -gétsu 'moon' and hári 'needle'. This deletion of the stem accent could be accounted for by assuming $k o$ - is inherently accented: if this prefix is accented, the loss of accent on the stem can be explained by the same pattern of edge orientation observed in minor phrases, namely the 'leftmost inherent accent wins'. If this analysis is the correct one, then it constitutes a clear counter-example to the Root-Controlled Accent hypothesis, which states that edge effects such as these are restricted to contexts where Faithfulness is not relevant. Faithfulness is surely relevant here because this prefix-stem sequence provides a situation of constraint conflict between Root and Affix Faithfulness, on a par with the analysis of these same sequences in Cupeño.

The analysis which uses a principle of leftward edge orientation, however, is almost certainly incorrect for these examples, and many others like them. As I will argue directly below, an analysis based on the theory of compound accent presented above is superior in several ways. Thus, the words which have the prefixes sin- and ko- behave exactly like compounds, according to an independently testable set of criteria. Therefore, the analysis in terms of compound accent is more explanatory than the alternative sketched above because it unifies this cluster of properties in a cogent analysis.

Before we can study the accentuation of prefixes, however, we require a set of criteria for diagnosing compounds. The following list is based on the criteria given in Otsu 1980 and Poser 1984, 1990a, modified to account for the new empirical generalizations clarified in Kubozono 1995.

\section{(62) Properties of Noun-Noun Compounds}

a. Semantic modification or complementation (MOD)

b. Rendaku Voicing (REND)

c. Distinction between short and long second members $(\mathrm{S} \neq \mathrm{L})$

d. Final syllable extrametricality $(\langle\sigma\rangle \#)$

e. Medial accent in $\mathrm{N}_{2}(\ldots \sigma o ́$ o...)

Noun-Noun compounds, ignoring dvandva compounds, involve the semantic modification or complementation of one member, distinguishing compounding from other types of morphological rules. This test is of course not a two-sided test, as noncompounds may also involve semantic modification, but I mention it here because this diagnostic correlates reasonably well with other test results. A second, more reliable test, is the existence of Rendaku voicing, i.e., the voicing of the first obstruent of the second member of the compound. I know of no other morphological rules which induce this pattern of obstruent voicing, so Rendaku is a very good test of the status of a derived form as a compound. Third, as exemplified in the above discussion, compounds are sensitive to the short/long distinction in the second position noun, and so differences in the accentual patterns dependent on this size distinction also provide a nice diagnostic for compound hood. Compounds are also distinguished from (some) non-compounds by final syllable extrametricality effects, and so this feature constitutes a fourth test for the status of a form as a compound. To clarify the diagnostic pattern of this test, if the second member of a compound has final syllable accent which defaults to initial accent, then the compound shows extrametricality effects of the right type. A final criterion for compounds, which will be shown to distinguish compounding from simply the attachment of a dominant suffix, is the existence of an accentual prominence on a medial syllable in the second 
member. Thus, if $\mathrm{N}_{2}$ has accent on a non-final, non-initial syllable, we cannot say that a prefix attached to this $\mathrm{N}_{2}$ is accent-deleting, as is observed in dominant, post-accenting prefixes like $m a$ - 'true' (examples given below), because this inherent accent would be lost in a word-medial position. With these tests in hand, I will now show how many productive prefixes in Japanese clearly behave like compounds.

Starting with the prefix sin- 'new', consider the following data with the set of tests presented above in mind. The examples presented below, and throughout this discussion, are sorted by the accentual properties and the prosodic size of the second member, as this organization is most revealing for our concerns. Specifically, compounds formed with an unaccented $\mathrm{N}_{2}$ are opposed to compounds with accented $\mathrm{N}_{2}$ 's, i.e., (a) and (c), versus the (b) and (d) examples. Also, compounds formed with short $\mathrm{N}_{2}$ 's, the (a) and (b) examples, are contrasted with forms that have long second members, i.e., the (c) and (d) forms.

(63) Examples with sin- as the First Member of a Compound

\begin{tabular}{|c|c|c|c|}
\hline a. kao & sin-gao & 'face' & $\left(-\operatorname{acc}\right.$, short $\left.\mathrm{N}_{2}\right)$ \\
\hline kyoku & sin-kyoku & 'song, music' & \\
\hline gara & sin-gara & ‘pattern’ & \\
\hline iri & sin-iri & 'enter, come' & \\
\hline -syo & sin-syo & ‘book’ & \\
\hline kabu & sín-kabu & 'stock' & \\
\hline án & sin-an & 'idea' & $\left(+\right.$ acc, short $\left.\mathrm{N}_{2}\right)$ \\
\hline géki & sin-geki & 'play' & \\
\hline mé & sin-me & 'bud' & \\
\hline katá & sin-gata & 'style' & \\
\hline -gétu & sín-getu & 'moon' & \\
\hline -nén & sín-nen & 'year' & \\
\hline -réki & sin-reki & 'year' & \\
\hline kabuki & sin-kábuki & 'kabuki' & $\left(-\operatorname{acc}\right.$, long $\left.\mathrm{N}_{2}\right)$ \\
\hline kansen & sin-kánsen & 'kansen' & \\
\hline keikoo & sin-kéikoo & 'tendency' & \\
\hline kenzai & sin-kénzai & 'material' & \\
\hline gakki & sin-gákki & 'semester' & \\
\hline kiroku & sin-kíroku & 'record' & \\
\hline zidai & sin-zídai & 'time, period' & \\
\hline seikatu & sin-séikatu & 'life' & \\
\hline tairiku & sin-táiriku & 'continent' & \\
\hline hakken & sin-hákken & 'discovery' & \\
\hline hatumei & sin-hátumei & ‘invention’ & \\
\hline hossoku & sin-hóssoku & 'start' & \\
\hline kufuu & sin-kúfuu & 'device, gadget' & \\
\hline
\end{tabular}




$\begin{array}{lll}\text { d. kénpoo } & \text { sin-kénpoo } & \text { 'constitution' } \\ \text { zítai } & \text { sin-zítai } & \text { 'situation' } \\ \text { syotái } & \text { sin-zyótai } & \text { 'household' } \\ \text { séido } & \text { sin-séido } & \text { 'regulation' } \\ \text { séifu } & \text { sin-séifu } & \text { 'government' } \\ \text { kanazúkai } & \text { sin-kanazúkai } & \text { 'kana usage' } \\ \text { yasúne, yasune } & \text { sin-yasúne } & \text { 'low (finance)' } \\ \text { tísiki } & \text { sin-tísiki } & \text { 'knowledge' } \\ \text { hikúne } & \text { sin-hikúne } & \text { 'low (finance)' } \\ \text { takáne } & \text { sin-takáne } & \text { 'high (finance)' } \\ \text { kígen } & \text { sin-kígen } & \text { 'epoch' } \\ \text { kotengákuha } & \text { sin-kotengákuha } & \text { '(Neo-)Classical School' }\end{array}$

The finding here is that these forms pass all the tests for compounds. Thus, sinclearly modifies the second member in a way that is consistent with other compounds. For example, the attachment of sin- to kao only changes the meaning of kao in that the resulting word sin-gao refers to those faces which are new. Furthermore, there are some examples showing Rendaku voicing, as in sin-gao, sin-basi in(63a), and an example from (63d), sinzyótai. It is important to note here the existence of forms where Rendaku is not observed, but predicted on a purely phonological basis, e.g., sin-kéikoo in (63c), does not confound this result because Rendaku is actually not predicted here. As noted in Itô \& Mester 1986, Rendaku is only observed in compounds formed with components from the Yamato stock, and so cases like these do not tell us anything, as -keikoo is a Sino-Japanese stem. The existence of native stems which undergo Rendaku, however, is revealing because they are only consistent with an analysis as the second member of a compound.

Moving now to the tests which assess accentual features, sin- passes all of these tests too. Hence, by comparing the forms in (63a) and (63b) with those in (63c) and (63d), one observes a clear contrast in the accentual patterns which is dependent on the short/long distinction: the compounds with short $\mathrm{N}_{2}$ 's are either unaccented or have accent on the prefix, whereas the forms with long second members always have a non-final accent on $\mathrm{N}_{2}$. Furthermore, these patterns are clearly consistent with a treatment in terms of the accentual defaults observed above in compounds. The forms with short $\mathrm{N}_{2}$ 's have a default word-final accent in $\mathrm{N}_{1}$, or no accent at all, which is also an independently observed pattern with compounds of this type. In the forms with long second members, on the other hand, the default position is clearly the initial syllable of $\mathrm{N}_{2}$, as shown by the examples in (63c) with unaccented bases, and one form in (63d) which has final accent on the base, but initial accent in the compound, namely sin-zyótai from syotái. This last fact is evidence for final syllable extrametricality, the fourth test for compounds. Finally, it is clear that sin-is not simply a dominant prefix, which would destroy the inherent accent of the second word, as there are several examples in (63d) with a non-initial accent in both the base and the derived form, e.g., sin-takáne from takáne. If sin- was a dominant prefix, we would expect either accent on the prefix if the prefix itself was accented, or accent on the initial syllable if it were dominant, post-accenting. However, neither of these patterns are observed, and so it is clear that sin-behaves like the first member of a compound.

Many of the features of compounds, like those found in words with sin-, overlap with words formed with dominant, post-accenting prefixes, and so it is worthwhile contrasting what appears to be a case of the latter with the sin-words from above, in an effort to tease these two patterns apart. Consider the data below for the prefix $m a$ - 'true', which is argued in Poser 1984 to be a dominant (i.e., accent-deleting), post-accenting prefix. 
(64) Dominant Post-accenting $m a-$ (examples from Poser 1984: 57, with some additions)

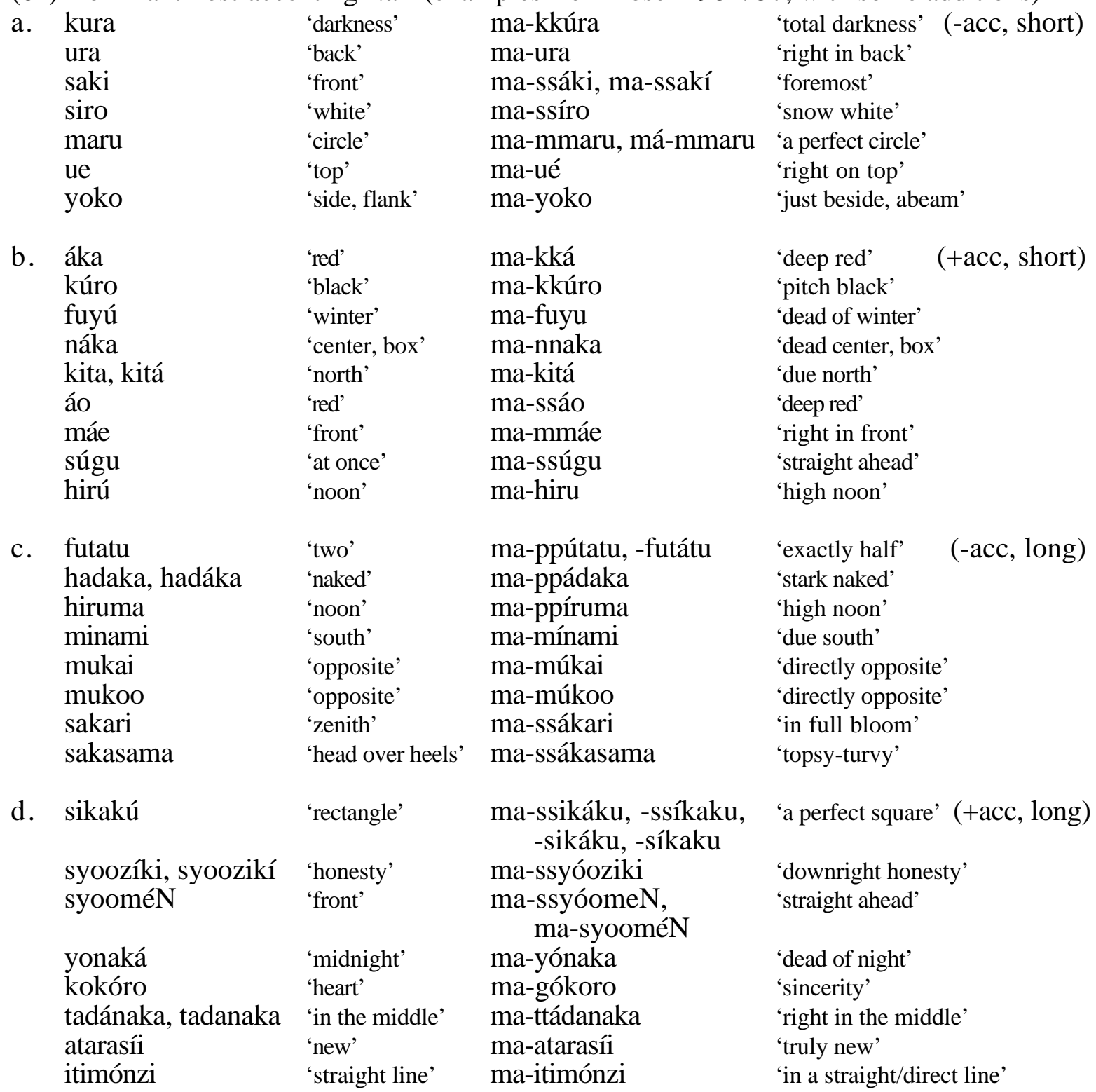

First off, the absence of any examples showing Rendaku voicing is suggestive that these words are not compounds. Furthermore, several examples have accent on the final syllable, e.g., ma-kitá in (64b) and ma-atarasí in (64d), which is another clue that these cannot be compounds because compounds are subject to final syllable extrametricality. This last fact is also not consistent with $m a$ - as a dominant post-accenting morpheme, as the analysis predicts stem-initial accent across the board, but as noted by Poser, there are some exceptions which show that this prefix vacillates between being recessive or dominant. Finally, there is one form in (64d), namely ma-gókoro from kokóro, that shows deletion of a medial accent, which is only consistent with an analysis in terms of a dominant prefix, a conclusive piece of evidence. Together with the other body of facts, this fact shows that words with $m a$ - are not compounds, but rather the result of dominant post-accentuation.

Much of the discussion that follows provides further evidence for the type of prefixes exemplified above with sin-; the reader more directly interested in the argument for 
the RCA hypothesis may press on to the chart below in (69) which summarizes this evidence.

Two additional prefixes which exhibit precisely the accentual behavior observed in compounds are the diminutive prefix $k o$ - and the augmentative prefix oo-. Both cases involve simple modification of the base to which they attach, and as can be seen from the examples in (65) and (66) below, both cases of prefixation correlate with instances of Rendaku voicing. Moreover, both $\mathrm{ko}$ - and oo- correlate with the accentual alternations predicted by the principles of compound accent. Starting first with $k o-$, there is a clear contrast between the forms with a short second member (65a-b) and those with a long second member $(65 c-d)$. The former cases have the defaults characteristic of this type of compound, namely final accent in the first component or they are unaccented.22 In contrast, the (65c-d) forms show the accentual patterns characteristic of their type, i.e., faithfulness to the lexical accent, unless the form is unaccented or accented on the final syllable, in which case you get initial accent in the second member.

${ }^{22}$ This is the main exceptional pattern in words with short second members. Though not treated in the previous section, this outcome is not unexpected given the role of DEP-PROM in yielding unaccented words. 
(65) Examples with ko- as First Member of Compound

\begin{tabular}{|c|c|c|c|}
\hline a. hati & kó-bati & 'bowl' & $\left(-\right.$ acc, short $\left.N_{2}\right)$ \\
\hline kabu & kó-kabu, ko-kabu & 'radish' & \\
\hline hako & kó-bako & 'box' & \\
\hline age & ko-age & 'unloading /dockhand' & \\
\hline eda & ko-eda & 'twig' & \\
\hline sika & ko-zika & 'deer/fawn' & \\
\hline sima & ko-zima & 'island' & \\
\hline nami & ko-nami & 'wave' & \\
\hline áyu & ko-ayu & ‘sweetfish’ & $\left(+a c c\right.$, short $\left.\mathrm{N}_{2}\right)$ \\
\hline áza & ko-aza & '(sub)section of village' & \\
\hline té & ko-te, kó-te & 'hand' & \\
\hline isí & ko-isi & 'rock' & \\
\hline imó & ko-imo & 'potato' & \\
\hline kikú & ko-giku & 'mum’ & \\
\hline báka & ko-baka & 'look down on' & \\
\hline hán & kó-ban & 'stamp/small oval gold coin' & \\
\hline hári & kó-bari & 'needle' & \\
\hline táti & kó-dati & 'sword' & \\
\hline áme & ko-same & 'rain' & \\
\hline atari & ko-átari & 'hit/beat around the bush' & $\left(-\operatorname{acc}\right.$, long $\left.\mathrm{N}_{2}\right)$ \\
\hline hanasi & ko-bánasi & 'story' & \\
\hline hasira & ko-básira & 'pillar' & \\
\hline saiku & ko-záiku & 'workmanship' & \\
\hline temari & ko-démari & 'hand ball/spirea' & \\
\hline doogu & ko-dóogu & 'stage props' & \\
\hline eguri & ko-éguri & 'scooping/cavetto' & \\
\hline kasira & ko-gásira & '(sub-)foreman' & \\
\hline katana & ko-gátana & 'knife' & \\
\hline kawase & ko-gáwase & 'postal note' & \\
\hline kitte & ko-gítte & 'postal stamp/check' & \\
\hline kizami & ko-kízami & 'chopping' & \\
\hline mawari & ko-máwari & 'turn' & \\
\hline midasi & ko-mídasi & '(sub-)title' & \\
\hline modosi & ko-módosi & 'recovery' & \\
\hline musubi & ko-músubi & 'closing/3rd ranking sumo' & \\
\hline musume & ko-músume & 'girl' & \\
\hline segare & ko-ségare & 'son' & \\
\hline akínai & ko-ákinai, -akínai & 'retail business' & $\left(+\right.$ acc, long $\left.\mathrm{N}_{2}\right)$ \\
\hline bóozu & ko-bóozu & 'bonze' & \\
\hline hyaku-shóo & ko-byáku-shoo & 'farmer' & \\
\hline takái & ko-dakái & 'high' & \\
\hline kárei & ko-gárei & ‘dap, plaice’ & \\
\hline génso & ko-génso & '(daughter) element' & \\
\hline hítuji, hitúzi & ko-hitúzi & 'lamb, hogling' & \\
\hline zyóoki & ko-zyóoki & 'steam (launch)' & \\
\hline nímotu & ko-nímotu & 'parcel' & \\
\hline nínzuu & ko-nínzuu & 'people' & \\
\hline tutumí & ko-zútumi & 'package' & \\
\hline tuzumí & ko-túzumi & 'type of drum' & \\
\hline
\end{tabular}


Likewise, words formed with $o o$ - show the accentual patterns found in compounds, though there are a few exceptions. Thus, while words with long $\mathrm{N}_{2}$ 's clearly have the predicted accentual default, as shown in (66c) and forms like oo-átama in (66d), the pattern is less transparent in forms with short second members. For example, a few forms in (65a) have initial accent on $\mathrm{N}_{2}$, despite the fact that they are unaccented, and should therefore either be unaccented or have accent on $o o-$. In sum, while the distinction is not perfect, there is a clear contrast between short and long second position nouns here, and this conclusion is consistent with the other facts, pointing in the direction that $o o$ - is the first member of a compound in these forms.

(66) Exc

\begin{tabular}{|c|c|c|c|c|}
\hline \multirow[t]{4}{*}{ a. } & kaze & oo-káze & ‘wind' & \multirow[t]{4}{*}{$\left(-\operatorname{acc}\right.$, short $\left.\mathrm{N}_{2}\right)$} \\
\hline & azi & oo-azi & 'insipid (taste)' & \\
\hline & atu-atu & oo-átu-atu & 'in love/deeply in love' & \\
\hline & mozi & oo-mozi & 'letter/capital letter’ & \\
\hline \multirow[t]{15}{*}{ b. } & áme & oo-áme & ‘rain’ & \multirow[t]{15}{*}{$\left(+\right.$ acc, short $\left.\mathrm{N}_{2}\right)$} \\
\hline & áse & oo-áse & 'sweat' & \\
\hline & aná & oo-ana & 'hole' & \\
\hline & asi & óo-asi, oo-asi & 'foot' & \\
\hline & húne & oo-bune & ‘ship’ & \\
\hline & kóe & oo-góe & 'voice/loud voice' & \\
\hline & hári & oo-hari & 'needle’ & \\
\hline & hasí & óo-hasi & 'bridge/large bridge' & \\
\hline & íki & oo-iki & 'breath/deep sigh' & \\
\hline & matá & oo-mata & 'long stride' & \\
\hline & kído & oo-kído & 'gate (of town)' & \\
\hline & mesí & óo-mesi & 'meal/hearty meal' & \\
\hline & monó & oo-mono & ‘man/great man’ & \\
\hline & túbu & oo-tubu & 'drop' & \\
\hline & uké & oo-uke & 'success' & \\
\hline \multirow[t]{15}{*}{ c. } & akubi & oo-ákubi & 'yawn’ & \multirow{15}{*}{$\left(-\operatorname{acc}\right.$, long $\left.\mathrm{N}_{2}\right)$} \\
\hline & atari & oo-átari & 'hit' & \\
\hline & hurosiki & oo-búrosiki & 'furoshiki' & \\
\hline & tigai & oo-tígai & 'difference' & \\
\hline & harai & oo-hárai & 'purification' & \\
\hline & hazure & oo-házure & 'failure' & \\
\hline & sikake & oo-jíkake & 'device' & \\
\hline & matsuri & oo-mátsuri & 'festival' & \\
\hline & mawari & oo-máwari & 'detour' & \\
\hline & midasi & oo-mídasi & 'headline' & \\
\hline & mikurai & oo-mikurai & '(Imperial) throne' & \\
\hline & sooji & oo-sóoji & 'house cleaning' & \\
\hline & tatimawari & oo-tátimawari & 'tumble/rough tumble' & \\
\hline & tokage & oo-tókage & 'lizard' & \\
\hline & yasuuri & oo-yásuuri & 'bargain' & \\
\hline
\end{tabular}



d. arasi
oo-árasi
'storm'
(+acc, long $\mathrm{N}_{2}$ )
atamá
toorí
oo-átama
'head'
hánabi
oo-dóori
híroma
oo-hánagi
ibiki
oo-híroma
'street/main street'
medamá
oo-íbiki
mooké
oo-médama
'fireworks'
'hall'
'snore'
'eye'
oo-móoke
'profit'

Another case exemplifying the same pattern is $s u$-, which applied to a stem gives the meaning 'bare $X$ '. As the following data show, there is a clear contrast between forms with short and long second members, (67a-b), cf. (67c-d), which accords generally with the predicted patterns. The abundant instances of Rendaku voicing and final syllable extrametricality effects in (67d) further point in the direction of the analysis of words with $s u$ - as compounds.

(67) Examples with $s u$ - as First Member of Compound

\begin{tabular}{|c|c|c|c|c|}
\hline a. & asi & sú-asi & 'foot' 1 ( & $\left(-\operatorname{acc}\right.$, short $\left.N_{2}\right)$ \\
\hline & kao & sú-gao & 'face' & \\
\hline & kaki & su-gaki & 'to draw/animation' & \\
\hline & yaki & su-yaki & 'to fire/unglazed pottery' & \\
\hline b. & té & su-dé, sú-de & 'hand' & $\left(+\operatorname{acc}\right.$, short $\left.\mathrm{N}_{2}\right)$ \\
\hline & hosí & su-bosi, su-bosí & ‘dry' & \\
\hline & utá & su-uta & 'soup' & \\
\hline & háda & sú-hada & 'skin' & \\
\hline c. & hadaka & su-ppádaka & 'naked' & $\left(-\operatorname{acc}\right.$, long $\left.\mathrm{N}_{2}\right)$ \\
\hline & toori & su-dóori & 'go through' & \\
\hline & tomari & su-dómari & 'stay' & \\
\hline & odori & su-ódori & 'dance' & \\
\hline & roonin & su-róonin & 'roonin' & \\
\hline & utai & su-útai & 'singing' & \\
\hline & zyooruri & su-zyóoruri & 'zyooruri' & \\
\hline & toonin & su-tóonin & 'townsman’ & \\
\hline & katari & su-gátari & 'to talk/recital of zyoruri music' & \\
\hline d. & awasé & su-áwase & 'a kind of clothes?' & $\left(+\right.$ acc, long $\left.\mathrm{N}_{2}\right)$ \\
\hline & hanasí & su-bánasi & ‘speech’ & \\
\hline & modorí & su-módori & 'to return/return empty handed' & \\
\hline & mogurí & su-móguri & 'diving' & \\
\hline & & & & \\
\hline
\end{tabular}

Another prefix which may form compounds is han-, which attaches to nouns and results in a derived noun meaning 'anti-X'. Han- may be used either as an Aoyagi prefix or a non-Aoyagi prefix, and we focus here on non-Aoyagi usage (which means the morphological word forms a single minor phrase). In support of han-as a component of a compound, there appears to be a contrast between words formed with short and long second members; contrast han-kaku, káku '(anti-)nuclear power' with the derived word han-táisei 'anti-regime', from the long base taisei 'regime'. My sample, however, is

\footnotetext{
${ }^{23}$ The lack of default initial accent here may be related to the fact that this derived form does not look like a straightforward case of modification.
} 
inconclusive, as there are no examples showing Rendaku voicing ${ }^{24}$, nor are there cases showing extrametricality effects or medial accent. Thus, prefixation of han- is consistent with either treatment of it as a compound, or as a dominant, post-accenting prefix.

Focusing more squarely on the issues at hand, while there are cases in which han-itself is accented, e.g., hán-so 'Anti-Soviet' and $h a ̂ N-i$ 'against one's will', these facts are consistent with an analysis in terms of compound accent, and since this analysis is still viable, it may be entertained as an alternative to the obvious analysis in terms of edge orientation.

Several other prefixes, typically Sino-Japanese in origin, may also be compoundlike, but again, the results are inconclusive given the lack of data. ${ }^{25}$ For example, $h i$ - 'non' consistently induces accent on the following stem when the stem is long, e.g., /hi + toosei/ $\rightarrow$ hi-tóosei 'uncontrolled' and /hi + nínzyoo/ $\rightarrow$ hi-nínzyoo 'inhuman(ity)'. Further, there is one example in which the stem accent is lost in favor of accenting $h i^{-}$, /hi + ún/ $\rightarrow$ hi-un 'unluckiness', which is again consistent with an analysis in terms of default accent on the first member in words with short second members. Thus, while these facts are consistent with an analysis in terms of compound accent, there are no instances of Rendaku voicing or final syllable extrametricality effects in my sample, and so the results are inconclusive. Likewise, the prefixes $h u$ - 'non-', $m u$ - 'lacking, un-', and $m i$ - 'not yet ...' all present the same pattern: they are consistent with an analysis in terms of the first member of a compound, but we lack the facts which would show this conclusively. The results of my findings for all of these prefixes are summarized in the chart in (69) below.

Another prefix of interest is $z i-$, which is used in making reflexives, because it shows that prefixes which are not simple modifiers of the base to which they attach can behave like an element of a compound. Thus, a clear contrast is observed between derivatives formed with short bases $(68 a-b)$ and those formed with long ones $(68 c-d)$ : the former are mostly unaccented, while the latter all have accent on the first syllable of the stem. ${ }^{26}$ Also, the form zi-hénsuu in (68d) shows that final syllable extrametricality is in force, as this form is derived from a base with final accent.

\footnotetext{
${ }^{24}$ The lack of forms showing Rendaku may be due to a morphological restriction which blocks attachment of han- to native stems, a restriction that is observed in many Sino-Japanese affixes.

${ }^{25}$ See Martin 1975: $389 \mathrm{ff}$. for a long list of examples of these prefixes, and the details of their semantic and morphological features.

${ }^{26} \mathrm{An}$ interesting fact here is that almost all of the forms in (68a-b) with short bases are unaccented, which is supposed to be the marked accentual default in compounds of this type. However, this fact may simply be an effect of sampling, as most of the examples here are derived from bound stems, which may account for this apparent aberration.
} 
(68) Examples with $z i$ - as First Member of Compound
-ri
saku zi-saku
syo zi-syo
-taku zi-taku
-ei zi-ei
a. kyoo zi-kyoo
'confession'
'self-interest'
'work/one's own work'
writing/one's own writing'
'one's own home'
'defense; run (e.g. a shop)'
b. kóku zi-koku
máe zi-mae
-mán zi-man
-méi zi-mei
-métu zi-metu
-món zi-mon
-nén zi-nen
-nín zi-nin
-réi zi-rei
-ríki zi-riki
-rítu zi-ritu
séi zi-sei
-séki zi-seki
-sén zi-sen
'country/one's own country'
'front/geisha living on her own'
$\left(-\right.$ acc, short $\left.\mathrm{N}_{2}\right)$
'pride, boasting'
'self-evidence'
'natural decay, self-destruction'
'one's own family'
'spontaneous conbustion'
'self-acknowledge, admission'
'self-excitation'
'one's own strength'
'self-reliance'
'self-control; self-examination'
'self-reproach'
'self-election'
c. doosi zi-dóosi
daraku zi-dáraku
syookai zi-syóokai
isiki zi-ísiki
senden ziko-sénden
saimin ziko-sáimin
'introduction'
'consciousness'
'advertisement'
saimin "zypnosis'
toosui ziko-tóosui 'absorption'
'verb/intransitive verb'
'slovenliness, untidiness'
$\left(-\operatorname{acc}\right.$, long $\left.\mathrm{N}_{2}\right)$
d. hensúu zi-hénsuu '(independent) variable'
kén'o ziko-kén'o 'hatred'
mánzoko zi-mánzoko 'contentment'
$\left(+\right.$ acc, short $\left.\mathrm{N}_{2}\right)$

A second example of a non-modifying prefix, but one which clearly behaves like the first member of a compound, is hi-, which is used in forming 'passive' nouns. The accentuation of $h i$ - depends on the size of the base to which it attaches. If the base is short, the result is loss of accent, as in /hi + koku/ $\rightarrow$ hi-koku 'defendant', or accent on hi-, e.g., /hi + gi/ $\rightarrow$ hí-gi 'suspect'. But if the base is long, the result is medial accent in bases with non-final accent, as in /hi + senkyóken/ $\rightarrow$ hi-senkyóken 'right to be elected', cf. senkyóken 'right to vote', or default initial accent, as for example in /hi + zyoosúu/ $\rightarrow$ $h i$-zyóosuи 'dividend'. Indeed, as shown by the following paradigm, one finds the two different accentual default patterns when affixation to the base alters its size, hí-gai 'damage', but hi-gáisa 'victim' and hi-gáiti 'place damaged'. Since these facts are only consistent in terms of compound accent, I conclude that $h i$ - is the first member of a compound in these examples, on a par with many others. 
The following chart summarizes the basic findings for the prefixes discussed above.

(69) Summary of Results 27

\begin{tabular}{l|c|c|c|c|c|c|c}
\multicolumn{1}{c|}{ Prefix } & Result & MOD & REND & $\mathrm{S} \neq \mathrm{L}$ & $\langle\sigma\rangle \#$ & $\ldots \sigma o ́$ & $n=$ \\
& & & & & & $\sigma \ldots$ & \\
\hline \hline sin- 'new' & comp & $\mathrm{Y}$ & $\mathrm{Y}$ & $\mathrm{Y}$ & $\mathrm{Y}(1 / 12)$ & $\mathrm{Y}(5 / 12)$ & 41 \\
$m a$ - 'true' & dom & $\mathrm{Y}$ & $\mathrm{N}$ & - & $\mathrm{Y} / \mathrm{N}$ & $\mathrm{Y} / \mathrm{N}$ & 32 \\
$k o$ - 'little' & comp & $\mathrm{Y}$ & $\mathrm{Y}$ & $\mathrm{Y}$ & $\mathrm{Y}$ & $\mathrm{Y}(3 / 12)$ & 49 \\
oo- 'big' & comp & $\mathrm{Y}$ & $\mathrm{Y}$ & $\mathrm{Y}$ & $\mathrm{Y}$ & - & 42 \\
$h a n$ - 'anti-' & comp & $\mathrm{N}$ & $\mathrm{N}$ & $\mathrm{Y}$ & - & - & 22 \\
su- 'bare' & comp & $\mathrm{Y}$ & $\mathrm{Y}$ & $\mathrm{Y}$ & $\mathrm{Y}$ & - & 22 \\
$h i$ - 'non-' & comp & $\mathrm{N}$ & $\mathrm{N}$ & $\mathrm{Y}(?)$ & - & - & 12 \\
$h u$ - 'non-' & comp & $\mathrm{N}$ & $\mathrm{Y}(?)$ & $\mathrm{Y}(?)$ & $\mathrm{Y}(3 / 11)$ & - & 12 \\
$m u$ - 'un-' & comp & $\mathrm{N}$ & $\mathrm{N}$ & $\mathrm{Y}(?)$ & $\mathrm{Y}$ & - & 8 \\
$m i$ - 'no yet' & comp & $\mathrm{N}$ & $\mathrm{N}$ & $\mathrm{Y}(?)$ & $\mathrm{Y}(?)$ & - & 10 \\
$z i$ - Reflexive & comp & $\mathrm{N}$ & $\mathrm{N}$ & $\mathrm{Y}$ & $\mathrm{Y}(?)$ & - & 30 \\
$h i$ - Passive & comp & $\mathrm{N}$ & $\mathrm{N}$ & $\mathrm{Y}$ & $\mathrm{Y}(?)$ & - & 15
\end{tabular}

Working with a substantial corpus of facts, the finding is that most of the nominal prefixes examined here exhibit patterns that are characteristic of the first member of a compound. This finding is significant because it strongly suggests that these prefixes should be treated in terms of the analysis presented above for noun-noun compounds. An analysis which treats these prefixes as the first member of a compound goes a long way towards explaining the complex and varied set of patterns here. When compared with the alternative analysis in terms of edge orientation sketched at the onset of this subsection, it is clear that the compound analysis is superior because it gives a unified analysis of these patterns.

Moreover, the compound analysis is fully consistent with the Root-Controlled Accent hypothesis. If these prefixes are treated as the first member of a compound, then their accentuation is predictable by the principles governing accentual defaults and not their underlying prominence specifications. The inherent accent of the prefix is irrelevant to the accentuation of the larger compound because, as argued above, the first member of a compound is consistently de-accented in this word-formation process. Thus, the prefixstem sequences do not bring about a situation of constraint conflict between Root and Affix Faithfulness. The various edge effects observed in compounds, therefore, are compatible with the RCA hypothesis because Faithfulness is irrelevant here.

As for dominant, post-accenting prefixes such as $m a-$, this prefix type is also consistent with an analysis of Japanese accent in terms of root-control. As shown in detail

${ }^{27}$ In this chart, if a test result is only supported by one or two examples, a ' $\mathrm{Y}($ ?)' appears in the appropriate cell. Also, the number of examples for each prefix is given in the last column, and where possible, the percentages of prefixes testing positively for a given diagnostic is shown. For example, 'Y(1/12)' for sinunder $\langle\sigma\rangle \#$ means that one out of twelve relevant words with this prefix show final syllable extrametricality effects. 
in $\$ 4.1$, dominant, i.e., accent-deleting affixes, do not counter the RCA hypothesis because they do not compete for prominence with a root accent. Dominant affixes may be accented or unaccented, as is the case with $m a-$, and therefore the deletion of a stem accent cannot always be attributed to a competition between a root accent and an affix accent: dominant unaccented affixes do not have an accent to participate in such a competition. Dominance effects therefore require some additional principles to account for the observed deletion of base prosody, and chapter 5 is dedicated to the development of these principles. The important point for the matters at hand, however, is that dominant affixes do not contradict the RCA hypothesis because they do not compete for prominence with a stem accent.

The last type of prefix which has an influence of word accentuation are the so-called 'Aoyagi prefixes' (after Aoyagi 1969; see Poser 1990b and Martin 1975: 751 for a list of these prefixes). In a detailed study of these prefixes, Poser 1990b points out two peculiar properties of these prefixes. These properties are exhibited in the data below, which appear to represent exceptions to the basic principles of accent in Japanese words. (The horizontal lines above and below the segments below represent approximations of the f0 profiles.)

(70) Words with Aoyagi Prefixes

\begin{tabular}{|c|c|c|c|}
\hline $\begin{array}{l}\text { a. moto- } \\
\text { han- }\end{array}$ & $\begin{array}{l}\text { 'former' } \\
\text { 'anti-' }\end{array}$ & $\begin{array}{l}\overline{\mathrm{m}} \text { óto- } \underline{\text { dáazin }} \\
\overline{\mathrm{h} \text { án-n- sh_ákai }}\end{array}$ & $\begin{array}{l}\text { 'former minister' } \\
\text { 'anti-social' }\end{array}$ \\
\hline $\begin{array}{l}\text { zeN- } \\
\text { ki- }\end{array}$ & $\begin{array}{l}\text { 'former' } \\
\text { 'your (honorific)' }\end{array}$ & 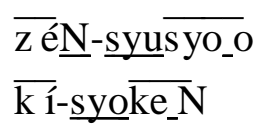 & $\begin{array}{l}\text { 'former Prime Minister' } \\
\text { 'your letter' }\end{array}$ \\
\hline
\end{tabular}

First, words with Aoyagi prefixes may have two pitch accents, as exemplified in (70a) above in the words with the HLHL pitch excursions, apparently contradicting the general pattern of one accent per word. The second important property is that these words may have a fall in pitch, followed by a rise after the stem-initial mora, as shown the forms with the HLH profiles in (70b). This last pattern is of course aberrant as well because Japanese words typically have a level low tonal pattern after a fall in pitch (see $\$ 3.3 .1$ for background discussion).

Clearly, unless some special provision is made for these prefixes, the basic descriptive assumptions underlying the analysis of Japanese accent must be called into question. The approach to these two problems taken in Poser 1990b is that Aoyagi prefixes, through a pair of subcategorization requirements, bring about a parse of the larger word which has two minor phrases, as illustrated below.

(71) Word-Internal Phrase Boundaries (Poser 1990b)

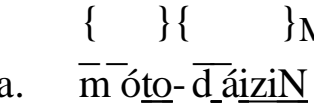
b. $\quad \frac{\{\} \text { z } \underline{N} \text {-syus yo_o }}{\}}$

To give a brief sketch, Aoyagi prefixes have subcategorization requirements which refer to both morphological and prosodic structure (an idea inspired by the dual structures proposed in Inkelas 1989). Thus, they must attach to stems in the morphology, but, in their prosodic analysis, these prefixes select a minor phrase, in effect inducing the word-internal phrase boundaries shown above. This analysis therefore explains the apparently aberrant properties of words with Aoyagi prefixes in terms of the special phrasing required by said prefixes. Words with accented stems may have a second accentual prominence because it 
is parsed by a minor phrase which excludes the prefix (71a); words with unaccented stems have a rise after the first mora because this profile is typical of minor phrases which do not have an inherent accent (71b).

The importance of this analysis is that it demonstrates that the accentuation of words with Aoyagi prefixes is fully consistent with the Root-Controlled Accent hypothesis. The logic of the argument runs as follows. The RCA hypothesis favors retention of a root accent when it competes with an affix accent for the unique word prominence. In Poser's analysis, words with Aoyagi prefixes have two separate minor phrases - this assumption is absolutely crucial to account for the HLHL and HLH tonal contours found in these words. From this it follows that an accent in the prefix does not compete for prominence with the stem accent because the prefix and the stem are in different minor phrases. The up-shot then is that the unusual accentual patterns brought about by Aoyagi prefixes are consistent with the analysis that accent in Japanese words is root-controlled, on a par with root-controlled accent in Cupeño.

To summarize the above discussion, three sets of prefixes were examined and all of them were shown to be consistent with the thesis that accent in Japanese is root-controlled. First, prefixes which act as the first member of a compound are consistent with the RCA hypothesis because their accentuation is predicted by the principles of compound accent, and not their inherent prominence specifications. Second, dominant post-accenting prefixes do not contradict RCA because dominant affixes do not compete for prominence with a root accent (chapter 4 and 5 continues the development of this argument). Finally, Aoyagi prefixes are fully compatible with a RCA analysis of Japanese accent because their special behavior requires the introduction of word-internal phrase boundaries which separate the prefix and the stem. This phrasing of the word has the effect of parsing the prefix and stem into separate prosodic domains and hence they too do not compete for the unique word prominence.

In conclusion, an apparent challenge for the restricted theory of edge effects developed in $\$ 3.1$ turns out to lend some support to the analysis of Japanese accent in terms of root-control. The prefixes which are known to have an effect on word accent were classified into three distinct types, and it was shown that all of these types are in fact compatible with the RCA-driven analysis. Considering the number of prefixes in the study, approximately 50 when the Aoyagi prefixes from Poser 1990b and Martin 1975 are included, the absence of a class of prefixes which uniformly override root accent supports the Root-Controlled Accent hypothesis, and casts serious doubt on an analysis in terms of edge orientation. In addition to this empirical argument, there are at least two theoretical arguments in support of approaching Japanese accent in terms of root-control. First, the system can be treated on a par with Cupeño, and hence overriding root accent can be explained as a general pattern of root privilege. Second, this analysis is consistent with a restrictive theory of edge effects which significantly reduces the patterns of edge orientation in accent systems. In chapter 5, additional motivation for the RCA analysis is given which involves showing the role of root accentedness in blocking the application of accentual processes like pre- and post-accentuation and accentual shifts.

\subsection{Summary and Conclusion}

Accent Resolution (AR), the deletion of all but one lexical accent, is root-controlled if it shows a preference for retention of a root accent over an affix accent. This pattern of accent retention is distinct from directional AR, where it is the accent which appears closest to a designated edge that is retained. The intrinsic ordering of Root and Affix Faithfulness 
that underlies the analysis of root-controlled AR, namely MAX-PROMRoot $>$ MAX-

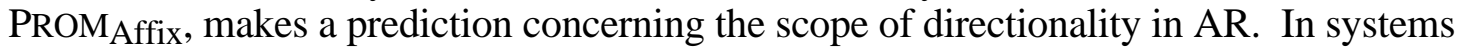
with an accentual contrast in both roots and affixes, directional AR only emerges in contexts where the Prosodic Faithfulness constraints are not decisive.

Two systems previously described in terms of directional AR, Russian and Japanese, were studied with this prediction in mind. A close examination of these systems showed that there is no crucial evidence which necessitate an analysis described solely in terms of directionality. In both Russian and Japanese, a stem accent generally takes precedence over accent in a following suffix, which is consistent with both root-controlled and directional AR (where the latter type set for leftward edge orientation). Furthermore, it was found that accent is predictable in the affixed structures which can distinguish these two analyses, namely prefix + root sequences. Thus, in both of these systems, there is an absence of a class of prefixes which consistently override accent in the following stem. This finding casts doubt on the analysis of AR in Russian and Japanese with phonological directionality. This gap is fully consistent, however, with an analysis of these systems with root-controlled AR because, if roots take precedence over affixes in AR, this is exactly the predicted pattern. At the very least then, the notion of root-controlled accent has some currency outside the analysis of overriding root accent in Cupeño; it is crucial in explaining this distributional gap.

In addition to this empirical issue, there are further reasons to extend the scope of root-controlled accent. First, the analysis of root-controlled accent obtained via the intrinsic ordering of Root and Affix Faithfulness makes a substantive restriction on the range of edge effects in accent systems. If this theory is correct, certain logically possible patterns of edge orientation are systematically ruled out. Second, as suggested in $\S 3.1$, the analysis of AR with morphologically-dispersed Faithfulness may make sense of an apparent directional asymmetry in AR. The pervasiveness of systems described with a principle of 'the leftmost accent wins' may be explained as a general preference for suffixing morphology; the apparent pattern of leftward edge orientation is really due to the privileged faithfulness status for roots, which tend to be word-initial. Third, the analysis of AR with root-control explains the observed pattern of root retention in terms of a general pattern of root privilege. Thus, the analysis of RCA in Cupeño, and by extension, Russian and Japanese, makes a connection to segmental phenomena like root-controlled vowel harmony in Akan. Fourth, as demonstrated in Cupeño (see \$2.4.3), this approach to AR clarifies a role for root accentedness in the analysis of morpho-accentual processes like pre- and postaccentuation. The imperative to preserve a root accent may have the effect of blocking morphological processes which change the prosody of the base, a very common pattern which is discussed in detail throughout chapter 5. From these considerations, there is reason to entertain the hypothesis that root-controlled accent is indeed a universal property; it explains a host of properties which appear to hold of all languages.

As for restricted edge effects, further typological work is still needed to determine the scope of directionality in accent systems. While it has been shown that two cases, formerly analyzed with directional $A R$, are in fact consistent with the restricted edge effects, a cross-linguistic study of AR has yet to be done. However, if it turns out that further investigation turns up a system with an unrestricted type of directional AR, which completely ignores word structure, it is important to emphasize that this finding would not necessitate a re-analysis of root and affix accent in Cupeño, Russian, and Japanese. Rootcontrolled accent in these systems can be analyzed in a way that has all of the advantages of this analysis listed above, but without restricted directionality. In particular, such a finding would support a more recent instantiation of Root Faithfulness in Prince 1997, Beckman 1997 [1998]. In this approac, the privileged status of roots is characterized by a set of 
Root Faithfulness constraints, but there are no corresponding Affix Faithfulness constraints. Thus, Root Faithfulness is not inherently ordered, and it may be ranked on a language particular basis in relation to context-free Faithfulness, or Faithfulness which is sensitive to whole words. Directional AR, then, is the result of Prince's 'Anti-Paninian' ranking, where the general or context-free Faithfulness constraint outranks the specific Root Faithfulness constraint, and the edge orientation constraint LEFTMOST is ranked between these two Faithfulness constraints, as shown below.

(72) Directional Accent Resolution with Anti-Paninian Ranking

\begin{tabular}{|cc|c|c|c|}
\hline Input & Output & MAX-PROM & LEFTMOST & MAX-PROMRoot \\
\hline \hline a. /áf + róot/ $\rightarrow$ áf-root & $*$ & & $*$ \\
\cline { 2 - 6 } & $*$ af-róot & $*$ & $* !$ & \\
\hline \hline b. /af + róot/ $\rightarrow$ af-róot & & $*$ & $*$ \\
\cline { 2 - 6 } & $*$ áf-root & $* !$ & & \\
\hline
\end{tabular}

In such a constraint system, the general constraint is only active in contexts where a lexical accent is not in competition with another accent that would be faithfully parsed wordinitially (72b). In words with more than one inherently accented morpheme, the leftmost one wins, regardless of whether it is in a root or an affix (72a).

It is clear, therefore, that it is possible to describe directional AR in a theory which assigns privileged Faithfulness to roots. The findings of this chapter, however, question whether AR is ever governed by directionality alone. The ultimate answer to this question will bear directly on the formulation of the special Faithfulness status of roots, as restricted directionality effects are only accounted for in a theory with distinct Faithfulness constraints for roots and affixes. 


\section{Chapter 4.}

\section{Transderivational Faithfulness and Anti-Faithfulness}

The thesis of Root-Controlled Accent clarifies the distinction between two classes of morpho-accentual phenomena: root-controlled accent (RCA) and affix-controlled accent (ACA). As in root-controlled vowel harmony, RCA entails that the accentual properties of the larger word are predictable from the properties of the word-internal root. Therefore, root-control in both kinds of phonological systems is systematic and applies across the board. Affix-control in accent systems, by contrast, is more sporadic and is predictable from the properties of individual morphemes. As illustrated in the next section, morphologically conditioned de-accentuation, the result of attaching a so-called 'dominant' affix, is affix-controlled because it requires the lexical specification for this de-accentuation on an affix-by-affix basis. The properties of affix-controlled processes will be studied in more detail in chapter 5, but from this first look at the problem, it will be clear that ACA forms a class of phenomena that excludes RCA.

The observed differences between these two phenomena suggests that they require a different analysis, and the last two chapters are dedicated to developing an integrated theory of affix-controlled accent. The current chapter provides the theoretical background for this theory, which has two basic components. The first involves a set of mechanisms which encode a formal relationship between morphologically related words. In Optimality Theory, this relationship is established through a set of Transderivational Faithfulness constraints which compare a base form with its related derivative and assess the pair for their phonological similarity. Following many recent proposals, I employ this basic approach in the analysis of accent-neutral morphology, i.e., cases where the derived form mimics the prosody of its base. The nuts and bolts of this theory, dubbed Transderivational Correspondence Theory (TCT) in Benua 1997 [1998], is presented in $\S 4.2$.

The second main ingredient in the theory of ACA involves a further development of Faithfulness in OT, namely the introduction of the related notion, Anti-Faithfulness. AntiFaithfulness demands a phonological change in related strings where Faithfulness requires phonological inertness. As will be shown in $§ 4.3$, Anti-Faithfulness constraints solve a significant problem in the analysis of exchange processes. After motivating these two basic ingredients, transderivational relations between words and the notion of Anti-Faithfulness, they will be applied jointly in the development of a theory of morpho-phonological alternations, which has broad implications for the morphology-phonology interface. Within this theory, the properties of affix-controlled accentual processes will be explained as a special type of a more general kind of morpho-phonological operation. 


\subsection{Morphemic Sources of Accentual Regularity}

The focus of much of the discussion up to now has been on what might be called 'culminativity effects' for inherent accent, i.e., the resolution of a sequence of lexical accents in the competition for a unique word accent. For example, in the study of word accent in Cupeño, we have seen a role for edge orientation in culminativity effects: in words with a sequence of inherently accented affixes, the rightmost accent wins. Another important means of resolving this competition is achieved by an ordering of Root and Affix Faithfulness. In root-controlled accent systems such as Cupeño, the competition between root and affix accent is resolved as the satisfaction of MAX-PROMRoot Over MAXPROM$_{\text {Affix }}$, as predicted by the RCA hypothesis, repeated below from chapter 2, section 2 .

\section{(1) Root-Controlled Accent Hypothesis}

In lexical-to-surface mappings of a word with more than one inherent accent, if accent is deleted, accent in the root is realized over accent elsewhere in the word.

If a sequence of accented morphemes do not differ in morphological class membership, then the thesis of Root-Controlled Accent (RCA) says nothing, and additional principles, e.g., edge orientation, may apply. However, if the accented morphemes do in fact differ in the relevant way, i.e., some are roots and some are affixes, the universal ordering of Root and Affix Faithfulness eliminates non-root accents from the competition. Further, a straightforward extension of this idea is to include a position for Stem Faith, or Faithfulness to derivational affixes (as in Revithiadou 1997) in the meta-constraint, in which case a more articulated 'chain of command' can be established (see the analysis of derived nouns in Russian in $§ 5.2 .3$ for a relevant example).

The RCA hypothesis is a restrictive claim in that it rules out certain logically possible types of culminativity effects, the obvious one being a case where accented affixes systematically win out over accented roots. This result is a necessary consequence of the overall theory because, as emphasized throughout, the explanation of overriding root stress derives directly from the assumption that Root Faith is universally ranked above Affix Faith. With this inherent ranking, the facts could not be otherwise. A possible challenge to this claim comes from the behavior of certain special affixes, often called 'dominant affixes' because they appear to win in competitions with a root accent. For example, the adjective-forming suffix -ppó in Japanese is a dominant suffix as all words that contain this suffix have accent on -ppó, even when it combines with an accented stem (2b). Another example is the noun-forming suffix in Russian -úx, which likewise ignores the accentedness of the base to which it attaches and is always accented (3).

(2) Dominant Accented Suffix -ppó in Japanese

$\begin{array}{lllll}\text { a. } \quad \text { /abura }+ \text { ppó }+\mathrm{i} / & \rightarrow & \text { abura-ppó-i } & \text { 'oily' } \\ \text { /kaze }+ \text { ppó }+\mathrm{i} / & \rightarrow & \text { kaze-ppó-i } & \text { 'sniffly' } \\ \text { b. } \quad \text { /adá }+ \text { ppó }+\mathrm{i} / & \rightarrow & \text { ada-ppó-i } & \text { 'coquettish' } \\ \text { /kíza }+ \text { ppó }+\mathrm{i} / & \rightarrow & \text { kiza-ppó-i } & \text { 'affected' }\end{array}$


(3) Dominant Accented Suffix -úx in Russian
a.

$$
/ \text { skak }+ \text { úx }+ \text { a/ }
$$$$
\rightarrow \quad \text { skak-úx-a }
$$$$
\text { /vos'm + úx }+\mathrm{a} /
$$$$
\rightarrow \quad \text { vos'm-úx-a }
$$
'frog'
b.

$$
\begin{array}{llll}
\text { /s'ív }+ \text { úx }+ \text { a/ } & \rightarrow & \text { s'iv-úx-a } & \text { 'raw alcohol' } \\
\text { /gólod }+ \text { úx }+ \text { a/ } & \rightarrow & \text { golod-úx-a } & \text { 'hunger' }
\end{array}
$$

The problem posed by these examples is that a root accent appears to be in competition with an affix accent, and so the thesis of RCA predicts that the root accent should win, contrary to fact.

If these examples represent valid cases of culminativity effects, then they constitute truly lethal counter-examples to the thesis of RCA, and accordingly, they would lead to a serious re-thinking of the patterns attributed here to RCA. However, the behavior of dominant morphemes is not standardly treated as an effect of culminativity. Rather, they are typically handled as a morphologically conditioned deletion of the accent in the base to which the dominant affix is attached. The reason for this assumption is that dominant morphemes often show a contrast in accentedness, and dominant unaccented morphemes also show evidence of a deletion. Dominant unaccented affixes, as exemplified below in Japanese and Russian, trigger a deletion of base prosody and the emergence of a default accentual pattern, or no accent at all in the case of Japanese. Thus, when the dominant suffix - $k k o$ attaches to accented stems, as shown in (4b), the result is a deletion of the stem accent and a completely unaccented word, which is a default pattern for accent in Japanese. Likewise, when noun-forming -ač attaches to accented stems, the stem accent is deleted and the larger word receives default ending stress (5b).

(4) Dominant Unaccented Suffix - $k k o$ in Japanese
/niigata $+\mathrm{kko} /$
a.
/edo + kko/
$\rightarrow \quad$ edo-kko
$\rightarrow \quad$ niigata-kko
'Native of Tokyo'
b.

$$
\begin{aligned}
& \text { /kóobe + kko/ } \\
& \text { /nyuuyóoku + kko/ }
\end{aligned}
$$
'Native of Kobe'
'Native of New York'

(5) Dominant Unaccented Suffix - $a \check{c}$ in Russian
a.

$$
\begin{aligned}
& / \text { borod }+ \text { ač }+\mathrm{u} / \\
& / \mathrm{gorb}+\mathrm{ač}+\mathrm{u} /
\end{aligned}
$$$$
\rightarrow \quad \text { borod-ač -ú }
$$$$
\text { 'man with beard' }
$$$$
\rightarrow \quad \text { gorb-ač -ú }
$$$$
\text { 'hunchback' }
$$
b. $\quad / p u ́ z+a \check{c}+\mathrm{u} /$
$\rightarrow \quad$ puz-ač -ú
'man with paunch'

$$
/ \text { tr'úk }+\mathrm{ač}+\mathrm{u} /
$$

$$
\rightarrow \quad \text { tr'uk-ač -ú }
$$
'stuntman'

Returning to the main point, dominant unaccented affixes show that dominance effects are clearly not culminativity effects: unaccented affixes cannot compete for the realization of an inherent accent. The more general point is that the behavior of dominant morphemes does not counter-exemplify RCA because dominance effects are not to be attributed to culminativity. 
As will be argued in detail in $\$ 5.1$, dominance effects are one type of a more general class of so-called 'affix-controlled' morpho-accentual processes. Some canonical examples of these affix-controlled processes are illustrated below with some further examples from Japanese.

(6) Affix-Controlled Morpho-Accentual Processes in Tokyo Japanese

a. Dominance effects require a deletion of base prosody

$$
\begin{array}{llll}
/ \text { edo }+\mathrm{kko} / & \rightarrow & \text { edo-kko } & \text { 'Native of Tokyo' } \\
/ \text { kóobe }+\mathrm{kko} / & \rightarrow & \text { koobe-kko } & \text { 'Native of Kobe' }
\end{array}
$$

b. Pre-accentuation requires an insertion of accent into the base

$$
\begin{array}{llll}
\text { /yosida }+\mathrm{ke} / & \rightarrow & \text { yosidá-ke } & \text { 'the Yoshida family' } \\
\text { /nisímura }+\mathrm{ke} / & \rightarrow & \text { nisimurá-ke } & \text { 'the Nishimura family' }
\end{array}
$$

c. Accent shifts require a shift of base prosody

$$
\begin{array}{llll}
/ \text { kúzu }+ \text { ya/ } & \rightarrow & \text { kuzú-ya } & \text { 'junkman' } \\
\text { /toma + ya/ } & \rightarrow & \text { toma-ya } & \text { 'mat seller' }
\end{array}
$$

Affix-controlled processes are so named because they correlate with the application of a morphological process, which is illustrated here with affixation. Linked to the morphology in a fundamental way, these processes in a sense support a contrast between the base of a process and its related derived form. Thus, dominance effects induce a deletion of the base accent, which supports a contrast between accented bases and unaccented derivatives, as in kóobe versus koobe-kko. Likewise, pre-accentuation, shown in (6b) with the suffix -ke, supports a contrast between base-derivative pairs by inserting an accent in the base of the derived form: nisimurá-ke, cf. nisimura. A different class of affix-controlled accent involves a shift in the position of base prosody, as exemplified in (6c) with the suffix -ya. In this case, a contrast is achieved through a shift of the base accent in the derived form. In sum, the three different types of affix-controlled processes form a class in that they serve to mark a contrast between a base and the form derived from that base.

Another important property of affix-controlled processes is that they are lexically idiosyncratic and must therefore be specified in the lexical entry of each affix. Thus, it is an idiosyncratic property of the morpheme -kko that it conditions a deletion of base prosody, which contrasts this affix from others that do not trigger a deletion. Likewise, both -ke and -ya must be set apart from other affixes on the basis of the accentual patterns they bring about. This property of affix-controlled processes contrasts sharply with the behavior of roots in accent systems, which, as we have seen throughout the case studies in chapters 2 and 3, show the systematic behavior of overriding accent in neighboring affixes. Hence, while the accentual regularities caused by affixes are lexically idiosyncratic, the accentual regularities induced by roots are systematic and apply across the board, i.e., in all the words containing accented roots.

The conclusion that can be drawn from this discussion is that affix-controlled processes such as dominance effects have a morphemic source. That is, it is an idiosyncratic property of the morpheme - $k k o$ that it triggers a deletion of base prosody, and the specification of this property is the key to explaining the morphological contrast induces about in base-derivative pairs. The same holds for the morphemes -ke and -ya, which must also be lexically specified for the accentual processes they trigger. In contrast to these 
morphemic sources of regularity, overriding root accent comes under grammatical control. In particular, the culminativity effects found in words with accented roots follow from a general property of Universal Grammar, namely that the Faithfulness constraints sensitive to roots are ordered above the Faithfulness constraints sensitive to affixes.

The rest of this thesis is thus dedicated to arriving at a principled explanation of the properties of affix-controlled accent and an explanation for the properties like those mentioned above which set ACA apart from RCA. Since a general explanation of these properties is sought, i.e., one which applies to non-accentual morpho-phonological processes, the next two sections develop and motivate a formal theory of morphophonological operations. Once this theory has been fully developed, then it will be applied to the analysis of affix-controlled morpho-accentual phenomena in chapter 5.

\subsection{Transderivational Correspondence Theory}

\subsubsection{Introductory Remarks}

Morphologically related words may be phonologically similar, even in ways that cannot be attributed to the fact that these words share morphemes. A brief look at English stress in suffixed words (to be revisited below) will illustrate this basic fact. English suffixes fall into two classes concerning their interaction with the stress of the base to which they attach (Siegel 1974). Class 2 suffixes contrast with class 1 suffixes in that only the former requires preservation of base prosody, yielding the surface contrast in the morphologically complex forms given below.

(7) Class 1 versus Class 2 Suffixation

$\begin{array}{lll}\text { Class 1 párent } & \neq & \text { parént-al } \\ \text { Class 2párent } & \approx & \text { párent-hood }\end{array}$

The first pair shows the regular pattern of stress in English nouns and adjectives, i.e., penultimate heavy, otherwise antepenultimate stress, which effectively causes a stress shift in the derived form. Yet suffixation of a class 2 affix like -hood does not trigger the same stress shift, even when the phonological composition of the string predicts penultimate stress. Therefore, for the second pair of words, it is said that class 2 suffixes require preservation of the prosodic information of the base to which they attach. The complex word párenthood mimics the prosody of its base párent, even if this results in an otherwise irregular stress pattern.

A classical approach to this problem, often called the Cyclic Approach, is to derive the similarity effects by embedding the simplex form in the derivation of the complex form (Chomsky \& Halle 1968, Siegel 1974, Allen 1978). Thus, stress sensitive suffixes are attached before Stress Assignment, predicting that they are counted in the placement of stress. Stress neutral suffixes, on the other hand, are attached after Stress Assignment, and as a result, they do not affect stress in the larger word. 
(8) Preservation of Base Prosody with the Cycle

$\begin{array}{lll} & \text { /parent/ } & \text { /parent/ } \\ \text { Class 1 Affixation } & \text { parent-al } & \\ \text { Stress Assignment } & \text { parént-al } & \text { párent } \\ \text { Class 2 Affixation } & - & \text { párent-hood } \\ & \text { [ paréntal ] } & \text { [ párenthood ] }\end{array}$

The derived form párenthood is not truly exceptional in this analysis because surface stress is the product of a valid application of the stress rules.

The Cyclic Approach to the preservation of base prosody may be characterized as strongly derivational because it relies crucially on the interleaving of the stress rules with an ordered set of morphological rules. It is on this basis that the Cyclic Approach has been criticized and a fresh alternative has been developed. With the advent of Optimality Theory (Prince \& Smolensky 1991, 1993) and other non-derivational frameworks (see Goldsmith 1993), the role of derivations in phonology has been seriously challenged, leading to a growing body of evidence that serial derivations may in fact be unnecessary and that the quality of the explanation is enhanced in non-derivational theories (see McCarthy 1993, 1997, Benua 1995, 1997, Alderete 1995, 1997, Kager 1995). Against this background, a number of researchers have proposed to derive similarity effects via a form of Faithfulness that holds between the morphologically related words (Benua 1995, 1997 [1998], Burzio 1994, 1996, 1998, Kenstowicz 1996, 1997). ${ }^{1}$ These works differ in many of the formal details of encoding Faithfulness relations between words, and so a choice of a specific model must be made before applying this idea to stress-neutral affixation. Since Benua's Transderivational Correspondence Theory (TCT) employs the basic tenets of Correspondence Theory fundamental to this problem and the larger thesis, I will employ this model, though many of the same arguments made below carry the same weight in the other theories.

In TCT, morphologically related words stand in correspondence in a way that is formally similar Input-to-Output Correspondence. Class 1 and 2 suffixes are then distinguished lexically by the type of correspondence relation they enter into: class 1 suffixes like -al trigger the output-to-output correspondence relation $\mathrm{OO}_{1}$, while class 2 suffixes like -hood trigger the relation $\mathrm{OO}_{2}$ (see also Urbanczyk 1995, 1996 for a parallel case with multiple reduplicative morphemes). The differences in phonological behavior may in turn be treated as a differences in ranking: $\mathrm{OO}_{2}$-Faithfulness is ranked above the Markedness constraints which are responsible for the regular stress pattern, call them STRESS, effectively requiring similarity in stress where the regular stress patterns predict alternation. Class 1 suffixation, in contrast, does not require preservation of base prosody because the Faithfulness constraint regulating similarity between simplex-complex words of this type is ranked below STRESS.

\footnotetext{
${ }^{1}$ For further discussion and developments of this notion of Transderivational Faithfulness see Buckley 1995, Crosswhite 1997, Duanmu 1995, Flemming 1995, Itô \& Mester 1997, Kager 1995, 1996, Lin 1998, McCarthy 1995, 1997, Orgun 1995, 1997, and Steriade 1998.
} 
(9) Transderivational Approach to Preservation of Base Prosody (Benua 1997 [1998])

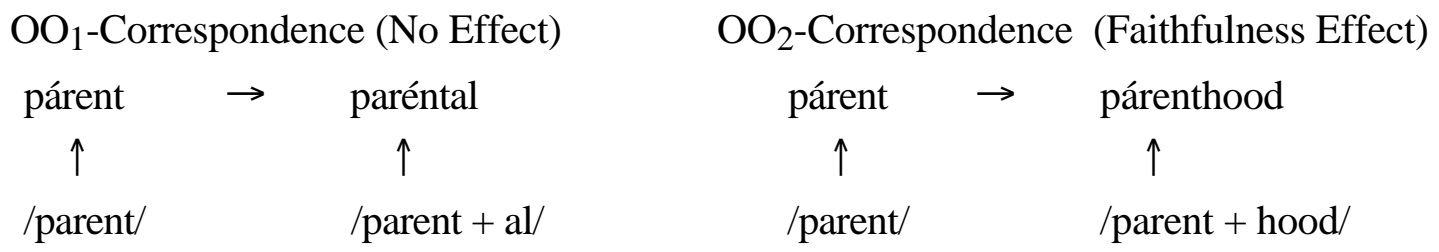

The transderivational approach does not differ from the cyclic approach in the proposed differences between class 1 and class 2 phonology: some measure of morpheme segregation is necessary on any analysis. The cyclic analysis needs a ordered block of phonological and morphological rules, while the transderivational analysis needs a ranking of distinct Faithfulness constraints to distinguish the two classes. The two theories differ sharply, however, on other matters of the analysis of similarity effects. As argued extensively in Burzio 1994 et seq., Benua 1997 [1998], and Kenstowicz 1996, the transderivational approach provides a natural account of the fact that similarity effects generally arise when the base of affixation is an independently occurring word (an observation originally due to Brame 1974). In TCT, this fact is explained by the very nature of Transderivational Correspondence - a phonological relation between words. This point will be developed directly below in the analysis of stress neutral affixation in English.

A second class of arguments that can be made in favor of the transderivational approach is that it provides a cogent explanation of non-uniform applications of a phonological process, i.e., the 'do something, except if...' patterns. Because the constraints responsible for the similarity effects, i.e., the Transderivational Faithfulness constraints, are ranked in an OT constraint hierarchy, these constraints may interact directly with other constraints in the grammar. This constraint interaction describes non-uniform phonological patterns in a direct and natural way, which distinguishes it from the rulebased approach to non-uniformity (see Prince \& Smolensky 1993, Prince 1993, Pater 1996, and Alderete 1997a for a related set of arguments). This argument will be developed below in the analysis of the 'semi-neutrality' of various affixes.

A third class of arguments presented in detail in Benua 1997 [1998] is that the transderivational model of phonological similarity is more restrictive. As will be shown in the analysis below, the behavior of both the base and its derivative is predicted by the same grammar, which in OT is an ordered ranking of universal constraints. Because of this requirement, the grammar puts substantive limits on the range of variation between the phonological patterns in base-derivative pairs. Derivational approaches to similarity effects such as the cyclic approach, on the other hand, allow the possibility that different levels of derivation be drastically different, and as a result, these theories are less restrictive than TCT.

Now that we have seen a glimpse of the motivation behind Transderivational Correspondence, let us proceed to apply this theory to the problem of stress neutral affixation and see how the approach is justified in the context of an explicit analysis. ${ }^{2} \mathrm{We}$ will start with a brief review of some familiar examples in English as a means of establishing the distinction between class 1 and class 2 affixes for stress. This review will then be followed by a presentation of the analysis given in Benua 1997 [1998] as an illustration of how TCT applies to stress-neutral affixation, a very common phenomenon in morphologically governed accent systems.

${ }^{2}$ For further reading on stress neutral affixation, see Crosswhite 1997, Kager 1996, and Kenstowicz 1997. 


\subsubsection{Transderivational Faithfulness in Stress Neutral Affixation}

\subsubsection{Theoretical Assumptions}

Preservation of base prosody presents a nice context for introducing the theoretical tools of TCT which will be used in subsequent analyses. The following set of assumptions together comprise TCT.

(10) Theoretical Assumptions in TCT (Benua 1997 [1998])

a. Transderivational (OO) Correspondence

Morphologically related words stand in correspondence; phonological similarity is regulated by OO-Faithfulness constraints.

\section{b. Recursive Hierarchies}

The constraint hierarchy is duplicated and ranked with respect to other hierarchies; morphological processes are associated with a given recursion of the recursive hierarchy.

\section{c. Subcategorization of OO-Correspondence Relations}

Subcategorization frames specify the OO-correspondence relation that links the affixed form with its base in a paradigmatic identity relation.

The first assumption (10a) forms the crux of the theory. In addition to relations between input-output pairs, correspondence relations hold between related outputs, e.g., párent $\approx$ párenthood. That is, correspondence relations may be 'transderivational' in nature, establishing a bond between two forms which share the same base. ${ }^{3}$ Furthermore, it is upon this correspondence relation that certain Faithfulness constraints are defined and which may bring about phonological similarity through constraint ranking.

A second basic assumption (10b) is that morphological concatenation is accompanied by the recursion of the constraint hierarchy which is ranked with respect to other hierarchies. The innermost input-output mapping establishes the 'base' of affixation for derived forms. This base is the form to which the morphologically complex forms must be faithful (to be elaborated on below) ${ }^{4}$ Thus, the first recursion of the constraint hierarchy gives penultimate stress on the monomorphemic form párent, establishing this form as the base for more complex words. Attachment of a class 2 suffix like -hood is derived in a second recursion, and it is on this input-output mapping that Faithfulness to the base becomes active, yielding preservation of base prosody in párenthood.

Finally, since the phonological behavior of affix-controlled phenomena is idiosyncratic, it must be lexically specified. In Benua's theory, this effect is achieved through subcategorization of the output-to-output correspondence relation that links the derived form with its base. Hence, class 1 suffixes like - $a l$ subcategorize for the correspondence relation $\mathrm{OO}_{1}$, while a class 2 suffix such as -hood selects a base specified for $\mathrm{OO}_{2}$-Correspondence. An important point is that the notion of subcategorization here is different that the notion commonly used in syntax. It is not the case that the affix selects a base with certain inherent properties of that base, e.g., it being a noun or bearing some feature. Rather, the affix imposes a property on the base to which it attaches, namely an

\footnotetext{
${ }^{3}$ The notion of transderivational relations between words is not new to OT; see for example Hock 1973, Hooper 1976, and Chung 1983.

${ }^{4}$ See also Benua 1995, 1997 [1998], Urbanczyk 1996, Kager 1995, Odden 1996, Itô \& Mester 1997, and Steriade 1998 for more discussion on this notion of a 'base' for other morphological processes.
} 
OO-correspondence relation. The differences in behavior between these two classes of suffixes are thus modelled as the differences in rankings of $\mathrm{OO}_{1}$ - and $\mathrm{OO}_{2}$-Faithfulness. These effects will be made explicit in the analysis that follows.

In most of the case studies developed below, the base of affixation stands in the simplex-complex relation, i.e., the bare stem or root forms the base for further affixation. In languages with obligatory inflections, however, the characterization of the base is a more subtle matter. Describing a pattern of analogy in Sanskrit, Green 1997 posits singular nouns as the base for the corresponding plurals; but there is no straightforward sense in which the inflected singular is the morphological base for the plural. A related case is found in Russian (\$5.2.3) where the singular forms the base for a shifting pattern of stress in the plural, e.g., kolbas-é versus kolbás-am 'sausage (singular/plural)'; yet again, the obligatory inflections show that the base-output relations go beyond the usual simplexcomplex relation. What principles are at work in predicting the base in these cases?

The answer to this question, I believe, involves considering traditional notions of markedness in morphology. It is common in linguistic studies to come across statements like 'the singular is the unmarked number' or 'the masculine is the unmarked gender', observations which are sometimes based on elicitation data, but also rooted in the linguistic processes of a given system. Interestingly, the base of an OO-correspondence relation is often the unmarked member of the pair in precisely this sense. In the Russian and Sanskrit examples given above, it is the singular which forms the base for the plural, where the former is less marked than the latter. Extending the case of Russian, masculine nouns form the base for corresponding feminine forms (see $\$ 3.2$ for discussion), a fact which follows the same basic principle: the base is the unmarked member of the pair. Clearly, the key to these cases is to make more precise the role of markedness in determining the base.

The problem posed by obligatory inflecting languages is analogous in a way to the problem posed by language learners when more than one input-output pair converge on the same output. Which of the inputs is learned as the one to which the actual output is faithful? Compare this problem with the situation in obligatory inflecting languages: various base-output relations are conceivable, but only one is operative in a given coupling of output forms. Prince \& Smolensky 1993 (see also Tesar \& Smolensky 1998) propose that constraint-based optimization has a role in solving this problem. According to the principle of Lexicon Optimization, the learned input is the one which leads to the most harmonic mapping relative to the language particular constraint hierarchy (see §1.2.2.3 for a definition and application of this principle). In other words, the input of the input-output pair which fares the best with respect to the grammar is the one which is posited as the lexical form.

My proposal is that the selection of the base is also the result of constraint-based optimization, as defined by the following principle.

(11) Base Optimization (Alderete 1997c, cf. Prince \& Smolensky 1993)

If a set of words created by some morphological process stands in the correspondence relation $R$, then the base for $R$ is the member of the base-output pair which is most harmonic with respect to the constraint hierarchy.

The idea is thus that the base is chosen as the word which leads to the best base-output pair, as prescribed by morphological markedness theory. Concretely, the observation that the singular is unmarked relative to the plural supports the ordering of constraints given below. Furthermore, these markedness relations are at work in determining the base for an output-to-output correspondence relation, as they are fundamental to establishing harmony 
relative to the constraint hierarchy. The following tableau illustrates this result for the singular-plural relations in Russian. The Markedness constraints here assess basederivative pairs as a whole - a paradigm in effect - and the resulting data structure is an ordered pair of the constraint violations incurred by the base and its related output. In evaluating these marks, violations incurred by the base take precedence over those of the output, consistent with the ordering implicit in the notion of Base Priority in Benua 1997 [1998].

(12) Selecting the Base with Base Optimization

\begin{tabular}{|c|c|c|}
\hline (Base, Output) & *PLURAL & *SINGULAR \\
\hline \hline (kolbas-amPL, kolbas-e $\mathrm{SG})$ & $(* !, \varnothing)$ & $(\varnothing, *)$ \\
\hline$\rightarrow($ kolbas-e $\mathrm{SG}$, kolbas-am $)$ & $(\varnothing, *)$ & $(*, \varnothing)$ \\
\hline
\end{tabular}

With this ordering of constraints, the singular is unmarked relative to the plural and not the other way around. As a result, Base Optimization chooses the singular form as the base because this option leads to better well-formedness in the paradigm overall. I speculate that the same principles are also at work in selecting other morphological classes, for example the masculine base in masculine-feminine pairs in Russian, and the case studies of languages with obligatory inflections will establish the base with the principle of Base Optimization. ${ }^{5}$ While there are still some details to be worked out concerning the evaluation of constraint violations, the important point here is that Markedness for morphological structure is what is fundamental, an idea that can be formalized in a variety of ways.

One important difference between the theory of OO-Correspondence developed here and that of Benua 1997 [1998] is that the base does not stand in correspondence with the entire derived form in the current theory. Rather, only the segments of the shared morphemes stand in correspondence. This model of OO-Correspondence is crucial, as will become clear in the analysis of various patterns of Anti-Faithfulness. Formally, what this assumption entails is that only the subconstituent of the derived form which has correspondents in the base stand in correspondence. If an affix is attached in an output and is not also present in the base, then the affix does not stand in correspondence with the base. Thus, the plural inflection -am does not stand in correspondence with anything in the base form kolbas-e above in (12). I show in chapter 5 that this model is important in explaining certain properties of pre- and post-accentuation and accentual shifts.

\subsubsection{Application to Stress Neutral Affixation}

Let us now apply these ideas to the case of stress neutral affixation. In English nouns and adjectives, primary stress is oriented to the right edge of the word, typically falling on a penultimate heavy syllable, otherwise the antepenultimate syllable. ${ }^{6}$

Suffixation may thus give rise to alternations in stress, depending on the sensitivity of the suffix to stress. A basic distinction that is necessary in any treatment of English stress is

\footnotetext{
${ }^{5}$ Indeed, the same principle of markedness may be at work in deriving the simplex-complex relation often found in base-output relations. Affixation categorically leads to a mismatch between the edges of the stem and larger prosodic structure like the PrWd; simplex forms may thus be chosen as the base for an affixed form by Anchoring constraints which evaluate the edge-anchoring properties of a stem relative to a prosodic category.

${ }^{6}$ On the principles of English Stress, see especially: Chomsky \& Halle 1968, Halle \& Keyser 1971, Ross 1972, Goyvaerts \& Pullum 1974, part II, Hayes 1980, 1982, Selkirk 1980, 1984, Fudge 1984, Kager 1989, Hammond 1989, Burzio 1994, and Pater 1995.
} 
one between STRESS SHIFTING and STRESS NEUTRAL affixes (Siegel 1974). Stress shifting suffixes are typically not themselves stressed, but are counted in the determination of stress and may also trigger certain non-automatic processes like vowel shortening. The examples given below show a shift in primary stress with attachment of this class of suffixes.

(13) Stress Shifting Suffixes ${ }^{7}$

$$
\begin{aligned}
& -a l_{\mathrm{A}} \\
& \text { órigin - oríginal } \\
& \text { médicine - medícinal } \\
& \text { úniverse - univérsal } \\
& \text { párent - paréntal }
\end{aligned}
$$

$$
\begin{aligned}
& \text {-ity } \\
& \text { tránquil — tranqúillity } \\
& \text { uniform - ùnifórmity } \\
& \text { pópular - populárity } \\
& \text { contínue — continúity }
\end{aligned}
$$

$$
\begin{aligned}
& \text {-ic } \\
& \text { áthlete - athlétic } \\
& \text { mícroscope - microscópic } \\
& \text { hý giene - hygénic } \\
& \text { álgebra - algebráic }
\end{aligned}
$$

The second class of suffixes, the stress neutral suffixes, are also themselves unstressed, but they differ from the previous class in that they do not affect the prosody of the base to which they are attached.

\section{(14) Stress Neutral Suffixes}

$$
\begin{array}{lll}
\text {-hood } & \text {-dom } & \text {-less } \\
\text { párent - párenthood } & \text { free - freedom } & \text { bóttom - bóttomless } \\
\text { child - childhood } & \text { mártyr - mártyrdom } & \text { defénse - defénseless } \\
\text { néighbor - néighborhood } & \text { báchelor - báchelordom } & \text { expréssion - expréssionless } \\
& \text { héathen - héathendom } & \text { méaning - méaningless }
\end{array}
$$

Stress neutral suffixes typically attach to independently occurring words (though there are some exceptions) and they may not trigger non-automatic processes. In summary, there is a fundamental distinction between suffixes which require Faithfulness to base prosody (class 2) and suffixes which do not (class 1), and any analysis of affixed words in English must recognize and analyze this basic distinction.

Before dealing with the difference between class 1 and class 2 suffixes, we require an analysis of stress in monomorphemic words. Assuming what may be characterized as the 'standard' analysis, primary stress is assigned by laying down moraic trochees from right-to-left, ignoring the final syllable; secondary stress is then derived by assigning trochees from the left edge of the word (see especially Hayes 1980, 1982, Selkirk 1984, Pater 1995, cf. Burzio 1994). Following Pater, this analysis is characterized in the partial ranking of constraints given below. ${ }^{8}$

(15) Stress in Monomorphemic English Words (Pater 1995)

FTBIN, TROCH, NonFinAlity $\gg$ ALIGN-HEAD-RIGHT $\gg$ ALL-FT-LEFT

\footnotetext{
${ }^{7}$ Two of these suffixes, namely -ity and -ic also trigger a non-automatic process of vowel shortening, but this fact will be ignored here because it is orthogonal to the Faithfulness effect discussed here. See Myers $1987 \mathrm{~b}$ for an attractive analysis, however, in terms of prosodic foot structure.

${ }^{8}$ The role of quantity sensitivity is ignored here because it does not bear directly on the arguments which follow.
} 
(16) The Operative Constraints

FTBIN: Feet are binary as some level of analysis $(\mu, \sigma),(\mathrm{P} \& \mathrm{~S}$, see also McCarthy \& Prince 1986, Hayes 1987).

TROCH: feet are left-headed.

NONFINALITY: No head of PrWd is final in PrWd (P\&S, OT constraint deriving extrametricality effects).

Align-HEAD-RIGHT (ALIGN-HD-RT): The foot head of the PrWd must coincide with the right edge of PrWd (McCarthy \& Prince 1993a).

ALL-FT-LEFT (ALL-FT-LT): The left edge of all feet must coincide with the left edge of PrWd (McCarthy \& Prince 1993a).

Because of the ranking of NONFINALITY, the final syllable is ignored, and so in a form like agénda, a moraic trochee is built over the penultimate heavy syllable: [a(gén) $\langle\mathrm{da}\rangle]$. In words with penultimate lights, e.g., Cánada, the stress is assigned to the antepenultimate syllable, as in: [(Cána)〈da $\rangle]$. In longer forms, a second foot is possible, yielding secondary stresses iterated from the beginning of the word, [(Àle)(xán)〈der $\rangle]$, which may result in an initial dactyl, e.g., [(Táta)ma(góu)〈chi〉], because of low-ranking ALL-FTLEFT.

Moving now to the analysis of stress in derived words, we employ the notion of Transderivational Correspondence to derive preservation of base prosody. To begin, in word pairs like párent- párentage, the attachment of a class 2 suffix like -age triggers a specific correspondence relation. Further, the Prosodic Faithfulness constraints defined upon this relation, an encapsulation of OO-MAX-PROM, OO-DEP-PROM, OO-NOFLOPPROM (see $§ 1.2)$, require similarity in stress. ${ }^{9}$ Thus, the output pairs [(pá)〈rent $\left.\rangle\right] \approx$ $[($ párent $)\langle$ hood $\rangle]$ match in prominence structure in that there are corresponding grid marks in each head of the stress foot, and so this pair of words satisfies OO-PROS-FAITH. However, a stress shift to improve the overall prosodic well-formedness of the word, as in *[pa(rént)〈hood $\rangle$, violates OO-PROS-FAITH because of the mismatch in prosody, and so this pairing can be ruled out through constraint interaction.

The distinction between class 1 and class 2 suffixes may now be modelled in terms of familiar kinds of constraint interaction. Hence, the stress-shifting/stress-neutral behavior of various affixes may be modelled by the interspersing of the OO-Faithfulness constraints relative to the constraints also active in underived words, as shown in (17). As Benua makes clear, it is significant that the ranking of the two Alignment constraints, ALIGN-HEAD-RIGHT and ALL-FT-LEFT, must be consistent with their ranking in (15). On the assumption that the grammar of English stress is a total ordering of constraints, the rankings could not be otherwise.

(17) Stress in Derived Words (Benua 1997 [1998])

$\mathrm{OO}_{2}$-PROS-FAITH $>$ ALIGN-HEAD-RIGHT $>\mathrm{OO}_{1}$-PROS-FAITH $>$ ALL-FT-LEFT

\footnotetext{
9In Benua's analysis, the Faithfulness constraint responsible for preserving base prosody is a Prosodic Anchoring constraint (after McCarthy 1997), but for the sake of consistency with the analyses developed elsewhere in this thesis, I employ the Prosodic Faithfulness constraints argued for in $§ 1.2$.
} 
Running through the ranking here, the Faithfulness constraint for class 2 suffixes, $\mathrm{OO}_{2}-$ PROS-FAITH ranks above ALIGN-HEAD-RIGHT, with the effect that the attachment of a class 2 suffix will not affect the prosody of the base. However, the corresponding Faithfulness constraint for class 1 affixes, $\mathrm{OO}_{1}$-PROS-FAITH, is dominated by the constraint responsible for the placement of main stress, and therefore class 1 suffixes like $a l$ will not induce preservation of base prosody. Finally, $\mathrm{OO}_{1}$-PROS-FAITH does dominate the constraint responsible for secondary stress assignment, namely ALL-FT-LEFT, and this ranking yields preservation of a base prosody in a non-primary stress foot, a typical kind of 'non-uniformity' effect. These results will be illustrated in a tableau given below.

Moving next to the individual affixes, consider the differences between class 1 and class 2 suffixes encoded in the lexical entries sketched below. Class 1 suffixes may differ from some class 2 suffixes in that they may attach to a bound stem, which, following Chomsky \& Halle 1968, is represented with different boundaries: ' + ' is used with bound stems and '\#' with free stems. More importantly, however, the two classes of suffixes are distinguished by their subcategorized correspondence relations, which is specified by the numerical index. Hence, class 1 suffixes trigger a $\mathrm{OO}_{1}$-Correspondence relation between the base and the derived form containing the suffix, while class 2 suffixes trigger $\mathrm{a} \mathrm{OO}_{2}$ correspondence relation. ${ }^{10}$

(18) Lexical Entries for Class 1 Suffixes

$$
\begin{array}{ll}
- \text { al } & {[+ \text { Noun+OO1_ }] \rightarrow \text { Non-Gradable Adjective }} \\
\text {-ity } & {[+ \text { Adjective+ }+ \text { OO1 }] \rightarrow \text { Noun: 'State/Quality of Adjective' }} \\
\text {-ic } & {[+ \text { Noun+OO1__ }] \rightarrow \text { (Non-Gradable) Adjective }}
\end{array}
$$

(19) Lexical Entries for Class 2 Suffixes

$$
\begin{aligned}
& \text {-hood [ \#Noun\# OO2 __ ] } \rightarrow \text { Abstract Noun: 'Status of Noun' } \\
& \text {-dom [ \#Noun\# } \mathrm{OO} 2 \text { _ _ ] } \rightarrow \text { Abstract Noun: 'Domain/Condition of Noun' } \\
& \text {-less [ \#Noun\# } \left.\text { OOO }_{2}{ }_{\text {_ }}\right] \rightarrow \text { Adjective: 'Without Noun' }
\end{aligned}
$$

The index on the host of the affix is the subcategorized correspondence relation which indicates the type of Faithfulness which relates the base to the derived form. Thus, consistent with other idiosyncratic features of the affix, subcategorization frames are used as a means of encoding the specific correspondence relation triggered by a given affix.

All of the basic assumptions inherent to TCT are illustrated in the following OT tableau. The prosody of the monomorphemic form parent is computed in the first recursion, yielding [(pá)〈rent $\rangle]$ as the base for further word derivation.

\footnotetext{
${ }^{10}$ The interpretations and morphological restrictions on these affixes are based on the description given in Quirk \& Greenbaum 1973, but nothing crucial hinges on this information.
} 
(20) Preservation of Base Prosody with Class 2 Suffixation

$i$. Recursion (a)

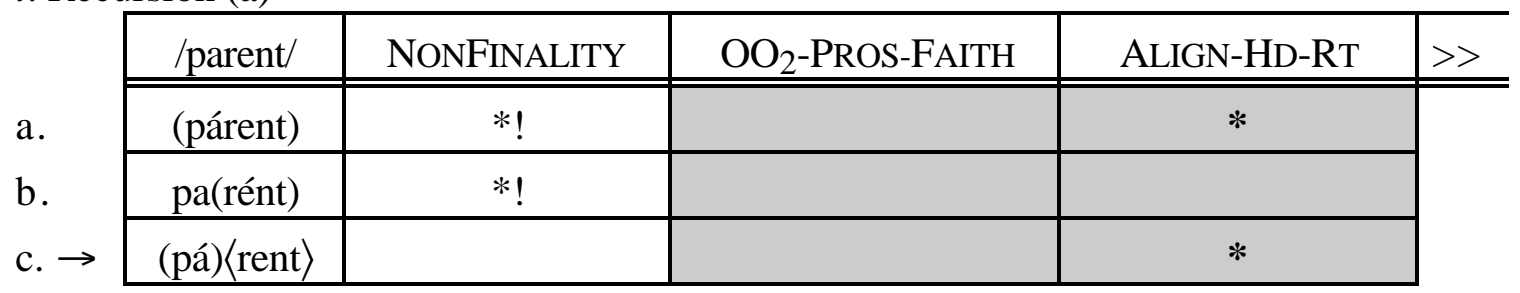

ii. Recursion (b)

\begin{tabular}{|c|c|c|c|c|c|}
\hline$>$ & Base & /parent + hood/ & NONFINAL & $\mathrm{OO}_{2}$-P-FAITH & HD-RT \\
\hline a. & (pá) $\langle$ rent $\rangle$ & $\mathrm{pa}($ rént $)-\langle$ hood $\rangle$ & & $* !$ & \\
\hline b. $\rightarrow$ & (pá) $\langle$ rent $\rangle$ & (párent)-〈hood $\rangle$ & & & $* *$ \\
\hline
\end{tabular}

On the second recursion, the class 2 suffix -hood is attached, triggering the Faithfulness constraint $\mathrm{OO}_{2}$-PROS-FAITH. Base-derivative pairs are therefore compared for their prosodic faithfulness, and the output pair which preserves the prosody of the base wins because of the rank of $\mathrm{OO}_{2}$-PROS-FAITH in the system.

Class 1 suffixes, on the other hand, subcategorize for $\mathrm{OO}_{1}$-correspondence, and as a result they are only sensitive to the Faithfulness constraints defined on this correspondence relation. Together with the constraint hierarchy in (17), this lexical property accounts for their non-neutral behavior in relation to base prosody. Thus, because the Alignment constraint responsible for the rightward orientation of stress outranks $\mathrm{OO}_{1}$ PROS-FAITH, the regular pattern of heavy penultimate stress is assigned in a form like paréntal, as illustrated in the following tableau.

(21) Stress Shift with Class 1 Suffixation

i. Recursion (a)

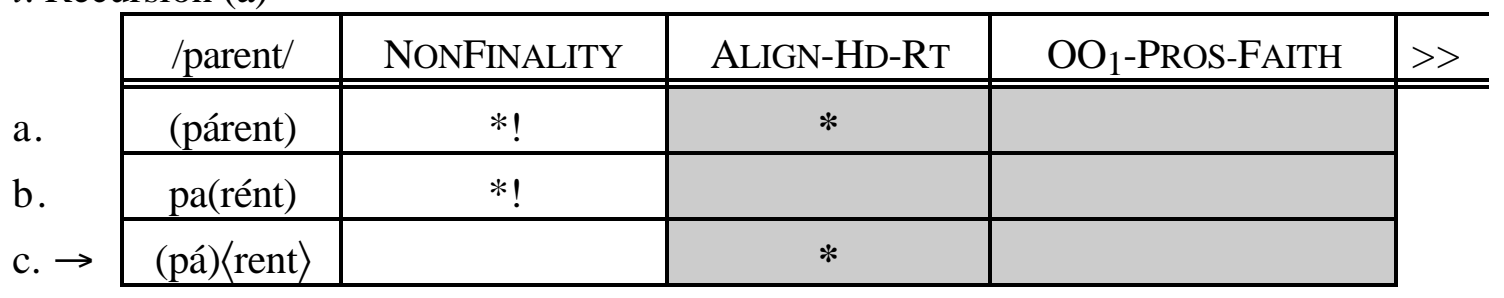

ii. Recursion (b)

\begin{tabular}{c|c|c|c|c|c|}
\hline$>>$ & Base & $/$ parent $+\mathrm{al} /$ & NONFINAL & HD-RT & OO $_{1}$-P-FAITH \\
\hline \hline \multirow{2}{*}{$\begin{array}{l}\text { a. } \rightarrow \\
\text { b. }\end{array}$} & (pá) $\langle$ rent $\rangle$ & pa(rén) $\langle$ t-al $\rangle$ & & $*$ & $*$ \\
\cline { 2 - 6 } & (pá) $\langle$ rent $\rangle$ & $($ páren $)\langle\mathrm{t}-\mathrm{al}\rangle$ & & $* * !$ & \\
\hline
\end{tabular}

While a form like párent receives initial stress, in the second recursion, $\mathrm{OO}_{1}$-PROS-FAITH has no force because of its rank in the system. As a result, párent cannot influence the prosody of the derivative, and we predict regular penultimate stress. 
Now that the basic analysis to stress-neutral affixation in TCT has been illustrated, we can make the first argument in favor of this approach more forcefully. This argument is based on the empirical observation that class 2 suffixes in English only exhibit base identity effects for stress when the affixed word stands in correspondence with an independently occurring word. Thus, while many class 2 suffixes only attach to free stems, some suffixes may also attach to bound stems (this tendency often correlates with different phonological behavior). For example, the suffixes exemplified below are stress neutral when they attach to a free stem, but counted in the placement of primary stress when the base to which they attach is not an independent word.

(22) Stress Neutral Suffixation with(out) Base Identity Effects

\begin{tabular}{|c|c|}
\hline $\begin{array}{l}\text {-age } \\
\text { párent — párentage } \\
\text { bróker — brókerage } \\
\text { hérmit — hérmitage } \\
\text { báronet — báronetage }\end{array}$ & $\begin{array}{l}\text {-or } \\
\text { conféss - conféssor } \\
\text { góvern - góvernor } \\
\text { prósecute - prósecutor } \\
\text { cf. cóntribute - contríbuto }\end{array}$ \\
\hline $\begin{array}{l}\text { advántage } \\
\text { vérbiage } \\
\text { fóliage } \\
\text { cámouflage } \\
\text { cf. éspionage }\end{array}$ & $\begin{array}{l}\text { ambássador } \\
\text { wárrior } \\
\text { interlócutor }\end{array}$ \\
\hline
\end{tabular}

-ist
extréme - extrémist
séparate - séparatist
módern - módernist
propagánda - propagándist

Báptist

recídivist

ventríloquist

opportúnist

Drawing on the classification system of Fudge 1984, these suffixes are stress neutral when they attach to words, but 'Pre-Stressed 1/2' (pre-stressing when they follow a strong syllable, otherwise pre-pre-stressing) if they attach to a bound stem. In other words, the regular pattern of 'penultimate heavy, otherwise antepenultimate stress' takes effect in the latter case.

The analysis of stress-neutral affixation in terms of Transderivational Faithfulness explains this split effect with the same basic premises of the theory. ${ }^{11}$ Assuming that these suffixes are class 2 , they will trigger $\mathrm{OO}_{2}$-correspondence, and as a result, the preservation of base prosody in base-derivative pairs such as párent-párentage is fully consistent with the analysis of this same fact in párent-párenthood. Moreover, in derived words which do not have an independently occurring free base, $\mathrm{OO}_{2}$-PROS-FAITH does not have an effect because there is no word for the derived form to be faithful to. The decision in a case like advantage falls to the lower ranking constraint, ALIGN-HEAD-RT, which gives the observed Latin-like stress pattern, as in [ad(ván)〈tage $\rangle$. In sum, one of the fundamental tenets of the theory, namely that Faithfulness relations specifically hold between related words, explains the curious dual behavior of some class 2 suffixes.

A second argument in favor of the transderivational approach can be constructed by considering the mixed behavior of class 1 suffixes. As noted in both Burzio 1994 and Benua 1997 [1998], while class 1 suffixes do not affect the placement of main stress, they may bring about an apparently exceptional pattern of secondary stress they are compared with a base form. As exemplified below, the prosody of the base may be preserved as a secondary stress in a derived form with a class 1 suffix, yielding irregular second syllable stress in words like orìginálity.

\footnotetext{
${ }^{11}$ This argument was first put forth in Burzio 1994, though it is couched in a different theoretical framework.
} 
(23) Preservation of Base Prosody in Class 1 Paradigms

oríginal orìginálity cf. Tàtamagóuchi

arístocrat arìstocrátic

theátrical theàtricálity

The analysis of this fact in TCT is again established through constraint ranking. While $\mathrm{OO}_{1}$-ANCHOR is dominated by some constraints, it is ranked above the constraint responsible for positioning secondary stress feet, namely ALIGN-FT-LT. By giving $\mathrm{OO}_{1^{-}}$ PROS-FAITH intermediate rank in the system, the non-uniform application of prosodic faithfulness is achieved simply and directly, as illustrated below (from Benua 1997 [1998]).

(24) Non-Uniform Preservation of Base Prosody with Class 1 Suffixes

i. Recursion (a)

\begin{tabular}{|c|c|c|c|c|c|}
\hline /origin/ & NONFIN & ALIGN-HD-R & $\mathrm{OO}_{1}$-P-FAITH & ALL-FT-LT & $>$ \\
\hline $\mathrm{o}$ (rígin) & $* !$ & & & $*$ & \\
\hline (óri) $\langle$ gin $\rangle$ & & $* *$ & & & \\
\hline
\end{tabular}

ii. Recursion (b)

\begin{tabular}{l|c|c|c|c|c|c|}
\hline$\gg$ & Base & /origin $+\mathrm{al} /$ & NONFIN & HD-R & OO $_{1}$-FAITH & ALL-FT-LT \\
\hline \hline \multirow{2}{*}{$\begin{array}{l}\text { a. } \\
\text { b. } \rightarrow\end{array}$} & (óri) $\langle$ gin $\rangle$ & (óri)gi $\langle$ n-al $\rangle$ & & $* * * !$ & & \\
\cline { 2 - 7 } & (óri) $\langle$ gin $\rangle$ & o(rígi) $\langle\mathrm{n}-\mathrm{al}\rangle$ & & $* *$ & $*$ & $*$ \\
\hline
\end{tabular}

iii. Recursion (c)

\begin{tabular}{l|c|c|c|c|c|c|}
\hline$>>$ & Base & /original + ity/ & NONFIN & HD-R & OO $_{1}$-FAITH & ALL-FT-LT \\
\hline \hline \multirow{2}{*}{$\begin{array}{l}\text { a. } \\
\text { b. } \rightarrow\end{array}$} & o(rígi) $\langle$ n-al $\rangle$ & (òri)gi(n-ál-i) $\langle$ ty $\rangle$ & & $*$ & $* !$ & $* * *$ \\
\cline { 2 - 7 } & o(rígi) $\langle$ n-al $\rangle$ & o(rìgi $)($ n-ál-i) $\langle$ ty $\rangle$ & & $*$ & & $* * * *$ \\
\cline { 2 - 7 }
\end{tabular}

As with the case of paréntal, when -al is attached on the second recursion, the prosody of the base is not preserved, and we find regular penultimate stress (24ii). However, when a second class 1 suffix is attached on the third recursion, the base prosody is matched in the derivative by a shifting of the secondary stress foot away from its canonically left-aligned position (24iii). Again, this result follows from the interleaving of $\mathrm{OO}_{1}$-Faithfulness with the constraints responsible for primary and secondary stress, ALIGN-HD-R and ALIGN-FTLT respectively. To summarize, the non-uniform application of $\mathrm{OO}_{1}$-PROS-FAITH derives directly from a basic assumption in OT, namely that constraints are ranked and violable.

The theory of Transderivational Correspondence developed in Benua 1997 [1998] was introduced as a formal theory of preservation of base prosody between morphologically related words. It was also shown that the principles inherent to this theory go a long way towards explaining the preservation of base prosody. First, some suffixes exhibit mixed phonological behavior, showing stress neutrality with a free stem but regular stress with bound stems. This fact follows from the nature of Transderivational Correspondence because this is a relation that only holds between independently occurring words. Second, the transderivational approach offers a cogent account of class 1 suffixation which shows a different species of non-uniform base identity effect, namely the 
preservation of base prosody only with secondary stresses in cases like

oríginal-originálity, but not with primary stress, e.g., órigin—oríginal. This fact follows from the parallelist interpretation of TCT in which Faithfulness constraints evaluating output pairs interact directly with the other constraints in the grammar. When OOFaithfulness is ranked between two constraints responsible for a given pattern, nonuniformity in base identity may result.

\subsection{Tranderivational Anti-Faithfulness}

In this section, the notion of Transderivational Anti-Faithfulness (TAF) is developed as a cross-linguistic theory of morpho-phonological alternations. The section starts by introducing exchange rules as a problem for both traditional generative phonology and Optimality Theory. The theory of TAF is then motivated as a means of describing morphologically governed exchange processes, and subsequently, the importance of TAF to explaining the properties of morpho-accentual processes is brought to the fore.

\subsubsection{Introduction}

It is a common observation that phonological processes have a morphological function. The voicing alternation in $h o u[\mathrm{~s}] e \sim h o u[\mathrm{z}] e s$ may be viewed as a phonological process that serves to mark a relationship between a singular and plural form. This type of morpho-phonological process is quite widespread and encompasses a variety of phonological structures. Indeed, a careful study of any given language is likely to turn up non-automatic alternations that are only found in particular morphological environments. For example, there are abundant examples in which a length alternation is only observed in specific affixed forms: Yidiñ (Dixon 1977), Slovak (Rubach 1993), and Gidabal (Geytenbeek \& Geytenbeek 1991). Similar examples can be given for features as different as vowel quality, laryngeal settings, nasality, stress, and tone (see Spencer 1998 for a survey and discussion of such morpho-phonological alternations).

The standard approach to this type of observation in the generative program is Lexical Specification (LS). In particular, morpho-phonological alternations are said to follow from a specification for the alternating feature in the lexical representation of the morphemes involved. For the English case, LS requires a lexical [+voice] specification for a consonantal position in the affixes which trigger the alternation (essentially the plural suffix and -ing), which, when occupied by the stem-final obstruent, yields the result depicted below.

(25) English Morpho-Phonology as Lexical Specification

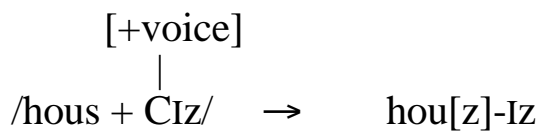

The 'frozen' character of the alternation therefore follows from an assumption about the input: the alternation is not automatic because it derives from a lexical listing. While there are some interesting puzzles for this approach, for example dominant affixes in Russian and Japanese, LS accounts for the spotty distribution of the morpho-phonological pattern with one of the most basic assumptions in generative phonology, the underlying representation.

Often morpho-phonology is 'one-way', effecting a change of one class of segments to another, as in the English case. But it may also be a two-way operation, yielding a full 
reversal of the lexical properties of the targeted element. A well-known example with this character is the voicing exchange found in the Nilotic language Luo. In this language, the alternation in voicing goes both ways: stem-final obstruents generally reverse their underlying [voice] specification in the plural.

(26) Consonantal Polarity in Luo (Gregerson 1972, Okoth-Okombo 1982) (2 $^{12}$

$\begin{array}{lll}\begin{array}{l}\text { Singular } \\ \text { bat }\end{array} & \begin{array}{l}\text { Plural } \\ \text { bed-e } \\ \operatorname{lu} \theta\end{array} & \text { 'arm' } \\ \text { reč } & \text { luD-e } & \text { 'walking stick' } \\ & \text { reǰ -e } & \text { 'fish' } \\ \text { č ogo } & \text { č ok-e } & \\ \text { owadu } & \text { owet-e } & \text { 'bone' } \\ \text { luedo } & \text { luet-e } & \text { 'brother' }\end{array}$

As with $h o u[\mathrm{~s}] e \sim h o u[\mathrm{z}] e s$, the alternation has a morphological function, again supporting the opposition between singular and plural forms. This morphological contrast also extends to singular/singular appertentive alternations (a construction similar to the Semitic construct state): got 'mountain', gode 'mountains', god 'mountain of', which demonstrates that the process is truly morphologically governed, and not, for example, a dual process of intervocalic voicing and (opaque) final devoicing. Another parallel case is plural formation in Diegueño verbs, which also involves a full rotation of the vowel length of a stem vowel (Langdon 1970; see Anderson \& Browne 1973, Anderson 1975, 1991, Moreton 1996, and Spencer 1998 for discussion of additional examples).

The importance of the voicing exchange in Luo is that it shows that morphophonological alternations are not simply additive functions requiring the addition of a given feature in a particular environment. Rather, they may encode a full exchange of two segment classes or other phonological elements. Alternations of this type therefore cannot be described with LS because it is inherently additive. If part of the exchange is lexically specified, then the other is left unaccounted for. Concretely, if the voicing of bed-e is due to a lexical marking [+voice] in the plural suffix, then this assumption predicts a different result than the observed pattern with $\check{c}$ ok-e.

Exchange processes also pose a challenge to some versions of Optimality Theory. As demonstrated in Moreton 1996, if an OT grammar is constructed by ranking Markedness constraints relative to Faithfulness constraints, then no grammar of this type will result in an exchange process or a circular exchange of any kind. To flesh out this point, a phonological process results when a Markedness constraint $\mathbb{M}$ compels the lexical form to change at the surface, that is, a violation of a Faithfulness constraint $F$. Thus, if the ranking $\mathbb{M} \gg \mathbb{F}$ gives in $/ \mathrm{A} / \rightarrow[\mathrm{B}]$, then $[\mathrm{B}]$ must be less marked than $[\mathrm{A}]$; /A/ changed to $[\mathrm{B}]$ in order to do better on $\mathbb{M}$. This same grammar will not then change $/ \mathrm{B} /$ to [A] (in the same context): to do so would result in a form which fares worse on $\mathbb{M}$. Moreton's finding is indeed an interesting one as exchanges of the kind found in Luo are always morphological (Anderson \& Browne 1973). It would appear therefore that there is a goodness of fit between the set of possible grammars predicted in OT and the phonological patterns observed in the world's languages.

${ }^{12} \mathrm{~A}$ note on transcription: /D/ is a (inter)dental fricative. 
How then are morpho-phonological exchanges accounted for in OT? The proposal made here is that, in addition to Markedness and Faithfulness, UG contains a set of rankable constraints which actively enforce an alternation in morphologically related words. These constraints, the so-called Transderivational Anti-Faithfulness (TAF) constraints, induce an alternation by requiring a violation of Faithfulness in base-derivative pairs. Applying this idea to the case of Luo, singular and plural forms are assumed to stand in correspondence, with the singular forming the base of this correspondence relation. Both parts of the exchange are thus accounted for as an effect of Anti-Faithfulness, which requires a reversal of the [voice] specification of the base segments.

(27) Morpho-Phonology as Transderivational Anti-Faithfulness

$\begin{array}{lll}\text { Base } & & \text { Output } \\ \text { bat } & \neq & \text { be[d]-e } \\ \text { chogo } & \neq & \text { cho[k]-e }\end{array}$

This notion of Anti-Faithfulness is not totally new. It has been applied in the analysis of repetition avoidance in reduplication (Yip 1996) and certain patterns of quantitative enhancement in Yupik languages (Baković 1996). Also, the notion of 'AntiCorrespondence' employed in Hayes 1997 to account for apparent language particular processes derives from the same underlying idea, namely that morpho-phonology supports an overt contrast (see also Blevins 1997). The novel aspect of the proposal here will be to give a formal characterization of a general set of Anti-Faithfulness constraints, and then to apply them to a wide range of examples, including the affix-controlled accentual process surveyed in $\$ 4.1$.

In the discussion which follows, there will be three main arguments in favor of TAF. First, TAF theory solves the problem confronted by the Lexical Specification theory of morpho-phonology by accounting for exchange processes as Faithfulness reversals. Both parts of the voicing exchange in Luo may be described as an effect of constraints which require dissimilarity in a base and its related derivative. A second type of argument is that TAF theory successfully explains phonological 'conspiracies' in morphophonological alternations (in the sense of Kisseberth 1970). As will be shown below, morpho-phonological patterns are often GRAMMAR DEPENDENT; their output is constrained by the independently motivated grammatical principles in the language on a whole. In the theory proposed here, the TAF constraints simply require that a form be unfaithful to its base, but they do not always specify exactly how the resulting unfaithfulness should be realized. For example, dominant unaccented suffixes in Russian trigger a deletion of a stem accent and bring about default ending stress, as in /púz + ač $+\mathrm{u} / \rightarrow p u z-a \check{c}-\bar{u}$ 'man with paunch (dative singular)'. The TAF account for this pattern requires a deletion of base stress, but it does not prescribe the ultimate outcome resulting from this deletion. Grammar dependence is the phenomenon whereby the rest of the constraint hierarchy - the independently motivated defaults - says how to be unfaithful. As shown in $\S 3.2$, the default pattern for stress in Russian is stress on the inflectional ending, and so the larger grammar of stress has a say here in predicting the pattern which results from the deletion. TAF theory explains grammar dependence by simply requiring a change, and the rest of the grammar dictates how the change is realized.

A third and important type of motivation for TAF theory is that it provides exactly the right tools for describing affix-controlled accent (ACA). As with the cases examined above, morpho-accentual processes are commonly characterized in terms of a morphological opposition. Russian, for example, has two patterns of mobile stress where 
singular inflected nouns show a pattern which is different than the plural inflected forms, e.g., kolbas-é versus kolbás-am 'sausage (dative singular/plural)'. Stankiewicz 1962 et seq. argues cogently that these patterns support an overt opposition between singular and plural pairs, a pattern of opposition which extends to other morphological classes (see $\$ 3.2$ for discussion). Likewise, in Getxo Basque, the morpho-accentual process of preaccentuation serves to mark salient morphological distinctions, also including the plural (Hualde \& Bilbao 1993). Singular-plural pairs in words with unaccented stems are distinguished by the presence of an accent in the final (or penultimate) syllable of the stem, as in gison-a, cf. gisón-ak 'man (absolutive singular/plural)', and gison-ak versus gisón-ak (ergative singular/plural); in the latter case, the only phonetic difference between the two words is the presence of an accent in the plural. In sum, accentual processes also have a morphological function, realizing a contrast between related words, which leads to a comparison with other morpho-phonological alternations in terms of Faithfulness reversals.

The principal goal of chapter 5 is to show that TAF constraints provide the right tools for explaining the properties of affix-controlled accentual processes. TAF theory will derive the fact that said processes are 'affix-controlled' because the TAF constraints operate between a base and its derivative, effectively accounting for the morphological nature of the alternation. Furthermore, a significant and important property of ACA is that it is grammar dependent. For example, a fundamental property of dominant affixes is that they trigger a deletion of accent and produce a default accentual pattern. In the TAF theory of ACA, dominant affixes trigger an obligatory deletion of accent and the rest of the grammar kicks in to yield the default accentual pattern, as with other morpho-phonological processes. A final important consequence of the TAF theory of ACA is that it makes a testable prediction concerning the range of affix-controlled accentual processes. Succinctly, if ACA is due to Faithfulness reversals, the range of possible accentual processes is limited by the set of Faithfulness constraints governing accent. As is shown in detail in chapter 5, the typology of affix-controlled processes is correctly correlated with the negation of the independently attested Faithfulness constraints for accent; reversals of the Prosodic Faithfulness constraints motivated in $\$ 1.2$ produces precisely the set of affix-controlled accentual phenomena found cross-linguistically.

\subsubsection{Transderivational Anti-Faithfulness}

\subsubsection{Theoretical Assumptions}

Morpho-phonological alternations may support a contrast between morphologically related words. The theory of Transderivational Anti-Faithfulness works in tandem with other independently motivated principles to derive the observed morphological oppositions. In line with Transderivational Faithfulness, this theory builds on the existing notion of Output-to-Output Correspondence (28a). As will become clear in the applications below, the TAF constraints compare a base and its related output, like the Transderivational Faithfulness constraints. The principal difference between these two classes of constraints is that the TAF constraints require a violation of Faithfulness, yielding the observed contrast between related words.

Another aspect of the theory, to be developed more explicitly below, concerns the analysis of locality effects sometimes observed in morpho-phonological alternations. It is often the case that the affected element is 'close' in some sense to the affix triggering the alternation. Following Lubowicz 1998, I treat this type of locality effect with the same tools used to account for locality in so-called derived environment effects (28b). The principle of Local Conjunction provides the descriptive framework for deriving a range of 
locality effects, all of which are attested in the morpho-accentual processes discussed in chapter 5.

(28) Morpho-Phonological Alternations as Anti-Faithfulness

a. Output-to-Output Correspondence (Benua 1997 [1998], Kenstowicz 1996, Burzio 1996; see $\$ 4.2$ for background) Morphologically related words may stand in correspondence.

b. Constraint Activation Through Local Conjunction (Lubowicz 1998, see also Smolensky 1993, 1995) Derived environment effects are explained as the Local Conjunction of a Faithfulness constraint with a Markedness constraint: violation of a Faithfulness constraint activates the Markedness constraint in a local context.

c. Anti-Faithfulness

Anti-Faithfulness constraints are the negation of the corresponding Faithfulness constraints, encouraging dissimilarity where Faithfulness constraints require similarity.

These independently necessary principles work to further refine the a new type of constraint, Anti-Faithfulness. Anti-Faithfulness, as stated below, is the negation of Faithfulness, instantiated through wide scope negation of the proposition expressed by the corresponding Faithfulness constraint.

(29) Anti-Faithfulness

Given the Faithfulness constraint $F, \neg F$ is the related Anti-Faithfulness constraint which is satisfied in a string $S$ iff $S$ has at least one violation of $F$.

To see how a Faithfulness constraint is converted to a corresponding Anti-Faithfulness constraint, let us consider some logical statements of the two classes of constraints.

(30) The Logic of Faithfulness Constraints

MAX-X: $\forall \mathrm{x} \exists \mathrm{x}^{\prime}\left[\mathrm{x} \in \mathrm{S}_{1} \rightarrow \mathrm{x}^{\prime} \in \mathrm{S}_{2} \& \mathrm{x} R \mathrm{x}^{\prime}\right]$

'Every $X$ in $S_{1}$ must have a correspondent in $S_{2}$.'

DEP-X: $\forall \mathrm{x}^{\prime} \exists \mathrm{x}^{\prime}\left[\mathrm{x} \in \mathrm{S}_{2} \rightarrow \mathrm{x}^{\prime} \in \mathrm{S}_{1} \& \mathrm{x} R \mathrm{x}^{\prime}\right]$

'Every $\mathrm{X}$ in $\mathrm{S}_{2}$ must have a correspondent in $\mathrm{S}_{1}$.'

$\operatorname{IDENT}(\mathrm{F}): \quad \forall \mathrm{y} \forall \mathrm{y}^{\prime} \forall \mathrm{F}\left[\mathrm{y} R \mathrm{y}^{\prime} \rightarrow \mathrm{y}=\mathrm{F} \mathrm{y}^{\prime}\right]$

'Correspondent segments must be identical for the feature F.'

By introducing a simple negation to the Faithfulness constraints above, and giving the negation highest scope, we arrive at the following Anti-Faithfulness constraints. 
(31) The Logic of Anti-Faithfulness Constraints

$\neg$ MAX-X: $\neg\left[\forall \mathrm{x} \exists \mathrm{x}^{\prime}\left[\mathrm{x} \in \mathrm{S}_{1} \rightarrow \mathrm{x}^{\prime} \in \mathrm{S}_{2} \& \mathrm{x} R \mathrm{x}^{\prime}\right]\right]$

'If there is one, delete (at least) one $X$ in the $S_{1} \rightarrow S_{2}$ mapping.'

$\neg$ DEP-X: $\neg\left[\forall \mathrm{x}^{\prime} \exists \mathrm{x}^{\prime}\left[\mathrm{x} \in \mathrm{S}_{2} \rightarrow \mathrm{x}^{\prime} \in \mathrm{S}_{1} \& \mathrm{x} R \mathrm{x}^{\prime}\right]\right]$

'Insert (at least) one $X$ in $S_{2}$ not present in $S_{1}$.'

$\neg \operatorname{IDENT}(\mathrm{F}): \quad \neg\left[\forall \mathrm{y} \forall \mathrm{y}^{\prime} \forall \mathrm{F}\left[\mathrm{y} R \mathrm{y}^{\prime} \rightarrow \mathrm{y}=\mathrm{F} \mathrm{y}^{\prime}\right]\right]$

'(At least) one pair of correspondent segments must differ in feature F.'

Working through the first 'Anti-MAX' constraint, the logic spelled out here entails the following: 'it is not the case that, for all $X$ in the input, there is a corresponding $X$ in the output', which in effect requires at least one violation of MAX-X. In general, by introducing a negation which takes scope over the proposition expressed by a given Faithfulness constraint, Anti-Faithfulness constraints demand at least one breach of the corresponding Faithfulness constraint.

The relative scope of the negative operator is actually an empirical issue; an alternative to giving the negation wide scope is clearly to give it narrow scope with respect to the quantifiers by introducing it at the beginning of the consequent of the implication. The latter formulation of Anti-Faithfulness would require a total lack of Faithfulness, forcing a complete reversal of the linguistic properties of the base. The morphophonological alternations discussed here, however, do not involve such a rampant breach of Faithfulness, and so it seems that giving the negation wide scope is best supported by the data.

Another empirical issue concerns the claim that there is an Anti-Faithfulness constraint for every Faithfulness constraint. This assumption predicts that affixes will bring about a change in a particular aspect of linguistic structure, e.g., the change of the [voice] specification through the negation of IDENT(voice). A plausible alternative to this approach is that the oppositions like those in Luo derive from a general Anti-Faithfulness constraint which simply requires an overt contrast, regardless of what aspect of linguistic structure yields the opposition. This approach also seems to be empirically unmotivated, as it makes the prediction that there can be more than one way of satisfying the AntiFaithfulness constraint in a given construction. While the present chapter is not an exhaustive study of segmental morpho-phonology, affix-controlled accentual processes are always limited to a single aspect of accentual structure. For example, there are accentdeleting affixes and accent shifting affixes, but there appear to be no cases where an affix either triggers a deletion or a shift of accent. The proposed symmetry between Faithfulness and Anti-Faithfulness constraints therefore seems to be on the right track too.

If there is an Anti-Faithfulness constraint for every Faithfulness constraint, then this assumption also predicts that Faithfulness reversals will be found in other correspondence relations, not just transderivational correspondence. In particular, Anti-Faithfulness is also implicated in base-reduplicant and input-output correspondence. It would appear that this prediction runs counter to the finding in Anderson \& Browne 1973, and supported further in Moreton 1996, that segmental exchange rules are always morphological. The TAF constraints are needed to account for exchanges between morphologically related words, but a completely unconstrained theory predicts non-morphological exchanges as well. Are all types of Anti-Faithfulness empirically motivated? 
In support of the general approach taken here is the fact that Faithfulness reversals are quite prevalent in base-reduplicant relations. As pointed out in McCarthy \& Prince 1986, and explored further in McCarthy \& Prince 1995, Yip 1992, 1995, and Alderete et al. 1998, reduplicative constructions and echo words frequently require an overt phonological difference between the base and the copied part. For example, echo words in English formed with shm- are blocked when the base word also begins with this sequence, as in *shmuck-shmuck. Reduplication of adjectives in Turkish likewise shows an avoidance of repetition between base-reduplicant pairs: the coda may be one consonant from the set $/ \mathrm{p} \mathrm{s} \mathrm{m} \mathrm{r/,} \mathrm{but} \mathrm{certain} \mathrm{consonants} \mathrm{are} \mathrm{blocked} \mathrm{when} \mathrm{they} \mathrm{would} \mathrm{mimic} \mathrm{the}$ consonantism of the base, e.g., kap-kara 'jet black', not *kar-kara. And as argued in detail in Yip 1996, the mutations observed in Javanese elatives also involve repetition avoidance. In sum, there seems to be ample cross-linguistic support for phonological mutations in reduplicative constructions as well, and this observation can be captured by extending AntiFaithfulness to base-reduplicant correspondence.

An unconstrained process of constructing Anti-Faithfulness constraints also yields input-to-output Anti-Faithfulness, a set of constraints which would yield purely phonological mutations. In contrast to the two types of Anti-Faithfulness examined above, there is not much support for this type of Anti-Faithfulness. Indeed, if Anderson \& Browne's generalization is correct, then segmental exchanges in lexical-to-surface mappings should be completely ruled out. For these reasons, it appears to be necessary to be stipulate that Anti-Faithfulness operates exclusively in surface-to-surface correspondence, defined in a way to include base-output and base-reduplicant correspondence, but to exclude input-output correspondence (see Benua 1997 [1998], McCarthy \& Prince 1995, 1997). Such a move would not be unprecedented, as Faithfulness to syllabic positions appears to be limited to surface-to-surface correspondence as well. While Faithfulness to a segment appearing in the onset or coda of a syllable appears to be crucial in the analysis of blocking effects in re-syllabification, it is never a contrastive feature in the syllable inventory of a given language, which would require input-output correspondence (see McCarthy \& Prince 1994a). Thus, as with Faithfulness to syllabic positions, Anti-Faithfulness appears to be limited to related structures which have an overt surface realization in both members of the pair.

There may be a deeper reason for this fact, however, stemming from the properties inherent to Optimality Theory or the way morpho-phonology is learned generally. The parallelist inclination in Optimality Theory entails that there are no intermediate steps or levels in the mapping from the lexical to the surface form. With this assumption, a purely phonological exchange is in fact indistinguishable from a fully faithful mapping from input to output. Thus, if /A/ goes to [B] and /B/ to [A], and there is not an intermediate step which can further apply to the output of this exchange, then the result is an inventory that contains both [A] and [B]. This result is of course the same in the absence of a phonological exchange: a fully faithful mapping of /A/ and /B/ yields the same inventory. Further, the same result holds for circular chain shifts as well; as long as candidate forms are evaluated in parallel, the result of a shifted series of sounds will have the same consequences for the inventory as if they are unshifted. The question one must ask at this point is, why would a child learning a language bother to reverse the specification of a given segment class? If there are no overt alternations showing that the lexical form has changed, why would the learner go to the trouble of undoing an exchange in positing lexical forms when a far more simple alternative is available, namely to assume that the overt structure is the actual input? These questions need to be answered in a specific model of language acquisition, but the basic point is clear: in the absence of overt structure showing an exchange, there is little, if any, incentive to learn a purely phonological 
exchange, which may explain the apparent gap in the generality of Anti-Faithfulness constraints observed here.

\subsubsection{Application to Exchange Processes}

Let us return now to the facts of Luo and see how Anti-Faithfulness applies to the morphological pattern of [voice] exchange. In the plural, voiceless consonants become voiced and voiced consonants become voiceless. This pattern is accounted for by negating the garden variety IDENT(VOI) constraint and restricting its application to the output-tooutput dimension of Faithfulness. As with in the analysis of stress neutral affixation in $\$ 4.2 .2$, affixes and morphological processes in general may impose on their base the OOcorrespondence relation upon which the TAF constraint is defined. Thus, the constraint given below is only operative in the plural and the appertentive because only these categories are lexically specified for this OO-correspondence relation. ${ }^{13}$

\section{(32) ᄀOO-IDENT(VOI)}

If a pair of words stand in an OO-correspondence relation, at least one pair of correspondent segments must be non-identical for the feature [voice].

The next step in the analysis is to say that the singular forms the base of the plural, which is a straightforward case of the simplex - complex relation in the case of the plural. Lastly, $\neg$ OO-IDENT(VOI) is ranked above the OO-Faithfulness constraint for voicing, which in turn yields both sides of the [voice] exchange. This result is depicted below. ${ }^{14}$

\section{(33) Consonantal Polarity in Luo as Transderivational Anti-Faithfulness}

a. bat $\neq$ bede

\begin{tabular}{|c|c|c|c|}
\hline Base & $/$ bat + e/ & $\neg$ OO-IDENT(VOI) & OO-IDENT(VOI) \\
\hline \hline bat & bet-e & $* !$ & $*$ \\
\hline$\rightarrow$ bat & bed-e & & $*$ \\
\hline
\end{tabular}

b. $\check{c}$ ogo $\neq$ č oke

\begin{tabular}{|c|c|c|c|}
\hline Base & č ogo $+\mathrm{e} /$ & $\neg$ OO-IDENT(VOI) & OO-IDENT(VOI) \\
\hline č ogo & č og-e & $* !$ & \\
\hline$\rightarrow \check{c}$ ogo & č ok-e & & $*$ \\
\hline
\end{tabular}

It is important to emphasize that the result here is quite uncharacteristic of the general treatment of phonological processes in OT. Phonological processes are unfaithful mappings which are typically modelled as the domination of Faithfulness constraints by Markedness constraints. In this case, there is no Markedness constraint which compels a different [voice] specification in the stem-final consonant. Rather, it is the negation of Faithfulness which derives this result, and because of this approach, the circular nature of

\footnotetext{
${ }^{13}$ The Anti-Faithfulness constraint could be defined for an input-to-output correspondence relation in this case, and if a distinct set of IO-correspondence relations could be motivated, the application of this constraint would be limited to just the right morphological contexts. However, as will be shown in $\S 4.3 .3$, the transderivational approach predicts that the effect of the Anti-Faithfulness constraint will only be observed in the base of affixation, a claim that has robust empirical support in the study of morphoaccentual phenomena conducted in chapter 5 .

${ }^{14}$ The first recursion of the constraint hierarchy is left out of the tableau here because it is irrelevant to the result being illustrated.
} 
the alternation is directly explained. Said another way, since the Markedness constraints attract a particular target, they are unidirectional. Since Anti-Faithfulness constraints, on the other hand, simply demand a phonological difference, they may be bi-directional, as observed in Luo (though Anti-Faithfulness effects can be uni-directional too, as explained below).

The theory of TAF as yet does not describe which segments of the string will be affected by the Anti-Faithfulness constraint. In other words, as far as $\neg$ OO-IDENT(VOI) is concerned, the plural form of [bat] could either be bed-e, or the non-occurring * pet-e both incur a violation of IDENT(VOI), and so both equally satisfy $\neg$ OO-IDENT(VOI) as it is construed. There are a wide range of choices in approaching this problem. One idea is to employ Positional Faithfulness constraints (see Beckman 1997 [1998]) as a means of predicting the target of the process. For example, in Luo, the fact that the word-initial consonant is inert to the mutation could be handled with high-ranking Faithfulness for rootinitial segments. Another possibility is to let the independently attested well-formedness constraints dictate the target of the phonological change. In the analysis of pre- and postaccentuation and accentual shifts in $\S 5$, I will show how Alignment constraints play a role in deriving accentual changes close to a designated edge of a word.

I will not explore these approaches at this point in the discussion because I believe there is a more basic fact to be accounted for, namely that the target of Anti-Faithfulness is often local in some sense to the triggering morpheme. Thus, in comparing [bed-e] versus *[pet-e], the changed consonant in the good form is 'closer' to the plural suffix, and this fact seems to be rather common. The problem of predicting the locality of target and trigger is of course a very general one, as a great deal of research in generative phonology has been concerned with the issue of explaining locality effects in phonological processes. Indeed, many developments in metrical and autosegmental phonology may be understood as a means of solving certain problems that arise in a strictly linear approach to stress and tone systems (see Hayes 1995, Odden 1995, and Goldsmith 1990 and references therein). ${ }^{15}$ In this line of research, however, the central focus is on the observed closeness between two phonological objects. The issue faced in Luo, by contrast, concerns the proximity of the affected element with a morphological entity, e.g., the base-mutating plural suffix. In sum, Luo shows that phonological units like segments may interact with morphological categories like affixes; we therefore require a notion of locality between morphological and phonological categories.

This qualification invites a comparison with so-called derived environment effects (DEE) of the morphological type (Kiparsky 1982b). In morphological DEE, the application of a morphological process $\mathrm{P}_{\mathrm{m}}$ feeds a phonological one $\mathrm{P}_{\mathrm{p}}$, which entails that they be local in some sense. Concretely, since the elements introduced by $\mathrm{P}_{\mathrm{m}}$ must be in the structural conditions of $\mathrm{P}_{\mathrm{p}}$, and further, since there are substantive restrictions on the distance between the target and trigger of $\mathrm{P}_{\mathrm{p}}$, it follows that the introduced morphological category will be 'close' enough to the element affected by the process. To make this logic explicit, consider the morphological conditions on First Velar Palatalization (FVP) in Polish. FVP turns velars into post-alveolars before high vocoids. FVP only applies in heteromorphemic words, however, because its structural conditions must be met by morpheme concatenation, as shown below.

\footnotetext{
${ }^{15}$ Building on these results, a number of researchers have worked towards a set of conditions governing the proximity of various formal objects in a phonological process (Archangeli \& Pulleyblank 1987, Selkirk 1988, Myers 1987a, Odden 1994, Suzuki 1998), leading to highly restrictive claims on spreading processes (Ní Chiosáin \& Padgett 1997, Gafos 1996a, Walker 1998). See also Frisch, Broe, \& Pierrehumbert 1996 for the use of probabilistic functions in the analysis of locality effects, and Bailey 1995 for various 'edge biases' in stress rules.
} 
(34) Morphologically Derived Environment Effects in Polish (Rubach 1984)
a. Tautomorphemic: FVP does not apply
b. Heteromorphemic: FVP applies
/xemik/
$\downarrow$
[xemik]
/xemik + ek/
$\downarrow$
[xemič -ek]

It is the velar's closeness to the affix which allows palatalization in (34b), showing the importance of a locality condition relative to morphological category. While it is difficult to see consonantal polarity in Luo in precisely these terms, as there is no straightforward sense in which the conditions for the voicing exchange are met through rule prior application, the fundamental ideas are still at work. In Luo, the voicing exchange is predicated on the presence of morphological categories, namely the plural and appertentive. Further, the affected element appears to be a neighbor to these morphological categories (though the appertentive has no overt realization). It seems fruitful, therefore, to approach these two types of morpho-phonological alternations with the same basic toolbox.

I will not pursue this connection within Lexical Phonology (LP) for the following reasons. The LP approach to DEE is inherently derivational, as it involves an interleaving between morphological and phonological processes; as such, LP is inconsistent with the program set forth in TCT for cyclic effects. Furthermore, an analysis of Luo in LP is inherently problematic because the phonological alternation must be described in terms of a possible rule in a cross-linguistic theory of rule types. But no language has phonological exchange rules of the type encountered in Luo. While it is common in the early generative literature to encounter alpha-switching or 'flip-flop' rules in descriptions of thorny problems, Anderson \& Browne 1973 (see also Anderson 1975, 1991 and Moreton 1996) argue convincingly that such exchanges always mention a morphological or morphosyntactic environment. For Luo, therefore, the LP theory requires a phonological rule which is otherwise unmotivated in the world's languages.

A new approach to DEE has recently been proposed in Lubowicz 1998, which provides a non-derivational alternative to LP and provides the principles needed to account for the observed locality restrictions on morpho-phonology. Roughly speaking, the idea is that DEE, of both the phonological and morphological type, involves a 'piling up' of the constraint violations in a local context (with obvious functional benefits for recoverability). In particular, morphological DEE involve the combination of an Anchoring constraint and a Markedness constraint through a process of Local Conjunction (36); when affixation triggers a violation of Anchoring, this violation leads to the activation of the Markedness constraint in the neighboring environment of the Anchoring violation. In essence, DEE can be characterized as avoiding 'the worst of the worst' (Prince \& Smolensky 1993), by bundling two constraints and limiting their application to a local environment.

These ideas are applied to DEE in Polish in Lubowicz 1998 in the following way. The constraint VPAL, which prohibits dorsals before high vocoids, is locally conjoined with a constraint demanding stem-to-syllable anchoring at the right edge of the stem (37). The formula for Anchoring constraints given in (35) below entails that the right edge of the syllable has a correspondent in the right edge of the stem (see McCarthy \& Prince 1993a,b for motivation of such a constraint and McCarthy \& Prince 1995 for subsequent reinterpretation of Alignment constraints in terms of Anchoring). 
(35) Formula for Anchoring Constraints (McCarthy 1997, McCarthy \& Prince 1995)

ANCHOR(Cat $\left.1, \mathrm{Cat}_{2}, \mathrm{P}\right)$, where $\mathrm{P}$ is one of $\{$ Initial, Final, Head $\}$.

If $\xi_{1} \in \mathrm{S}_{1}$, and $\xi_{2} \in \mathrm{S}_{2}$, and $\xi_{1} R \xi_{2}$, and $\xi_{1}$ stands in position $\mathrm{P}$ of Cat $\mathrm{C}_{1}$, then $\xi_{2}$ stands in position $\mathrm{P}$ of Cat2.

(36) Local Conjunction of $\mathrm{C}_{1}$ and $\mathrm{C}_{2}$ in Domain $D$ (Smolensky 1993, 1995, 1997)

$\mathrm{C}_{1} \&_{1} \mathrm{C}_{2}$ is violated when there is some domain of type $D$ in which both $\mathrm{C}_{1} \&_{1} \mathrm{C}_{2}$ are violated.

Following Smolensky's formulation of Local Conjunction, the conjoined constraint has the effect of shunning the banned sequence specifically at the stem + affix juncture, as it is only in this context that stem-to-syllable Anchoring is violated. The local context here is characterized in terms of the notion of Root Adjacency (RA), i.e., adjacent root nodes or segments. ${ }^{16}$

(37) (VPAL \& ANCHOR(Stem, $\sigma$, Final) $)_{\mathrm{RA}} \equiv$ VPALAdj Seg

Avoid dorsals before high vocoids (VPAL) \& a violation of stem: syllable anchoring in adjacent segments.

As depicted below, when the attachment of a suffix yields a dorsal following by a high vowel, faithful treatment of this sequence leads to a violation of the conjoined constraint in adjacent segments (38a), thereby motivating the alternation. In tautomorphemic words, however, there is no violation of Anchoring, and therefore VPAL is inactive (38b).

(38) Morphological Derived Environment Effects as Local Conjunction (Lubowicz 1998)

\begin{tabular}{|c|c|c|c|c|c|}
\hline Input & & Output & VPALAdj Seg $_{\text {A }}$ & IDENT(cor) & VPAL \\
\hline \multirow[t]{2}{*}{ a. /xemik + ek/ } & $\rightarrow$ & xe.mi. $[\check{c}-i] k$ & & $*$ & \\
\hline & & *xe.mi. $[\mathrm{k}-\mathrm{i}] \mathrm{k}$ & $* !$ & & $*$ \\
\hline \multirow[t]{2}{*}{ b. /xemik/ } & $\rightarrow$ & xe.mik & & & $*$ \\
\hline & & *š e.mik & & $* !$ & \\
\hline
\end{tabular}

In sum, the approach taken in Lubowicz 1998 is a fully non-derivational approach to DEE, achieved as an effect of constraint activation through Local Conjunction. Furthermore, this theory gives the required type of locality effects, i.e., a notion of locality between morphological and phonological categories.

A related approach can be applied to the analysis of the morphologically conditioned exchange in Luo, though the Markedness constraint must be substituted for an AntiFaithfulness constraint. ${ }^{17}$ Thus, while morphological DEE derives from the conjunction of $\mathbb{M} \& \mathbb{A}$ (for $\mathbb{M}$ a Markedness constraint and $\mathbb{A}$ an Anchoring constraint), morphologically conditioned exchanges derive from the conjunction $\neg \mathbb{F} \& \mathbb{A}$. Both types of constraint conjunction lead to the piling up of Faithfulness violations in discrete domains. With

\footnotetext{
${ }^{16}$ See Suzuki 1998 on the incorporation of adjacency relations such as this in the definition of Local Conjunction.

${ }^{17}$ The inadequacy of the treatment of Luo as a morphological DEE follows from Moreton's theorem (Moreton 1996): no ranking of just Markedness and Faithfulness constraints will yield the exchange.
} 
affixation, for example, the attachment of a suffix will incur a violation of ANCHOR(Stem, PrWd, Final) because suffixation entails that the stem-final element is not longer final in the prosodic word. Conjunction of an Anti-Faithfulness constraint with ANCHOR(Stem, PrWd, Final) therefore correctly predicts the application of an exchange process in the local environment of the affix, as spelled out in the following constraint.

(39) ( $\neg$ OO-IDENT(VOI) \& ANCHOR(Stem, PrWd, Final)) Seg $\equiv \neg O O-I D E N T(V O I)$ Fin Seg In morphologically related words, attachment of an affix must be accompanied by a violation of IDENT(VOI) in the stem-final segment.

Working through the complex constraint, the Anti-Faithfulness constraint $\neg$ IDENT(VOI)OO requires a violation of Faithfulness. The conjunction of this constraint with

ANCHOR(Stem, PrWd, Final) entails that a violation of Anti-Faithfulness is not tolerated in the same local domain, namely the segment, as a violation of Stem: PrWd Anchoring. Since suffixation generally induces a violation of the Anchoring constraint, then the attachment of a plural suffix will trip a violation of ANCHOR(Stem, PrWd, Final), thereby activating the Anti-Faithfulness constraint in the stem-final segment. ${ }^{18}$ This effect is illustrated below.

(40) Locality of Anti-Faithfulness Effect through Local Conjunction

\begin{tabular}{|c|c|c|c|}
\hline Base & /bat $+\mathrm{e} /$ & $\neg \mathrm{OO}$-IDENT(VOI)Fin Seg & IDENT(VOI)IO \\
\hline bat & $\mathrm{pe}[\mathrm{t}]-\mathrm{e}$ & $* !$ & $*$ \\
\hline bat & $\underline{\mathrm{pe}}[\underline{\mathrm{d}}]-\mathrm{e}$ & & $* * !$ \\
\hline$\rightarrow$ bat & $\mathrm{be}[\underline{\mathrm{d}}]-\mathrm{e}$ & & $*$ \\
\hline
\end{tabular}

In the above tableau, all of the derived words incur a violation of ANCHOR(Stem, PrWd, Final) because all of them have the plural suffix $-e$, which leads to a mis-match between the right edges of the stem and the PrWd. But only the last two satisfy Anti-Faithfulness by mutating the segment that also incurs the Anchoring violation (which is in square brackets). The first candidate, therefore, violates the conjoined constraint because it violates both of the constraints in the conjunction specifically in the stem-final segment. Underlining here indicates a mutated consonant, which therefore leads to the satisfaction of the TAF constraint. Furthermore, the doubly mutated candidate, ped-e, with a total reversal of consonant voicing, is ruled out because it has a gratuitous violation of low-ranking Faithfulness. With the characterization of Anti-Faithfulness in which the negation takes wide scope over the Faithfulness requirement, satisfaction of the constraint simply involves a single violation of Faithfulness; additional Faithfulness violations do not help in any way and are therefore ruled out by Faithfulness. To summarize, the conjunction of an AntiFaithfulness constraint and Stem-to-PrWd Anchoring correctly defines the locality requirements on the exchange process.

This theory of the locality conditions on morpho-phonological alternations leads to an interesting question: since the Anti-Faithfulness constraint is activated by the attachment of an affix (which results in a violation of Anchoring), how are morpho-phonological alternations to be modelled which do not involve affixation at all? For example, eclipsis mutation in Irish affects the initial consonant of words appearing in certain morphosyntactic environments, but these words do not reliably receive a set of affixes. And yet

\footnotetext{
${ }^{18}$ It is important to be clear that the role of ANCHOR(Stem, PrWd, Final) is not a morphological one this is the function of the TAF constraint. The conjunction of ANCHOR(Stem, PrWd, Final) with the TAF constraint, in this case, simply accounts for the locality effects observed in the exchange.
} 
the mutation consistently appears on the initial consonant of the word. ${ }^{19}$ How is the target of the mutation process to be localized in this case, where there is no neighboring affix to predict the locus of the mutation? The current theory affords several options. First, one obvious tack is to assume that some mutations of this kind simply involve Transderivational Anti-Faithfulness, unconjoined with an Anchoring constraint. Thus, the Anti-Faithfulness constraint requires an overt difference in the form and other constraints in the grammar ensure that it is the initial syllable which undergoes the process. While several examples examined below are approached in precisely this way, this line of analysis does not look so attractive for Irish word-initial mutation as it is common for languages to actually be more faithful for word-initial segments (see especially Beckman 1997 [1998]). Another option is to posit a non-overt affix in the neighborhood on the mutation, which, through the means described above, incurs a Faithfulness violation and thereby activates Anti-Faithfulness. This approach is clearly not highly explanatory, given that there is no independent means of testing the position of the affix (beyond historical studies), but it appears to be a prudent approach to Irish, given the bizarre nature of the initial mutation.

A third option, more in line with the first, is to assume that the Anti-Faithfulness constraint is likewise unconjoined, and that the target of the mutation is achieved through the negation of a Positional Faithfulness constraint in the sense of Beckman 1997 [1998]. In this theory, a set of Faithfulness constraints target specific locations in a form, effectively accounting for the fact that these positions generally license a wider range of contrasts (and therefore require higher-ranking Faithfulness). Taking the null hypothesis given above, namely that there is a Anti-Faithfulness constraint for every Faithfulness constraint, $C O N$ will also have Anti-Positional Faithfulness constraints which in effect require a mutation in the privileged position targeted by the Positional Faithfulness constraint. Returning to Irish eclipsis, the mutation in the initial segment can be explained straightforwardly as a response to the negation of the word-initial segment Faithfulness constraint, i.e., ᄀFAITH-SEG 1 . Indeed, such mutations may even have the same functional basis as Positional Faithfulness: by requiring the phonological change in a phonologically salient position, the coding properties of the mutation will be more reliably heard. Extending the application of Anti-Positional Faithfulness, this line of analysis may prove very useful in the analysis of spreading from an affix to a stressed syllable, as found in languages like Chamorro and Montañes Spanish (see Chung 1983 and McCarthy 1984 and references therein). The motivation for the process in these cases involves, by hypothesis, the negation of Faithfulness in stress syllables, effectively requiring an alternation in this salient position in the word.

To summarize these ideas concerning mutation without affixation, there are several possible approaches to this type of mutation within the theory developed here. Such mutations can be modelled in one of the three following scenarios: (i) pure unconjoined Anti-Faithfulness where the other constraints in the grammar give the target of the mutation, (ii) Anti-Positional Faithfulness, or more conservatively, (iii) an abstract segment may be posited to predict the location of the change. Each option of course has different empirical predictions, and so each case will require careful study before choosing among these alternatives.

\footnotetext{
${ }^{19}$ The set of meaningful phonological processes described in Woodbury 1987 for Central Alaskan Yupik may also be cases of 'affix-free' Anti-Faithfulness. While the expressive aspect of these processes is rather different than the truth-conditional meanings typical of the cases examined here, the observed changes are correlated with a change in meaning, and yet there is no consistent set of affixes involved.
} 


\subsubsection{Implications of Transderivational Anti-Faithfulness}

There are a number of predictions that TAF theory makes which distinguish it from plausible alternatives to morphologically governed phonology. These predictions will be reviewed here, in abstract form, before they are confronted in real life examples in subsequent discussions.

The first kind of pattern predicted in the TAF model developed here has to do with the target of the structural change in the morpho-phonological process. One general prediction, codified in the following thesis, is that mutation processes specifically affect the 'base' of the morphological process, i.e., the root or stem.

\section{(41) Thesis of Strict Base Mutation (SBM)}

Transderivational Anti-Faithfulness may only affect the base of affixation.

To see this effect, consider the following hypothetical example. With simple suffixation, the bare root forms the base for the derived form [root + af]. If the affix is changed, indicated here with capitalization, then the base of affixation is no different than the simplex base, and therefore Anti-Faithfulness is violated. On the other hand, if the root is mutated, as in the first candidate, it does incur a violation of OO-Faithfulness, and as a result, AntiFaithfulness is satisfied.

(42) Illustration of Strict Base Mutation Effect

\begin{tabular}{|c|c|c|c|}
\hline Base & Derivative & $\neg$ OO-FAITH & OO-FAITH \\
\hline \hline$\rightarrow$ root & ROOT-af & & $*$ \\
\hline root & root-AF & $* !$ & \\
\hline
\end{tabular}

SBM effects such as these are very general, and this result holds regardless of the character of the Faithfulness constraint. Thus, if $\neg \mathrm{OO}-\mathrm{FAITH}$ is the negation of a Featural Identity constraint, as with the case of Luo, then the Anti-Faithfulness constraint will demand lack of Faithfulness in the segmental make-up of the base. Likewise, if $\neg$ OO-FAITH is a constraint of the MAX variety, then the negation of OO-FAITH will necessarily bring about a deletion of some element in the base. Finally, if $\neg \mathrm{OO}$-FAITH is a DEP-type constraint, then the Anti-Faithfulness constraint is only satisfied by the insertion of some feature in the base. This last point follows from the assumption that only the base stands in correspondence with the base subconstituent in the derived form (see $\$ 4.2$ ). Thus, if a feature is inserted into the affix, as in the loser above, then $\neg \mathrm{OO}$-FAITH is violated because the segments which stand in correspondence with the base have not changed.

This result is by no means trivial and it will be crucial in the formal analysis of various types of morpho-accentual processes. For example, it is a common finding that pre- and post-accentuation is a property of affixes and not of bound roots and stems. ${ }^{20}$ Also, dominant morphemes seem to be linked exclusively to morphological processes like affixation. Thus, it is common to find accent-deleting affixes, but I know of no language with roots which idiosyncratically cause the deletion of an accent of a neighboring affix (see Inkelas 1996 for a consistent view), a point which is returned to in \$5.3.4. Also,

${ }^{20} \mathrm{An}$ apparent counterexample to this claim is the existence of so-called post-accenting stems in Russian. As argued in chapter 3, however, there is a more insightful analysis of these stems as unaccented bases, and so Russian does not counter-exemplify this claim. 
affix-controlled accentual shifts are always triggered by an affix and have the effect of shifting the base accent. Affix-controlled accentual processes are manifestly base-mutating, and this observation receives a natural explanation in TAF theory.

A second important prediction also concerns the target of the morpho-phonological process. All things being equal, the structural change induced by the process should be towards a language particular default structure (see Alderete et al. 1998 on the characterization of default structure). For example, dominant affixes in Russian cause a deletion of the stress of the base, and if they are themselves unaccented, the result is default ending stress. In other words, the output of this type of dominance effect is the same as the output of a word with no underlying accent: /púz + ač Dom $+\mathrm{u} / \rightarrow$ puz-ač $-u$ ' $m a n$ with paunch', cf. /stol $+\mathrm{u} / \rightarrow$ stol-ú 'table (dative singular)'. Dominance effects in Russian are thus grammar dependent in the sense that they are governed by the independently attested constraints giving a default stress pattern, in this case POST-STEMPROM (see $\$ 3.2$ for the definition and motivation of this constraint).

Grammar dependence follows directly from the assumptions inherent to TAF theory. The TAF constraints require a change in the base, in this case a deletion of base prosody, and the rest of the constraint system predicts how the change is accommodated. Thus, the attachment of the dominant suffix -ač triggers the activation of the TAF constraint $\neg$ OO-MAX-PROM, but the stress pattern resulting from this deletion is due to an additional constraint in the system, namely POST-STEM-PROM, as illustrated below.

(43) Dominance Effect with Language Particular Default

\begin{tabular}{|c|c|c|c|c|}
\hline Base & /púz + ač + u/ & $\neg$ OO-MAX-PM & OO-MAX-PM & POST-STEM-PROM \\
\hline \hline a. púz-u & [púz-ač ]-u & $* !$ & & \\
\hline b. púz-u & [puz-áč ]-u & & $*$ & $* !$ \\
\hline c. $\rightarrow$ púz-u & [puz-ač ]-ú & & $*$ & \\
\hline
\end{tabular}

In this way, the direction of the mutation is predicted to be towards a language particular default pattern. In Russian, the default pattern is for words to have ending stress; dominance effects also bring about default ending stress because their output is governed by the same constraint system. Several additional examples can be explained in this way. Pre-accentuation in Cupeño, for example, yields an accent on the rightmost syllable of the root; the rightward orientation of the inserted accent is consistent with the general trend for rightmost accent in the language, as evidenced by the fact that in words with more than one accented affix, the rightmost one realizes its inherent accent. Another interesting example, studied in $\$ 5.4$, is accent shift in the Jivoroan language Aguaruna. In this language, certain suffixes cause a shift of the stem accent one mora to the right. Moreover, bounded rightward accent shift is in fact the default pattern, as the accent of vowels which are deleted by a regular rule of syncope also shift one mora to the right. In sum, a wide range of morpho-accentual alternations involve a change towards a language particular default pattern. This fact is explained here with the assumptions intrinsic to TAF theory: the negation of Faithfulness requires a change, yet the independently active constraints in the language dictate how the change is rendered.

A third important prediction of this approach is not inherent to TAF, but rather a general prediction in Optimality Theory. The prediction is that Anti-Faithfulness effects may apply non-uniformly across types of strings. Anti-Faithfulness constraints are wellformedness constraints which are ranked relative to a whole ensemble of constraints in a language particular grammar. When the Anti-Faithfulness constraint has high rank in the 
system, the result is morphologically triggered phonological processes. In contrast, when Anti-Faithfulness is low ranking, no such process is predicted, and morphologically related forms are phonologically similar. However, some grammars will rank the Anti-

Faithfulness constraint between two sets of constraints, as shown below, which results in a different type of grammar dependence where Anti-Faithfulness is blocked in certain contexts.

(44) Non-Uniformity of Anti-Faithfulness

$$
\mathbb{C}_{1} \gg \text { Anti-Faithfulness } \gg \mathbb{C}_{2}
$$

With a word type which is not subject to $\mathbb{C}_{1}$, Anti-Faithfulness will get its way and induce a mutation. But Anti-Faithfulness will not have an effect in forms where $\mathbb{C}_{1}$ is relevant, which yields the non-uniform morpho-phonological pattern. Put differently, the above ranking results in 'structure-preserving' grammar dependence: a mutation is predicted, but not if it would result in structures or mappings that are prohibited in the language as a whole.

As will be shown in the next chapter, morpho-accentual rules abound in such patterns of non-uniformity. An example that is observed in languages as different as Cupeño, Russian, and Basque, is pre-accentuation that only in words with unaccented stems. Hence, when the pre-accenting suffix in Cupeño, $-i$ 'objective', is attached to a root with no inherent accent, the suffix posits an accent on the root, e.g., /né-s ula-?a-'i// $\rightarrow$ [ne-s ulá-?ai] 'my fingernails'. When the same suffix attaches to an inherently accented root, however, the root accent overrides the accent contributed by the pre-accenting suffix as in: /?ísi-ly-'i/ $\rightarrow$ [?ísi-lyi] 'coyote'. This pattern of non-uniformity can be explained if we simply substitute MAX-PROM Root for $\mathbb{C}_{1}$ in (44) above. Thus, in words with unaccented stems, Anti-Faithfulness has an effect and causes the insertion of an accent in these forms because MAX-PROMRoot is irrelevant. Anti-Faithfulness is kept in check, however, in words with accented roots because it is dominated by Root Faithfulness. The heterogeneous behavior of these pre-accenting suffixes is therefore derived directly through constraint domination.

A rather different type of non-uniformity effect can be modelled by ranking two related Faithfulness constraints differently relative to Anti-Faithfulness. To fully understand this point, it is necessary to give a bit of background on features in OT. It is common to distinguish between two kinds of Faithfulness constraints which make reference to the same feature. Thus, MAX and DEP may govern the behavior of the same features, but they are independently rankable in the constraint hierarchy. Likewise, a number of researchers, including Pater 1996, Urbanczyk 1996, and McCarthy 1997 have proposed different dimensions of IDENT-type constraints. For example, Pater distinguishes between IDENT[-voi $\rightarrow+$ voi] and IDENT[+voi $\rightarrow$-voi] in his theory of postnasal voicing alternations. Now, if two related Faithfulness constraints, e.g., $F(+A \rightarrow-A)$ and $F(-A \rightarrow+A)$, are ranked differently relative to the corresponding Anti-Faithfulness constraint, the prediction is that the mutation effected by the Anti-Faithfulness constraint will only go in the direction allowed by the relevant Faithfulness constraint(s). The ranking for this type of mutation is given below. ${ }^{21}$

\footnotetext{
${ }^{21}$ For ease of exposition, I have grouped the two Anti-Faithfulness constraints together in a single constraint. But technically speaking, the complex constraint $\neg \mathrm{F}(+\mathrm{A} \rightarrow-\mathrm{A} ;-\mathrm{A} \rightarrow+\mathrm{A})$ represents two independent constraints which correspond to the related Faithfulness constraints given in the same ranking.
} 
(45) Uni-Directional Mutations: $-\mathrm{A} \rightarrow+\mathrm{A}$, but not $+\mathrm{A} \rightarrow-\mathrm{A}$

$$
\mathrm{F}(+\mathrm{A} \rightarrow-\mathrm{A})>>\mathrm{F}(+\mathrm{A} \rightarrow-\mathrm{A} ;-\mathrm{A} \rightarrow+\mathrm{A})>>\mathrm{F}(-\mathrm{A} \rightarrow+\mathrm{A})
$$

This ranking predicts that /-A/ will go to $[+\mathrm{A}]$ in the relevant morphological contexts because the Anti-Faithfulness constraint requiring this mutation dominates $F(-A \rightarrow+A)$. The opposite pattern of mutation, however, is not allowed: high-ranking $\mathbb{F}(+\mathrm{A} \rightarrow-\mathrm{A})$ rules out this possibility. In sum, 'one-way' mutations can be successfully modelled in this way, and that is exactly the way I will approach such cases below.

It is worthwhile illustrating this last prediction with some well-known vowel length alternations. Many languages with contrastive vowel length also have a set of suffixes which induce lengthening on the preceding vowel, rather similar to pre-accentuation in accent systems. For example, Slovak has a contrast between long and short vowels, but this contrast is neutralized before certain pre-lengthening suffixes (see Rubach 1993 and references therein). Two aboriginal languages of Australia also display these same features, namely Gidabal (Geytenbeek \& Geytenbeek 1971) and Yidiñ (Dixon 1977). Consider the following examples from Yidiñ in which the presence of the anti-passive ending, - $D i-n$, triggers lengthening of the preceding vowel.

(46) Morphologically Conditioned Lengthening in Yidiñ (Dixon 1977) 22

$$
\begin{aligned}
& \text { a. wawa-l 'see, look', cf. wawa:-Di-n, wawa:-Diñu (past), wawa:-DiN (present) } \\
& \text { b. wuNaba-n 'hunt', cf. wuNaba:-Di-n }
\end{aligned}
$$

The important point here is that the anti-passive suffix induces lengthening of short vowels but no shortening of long vowels. We have here a uni-directional mutation, and equipped with the schematic ranking given above, we can explain this case in terms of constraint domination of the Anti-Faithfulness constraint.

Following Urbanczyk 1996, we distinguish between two kinds of Length Faithfulness constraints, i.e., *SHORTENING and *LENGTHENING. If Anti-Faithfulness for vowel length is ranked between these two constraints, as shown below, then the mutation triggered by the Anti-Faithfulness constraint will only induce lengthening.

(47) Uni-Directional Length Mutation in Yidiñ

*SHORTENING > ᄀLENGTH-IDENT $>$ *LENGTHENING

Walking through the ranking, morphologically triggered lengthening is allowed because *LENGTHENING is dominated by the Anti-Faithfulness constraint. But shortening is not permitted because this component of Length Faithfulness is top-ranked in the hierarchy. In sum, the fact that some morphologically induced alternations only go in one direction is explained as a consequence of two basic tenets in OT: constraint ranking and violability.

In this way, Anti-Faithfulness can take over some, or even all, of the work of constraints encouraging the overt realization of a morphemic unit. Commonly used constraints include MORPH-REAL from Samek-Lodivici 1993 and MORPH-DIS from McCarthy \& Prince 1995. In traditional Item-and-Arrangement-style morphology, cases like the length-inducing suffixes in Yidiñ are said to involve a floating unit of length,

\footnotetext{
${ }^{22}$ The comparison between a disyllabic and a trisyllabic stem shows that the length alternation is not rhythmically governed, another important factor in length alternations. Also, /D/ is a laminal stop.
} 
which, because it cannot be realized on the suffix itself, docks to a nearby vowel. The role of MORPH-REAL in such an analysis is thus to ensure that the floating mora is overtly realized, effectively distinguishing the base from the derived form with the lengthening suffix.

The functional purpose of the lengthening is of course to mark a contrast between the base and its derivative, and Anti-Faithfulness therefore offers an interesting alternative to the Floating Feature analysis. Thinking of Yidiñ again in terms of TAF, there is no need to posit a floating mora as the morphological exponence of 'derivedness'. Rather, by employing Anti-Faithfulness, the alternation in length can be induced through constraint interaction. In other words, the phonological alternation is a response to well-formedness constraints requiring difference, rather than as the realization of an underlying element. The analysis in terms of TAF is therefore less abstract, which is an argument in its favor. ${ }^{23}$

A further important implication of the theory of morpho-phonological alternations developed here is that it predicts that said alternations may be subject to locality conditions which are specified in terms of different prosodic (and potentially morphological) categories. The TAF constraints which bring about a mutation of the base may operate independently, in which case there are no locality restrictions and the target of the morphophonological operation is towards a language particular default (discussed above). Or, following Lubowicz 1998, the TAF constraints may be locally conjoined with an Anchoring constraint, which has the effect of 'activating' the TAF constraint in a unit which appears at the edge of the base which borders the base-affix juncture (see $\$ 4.3 .2$ for details). The prediction of this theory is that different specifications for domain of Local Conjunction may result in different locality domains for the pattern of Anti-Faithfulness. For example, in contrast to the segment-based locality restriction found in Luo, if the conjunction of the TAF and Anchoring constraint is defined for the syllable, then the mutation must be in an edgemost syllable of the base. The examination of morphoaccentual phenomena in the next chapter shows that this prediction is indeed borne out, as the range of possible locality domains are attested. All of the prosodically defined locality restrictions (excluding the segment) ${ }^{24}$ are integral in the analysis of specific affix-controlled processes.

(48) Locality Effects in Affix-Controlled Accent

a. Mora-based locality: the dominant enclitic no in Japanese (\$5.2.4)

b. Syllable-based locality: pre- and post-accentuation in Japanese (§5.3.3.1), dragging tone mutation in Limburg Dutch (\$5.4.2)

c. Foot-based locality: pre-accentuation in Cupeño (\$5.3.2), accent shift in Aguaruna $(\S 5.4 .4)$

As discussed in detail in Poser 1984, the dominant morpheme no in Tokyo Japanese is subject to a locality restriction, namely that it only causes the deletion of accent in a neighboring mora. Moving up the Prosodic Hierarchy, ACA may also have syllable-based locality restrictions, essentially entailing a mutation of the base prosody in the stem syllable

\footnotetext{
${ }^{23}$ It is important to emphasize that this argument applies even if a null morpheme is required in conjunction with a TAF constraint, which was one of the possibilities entertained above in the analysis of Irish. In this case, the null morpheme does not have a phonological function because it does not sponsor a phonological feature.

${ }^{24}$ The absence of segment-based locality in ACA is not at all a surprise, given that segments do not typically sponsor accentual categories like stress and tone.
} 
which is adjacent to the relevant affix. In addition to the syllable-based locality in Japanese post-accenting prefixes and pre-accenting suffixes, there is the striking case of the dragging tone mutation in certain Dutch dialects where a retraction of the (non-stress) accent is only observed in stem-final syllables which abut the accent-shifting suffixes. Finally, two other affix-controlled accentual processes seem to be limited to the final foot of the stem which shares the base + affix border with a special suffix. Taken together, these cases present a strong case of the proposed theory of locality used in restricting the application of morphophonological operations because they attest to the full range of locality domains predicted by Smolensky's theory of Local Conjunction.

The following list summarizes the implications of TAF theory fleshed out above.

(49) Predictions of Transderivational Anti-Faithfulness

a. Strict Base Mutation Effects: TAF constraints encourage dissimilarity specifically in the base of the morphological process.

b. Outputs as Language Particular Defaults: in cases where Anti-Faithfulness is not decisive, other constraints in the system dictate the direction of structural change to a language particular default structure.

c. Non-Uniformity Effects: Anti-Faithfulness effects may be non-uniform, applying to only a subset of the range of possible word types.

d. Locality Effects: Anti-Faithfulness effects may be required to be 'close enough' to the base-mutating affix.

The theory of Transderivational Anti-Faithfulness has a number of properties which make it special and distinguish it from alternative approaches to morpho-phonology. First, TAF constraints induce changes to the base of a morphological process - this result stems from the basic premise of the theory, namely that TAF constraints operate in base-derivative pairs. Second, all else being equal, the direction of the change triggered by TAF constraints will be towards a language particular default structure. Thus, dominance effects in Russian require a deletion of stress, and the independently needed constraint POSTSTEM-PROM dictates the stress pattern resulting from this deletion. Third, AntiFaithfulness may apply non-uniformly across word types. That is, one class of words may undergo the mutation, while certain others may not, and the dividing line between these two classes of phonological behavior is negotiated through constraint ranking. The last two effects fall under the general rubric of grammar dependence, where the independently needed constraints in the grammar interact with the Anti-Faithfulness constraint, predicting the locus of the mutation, the patterns resulting from deletion, or whether or not the morpho-phonological operation is blocked in a specific context. Finally, Anti-Faithfulness effects may be subject to locality requirements which entail that the mutation occur in a position which is in the proximity of the base-mutating affix. 


\section{Chapter 5.}

\section{The Role of Transderivational Anti-Faithfulness \\ in Morpho-Accentual Phenomena}

In chapters 2 and 3, the phenomenon of root-controlled accent was studied and analyzed in terms of the interaction of Faithfulness constraints in Optimality Theory. This analysis draws on an important development in OT, namely the notion of Root Faithfulness, and explains the very common pattern of overriding root accent in terms of a universal ordering of Root and Affix Faithfulness constraints.

This approach to root-controlled accent also clarifies an independent body of facts which might be dubbed 'affix-controlled accent', i.e., morpho-accentual processes which correlate with the attachment of an affix. Root-controlled accent and affix-controlled accent (RCA and ACA respectively) are clearly different phenomena because they exhibit strikingly different formal properties. As brought to the fore in $\$ 4.1$, a basic difference is that RCA is systematic and applies across the board, while affix-controlled phenomena are non-systematic and triggered by particular morphemes. This distinction, among several others examined here, leads to the conclusion that the two types of morphologically governed accentual phenomena must receive separate treatment. This conclusion is further supported directly below with a review of some basic properties of affix-controlled phenomena. In the following sections, I will develop a theory of affix-controlled morphoaccentual processes which shows a clear role for Prosodic Anti-Faithfulness constraints in each case. This theory explains the properties of affix-controlled accentual processes and unifies ACA as a class that distinguishes itself from RCA.

\subsection{Towards an Integrated Theory of Affix-Controlled Accent}

\subsubsection{Properties of Affix-Controlled Accent}

(A) Lexically Idiosyncratic To begin where I left off in $\S 4.1$, it is an idiosyncratic feature of a given affix whether or not it induces a morpho-accentual process, and therefore, this feature must be lexically listed. For example, the dominant/recessive distinction in Russian suffixes must be lexically-specified because this distinction does not always correlate with a given phonological or morphological property. Thus, the accented/unaccented contrast is orthogonal to the distinction between dominant/recessive affixes, as shown by the fact that in Tokyo Japanese, and several Indo-European languages, there are both dominant accented and dominant unaccented affixes. Also, while dominant affixes are sometimes derivational, this morphological property is not a reliable predictor of dominance. In Russian, for example, the plural suffix - $a$ used in technical jargon is dominant, and yet it is clearly inflectional; furthermore, many derivational suffixes in Russian are recessive, showing that dominance is not always a property of category-changing affixes.

Other common affix-controlled processes, such as pre- and post-accentuation, are likewise lexically idiosyncratic. In Cupeño, accented suffixes are either auto-stressed or 
pre-stressing, requiring a lexical distinction between the two classes of accented suffixes. Moreover, the fact that there are both dominant pre-accenting and recessive pre-accenting suffixes in Japanese shows that this contrast is orthogonal to the dominant/recessive distinction. The same point holds for processes of accentual shift: in languages like Japanese and Aguaruna, accent-shifting suffixes must be distinguished from auto-accented and accent-neutral suffixes, and this contrast is clearly a lexical property.

(B) Morphologically Triggered A second important property of affix-controlled accentual processes is that they always correlate with the application of a morphological process, and as a result, these processes may serve as an important cue for 'derivedness'. While affix-controlled processes are often correlated with the attachment of an affix, the morpho-accentual phenomena examined here may also correlate with non-affixal morphology, such as compounding and root-and-pattern morphology. Stated differently, a relationship is established between the base and its derivative in which certain prosodic patterns obtain; different affix-controlled processes specify different types of prosodic requirements.

The dominant/recessive distinction in affixes may be captured in terms of this relationship between a base and its related derived form. Dominance effects thus cause an obligatory deletion of the base prosody, which yields an opposition in base-derivative pairs when the base itself has an accent. Compare this requirement to the Faithfulness effect by stress-neutral affixation which enforces preservation of the base prosody in derived forms. Also, pre- and post-accentuation and accentual shifts are described straightforwardly in terms of this morphological relationship. Pre-accenting suffixes, for example, require the insertion of an accent on a nearby syllable in the derived form, thereby distinguishing the derivative from its base with an epenthetic accent. Likewise, accent-shifting affixes require an overt shift in the derived form, again instantiating a morphological contrast between a base and its derivative. ${ }^{25}$

(C) Base Mutating A third important property, which is a general property of many morpho-phonological alternations, is that the affected element in an affix-controlled process is always the base of the morpho-accentual process, i.e., the basic formative to which the process applies. Since roots and stems are generally the bases for these operations, they are always the target of the phonological change. Thus, as observed in Inkelas 1996, the dominant/recessive contrast cross-classifies affixes which induce a change in the base to which they attach, but there are no roots which idiosyncratically cause the deletion of an accent in a neighboring affix. Furthermore, while pre- and post-accenting affixes abound in the case studies examined in this thesis, pre- and post-accenting stems are far less common, and perhaps completely unattested. In Cupeño, for example, many suffixes are pre-accenting but no roots trigger the insertion of accent on a neighboring affix. Finally, the morphologically triggered accent shifts in Tokyo Japanese and Aguaruna always affect the base of affixation, and so they pattern with the other two types of affix-controlled accentual processes.

(D) Grammar Dependent Fourth, the output of affix-controlled processes is often constrained by the independently necessary constraints on accent. While these processes appear to give rise to otherwise exceptional accentual patterns, the affected element is not

\footnotetext{
${ }^{25}$ Of course there are numerous accentual shifts, typically involving a pitch accent, which are purely phonological, as exemplified in Kikuyu (Clements \& Ford 1979), Shona (Myers 1987a), Winnebago (Miner 1979), and various Micronesian languages (Rehg 1993), and these processes are in fact crucial to the synchronic description of the surface prosody. As phonological shifts, however, these cases can be straightforwardly treated through the domination of Faithfulness by Alignment constraints which favor accent at a given edge (see Myers 1997a and Bickmore 1996 for some leading ideas).
} 
completely outside the bounds of the system. Indeed, there are often components of the processes which are directly predicted by the independently needed constraints on the distribution of accent. Thus, dominant morphemes idiosyncratically trigger deaccentuation, but the pattern which emerges from this process is invariably a default pattern. In Russian, for example, the grammar of accent characterizes ending stress as a default position. Further, when dominant suffixes delete the stem accent, default ending stress emerges. Similar patterns arise in Tokyo Japanese where the dominant affixes bring about a default accentual pattern.

Pre- and post-accentuation and accentual shifts are also grammar dependent in that the output of these processes is often governed by the independently required constraints on accent. In Cupeño, for example, the accent introduced by the pre-stressing suffixes is preferentially aligned with the right edge of the stem. This fact accords with the general rightward orientation of stress in the language, as evidenced by the fact that in words with two inherently accented affixes, the rightmost affix wins. Accent shift in Aguaruna is particularly interesting in this light: accent-shifting suffixes cause a rightward shift of the base accent. Furthermore, there is a general rightward orientation for accent in the language, as shown by the fact that the accent of a deleted vowel typically follows the same pattern, shifting one mora to the right. The same pattern of grammar dependent accent shift is found in Tokyo Japanese, where accent-shifting suffixes induce a rightward shift in accent, which, as I argued in $\S 3.3$ on the basis of compound accent, is the default edge orientation for accent in the language.

(E) Subject to Locality Requirements A final important property of morpho-accentual processes is that there may be locality conditions on the triggering affix and the element undergoing the structural change of the process, though this is not a necessary condition for ACA. For example, pre- and post-accentuation often have locality requirements. Thus, pre- and post-accenting affixes in Tokyo Japanese only insert an accent on an immediately adjacent syllable (see Poser 1984 for discussion of the bounded nature of morpho-accentual rules). Locality conditions are also in effect in morphologically triggered accent shifts. In Aguaruna, there is a threshold on the triggering effect of the accent-shifting suffixes noted in Payne 1990, namely the stem accent must be 'close enough' to the accent-shifting suffix in order to trigger the process, showing the need for locality in morphologically triggered shifts as well. A similar restriction is found in Limburg Dutch where the retraction of certain tonal types is limited to the syllable directly preceding the base-mutating suffix.

As for dominance effects, such locality requirements are perhaps less common, but there is one clear example in Tokyo Japanese which is subject to a condition on the distance between the mutating morpheme and the accent to be deleted. The genitive particle no triggers de-accentuation specifically in the final mora of a disyllabic stem; non-final accents are left alone. Thus, this particle clearly induces a dominance effect that is limited to the mora closest to no. In sum, while locality effects are not a necessary condition for diagnosing an affix-controlled accentual process, they constitute a property of ACA which unifies a heterogeneous set of patterns as a class.

The following list summarizes the properties of affix-controlled phenomena discussed above. 
(1) Formal Properties of Affix-Controlled Accent

a. Lexically idiosyncratic: the application of affix-controlled processes is unpredictable and must be specified in the lexical entry of individual morphemes.

b. Morphologically triggered: affix-controlled processes correlate with the application of a morphological process.

c. Base-mutating: affix-controlled processes affect the base of a morphological process.

d. Grammar dependent: the output of an affix-controlled accentual process may be predicted by independently attested grammar of accent.

e. Subject to locality requirements: in some affix-controlled processes, the target of the process must be 'close enough' to the triggering morpheme.

Before developing the theory of these phenomena, it is important to emphasize that this list of features shows that affix-controlled accent forms a class that is distinguished from root-controlled accent. Thus, while ACA is lexically idiosyncratic, RCA is fully predictable from the morphological structure of the word. In contrast to affix-controlled accent which must be lexically listed, root-controlled de-accent applies across the board in all words containing accented roots.

Furthermore, ACA is clearly associated with the application of a morphological process, which is not necessarily the case with RCA. As clarified above, affix-controlled processes have a morphological function in that they create oppositions in base-derivative pairs. RCA clearly works against this pressure to realize morphological contrast, bringing about uniformity within a paradigm. Indeed, one of the basic predictions of the RCA hypothesis is that root accentedness results in fixed accent within a paradigm (see §3.1). Therefore, the underlying functions of these two types of morpho-accentual processes underscore the fundamental difference between RCA and ACA. A related difference between the two is that ACA specifically requires mutation in the base of affixation, while RCA actively suppresses such mutations because the basic constraints involved in RCA assert special Faithfulness privileges to roots, which are typical bases of affixation. Thus, the basic function of RCA is again at odds with the morphological function of ACA.

The properties of grammar dependence and locality effects also distinguish ACA from RCA. Grammar dependent affix-controlled processes trigger a change which often leads to a default or unmarked accentual pattern. This improvement of the overall markedness of a form in ACA is to be contrasted with the greater markedness resulting from RCA. Succinctly, RCA assigns special Faithfulness properties to roots, which allows marked structures to emerge. Moreover, while ACA may be subject to locality conditions, this is not the case with RCA. Overriding root accent, like that found in Cupeño, affects all accented affixes, regardless of their proximity to the root accent.

\subsubsection{Affix-Controlled Accent as Prosodic Anti-Faithfulness}

Affix-controlled accent behaves in a way that sets it apart from RCA. The explanation of this finding offered here is that the constraints responsible for these different types of morpho-accentual phenomena are completely different. RCA is due to the effects of high-ranking Prosodic Faithfulness constraints for roots, which are generally felt in the 
input-output dimension of Faithfulness. ACA, on the other hand, derives from constraints which have a very different character, namely Transderivational Anti-Faithfulness constraints. However, before implementing these constraints, it is instructive to point out an interesting parallel between ACA and another affix-controlled phenomenon which we have already examined in detail, namely accent-neutral affixation.

As illustrated in chapter 4 , section 1 , there is a basic difference between stressneutral and non-neutral affixes in English, and this difference must be lexically represented. Moreover, the Faithfulness effects induced by stress-neutral affixation (SNA) may be characterized as a relationship between base-derivative pairs: these affixes require that the prosody of the base be the same as the prosody of the derived form. Comparing this type of affix-controlled phenomenon with dominance effects, the differences between the two are readily statable in terms of a base-derivative coupling: dominance effects require deletion of base prosody, while stress-neutral affixation requires preservation of base prosody. Thus, consistent with other affix-controlled phenomena, the differences lie in the statement of the prosodic requirement. In sum, it appears that SNA should be grouped with the affix-controlled phenomena examined here.

The explanation for these related facts, namely that ACA differs formally from RCA, but resembles stress-neutral affixation, I argue, lies in the analysis of ACA in terms of Transderivational Anti-Faithfulness (TAF). To begin, the fact that affix-controlled processes are lexically idiosyncratic follows from the assumption that they are a response to TAF constraints. Thus, on a par with stress-neutral affixes, affixes which trigger a morpho-accentual process subcategorize for a specific surface-to-surface correspondence relation, and the TAF constraints defined on this relation bring about the observed pattern of Anti-Faithfulness. The lexical idiosyncrasy of both ACA and SNA derives from subcategorization in the lexicon.

The fact that both ACA and SNA are morphologically triggered also follows from the general theoretical assumptions in Transderivational Correspondence Theory. Both Transderivational Faithfulness and Anti-Faithfulness operate between morphologically related words; thus, from this basic premise, it follows that the effects derived from these constraints are associated with morphological processes. Moreover, as will be demonstrated shortly, the various phonological patterns observed in ACA can be directly described in terms of the negation of the already existing Prosodic Faithfulness constraints, and so there is a symmetry in the theoretical mechanisms used to describe both Faithfulness and Anti-Faithfulness effects. The observed symmetry is significant because the premise that there is an Anti-Faithfulness constraint for every Faithfulness constraint predicts this very outcome.

Two of the remaining properties of ACA follow from the basic principles of the theory of Transderivational Anti-Faithfulness. Thus, the fact that ACA is generally basemutating follows from Strict Base Mutation (spelled out in chapter 4). Affix-controlled processes generally affect the base of a morphological process because ACA is a special type of morpho-phonological operation, and such operations generally affect the base. Furthermore, the locality effects observed in ACA are again a consequence of the general fact that morpho-phonological operations may be subject to locality constraints. Therefore, in regard to these two last properties, ACA is just a special kind of morpho-phonological alternation.

Lastly, the fact that the output of an affix-controlled process is grammar dependent follows from the nature of Anti-Faithfulness and the basic tenets of Optimality Theory. On par with the morpho-phonological operations discussed in $\S 4.3$, the TAF constraints generally induce a phonological change in base-derivative pairs, but often other constraints 
active elsewhere in the system will have a say in how this change is realized. This result, where the entire system of constraints conspire together to achieve a larger result, is a direct consequence of the basic assumption in OT that constraints are ranked and violable. Thus, in the analysis of dominance effects presented below, we will see that a high-ranking TAF constraint triggers de-accentuation of base accent, but it is the low-ranking Faithfulness and Markedness constraints which determine the accentuation of a de-accented word.

Furthermore, the output of pre-/post-accentuation and accent shifts are likewise governed by dominated constraints, which explains the fact that these morpho-accentual processes are also grammar dependent. Finally, undominated constraints in the system may also influence the direction of the structural change, as we will see in the analysis of the dragging tone mutation in Limburg Dutch. In this dialect of Dutch, certain suffixes condition a flop of the high tone in the preceding syllable, but this mutation never results in a rising tone structure because this structure is ruled out in the tonal inventory of the system as a whole.

To recapitulate the above discussion, the theory of Transderivational AntiFaithfulness explains the basic properties of ACA in a way that shows how ACA is similar to accent-neutral affixation but different from root-controlled accent. A very important point here is that this accomplishment is achieved without appeal to mechanisms or constraints that are specific to the theory of accent. That is, the formal properties of ACA are explained in exactly the same way as the affix-controlled segmental processes discussed in chapter 4 are treated. The theory provides the necessary constraints for describing morpho-phonological alternations, namely Transderivational Anti-Faithfulness constraints, and when these constraints are applied in the domain of prosody, the result is a fully formal theory of morpho-accentual processes. In the remainder of this section, I will present the Prosodic Anti-Faithfulness constraints which define this theory, giving structure to the analyses that will be proposed throughout the rest of the chapter.

An important assumption made in $\$ 4.3$ is that there is an Anti-Faithfulness constraint for every Faithfulness constraint. Anti-Faithfulness constraints involve wide scope negation of the proposition expressed by the corresponding Faithfulness constraint. With these two assumptions, the theory proposed here predicts the following Prosodic Anti-Faithfulness constraints.

(2) Prosodic Anti-Faithfulness

$\neg$ MAX-PROM $\rightarrow$ Obligatory deletion of prominence It is not the case that every prominence in $\mathrm{S}_{1}$ has a correspondent in $\mathrm{S}_{2}$.

$\neg$ DEP-PROM $\rightarrow$ Obligatory insertion of prominence It is not the case that every prominence in $S_{2}$ has a correspondent in $S_{1}$.

$\neg$ NO-FLOP-PROM $\rightarrow$ Obligatory shift in prominence It is not the case that every corresponding prominence must have a corresponding sponsor.

Thus, the three Prosodic Faithfulness constraints argued for in $§ 1.2 .2 .1$, MAX-PROM, DEP-PROM, and NO-FLOP-PROM, each have a negated counterpart, and these negated constraints, when defined for the correct correspondence relation, give the basic ingredients for describing the phonological patterns observed in ACA.

To sketch how these constraints will be employed in subsequent analyses, consider the following schematic rankings, which characterize the constraint hierarchies used below. 
(3) Schematic Rankings for Affix-Controlled Accentual Processes
a. ᄀOO-MAX-PROM >> OO-MAX-PROM
Dominance Effects
b. ᄀOO-DEP-PROM >> OO-DEP-PROM
Pre-/Post-Accentuation
c. $\neg \mathrm{OO}-\mathrm{NO}-\mathrm{FLOP}-\mathrm{PROM}>>$ OO-NO-FLOP-PROM
$\sim$ Accentual Shifts

With the obligatory deletion constraint, $\neg$ OO-MAX-PROM, ranked above the corresponding Transderivational PROS-FAITH constraint, OO-MAX-PROM, a deletion of prominence in the base of affixation is predicted, and so this ranking will be at the core of the analysis of dominant morphemes. Likewise, when $\neg$ OO-DEP-PROM is ranked above OO-DEP-PROM , the result will be the obligatory insertion of accent in base-derivative pairs, which is the morpho-accentual pattern observed in pre- and post-accentuation. Finally, with the AntiFaithfulness constraint $\neg$ OO-NO-FLOP-PROM top-ranked, an accentual shift is predicted in morphologically related words. In $§ 5.3 .3$, a wider range of constraint permutations will be illustrated and the predictive factorial typology will be given.

The rest of this chapter is organized as follows. In the next section, the problem posed by dominant morphemes will be studied in more detail and a theory of dominance effects will be proposed which shows a fundamental role for Transderivational AntiFaithfulness. This theory will be applied in an analysis of dominant affixes in Tokyo Japanese and Modern Russian, which will distinguish the TAF theory of dominance effects for other plausible alternatives. In $\$ 5.3$, I will consider the affix-controlled process of preand post-accentuation from various angles, focusing in particular on pre-accenting suffixes in Cupeño, and I will also conclude in favor of an analysis in terms of TAF. The discussion in $\$ 5.4$ extends the scope of TAF constraints to morphologically triggered accent shifts, arguing that this approach again has some major advantages over the available alternatives and that this last body of facts shows the pervasiveness of Transderivational Anti-Faithfulness in morpho-accentual processes.

\subsection{Dominance Effects as Transderivational Anti-Faithfulness}

In this section, the problem posed by morphologically induced dominance effects is studied and analyzed in terms of Transderivational Anti-Faithfulness. Following a brief introduction to the problem, an analysis of dominant suffixes in Tokyo Japanese is presented in $\S 5.2 .2$ as an illustration of the basic theory, which is followed in $\S 5.2 .3$ by a case study of dominant morphology in Russian as further empirical support for the overall approach. Next, some important implications of the basic approach are examined in $\S 5.2 .4$, which will enable me to contrast the TAF analysis of dominance effects with some plausible alternatives in $\$ 5.2 .5$.

\subsubsection{The Problem}

Dominant, or accent-deleting, affixes are very common in accentual systems. Dominant affixes trigger the deletion of an accent in the base to which they attach, and if they are themselves unaccented, they bring about a default, or unmarked, accentual pattern. To begin with a now familiar system, dominant suffixes in Tokyo Japanese are of two basic types. As exemplified in (4) below with the adjective-forming suffix -ppó, dominant accented suffixes are always accented, regardless of the accentedness of the base to which they attach. Dominant unaccented suffixes, on the other hand, trigger a de-accentuation of the stem and create default unaccented words. This behavior is illustrated in (5) with the 
suffix $-k k o$, which forms words with the meaning 'indigène of $\mathrm{X}$ ', where $\mathrm{X}$ stands for the base toponym.

(4) Dominant Accented Suffix

$\begin{array}{lllll}\text { a. } \quad \text { /abura }+ \text { ppó }+\mathrm{i} / & \rightarrow & \text { abura-ppó-i } & \text { 'oily' } \\ / \text { kaze }+ \text { ppó }+\mathrm{i} / & \rightarrow & \text { kaze-ppó-i } & \text { 'sniffly' } \\ \text { b. } \quad \text { /adá }+ \text { ppó }+\mathrm{i} / & \rightarrow & \text { ada-ppó-i } & \text { 'coquettish' } \\ \text { /kíza }+ \text { ppó }+\mathrm{i} / & \rightarrow & \text { kiza-ppó-i } & \text { 'affected' }\end{array}$

(5) Dominant Unaccented Suffix
a. /edo $+\mathrm{kko} /$
$\rightarrow \quad$ edo-kko
'Native of Tokyo'
/niigata $+\mathrm{kko} /$
$\rightarrow \quad$ niigata-kko
'Native of Nigata'

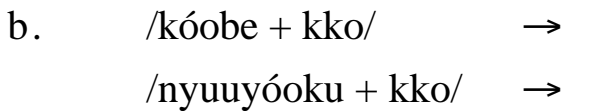
koobe-kko
'Native of Kobe'
nyuuyooku-kko
'Native of New York'

The two suffixes, therefore, have in common the property of causing a deletion of base prosody, often referred to as a 'dominance effect', and differ only in whether or not they carry their own inherent accent.

A basic property of dominance effects is that they are grammar dependent; the structures resulting from the process are governed by independently attested constraints in the accent system. Grammar dependence is most perspicuous in structures resulting from dominant unaccented affixes because the affix itself does not have an accent of its own to realize. For example, the suffix -kko creates completely unaccented words, which as we have seen in $\$ 3.3$, is the accentual default in Japanese, i.e., the structure assigned by the grammar to unaccented words. Thus, the anti-insertion constraint, DEP-PROM, which is responsible for default unaccented words, is in full force here, ensuring that de-accented words are likewise unaccented at the surface. This pattern of deletion plus default accent assignment is in fact a very general property of dominant morphemes, as exemplified in several cases throughout this dissertation. For example, dominant (unaccented) affixes in Russian yield words with stress on the inflectional ending, as shown by the behavior of -ač in /púz + ač $+\mathrm{u} / \rightarrow$ puz-ač $-\breve{u}$ 'man with paunch (dative singular)'. That stress on the ending is the default position is supported by the fact that words with unaccented stems generally receive stress in this position (see $\$ 3.2$ for detailed argumentation).

To summarize this discussion, the following two questions are central in the treatment of dominance effects:

1. How is the morphologically conditioned deletion achieved?

2. How is the default accentual pattern predicted as the result of this deletion?

The proposal I will argue for here is that dominance effects are explained as a response to the Transderivational Anti-Faithfulness constraint $\neg$ OO-MAX-PROM, which specifically requires a deletion of base prosody in morphologically related words. When properly integrated in an OT grammar, the TAF constraint $\neg$ OO-MAX-PROM predicts the 
observed deletion, but the independently necessary constraints in the grammar give the accentual default, as illustrated in the following tableau. ${ }^{26}$

(6) Dominance Effects as Transderivational Anti-Faithfulness

\begin{tabular}{|c|c|c|c|c|}
\hline Base & /kóobe + kko/ & ᄀOO-MAX-PROM & OO-PROS-FAITH & DEP-PROM \\
\hline \hline a. kóobe & kóobe-kko & $* !$ & & \\
\hline b. kóobe & koobé-kko & & $*$ & $*$ ! \\
\hline c. $\rightarrow$ kóobe & koobe-kko & & $*$ & \\
\hline
\end{tabular}

The TAF constraint $\neg$ OO-MAX-PROM is transderivational; it evaluates base-derivative pairs in a way similar to OO-Faithfulness constraints, with the important difference that $\neg \mathrm{OO}$ MAX-PROM requires a deletion in derived forms. The first base-derivative pair is faithful to the prosody of the base, and it therefore incurs a fatal violation of the TAF constraint. The remaining two candidates satisfy this constraint by mutating the base through deletion of the base accent. Only the last candidate, however, achieves the required deletion without a violation of the independently motivated constraint, IO-DEP-PROM, and so the derived word is unaccented by default.

While there are still some formal issues to be addressed here, it is clear from this brief sketch how the TAF theory of dominance effects accounts for the observed grammar dependence. A Transderivational Anti-Faithfulness constraint requires a change in the derived form, in effect nullifying the force of the otherwise undominated Faithfulness constraints. The ultimate result of the phonological change, however, is dictated by the independently necessary constraints on the distribution of accent, which results in the observed accentual default. Moreover, it should be clear how the analysis of dominance effects in TAF theory will explain additional properties of dominant morphemes in a way that relates this phenomenon to other kinds of affix-controlled accentual processes. Highlighting three important properties, affix-controlled accentual processes are morphologically triggered, base-mutating, and lexically idiosyncratic. Dominance effects are morphologically triggered on this theory because the Anti-Faithfulness constraints which bring about the change are transderivational, and thus they necessitate a change specifically in base-derivative pairs. Furthermore, dominance effects are base-mutating because TAF constraints can only bring about a change in the base, which is a direct consequence of the thesis of Strict Base Mutation (see chapter 4, section 2). Finally, the fact that dominance effects are lexically idiosyncratic, i.e., must be specified in the lexicon, will follow from the assumption that dominant morphemes subcategorize for a OOcorrespondence relation upon which a high-ranking TAF constraint is defined. In short, the proposed analysis of dominance effects is quite on a par with the analysis of stressneutral affixation in English, and as we will see, all other affix-controlled phenomena.

The theory of dominance effects developed below represents the first step towards the larger goal of providing a general theory of affix-controlled accentual processes: it explains the behavior of dominant affixes with the same machinery used in the analysis of other affix-controlled morpho-accentual phenomena like pre- and post-accentuation and morphological accent shifts. This unification of the treatment of dominance effects and other affix-controlled phenomena will be made more explicit in the analysis of dominance

\footnotetext{
${ }^{26}$ In the tableaux throughout this chapter, base-derivative pairs are arranged vertically in a single complex tableau, as shown here. While these tableaux clearly illustrate the relationship between the base and its related output, they obscure the role of the constraint system in determining the base form. To clarify this role, each case study begins with a brief description of the formal account of the basic (underived) words.
} 
effects in Japanese and Russian, which is presented in the next two subsections.

Furthermore, the unified analysis of ACA will be central in distinguishing the analysis of dominance effects in terms of TAF from other possible alternatives, an issue which is addressed in the last subsection.

\subsubsection{The Proposal: Dominance Effects as the Negation of MAX-PROM}

We return now to the facts of dominant morphemes in Tokyo Japanese as an illustration of the analysis of dominance effects within TAF theory. The analysis builds on the constraint system developed in $\$ 3.3$, and explains grammar dependent dominance effects by incorporating a set of Transderivational Anti-Faithfulness constraints into this system. The discussion below starts with a brief recap of accent in underived words, and then moves to the analysis of the distinction between dominant and recessive affixes.

Accent is contrastive in two ways in Japanese: the position of accent may introduce contrast in otherwise identical words, and further, lexical accent contrasts accented and unaccented words. Therefore, in words with $n$ number of syllables, the number of possible contrasts is $n+1$, e.g., a three-way contrast in disyllabic words: hási 'chopsticks', hasí 'bridge', hasi 'edge'. Ignoring certain irrelevant ranking details, the following partial ordering of constraints accounts for the basic facts.

\section{(7) Japanese Word Accent}

\section{a. MAX-PROM, No-FlOP-PROM > Alignment, NONFINALITY \\ b. DEP-PROM >> HEADEDNESS(PrWd)}

The first ranking accounts for the fact that Faithfulness to lexical prosody outweighs the constraints effecting a left or right edge bias for accent and the constraint disallowing final accent. Together with these rankings, the second ranking accounts for the accented/unaccented contrast in underived words by requiring unaccented morphemes to remain unaccented, despite pressure from HEADEDNESS(PrWd), which requires every prosodic word to have a head foot, and thus an accent. This last ranking is important for the discussion which follows because it shows how the grammar of accent in Japanese treats words which are totally unaccented: they are left unaccented by default.

Moving next to derived words, affixes may be either accented or unaccented, and when an accented affix or particle combines with an accented stem, stem accent typically prevails (except with dominant morphemes, discussed below) because of the general pattern of root privilege. Thus, when the accented suffix -tára combines with an inherently accented verb stem, it loses its inherent accent, e.g., /yóm + tára/ $\rightarrow$ yón-dara 'if he reads', cf. /yob + tára/ $\rightarrow$ yon-dára 'if he calls'. Likewise, inherently accented enclitics such as kará 'from' may only realize their accent when they combine with an unaccented stem in the same minor phrase, as in /miyako + karál $\rightarrow$ miyako kará 'from the city', cf. línoti + karál $\rightarrow$ inoti kara 'from life'. Lastly, as argued extensively in §3.3, prefix + stem sequences are consistent with this pattern of root-control, as most prefixes are either the first member of a compound or create their own minor phrase.

These suffixes and enclitics are therefore recessive in that they lose to an accented stem in a competition for the unique word accent. This behavior contrasts with that of the dominant affixes which cause deletion of a stem accent. Dominant affixes are typically suffixes or enclitics in Japanese, and they can be either accented or unaccented. Dominant accented morphemes are generally accented in every word or phrase that contains them 
(except, of course, in words with more than one dominant accented affix). Thus, the adjective-forming suffix -ppó mentioned in the introduction always takes the accent of the word, even when it attaches to inherently accented stems (8b).

(8) Dominant Accented Suffix: - ppó (Poser 1984: 49)

\begin{tabular}{|c|c|c|c|}
\hline \multirow{4}{*}{$\begin{array}{ll}\text { a. } & \text { /abura }+ \text { ppó }+\mathrm{i} / \\
& \text { /kaze }+ \text { ppó }+\mathrm{i} / \\
& \text { /kodomo }+ \text { ppó }+\mathrm{i} / \\
& \text { /mizu }+ \text { ppó }+\mathrm{i} /\end{array}$} & $\rightarrow$ & abura-ppó-i & ‘oily’ \\
\hline & $\rightarrow$ & kaze-ppó-i & 'sniffly' \\
\hline & $\rightarrow$ & kodomo-ppó-i & 'childish' \\
\hline & $\rightarrow$ & mizu-ppó-i & 'watery' \\
\hline /adá + ppó + i/ & $\rightarrow$ & ada-ppó-i & 'coquettish' \\
\hline /netú + ppó + i/ & & netu-ppó-i & 'zealous' \\
\hline /honé + ppó + i/ & $\rightarrow$ & hone-ppó-i & 'bony' \\
\hline /kíza + ppó + i/ & $\rightarrow$ & kiza-ppó-i & 'affected' \\
\hline
\end{tabular}

Other dominant accented morphemes include: -más 'politeness marker', -rási 'seem', gúrai 'as much as a X', rasíi 'like a X' (see McCawley 1968: $140 \mathrm{ff}$. and Poser 1984 for more examples).

Dominant unaccented morphemes, on the other hand, do not carry an inherent accent, and as a result, they bring about a default pattern, which in Japanese means forming unaccented words. The suffix -kko shows this behavior, as all words with this suffix are unaccented.

(9) Dominant Unaccented Suffix - $k k o$ 'indigène of X' (Poser 1984: 72)

\begin{tabular}{|c|c|c|c|}
\hline \multirow{4}{*}{$\begin{array}{l}\text { /edo + kko/ } \\
\text { /niigata + kko/ } \\
\text { /oosaka + koo/ } \\
\text { /tookyoo + kko/ }\end{array}$} & $\rightarrow$ & edo-kko & 'Native of Tokyo' \\
\hline & $\rightarrow$ & niigata-kko & 'Native of Nigata' \\
\hline & $\rightarrow$ & oosaka-kko & 'Native of Osaka' \\
\hline & $\rightarrow$ & tookyoo-kko & 'Native of Tokyo' \\
\hline /kóobe + kko/ & $\rightarrow$ & koobe-kko & 'Native of Kobe' \\
\hline /kyóoto + kko/ & $\rightarrow$ & kyooto-kko & 'Native of Kyoto’' \\
\hline /nágoya + kko/ & $\rightarrow$ & nagoya-kko & 'Native of Nagoya' \\
\hline /nyuuyóoku + kko/ & $\rightarrow$ & nyuuyooku-kko & 'Native of New York' \\
\hline
\end{tabular}

Another dominant unaccented suffix is -teki which forms adjectival nouns (see Martin 1975 for the details).

The chart below summarizes the main facts of relevance here. Accent is rootcontrolled in Japanese, and as a result, stem accent wins out over accent in a recessive suffix (10b). Dominant accented suffixes run counter to this pattern, as their inherent accent beats the accent of an accented stem (10c). Dominant unaccented suffixes likewise steal stem accent, but since they are themselves unaccented, they yield unaccented words by default (10d), cf. (10a). 
(10) Summary of the Facts

a. Bare substantives without a lexical accent are unaccented at the surface:
/hasi/
$\rightarrow$ hasi
cf. /hási/
$\rightarrow \quad$ hási

b. Stem accent precludes the realization of accent in a recessive suffix:

$$
\begin{array}{lll}
\text { /yoN + dára/ } & \rightarrow & \text { yoN-dára } \\
\text { /yóN + dára/ } & \rightarrow & \text { yóN-dara }
\end{array}
$$

c. Dominant accented suffixes are always accented:

$$
\begin{array}{lll}
\text { /abura }+ \text { ppóDom }+ \text { i/ } & \rightarrow & \text { abura-ppó-i } \\
\text { /adá }+ \text { ppó Dom }+ \text { i/ } & \rightarrow & \text { ada-ppó-i }
\end{array}
$$

d. Dominant unaccented suffixes create words which are unaccented:

$$
\begin{array}{lll}
\text { /edo }+ \text { kkoDom } / & \rightarrow & \text { edo-kko } \\
\text { /kóobe }+ \text { kkoDom } / & \rightarrow & \text { koobe-kko }
\end{array}
$$

While the contrast between (10b) and (10c) appears to pose an empirical challenge for the analysis of Japanese as a root-controlled accent system, it is clear from the comparison of dominant accented and unaccented suffixes that once we have a good understanding of the pattern of deletion in (10d), the analysis of (10c) will come for free.

The phonological operation put into effect by dominant affixes (both accented and unaccented) is the deletion of an accent in the base to which they attach. Ignoring for the moment how the base is targeted for this phonological deletion, dominance effects may be explained as the negation of MAX-PROM, the anti-deletion Prosodic Faithfulness constraint. This result is shown below with the logical statement of $\neg$ MAX-PROM, consistent with the formulation of Anti-Faithfulness constraints developed in chapter 4.

(11) $\neg$ MAX-PROM: For $\mathrm{x}$ a prominence, $\neg\left[\forall \mathrm{x} \exists \mathrm{x}^{\prime}\left[\mathrm{x} \in \mathrm{S}_{1} \rightarrow \mathrm{x}^{\prime} \in \mathrm{S}_{2} \& \mathrm{x} R \mathrm{x}^{\prime}\right]\right]$

'It is not the case that every prominence in $S_{1}$ has a correspondent in $S_{2}$.'

The negation of MAX-PROM therefore has the effect of requiring deletion of (at least) one prominence. When this obligatory deletion constraint is defined on a transderivational correspondence relation, the deletion is required in the mapping from a base form to its derivative, which is exactly the phonological pattern observed in Japanese. The dominant/recessive distinction can therefore be straightforwardly modelled in terms of the following constraint rankings.

(12) Dominant/Recessive Distinction through Constraint Ranking

$$
\begin{aligned}
& \text { a. Dominant affixes: } \neg \text { OO-MAX-PROM > OO-MAX-PROM } \\
& \text { b. } \underline{\text { Recessive affixes: }} \text { OO-MAX-PROM > } \neg \text { OO-MAX-PROM }
\end{aligned}
$$

When $\neg$ OO-MAX-PROM is high-ranking, specifically ranked above MAX-PROM defined for OO-correspondence, this ranking will require deletion of the base prosody, as observed in the base-derivative pair [kóobe] $\approx[$ koobe-kko], where the base of affixation for $-k k o$, namely the stem kóobe, loses an accent in the derivative form. On the other hand, if the 
Prosodic Anti-Faithfulness constraint is ranked below OO-MAX-PROM, then stem stress is not deleted, as in the mapping from [yón-da] to [yón-dara]. This last point assumes that the inflected form yón-da forms for the base of affixation for derived words, but as discussed in $\$ 4.2$, in contexts where inflections are obligatory, inflected forms may serve as the base.

Assuming multiple OO-correspondence relations (see §4.2), the schematic rankings can be conflated into a total ordering of constraints, as shown below.

(13) Conflating the Schematic Rankings

$\neg \mathrm{OO}_{\text {Dom }}$-MAX-PROM $>$ OO-MAX-PROM $>>\mathrm{OO}_{\mathrm{Rec}-\mathrm{MAX}-\mathrm{PROM}}$

I use the subscripts 'Dom' and 'Rec' to differentiate the correspondence relations referred to by the different $\neg$ OO-MAX-PROM constraints. The top-ranked TAF constraint

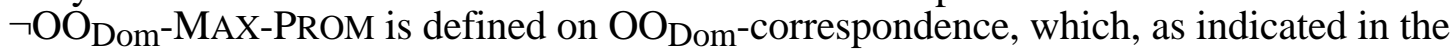
lexical entries in (14b-c), is the correspondence relation subcategorized for by dominant affixes. Likewise, the recessive affixes trigger $\mathrm{OO}_{\mathrm{Rec}}$-correspondence (14a), and therefore words with these affixes are governed by $\neg \mathrm{OO}_{\mathrm{Rec}}-\mathrm{MAX}-\mathrm{PROM}$. It should be emphasized that the subscripts are simply handy mnemonics whose only formal role in the theory is to link up the individual affixes with the TAF constraints which are sensitive to them. The dominant behavior of the different affixes is predicted purely on the basis of the inherent rank of their TAF constraint; thus, the real work in the analysis of dominance effects derives from a fundamental premise in OT, namely that constraints are ordered with respect to each other.

(14) Lexical Entries for Dominant and Recessive Suffixes
a. -tára $\quad \mathrm{V}_{\text {Cond }}$
kará
$\mathrm{P}$
[ Verb] OORec _ _ ]MWd
[ Noun] OORec __ ]
A
-más
Volite
rasíi
Comp
b. - $p p o ́$
[ [Verb] OODom __ ] $]_{\text {Stem }}$
[ [Noun] OODom __ ]
[ [ Verb]oODom __]Stem
[Dominant Accented]
c. $-k k o \quad \mathrm{~N}$
-teki $\mathrm{N}_{\text {Adjectival }}$
[ [ Noun] oODom __ ] $]_{M W d}$
[ [Stem $]_{\text {OODom __ }] \text { MWd }}$
[Dominant Unaccented]
[Recessive]

To sum up, the link between the individual affixes and the Anti-Faithfulness constraints responsible for the dominance effects is achieved in the lexicon through the subcategorization of OO-correspondence, very similar to Benua's 1997 [1998] approach to class 1 versus class 2 affixation in English. The dominant affixes are evaluated by the topranked TAF constraint, while recessive affixes are subject to the relatively low-ranking TAF constraint. The results of these assumptions will now be illustrated with a series of tableaux.

Starting first with -ppó, when dominant accented suffixes attach to unaccented stems, as in /abura + ppó $+\mathrm{i} / \rightarrow$ abura-ppó- $i$, their behavior is unremarkable as there is no stem accent to be deleted. On the other hand, when -ppó attaches to an accented stem, this suffix triggers deletion of the stem accent, as illustrated below, because this suffix 
subcategorizes for $\mathrm{OO}_{\mathrm{Dom}}$-correspondence, and the TAF constraint which operates on this

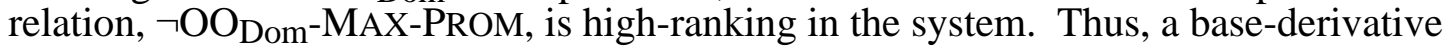
pair which preserves the base prosody, as in (15a), incurs a fatal violation of the TAF constraint, which rules out this option. The remaining candidates here satisfy $\neg \mathrm{OO}_{\mathrm{Dom}^{-}}{ }^{-}$ MAX-PROM by losing the accent of adá, but since the dominant suffix itself is accented, the winning candidate is the pair of outputs which preserves accent in -ppó, namely (15c).

(15) Dominance Effect with Dominant Accented -ppó-27

\begin{tabular}{|l|c|c|c|c|}
\hline \multicolumn{1}{|c|}{ Base } & /adá + ppó $+\mathrm{i} /$ & $\neg O_{\text {Dom-MAX-PM }}$ & OO-MAX-PM & IO-MAX-PM \\
\hline \hline a. $\quad$ adá & adá-ppo-i & $* !$ & & $*$ \\
\hline b. $\quad$ adá & ada-ppo-i & & $*$ & $* * !$ \\
\hline c. $\rightarrow$ adá & ada-ppó-i & & $*$ & $*$ \\
\hline
\end{tabular}

The application of TAF theory to dominant accented enclitics is directly parallel with this result. For example, when the dominant accented particle rasíi combines with an accented stem, e.g., inoti rasíi 'like a life', the correct results are obtained by extending the application of the TAF constraints to stem + enclitic structures. Thus, $\neg \mathrm{OO}_{\text {Dom }^{-M A X}-}$ PROM is active in the mapping of [ínoti] to [inoti rasíi], which results in the observed deletion here. Importantly, the dominance effect observed here cannot be attributed to the accentuation of compounds, as sometimes suggested, because compounds never preserve accent in the final syllable of the second member, which runs counter to the examples with rasíi.

Moving next to the behavior of recessive morphemes, because these morphemes subcategorize for $\mathrm{OO}_{\mathrm{Rec}}$-correspondence, they do not condition a deletion of base prosody: the TAF constraint defined on this correspondence relation is ranked below OO-MAXPROM. As shown below, therefore, derivatives formed with the recessive suffix -tára, lose to the accent of the stem because of high-ranking OO-Prosodic Faithfulness.

(16) Lack of Dominance Effect with Recessive Accented -tára ${ }^{28}$

\begin{tabular}{|c|c|c|c|}
\hline Base & /yóm + tára/ & OO-MAX-PROM & ᄀOORec-MAX-PROM \\
\hline \hline a. $\quad$ yón-da & yon-dára & $* !$ & \\
\hline b. $\rightarrow$ yón-da & yón-dara & & $*$ \\
\hline
\end{tabular}

In short, the distinction between dominant and recessive morphemes is determined by the rank of the TAF constraint assessing the Anti-Faithfulness properties of base-output pairs. Dominant suffixes such as - ppó trigger $\mathrm{OO}_{\mathrm{Dom}}$-correspondence, so they induce a

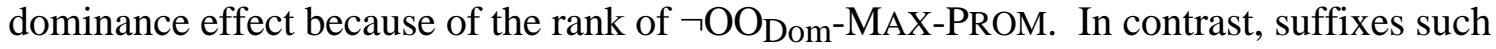

\footnotetext{
${ }^{27}$ The dominant suffix - ppó is attached simultaneously with the inflection $-i$ here for ease of exposition; the inflection could trigger an additional recursion. It turns out that this assumption is of little consequence here, as the dominance effect is predicted in both cases. This issue, however, raises the question of what the predictions of the TAF model are in words with sequences of dominant and/or recessive affixes, and this question is addressed in detail in §5.2.4.

${ }^{28} \mathrm{I}$ assume that the base of formation of words with -dára is the past tense form as this form has the same allomorph as the one found in the conditionals here. Using the notion of Base Optimization developed in $\S 4.2$, this assumption entails that the past is the unmarked tense in the system. Other bases, however, such as the present tense form, would achieve the same effect, as they preserve the lexical accent also, and so would require preservation of the accent in the base of the larger form.
} 
as -tára do not condition deletion of base prosody because they select $\mathrm{OO}_{\mathrm{Rec}^{-}}$ correspondence and the TAF constraint operating on this dimension of Faithfulness is too low-ranking to have an effect.

To complete the analysis, let us consider how the assumptions laid out so far apply to dominant unaccented morphemes. These morphemes are dominant, and therefore they subcategorize for $\mathrm{OO}_{\mathrm{Dom}}$-correspondence, which in turn accounts for the observed deletion of the accent of the base with these suffixes. As discussed above, the words resulting from this de-accentuation are always unaccented, which shows that the dominance effect is grammar dependent. This default pattern is therefore not the responsibility of the TAF constraints, but the larger constraint system on a whole. The accentual default therefore follows from the same ranking which is responsible for unaccented words in the inventory of underived words, namely IO-DEP-PROM > HEADEDNESS(PrWd), as shown in the following tableau. The fully faithful base-output pair in (17a) is eliminated from the candidate set because it fails to delete the base accent, and therefore violates top-ranked

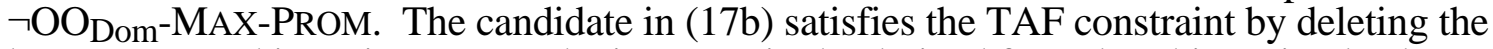
base accent and inserting an epenthetic accent in the derived form, but this option leads to a violation of IO-DEP-PROM by inserting an accent not present in the input. Because IODEP-PROM dominates the constraint which would encourage such an insertion, i.e., HEADEDNESS(PrWd), the base-derivative mapping in (17c) is chosen as the winner.

(17) Dominance Effect with Dominant Accented -kko

\begin{tabular}{|c|c|c|c|c|}
\hline Base & /kóobe + kko/ & $\neg \mathrm{OO}_{\text {Dom-MAX-PM }}$ & DEP-PROM & $\operatorname{HEAD}(\operatorname{PrWd})$ \\
\hline a. kóobe & kóobe-kko & $* !$ & & \\
\hline b. kóobe & koobé-kko & & $* !$ & \\
\hline c. $\rightarrow$ kóobe & koobe-kko & & & $*$ \\
\hline
\end{tabular}

In sum, the TAF constraint induces a deletion of base prosody in derivatives with accented bases, and since the suffix itself is unaccented, the default structure for words without a lexical accent is predicted, which is a completely unaccented form at the surface.

As summarized in the following chart, dominance effects in Japanese exhibit many of the formal properties characteristic of other affix-controlled morpho-accentual processes.

(18) Dominance Effects in Japanese

$\begin{array}{lll}\text { Lexically idiosyncratic: } & \rightarrow & \text { Subcategorized correspondence relations } \\ \text { Morphologically governed: } & \rightarrow & \text { Transderivational Anti-Faithfulness } \\ \text { Grammar dependent: } & \rightarrow & \text { Unitary grammars } \\ \text { Base-Mutating: } & \rightarrow & \text { Strict Base Mutation }\end{array}$

The de-accentuation triggered by the dominant suffixes was observed to be lexically idiosyncratic, and the analysis of this fact involved the specification of various correspondence relations in the subcategorization frame of individual affixes. Furthermore, the dominance effects observed here are morphologically governed, and this fact is explained in the above analysis as an effect the TAF constraint $\neg \mathrm{OO}_{\text {Dom}}$-MAX-PROM, which operates exclusively between a base and its derivative. 
Another very significant property of dominance effects in Japanese is that they are grammar dependent, meaning that the independently attested constraints on the distribution of prominence dictate the structure of the output resulting from de-accentuation. Grammar dependence is most clearly attested in structures with dominant unaccented morphemes, as the accentual default can be independently verified by examining underived forms which lack a lexical accent. Concretely, the accentual default for lexically unaccented words is to remain unaccented at the surface, and this pattern is exactly the observed pattern in words with the dominant unaccented suffix $-k k o$. Dominance effects brought about by dominant accented morphemes are also grammar dependent in that the independently necessary IOFaithfulness constraints demand realization of the accent in the affix itself. The main difference between these two types of grammar dependence is that only the type governing dominant unaccented morphemes predicts a correlation between the result of deaccentuation and the accentual default in the language as a whole. Grammar dependence with accented dominant affixes simply shows the emergence of an accentual contrast in affixes, which also has independently testable predictions, i.e., that dominant affixes will have a contrast in accentedness if recessive ones do as well.

This basic fact of grammar dependence is explained in the above analysis by assuming that there is one and only one constraint system governing accent. Thus, the fact that the accentual default is the same in derived and underived words follows from the assumption that derived and underived words are governed by the same grammar, i.e., the same language particular ranking of constraints. In Japanese, therefore, the ranking DEPPROM $\gg$ HEADEDNESS(Ft) holds in both morphological contexts, and as a result, unaccented words (either underlyingly or as an effect of Anti-Faithfulness) remain unaccented at the surface. If, on the other hand, this ranking of constraints was permuted in the analysis of the different word types, this result does not obtain.

Lastly, a final important property of dominant morphemes in Japanese is that they are always base-mutating. Thus, there are dominant suffixes which induce deletion of a stem accent, but no analogous dominant roots or stems. Concretely, what the latter state of affairs would entail is a dominant/recessive distinction which actually cross-classifies roots or stems, e.g., some roots steal an accent from a neighboring affix (even a dominant affix), while others do not. Contrast this type of idiosyncratic dominance with the systematic dominance of roots observed elsewhere in the system. This observation is, in fact, a very general one, as I will argue in some detail in \$5.2.4. Thus, Russian has a dominant/recessive contrast for suffixes, but not for roots. Likewise, in Spokane (Interior Salish), certain types of suffixes may be dominant or not, but roots do not show this contrast. This fact lends strong support to the overall approach here because on the analysis that dominance effects are due to Transderivational Anti-Faithfulness constraints, the facts could not be otherwise. The up-shot is that the fact that dominant morphemes are always base-mutating is explained as a Strict Base Mutation effect, which applies to all morpho-phonological operations.

We have seen how the TAF theory of dominance effects accounts for the observed properties of dominant morphemes in Tokyo Japanese. Furthermore, a peculiarity of this morpho-phonological system, namely that dominant unaccented affixes create unaccented words, is consistent with a finding in the larger phonological system, as unaccented words are the accentual default in bare substantives. This same correlation between default patterns in derived and underived words is also found in Modern Russian; as further support for the basic approach taken here, the next subsection studies the phenomenon of dominant affixes in this language. 


\subsubsection{Case Study: Dominance Effects in Russian}

To begin with a recap of the basic facts of Russian stress, accent is root-controlled in Russian, which means that underived words with inherently accented stems have fixed stem stress throughout the paradigm, as illustrated in (19a). If the word has an unaccented stem, on the other hand, the canonical pattern is fixed stress on the first vowel of the inflectional ending, as in (19b). Also, when the inflection is null, words with unaccented stems receive stem-final stress, as illustrated with the nominative singular form in (19c).

(19) Basic Patterns of Russian Stress

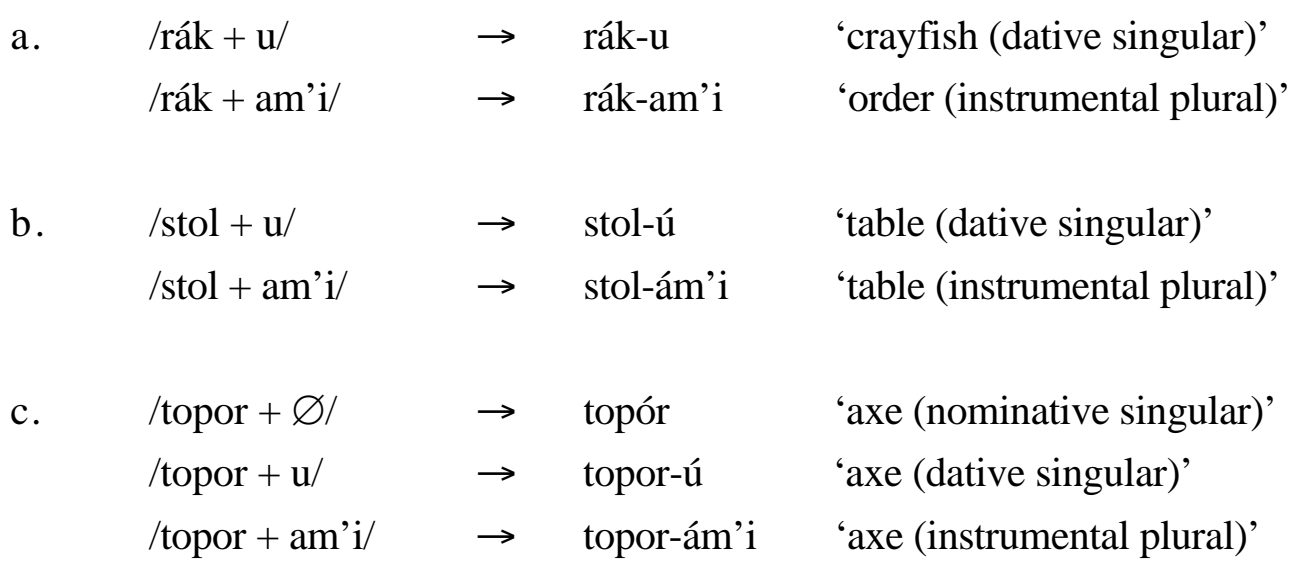

The analysis of these patterns given in $\S 3.2$ is characterized with the following ranking.

(20) Root-Controlled Accent in Russian

MAX-PROMRoot $_{\text {P POST-STEM-PROM }>\text { PAX-PROMAffix }}$

With MAX-PROMRoot top-ranked, the accentuation of the basic constituent of the stem, namely the root, determines the stress patterns observed in paradigms. Thus, if a form has an accented root, then MAX-PROMRoot requires realization of the lexical accent in all words with that root. In words with unaccented roots, in contrast, MAX-PROMRoot has no force, and therefore the next highest constraint in the hierarchy, POST-STEM-PROM, which specifically requires stress on the first vowel of the inflectional ending, yields default ending stress. Furthermore, this constraint has a role in mappings like /topor $+\varnothing / \rightarrow$ topór, where the absence of an overt ending leads to stem-final stress as a 'best attempt' at satisfying gradient POST-STEM-PROM. ${ }^{29}$

Extending the discussion now to derived words, one finds that certain suffixes require special attention because they run counter to the pattern of root-controlled accent just described. I will argue, however, that these special suffixes are dominant suffixes in the sense that they are sensitive to high-ranking TAF constraints; by incorporating the schematic rankings for dominance effects employed above into the constraint system for root-controlled accent, the properties of dominant suffixes in Russian can be explained in a natural way. Much of the data and analytical insights into word derivation in Russian

${ }^{29}$ This brief sketch of stress in paradigms does not account for the mobile stress patterns, but as argued in $\S 3.2$, these minor patterns are best understood in connection with constraints which require an opposition between singular and plural forms; the Prosodic Anti-Faithfulness constraints employed here make this connection in the analysis developed below. 
discussed here derive from two key works on the subject, namely Halle 1973a and Melvold 1990. I depart from these works, however, concerning certain underlying analytical assumptions which are very much a part of their descriptions, especially Melvold's characterization of the accented/unaccented contrast in dominant suffixes. These empirical issues will be made clear in the analysis presented below.

The first set of suffixes to be examined are recessive in the same sense as discussed above in connection with the inflectional endings. The behavior of recessive suffixes is exemplified below in words derived with the feminine noun-forming suffix -ic , which are compared with an underived form to determine the accentedness of the stem. This suffix is unstressed when it attaches to an inherent accented stem (21a), but realizes its inherent accent when it combines with an unaccented stem (21b). This behavior is further supported in (22) with nouns derived from adjectives (the feminine plural predicative, or "short", form of the adjective is given here), where we again observe stem stress with an accented stem (22a), otherwise suffix stress (22b).

(21) Diminutives with Recessive Suffix -íc
a. lúž -a
rý b-a
lúž -ic-a
rý b-ic-a
'puddle (diminutive)'
b. $\quad \check{c}$ ast'
č ast'-íc-a
vešč -íc-a
'particle'
'thing (diminutive)'

(22) Derived Nouns, with Recessive Suffix -ic
a. leníva, lenívy
lenív-ic-a
'lazy/lazy woman'
b. golá, góly
molodá, mólody
gol-íc-a
molod-íc-a
tupá, túpy
tup-íc-a
'naked/unlined leather mitten' 'young/married peasant woman' 'dull/dimwit'

The augmentative suffix -i $\check{s} \check{c}$ behaves similarly, in that it does not affect inherent accent on a stem, e.g., kníz -ǐ̌č - a 'book (augmentative)', cf. kníga 'book', but with an unaccented stem, it receives stress, as in gor- $\check{\imath} \check{S} \check{c}$ - $a$ 'big mountain', from gorá 'mountain'. An important empirical point here is that not all recessive suffixes are themselves accented. For example, Melvold 1990: $50 \mathrm{ff}$. argues in detail that the suffix -ost', which forms abstract nouns from adjectives, is recessive and unaccented. This fact shows that stemforming suffixes actually support a contrast in accentedness. To sum up then, derivational suffixes in Russian may be either accented or unaccented, but these suffixes behave like most of the inflectional suffixes in not causing deletion of the stem accent.

The behavior of the recessive suffixes contrasts with the behavior of the dominant suffixes, so-called because they dictate a particular stress pattern in all the words that contain them. The dominant suffixes in Russian are of two basic types: those which are themselves stressed, or 'auto-stressing', and those which require stress on the following vowel of the inflectional ending, hence the term 'post-accenting'. The first type is exemplified below with words formed with the derivational suffix -úx, which typically forms nouns from verbs or adjectives. 
(23) Auto-Stressed Dominant Suffix -úx

a. Accented Stem + úx

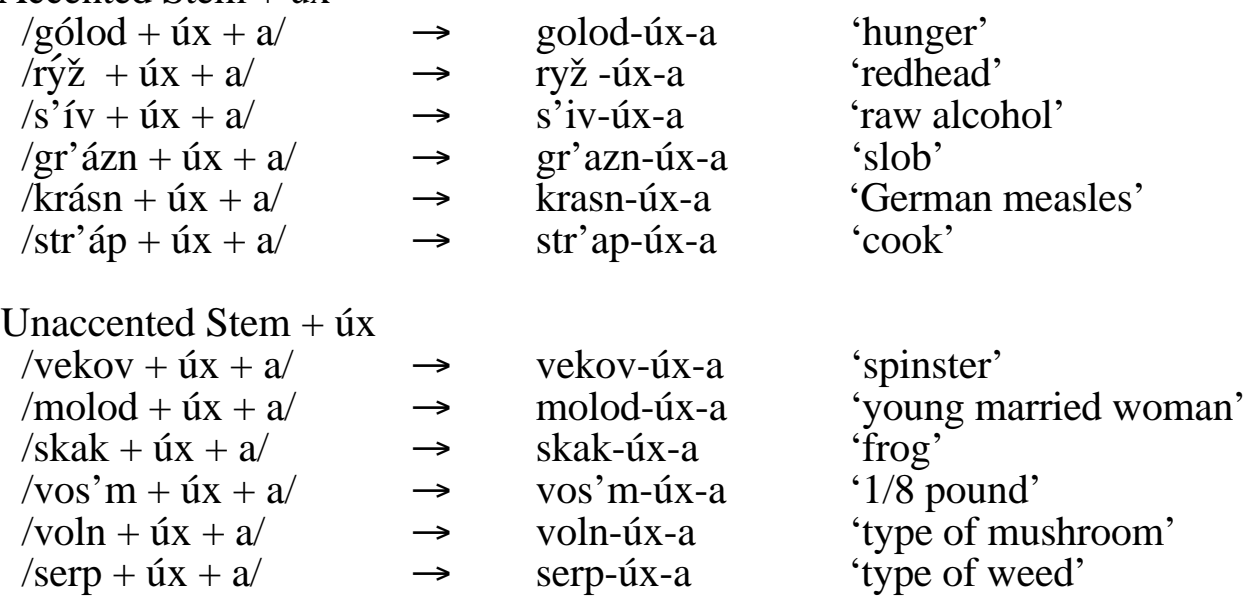

As with dominant accented -ppó in Tokyo Japanese, all words with auto-stressing -úx have stress on this suffix, regardless of the accentedness of the base to which it attaches. Other suffixes showing this behavior include the suffix -án, which creates nouns denoting a type of person (cf. -ánin, used in describing a person's nationality), and the suffix -jág. Lastly, the prefectivizing prefix $v i$ - 'out' is also dominant and accented as it causes deletion of the stem accent and surfaces with stress (see $\$ 3.2 .3$ for discussion); thus, it patterns with the above noun-forming suffixes.

The behavior of the auto-stressing suffixes contrasts with that of the post-accenting suffixes, such as the suffix - $a \check{c}$, which generally forms masculine nouns denoting a type of person. As a post-accenting suffix, $-a \check{c}$ requires a stress on the following inflectional ending, as illustrated with the examples below. In these examples, I distinguish between the so-called 'oxytone' (class 1 unaccented) and 'circumflex' stems (class 2 unaccented) to show that the dominance effect with consistent ending stress illustrated here is independent of this division employed in the traditional three-way classification of stems (in which 'oxytone' stems also give ending stress).

(24) Post-Accenting Dominant Suffix -ač

a. Accented Stem + ač

$\begin{array}{llll}/ \text { púz }+ \text { ač }+u / & \rightarrow & \text { puz-ač }-u ́ & \text { 'man with paunch' } \\ / \text { rífm }+ \text { ač }+u / & \rightarrow & \text { rifm-ač -ú } & \text { 'poetaster' } \\ / \text { tr'úk }+ \text { ač }+u / & \rightarrow & \text { tr'uk-ač }-u ́ & \text { 'stuntman' }\end{array}$

b. Unaccented Stem (Class 1) + ač

$$
\begin{array}{llll}
/ \mathrm{izb}+\mathrm{ac}+\mathrm{u} / & \rightarrow & \text { izb-ač }-\mathrm{u} & \text { 'village librarian' } \\
/ \mathrm{trub}+\mathrm{ac}+\mathrm{u} / & \rightarrow & \text { trub-ač }-\mathrm{u} & \text { 'trumpeter' } \\
/ \mathrm{gorb}+\mathrm{ac}+\mathrm{u} / & \rightarrow & \text { gorb-ać }-\mathrm{u} & \text { 'hunchback' }
\end{array}
$$

c. Unaccented Stem (Class 2) + ač

$$
\begin{array}{llll}
/ \text { borod }+\mathrm{ač}+\mathrm{u} / & \rightarrow & \text { borod-ač }-\mathrm{u} & \text { 'man with beard' } \\
/ \mathrm{golov}+\mathrm{ač}+\mathrm{u} / & \rightarrow & \text { golov-ač }-\mathrm{u} & \text { 'man with big head' } \\
/ \mathrm{nos}+\mathrm{ac}+\mathrm{u} / & \rightarrow & \text { nos-ač }-\mathrm{u} & \text { 'man with big nose' }
\end{array}
$$


The mildly productive or unproductive suffixes -un (which forms masculine nouns), $e \check{z}$ (which forms masculine nouns), and - $a k$, -jak (which form diminutives) behave similarly, inducing deletion of the stem accent and consistent ending stress.

An important factual point, which will be directly relevant for the discussion of alternatives in \$5.2.5, is that, while most dominant suffixes are derivational, this is not always the case. For example, the plural suffix $-a$, often used in technical jargon, is a dominant suffix, as shown by the examples below formed with inherently accented stems, and yet this suffix is clearly inflectional.

$\begin{array}{ccll}\text { Dominant Nominative Plural Suffix - } a & \\ \text { /rukáv }+ \text { a/ } & \rightarrow & \text { rukav-á } & \text { 'sleeves' } \\ \text { /máster }+ \text { a/ } & \rightarrow & \text { master-á } & \text { 'foremen' } \\ \text { /jákor' }+ \text { a/ } & \rightarrow & \text { jakor'-á } & \text { 'anchors' } \\ \text { /obš lág + a/ } & \rightarrow & \text { obš lag-á } & \text { 'cuffs' } \\ \text { /ókrug }+ \text { a/ } & \rightarrow & \text { okrug-á } & \text { 'regions' } \\ \text { /stórož }+ \text { a/ } & \rightarrow & \text { storož -á } & \text { 'watchmen' }\end{array}$

That this suffix is dominant is further supported by the fact that stems which take both $-a$ and the standard first declension plural ending $-i$, have ending stress with $-a$ but stem stress with $-i$, as in prómysl- $i$ 'trade, business (nominative plural)', cf. promysl-á. Thus, the pattern of 'stem stress in the singular, ending stress in the plural' observed in words with $a$ cannot be attributed to a mobile stress pattern (i.e., 'pattern $C$ ' stress discussed in §3.2), because the standard forms have fixed stem stress. Moreover, I have suggested that pattern $\mathrm{C}$ mobile stress in $\S 3.2$ is in fact a dominance effect, arising out of a need to realize a morphological contrast between singular and plural case forms. If this analysis is indeed correct, then the standard plural endings also trigger a deletion, which further substantiates the claim that inflectional suffixes may be dominant. To sum up, dominant suffixes in Russian are of two types: auto-stressing, as in -úx, or post-accenting, as exemplified by $a \check{c}$. Furthermore, there is no correlation between dominant suffixes and derivational suffixes: there are recessive derivational suffixes, such as -ic, and there are inflectional dominant suffixes, as exemplified above by the plural ending $-a$.

In approaching the distinction between auto-stressed and post-accenting dominant affixes, it would appear that the most natural assumption would be to treat this contrast like the related pattern observed in Japanese, namely in terms of a contrast between accented and unaccented dominant suffixes, and this is the tack I will take here. ${ }^{30}$ Thus, autostressing suffixes such as -úx, are dominant accented, and thus, because they bring about a deletion of the stem accent, they are always themselves accented. In contrast, the postaccenting suffixes, such as $-a \check{c}$, are assumed to be unaccented, and they bring about default ending stress as a consequence of deletion.

The following chart summarizes the facts of derived and underived words in Russian, including the underlying analytical assumptions concerning the accentedness of dominant suffixes. As shown in (26a), accent in Russian is root-controlled. That is, the accentuation of the basic formative in underived words determines the accentuation of the larger word: words with accented roots have fixed stem stress, while words with

\footnotetext{
${ }^{30}$ This analytical classification differs somewhat from the one given in Melvold 1990, which distinguishes among three classes of dominant affixes: dominant accented, such as -úx, dominant post-accenting, as in $a \check{c}$, and dominant unaccented affixes, the only representative of the latter case being -En'. Since this last suffix is unproductive and all of the examples seem to involve deverbal nouns from affix-stressed or initialstressed stems (Robert Rothstein, personal communication), it is not clear that there is robust empirical evidence in support of this third class, and so I will ignore it in the present discussion.
} 
unaccented roots have default ending stress. Certain derivational suffixes, such as -ic, pattern with most of the inflectional suffixes in that they also lose to an accented stem in the competition for the unique word accent (26b). However, the dominant suffixes idiosyncratically trigger deletion of the stem accent, either allowing the suffix to realize its own inherent accent, as with -úx in (26c), or bringing about a default stress pattern, as exemplified with $-a \check{c}$ in (26d).

(26) Summary of the Russian Facts

a. Underived nouns without a lexical accent receive ending stress by default:

$$
\begin{array}{lll}
/ \mathrm{stol}+\mathrm{u} / & \rightarrow & \text { stol-ú } \\
/ \text { rák }+\mathrm{u} / & \rightarrow & \text { rák-u }
\end{array}
$$

b. Root accent precludes the realization of accent in a recessive suffix:

$$
\begin{array}{lll}
\text { /lúž }+ \text { íc }+\mathrm{a} / & \rightarrow & \text { lúž }-\mathrm{ic}-\mathrm{a} \\
\text { /c } a s t '+1 \mathrm{c}+\mathrm{a} / & \rightarrow & \text { č ast'-íc-a }
\end{array}
$$

c. Dominant accented (='auto-stressed') suffixes are always accented:

$$
\begin{array}{lll}
\text { /s'îv }+ \text { úx }+ \text { a/ } & \rightarrow & \text { s’iv-úx-a } \\
\text { /skak }+ \text { úx }+ \text { a/ } & \rightarrow & \text { skak-úx-a }
\end{array}
$$

d. Dominant unaccented (='post-accenting') suffixes create words with default ending stress:

$$
\begin{array}{lll}
/ \text { púz }+ \text { ač }+\mathrm{u} / & \rightarrow & \text { puz-ač }-u ́ \\
/ \text { borod }+ \text { ač } / & \rightarrow & \text { borod-ač }-u ́
\end{array}
$$

The analysis of this body of facts is the same as the analysis of the parallel set of observations in Tokyo Japanese given above, with an additional well-motivated assumption concerning the role of stems in the characterization of Prosodic Faithfulness (discussed below). The distinction between dominant and recessive accentual behavior is derived through the constraint rankings given in (27a). Also, the top-ranked TAF constraint must also outrank the IO-Prosodic Faithfulness constraint for roots (27b), as dominant suffixes can bring about a deletion of the root accent.

(27) Dominant/Recessive Distinction in Russian through Constraint Ranking

$$
\begin{aligned}
& \text { a. } \neg \mathrm{OO}_{\text {Dom }} \text {-MAX-PROM }>\text { OO-MAX-PROM }>>\mathrm{OO}_{\mathrm{Rec}^{-M} \text {-MX-PROM }} \\
& \text { b. } \neg \mathrm{OO}_{\text {Dom-MAX-PROM }}>\text { MAX-PROMRoot }
\end{aligned}
$$

The lexical entries shown in (28) indicate which suffixes are dominant, and hence sensitive

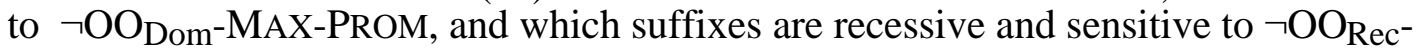
MAX-PROM. The distinction between auto-stressed and post-accenting dominant suffixes is encoded simply in terms of accentedness: auto-stressing suffixes are inherently accented, while the post-accenting suffixes are not. 
(28) Lexical Entries for Dominant and Recessive Suffixes ${ }^{31}$
-án $\quad \mathrm{N}_{\text {Masc }}$
-jág
$\mathrm{N}$
a. -úx $\mathrm{N}$
[ [ StemoODom _ _ ]Stem
[ [ StemoODom _ _ ]Stem
[ [ Adj/VerboODom _ _]Stem
b. $-a \check{c}$
-un $\quad \mathrm{N}_{\text {Masc }}$
$-a \quad$ NPlural
$-e \check{z} \quad \mathrm{~N}_{\text {Masc }}$
$\mathrm{N}_{\text {Masc }}$
[ [ N/V/AdjooDom ]Stem
[Dominant Unaccented]
[ [ VerboODom _ _ ] $]_{\text {Stem }}$
[ [ Noun OODom ] Stem _ ] ]
[ [ VerboODom _ _ ] $]$ Stem
c. $-i c$
$\mathrm{N}_{\text {Fem }}$
[ [N/AdjoORec ___ ] $]_{\text {Stem }}$
[Recessive Accented]
$-i \tilde{s} \check{c}$
$\mathrm{N}_{\mathrm{Fem} / \mathrm{Neut}}$
[ [N/AdjOORec ___ ]
[Dominant Accented]

With these lexical distinctions, the contrast in phonological behavior may now be modelled in terms of the rank of the relevant Anti-Faithfulness constraint, as I will illustrate below.

Before presenting the results, however, it is necessary to consider the accentual contrast found in stem-forming suffixes, i.e., the derivational suffixes examined above, as it is not clear at present which constraints account for the Faithfulness properties of these affixes. The Root Faithfulness constraints have no role here; derivational suffixes are not roots (at least not synchronically), and so the realization of inherent accent in these suffixes is not governed by MAX-PROMRoot. Furthermore, I claim in $\$ 3.2$ that the inflectional endings do not support a positional contrast in inherent accent, which is derived by the ranking POST-STEM-PROM > MAX-PROM ${ }_{\text {Affix }}$; thus, the accentual contrast found in the stem-forming suffixes cannot be due to low ranking MAX-PROMAffix because this ranking would neutralize the observed contrast. Furthermore, we cannot re-rank these constraints,

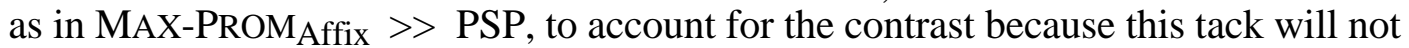
account for the fact that the derivational suffixes win out over (possibly) accented endings: these suffixes are of equal status by Affix Faith, which incorrectly gives e.g., /č ast' + íc + á/ $\rightarrow * c ̌ c$ ast'-ic-á, by low-ranking PSP.

Rather than posing an intractable formal problem, however, the presence of an accentual contrast exclusively in the stem-forming suffixes provides the crucial evidence for a different set of Positional Faithfulness constraints anticipated in chapter 2, section 2. In particular, the presence of a contrast in stem-internal suffixes shows that MAX-PROM for stems is sufficiently high-ranking in the grammar, as shown in the expanded ranking below. ${ }^{32}$

(29) Prosodic Faithfulness for Roots and Stems in Russian

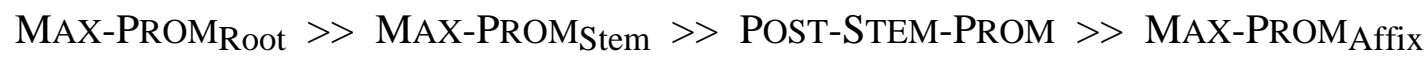

\footnotetext{
${ }^{31}$ The various morphological restrictions encoded in these entries are based on the characterization of these suffixes given in Townsend 1975, but nothing crucial depends on these restrictions.

${ }^{32} \mathrm{An}$ alternative approach is to endow the derivational suffixes themselves with special Faithfulness properties, as in Revithiadou 1997. While fully consistent with the facts of Russian, I adopt an analysis in terms of Stem Faithfulness because of the clear parallels between this notion and Root Faithfulness.
} 
With the appropriate Stem Faithfulness constraint correctly ranked, the analysis accounts for the observed 'chain of command' in the resolution of the competition for the unique word accent: a root accent beats out a (non-root) stem accent, and a stem accent (potentially outside the root), can cause non-ending stress. This result is illustrated in the following two tableaux.

First, when an accented derivational suffix such as -ic attaches to an accented root, the inherent accent in the root is realized: to do otherwise would result in a fatal violation of MAX-PROMRoot.

(30) Root-Controlled Accent in Derived Nouns

\begin{tabular}{|c|c|c|c|}
\hline /[ lúž + íc ] + a/ & MAX-PMRoot & MAX-PMStem & POST-STEM-PROM \\
\hline [luž -ic]-á & $* !$ & $* *$ & \\
\hline b. $\quad[$ luž -íc $]-\mathrm{a}$ & $* !$ & $*$ & $*$ \\
\hline c. $\rightarrow[$ lúž -ic $]-\mathrm{a}$ & & & $*$ \\
\hline
\end{tabular}

However, when the accented stem-internal suffix combines with an unaccented root, it may realize its inherent accent, despite the violation of POST-STEM-PROM this candidate incurs.

(31) A Role for Stem Faithfulness in Derived Nouns

\begin{tabular}{|l|c|c|c|}
\hline$/[\check{c}$ ast' + íc $]+\mathrm{a} /$ & MAX-PMRoot & MAX-PMStem & POST-STEM-PROM \\
\hline \hline a. $\quad[\check{c}$ ast'-ic]-á & & $* !$ & \\
\hline b. $\rightarrow[\check{c}$ ast'-íc]-a & & & $*$ \\
\hline
\end{tabular}

The presence of an accentual contrast in derivational suffixes differs from the behavior of the inflectional suffixes, which do not support a contrast; this observation motivates the introduction of an additional Positional Faithfulness constraint for stems. Below, we will see an additional role for MAX-PROMStem in the analysis of the dominant accented suffix $u x$, which is also stem-forming.

Returning now to the matters at hand, -ic is a recessive suffix, and so it does not bring about a deletion of the base accent, even as a means of satisfying the TAF constraint $\neg \mathrm{OO}_{\mathrm{Rec}}$-MAX-PROM. This pattern follows from the assumption that -ic subcategorizes for $\mathrm{OO}_{\mathrm{Rec}}$-correspondence, and so OO-MAX-PROM dominates the TAF constraint which evaluates words with this suffix. ${ }^{33}$

(32) Lack of Dominance Effect with -ic

\begin{tabular}{|c|c|c|c|c|}
\hline Base & /lúž + íc $+\mathrm{a} /$ & OO-MAX-PM & ᄀOORec-MAX-PM & PSP \\
\hline \hline a. $\quad$ lúž $-\mathrm{u}$ & {$[$ luž -ic]-á } & $* !$ & & \\
\hline b. $\rightarrow$ lúž -u & {$[$ lúž -ic]-a } & & $*$ & $* *$ \\
\hline
\end{tabular}

\footnotetext{
${ }^{33}$ The base for the derived word here is assumed to be the dative singular, but any of the case forms would yield the same result because stress is fixed throughout the paradigm when the stem is inherently accented; thus, there will always be a stem accent to mutate or be faithful to.
} 
The winning candidate is thus the form which preserves the prosody of the base in the derived form, even though doing so results in a violation of low ranking $\neg \mathrm{OO}_{\mathrm{Rec}}$-MAXPROM.

This recessive behavior is to be contrasted with the pattern brought about by the dominant accented suffix $-u x$. This suffix attaches to adjectival and verbal roots to form nouns, and so the base for OO-correspondence is the corresponding inflected adjective or verb. For concreteness, I assume that the base is the masculine singular form, which is consistent with the principle of Base Optimization if the masculine is the unmarked gender (as discussed in \$4.2, see also Halle 1973a). If the base is accented, as with the adjective $s$ ' $i v$ 'gray' below, this accent will not be preserved in the derived form because it will be deleted as a means of satisfying high-ranking $\neg \mathrm{OO}_{\text {Dom-MAX-PROM. The pattern }}$ resulting from this morphologically triggered de-accentuation is stress on the accented suffix because this is the pattern predicted by the grammar of accent. Specifically, because MAX-PROMStem dominates PSP, the suffix -úx must realize its inherent accent because failure to do so, for example to stress the inflection, leads to a fatal violation of MAXPROMStem; contrast (33b) with (33c).

(33) Dominance Effect with -úx

\begin{tabular}{|c|c|c|c|c|c|}
\hline Base & /s'ív + úx + a/ & $\neg \mathrm{OO}_{\text {Dom }}-\mathrm{MAX}-\mathrm{PM}$ & OO-MAX-PM & MAX-PMStm & PSP \\
\hline a. s'ív & [s'ív-ux]-a & $* !$ & & $* *$ & \\
\hline b. s'ív & [s'iv-ux]-á & & $*$ & $* * !$ & \\
\hline c. $\rightarrow$ s'ív & [s'iv-úx]-a & & $*$ & $*$ & $*$ \\
\hline
\end{tabular}

The same set of assumptions used for dominant accented -úx correctly predicts ending stress in words with dominant unaccented $-a \check{c}$. This suffix is like -úx in that it triggers $\mathrm{OO}_{\mathrm{Dom}}$-correspondence, and so $\neg \mathrm{OO}_{\mathrm{Dom}}-\mathrm{MAX}-\mathrm{PROM}$ evaluates words formed with $-a \check{c}$. In contrast to dominant accented suffixes, $-a \check{c}$ has no underlying accent, and so it does not exert any special Faithfulness privileges. When - $a \check{c}$ attaches to an accented root, therefore, it causes deletion of the root accent, but brings about a default accentual pattern, namely ending stress, as shown below.

(34) Dominance Effect with $-a \check{c}$

\begin{tabular}{|l|c|c|c|c|c|}
\hline Base & $\begin{array}{c}\text { /púz + ač }+ \\
\text { u/ }\end{array}$ & $\neg$ OODom-MAX-PM & OO-MAX-PM & MAX-PMStm & PSP \\
\hline \hline a. púz-u & [púz-ač ]-u & $* !$ & & & \\
\hline b. púz-u & {$[$ puz-áč ]-u } & & $*$ & $*$ & $* !$ \\
\hline c. $\rightarrow$ púz-u & {$[$ puz-ač ]-ú } & & $*$ & $*$ & \\
\hline
\end{tabular}

As with the analysis of dominant accented versus dominant unaccented affixes in Tokyo Japanese, both types of dominant suffixes cause a deletion of the stem accent. But dominant unaccented affixes also bring about a default accentual pattern because the result of the de-accented structure is determined by the larger grammar of accent.

The analysis of dominant suffixes in Russian presented above suggests a clear line of analysis for one of the patterns of mobile stress examined in §3.2. Recall from this discussion that there are two patterns of singular-plural opposition, repeated below. Thus, 
pattern $\mathrm{C}$ stress exhibits an opposition between initial stress in the singular and ending stress in the plural, while in pattern D, the contrast is between ending stress in the singular and stem-final stress in the plural. As noted in $\$ 3.2 .3$, these same patterns of mobile stress are also found in verb conjugations, and thus, the ultimate analysis of nouns will carry over to the verbal system as well.

(35) Stress Opposition between Singular and Plural
a. Pattern C
Singular
ó $\sigma \ldots-\mathrm{V}$
kólokol-u
Plural
$\sigma \sigma \ldots-\hat{V}$
kolokol-ám
b. Pattern D
Singular
Plural
$\ldots \sigma \sigma-\hat{V}$
kolbas-é

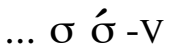
kolbás-am

Approaching these patterns in a pre-theoretical vein, the two patterns resemble quite closely two very common types of affix-controlled processes. First, pattern $\mathrm{C}$ looks like a dominance effect induced by the plural suffixes: the stem accent of the singular forms is deleted in the plural and default ending stress emerges. Russian has several derivational suffixes which show precisely this behavior, as we have just seen. Furthermore, the nominal plural ending - $a$ also fits this pattern: it deletes the accent of the stem (which may be lexical), and gives default stress on the inflectional ending. In short, there is a clear parallel between pattern $\mathrm{C}$ mobile stress and the independently attested dominance effects, and it seems wise therefore to establish this parallel in a formal way.

Moreover, pattern D stress also resembles a very common morpho-accentual process, namely pre-accentuation. The attachment of an affix often correlates with the insertion of an accent on a neighboring syllable, as we will see in several case studies discussed in chapter 5, section 3. The plural suffixes exhibit precisely this behavior in that they cause the insertion of an otherwise unmotivated accent on the stem-final syllable. It is therefore desirable, as with pattern $\mathrm{C}$, to approach this singular-plural opposition as this common morpho-accentual process; this is exactly the position I take here.

To give structure to the basic analysis, it appears that certain paradigms exhibit subregularities in the grammar, perhaps representative of a historically prior stage for the inflectional system, and these sub-regularities are dealt with as one of two patterns of AntiFaithfulness. Thus, stems showing pattern $\mathrm{C}$ stress have an inherent accent, which is realized in the singular (35a). Assuming that the singular forms are the base for the corresponding plural forms (which is predicted by the principle of Base Optimization, see $\S 4.2$ ), the stem accent will be deleted in the plural because the endings in these cases pattern with the plural ending $-a$, triggering a dominance effect (36a). More concretely, I assume that these stems select an allomorph of the same inflections used with other nouns, except this allomorph is a dominant suffix, and accordingly, triggers a deletion of the stem accent. The fact that plural forms with pattern $\mathrm{C}$ stress have ending stress therefore follows from the grammar dependent character of dominance effects: they receive a default stress pattern, which in Russian is ending stress. While the introduction of allomorphy brings additional complexity to the analysis, it appears to be unavoidable given the gross differences between the statistically important accentual patterns discussed in $\$ 3.2$ and the minor patterns showing mobile stress. Furthermore, the analysis sketch here brings formal rigor to the observation frequently made in the literature on Russian accent (see Stankiewicz 1962), namely that mobile stress in these cases is used as a means of enhancing the contrast between singular-plural pairs. The analysis of dominance effects as Anti-Faithfulness, and 
affix-controlled processes in general, entails that oppositions such as these are crucially linked to the morphology, requiring an opposition in base-output pairs.

The same pattern of allomorphy in the plural suffix is in effect in pattern D stress, except that a different TAF constraint is at work. Assuming again that the singular forms the base for OO-correspondence, the presence of ending stress in singular forms in (35b) is consistent with an analysis that such stems are unaccented and therefore receive default ending stress. As discussed above with pattern $\mathrm{C}$ stress, certain stems select an allomorph of the plural ending which is pre-accenting, and as a result, yield stem-final stress (36b). The analysis of pre-accentuation is developed in the next section and so the analysis can only be sketched informally here. But the parallels between pattern $\mathrm{C}$ and pattern $\mathrm{D}$ stress is striking: the main difference between $\mathrm{C}$ and $\mathrm{D}$ is that the analysis of $\mathrm{D}$ involves a ranking of a different TAF constraint, $\neg$ OO-DEP-PROM, which requires an insertion of accent into the stem. ${ }^{34}$ The fact that the inserted accent in plural forms is on the stem-final syllable is again a matter of grammar dependence: the inserted stress must appear on the stem, and the independently motivated constraint POST-STEM-PROM requires that the stress appear as close as possible to post-stem vowel, exactly on par with nouns with null inflections.

(36) Singular-Plural Oppositions as Anti-Faithfulness
Base
Derivative
a. Pattern C kólokol-u kolokol-ám = Dominance Effect by $\neg$ OO-MAX-PROM
b. Pattern D kolbas-é kolbás-am = Pre-Accentuation by $\neg$ OO-DEP-PROM

Thus, while the formal details of Anti-Faithfulness in the singular-plural pairs involve some additional complexities, it is clear that the same basic ideas used in the analysis of affixcontrolled accentual processes will apply here as well.

To summarize the analysis, stress in Russian, both in derived and underived nouns, is governed by the following set of constraint rankings.

(37) Summary Ranking for Stress in Russian

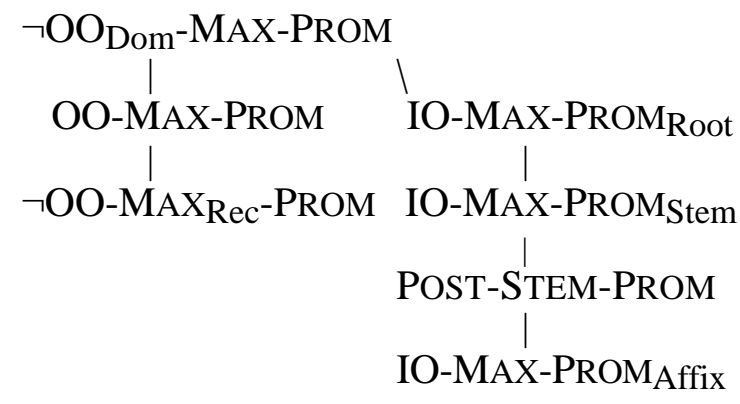

The top-ranking TAF constraint $\neg \mathrm{OO}_{\text {Dom }}$-MAX-PROM here assesses words which are lexically marked for the correspondence relation $\mathrm{OO}_{\mathrm{Dom}}$; as a result of its top-ranked

\footnotetext{
${ }^{34}$ Presumably the absence of pre-accentuation (=pattern D) with inherently accented stems is a consequence of root-control, which is again independently motivated in Russian. If an accented stem selects a preaccenting allomorph then the absence of pre-accentuation can be analyzed on a par with the same pattern in Cupeño: insertion of a non-lexical accent into the base leads to deletion of the stem accent, and hence a fatal violation of Root Faithfulness (see $\$ 2.4$ and $\$ 5.3 .2$ for discussion of pre-accentuation in Cupeño).
} 
position, this constraint can bring about deletion of a base accent, even if the base accent is lexically sponsored by a root because of the domination of IO-MAX-PROMRoot. Affixes which trigger $\mathrm{OO}_{\mathrm{Rec}}$-correspondence, on the other hand, are governed by $\neg \mathrm{OO}_{\mathrm{Rec}}$-MAXPROM, and because this constraint is dominated by the OO-Prosodic Faithfulness constraint, such suffixes do not condition deletion. Moving to the results established in the IO-dimension of Faithfulness, the column of constraints on the right describes the observed rank order in the Faithfulness properties of different morpheme classes in Russian. Thus, a root accent wins out over a non-root stem accent, which are both superordinate to an accent in an inflectional ending. Finally, words which are completely unaccented (either underlyingly or through de-accentuation) receive default ending stress as a consequence of the rank of POST-STEM-PROM, which prescribes stress on the first vowel of the inflectional ending.

To assess the larger set of results established here, TAF theory gives us the right set of tools for explaining grammar dependent dominance effects in Russian. Consistent with the analysis presented in the previous section for Japanese, the dominant/recessive distinction in affixes is derived through subcategorization in the lexicon, thus accounting for the lexically idiosyncratic nature of the dominant morphology. Also, dominance effects here have a morphological role; they bring about a morphological contrast between basederivative pairs that is exclusively base-mutating. This result again follows from the transderivational nature of the Anti-Faithfulness constraints. Finally, dominance effects in Russian are also like dominance effects in Japanese in that they bring about a default accentual pattern. This fact was explained in the analysis above by employing the independently necessary constraint, POST-STEM-PROM. This finding is significant because it lends strong support for the TAF theory of dominance effects developed here, and it further supports the use of this constraint in the analysis of words with no underlying accent.

\subsubsection{Implications}

The TAF theory of dominance effects proposed here makes a number of restrictive claims, which, as we will see in the next subsection, distinguish this theory from the previous approaches. Among these claims, two predictions stand out. First, TAF theory predicts that dominance effects are grammar dependent, which roughly speaking means that the constraint $\neg$ OO-MAX-PROM demands a deletion of base prosody, but the rest of the grammar determines the accentuation of the de-accented structure. Second, TAF theory, because of its transderivational character, predicts that dominance effects must be basemutating. In other words, there are affixes which trigger deletion of base prosody, but no bases, e.g., roots or stems, which idiosyncratically induce a deletion in the affixes with which combine. The first question one may ask therefore in assessing the TAF theory of dominance effects is, how does the theory hold up cross-linguistically? I will attempt to answer this question by examining a larger set of languages with the predictions of TAF in mind.

Before proceeding, however, a word of caution is in order. At this point, we have examined the accentuation of derived and underived words in two languages, Modern Russian and Tokyo Japanese, and a thorough study of these systems has permitted a conclusion concerning the properties of the dominant morphemes. It is incumbent on the researcher, however, to have an analysis of the larger system before making a conclusion about dominance effects in the various corners of the morphology, and I do not propose to do so in the present discussion for other languages. Thus, while I am confident about the conclusions I have made concerning accent in Russian and Japanese, the conclusions for 
the languages discussed below will have to be of a more speculative nature, as the present work does not bring all of these systems under the scrutiny of a rigorous formal analysis.

Having made this disclaimer, let us focus on the question of grammar dependent dominance effects. In discussions of dominance effects, it is sometimes noted that dominant (unaccented) affixes may bring about a default or unmarked pattern (see especially HV and Revithiadou 1997). Chapter 4, section 2 gives a characterization of grammar dependence in morpho-phonological alternations generally, but how does this apply to dominance effects? For the present purposes, we can be satisfied with the following statement: dominance effects are grammar dependent if the behavior of dominant unaccented affixes is mirrored in the language as a whole in lexically unaccented words, i.e., words which do not have an inherent accent. With this correlation in mind, consider the following chart which surveys some well-known accent systems.

(38) Grammar Dependent Dominance Effects

\begin{tabular}{|c|c|c|c|}
\hline & Language & Behavior of $[+$ dom, - acc $]$ affix & Behavior of $[-\mathrm{acc}]$ words \\
\hline a. & Japanese & unaccented & unaccented \\
\hline b. & Russian & ending & ending \\
\hline c. & Lithuanian & initial accent & initial accent (stem-final) \\
\hline d. & Sanskrit & initial accent & initial accent (?) \\
\hline e. & Getxo Basque & unaccented & unaccented \\
\hline f. & Moses Columbia Salish & accent on root & accent on root \\
\hline
\end{tabular}

We have already seen in Japanese and Russian that the default behavior of unaccented words is reproduced in dominance effects. Enlarging our empirical survey, the Indo-European languages Lithuanian and Sanskrit seem to by and large pattern with Japanese and Russian. HV's account of Lithuanian assigns default initial stress in both unaccented words and words created by dominant unaccented affixes. Furthermore, in Blevins 1993, a thorough review of the various accentual classes in this language turns up a similar default pattern, with the possible exception of a stem-final default in some stem classes (compare Blevin's tonal analysis of the Basic Accentuation Principle with her HTone Association rule which docks a floating high tone to the stem-final syllable). In general, however, it appears that the behavior of the dominant unaccented affixes is mirrored in most unaccented words, with the possible qualification that some accentual classes seem to have a different accentual default (which appears to have different defaults depending on accentual class). As for Sanskrit, HV characterize this system on a par with Lithuanian in positing default initial accent in both derived and underived unaccented words. ${ }^{35}$ Thus, in two additional Indo-European languages, the dominant unaccented affixes also bring about a default pattern.

Turning next to some non-Indo-European languages, Getxo Basque presents the same fundamental pattern as observed in Japanese: unaccented words are unaccented by

\footnotetext{
${ }^{35}$ As noted in Poser 1984: 67, however, the status of initial-accenting processes in pitch accent systems (and by extension, descendants of these systems, as in the case with these Indo-European languages) is controversial, and Poser cites Kiparsky 1982c as re-analyzing initial-accenting processes in Sanskrit as a byproduct of other necessary rules in Vedic. Thus, while there are some previous analyses which have analyzed dominance effects in Sanskrit as a grammar dependent morpho-accentual process, there is a possible alternative here that should be explored in more detail.
} 
default, and dominant unaccented suffixes create fully unaccented words (Hualde \& Bilbao 1992, 1993). Indeed, Hualde \& Bilbao 1993 present an analysis of de-accentuation which is fully consistent with the analysis I have presented above for Japanese (though the formal implementation is completely different). A final case are the dominant unaccented affixes found in Moses-Columbia Salish, which according to Czaykowska-Higgins 1993: $235 \mathrm{ff}$., yield words with accent on the root. This fact is of some interest because root accent is also the pattern observed in words with so-called weak roots and no following accented or dominant suffixes. While the analysis of this fact is a complex and interesting formal problem, the correlation here between default structures with weak roots and the default triggered by dominant unaccented suffixes is consistent with all of the above cases if we analyze the weak roots as unaccented, as proposed in Idsardi 1992: 68. Concretely, if weak roots are unaccented they will trigger a process of default accent assignment, in effect drawing accent to the root as observed.

The conclusion that I draw from this brief survey is that dominance effects are quite generally grammar dependent. That is, the independently necessary constraints on the distribution of accent play a crucial role in the accentuation of morphologically de-accented structures. This finding is strong support for the overall approach taken here because the operative constraint responsible for the deletion of base prosody, $\neg$ OO-MAX-PROM, says nothing about the structures resulting from the deletion. This TAF constraint simply requires a deletion of the base accent, and the rest of the grammar predicts the observed accentual default structure. When coupled with the assumption that derived words are subjected to the same grammar which is at work in the larger system, TAF theory directly explains the correlations observed above. As we will see in the discussion of alternatives, this result distinguishes the TAF theory of dominance effects from other plausible approaches to the problem.

A second important prediction of the TAF model is that dominance effects are always base-mutating. Thus, the Thesis of Strict Base Mutation (given in §4.3) entails that morpho-phonological alternations only affect the base of affixation. Since dominance effects are just a special type of morpho-phonological alternation, this claim makes a concrete prediction, namely that base elements, such as roots and stems, never support a dominant/recessive contrast like that found in Japanese and Russian affixes. In other words, roots or stems will never idiosyncratically steal an accent from a neighboring affix, and so dominance is not a feature which distinguishes one base from another one, as shown below with a hypothetical case.

(39) Dominant/Recessive Contrast in Base

$$
\begin{array}{llll}
\text { a. } / \text { róot }_{\operatorname{Rec}}+\text { áf }_{\operatorname{Red}} / & \rightarrow & {[\text { róot-af }]} & =\text { Effect of Root-Controlled Accent } \\
\text { b. } / \text { róot }_{\text {Rec }}+\text { áf }_{\text {Dom }} / & \rightarrow & {[\text { root-áf }]} & =\text { Dominance effect due to áf } \\
\text { c. } / \text { róot }_{\text {Dom }}+\text { áf } &
\end{array}
$$

If neither of the morphemes are dominant, as in (39a), the result is overriding root accent, consistent with many case studies in chapters 2 and 3. In (39b-c) however, the affix is dominant, and therefore the properties of the base become crucial. If roots have a dominant/recessive contrast, we would except to find a contrast here: a recessive root loses to a dominant affix (39b), while a dominant root will (potentially) win out over a dominant affix (39c). The assumption in the last case is that the two dominant morphemes are of equal 'strength', and therefore the root stress wins as a consequence of high-ranking Root Faithfulness (though other factors could be at work here as well). This type of root 
dominance should therefore be contrasted with intrinsic, or systematic, root dominance, as observed in Cupeño, because the property of overriding the accent in a dominant affix in (39c) is an idiosyncratic property of the root which distinguishes it from other roots.

Interestingly, Inkelas 1996 speculates that this type of root dominance is not attested cross-linguistically. That is, there are no accent systems which have a dominant/recessive contrast in roots like that described above. In support of this conclusion, consider the following list of languages which have a significant number of dominant morphemes.

(40) Base-Mutating Dominance Effects

\begin{tabular}{|c|c|c|}
\hline Language & Contrasts in Roots/Stems & Contrasts in Affixes \\
\hline a. Japanese & 土accent & \pm accent, \pm dominant \\
\hline b. Russian & 土accent & \pm accent, \pm dominant \\
\hline c. Lithuanian & 土accent & \pm accent, \pm dominant \\
\hline d. Sanskrit & 土accent & \pm accent, \pm dominant \\
\hline e. Interior Salish & \pm accent, $( \pm$ extrametricality) & $( \pm \text { accent },)^{36} \pm$ dominant \\
\hline
\end{tabular}

Remarkably, every system has a dominant/recessive contrast in affixes, but no parallel contrast in roots or stems. Thus, in every system, there is a two-way contrast in roots and stems, but a four-way contrast in affixes (subject to certain qualifications - see footnote 12).

A possible anomaly in this otherwise general trend is the presence of a contrast in the feature [ \pm extrametricality] documented by Czaykowska-Higgins 1993 for roots in Moses-Columbia Salish. In this language, certain roots may idiosyncratically make the following syllable ineligible for stress, even if the following suffix is dominant. While we have not yet seen a locality effect such as this on a dominant morpheme (though a parallel case is presented below for certain particles in Japanese), this fact could readily be treated as a dominant/recessive contrast in roots which is limited to an adjacent syllable. If such an analysis was the correct analysis, this case would surely counter-exemplify the strong claim that all dominance effects are base-mutating: dominant roots (i.e., Czaykowska-Higgins' [+extrametrical] roots), in this analysis, affect a non-root. Of course, facts such as this do not directly counter-exemplify this claim, as there are at least two possible alternative analyses that come to mind here. In addition to Czaykowska-Higgins' analysis in terms of extrametricality, Idsardi 1992: 70 suggests that this ineligibility for stress on the vowel following the root is due to a minor rule of syncope triggered by certain roots, an attractive idea given the intricate interplay between vowel deletion and stress assignment observed elsewhere in the language. I tentatively conclude therefore that, in all the languages in my survey, dominance effects are exclusively base-mutating. This finding will also feature in the comparison of alternatives discussed in the next subsection.

A third prediction, anticipated in $\S 5.1$, is that dominance effects may be subject to locality requirements. The reason for this is that dominance effects are, by hypothesis, derived as effects of Transderivational Anti-Faithfulness constraints; such constraints can be freely conjoined with Anchoring constraints under certain locality relations, which have

\footnotetext{
${ }^{36}$ My reading of Carlson 1989 and Bates \& Carlson 1989 does not demand a contrast in accentedness in variable and dominant suffixes, as they could be exclusively accented, but Czaykowska-Higgins 1993 and Idsardi 1992 both distinguish between accented and unaccented suffixes.
} 
the effect of limiting the scope of the Anti-Faithfulness constraint. Indeed, as we will see in $\$ 5.3$ and $§ 5.4$, this application of Local Conjunction plays a crucial role in describing locality effects in other affix-controlled accentual processes. How then do dominance effects compare to these other types of ACA?

At present, I have not found a robust set of examples which argue definitively for locality conditions in dominance effects, but one clear case in Tokyo Japanese shows a role for such a locality condition. As discussed in detail in Poser 1984: §4.1., two morphemes in Japanese having the segmental make-up no trigger de-accentuation of a neighboring stem accent, namely the genitive enclitic no and the prenominal allomorph of the copula no. Poser argues convincingly that this de-accentuation is a post-lexical phenomenon, essentially because this process requires reference to phrasal information. The fact of relevance to the present discussion is that no only triggers de-accentuation when the accent of the preceding noun is on a final light syllable (subject to certain qualifications discussed below). Thus, contrast the genitive forms below in (41a) with those in (41b-c). In (41a), the nominal accent appears on a final light, and this accent is lost in the genitive form; in contrast, in cases with non-final accent (41b) or accent on a final heavy syllable (41c), the accent of the base is not lost. This descriptive statement is subject to the following qualifications: (i) there are some exceptions, e.g., tugí no in (41a), where the accent of the final light syllable is not deleted, but in these cases it appears that the accent has been shifted to this position, and so it represents an opaque context not described by the principle given above; (ii) some nouns actually lose an accent from a final heavy syllable, as in nihon no in (41c), which simply appear to be lexical exceptions; (iii) finally, monosyllabic nouns never lose their accent, as shown by the examples in (41d).

(41) De-Accentuation with no (Poser 1984, Haraguchi 1977, Okuda 1971)

a. Accent on final light syllable

$\begin{array}{llll}\text { /kawá }+ \text { no/ } & \rightarrow & \text { kawa no } & \text { 'river' } \\ \text { /atamá }+ \text { no/ } & \rightarrow & \text { atama no } & \text { 'head' } \\ \text { /oNná }+ \text { no/ } & \rightarrow & \text { oNna no } & \text { 'woman' } \\ \text { cf. /tugí }+ \text { no/ } & \rightarrow & \text { tugí no } & \text { 'patch' }\end{array}$

b. Accent on non-final syllable

$\begin{array}{llll}\text { /úmi }+ \text { no/ } & \rightarrow & \text { úmi no } & \text { 'sea' } \\ \text { /árasi }+ \text { no/ } & \rightarrow & \text { árasi no } & \text { 'storm' } \\ \text { /utíwa + no/ } & \rightarrow & \text { utíwa no } & \text { 'fan' } \\ \text { /irógami + no/ } & \rightarrow & \text { irógami no } & \text { 'colored paper' }\end{array}$

c. Accent on final heavy syllable

$\begin{array}{llll}\text { /ehóN + no/ } & \rightarrow & \text { ehón no } & \text { 'illustrated book' } \\ \text { /seNséi }+ \text { no/ } & \rightarrow & \text { seNséi no } & \text { 'teacher' } \\ \text { hukóo }+ \text { no/ } & \rightarrow & \text { hukóo no } & \text { 'misfortune' } \\ \text { cf. /nihón + no/ } & \rightarrow & \text { nihon no } & \text { 'Japan' }\end{array}$

d. Accented monosyllabic stem

$\begin{array}{llll}\text { /há }+ \text { no/ } & \rightarrow & \text { há no } & \text { 'teeth' } \\ \text { /kyóo }+ \text { no/ } & \rightarrow & \text { kyóo no } & \text { 'today' } \\ \text { /hón }+ \text { no/ } & \rightarrow & \text { hón no } & \text { 'book' }\end{array}$

To give a brief summary, while there are both lexical and systematic exceptions to the process, no generally triggers a loss of accent on the final mora of the noun with which it combines. The restriction on the final mora thus accounts for de-accentuation in nouns with accent on the final mora (i.e., in a final light syllable), while exempting nouns with 
non-final accent or accent in the final heavy syllable (but not the final mora of the heavy syllable because Japanese generally prohibits accent on the second mora of a heavy syllable - see $\S 3.3$ ).

To re-state the observation above in a more theoretical vein, no triggers a dominance effect on the immediately adjacent mora, which therefore presents a clear role for locality conditions in this context. Pressing further, TAF theory gives us the right equipment to account for this type of observation, as I will now sketch. When locally conjoined with the Anchoring constraint, ANCHOR(MWd, MinP, R), which enforces right edge matching between the morphological word and minor phrase, the TAF constraint will yield a pattern of Anti-Faithfulness only in the final mora of the word (see $\$ 4.3$ for details of the interpretation of Anchoring constraints and Local Conjunction). Thus, by locally conjoining these two constraints in the domain of the mora, as shown below, the obligatory deletion constraint $\neg$ OO-MAX-PROM will only be active in the neighborhood of the Anchoring violation, i.e., in the final mora of a word that is separated from the right edge of the minor phrase.

(42) ( $\neg \mathrm{OO}_{\text {Dom }}$-MAX-PROM \& ANCHOR(MWd, MinP, R $)_{\mu} \equiv \neg \mathrm{OO}_{\text {Dom }}$-MAX-PROMFin- $\mu$ ) If a prominence $x$ in the base stands in correspondence with a prominence $x$ ' in the related output, and if $x^{\prime}$ is associated with the mora which is final in MWd, delete $x^{\prime}$.

As illustrated in the complex tableau below, when no combines with a noun containing an accent on the final mora, as shown in (43a), the conjoined TAF constraint is active and conditions a dominance effect. When the accent of the base appears on a non-final mora, as in (43b) and (43c), the TAF constraint is not active because the accent falls outside of the scope of this constraint. Since deletion of the accent in these last two contexts would lead to a gratuitous violation of OO-Faithfulness, these genitive forms preserve the lexical accent.

(43) Locality Conditions on Dominance Effects for no

\begin{tabular}{|c|c|c|c|}
\hline Base & Derivative & $\neg \mathrm{OO}_{\text {Dom-MAX-PROM }}$ Fin- $\mu$ & OO-MAX-PROM \\
\hline /kawá/ & /kawá + no/ & & \\
\hline kawá & kawá no & $* !$ & \\
\hline$\rightarrow$ kawá & kawa no & & * \\
\hline b. /utíwa/ & /utíwa + no/ & & \\
\hline$\rightarrow$ utíwa & utíwa no & & \\
\hline utíwa & utiwa no & & $* !$ \\
\hline c. /hukóo/ & /hukóo + no/ & & \\
\hline$\rightarrow$ hukóo & hukóo no & & \\
\hline hukóo & hukoo no & & $* !$ \\
\hline
\end{tabular}

This analysis leaves monosyllabic genitive-marked nouns unaccounted for. However, once an additional well-motivated constraint ranking is brought into the picture, this fact will have a principled explanation as well. Following Beckman 1997 [1998], I assume that there is a set of Positional Faithfulness constraints which specifically targets root-initial syllables. In particular, Japanese has an IO-Prosodic Faithfulness constraint, $\sigma_{1}$-MAX-PROM, which prohibits the deletion of an accent in the initial syllable of a root. 
There is in fact additional evidence for such a constraint in Japanese, as the honorific prefix $o$ - generally deletes a root accent, unless the accent is in the initial syllable (Martin 1975, Higurashi 1983, cf. Poser 1984). While I will not delve into the ranking details which achieve this result, it is clear from these facts that a constraint which 'protects' the accent of the root-initial syllable is independently needed.

The exemption of monosyllabic nouns can be readily explained by ranking $\sigma_{1^{-}}$ MAX-PROM above $\neg$ OO-Dom-MAX-PROMFin- $\mu$, as shown below. Thus, in just these cases, where the accent is both on the final mora and in the initial syllable, the TAF constraint will carry no force because it is dominated by top-ranked $\sigma_{1}$-MAX-PROM.

(44) Positional Faithfulness Effect in Monosyllabic Nouns

\begin{tabular}{|c|c|c|c|c|}
\hline Base & /há + no/ & $\sigma_{1}$-MAX-PM & ᄀOO-Dom-MAX-PMFin- $\mu$ & OO-MAX-PM \\
\hline \hline a. $\rightarrow$ há & há no & & $*$ & \\
\hline b. há & ha no & $* !$ & & $*$ \\
\hline
\end{tabular}

The interim conclusion I will draw from this analysis is that dominance effects may indeed be subject to locality requirements, as such a requirement is in fact crucial in the analysis of no constructions in Tokyo Japanese. Furthermore, such requirements provide another reason for grouping dominance effects with other types of affix-controlled accentual phenomena which also show locality effects. As for the attestation of this type of effect in other systems, further examples are not forthcoming, but this apparent gap may in fact be due to the sample of languages in this thesis. Many of the languages studied here, e.g., Russian, Tahltan, Salish languages, show a strong preference for monosyllabic roots; thus, in underived stems, such locality restrictions would be decidedly hard to spot because of a lack of crucial evidence showing a distributional gap. Furthermore, among the languages with an abundance of longer roots, only Japanese has a robust list of dominant affixes (Getxo Basque has only a handful; Aguaruna only has one 'accent-attracting suffix'). I speculate therefore that a wider survey of languages, specifically including languages with longer roots and stems, will turn up some additional cases of interest here.

The TAF theory of dominance effects also makes predictions concerning the behavior of a sequence of affixes, dominant and/or recessive, and as this type of behavior has played a role in forming different theories of dominance effects (see Inkelas 1996 for a recent review), it is worthwhile considering the implications of TAF on this issue. I show below in $\$ 5.2 .5$ that TAF theory is very much on a par with HV's cyclic approach to sequences of dominant affixes. A fundamental notion in the TAF analysis of these sequences is Benua's 1997 [1998] notion of an 'extended paradigm' (see also Buckley 1995), which will be reviewed directly below before turning to multiple dominance effects.

In §4.2, Benua’s 1997 [1998] analysis of the behavior of class 1 versus class 2 suffixes in English stress was reviewed. The finding in this discussion was that class 1 suffixes were stress-shifting, and thus that the OO-Prosodic Faithfulness constraints sensitive to words with these suffixes are low-ranked in the grammar. This assumption accounts for the fact that a class 1 suffix like -al typically does not induce a preservation of the base prosody in words with this suffix, as shown below in the mapping from [(óri)gin] to [o(rígi)n-al]. However, the attachment of a second class 1 suffix, e.g., -ity, does correlate with a preservation of secondary stresses, as shown in the mapping from [o(rígi)n-al] to [o(rìgi)(n-ál-i)ty]. These doubly derived forms have an irregular pattern of secondary stress on a non-initial syllable (cf. Tàtamagóuchi), and this result is established in Transderivational Correspondence Theory (TCT) with the notion of an extended paradigm. As sketched below, an extended paradigm is composed of a set of 'sub- 
paradigms', i.e., base-output pairs of the usual type. To point out the concrete results in the mapping from [o(rígi)n-al] to [o(rìgi)(n-ál-i)ty], primary stress is not preserved here because -ity is a class 1 suffix and OO-Prosodic Faithfulness for this class, i.e. OO-PROSFAITHI, is ranked below the constraints responsible for positing main stress. However,

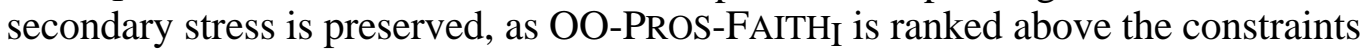
responsible for predicting secondary stress.

(45) An Extended Paradigm with Class 1 English Morphology (Benua 1997 [1998])

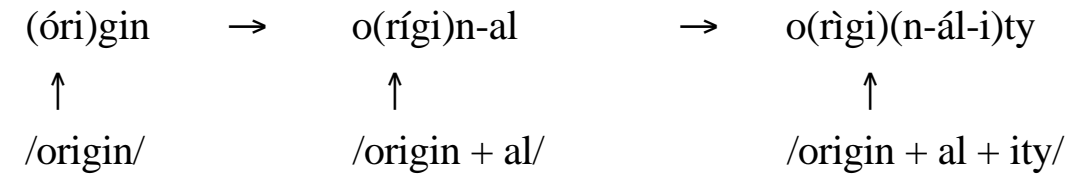

In general, the behavior of the larger word is dictated by the last affix attached in the string. The reason for this is that the type of OO-Faithfulness relating original and originality is determined by the subcategorization requirements of the affix attached at this recursion. Thus, stress is not preserved as a primary stress in the last leg of the extended paradigm here because -ity is a class 1 suffix, and OO-PROS-FAITH $H_{I}$ is not powerful enough to bring about Faithfulness to the primary stress. It is certainly the case, however, that internal affixes may have an influence on the prosody of the larger word, as indeed we have seen here with the preservation of the main stress of oríginal as a secondary stress in originálity. But the Faithfulness properties of the external affix are superimposed on the base established on an intermediate recursion, giving the effect that the external affix has the 'last crack' at enforcing a particular pattern.

The TAF theory of dominance effects is developed in TCT, and so the same basic principles at work in this theory apply to the analysis of dominant affixes as well. When a sequence of affixes are attached, the outermost affix will superimpose its Anti-Faithfulness properties onto the larger word. Thus, just as OO-Faithfulness relating original and originality is due to the subcategorization requirements of -ity, the type of OO-AntiFaithfulness applied in a doubly derived string is determined by the morphologically external affix. Innermost affixes may also condition a dominance effect, but this effect will be subordinated to the effect of an external dominant affix, as illustrated below. In the following set of schematic examples, all of the morphemes are inherently accented in order to make the 'power relations' among the subconstituents of the word more perspicuous, but the same patterns are also observed with dominant unaccented affixes. 
(46) Predictions for Sequences of Dominant/Recessive Affixes

a. Root + Recessive Affix + Recessive Affix: Root Controlled Accent

$$
\begin{array}{cccc}
\text { róot } & \rightarrow & \text { róot }+ \text { af } & \text { róot }+ \text { af }+ \text { af } \\
\uparrow & \uparrow & \uparrow \\
\text { / róot / } & & \text { / róot }+ \text { áf } & \\
\text { Rec } / & & \text { / róot }+ \text { áf } f_{\text {Rec }}+\text { áf } \\
\text { Rec }
\end{array}
$$

b. Root + Dominant Affix + Recessive Affix: Innermost Dominance Effect

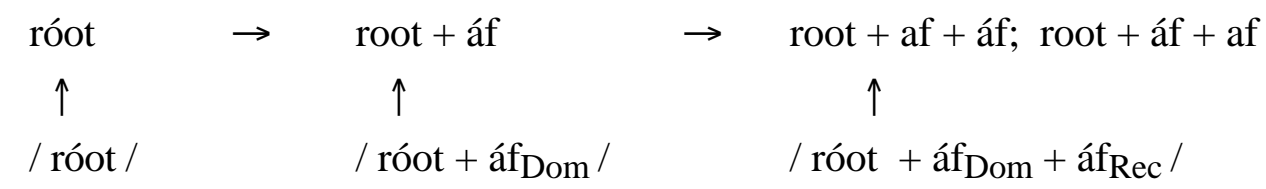

c. Root + Recessive Affix + Dominant Affix: Outermost Dominance Effect

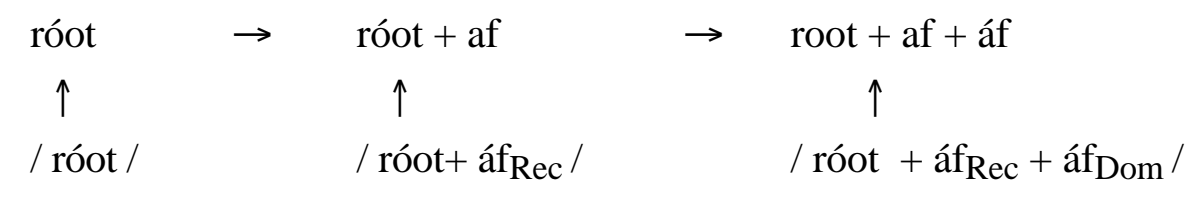

d. Root + Dominant Affix + Dominant Affix: Inner or Outermost Dominance effect

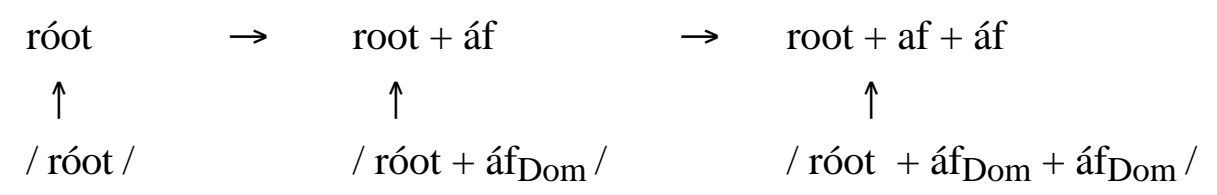

Starting in (46a) with the behavior of a sequence of recessive suffixes, this pattern is somewhat unremarkable in root-controlled accent systems. Because this word type has an accented root and no dominant affixes, the root accent always wins, in accordance with the Root-Controlled Accent Hypothesis. We have already encountered this type of behavior in Russian where the attachment of recessive -ic plus an inflection led to the deletion of affix accent with accented roots.

In the extended paradigm in (46b), the dominant affix in the first sub-paradigm brings about a deletion of the root accent, making possible two outcomes in the doubly derived word. Thus, the accent of the innermost affix or the accent of the outermost affix could be preserved; the choice here is not decided by the Anti-Faithfulness constraint, but rather the grammar as a whole. This is because the last affix is recessive, and therefore cannot cause a deletion of the base [root + áf] on its own. The predicted outcome is therefore a culminativity effect, governed by e.g., principles of edge orientation or morphologically sensitive Faithfulness constraints. The behavior of the dominant derivational suffixes $-u ́ x$ and $-a \check{c}$ followed by an inflection ending in Russian also pattern in this way (see the conflated tableaux in (33) and (34) above).

The extended paradigms in (46c) and (46d) are fundamentally the same in that the outermost dominant affix always dictates the observed pattern. Thus, regardless of whether the innermost affix is recessive (46c) or dominant (46d), the last affix attached causes a deletion of the base created in the first BO-mapping, resulting in an accent on the dominant affix if it is accented, as shown here, or in a default accent pattern if it is unaccented. The hypothetical patterns depicted here are consistent with the patterns described for English (Fudge 1984, Burzio 1994), Sanskrit (Halle \& Mohanan 1985, HV), and Spokane (with some interpretation of Bates \& Carlson 1989). Thus, it is clear that the 
TAF theory of dominance effects provides the right tools for describing this very common pattern in sequences of dominant and/or recessive affixes.

To summarize the larger results, while an internal dominant suffix may induce a deletion (46c), the general principle governing the behavior of sequences of suffixes is that the morphologically external affix generally dictates the accentuation of the larger word. If the external affix is recessive, then the grammar of accent is applied to yield the 'regular' accentual pattern, as in (46a) and (46b). If, on the other hand, the external affix is dominant, it causes a deletion of the accent in the complex stem, as in (46c) and (46d), and the emergence of affix accent or a default accentual pattern.

\subsubsection{Discussion of Alternatives}

Now that we have a better understanding of the predictions of the TAF theory of dominance effects, we may compare and contrast this theory to some previous approaches to the problem, and also explore some plausible alternatives that come to mind in an OT framework. In the review of the literature given below, two features of dominance effects separate the TAF theory from the alternative approaches. First, the property of grammar dependence, i.e., that the result of de-accentuation is predicted by the independently motivated grammar of accent, is critical in distinguishing the TAF analysis from its competitors. Equally important is the finding that dominance effects are base-mutating, as this feature too points to a liability of some of the alternative approaches.

An initially attractive theory of dominance effects appears in Prince 1983. Based on certain observations made in Kiparsky \& Halle 1977, Prince proposes that accented dominant morphemes involve a grid mark on the highest level of the metrical grid, as depicted below for the dominant accented suffix -úx in Russian. In this illustration, both of the lexically accented morphemes here project a grid mark at Level 2, but only the dominant suffix -úx projects an additional grid mark at the highest level, thereby accounting for its grid prominence in surface forms.

(47) Dominance Effects as Prominence on the Grid (Prince 1983)

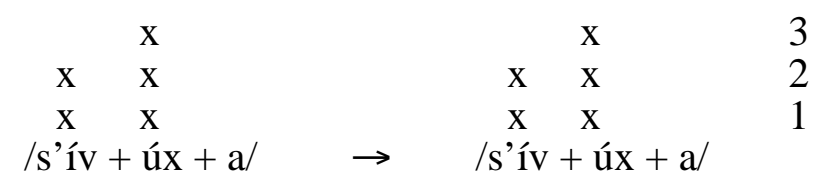

While this theory is attractive for its overall simplicity, a fundamental premise in this theory leads to two serious descriptive problems. As acknowledged by the author (p. 91), dominance is equated with accentedness because it is a lexical marking made with the same stuff that encodes accent. This theory runs into difficulty, therefore, in languages like Russian and Japanese which have a four-way contrast for affixes, i.e., a crossclassification for the properties dominant/recessive and accented/unaccented. Put differently, the Grid Prominence Theory of dominance effects treats dominance as a culminativity effect, but dominant unaccented affixes show that this is not the case (see $\S 4.1$ for explicit argumentation). Another problem with this theory is that, nothing else said, it does not account for Strict Base Mutation effects, i.e., the fact that dominance effects always affect the base of a morphological process. Succinctly, if dominance is purely equated with a lexical marking for prominence on the grid, why are roots not also lexically specified for this prominence, thereby putting a dominant accented root on a par 
with a dominant accented affix ? $^{37}$ In sum, the assumptions inherent to the Grid Prominence Theory confront serious empirical challenges when one looks to a wider range of cases. ${ }^{38}$

Another idea, which solves an important problem with the Grid Prominence Theory, is that dominance effects are linked to word derivation in a fundamental way. In particular, it is sometimes observed that there is a close correlation in some languages between dominant morphemes and derivational or category-changing morphemes (see e.g. Blevins 1993, fn 26). Revithiadou 1997 captures this correlation by endowing derivational affixes with special Faithfulness properties in an account of dominant accented affixes. Thus, building on the insights of Beckman 1997 [1998], Revithiadou proposes a set of Positional Faithfulness constraints for derivational affixes, or morphological 'heads' in the sense of Williams 1981. With the head-sensitive Faithfulness constraint top-ranked in the hierarchy, accented derivational affixes, such as Russian -úx $\mathrm{Hd}$, will realize their inherent accent over other competing morphemes, as illustrated below.

(48) Dominance Effects as Head Faithfulness (after Revithiadou 1997)

\begin{tabular}{|c|c|c|}
\hline$/$ s'ív + úx $_{\mathrm{Hd}}+\mathrm{a} /$ & PROS-FAITH $_{\text {Head }}$ & PROS-FAITH \\
\hline \hline s'ív-ux $\mathrm{Hd}_{\mathrm{H}} \mathrm{a}$ & $* !$ & \\
\hline$\rightarrow$ s'iv-úx & & $*$ \\
\hline
\end{tabular}

The proposal therefore accounts for one of the major problems with the Grid Prominence Theory, namely the observation that dominance effects are always base-mutating. If only derivational affixes are given special Faithfulness, only they will bring about dominance effects.

While this idea may be well-suited for some languages, the Head Faithfulness approach does not seem to provide a theoretical basis for a cross-linguistic theory of dominance effects. One initially troubling fact is that the correlation between dominance and derivational morphology captured in this theory simply does not hold true in all languages. Thus, as shown in the above case study of Russian, there are both dominant and recessive derivational suffixes, which leaves the recessive suffixes unaccounted for. Moreover, there are inflectional affixes which bring about dominance effects, as for example the plural ending - $a$ often used in Russian technical jargon, e.g., /máster + a/ $\rightarrow$ master-á 'foremen'. Finally, in the account of Hausa tonology given in Inkelas 1996, there are both derivational and inflectional dominant affixes, which presents a second case where the correlation between derivational and dominant affixes breaks down.

A more pressing problem for this theory, however, is the behavior of dominant unaccented affixes, which as shown in detail above, bring about a language particular

\footnotetext{
${ }^{37}$ Lexical specification for prominence in this theory is of course distinct for the intrinsic prominence typical of roots, as found in Cupeño. As discussed in \$5.2.4, roots systematically take precedence over affixes, which is distinct from lexically idiosyncratic prominence found with dominant accented morphemes. Thus, the inherent prominence of root accent is not the type of prominence which is predicted by the Grid Prominence Theory.

${ }^{38}$ One interesting insight one can make in this theory, however, is that dominant (accented) morphemes should have a greater impact on 'local' prominent syllables, a point which has been discussed above in \$5.2.4. This type of locality effect can be straightforwardly treated as an effect of Clash Avoidance constraints for grid prominences (though it is impossible to treat Tokyo Japanese no in this way, as it is a dominant unaccented morpheme).
} 
default pattern for accent. While this generalization is noted in Revithiadou 1997, the assumptions inherent to the Head Faithfulness theory do not account for this pattern. I will illustrate this point with the dominant unaccented suffix $-a \check{c}$ in Russian, which as we have seen in the case study above, gives default ending stress. ${ }^{39}$ Following Alderete 1996, Revithiadou treats this type of system as 'root-controlled', i.e., governed by high-ranking Root Faithfulness, which seems unavoidable given the role of roots in Russian stress. As shown below, even with the Head Faithfulness constraint top-ranked in the system, the ranking consequences of this analysis incorrectly lead to the preservation of the lexical root accent in words with an accented root and a dominant unaccented affix.

(49) Problem: Dominance Effects are not Culminativity Effects

\begin{tabular}{|c|c|c|c|}
\hline$/$ púz + ač $+\mathrm{u} /$ & PROS-FAITH $_{\text {Head }}$ & MAX-PROMRoot & PSP \\
\hline \hline$[$ puz-ač ]-ú & & $* !$ & \\
\hline$(*) \rightarrow[$ púz-ač ]-u & & & $*$ \\
\hline
\end{tabular}

The general problem here is the same as the one which afflicts the Grid Prominence Theory: dominant effects are not culminativity effects, and thus a theory which models the behavior of dominant morphemes in terms of a competition between morphemes of various types (i.e., through the constraint interaction shown here) will invariably fail to account for grammar dependent dominant morphemes.

To give an interim summary, I have discussed two previous proposals for dominance effects, namely the Grid Prominence Theory and the Head Faithfulness theory, and I have shown that the assumptions inherent to these proposals lead to significant problems which distinguish it from the TAF theory. The chief problem with these proposals is that they model dominance effects as the competition between two accented morphemes, which fails to adequately characterize the grammar dependent behavior of dominant affixes. Next, I will consider two further theories which solve this problem in different ways and compare the predictions of these theories with the TAF theory of dominance effects.

Inkelas 1996 proposes that dominance effects arise as an effect of the 'cophonology' of a dominant affix (see also Inkelas 1994, Inkelas, Orgun \& Zoll 1995 on the notion of a co-phonology). What this theory entails in OT is that the attachment of a dominant affix induces a re-ranking of Faithfulness and Markedness constraints, which in turn brings about the desired effect of grammar dependence if the re-ranking preserves certain ranking relations (made clear below). The ideas inherent to the Constraint ReRanking theory is illustrated below for Russian with a pair of tableaux which in a sense correspond to different levels or strata in the grammar. In the first tableau, PROS-FAITH enjoys a relatively high-ranking position in the hierarchy, and therefore is responsible for the realization of contrast at this stratum in the grammar. At a different level, Level Y, where derived words are formed, PROS-FAITH is demoted below POST-STEM-PROM (the constraint which is necessary to derive ending stress in unaccented words), which results in a default stress pattern at precisely this level. (The second tableau has dominant unaccented affix -ač , which as shown in $\$ 5.2 .3$, gives default ending stress).

\footnotetext{
${ }^{39}$ Revithiadou follows Melvold 1990 in assuming that dominant unaccented suffixes yield initial stress, but given the weak empirical support for such suffixes (see discussion in $\$ 5.2 .3$ above), I illustrate the problem here with a different dominant unaccented suffix. The choice of the affix does not effect the overall argument however; the default-inducing constraint PSP can simply be exchanged with a constraint requiring initial stress.
} 
(50) Dominance Effects as Constraint Re-Ranking

a. Level X Constraint Ranking: Prosodic Faithfulness is Active

\begin{tabular}{|c|c|c|}
\hline /púz $+\mathrm{u} /$ & PROS-FAITH & POST-STEM-PROM \\
\hline \hline púz-u & & $*$ \\
\hline puz-ú & $* !$ & \\
\hline
\end{tabular}

b. Level Y Constraint Ranking: Prosodic Faithfulness De-Activated

\begin{tabular}{|r|c|c|}
\hline$/$ púz + ač $+\mathrm{u} /$ & POST-STEM-PROM & PROS-FAITH \\
\hline \hline$[$ púz-ač ]-u & $* !$ & \\
\hline$\rightarrow$ [puz-ač ]-ú & & $*$ \\
\hline
\end{tabular}

It is clear from this brief sketch that the Constraint Re-Ranking theory has the descriptive power to bring about a deletion plus a default accentual structure. It is fair to say, however, that it differs from the TAF approach in that only the latter explains the property of grammar dependence. In TAF theory, the fact that the result of de-accentuation goes towards a default pattern is due to the de-activation of the relevant Faithfulness constraint and the consequent activation of lower-ranking well-formedness constraints. In other words, the default pattern is explained by the premise that there but is a single constraint system, and so, if a base accent is deleted because of high-ranking $\neg \mathrm{OO}_{\text {Dom }^{-}}$ MAX-PROM, the result will always be towards the language particular default for accent. In the Constraint Re-ranking theory, on the other hand, the result of the deletion of base prosody does not automatically go towards the default pattern because of the unrestricted nature of the re-ranking operation. Thus, it is not clear in this theory why the ranking of Markedness and Faithfulness constraints which characterize the default pattern in underived words is left intact in the grammar of derived words, and as a result, the Constraint ReRanking approach does not explain the fundamental property of grammar dependence. ${ }^{40}$ Furthermore, while it is certainly possible to introduce further restrictions on constraint reranking across levels in order to rectify this situation, e.g., an OT-equivalent to the Strong Domains Hypothesis (Kiparsky 1984b, Myers 1991), such restrictions come as an additional imposition on the basic theory, and so they will not help in explaining the phenomenon. ${ }^{41}$

A second important difference between the two theories is in the treatment of strict base mutation effects, i.e., the fact that roots do not idiosyncratically trigger the deletion of an accent in a neighboring affix (see discussion above). Inkelas 1996 accounts for the lack of dominant roots by stipulating that only affixes may be specified for a dominant cophonology without a principled basis for arriving at this result. In contrast, the transderivational nature of the TAF theory of dominance effects explains this observed gap: it is a direct consequence of the thesis of Strict Base Mutation (\$4.3), which follows

\footnotetext{
${ }^{40}$ See Benua 1997 [1998] for a related criticism of serialist OT approaches to cyclic effects.

${ }^{41}$ This conclusion raises the question of how other language internal sub-patterns attributed to constraint reranking are accounted for, like the lexical stratification in Japanese modelled in Itô \& Mester 1995a as constraint re-ranking. As argued in Fukazawa 1998 (see also Itô \& Mester 1998), the range of variation found across levels can be described as a strict ordering of Faithfulness constraints defined on different correspondence relations (compare this idea with the notion of multiple correspondence in Benua 1997 [1998]). Indeed, this approach explains Itô \& Mester's key insight into the problem, which is that lexical levels differ only in their Faithfulness properties.
} 
naturally from the Benua's notion of Base Priority. Therefore, we again find that the Constraint Re-Ranking requires an additional mechanism to account for a basic property of dominant affixes, while TAF gives a natural explanation of this property.

A second alternative to TAF theory that successfully accounts for grammar dependence is the proposal for dominance effects given in Halle \& Vergnaud 1987a (HV). In this work, dominant morphemes are distinguished from recessive ones through cyclicity. In particular, dominant affixes are 'cyclic' morphemes (cf. Kiparsky 1982b), which are represented on a metrical plane which is distinct from that of other morphemes, as illustrated in 'Cycle 2' below in (52). Furthermore, cyclic affixation triggers a copying process from one metrical plane to the plane of the cyclic affix. This copying is governed by the Stress Erasure Convention (SEC), given below, which essentially states that stresses generated on previous cycles are carried over only if the affixed constituent is not a domain for the cyclic stress rules.

(51) Stress Erasure Convention (Halle \& Vergnaud 1987a: 83)

In the input to the rules of cyclic strata information about stress generated on previous passes through the cyclic rule is carried over only if the affixed constituent is itself a domain for the cyclic stress rules. If the affixed constituent is not a domain for the cyclic stress rules, information about stresses assigned on previous passes is erased.

Applying these assumptions to the case of dominant affixes in Russian, a root accent will be consistently deleted when the root bearing this accent is combined with a dominant affix, as depicted below, regardless of whether the affix is accented or unaccented.

(52) Dominant Effects as Stress Erasure (Halle \& Vergnaud 1987a)

\begin{tabular}{|c|c|c|}
\hline & Stem + Affix [+dom, +acc] & Stem + Affix $[+$ dom, -acc $]$ \\
\hline INPUT & /s'ív/ & /púz/ \\
\hline Cycle 1 & $\begin{array}{c}\mathrm{X} \\
\text { S'ív- }\end{array}$ & $\begin{array}{l}\text { X } \\
\text { púz- }\end{array}$ \\
\hline Cycle 2 & $\underset{\substack{\mathrm{x} \\
\text { s'ív }}}{\mathrm{u} x}+\mathrm{a}$ & $\begin{array}{l}\text { púz }+ \text { ač }+u \\
\text { pula }\end{array}$ \\
\hline SEC & s’iv $_{x}+$ úx $+a$ & $\mathrm{puz}+\mathrm{ac}+\mathrm{u}$ \\
\hline $\begin{array}{l}\text { Default Accent } \\
\text { Assignment }\end{array}$ & N/A & $\mathrm{puz}^{\mathrm{acc}+\mathrm{u}_{\mathrm{x}}}$ \\
\hline OUTPUT & [s'iv-úx-a] & [puz-ač -ú] \\
\hline
\end{tabular}

In this illustration, the root accent is represented on a distinct metrical tier than the accent of the dominant affix -úx (the latter accent is placed directly below the relevant form). When information about the make-up of the root is copied at Cycle 2, the accent is lost because the larger constituent forms a domain for the cyclic stress rules (i.e., the Basic Accentuation Principle, see §3.1). Thus, the SEC neutralizes the accented/unaccented contrast in these 
roots and allows a default pattern to emerge. In the mapping from the second cycle to the output form, the inherent accent of the affix is preserved in s'iv-úx-a, but since words derived with - $a \check{c}$ are completely unaccented, they receive default ending stress, as in the case of $p u z-a \check{c}-u$.

As made clear by this illustration, the Stress Erasure approach resembles the TAF analysis in that both theories treat dominance effects as formal deletion, and the resulting structure is subject to the same principles of accentuation used elsewhere in the system. Thus, HV's theory successfully accounts for the fact that dominant morphemes are always grammar dependent. The two theories are therefore on a par with each other in this respect. Another important empirical domain where the predictions of the two theories intersect is in the treatment of sequences of dominant affixes. The cyclic theory with Stress Erasure also predicts that the morphologically external affix predicts the accentuation of the larger word, a necessary consequence of the serialist approach to affixation and the application of the SEC, which is fully compatible with the TAF approach. Indeed, HV: 86 point out that the SEC approach renders superfluous certain ad hoc deletion rules which were necessary in Halle \& Mohanan 1985 for some types of external dominant suffixes.

While the theory of dominance effects as SEC solves some important problems, the bases of this theory do not provide a straightforward means of relating dominance effects to other types of affix-controlled accentual phenomena. As argued at length in \$5.1, dominance effects have a host of properties which put them in a class with affix-controlled processes like morphologically triggered accent insertion and accent shift. Transderivational Anti-Faithfulness theory explains the similarities among these accentual processes as Faithfulness reversals; TAF constraints compare a base-derivative pair and the different morphological oppositions stem from reversals of the independently necessary Prosodic Faithfulness constraints. Moreover, because these processes involve forced violations of Faithfulness, they are all predicted to exhibit grammar dependence, as illustrated in detail in this chapter. Lastly, the assumptions inherent to TAF theory predict that affix-induced processes will be base-mutating and subject to locality effects, an empirical point with is also supported by the case studies here.

In contrast to this natural grouping of morpho-accentual processes, the SEC theory does not predict the clustering of properties, nor even the existence of certain accentual processes like the accentual shifts found in Japanese and Aguaruna (\$5.4). The SEC approach derives affix-triggered deletion through a multi-planar representation of prosody and certain restrictions on the copying of this prosody from one level to the next. What principles in this ensemble of assumptions predict the insertion of accent, or the shift of accent, between levels? Further, how do said principles ensure that these processes will have all of the properties characteristic of affix-controlled accent? Short of stipulating analogues to the SEC which yield the desired results, e.g., a Stress Insertion Convention, HV's model does not give cogent answers to these questions. Therefore, the notion of Anti-Faithfulness makes connections to other morpho-accentual phenomena that distinguish it from the SEC approach. This result is a significant point in its favor as it provides a fully integrated theory of a range of morpho-accentual processes.

As suggested to me by John Kingston, an alternative to affix-triggered AntiFaithfulness is for affix-triggered Markedness to act directly in base-derivative pairs, conditioning the change as a way of improving the overall harmony of the output form with respect to a given constraint. In particular, suppose that the deletion observed in the derived from is due to a constraint that bans a prominence, *PROM, and that this constraint refers to the appropriate correspondence relation to model the dominance effect. The best way to illustrate this idea is to consider the force of Affix-Triggered Markedness (ATM) as an operation in a two-level mapping, along the lines of the model developed in Lakoff 1993 
and implemented in OT in Orgun 1995 (though the latter model does not encompass twolevel Markedness constraints of this kind). In the mapping from Level 1 to Level 2 below, accent is lost because this mapping is characterized by an activation of the constraint *PROM, which actively suppresses a prominence that appears in a morpheme that is present at the two levels.

(53) Two-Level Approach to Dominance Effects

$$
\begin{aligned}
& \text { 1. } \\
& \text { púz-u } \\
& \text { 2. } \\
& \text { puz-ač -ú }
\end{aligned}
$$

The affix-induced Markedness effect here must be relational, assessing the Markedness of corresponding prominences in related forms. Otherwise, *PROM will simply ban a prominence in the base, contrary to many dominance effects which result in a default accentual pattern within the stem. The formulation below achieves the required relational aspect of the constraint through an OO-correspondence relation.

(54) OO-*PROM: For $\mathrm{x} \in$ prominence, $\exists \mathrm{x} \in \mathrm{S}_{1}$ (=base) $\rightarrow \neg \exists \mathrm{x}^{\prime} \in \mathrm{S}_{2}$ (=output) \& $\mathrm{x} R \mathrm{x}^{\prime}$ Avoid a prominence in the output which has a correspondent in the related base.

This constraint is formally distinct from the de-accenting TAF constraint, $\neg \mathrm{OO}-\mathrm{MAX}-$ PROM. Whereas the TAF approach uses Faithfulness reversals, the Affix-Triggered Markedness theory simply enhances the power of an existing Markedness constraint. Furthermore, the teleological purpose of these constraints underscores their differences: TAF constraints induce an alternation as a means of realizing a contrast. The purpose of ATM constraints, on the other hand, is to improve on the overall harmony of a derived form relative to a given constraint.

The tableau below illustrates how the ATM approach accounts for dominance effects. The last two base-output pairs are separated from the first in that they satisfy OO*PROM by deleting the lexical accent of the base. The winner is thus the form which deletes this accent and also satisfies low-ranking POST-STEM-PROM, (55c), cf. (55b).

(55) Dominance Effect as Affix-Triggered Markedness

\begin{tabular}{|l|c|c|c|c|}
\hline \multicolumn{1}{|c|}{ Base } & $/$ púz + ač +ú/ & OO-*PROM & OO-MAX-PM & POST-STEM-PROM \\
\hline \hline a. púz-u & [púz-ač ]-u & $* !$ & & \\
\hline b. púz-u & [puz-áč ]-u & & $*$ & $* !$ \\
\hline c. $\rightarrow$ púz-u & {$[$ puz-ač ]-ú } & & $*$ & \\
\hline
\end{tabular}

This example also illustrates the chief advantages of the ATM approach. First, dominance effects are base-mutating in this theory because, like TAF theory, the operative constraints are transderivational in nature. As a result, the emergent unmarkedness observed in the derivative is dependent on certain properties of the base, like the presence of an accent in this case. Second, ATM theory also has an angle on grammar dependent dominance effects. As with the TAF theory, the ATM constraint OO-*PROM requires a deletion and the independently needed grammar of accent predicts the result of this de-accentuation (though this is not a natural consequence of this theory - see below).

The advantages of ATM, however, do not outweigh its disadvantages, and so it does not represent a viable alternative to TAF. One significant problem in this approach to 
dominance effects is that it appears to require constraints which are not independently motivated. Thus, while other morpho-accentual processes may be brought about by beefing up well-motivated Markedness constraints, like Alignment constraints for pre- and post-accentuation (see \$5.3.4), this approach to dominance effects requires a Markedness constraint which prohibits a stress, *PROM. It is not clear, however, that such a constraint is truly necessary in the description of stress. For example, it is sometimes proposed that word-internal clash or line conflation effects (in the sense HV) are due to constraints which ban stress or, similarly, a stress foot (see e.g. Baerman 1998). There are plausible

alternatives to these analyses, however, which use independently necessary constraints on the alignment of prosodic categories or the rhythmic distribution of prominence structure (Hung 1994, McCarthy \& Prince 1993a, Baković 1998; cf. Crowhurst 1996). Therefore, the underlying function of this approach, i.e., improved Markedness in base-output pairs, has not yet been solidly established.

There is a more basic problem with the ATM theory, however, which stems from the extension of correspondence to the formulation of Markedness constraints. In a way, ATM is a much more radical departure from classical Optimality Theory than TAF theory. TAF theory introduces a new constraint for every Faithfulness constraint by proposing negated Faithfulness constraints. The ATM theory, on the other hand, innovates in a different way, allowing Markedness constraints to refer to correspondence relations. The latter move is clearly a real weakening of the theory because it enables affixes to bring about any type of Markedness effect. Thus, while the enhanced Markedness effect with OO-*PROM illustrated above leads to a deletion plus default stress, grammar dependent affix-controlled processes are not ensured by ATM theory. An activated Markedness constraint may directly dictate the outcome in the derivative. For example, the logically possible OO-Markedness constraint OO-WSP simply requires stress on a heavy syllable in the derivative, regardless of whether or not this constraint played any role in the larger system. To conclude then, ATM is not a likely theory of dominance effects because it is less restrictive than the TAF theory, and the operative constraint in this theory, *PROM, is not well-motivated outside of the analysis of dominant morphemes. 


\subsection{Pre-/Post-Accentuation as Transderivational Anti-Faithfulness}

In this section, the affix-controlled phenomenon of pre- and post-accentuation (PPA) is examined and analyzed in terms of Transderivational Anti-Faithfulness, on a par with the analysis of dominance effects presented in the previous section. The discussion begins with an introduction to the problem, and then moves to an analysis of pre-accenting suffixes in Cupeño in $\$ 5.3 .2$ as an illustration of the basic analysis. Next, some further implications of the overall approach are examined (\$5.3.3) and used as a means of contrasting the TAF theory of pre- and post-accentuation with some plausible alternatives $(\S 5.3 .4)$.

\subsubsection{The Problem}

An affix may trigger the insertion of an accent into the stem to which it is attached. When this insertion of an accent happens at the same time as prefixation, as exemplified in (56), it is referred to as POST-ACCENTUATION. When this morpho-accentual process correlates with suffixation, as shown by some familiar examples from Cupeño in (57), it is referred to as PRE-ACCENTUATION. The Cupeño examples illustrate a further property commonly found with PPA, namely that this process may not apply when the affix combines with an inherently accented root, as shown in (57b). (The pre-accenting suffixes in Cupeño are lexically marked with a subscript 'pre'; roots are underlined.)

(56) Post-Accenting $m a$ - in Tokyo Japanese (Poser 1984)
a. /ma + futatu/
/ma + usiro/
$\rightarrow \quad$ ma-ppútatu
'exactly half'
$\rightarrow \quad$ ma-úsiro
'right behind'
b. /ma + yonaká/
$\rightarrow \quad$ ma-yónaka
'dead of night'
'straight ahead'

(57) Pre-Accenting Suffixes in Cupeño (Hill 1967, Hill \& Hill 1968)
a. /wena + nukprel $^{\prime}$
/né $+\underline{\mathrm{ma}}+\check{c} \mathrm{i}_{\text {pre }}$
$\rightarrow \quad$ wená-nuk
$/$ né $+\underline{\mathrm{s}} \underline{\text { ula }}+? \mathrm{a}+\mathrm{i}_{\text {pre }} / \quad \rightarrow \quad$ ne-ș $\underline{\text { ulá}}-? \mathrm{a}-\mathrm{i}$
'having put in'
'with my hand(s)'
'my fingernails (object)'
b. /? $\underline{\text { ísi }}+1 \mathrm{ye}_{\mathrm{e}}+\mathrm{i}_{\mathrm{pre}} / \quad \rightarrow \quad \underline{\text { ?ísi }}-1 \mathrm{y}-\mathrm{i}$
/méme + yke $_{\text {pre }} / \quad \rightarrow \quad$ méme-yke
/tívii?e + maa $_{\text {pre }}+$ le/ $\rightarrow \quad \underline{\text { tívi? }}-m e-1$
'coyote (objective case)'
'to the ocean'
'small round basket'

In Item-and-Arrangement-style frameworks, PPA is commonly analyzed as a floating auto-segment that is sponsored by the affix with which it co-occurs, but for various reasons ends up on a neighboring syllable. Assuming that pitch accents in Japanese are represented tonally, post-accenting $\mathrm{ma}$ - can be treated as a floating $\mathrm{H}$ tone in the lexical representation of this prefix; when this prefix combines with other morphemes, the result is an association of the $\mathrm{H}$ to a favored position in the word, for example steminitially, as shown below. 
(58) Floating Feature Approach to Pre- and Post-Accentuation

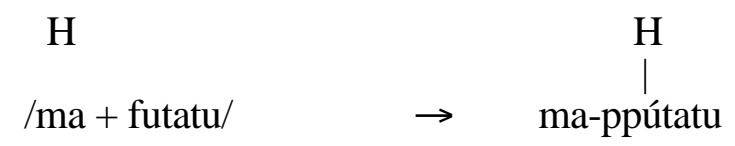

There are, however, some problems with this analysis, which I will discuss here in order to highlight some issues that any theory of PPA must address. First, an important property of PPA is that the inserted accent tends to be placed on a syllable which is 'close' phonologically to the accent-inserting affix. The example of Japanese above shows that the inserted accent must be on an adjacent syllable, which is common cross-linguistically. There are also cases of PPA which involve counting 'by twos', i.e., inserting an accent two syllables or moras from the affix in question, as in post-post-accentuation in the Papuan language Fore (Pike \& Scott 1963), and pre-pre-accentuation found in some contexts in Getxo Basque (Hualde \& Bilbao 1993) and in Tokyo Japanese (Poser 1984). More often than not, therefore, it seems that the site of insertion is at a morphological boundary, assigned either on the unit which appears at that boundary, or on a constituent bound to that edge, like a binary stress foot (though see discussion in $§ 5.3 .4$ for some interesting opposite-edge insertion sites). At first glance, the Floating Feature approach does not predict such locality effects; why should an accent, with no link to its lexical sponsor, be required to be close to its sponsor?

An additional empirical issue for the Floating Feature analysis is that PPA is always base-mutating. As discussed in $§ 5.1$, there are abundant cases of affixes which induce an insertion of accent into the base, but arguably no instances of pre- or post-accenting roots or stems. Concretely, if PPA is a property invested in a root, roots should idiosyncratically cause the insertion of an accent on a neighboring affix, but I know of no clear cases where some roots trigger PPA, while others do not. (Russian stress is a possible counterexample, discussed below.) This fact is important because it foregrounds an important similarity between PPA and dominance effects, which are likewise always basemutating. If PPA involves a floating feature, however, there is no straightforward analysis of this fact: why should unassociated auto-segments be limited to just affixes? ${ }^{1}$ Indeed, this assumption would entail a rather bizarre typological prediction, namely that affixes have a three-way contrast for accent, i.e., accented, unaccented, and accented but floating, while roots only have the standard two-way contrast. This state of affairs is clearly at odds with the important cross-linguistic trend that roots sponsor a wider range of contrasts than affixes (see discussion in §2.2). A related problem involves languages, like Tokyo Japanese, that have both post-accenting prefixes and pre-accenting suffixes. Thus, in addition to post-accenting prefixes like $m a$-, Japanese has several pre-accenting suffixes, e.g., -ke in nisimurá-ke 'the Nishimura family', cf. nisimura. The problem with cases like these is that the association of the floating feature is determined by rule in this analysis; therefore, the fact that the floating feature has two distinct edge orientations requires two separate rules for the docking of the accent at the surface. The Floating Feature analysis thus misses the generalization that these affixes are base-mutating, and must therefore be realized on the base of affixation, a point to which we will return below.

\footnotetext{
${ }^{1}$ One clear line of analysis here is to ascribe affixes with the property of Invisibility, putting them outside of the domain for accentual processes (Poser 1984). This approach is discussed in $\$ 5.3 .4$, along with other plausible alternatives.
} 
These problems with the Floating Feature analysis bring to the fore two important questions that an adequate theory of pre- and post-accentuation must address:

1. Why is the affected syllable typically a neighbor to the triggering affix?

2. Why is pre- and post-accentuation always base-mutating?

The tack taken directly below is to develop an alternative to the Floating Feature analysis which makes crucial use of Transderivational Anti-Faithfulness constraints. As I show in this section, the analysis of PPA in TAF theory provides principled answers to these questions.

On a par with dominant affixes, pre- and post-accenting affixes subcategorize for a given correspondence relation, and upon this relation, high-ranking Transderivational AntiFaithfulness constraints are formulated. Further, the anti-insertion constraint, DEP-PROM, has a counterpart in the set of Anti-Faithfulness constraints, namely $\neg$ DEP-PROM, which specifically forces insertion of a prominence in the base. The basic features of PPA are thus explained as the negation of an existing Faithfulness constraint, together with the assumption that Anti-Faithfulness effects must be felt in the neighboring environment of the triggering affix. One of the locality requirements defined in $\$ 4.3$ prescribed an alternation on an adjacent syllable, which, when applied to the Anti-Faithfulness constraint demanding an insertion of accent, triggers an epenthetic accent on the syllable directly following or preceding the affix involved, as sketched below. The square brackets indicate the scope of the Anti-Faithfulness effect.

(59) Pre- and Post-Accentuation as the Negation of DEP-PROM

\begin{tabular}{|l|c|c|c|}
\hline Base & /ma + futatu/ & ᄀOO-DEP-PROM & OO-DEP-PROM \\
\hline \hline a. $\quad$ futatu & ma-[ppu]tatu & $* !$ & \\
\hline b. $\quad$ futatu & ma-[ppu]tátu & $* !$ & $*$ \\
\hline c. $\rightarrow$ futatu & ma-[ppú]tatu & & $*$ \\
\hline
\end{tabular}

Thus, the last candidate ma-ppútatu is the winner because only it mutates the base by inserting an accent on a syllable close enough to the triggering affix.

From this brief sketch, it is clear how the TAF approach to PPA addresses the two questions given above. First, the locality conditions on PPA are accounted for in a general theory of locality effects, namely the set of locality conditions which are attested in one form or another in all affix-controlled processes. Second, PPA is base-mutating because it is affix-controlled, and all affix-controlled accentual processes must conform to Strict Base Mutation (see \$4.3.3). Rather on a par with the treatment of these same properties found in dominance effects, therefore, the basic properties of PPA are explained with a unified theory of Transderivational Anti-Faithfulness. The overall cohesiveness of the theory is a further important advantage and will be used in the final subsection as a means of contrasting the TAF theory of pre- and post-accentuation with some previous approaches to the problem. 


\subsubsection{The Proposal: Pre- and Post-Accentuation as Negation of DEP-Prom}

The Transderivational Anti-Faithfulness constraint, $\neg$ OO-DEP-PROM, will be employed below in the formal analysis of morphologically conditioned accent insertion, or pre- and post-accentuation. Specifically, I will argue that the symmetric counterpart to $\neg \mathrm{OO}-\mathrm{MAX}$-PROM in the TAF constraints has a role in the analysis of affix-controlled accentual processes. To illustrate the basic features of this theory, we examine preaccentuation in Cupeño in more detail, complementing the analysis presented in chapter 2 with a complete account of this important morpho-accentual process. A review of the data comes directly below, followed by the analysis within TAF theory.

Accent is culminative in Cupeño, and so when more than one inherently accented morpheme combines in word formation, only one of the accents can be realized. Like many morphologically governed accent systems, Cupeño shows a preference for realizing inherent accent in roots over affixes, as shown by the input-output mappings in (60a). Inherent affix accent is therefore only observed in words with unaccented roots, as exemplified in (60b). Finally, in words with more than one accented affix, the rightmost inherently accented affix surfaces with stress (60c).

(60) Cupeño Accent (Hill \& Hill 1968, Crowhurst 1994, Alderete 1996)

a. Overriding Root Accent

$$
\begin{array}{llll}
/ \text { né }+\underline{\text { Níy }}+\text { qál }+1 \text { i pe/ } & \rightarrow & \text { ne-Níy-qal-i-pe } & \text { 'When I go away' } \\
/ p e ́ ~+\underline{N i ́ y}+\text { pi/ } & \rightarrow & \text { pe-Níy-pi } & \text { 'S/he would go away' }
\end{array}
$$

b. Inherent Affix Accent with Unaccented Root

$$
\begin{array}{llll}
\text { /pé + yax/ } & \rightarrow & \text { pé-yax } & \text { 'S/he said' } \\
/ \text { pé }+ \text { yax }+ \text { qál/ } & \rightarrow & \text { pe-yax-qál } & \text { 'S/he was saying' }
\end{array}
$$

c. Rightmost Inherent Accent Wins

$$
\begin{array}{llll}
\text { /yax }+ \text { qál }+ \text { í/ } & \rightarrow & \text { yex-qel-í } & \text { 'While s/he was saying' } \\
\text { /né }+\underline{\text { wen }}+\text { qál/ } & \rightarrow & \text { ne-wen-qál } & \text { 'I was putting' }
\end{array}
$$

This last fact illustrates the rightward orientation of inherent accent. In the absence of any inherently accented morphemes, however, words receive initial stress, e.g., /yax $+\mathrm{em} / \rightarrow$ yáx-em 'Speak (2nd Plural)'. In chapter 2, this pattern of conflicting edge orientations is analyzed in terms of conflicting Alignment constraints at different levels of analysis in the prosodic hierarchy. Words with no inherently accented morphemes are analyzed as lacking a level 3 grid mark, and as a result, receive default initial stress. I maintain this assumption in the assessment of base-output relations, but as explained below, this assumption is not crucial to the analysis of pre-accentuation in Cupeño.

Cupeño also has a set of pre-accenting suffixes which impose root-final stress. For example, the words in (61a) have pre-accenting suffixes (which are marked with the subscript 'pre'), and these words all have root-final stress (see $\$ 2.4 .3$ for further exemplification). ${ }^{2}$ Importantly, pre-accentuation is blocked in words with accented roots

${ }^{2}$ It appears that these suffixes do not simply require accent on the syllable directly preceding them, as Hill 1967 and Hill \& Hill 1968 describe a pattern of pre-pre-accentuation in cases where the pre-accenting suffix is separated from the base by another affix, e.g., /né $+\underline{s} \underline{\text { ula }}+? \mathrm{a}+\mathrm{i}$ pre $/ \rightarrow n e-\underline{s} \underline{\text { ulá }}-? a-i$ 'my fingernails (obj.)'. Because the conditions on this pattern are still unclear descriptively, I address it in a speculative way at the end of this subsection. 
(61b). Thus, consistent with the general pattern in the language, inherent accent in roots impedes the realization of accent contributed by the pre-accenting suffixes.

(61) Pre-Accentuation in Cupeño (Hill 1967, Hill \& Hill 1968)

a. Inherent Affix Accent with Unaccented Root

$$
\begin{array}{llll}
/ \text { né }+\underline{\text { ma }}+\check{c} i_{\text {pre }} / & \rightarrow & \text { ne-má-č i } & \text { 'with my hand(s)' } \\
\text { /wena }+ \text { nuk } & \rightarrow & \text { wená-nuk } & \text { 'having put in' }
\end{array}
$$

b. Overriding Root Accent

$$
\begin{aligned}
& \text { /méme }+ \text { 'yeke } \text { prel } \rightarrow \text { méme-yke 'to the ocean' } \\
& \text { /tívii?e + 'maapre-le/ } \rightarrow \text { tívi?-mel 'small round basket' }
\end{aligned}
$$

While pre-accenting suffixes are abundant in the language, not all of the suffixes are preaccenting. Suffixes may be unaccented, and therefore they do not surface with accent, as in the plural imperative suffix -em in yax-em 'Say! (2nd Plural)'. Alternatively, they may be accented, and therefore surface with stress when they attach to unaccented roots, e.g., pe-yax-qál 'He was saying'. In summary, the pre-accenting suffixes differ from other affixes in the language in that the former trigger insertion of an accent into an unaccented stem.

The analysis of the first body of data given in chapter 2 is that Root Faith generally outranks Affix Faith (McCarthy \& Prince 1995); as a result, this ordering is respected in the MAX-PROM constraints, as shown below.

(62) Accent in Cupeño

$$
\text { MAX-PROM Root }_{\text {P MAX-PROMAffix }} \gg \text { ALIGN-R(PK, PrWd) }
$$

The above ranking correctly accounts for the observed patterns: a root accent takes precedence over an affix accent because the Prosodic Faithfulness constraint for roots is top-ranked. The rightmost inherently accented affix is realized only in the absence of accented roots.

The pre-accenting suffixes induce an insertion of accent somewhere in the root to which they attach. The phonological operation observed in pre-accentuation is therefore the insertion of an accent which is not present in the related form. The observed phonological pattern may be explained in terms of an Anti-Faithfulness constraint which negates the logical statement of DEP-PROM, as shown below.

(63) $\neg$ DEP-PROM: For $\mathrm{x}$ a prominence, $\neg\left[\forall \mathrm{x} \exists \mathrm{x}^{\prime},\left[\mathrm{x} \in \mathrm{S}_{2} \rightarrow \mathrm{x}^{\prime} \in \mathrm{S}_{1} \& \mathrm{x} R \mathrm{x}^{\prime}\right]\right]$ 'It is not the case that every prominence in $S_{2}$ has a correspondent in $S_{1}$.

Satisfaction of $\neg$ DEP-PROM entails the appearance of (at least) one epenthetic prominence. Formulated as a TAF constraint, $\neg$ OO-DEP-PROM requires accent insertion in morphologically related words. On par with the analysis of dominance effects, therefore, the contrast between pre-accenting and non-pre-accenting affixes is established through constraint ranking, as shown below with two schematic rankings. 
(64) Accent Insertion/No Accent Insertion through Constraint Ranking

a. Accent Insertion: $\neg$ OO-DEP-PROM >> OO-DEP-PROM

b. No Accent Insertion: OO-DEP-PROM >> ᄀOO-DEP-PROM

With $\neg$ OO-DEP-PROM ranked above OO-DEP-PROM, affixation will trigger an insertion of prominence in the base, as observed with the attachment of -nuk in [wena] $\approx$ [wená-nuk]. On the other hand, if $\neg$ OO-DEP-PROM is ranked below OO-DEP-PROM, then affixation does not bring about a change in the base, as we have seen with the suffix -em, e.g., $[\operatorname{yax}(\mathrm{e})] \approx[\mathrm{yax}-\mathrm{em}]$. Conflating these rankings in a single hierarchy yields the following constraint system.

(65) Pre-Accentuation in Cupeño

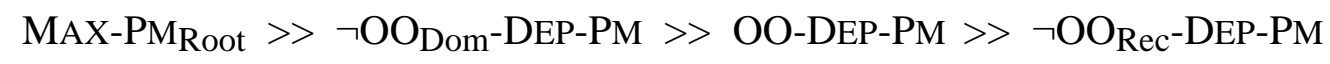

In this system, the same mnemonics from $§ 5.2 .2$ are used. The pre-accenting suffixes trigger $\mathrm{OO}_{\mathrm{Dom}}$-correspondence, and because the Anti-Faithfulness constraint defined on this relation is high-ranking, these suffixes will bring about overt effects in base-derivative pairs. In contrast, words with non-pre-accenting suffixes are assessed by $\neg \mathrm{OO}_{\mathrm{Rec}}$-DEPPROM, which, because of its low-ranking position in the constraint system, accounts for the lack of accent insertion. Finally, in this ranking, $\neg$ OO-DEP-PROM is ranked below the Root Faithfulness constraint, MAX-PROMRoot, and as a result, words with accented roots will always be faithful to the accentuation of the root.

The following lexical entries distinguish the pre-accenting suffixes and non-preaccenting suffixes through the subcategorization of correspondence relations discussed above.

(66) Lexical Entries for Pre-Accenting and Non-Pre-Accenting Suffixes in Cupeño 3
a. -nuk $\mathrm{V}_{\text {Punc }}$
-ye $\quad \mathrm{N}_{\mathrm{Obj}}$
-yeke $\mathrm{N}_{\mathrm{Dir}}$
[ [ Verb ]oODom __ ]
[Pre-Accenting]
$-\check{c} i \quad \mathrm{~N}_{\text {Instr }}$
[ [ Noun ]oODom __ ]
[ [ Noun ]oODom __ ]
[ [ Noun ]oODom __ ]
b. qál
-em

$$
\text { V Past-dur }
$$$$
\text { [ [Verb ]oORec __ ] }
$$$$
\text { [ [Verb ] JOORec __ ] }
$$
[Non-Pre-Accenting]

With the contrast between the two affix classes established in the lexicon, the various patterns of Faithfulness and Anti-Faithfulness for accent may be modelled in terms of familiar types of constraint interaction, which I now illustrate.

Words with the suffix -nuk are sensitive to $\mathrm{OO}_{\mathrm{Dom}}$-correspondence. Therefore,

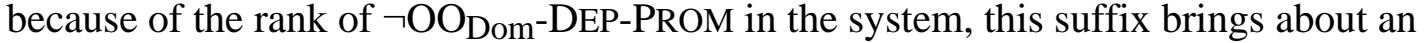
insertion of accent in the derived form, specifically in the interval of the word which is also

\footnotetext{
${ }^{3}$ The morphological restrictions given in these entries are based on the grammatical sketch in Hill \& Nolasquez 1973.
} 
present in the base, as illustrated below (see $\$ 4.2 .1$ for discussion of the role of Stem-toStem correspondence in DEP-type constraints). ${ }^{4}$

(67) Pre-Accentuation with Suffix -nuk

\begin{tabular}{|l|c|c|c|}
\hline Base & $/$ wena + nuk $_{\text {pre }} /$ & $\neg$ OO $_{\text {Dom-DEP-PROM }}$ & OO-DEP-PROM \\
\hline \hline a. $\quad$ wena & wena-núk & $* !$ & \\
\hline b. $\rightarrow$ wena & wená-nuk & & $*$ \\
\hline
\end{tabular}

The winner here is thus the base-derivative pair which achieves an overt opposition by inserting a non-lexical accent into the base, similar to the opposition realized as a deletion of accent found in words with dominant morphemes.

Pre-accenting suffixes must affect the base, but if the base of affixation is greater than one syllable, how is the site of prominence insertion predicted? As noted in \$5.3.1, in many cases, the insertion site may be predicted from the locality requirements inherent to the process, e.g., Japanese pre-accenting suffixes must affect a neighboring syllable, and so these suffixes posit a threshold on the scope of Anti-Faithfulness (achieved though Local Conjunction in the domain of the syllable — see the analysis in \$5.3.3.1 below). In Cupeño, however, the locality restrictions are less stringent (see footnote 19), and so the precise pattern of base mutation becomes an empirical issue. In such a context, lowerranking constraints in the system, which are needed on independent grounds, become active and predict a specific pattern of Anti-Faithfulness, as illustrated below.

(68) Grammar Dependent Pre-Accentuation

\begin{tabular}{|l|c|c|c|c|}
\hline \multicolumn{1}{|c|}{ Base } & /wena + nuk $_{\text {pre }}$ & $\neg$ OODom-DEP-PM & OO-DEP-PM & ALIGN-R \\
\hline \hline a. wena & wena-nuk & $* !$ & & \\
\hline b. $\quad$ wena & wéna-nuk & & $*$ & $* * !$ \\
\hline c. $\rightarrow$ wena & wená-nuk & & $*$ & $*$ \\
\hline
\end{tabular}

The fully faithful candidate (68a) is ruled out because the suffix -nuk is evaluated by

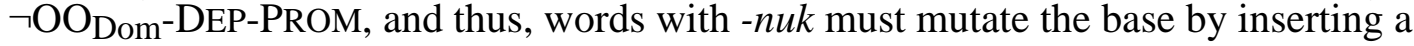
prominence. The remaining two candidates satisfy the Prosodic Anti-Faithfulness constraints in this way, and since they both violate OO-DEP-PROM equally, a different constraint is needed to separate them. The required constraint is the independently needed Alignment constraint, ALIGN-R, which favors final accent over non-final accent in the root, as this option better satisfies the gradient Alignment constraint. In sum, the inserted accent must appear in the base because TAF constraints always require base mutation, and the inserted accent must be stem-final because of the independently attested edge orientation for word accent.

\footnotetext{
${ }^{4}$ For ease of exposition, I assume that the bases here are not marked for an accent, i.e., a grid mark at the level on the grid where lexical accent is given (see $\$ 2.4 .3$ for discussion). While these unaccented words do have a prominent syllable (at level 2), they do not have a stress-accent (at level 3), which is the level upon which the Faithfulness and Anti-Faithfulness constraints operate. If it turns out there is a better analysis in which the bases here are in fact phonologically accented on the initial syllable, this would not affect the ranking argument here because the high-ranking Anti-Faithfulness constraint will still require an inserted accent, different from the one present in the base.
} 
This last result highlights an important feature of the analysis, namely the treatment of grammar dependent morpho-accentual processes. Paralleling the treatment of dominant suffixes, pre-accenting suffixes in Cupeño require a change in the base, but the independently needed grammar of accent helps, in some contexts, to predict the overall character of the accentual change. In Russian, for example, the TAF constraint $\neg \mathrm{OO}-$ MAX-PROM requires a loss of accent in the base, but the dominated constraint POST-STEMPROM determines the stress of de-accented words. Likewise, in Cupeño, $\neg$ OO-DEPPROM requires an insertion of accent in the base, but the lower-ranking constraint, ALIGN$\mathrm{R}$, is responsible for predicting where this epenthetic accent is placed in the base. Thus, there is a symmetry in the patterns of morphologically triggered Anti-Faithfulness in that they are both grammar dependent.

Returning to the details of the analysis, a different set of suffixes subcategorize for $\mathrm{OO}_{\mathrm{Rec}}$-correspondence, and as a result of the rank order of $\neg \mathrm{OO}_{\mathrm{Rec}}-\mathrm{DEP}-\mathrm{PROM}$, these suffixes do not condition pre-accentuation. The imperative plural suffix -em is one such suffix, and as illustrated in the following tableau, derivatives with this suffix are like their simplex bases.

(69) Lack of Pre-Accentuation with -em

\begin{tabular}{|l|c|c|c|}
\hline Base & /yax $+\mathrm{em} /$ & OO-DEP-PROM & ᄀOORec-DEP-PROM \\
\hline \hline a. $\quad$ yax $(\mathrm{e})$ & yáx-em & $* !$ & \\
\hline b. $\rightarrow \operatorname{yax}(\mathrm{e})$ & yax-em & & $*$ \\
\hline
\end{tabular}

Because -em is itself unaccented, the larger word surfaces without an accent as a means of satisfying the Transderivational Prosodic Faithfulness constraints.

The next fact of interest here concerns the combination of a pre-accenting suffix with an accented root. As exemplified above, the inherent accent of the root wins out over the pre-accenting suffix, and this fact is also correctly predicted through constraint domination. Thus, employing the well-motivated distinction between Root and Affix Faithfulness, the constraint which requires realization of inherent accent in roots, namely MAX-PROMRoot, is ranked above the constraint which calls for pre-accentuation in pre-

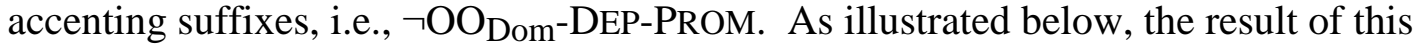
ranking is that it is more harmonic to realize root accent than to mutate the base with an insertion of accent, even with the suffixes which are sensitive to $\neg \mathrm{OO}_{\text {Dom-DEP-PROM. }}$

(70) Blocking Effect of RCA

\begin{tabular}{|c|c|c|c|}
\hline Base & /méme + yeke prel & IO-MAX-PMRoot & $\neg \mathrm{OO}_{\text {Dom }}$-DEP-PM \\
\hline méme-t & memé-yke & $* !$ & \\
\hline b. $\rightarrow$ méme-t & méme-yke & & * \\
\hline
\end{tabular}

The loser here satisfies the Anti-Faithfulness constraint, as the accentuation of the base is changed in base-output pairs, but this pattern is of little help because base-mutation in this context requires being unfaithful to the root accent on the IO-dimension. The winner is thus the candidate which fails to change the base as a means of satisfying high-ranking Root Faith.

Non-uniformity effects such as this were mentioned as a conceptual possibility in chapter 4: the demands placed on an affixed form by a TAF constraint can be stymied by 
the force of an higher-ranking constraint. Pre-accentuation in Cupeño is thus a real life example of such an effect, providing empirical evidence for the approach taken here where constraint ranking plays a crucial role. Interestingly, many systems which have preaccentuation exhibit this type of blocking effect. For example, in Getxo Basque, there are several pre-accenting suffixes, but these suffixes only trigger an insertion of accent in words with unaccented stems, again showing a role for Root Faithfulness in preaccentuation. Another relevant example is metatony, or 'pattern D mobile stress' in Russian. As argued in \$5.2.3, this pattern is best treated as a case of pre-accentuation; if this analysis is correct, it is significant that this pattern of mobile stress is only available in words with unaccented roots because only such forms will be able to insert a new accent into the stem.

Returning to the locality restriction on the site of accent insertion noted above, as this type of effect is one of the stated goals of this section, it is necessary to consider how to analyze this restriction. Unfortunately, the available evidence is rather inconclusive, so I can only make a speculative hypothesis at this time. Starting with my primary source, Hill \& Hill 1968: 236 discuss a morphological restriction on the range of pre-accentuation, namely that the pre-accenting suffix may not be separated from the base by more than one affix. Megan Crowhurst (personal communication) suggests that this apparent morphological restriction may in fact be phonological. Given that almost all of the suffixes are monosyllabic at the surface, it may be possible to state this restriction in terms of a two syllable window: the accent contributed by the pre-accenting suffix must not be farther than two syllables from the triggering suffix. This kind of restriction would not come as a surprise, because, as noted in the introduction, there are a number of languages with PPA which exhibit a two syllable threshold.

The observed disyllabic threshold on pre-accentuation invites a foot-based interpretation: the inserted accent must appear in the foot directly preceding the preaccenting suffix. To make this proposal more concrete, I assume that there is a relatively high-ranking constraint in the grammar which aligns a stress foot at the right edge of the stem (excluding the pre-accenting suffix). This constraint is of course subordinate to the Prosodic Faithfulness constraints which may bring about non-right-aligned feet and the Alignment constraint governing the edge properties of affix accent (see \$2.4.3). In words with unaccented roots, however, this foot will always be right-aligned, providing the basis for the following restriction: the accent contributed by the pre-accenting suffix must be in the foot which abuts this suffix. In a case like the one given above, pre-pre-accentuation places an accent on the head of a trochaic foot, as in: [ne-[su(lá-?a)]Stem-i], while preaccentuation inserts an accent into the head of an iambic foot, as in [(wená) $]_{\text {Stem-nuk]. }}$ However, the inserted accent cannot be pre-penultimate in the stem because such a pattern would put an accent outside of the stress foot: [[ó $(\sigma \sigma)]$-af pre]. Within the framework for locality effects developed in $\$ 4.3$, this type of restriction is described by locally conjoining the TAF constraint with an Anchoring constraint in the domain of the prosodic foot, i.e., $\left(\neg \mathrm{OO}-\mathrm{DEP}-\mathrm{PROM} \& \mathrm{ANCHOR}(\mathrm{Stem}, \mathrm{PrWd}, \mathrm{R})_{\text {Foot}}\right.$. In the case study of Aguaruna presented in $\$ 5.4 .3$, there appears to be a similar type of restriction on accent shifts, namely that the shifted accent must be within the foot directly preceding the accent-shifting suffix. Thus, while there are still some formal details to be worked out, the facts here seem to pattern with other types of affix-controlled processes. 
The following constraint hierarchy summarizes the ranking arguments given above in the analysis of pre-accentuation in Cupeño.

(71) Summary Ranking 5

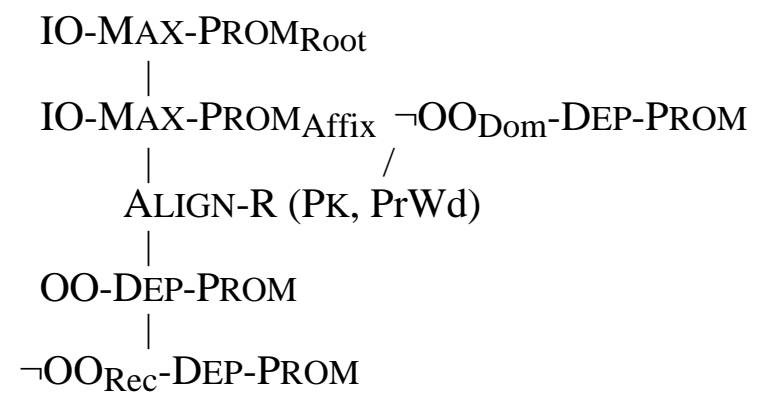

Reviewing the essential features of the analysis, with the IO-Root Faithfulness constraint top-ranked, words with accented roots will always be paired with derived forms which are faithful to this root accent. However, in derived words with an unaccented root and a preaccenting suffix such as -nuk, the result is insertion of accent in the base, because the TAF constraint $\neg$ OO Dom-DEP-PROM outranks OO-DEP-PROM. The constraint ranking here predicts a very specific pattern of base mutation, namely insertion of accent in the root-final syllable, as this pattern fares better on the relatively low-ranking Alignment constraint, ALIGN-R. Furthermore, suffixes such as -em subcategorize for $\mathrm{OO}_{\mathrm{Rec}}$-correspondence, and because the Anti-Faithfulness constraint defined on this relation is low-ranking, specifically ranked below the Transderivational Prosodic Faithfulness constraints, these suffixes contrast with the pre-accenting suffixes in not causing insertion of accent into the base. Finally, inherently accented affixes, such as -qál, may realize their inherent accent because IO-MAX-PROM ${ }_{\text {Affix }}$ dominates the Anti-Faithfulness constraint sensitive to this non-pre-accenting suffix.

Now that I have constructed an analysis of a particular language, it is possible to establish more explicitly the relation between the TAF analysis of PPA and the analysis presented in the previous section for another type of affix-controlled accentual process, dominance effects. First, in the analysis of both dominance effects in Russian and of preaccentuation in Cupeño, the affix-controlled process is lexically specified through subcategorization of correspondence relations. Thus, dominant suffixes and pre-accenting suffixes select a correspondence relation upon which a high-ranking Anti-Faithfulness constraint is defined, and as a result, only these suffixes trigger the process. Furthermore, in the TAF analysis for both types of affix-controlled processes, the observed processes are morphologically triggered and base-mutating. That is, in both analyses, the observed accentual change is a response to an OO-Anti-Faithfulness constraint, and because of their transderivational nature, the constraints only affect base-derivative pairs. The observed changes always affect the base, consistent with Strict Base Mutation.

An additional parallel between the TAF analyses of these two affix-controlled phenomena is that in both cases the morpho-accentual process is grammar dependent. That is, a TAF constraint requires an accentual change between a base and its derivative, and other constraints operative in the system play a role in predicting the precise nature of the

\footnotetext{
${ }^{5}$ The ranking of ALIGN-R above OO-DEP-PROM, though not apparent from the above discussion, is required to account for the fact that the base accent is not preserved in the base-output pair, pé-yax/pe-yaxqál 'He says, was saying'; if the reverse ranking held, then prefix accent would be preserved in the derivative, instead of the observed pattern of rightward edge orientation.
} 
change. In Russian, the constraint POST-STEM-PROM was responsible for the fact that deaccented words always surface with ending stress. In Cupeño too, the constraint ALIGN-R had a say in the pattern of Anti-Faithfulness observed in derived forms, namely that the inserted accent must be rightmost in the root. In both analyses, therefore, independently attested constraints on the distribution of accent have a formal role in characterizing the outcome of the morpho-accentual process.

Another important similarity between the TAF approaches to dominance effects and PPA, though not made explicit in these particular analyses, is that both approaches account for certain locality conditions on the range of an Anti-Faithfulness constraint. Thus, in the analysis of phrases with no in Tokyo Japanese (\$5.2.4), the observed locality condition on de-accentuation is handled with the same machinery employed in characterizing locality effects in pre-accenting suffixes and post-accenting prefixes (discussed in more detail below). In both cases, accent deletion or accent insertion only affects a prosodic category which is adjacent to the relevant affix. This fact is described by locally conjoining a TAF constraint with an Anchoring constraint within the domain of the relevant prosodic unit. On this count too, therefore, TAF theory gives an integrated analysis of a related fact.

To conclude this subsection, I have shown in the context of a set of problems in Cupeño that the TAF theoretic analysis of pre- and post-accentuation accounts for all of the formal properties observed in other affix-controlled processes. This result is significant because it is the second step in establishing a fully integrated theory of ACA, a program which is to be continued in the next section. Furthermore, this result will also enable us to distinguish the TAF approach to PPA from other possible alternatives, which are studied in the last subsection.

\subsubsection{Implications}

\subsubsection{Strict Base Mutation in Pre- and Post-Accentuation}

Before we study to approaches pre- and post-accentuation in a wider theoretical context, I would like to flesh out some further implications of the TAF approach which will serve to distinguish it from various plausible alternatives. The first point involves the treatment of locality conditions and how the account in TAF theory extends to languages like Tokyo Japanese which have both pre-accenting suffixes and post-accenting prefixes. As I show below, the apparent bi-directional orientation for the accents introduced by these affixes follows quite naturally as an effect of Strict Base Mutation.

As illustrated with the following examples, Japanese has both prefixes which give stem-initial accent and suffixes which cause stem-final accent.

(72) Pre- and Post-Accentuation in Tokyo Japanese (Poser 1984: 54 ff.)
a. /ma' + futatu/
$\rightarrow \quad$ ma-ppútatu
'exactly half'
/ma’ + yonakál
$\rightarrow \quad$ ma-yónaka
'dead of night'
b. /yosida + 'ke/
/nisímura + ke/
$\rightarrow \quad$ yosidá-ke
$\rightarrow$ nisimurá-ke
'the Yoshida family'
'the Nishimura family'

The two patterns above are clearly connected by the fact that they are both base-mutating and subject to a locality requirement dictating that the insertion of accent be in an adjacent syllable. 
How are these two related facts to be accounted for? The only mechanism for characterizing locality conditions in TAF theory is through Local Conjunction defined within certain morphological or prosodic categories, and thus the locality conditions on Japanese PPA must fall out from this theory. Consistent with the set of assumptions developed in $\$ 4.3$, therefore, the observed locality conditions here may be characterized through local conjunction in the domain of the syllable, as in the constraint below.

(73) ( $\neg$ OO-DEP-PROM \& ANCHOR-R/L(Stem, PrWd) $)_{\sigma} \equiv \neg$ OO-DEP-PROMEdge- $\sigma$ In base-derivative pairs, a prominence which is not present in the base must be inserted in the derived form in the syllable which is de-aligned through affixation.

$\neg$ OO-DEP-PROM is violated in a pair of related words where the derived word does not have an inserted accent in the base, while ANCHOR-R/L is violated in all affixed words because such words introduce material which stands in the way of perfect Stem-to-PrWd Alignment. On a strictly formal level, ANCHOR-R/L is a combination of two constraints, so the conjoined constraint here actually involves a conjunction of $\neg$ OO-DEP-PROM with an already conjoined constraint; since the two Anchoring constraints have the same overall effect, I refer to them jointly as ANCHOR-R/L. 6 The conjunction of the TAF and Anchoring constraints in the domain of the syllable therefore entails that it is not allowed to de-align the stem through affixation and at the same time fail to insert an epenthetic prominence into the stem at the de-aligned edge. The effect of this complex constraint is illustrated in the following two sets of tableaux.

(74) Post-Accentuation in Tokyo Japanese

\begin{tabular}{|c|c|c|c|c|}
\hline Base & & Output & $\neg$ OO-DEP-PROMEdge- $\sigma$ & OO-PROS-FAITH \\
\hline \multirow[t]{2}{*}{ a. futatu } & \multirow[t]{2}{*}{$\approx$} & ma-[ppú]tatu & & $*$ \\
\hline & & $*_{\text {ma-}}[\mathrm{ppu}] \mathrm{tatu}$ & $* !$ & \\
\hline \multirow[t]{2}{*}{ b. yonaká } & \multirow[t]{2}{*}{$\approx$} & ma-[yó]naka & & $*$ \\
\hline & & *ma-[yo]naká & $* !$ & \\
\hline \multirow[t]{2}{*}{ c. yosida } & \multirow[t]{2}{*}{$\approx$} & yosi[dá]-ke & & $*$ \\
\hline & & $*_{\text {yosi[da]-ke }}$ & $* !$ & \\
\hline \multirow[t]{2}{*}{ d. nisímura } & \multirow[t]{2}{*}{$\approx$} & nisimu[rá]-ke & & $*$ \\
\hline & & *nisímu[ra]-ke & $* !$ & \\
\hline
\end{tabular}

In the candidates above, the bracketed syllable identifies the locus of the Anchoring violations, and hence, the syllable in which the TAF constraint is active. As can be seen from each base-output pair, this constraint always rules out the fully faithful form in favor of a pattern of Anti-Faithfulness with insertion in a neighboring syllable. For example, in the losing base-output pairs in (74a) and (74b), failure to insert an accent in the first syllable of the stem results in a violation of the Anti-Faithfulness constraint $\neg$ OO-DEPPROM. Since this constraint violation is local to the violation of Anchoring incurred by

${ }^{6}$ Perhaps the combined force of these two constraints can be achieved with the Wrap constraints developed in Truckenbrodt 1995 and applied specifically to word-level units in Peperkamp 1997. The idea behind these constraints is the edge of the morphological stem must be 'wrapped' by, or co-extensive with, the edge of the PrWd (see also McCarthy \& Prince 1994 for a related idea in the analysis of recursive stem structure in Diyari). 
prefixation, these pairs are ruled out. The same holds with the pre-accenting suffixes shown in (74c) and (74d), except the insertion of a suffix in the derived form calls for accent insertion in the final syllable of the stem. As illustrated with the losing candidates, failure to insert an epenthetic accent in this context leads to a violation of the conjoined constraint, which effectively eliminates these base-output pairs as well.

To summarize the result here, the conjunction of the two constraints in the domain of the syllable requires a breach of Faithfulness in the syllable which is adjacent to the relevant affix. With this approach to the locality condition, the fact that prefixes are postaccenting and suffixes are pre-accenting is explained as an effect of Strict Base Mutation. Since TAF constraints require a mutation of the base of a morphological process, the directionality effects of the two types of affixes are explained by the subcategorization requirements which define the edge properties of the individual affixes. In this theory, therefore, there can be no pre-accenting prefixes nor post-accenting suffixes because, without a rather non-standard morphological analysis, such morpho-accentual processes are not base-mutating. This following statement foregrounds this prediction, which is a special type of SBM, and hence does not need to be stipulated in the analysis of PPA.

\section{(75) Strict Base Mutation in Pre- and Post-Accentuation}

Morphologically triggered insertion of accent always affects the base of affixation.

This prediction can be counter-exemplified in two logically possible scenarios: (i) an affix which causes insertion of an accent into a non-base affix, and (ii) a stem which inserts an accent into a neighboring non-base affix. The constraint behind the scenario in (i) is at work in the explanation of the two patterns of base-mutation in Japanese PPA. Concerning scenario (ii), however, it is interesting to review the stress system in Russian inflected nouns, as a common approach to the analysis of stress in Russian involves recognizing a set of 'post-accenting stems', so-called because they are claimed to trigger a shift of accent to the following vowel in the inflectional ending (see e.g. Melvold 1990 and references therein). If such a category was indeed required in the analysis of Russian stress, this system would constitute a clear counter-example to the restrictive claim established above. It turns out that this approach is unsatisfactory for several reasons, and so it does not refute the application of SBM to pre- and post-accentuation, as I will now show. ${ }^{7}$

Russian has two productive patterns of stress in nominal paradigms (which is mirrored in verbs and adjectives as well, as discussed in §3.2.3). These two patterns are exemplified below with two second declension nouns.

(76) Stress in Russian Noun Paradigms
a. Fixed Stem Stress
b. Fixed Inflection Stress
kómnat-a
kómnat-e
kómnat-am

$\begin{array}{ll}\text { č 'ert-á } & \text { Nominative Singular } \\ \text { č 'ert-é } & \text { Dative Singular } \\ \text { č 'ert-ám } & \text { Dative Plural }\end{array}$

${ }^{7}$ Another interesting case in regard to SBM is the Hare dialect of Slave, which, according to Rice 1989, 1990 has a two-way contrast between unmarked stems and stems which trigger the insertion of tone into the preceding syllable in verbs; in nouns, the marked tone stays on the stem. Following Rice 1990 (see also Gessner 1999), however, the retraction of tone specifically in verbs can be accounted with the special prosody characteristic of this word class, whereby the stem tone shifts to the preceding syllable to appear in the head of a trochaic foot. 
The first pattern, which is by far more common, is fixed stress on a stem vowel (76a). The second pattern, shown in $(76 \mathrm{~b})$, constitutes roughly six percent of the total noun inventory and has fixed stress on the first vowel of the inflection ending (which is disyllabic in instrumental forms). Also, there are two minor stress patterns which involve mobile stress, but since they are unproductive, they will be treated later.

The analysis alluded to above, which I will call the Post-Accenting Analysis, accounts for these two basic patterns by assuming that the stems which form these paradigms are both inherently accented. Further, stems which give fixed ending stress are accented on the final syllable and lexically marked for the feature [+Post-Accenting], which systematically yields ending stress by triggering a rule of stress shift. Thus, a stem like $\sqrt{k o ́ m n a t}$ is accented on the first syllable which is preserved in surface inflected forms (because this stem is not post-accenting); the stem $\sqrt{\tilde{c}}$ 'ért is likewise inherently accented, but because it is post-accenting, its inherent accent always ends up on the first vowel of the inflectional ending.

This analysis is of course one among many, and the alternative analysis developed in $\S 3.2$ has a number of advantages over this approach. This alternative analysis is quite simple: paradigms with fixed stem stress have an inherently accented stem which is consistently preserved in surface forms because of high-ranking Root Faithfulness (on a par with Cupeño); paradigms with fixed inflection stress have unaccented stems and receive ending stress by default because there is a constraint in the grammar, POST-STEM-PROM, which actively requires stress in this position. I will refer to this analysis as the RootControlled Accent (RCA) analysis.

One advantage of the RCA analysis is that the constraint needed in the characterization of the default position for stress, POST-STEM-PROM, provides the right tool for explaining other stress patterns in which the post-stem vowel is the default position for stress. First, certain inflectional categories in some declension classes do not have an ending, e.g., the nominative and accusative singular in I and III declension nouns. In such contexts, if the noun has fixed inflection stress throughout the rest of the paradigm, these inflectional categories have stem-final stress, e.g., topór, cf. topor-ú 'axe (nominative/dative singular)', showing that POST-STEM-PROM sets the post-stem vowel as a target that is approximated in these forms.

The second form of evidence for the post-stem vowel as a default position involves the analysis of the two patterns of mobile stress in words with unaccented stems. One pattern, often called 'pattern D', has ending stress in the singular, but stem-final stress in the plural, e.g., kolbas-á 'sausage (nom. sg.)', cf. kolbás-y (nom. pl.). Another mobile pattern ('pattern C') involves initial stress in the singular, but ending stress in the plural, e.g., kólokol 'bell (nom. sg.), cf. kolokol-á (nom. pl.). In §5.2.3, I explain both of these mobile stress patterns as an effect of an Anti-Faithfulness constraint requiring an overt difference between singular and plural forms. In particular, the plural suffixes are analyzed as pre-accenting and the change brought about in the plural is thus due to the base-mutating effects of the TAF constraint yielding pre-accentuation. For example, in the pattern D mutation, the singular form has default ending stress, as in kolbas-á, because that is the default position for stress in words with unaccented stems, as determined by POST-STEMPROM. The TAF constraint therefore requires a minimal difference in the plural, which correctly predicts stem-final stress in kolbás-y as a minimal violation of POST-STEMPROM. The same principles of morphological opposition are at work in the analysis of pattern $\mathrm{C}$ mobile stress, except this pattern is treated as a dominance effect. The singular 
has lexically specified initial stress, as in kólokol, and the TAF constraint $\neg$ OO-MAXPROM therefore requires a deletion, with the result of giving ending stress in the plural, e.g., kolokol-á, as a means of satisfying POST-STEM-PROM. To summarize, the same constraint which is operative in the analysis of default ending stress also has a role in the analysis of these unproductive patterns of mobile stress, which provides further support for the RCA approach.

In contrast to the RCA analysis, the connection between the accentual defaults in the productive and unproductive stress patterns is unexplained within the Post-Accenting analysis. For example, pattern D mobile stress is analyzed as evidence for an additional rule, Stress Retraction, which targets an accent shifted to the inflectional ending, and moves it back to its lexical stem-final position, a 'Duke of York' derivation in the sense of Pullum 1976. The derivation for the plural form kolbás-y runs as follows: /kolbás $+\mathrm{y} / \rightarrow$ [kolbas-ý ] $\rightarrow$ [kolbás-y]. Why is stress retracted specifically in the plural, and why is it retracted only one syllable? These are questions which arise in the Post-Accenting analysis but receive no explanation. In contrast, a straightforward response to these questions is given in the RCA analysis sketched above. The stress is mutated in the plural as a means of supporting a morphological contrast; the shift is only one syllable because this shift minimally violates POST-STEM-PROM.

The overall argument here is thus that the Post-Accenting analysis is inferior to the Root-Controlled Accent analysis because the latter explains a wider range of data with a cogent analysis. The superiority of the RCA analysis therefore vitiates the assertion that Russian counter-exemplifies the application of the thesis of Strict Base Mutation to pre- and post-accentuation. Since there is an alternative to the Post-Accenting analysis which is superior, it does not follow that Russian must be analyzed as having post-accenting stems.

\subsubsection{Dominant/Recessive Pre-Accentuation in Tokyo Japanese}

The next set of implications to be examined involves the treatment of dominant preand post-accenting affixes. The languages studied thus far, namely Cupeño, and tangentially Russian, all involve a non-uniform pattern of pre-accentuation in which the pre-accenting suffixes only induce an accentual change in words with unaccented roots. However, PPA may apply across the board in some instances, causing deletion of a stem accent in derived forms. Thus, one finds cases of dominant, pre-accenting suffixes, such as the suffix - $k e$ in Tokyo Japanese, ${ }^{8}$ where both accented and unaccented stems receive an epenthetic accent.

\footnotetext{
${ }^{8}$ There is a possible analysis of dominant pre-accenting suffixes as the second member of a compound: short second members typically give final accent in the first member (see chapter 3, section 3 for data and analysis), which is consistent with the observed pattern here. Recessive pre-accenting suffixes, however, cannot be treated this way, as compounding is generally insensitive to the accentuation of the first member, i.e., it deletes the accent if there is one. Since this fact shows that there are at least some pre-accenting suffixes, I follow Poser 1984 in assuming that dominant pre-accentuation is a linguistically significant morpho-accentual pattern in Japanese.
} 
(77) Dominant Pre-Accenting suffix -ke (Poser 1984: 55)
a. /yosida $+\mathrm{ke} /$
$\rightarrow \quad$ yosidá-ke
/ono + ke/
$\rightarrow \quad$ onó-ke
/matumoto $+\mathrm{ke} /$
$\rightarrow \quad$ matumotó-ke
'the Yoshida family'
'the Ono family'
'the Matsumoto family'
b. /nisímura $+\mathrm{ke} /$
$\rightarrow \quad$ nisimurá-ke
/ándoo + ke/
$\rightarrow \quad$ andóo-ke
/sátoo + ke/
$\rightarrow \quad$ satóo-ke
'the Nishimura family'
'the Ando family'
'the Sato family'

The introduction of dominant pre-accenting - $k e$ leads to a situation of constraint conflict that must be resolved through constraint interaction. Thus, it is a consistent fact that accent is culminative in Tokyo Japanese in that every minor phrase has at most one pitch accent. In $\S 3.3$, this fact is treated as a consequence of the building up of prominence structure, but here we will simply refer to the generic constraint CULMINATIVITY to account for the 'one accent per phrase' fact (see $\$ 1.2 .2 .3$ for the formal details). Because this constraint is undominated in the language, attachment of -ke leads to the following question: should accented stems preserve their inherent accent, or should this accent be lost in favor of the epenthetic accent called for by the pre-accenting suffix? As illustrated below, this competition for the unique word accent can be successfully resolved by ranking $\neg \mathrm{OO}_{\text {Dom-DEP-PROM}}$ Edge- $\sigma$ (an abbreviation of the complex constraint in (73)) above the IO-Faithfulness constraint calling for faithful realization of stem accent.

(78) Dominant Pre-Accenting -ke (with accented base nisímura)

\begin{tabular}{|c|c|c|c|}
\hline /nisímura + ke/ & CULMIN & $\neg \mathrm{OO}_{\text {Dom}}$-DEP-PMEdge- $\sigma$ & IO-MAX-PMRoot \\
\hline a. nisímurá-ke & $* !$ & & \\
\hline nisímura-ke & & $* !$ & \\
\hline c. $\rightarrow$ nisimurá-ke & & & $*$ \\
\hline
\end{tabular}

The loser in (78a) attempts to satisfy both of the constraints which call for a surface accent, but by doing so, this candidate violates the high-ranking constraint in the language requiring a unique accent. The remaining two candidates have a single accent, but only the form in (78c) satisfies the TAF constraint requiring an accent adjacent to the pre-accenting suffix, and since this constraint is ranked above the IO-Faithfulness constraint, this candidate is the winner.

Applying the same logic, pre-accentuation is predicted in forms with unaccented stems. Thus, if the TAF constraint outranks the anti-insertion constraint in roots, an inserted accent will appear on the stem-final syllable, despite the fact that this candidate violates a different form of Root Faithfulness.

(79) Dominant Pre-Accentuation with -ke (with unaccented base yosida)

\begin{tabular}{|c|c|c|}
\hline /yosida + ke/ & $\neg \mathrm{OO}_{\text {Dom-DEP-PROM}}$ Edge- $\sigma$ & IO-DEP-PROMRoot \\
\hline a. yosida-ke & $* !$ & \\
\hline b. $\rightarrow$ yosidá-ke & & $*$ \\
\hline
\end{tabular}


This ranking is of course crucial in the account of pre-accentuation with accented stems because stems receive an inserted accent in these contexts as well.

The summary ranking for dominant pre-accentuation is given below, which will later be subsumed in a larger ranking accounting for recessive pre-accentuation.

(80) Dominant Pre-Accentuation in Tokyo Japanese

$$
\text { CULMIN, } \neg \mathrm{OO}_{\text {Dom-DEP-PROM}} \text { Edge- } \sigma \text { > IO-MAX-PROMRoot, IO-DEP-PROMRoot }
$$

In this ranking, words may only have one surface accent, and since the TAF constraint is ranked above the two Root Faithfulness constraints, pre-accentuation is predicted across the board.

A different pattern of pre-accentuation is observed with the suffix -si, which is characterized as recessive pre-accenting because it fails to insert an accent in words with accented stems. Consider the following examples, which are organized by accentual type.

(81) Recessive Pre-Accenting suffix -si (Poser 1984: 54)
/ono + si/
/matumoto + si/
a. /yosida + si/
$\rightarrow$ yosidá-si
$\rightarrow \quad$ onó-si
$\rightarrow \quad$ matumotó-si
'Mr. Yoshida'
'Mr. Ono'
'Mr. Matsumoto'
b. /nisímura + si/
/ándoo + si/
/sátoo + si/
$\rightarrow \quad$ nisímura-si
$\rightarrow \quad$ ándoo-si
'Mr. Nishimura'
$\rightarrow \quad$ sátoo-si
'Mr. Ando'
'Mr. Sato'

The different phonological behavior observed with -si is further evidence that affix classes may be defined by OO-correspondence relations. Thus, the distinction between pre-accenting and non-pre-accenting suffixes in Cupeño involves subcategorization of distinct OO-correspondence relations. Here too, it appears that different correspondence relations define two affix classes, both of which happen to be pre-accenting. Thus, in contrast to the dominant pre-accenting suffixes such as -ke, which subcategorize for

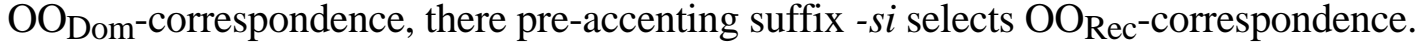
When properly ranked in relation to the IO-MAX-PROMRoot, the TAF constraint sensitive to words with $-s i$ will give the correct output. As shown in the following tableau, only derived words with unaccented stems will receive an epenthetic accent (82a), cf. (82b) (the base forms here correspond to the underlying forms for each stem).

(82) Recessive Pre-Accentuation with -si

\begin{tabular}{|cc|c|c|}
\hline Base & Output & IO-MAX-PROMRt & $\neg O_{\text {Rec-DEP-PROMEdge- }}$ \\
\hline \hline a. yosida $\approx$ yosidá-si & & \\
\hline *yosida-si & & $* !$ \\
\hline \hline b. nisímura $\approx$ nisímura-si & & $*$ \\
\hline & $*$ nisimurá-si & $* !$ & \\
\hline
\end{tabular}


Thus, on a par with the analysis of a parallel set of facts in Cupeño, the domination of a TAF constraint by IO-MAX-PROMRoot gives the recessive behavior of the pre-accenting suffixes. Thus, the root-controlled nature of Japanese surfaces here as well, as Root Faithfulness also has a role in blocking pre-accentuation in some forms.

To summarize, the ranking for recessive pre-accentuation is given below.

(83) Recessive Pre-Accentuation in Tokyo Japanese

$$
\text { CULMIN, IO-MAX-PROMRoot }>\neg \mathrm{OO}_{\text {Rec }} \text {-DEP-PROMEdge- } \sigma \text { > IO-DEP-PROMRoot }
$$

Incorporating the subhierarchies argued for above gives the following totally ordered ranking.

(84) Summary Ranking

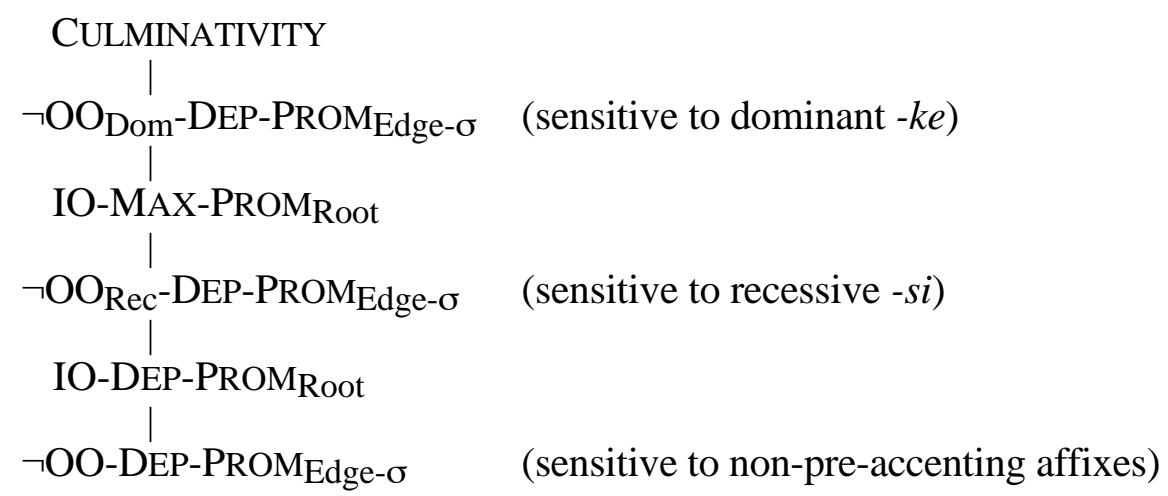

The suffix - $k e$ triggers $\mathrm{OO}_{\mathrm{Dom}}$-correspondence, and as a result of the rank of $\neg \mathrm{OO}_{\mathrm{Dom}^{-}}$

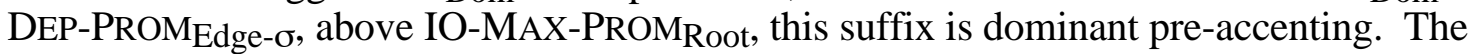
suffix -si subcategorizes for $\mathrm{OO}_{\mathrm{Rec}}$-correspondence, and thus, because of the intermediate rank of the TAF constraint defined on this relation, -si is recessive pre-accenting. Finally, some affixes subcategorize for a generic OO-correspondence relation, and since $\neg$ OO-DEP-

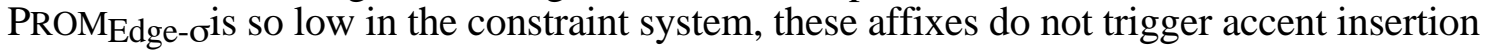
in any environments.

\subsubsection{Factorial Typology}

It is worth considering a wider range of possible rankings at this time to see what kinds of patterns TAF theory actually predicts. It turns out that we have already encountered all of the predicted patterns in the discussions developed thus far in this section and in $\$ 5.2$. To prove this assertion, however, it is necessary to construct a factorial typology with all of the relevant Faithfulness and Anti-Faithfulness constraints, which we turn to below.

To simplify the constraint permutations, I will limit the re-rankings to four constraints, namely OO-DEP-PROM and OO-MAX-PROM, and their corresponding AntiFaithfulness constraints $\neg$ OO-DEP-PROM and $\neg$ OO-MAX-PROM. While certainly the relative ordering of other prosodic well-formedness constraints is relevant in predicting the precise character of an accentual process, the rank of these constraints will only give the default patterns (or possibly certain kinds of blocking effects), which are not the focus of this experiment. The Prosodic Faithfulness constraint OO-NO-SHIFT-PROM and its corresponding TAF constraint are also relevant to studying the range of affix-controlled 
accentual processes. Because of the nature of these constraints, however, they do not interact crucially with the MAX and DEP constraints examined here. Concretely, accentual shifts are only possible when a lexical accent has been preserved; since reversals of MAXPROM and DEP-PROM require deletion of accent and insertion of a non-lexical accent, constraints on the migration of a base accent do not enter into the factorial typology of these constraints. Thus, these two sets of constraints can be studied separately.

A factorial typology with four constraints yields twenty-four possible grammars. It is unnecessary to examine each grammar in detail, however, as there are certain regular patterns which emerge with the constraint rankings we have already examined. Thus, the schematic rankings for pre- and post-accentuation and dominance effects discussed previously are given in (85a) and (85b) below. The remaining rankings order an AntiFaithfulness constraint relative to MAX-PROM, which determines whether or not an AntiFaithfulness effect is found in a word with a lexical accent. Thus, a process is characterized as dominant if MAX-PROM is dominated (85c), and hence, the lexical accent is deleted. By contrast, if MAX-PROM is ranked above a TAF constraint (85d), the process is recessive, and accordingly is blocked by the presence of a lexical accent. Of course, if the Anti-Faithfulness constraint in (85d) is $\neg$ MAX-PROM, then there is no process at all, but the affix in question is still characterized as recessive in this typology. As all AntiFaithfulness effects are derived in surface-to-surface correspondence, I will leave out 'OO-' because it is redundant.

(85) Decisive Rankings for Prosodic Faithfulness and Anti-Faithfulness
a. $\neg$ DEP-PROM > DEP-PROM
$\rightarrow \quad$ Accent Insertion
b. $\neg$ MAX-PROM >> MAX-PROM $\rightarrow$
Accent Deletion
c. $\neg \mathbb{F} \gg$ MAX-PROM
$\rightarrow \quad$ Process is Dominant
d. MAX-PROM > $\neg \mathbb{E}$
$\rightarrow \quad$ Process is Recessive

With these characteristic rankings, we may describe all of the patterns predicted in the factorial typology, which are shown in the following implicational statements.

(86) Typology of Morpho-Accentual Processes
a. If (85a) and (85c)
$\rightarrow \quad$ Dominant PPA
b. If (85a) and (85d)
$\rightarrow \quad$ Recessive PPA
c. If $\neg(85 a)$, and (85b)
$\rightarrow \quad$ Dominant Default
d. If $\neg(85 a)$, and $\neg(85 b)$
$\rightarrow \quad$ Faithful Mapping

Starting with (86a-b), if the ranking for accent insertion holds, namely $\neg$ DEPPROM > DEP-PROM, then in all the grammars generated in this theory, the result is preand post-accentuation. This statement holds, even if $\neg$ MAX-PROM is top-ranked in the constraint system. The reason for this is that $\neg$ DEP-PROM demands an insertion of a nonlexical accent, while $\neg$ MAX-PROM requires a deletion. In a language where both are topranked, the two constraints work together to achieve a deletion in words with a lexical accent in the base, as shown in (87a) below. Furthermore, in a language in which (85a) holds, unaccented bases will receive an epenthetic accent in the derived form, as shown in 
(87b). The indices here show the correspondence relations between prominences in baseoutput pairs.

(87) Obligatory Accent Insertion with $\neg$ DEP-PROM and $\neg$ MAX-PROM Top-Ranked

\begin{tabular}{|c|c|c|c|c|c|c|}
\hline & Base & Output & $\neg \mathrm{DEP}$ & $\neg \mathrm{MAX}$ & DEP & MAX \\
\hline \multirow[t]{3}{*}{ a. } & $\begin{array}{l}\mathrm{x}_{1} \\
\text { root }\end{array}$ & $\approx \begin{array}{c}\mathrm{x}_{2} \\
\text { root }+ \text { af }\end{array}$ & & & $*$ & \\
\hline & $\begin{array}{l}\mathrm{x}_{1} \\
* \text { root }\end{array}$ & $\begin{array}{c}\mathrm{x}_{1} \\
\text { root }+ \text { af } \\
\end{array}$ & $* !$ & * & & * \\
\hline & $\begin{array}{r}\mathrm{x}_{1} \\
\text { * root } \\
\end{array}$ & root + af & $* !$ & & & $*$ \\
\hline b. & root & $\approx \quad \begin{array}{c}x_{2} \\
\text { root }+ \text { af }\end{array}$ & & & $*$ & \\
\hline & * root & root & $* !$ & & & \\
\hline
\end{tabular}

Examination of the base-output pairs in (87) reveals an interesting property of the constraint interaction with these two TAF constraints: a violation of $\neg$ MAX-PROM always entails a violation of $\neg$ DEP-PROM. Thus, the rank of the Anti-Faithfulness constraint $\neg$ MAXPROM is not relevant in distinguishing the different types of PPA as the effects of $\neg$ MAXPROM are eclipsed by $\neg$ DEP-PROM, a point which will be returned to below. The differences in the type of PPA thus derive exclusively from the rank of $\neg$ DEP-PROM relative to MAX-PROM. If MAX-PROM is ranked above $\neg$ DEP-PROM, then a recessive pattern of PPA is predicted (86b), as we have seen in the case study of Cupeño and with certain suffixes in Japanese. If, on the other hand, the reverse ranking holds (86a), the predicted outcome is dominant PPA, as with pre-accenting -ke in Japanese. In sum, if $\neg$ DEP-PROM $>>$ DEP-PROM, then an accent is inserted in the base, and the rank of MAXPROM determines the dominant or recessive nature of the affix triggering the insertion.

The remaining twelve grammars have DEP-PROM $>\neg$ DEP-PROM, and as a result, an accent is not obligatorily inserted. As shown in (86), there are basically two types of patterns which can result in such a scenario. First, if the accent deletion ranking holds (85b), then the result is a 'dominance effect', i.e., deletion of an accent with the emergence of a default pattern, as we have encountered in Russian and Japanese in \$5.2. In this type of system, the rank of DEP-PROM is irrelevant because $\neg$ DEP-PROM is crucially dominated, so new accents do not have to be introduced (though they may, for phonological reasons). Furthermore, the dominance effect here is the deletion of an accent, so the anti-insertion constraint has no role in defining the morpho-accentual process. Of the twelve remaining grammars, six will have $\neg$ MAX-PROM ranked above MAX-PROM, and all of these grammars will exhibit dominance effects.

In the last six grammars, both of the Anti-Faithfulness constraints are dominated by their related Faithfulness constraints, and so these grammars will always give faithful treatment of the prosody of the base form. Thus, the remaining quarter of the rankings have no process at all. The following chart summarizes the patterns which are possible by the typology developed here and relates them to particular examples that we have seen so far. 
(88) Typology of Morpho-Accentual Processes
a. Dominant PPA
Japanese $-k e(\$ 5.3 .3)$
b. Dominant Default
Russian -ač (\$5.2.2), Japanese -kko (\$5.2.3)
c. Recessive PPA
Cupeño -nuk (\$5.3.2), Japanese -si (§5.3.3)
d. Faithful Mapping
Russian -ic (§5.2.3), Cupeño -em (§5.3.2)

The first pattern examined above is dominant PPA, as exemplified by Japanese -ke. This type of affix-controlled process is analyzed as the result of a grammar with $\neg$ DEP-PROM ranked above the related constraint DEP-PROM, and MAX-PROM is crucially dominated. The second affix-controlled accentual process involves de-accentuation with an accentual default, i.e., dominance effects as observed with some derivational suffixes in Russian. This pattern of Anti-Faithfulness is accounted for by a grammar in which $\neg$ MAX-PROM dominates MAX-PROM, and additionally, ᄀDEP-PROM is ranked below DEP-PROM (if the reverse ranking holds, the result is dominant PPA). The third affix-controlled process is recessive pre- and post-accentuation, as observed with many suffixes in Cupeño. This non-uniform pattern of Faithfulness and Anti-Faithfulness is approached as a consequence of a ranking ᄀDEP-PROM above DEP-PROM, but below MAX-PROM, an interspersing of constraints which is unavoidable in this theory. Lastly, the final kind of morpho-accentual phenomena is not a process at all, but rather a fully faithful mapping from the base to its derivative; this common pattern of output-to-output Faithfulness is accounted for with the complement set of rankings, i.e., those rankings in which the both Faithfulness constraints outrank their related Anti-Faithfulness constraint.

\subsubsection{Discussion of Alternatives}

Now that the implications of the TAF theory of affix-induced accent insertion has been outlined, we may consider a set of alternatives to this theory. Two important issues in the discussion that follows concern how these alternative theories account for grammar dependence and Strict Base Mutation effects. Also, while some alternatives successfully account for these properties of ACA, they do so in a way that fails to make the connections with other affix-controlled accentual processes. Thus, similar to the conclusion reached in $\S 5.2 .5$, the TAF theory of pre- and post-accentuation (PPA) prevails over the alternatives on theoretical grounds, relating a heterogeneous body of facts with an integrated theory.

A common approach to accent-inserting morphemes in metrical stress theory is that they are lexically specified for a structure that would cause a stress (or a tonal accent indirectly) to appear on a nearby syllable. This general mode of analysis is assumed by many (HV, Kager 1989, Halle \& Kenstowicz 1991, Idsardi 1992, McCarthy 1995, Itô, Kitagawa, \& Mester 1996, among others), and since each implements the basic idea differently, it is necessary to examine a particular theory in order to develop a careful argument. Therefore, I will discuss the Lexical Edge Marking (LEM) theory of Idsardi 1992 as the representative of this group, though the same basic arguments can be made for the other approaches.

In Idsardi 1992, lexical edge markings for certain morphemes conspire with other rules which assign metrical constituency to give the effect of PPA. In particular, a lexical bracket is posited for pre-accenting suffixes, which guides the construction of other higher level metrical constituents, assigned by other rules of edge marking and head specifications. Applying these ideas to Cupeño, the pre-accenting suffixes '-nuk and ' $-\check{c} i$ are pre-specified for a left bracket to their left. Considering the example in (89a), root-final stress is derived by building a right-headed constituent at the next level, as shown in (90). 
Furthermore, an example like that in (89b), with an inherently accented prefix and a preaccenting suffix, shows that an additional layer of structure is needed in order to derive the preference for stress on the rightmost head, as shown below.
a. /wena +'nuk/ $\rightarrow$ wená-nuk 'having put in'
b. /né $+m a+` \check{c} \mathrm{i} / \quad \rightarrow \quad$ ne-má-č $\mathrm{i} \quad$ 'with my hands'

(90) Cupeño Pre-Accentuation with Lexical Edge Marking (after Idsardi 1992)

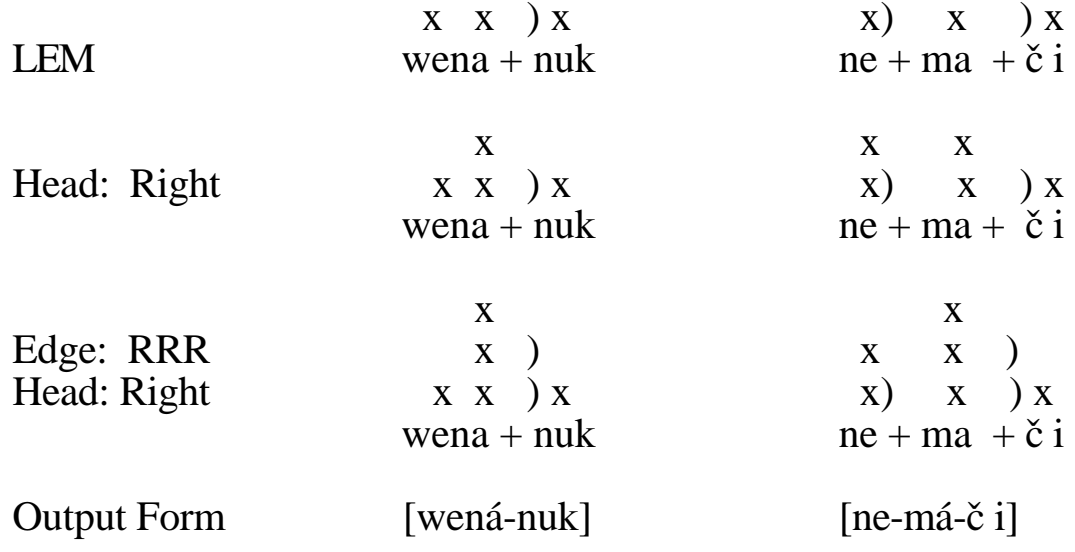

The assumption that metrical constituents are right-headed, together with a final pass of End Rule Right (= the edge marking parameter RRR), correctly describes the observed facts. ${ }^{9}$

A significant problem with LEM theory is that it does not account for Strict Base Mutation effects. Thus, if pre- and post-accentuation is the result of a lexical edge marking, nothing stands in the way of positing a bracket at the edge of a stem, yielding a post-accenting or pre-accenting stem (as is employed in the analysis of Russian oxytones, Idsardi $1992 \$ 4.2$; see arguments in $\$ 3.2$ and $\$ 5.3 .3 .1$ in the present work, however, that Russian post-accenting stems are spurious). Such effects are ruled out in TAF theory as a matter of principle, and so this theory makes a substantive restriction on this morphoaccentual process that is not made in LEM theory. To develop this point further, consider the assumptions that need to be made to account for pre- and post-accentuation in Japanese. Recall from \$5.3.3.1 that the post-accenting prefix $m a$ - yields an accent on the first syllable of the stem, as in ma-ppútatu 'exactly half', while the pre-accenting suffix produces stemfinal accent, e.g., yosidá-ke 'the Yoshida family'. Assuming the necessary lexical edge markings shown below, it is impossible to describe both pre- and post-accentuation in the same system without extra derivational steps. ${ }^{10}$ Thus, if right-headed constituents are built at the next level, then $m a$ - incorrectly yields stem-final accent (as indicated with the " $\downarrow$ " arrows); conversely, if a left-headed constituent is built, then $k e$-yields stem-initial accent (shown by the " $\uparrow "$ arrows), contrary to fact.

\footnotetext{
${ }^{9}$ I will ignore the problem posed by overriding root accent here, which introduces an additional wrinkle, because it is orthogonal to the argument

${ }^{10}$ And further, other lexical edge markings will not bring more straightforward results here; if a right bracket is switched for a left bracket, or vice versa, this will not bring about the desired effect of placing an accent on the stem.
} 
(91) Pre- and Post-Accentuation in Japanese with Lexical Edge Marking

\begin{tabular}{|c|c|c|}
\hline LEM & 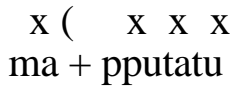 & $\begin{array}{c}x \times x) x \\
\text { yosida }+ \text { ke }\end{array}$ \\
\hline Head: Right & $* \downarrow$ & $\downarrow$ \\
\hline & $\begin{array}{c}\text { X }\left(\begin{array}{r}\text { X X } \\
\text { ma + pputatu }\end{array}\right.\end{array}$ & $\begin{array}{c}x \quad x \quad x \quad x \\
\text { yosida }+k e\end{array}$ \\
\hline Head: Left & $\uparrow$ & $* \uparrow$ \\
\hline
\end{tabular}

Succinctly, since pre- and post-accentuation is a combination of the edge markings of a particular morpheme and the head parameters of higher level structure, a single specification for the head parameter cannot account for the conflicting edge properties of these affixes. It is of course true that additional mechanisms can be introduced to rectify this situation. For example, a bounded constituent can be built at the next level with an opposite specification for the head parameter at the level below it; or alternatively, these affixes could be handled with additional representational levels, making different metrical constituents possible. However, the analysis of Strict Base Mutation effects such as these in TAF theory presents a simpler alternative. As shown by the account given in \$5.3.3.1, both affixes must mutate the base of affixation, so the fact that accent appears on the stem in both cases, follows from the assumptions inherent to TAF theory. The only additional step needed in this analysis is to limit this mutation to the syllable neighboring the basemutating affix. The tool for describing this type of locality effect, Local Conjunction, is shown in $\$ 4.3$ to apply to a variety of affix-controlled processes, both accentual and nonaccentual ones, and thus it is clear that this mechanism is needed independently. To summarize, LEM theory does not account for Strict Base Mutation as the assumptions of this theory do not rule out pre- and post-accenting stems, nor does it give a principled account of post-accenting prefixes and pre-accenting suffixes in the same language.

A different approach, which solves some of the problems clarified above, involves the assignment of the property of Invisibility to a morpheme, making it outside the domain of an accentual process (see Poser 1982, 1984, cf. Archangeli \& Pulleyblank 1984 and Barker 1989). As shown in Poser 1984, this mode of analysis provides a means of characterizing processes which apply specifically to the base of affixation, in effect deriving what I am calling Strict Base Mutation effects. Thus, suppose that the pre-accenting suffix $k e$ - and the post-accenting prefix $m a$ - discussed above are invisible to processes assigning accent. The insertion of accent triggered by these affixes - however this is achieved must be base-mutating, because the affix itself is invisible, as illustrated below.

(92) Pre- and Post-Accentuation in Japanese with Invisibility
a. $/\langle\mathrm{ma}\rangle+$ futatu $/\langle\mathrm{ma}\rangle-$ ppútatu
b./yosida $+\langle\mathrm{ke}\rangle / \quad \rightarrow \quad$ yosidá- $\langle\mathrm{ke}\rangle$

Some further details are needed to account for the fact that the inserted accent appears on a neighboring syllable, but if the affixes themselves are inherently accented, then substantive restrictions on the migration of this lexical accent will account for the observed locality effects (see Myers 1997a, Bickmore 1996, and the case study of Aguaruna in \$5.4.4 for some leading ideas). To summarize, there is an interesting point of intersection here between the TAF theory of PPA and a theory which espouses Invisibility; both derive basemutating accent insertion from their inherent assumptions.

The role of Invisibility does not extend to Strict Base Mutation in dominance effects, however. As discussed in detail in $\$ 5.2 .4$, the TAF theory of dominance effects 
rules out the possibility of a root idiosyncratically triggering a deletion of accent in a nearby affix; since TAF constraints are transderivational, accent deletion must be within the base of affixation. In contrast, the observation that the base is always targeted in dominance effects does not follow from the lexically idiosyncratic assignment of Invisibility. Concretely, lexical assignment of Invisibility will not preclude a deletion of affix accent caused by a root; to do so would entail marking every affix as invisible, which is clearly not a valid option. ${ }^{11}$ From these considerations, it appears that Invisibility does make an interesting connection with base-mutating TAF constraints, but it does not provide the basis for a restrictive theory of affix-controlled accentual processes in general, excluding certain logically possible insertion or deletion processes.

As discussed throughout this section, pre- and post-accenting affixes tend to show locality effects: the inserted accent is often on a nearby syllable. ${ }^{12}$ A recent approach to pre- and post-accentuation derives this fact from the alignment of prosodic and morphological categories (McCarthy \& Prince 1993a). In particular, Kager 1996 proposes that pre-accentuation is due to a lexical marking for an alignment property, namely alignment to a base-final stressed syllable. Spelling out these assumptions for Cupeño entails a complex constraint like the one given below.

(93) PRE-ACCENT $\equiv$ Align(Affix pre $_{\text {, L }}$, Root, R) $\&_{\text {or }}$ Align(Affix $_{\text {pre }}$, L, PROM, R) The left edge or pre-accented suffixes (a lexically marked class) must coincide with both the right edge of the root and the right edge of a prominent syllable.

In addition to being suffixes, i.e., affixes which appear to the right of a root, pre-accenting suffixes must be aligned to the right edge of a prominent syllable. ${ }^{13}$ When ranked properly in the constraint hierarchy (see $\$ 2.4 .3$ for more details), this constraint derives the desired result of ensuring the attachment of pre-accenting suffixes to a root with final stress, as depicted below.

(94) Pre-Accentuation in Cupeño through MCat-PCat Alignment

\begin{tabular}{|c|c|c|}
\hline$/$ wena + nuk $_{\text {pre }} /$ & PRE-ACCENT & ALIGN-R(PEAK, PrWd) \\
\hline \hline wéna-nuk & $* !$ & \\
\hline$\rightarrow$ wená-nuk & & $*$ \\
\hline
\end{tabular}

The Alignment theory therefore accounts for one of the basic properties of PPA, namely that the inserted accent appears on a syllable close to the affix. Furthermore, a natural extension of this idea can account for pre-pre-accentuation, as is found in Japanese (Poser 1984) and some limited contexts in Getxo Basque (see Hualde \& Bilbao 1993); requiring the affix to attach to a trochaic foot gives the effect of pre-pre-accentuation.

The Alignment theory therefore seems to have a possible advantage over the TAF theory of PPA because it derives locality effects with its most basic premise, namely that morphological and prosodic categories must coincide (though the Alignment theory has some problems with non-local morpho-accentual phenomena, discussed below). Furthermore, the Alignment theory makes do with constraints that are independently

\footnotetext{
${ }^{11}$ Though certain cases can be ruled out as Extended Invisibility effects — see the discussion of Turkish stress in Poser 1984 for details.

12Thanks to John Kingston for encouraging me to consider this alternative.

${ }^{13}$ The combination of requirements here is the meaning of the 'or' in the conjunction of these two constraints; if either of the two constraints are violated, the conjoined constraint is too (Hewitt \& Crowhurst 1996, Crowhurst \& Hewitt 1997, cf. Smolensky 1993).
} 
needed elsewhere in morpho-phonology. For example, the subcategorization-type Alignment constraints are needed in the characterization of the alignment properties of affixes. Likewise, the second MCat-PCat Alignment constraint appearing in the complex

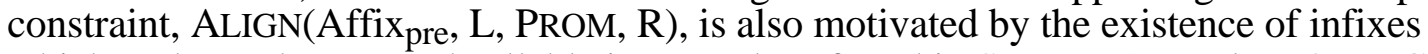
which seek out the stressed syllable in a word, as found in Samoan (Broselow \& McCarthy 1982) and the Nicaraguan language Ulwa (see McCarthy \& Prince 1993b for discussion and analysis). The Alignment theory is therefore equal to the TAF theory of pre- and postaccentuation in this respect (see $\$ 4.3$ for motivation of Anti-Faithfulness outside the domain of accentual systems).

The Alignment theory does not account for two basic properties of pre- and postaccentuation, however, which distinguishes it from the TAF theory. First, Alignment does not exclude pre- or post-accenting stems or roots. The distinction between pre-accenting and normal affixes in this theory is established with a lexical marking for the two affix classes. The assumptions inherent to this theory, therefore, do not rule out the same lexical distinction in the analysis of pre- or post-accenting roots or stems. Indeed, such a constraint is employed in $\S 3.2$ in the analysis of stress in Russian inflections, though the effects of this constraint are not lexical. Thus, while the Alignment theory can describe base-mutating PPA by specifying the proper arguments in an Alignment constraint, the theory does not provide a basis for ruling out certain unattested types of PPA, as TAF does, which is a major flaw of this theory.

Second, the Alignment theory does not account for grammar dependent accent insertion. The site of accent insertion in this theory is purely the result of the forced alignment of an affix with a root-final stressed syllable. The connection with other constraints in the grammar is not made, as it is in TAF theory through the use of independently needed constraints on the distribution of accent. In the analysis of Cupeño pre-accentuation sketched above, it is an accident that the accent contributed by the preaccenting suffix is rightmost in the root. In other words, PPA has very limited scope in the Alignment theory, essentially restricted to an adjacent prosodic category, or two units from the accent-inserting affix through the use of metrical structure discussed above. If there is a overlap between the location of the inserted accent and the Alignment properties of accent found elsewhere in the language, this result does not follow from the assumptions inherent to the theory, and this constitutes a major problem for this approach as well.

In connection to this last point, there is a clear dividing line between the Alignment theory, on the one hand, and the three other theories discussed here, namely, TAF, LEM, and Invisibility. The former does not provide a means of describing non-local accentual insertions, i.e., affixes which trigger an insertion of accent in a unit which is not restricted to the morpheme boundary separating the base and affix. If PPA is due to the alignment of an affix and a prosodic category, then the affix in question must be bound by that prosodic category. This requirement does not hold, however, of the other three theories, though in the TAF theory, non-local accent insertions occur under very special circumstances.

Specifically, if an accentual change is not subject to locality conditions, then it will arise as a consequence of the independently motivated constraints on the distribution of accent. Thus, a logical possibility in this theory, as with LEM and theories with Invisibility, is a suffix which triggers an insertion on an initial syllable, regardless of the size of the base. Other possibilities are prefixes which trigger insertion of an accent on the penultimate or final syllable. It is crucial, however, in the TAF theory that the site of insertion be default positions in some sense; this is because when affix-controlled accentual processes are not local, they must be grammar dependent. 
At present, a robust set of examples in accent systems showing the 'opposite-edge' effects described above is not forthcoming. There are a few relevant examples, however, which suggests that further investigation may turn up a larger set of examples. For example, Hill \& Hill 1968: 236 mention two suffixes, namely -wee 'present imperfect (plural subject)' and -weene 'past imperfect (plural subject)' which specifically trigger an insertion of stress into the first syllable of the root. Also, Melvold 1990 discusses one unproductive suffix, -En, which is claimed to bring about stem-initial stress (though see discussion in \$5.3). While these examples are not solid, one clear case of an opposite-edge effect brought about through affixation involves certain length alternations commonly found in Wakashan languages. In Nuucha' anulh, for example, certain suffixes trigger a lengthening of the word-initial syllable, regardless of the distance separating them from the beginning of the word. As suggested to me by Douglas Pulleyblank and Darin Howe (personal communications), it appears that this pattern of lengthening makes sense in the system as a whole because independently needed constraints on initial prosodic feet dictate that a long vowel on the initial syllable is a preferred quantitative pattern. It seems, therefore, that a more careful look at the edge effects brought about by special affixes may lead to the discovery of the predicted patterns here, and I so leave this empirical issue for future research. 


\subsection{Accent Shifts as Transderivational Anti-Faithfulness}

Up to this point, we have studied two patterns of Anti-Faithfulness: dominance effects brought about by the TAF constraint $\neg$ MAX-PROM and accent insertion due to $\neg$ DEP-PROM. The proposal is thus that two of the Prosodic Faithfulness constraints have corresponding Anti-Faithfulness constraints, and that these constraints explain the properties of two known morpho-accentual processes. If the TAF theory of affixcontrolled processes is fully symmetric, meaning there is an Anti-Faithfulness constraint for every Faithfulness constraint, it follows that there should be a third pattern of AntiFaithfulness, namely a shift in accent predicted by the negation of the remaining Prosodic Faithfulness constraint, NO-FLOP-PROM. In this section, I show that some accent systems do indeed exhibit this pattern, and that this type of affix-induced accentual process therefore provides further empirical confirmation of the overall approach taken here.

\subsubsection{The Problem}

Affixes may trigger a shift of the accent in the base to which they attach. For example, in Tokyo Japanese, certain suffixes require a shift of the base accent, as shown by the words in (95a) formed with the suffix -mono 'thing'. In (95b), the same suffix is used with an unaccented stem and the result is an unaccented word, showing that the suffixes specifically shift a lexical accent.

(95) Accent Shift in Tokyo Japanese (McCawley 1968, Poser 1984)
a. /káki + mono/ $\rightarrow$ kakí-mono 'scroll'
yómi + mono/ $\quad \rightarrow \quad$ yomí-mono $\quad$ 'reading matter'
b. /nori + mono/ $\rightarrow$ nori-mono 'vehicle'
/wasure + mono/ $\rightarrow$ wasure-mono 'forgotten item'

A second example of this morpho-accentual process is found in the Jivaroan language Aguaruna. This language has a large number of 'accent-shifting' suffixes that shift the lexical accent of the stem one mora to the right in the derived form. The accusative and possessive forms in (96) illustrate this morphologically triggered shift, which can be seen by comparing the position of accent in these derivatives with its position in the related nominative base forms.

(96) Accent Shift in Aguaruna (Payne 1990)

$\begin{array}{llll}\text { Nominative } & \text { Accusative } & \text { Possessive } & \\ \text { núka } & \text { nuká-n } & \text { nuká-nuu } & \text { 'leaf' } \\ \text { kawáu } & \text { kawaú-n } & \text { kawaú-nuu } & \text { 'parrot' } \\ \text { káš ai } & \text { kaš ái-n } & \text { kaš ái-nuu } & \text { 'paca' }\end{array}$

The accent shifts illustrated above in Japanese and Aguaruna have all of the properties of affix-controlled accentual processes laid out in $\$ 5.1$. First, the accent shifts are lexically idiosyncratic: certain suffixes trigger the shift, while others do not, e.g., the negation suffix - $\check{c} u u$ in Aguaruna is not accent-shifting: núka- ̌̌ $и и$ 'not a leaf'. Second, the accentual shift is morphologically triggered; it correlates with the application of the morphological process of affixation. Thus, these shifts differ from stress shift in English 
which is governed purely by phonological principles. Third, they are base-mutating in that they shift the accent of the base. Fourth, Aguaruna provides good evidence for a locality requirement on accent shift. As noted in Payne 1990: 181, the base accent must be "close enough' to the suffix in order to trigger the shift; compare the forms above with the nominative-accusative pair, ámuntai and ámuntai- $n$ 'buzzard', where the base accent does not shift because it is further from the suffix than is acceptable to condition the process.

A fifth important property of accent shift in these languages is that it is grammar dependent. In Japanese, the shift is to the rightmost syllable of the stem, which is the default edge for accent, as the analysis of noun-noun compounds given in $§ 3.3$ demonstrates. Likewise, in Aguaruna, the direction of the shift triggered by suffixation is to the right, and there is additional evidence in the language that accent is oriented to the right edge of the word. Thus, when accented vowels are deleted by the regular rules of syncope, the accent typically shifts to the right, as in /uNuš ínumi-š akam/ $\rightarrow u N u \check{s}$ núm-š akam 'tree species'. The fact that accent shift in the derived words above patterns with accent shift under syncope shows that the same principles are at work, which is the definition of grammar dependence.

These facts show that the morphologically triggered accent shifts in Japanese and Aguaruna have all of the formal properties of other affix-controlled processes like dominance effects and pre- and post-accentuation, and the central goal of this section is to provide a principled explanation of this finding. The larger theoretical framework for achieving this result is already in place: morphologically conditioned shifts are due to Transderivational Anti-Faithfulness. The only new element in the theory is to propose that the Prosodic Faithfulness constraint NO-FLOP-PROM has a counterpart in the set of TAF constraints, which is clearly an expected consequence of the theory of Anti-Faithfulness. The application of this new constraint to Aguaruna sketched below.

(97) Accent Shift in Aguaruna as Transderivational Anti-Faithfulness

\begin{tabular}{|c|c|c|c|c|}
\hline Base & /kawáu + na/ & ᄀOO-NO-FLOP-PM & OO-P-FAITH & ALIGN-R \\
\hline \hline a. kawáu & kawáu-n & $* !$ & & \\
\hline b. kawáu & káwau-n & & $*$ & $* *$ ! \\
\hline c. $\rightarrow$ kawáu & kawaú-n & & $*$ & \\
\hline
\end{tabular}

The accusative suffix is accent-shifting, and thus triggers an OO-correspondence relation upon which high-ranking $\neg$ OO-NO-FLOP-PROM is defined. This high-ranking TAF constraint rules out the fully faithful base-output pairing in (97a) because the two related outputs have identical prosody. The remaining two candidates therefore satisfy $\neg \mathrm{OO}-\mathrm{NO}-$ FLOP-PROM by shifting the accent in the derived form, but only the form in (97c) satisfies this constraint at the same time that it satisfies the lower-ranking constraint ALIGN-R. Since this constraint also has a role in the analysis of shift under syncope, the outcome here is predicted by an independently motivated constraint in the grammar. In this way, the analysis of morphological accent shifts in TAF theory explains the properties of this affixcontrolled process on par with the same explanation offered for other grammar dependent affix-controlled processes.

The remainder of this section is structured as follows. In the next subsection, a peculiar pattern of tone retraction in Limburg Dutch is studied and used as a means of motivating the basic constraint formula that is at work in accent shifts. Subsequently, $\$ 5.4 .3$ looks at various accent-shifting suffixes in Tokyo Japanese, and it is argued that this case provides additional empirical support for the overall approach because of the 
grammar dependent nature of the morpho-accentual process. Finally, an extended case study of accent in Aguaruna is given in §5.4.4, and an analysis of grammar dependent accent shift is presented as further support for the TAF approach.

\subsubsection{Dragging Tone Mutation in Limburg Dutch: Evidence for $\neg$ No-FLOP}

The two examples sketched above involve rightward accentual shifts which are triggered by suffixation. While a purely phonological account of these shifts is highly problematic (discussed in detail below), this perhaps more standard approach is not totally ruled out. In the case study that follows, an affix-controlled process in Limburg Dutch is examined which actually involves a retraction of an accent with suffixation. I show that this pattern of accentual mutation is an expected pattern of accent shift, given that the TAF constraint responsible for accent shifts does not specify a direction for the shift. I then discuss the problems which arise if one treats this pattern in purely phonological terms and compare this pattern with some other types of accentual shifts that can be attributed to negations of NO-FLOP constraints.

The morpho-accentual process in Limburg Dutch is clearly tonal in nature, and so the analysis requires the use of Faithfulness and Anti-Faithfulness for tone structure, which is independent of prominence structure. However, the same basic principles are at work in this case as those which will be employed in Japanese and Aguaruna below, and so it is appropriate to compare these different accentual systems. A final word before we begin; all of the data and many important analytical insights into the system come from Hermans 1991, which presents and analyzes the results of extensive fieldwork on the Maasbracht dialect of Dutch Limburg.

\subsubsection{Data and Observations}

Most dialects of Limburg Dutch (LD), including the Maasbracht dialect discussed here, show a contrast between a 'falling tone' and a 'dragging tone', which is exemplified in (98). Phonetically, the falling tone has a high tonal target at the onset of its syllable, which falls swiftly. The realization of the dragging tone is context-dependent. Utterancemedially, it is a level high; but utterance-finally it has a 'concave' structure, meaning that the first component is falling, while the second component is rising.

(98) Contrastive Accent in the Maasbracht dialect of Limburg Dutch

$\begin{array}{cll}\text { a. Falling } & \text { Tone } & \text { b. Dragging Tone } \\ \text { bíi } & \text { 'bee' } & \text { bíí 'at' } \\ \text { vríi } & \text { 'to court' } & \text { vríi 'free' } \\ \text { zúa } & \text { 'right!' } & \text { zúá 'like that' } \\ \text { bǽ } 1 & \text { 'to ring' } & \text { bǽí 'last name' } \\ \text { dǽ n } & \text { 'fir' } & \text { dǽń 'then' } \\ \text { píip } & \text { 'to squeak' } & \text { pííp 'pipe' } \\ \text { wíis } & \text { 'melody' } & \text { wíis 'wise' }\end{array}$

The main constraint on the distribution of this tonal contrast which is relevant here is that falling tones only contrast with dragging tones if the syllable has at least two sonorants beyond the onset cluster. In the examples above, the contrast is observed in syllables with two vowel slots or in syllables with a vowel plus a sonorant, but not in syllables with a 
single sonorant. Hermans therefore characterizes LD as a 'mora-accenting' language like Lithuanian because the distribution of accent in moraic subconstituents of the syllable is distinctive. ${ }^{14}$

In underived words, therefore, one observes a contrast between falling and dragging tones which is subject to certain phonological restrictions. This contrast is also governed by a morphological restriction. As exemplified below in (99a), certain suffixes cause the dragging tone of the base to become a falling tone. These suffixes include the masculine singular suffix - $\partial$ (a schwa-like vowel) which is added to adjectives (Masc.), the feminine counterpart to this suffix (Fem.), which is often segmentally null, and the comparative suffix - $\partial r$. The mutation affecting the dragging tone is blocked, however, when the stem-final consonant is (underlyingly) a voiceless obstruent, as shown by the examples in $(99 b) .{ }^{15}$

(99) Accent Shift in Derived Environments

\begin{tabular}{|c|c|c|c|c|c|}
\hline \multirow[t]{7}{*}{ a. } & wiís & $\begin{array}{l}\text { Masc. } \\
\text { wíiz- } \partial\end{array}$ & $\begin{array}{l}\text { Fem. } \\
\text { wíis }\end{array}$ & 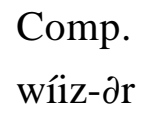 & 'wise' \\
\hline & stíif & stíiv- $\partial$ & stíif & 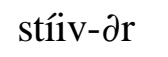 & 'stiff' \\
\hline & káál & káal-d & káal & káal-ər & 'bald' \\
\hline & láám & láam-d & láam & 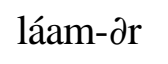 & 'paralyzed' \\
\hline & brúún & brúun- $\partial$ & brúun & brúun- $\partial \mathrm{r}$ & 'brown' \\
\hline & fiín & fíin- $\partial$ & fíin & fíin- $\partial \mathrm{r}$ & 'refined' \\
\hline & táám & táam- $\partial$ & táam & 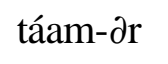 & 'tame' \\
\hline \multirow[t]{2}{*}{ b. } & ríík & ríík- $\partial$ & ríík-ə & 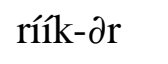 & 'rich' \\
\hline & záát & záát-d & záát-d & 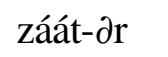 & 'drunk' \\
\hline
\end{tabular}

An important point is that this mutation is one-way. That is, while the dragging tone becomes a falling tone in this morphological context, the falling tone does not become the dragging tone, or a different tone, in the same context. Also, Ben Hermans (personal communication) points out that there is a locality restriction on the dragging tone mutation: in inflected nouns, the dragging tone only mutates to the falling tone if it appears in the stem-final syllable. Thus, in disyllabic nouns such as vóóX $l$ 'bird' and káám $\partial r$ 'room', inflected forms retain the dragging tone because it is not stem-final. ${ }^{16}$ This observation is

\footnotetext{
${ }^{14}$ In addition to this restriction, there are a number of distributional gaps which show certain segmental restrictions on the different tones. For example, the falling tone is not found in syllables ending in consonant clusters in which the final member is a (voiceless) obstruent. As these restrictions do not bear directly on the matters at hand, I refer the readers to Hermans' discussions, pp. 300, $302 \mathrm{ff}$., $312 \mathrm{ff}$. ${ }^{15}$ While many of the endings in this dialect trigger the tonal mutation exemplified here, it is not a consistent property of inflectional suffixes. For example, Ben Hermans (personal communication) states that the two overt allomorphs of the plural suffix do not trigger the change from a dragging to a falling tone, as shown by the word pairs: bláát/blááj- $\partial r$ 'leaf (singular/plural)' and búúr/búúr- $\partial$ 'peasant (singular/plural)'. So, it appears that whether or not an affix triggers the mutation is a property that must be lexically specified.

${ }^{16}$ Though there are some noun-verb pairs which show tonal slip on a non-final syllable; for example, the minimal pair $v A ́ E ́ r s ' I l$ 'difference' and $v A ́ E r s ' I l$ 'to differ' contrasts only in the tonal event in the steminitial syllable. This morpho-accentual process is clearly distinct, however, from the cases involving
} 
significant because it classifies the morpho-accentual process here with other affixcontrolled accentual processes which may also exhibit locality effects. To summarize the larger set of patterns, the inventory of underived words countenances a contrast between falling and dragging tones. This contrast is, however, neutralized in certain derived environments because of the mutation of the dragging tone in stem-final syllables.

\subsubsection{Tone in Monomorphemic Words}

Hermans' analysis of this mutation assumes that the accent-shifting suffixes have a underlying low tone which shifts to the second mora of the preceding syllable, effectively accounting for the loss of the dragging tone in exactly these contexts. While Hermans' analysis has it merits, especially in the analysis of the segmental restrictions on the mutation, I will pursue an alternative to this analysis here which establishes a relationship between the dragging tone mutation and the accent shifts found in Japanese and Aguaruna. Furthermore, there is another, more substantive, reason for developing this alternative. I seek to develop an analysis of LD accent which does not require a lexical contrast for low (L) tones, with the obvious advantage that lexical accent in LD would be comparable to other pitch accent languages which are described purely in terms of the distribution of a high $(\mathrm{H})$ tone (as argued in Pulleyblank 1986, Myers 1987a, Blevins 1993, among others). The analysis I develop here makes crucial use of a TAF constraint which requires an overt 'flop' of a tone as observed in the mutation of the dragging tone. Since this analysis is transderivational in nature, however, it requires first an analysis of the falling/dragging tone contrast. I present this analysis directly below, followed by the analysis of the dragging tone mutation.

In the analysis of the tonal inventory, I assume that the basic contrast is represented through the association of a H tone. Thus, following Blevin's 1993 analysis of Lithuanian, the falling tone has a $\mathrm{H}$ tone over the first sonorant, as shown below. On the other hand, the dragging tone is represented as a doubly-linked $\mathrm{H}$ tone, a structure argued for in many Bantu languages (see Odden 1995 and references therein) and for the Kyungsang dialects of Korean (Kim 1996). The tone-bearing units (TBUs) here are moras, and so I assume that sonorants which receive a $\mathrm{H}$ tone are moraic.

(100) Tonal Inventory in Underived Words

Falling Tone Dragging Tone

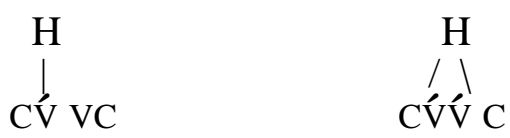

Concerning the context-dependent realization of the dragging tone, I assume that the observed concave structure utterance-finally is due to a boundary tone, presumably a phrase-final $\mathrm{H}$, which accounts for the elevated pitch curve phrase-finally.

One type of accentual pattern commonly found in mora-counting languages such as Greek and Lithuanian is missing in LD, namely a 'rising' accent in which an $\mathrm{H}$ target appears only on the second TBU of an accented syllable. The following constraint, formulated in the theory of Generalized Alignment (McCarthy \& Prince 1993a), accounts for this distributional gap.

inflectional suffixes examined here, as it is derivational. Further, it does not involve overt affixation, so the difference in the locus of the mutation does not come as a surprise. 
(101) Align-L(H, б)

The left edge of every $\mathrm{H}$ tone must coincide with the left edge of some syllable.

The representations for the falling and dragging tones will not violate ALIGN-L(H, $\sigma)$ because both accentual types have a left-aligned $\mathrm{H}$ tone. On the other hand, rising tones such as CVV́ C are systematically ruled out because they require an initial mora without a $\mathrm{H}$ tone. This same constraint is probably at work in Tokyo Japanese, where accented syllables always have accent on the initial mora. In mora-accenting languages which have rising tones, I assume that this constraint is dominated by the relevant Tone Faithfulness constraints.

The tonal specifications yielding the contrast here are tropic to a single syllable in a word - call it the tonic - and so in polysyllabic forms there is only one contrast between falling and dragging tones. While this issue is not central to our chief concerns here, I follow Hermans in assuming that there is a separate constraint in the language which requires an accent (realized tonally) on the tonic, which is the head of the main stress foot. Thus, within the framework of ideas in Hermans 1991, accent in Limburg Dutch is 'composite' in nature; an independent constraint requires the head position in the word to have a tonic, but the actual realization of the accent is characterized by the tonal structure of the head position. Also, it is clear that there is also a role for head syllable Tonal Faithfulness here (see Alderete 1995, 1997a, and Beckman 1997 [1998]), in order to account for the fact that specifically this syllable supports a tonal contrast, but non-head syllables do not. Following Yip 1996, who accounts for a related pattern of tonal reduction in Chinese dialects, I assume without argument that the Tonal Faithfulness constraints are relativized to the head of a stress foot, and that this accounts for the privileged Faithfulness properties of these positions in the word.

The analysis as it has been sketched thus far is consistent with a number of analyses of Bantu languages (see e.g., Pulleyblank 1986, Myers 1987a, 1997a, Bickmore 1996, 1997, Zoll 1996b) in which the tone structure of a form is described purely in terms of high tonal targets, with L tones, if needed at all, being filled in 'by default'. Conceived in terms of Markedness and Faithfulness constraints, a language with only surface $\mathrm{H}$ tones entails that $\mathrm{L}$ tones are more marked than $\mathrm{H}$ tones, as derived by the following Markedness subhierarchy.

(102) Tonal Markedness Subhierarchy (see Zoll 1996b and Myers 1997a)

$$
* \mathrm{~L} \gg * \mathrm{H}
$$

The grammar of a tone system with only surface H's is constructed by ranking the Tone Faithfulness constraints between these two Markedness constraints (illustrated below for LD). The Tone Faithfulness constraints (TONE-FAITH) which are ranked relative to these markedness constraints are given below, following some leading ideas in McCarthy 1997's formulation of Moraic Faithfulness constraints. 
MAX-TONE: 'No deletion'

Every tone in $\mathrm{S}_{1}$ has a correspondent in $\mathrm{S}_{2}$.

DEP-TONE: 'No insertion'

Every tone in $\mathrm{S}_{2}$ has a correspondent in $\mathrm{S}_{1}$.

NO-FLOP-TONE: 'No loss of links'

$\forall \mathrm{x} \forall \mathrm{y} \forall \mathrm{z}, \mathrm{x} \in$ tone, $\mathrm{y} \in$ sponsor, $\mathrm{z} \in$ link, if $\mathrm{x}$ and $\mathrm{y}$ are associated by $\mathrm{z}$ in $\mathrm{S}_{1}$, then $\exists \mathrm{x}^{\prime} \exists \mathrm{y}^{\prime} \exists \mathrm{z}$ ' s.t. $(\mathrm{x}, \mathrm{y}, \mathrm{z}) R\left(\mathrm{x}^{\prime}, \mathrm{y}^{\prime}, \mathrm{z}^{\prime}\right)$ and $\mathrm{x}^{\prime}$ and $\mathrm{y}^{\prime}$ are associated by $\mathrm{z}^{\prime}$ in $\mathrm{S}_{2}$.

NO-SPREAD-TONE: 'No insertion of links'

$\forall \mathrm{x} \forall \mathrm{y} \forall \mathrm{z}, \mathrm{x} \in$ tone, $\mathrm{y} \in$ sponsor, $\mathrm{z} \in$ link, if $\mathrm{x}$ and $\mathrm{y}$ are associated by $\mathrm{z}$ in $\mathrm{S}_{2}$, then $\exists \mathrm{x}^{\prime} \exists \mathrm{y}^{\prime} \exists \mathrm{z}$ ' s.t. $(\mathrm{x}, \mathrm{y}, \mathrm{z}) R\left(\mathrm{x}^{\prime}, \mathrm{y}^{\prime}, \mathrm{z}^{\prime}\right)$ and $\mathrm{x}^{\prime}$ and $\mathrm{y}^{\prime}$ are associated by $\mathrm{z}^{\prime}$ in $\mathrm{S}_{1}$.

The first three constraints are familiar since they have counterparts in the family of Prosodic Faithfulness constraints used throughout this thesis. A substantive difference between prominence structure and tonal structure, namely that tones may spread and be doubly linked, requires an additional constraint, NO-SPREAD-TONE, which militates against the spreading of a tone.

The rankings of these constraints required from Limburg Dutch are given below.

(104) Rankings for Limburg Dutch

a. *L > MAX-TONE: no lexical contrast for $\mathrm{L}$ tones.

b. MAX-TONE, NO-FLOP-TONE >> *H: lexical associations for $\mathrm{H}$ tones are faithfully mapped onto output forms (in head syllables).

c. ALign-L(H, $\sigma)$ > No-FLOP-TONE: $\mathrm{H}$ tones not linked to the first mora of syllable will be linked to the first more in the output.

The effects of these constraint rankings are illustrated in the large tableau in (105). First, with the Tonal Markedness constraint *L ranking above TONE-FAITH, specifically above MAX-TONE, surface forms only have $\mathrm{H}$ tones. Therefore, if an input has a lexical $\mathrm{L}$ tone, as in (105a) below ( $\mathrm{L}$ tones here are marked with a grave accent), then this tone is deleted in the output because of high-ranking *L. Second, the Alignment constraint ALIGN-L(H, $\sigma)$ rules out syllables without an initial $\mathrm{H}$ tone, as shown in the IO-mapping in $(105 \mathrm{c})$. Finally, the ensemble of TONE-FAITH constraints outrank $* \mathrm{H}$, and as a result, doubly associated $\mathrm{H}$ tones (i.e., the dragging tones), are faithfully mapped onto related outputs. In particular, because NO-FLOP-TONE dominates *H, the association of the lexical $\mathrm{H}$ to the second TBU is lost in the surface form, as shown in (105b). ${ }^{17}$

\footnotetext{
${ }^{17}$ This last ranking argument assumes that multiple associations of a single feature can lead to multiple violations of the related Featural Markedness constraint. If Beckman 1997 is right, and this type of association leads to a single violation of Featural Markedness, then this constraint ranking is unnecessary. A different constraint ranking is nonetheless required, in which the Tone Faithfulness constraints outrank the constraint prohibiting double association.
} 
(105) Illustration of Results for Inventory of Underived Words

\begin{tabular}{|c|c|c|c|c|c|c|}
\hline Input & & Output & $\operatorname{ALIGN-L}(\mathrm{H}, \sigma)$ & $* \mathrm{~L}$ & TONE-FAITH & $* \mathrm{H}$ \\
\hline \multirow[t]{2}{*}{ a. /b̂̂1/ } & $\rightarrow$ & bíi & & & $*($ MAX-TONE $)$ & \\
\hline & & *bî̀ & & $* !$ & & $*$ \\
\hline \multirow[t]{2}{*}{ b. /bíí/ } & $\rightarrow$ & bíí & & & & $* *$ \\
\hline & & *bíi & & & *(NO-FLOP-TONE)! & $*$ \\
\hline \multirow[t]{2}{*}{ c. /bií/ } & $\rightarrow$ & bíi & & & *(NO-FLOP-TONE) & $*$ \\
\hline & & *bií & $* !$ & & & $*$ \\
\hline
\end{tabular}

To give an interim summary, an accent is required in the head syllable of the main stress foot, which accounts for the observed distribution of accent. The realization of this accent tonally is governed further by $\operatorname{ALIGN-L}(\mathrm{H}, \sigma)$, the Tonal Markedness constraints, and a set of Tone Faithfulness constraints. The interaction of these constraints, shown in (105) above, accounts for the inventory of tonal contrasts in the head position of the word.

\subsubsection{Tonal Mutation in Derived Environments as $\neg$ NO-FLOP-TONE}

Now that I have given the constraint rankings necessary for the tonal inventory in underived words, we can move to the treatment of derived forms, which is relevant to the character of the Anti-Faithfulness constraint employed in accent shifts. As illustrated below, the tonal inventory in certain derived words is more restricted because of the mutation of the dragging tone.

(106) Tonal Inventory in Derived Words (with Accent-Mutating Suffixes)
a.
CV́V́ C
$\approx \quad \quad \quad C V^{\prime}$ VC-V
(Mutation: Dragging $\rightarrow$ Falling)
b. $\quad$ C V́ VC
$\approx \quad \quad \quad \quad V^{\prime} \mathrm{VC}-\mathrm{V}$
(No Mutation)

Any analysis of accent in LD will therefore need to account for the observed neutralization of contrast in these derived forms.

The phonological operation observed in the dragging tone mutation is the obligatory loss of an autosegmental link. Since NO-FLOP-TONE governs Faithfulness to this tone to sponsor affiliation, it follows that the negation of NO-FLOP-TONE will give the desired outcome, as spelled out below.

(107) $\neg$ NO-FLOP-TONE

$\neg\left[\forall \mathrm{x} \forall \mathrm{y} \forall \mathrm{z}, \mathrm{x} \in\right.$ tone, $\mathrm{y} \in$ sponsor, $\mathrm{z} \in$ link, if $\mathrm{x}$ and $\mathrm{y}$ are associated by $\mathrm{z}$ in $\mathrm{S}_{1}$, then $\exists \mathrm{x}^{\prime} \exists \mathrm{y}^{\prime} \exists \mathrm{z}$ ' s.t. $(\mathrm{x}, \mathrm{y}, \mathrm{z}) R\left(\mathrm{x}^{\prime}, \mathrm{y}^{\prime}, \mathrm{z}^{\prime}\right)$ and $\mathrm{x}^{\prime}$ and $\mathrm{y}^{\prime}$ are associated by $\mathrm{z}$ ' in $\mathrm{S}_{2}$.]

While $\neg$ NO-FLOP-TONE can be satisfied by a complete shift of a tone to a neighboring TBU, this constraint is also satisfied when a doubly-linked structure loses a link, which is 
exactly the observed pattern in LD. This result is illustrated below with explicit representations of the base-derivative mapping.

(108) Structural Characteristics of Dragging Tone Mutation

Base Derivative<smiles>[Al]C=[Al]</smiles>

The second mora (dominating the second vowel) in the base stands in correspondence with the second mora of the derivative. The $\mathrm{H}$ tones likewise stand in correspondence; thus, since the base $\mathrm{H}$ is associated with the second mora, the loss of a link to the corresponding mora in the derivative violates NO-FLOP-TONE as defined above. Therefore, this loss of a link to a base $\mathrm{H}$ tone satisfies the negation of this Tone Faithfulness constraint, $\neg$ NoFLOP-TONE. Another important role for correspondence of links is that it allows for nonovert satisfaction of a TAF constraint, as needed in the analysis of accent shifts in Japanese (discussed in §5.4.3).

Formulated as a TAF constraint, $\neg$ OO-NO-FLOP-TONE will operate on basederivative pairs. Using the usual ranking logic, the following schematic rankings predicts the presence or absence of a tone flop mutation.

(109) Schematic Rankings

\section{a. Obligatory Tone Flop: $\neg$ OO-NO-FLOP-TONE >> OO-NO-FLOP-TONE \\ b. No Tone Flop: OO-NO-FLOP-TONE >> $\neg$ OO-NO-FLOP-TONE}

The dragging to falling tone alternation can thus be explained by stipulating that the accentmutating suffixes shown above subcategorize for an OO-correspondence relation, and upon this relation, the ranking in (109a) holds. As noted in \$5.4.2.1, the dragging tone mutation is only found in the stem-final syllable, showing that the Anti-Faithfulness effect is restricted to the syllable adjacent to the base-mutating affix. In the framework for locality effects developed in $\S 4.3$, this restriction is described by locally conjoining the TAF constraint with ANCHOR(Stem, PrWd, R) in the domain of the syllable, i.e., ( $\neg \mathrm{OO}-\mathrm{NO}-$ FLOP-TONE \& ANCHOR(Stem, PrWd, R) $)_{\sigma}$, which I will abbreviate as $\neg$ OO-NO-FLOP-

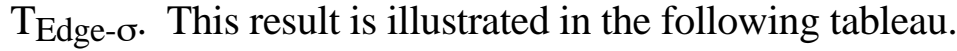

(110) Tone in Derived Words Part 1: Mutate Dragging to Falling

\begin{tabular}{|c|c|c|c|c|}
\hline Base & /CV́V́ C + V/ & ALIGN-L & $\neg \mathrm{OO}-\mathrm{NO}-\mathrm{FLOP}-\mathrm{T}_{\text {Edge- } \sigma}$ & OO-NO-FLOP-T \\
\hline a. $\rightarrow$ CV́v C & CV́ VC-V & & & * \\
\hline b. CV́f C & CV́V C-V & & $* !$ & \\
\hline c. Cúv C & CVV́ C-V & $* !$ & & \\
\hline
\end{tabular}

The faithful mapping shown in (110b) is ruled out because it violates the TAF constraint $\neg$ OO-NO-FLOP-TONE, and this constraint dominates the OO-Tone Faithfulness constraint OO-NO-FLOP-TONE. The remaining candidates mutate the tonal structure of the base in different ways: candidate (110a) loses the link between the $\mathrm{H}$ tone and the second mora, while candidate (110c) loses the association with the first mora. The latter option can be 
ruled out in a principled fashion because this candidate violates an independently attested constraint in the grammar, namely $\operatorname{ALIGN}-\mathrm{L}(\mathrm{H}, \sigma)$. In other words, the mutation of the dragging tone to a rising tone is ruled out because this mapping would produce a structure that is generally avoided in the language. Finally, words which have a dragging tone on a non-final syllable do not experience a change because the tonal unit in this case is outside the scope of the complex Anti-Faithfulness constraint.

The next step in the analysis is to account for the lack of mutation in mappings in which the base has a falling tone. The same basic constraints are at work in this case too, except a lower-ranking OO-TONE-FAITH decides the final outcome. Thus, given a base with a falling tone, the derivative cannot shift the $\mathrm{H}$ tone one mora to the right, as in (111c), because this mapping gives a rising tonal contour, and such configurations are not allowed. In particular, the absence of such a mutation shows that ALIGN-L(H, $\sigma)$ dominates the TAF constraint in this system. The remaining two candidates, (111a) and (111b), fail to mutate the derived form in the required way as neither brings about a loss of an association with the base sponsor of the $\mathrm{H}$ tone. Lower-ranking Tone Faithfulness therefore decides the competition between these two candidates, favoring the faithful candidate because it avoids a violation of the anti-spreading constraint for tones.

(111) Tone in Derived Words Part 2: Don't Mutate Falling to Dragging

\begin{tabular}{|c|c|c|c|c|}
\hline Base & /CV́ VC + V/ & ALIGN-L & ᄀOO-NO-FLOP-T Edge- & OO-NO-SPREAD-T \\
\hline \hline a. $\rightarrow$ CV́ VC & CV́ VC-V & & $*$ & \\
\hline b. $\quad$ CV́ VC & C'V́V C-V & & $*$ & $* !$ \\
\hline c. $\quad$ CV́ VC & CVV́ C-V & $* !$ & & $*$ \\
\hline
\end{tabular}

In other words, this ranking of TAF constraint relative to the Tone Faithfulness constraints yields a flopping mutation only when the change felt in the base-derivative pair does not violate the high-ranking Alignment constraint. When the mutation would require a violation of this constraint, the system opts not to change the derivative at all. The analysis therefore shows another type of grammar dependent mutation in that high-ranking constraints in the grammar predict both the pattern of Anti-Faithfulness observed in the system, as observed in the tableau in (110), and, whether or not the mutation takes place at all, as shown in (111). Thus, in addition to governing the ultimate outcome of a process, a top-ranked constraint may actually block a process altogether.

These results are reflected in the constraint rankings given below in which the Alignment constraint is top-ranked in both subsystems.

(112) Summary Rankings for Accent Shift in Limburg Dutch

Rankings for Derived Words

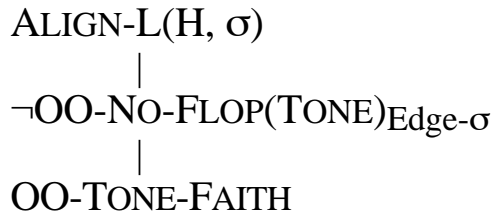

\section{Rankings for Underived Words}

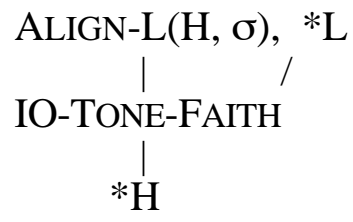

To summarize the results reached above, I have proposed an analysis of a morphological pattern of tonal mutation with the use of Faithfulness and Anti-Faithfulness constraints for tone structure. The proposed analysis explains all of the basic properties of 
this morphological pattern, i.e., that it is lexically idiosyncratic, morphologically triggered, base-mutating, grammar dependent, and subject to locality effects. Furthermore, the same basic mechanisms at work in the analysis of tone flop in Limburg Dutch are also employed in the analysis of accent shifts in Japanese and Aguaruna developed below, and so the proposed analysis explains these properties in a way that extends to other languages.

Before bringing this case study to a close, it is important to contrast the analysis of accent shift given here with a purely phonological analysis of the problem. Hermans 1991 proposes that the suffixes which have the effect of mutating the dragging tone are specified for a $\mathrm{L}$ tone, which, when it docks to the second component of an accented syllable, triggers a de-linking of doubly-linked $\mathrm{H}$ tone, as sketched below

\section{(113) Phonological Account of Dragging Tone Mutation}

Input Output

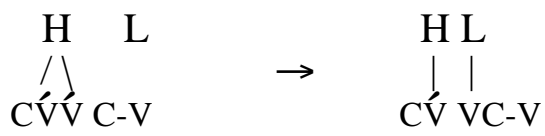

It is difficult to compare Hermans' actual analysis with the one made here as they are formulated in very different frameworks, and so I will not enter into the formal details. The chief difference is that Hermans' analysis is phonological, while the TAF analysis treats the dragging tone mutation as a morphological phenomenon. This larger theoretical difference between the two theories leads to a substantive formal difference, however, which is that the phonological account entails lexical specification of a $\mathrm{L}$ tone to account for the retraction of the doubly-linked structure. The analysis presented here accounts for accent purely in terms of $\mathrm{H}$ tones, and as emphasized above, the analysis is in line with recent approaches to tonal accent systems like the one given in Pulleyblank 1986 for Tonga. The advantages of the pre-linked $\mathrm{H}$ tone analyses of accent therefore apply to this case, tipping the scales in favor of the TAF analysis.

A second point in favor of the TAF analysis is that it relates a wide range of morphologically triggered shifts as effects of a specific type of Anti-Faithfulness. Thus, the loss of a link in the dragging tone mutation is treated on a par with the obligatory shifts found in Japanese and Aguaruna. Both of these cases are modelled as responses to a constraint type that requires a severing of the ties between a suprasegmental and its sponsor, namely $\neg$ NO-FLOP. This analysis differs from the phonological account here which is clearly designed for Limburg Dutch alone. For example, purely phonological accounts of Japanese involve the insertion of an autosegmental link with affixation (Poser 1984), which is clearly inappropriate for the dragging tone mutation.

A final argument in favor of the account of the accentual mutation in terms of AntiFaithfulness is that it explains the relation between the properties of the accent shift with independently needed constraints. The Anti-Faithfulness constraint $\neg$ NO-FLOP says literally nothing about the output of the accentual shift; it simply states that an existing accent must change in some way. As is common in ACA, the rest of the grammar then kicks in and determines the actual shape of the mutation. In LD, we have found that independently needed constraints had a role in both predicting the outcome of the change and blocking another logically possible shift. This aspect of the analysis truly distinguishes the TAF theoretic approach to accent shifts from purely phonological analyses which simply posit the necessary elements to get the facts right. In summary, the TAF approach clarifies the role of the larger grammar in the process, while the phonological analysis masks this fundamental property of accentual shifts. 
One interesting question that arises in the comparison of these two analyses is whether or not one finds a morphological construction in which accent is shifted in two different directions. The phonological analysis, with a lexical L tone, predicts that shifts will only flop, or be retracted, in one direction, basically shifting away from the lexically specified tone in the affix. In the morphological analysis, on the other hand, the unidirectional pattern is only due to the language particular ranking of ALIGN-L(H, $\sigma)$ over the TAF constraint. If $\neg$ NO-FLOP-TONE was undominated, a shift in both directions would be possible because the Anti-Faithfulness constraint does not specify the direction of the shift. The existence of a morphologically triggered bi-directional shift would therefore constitute a further argument in favor of the theory proposed here.

Stress shift in two related consecutive constructions in Tiberian Hebrew appears to be a case in point (see McCarthy 1979a for discussion and analysis of the relation between these shifts and other phonological patterns in Hebrew). In imperfect consecutives, stress shifts from the final to the penultimate syllable, as in wayyáaqom 'and he arose', from the related jussive yaaqóom 'let him arise' (the final vowel is underlyingly short); this shift is blocked, however, if the penultimate syllable does not contain a long vowel, as shown by wayyabdéel 'and he divided'. In perfect consecutives, stress shifts from the penult to the ultima if the penultimate syllable has a short vowel, as in wdkaatabtáa 'and you will write', cf. wdqaaráataa 'and you will read' (the underlined vowel shows the position of stress in the basic finite verb). While these are not identical morphological constructions, they are related in that they both produce verbs with consecutive meanings. Therefore, the fact that they exhibit similar phonological patterns, yielding a shift in stress and showing sensitivity to vowel length in the penultimate syllable, suggests that they should be treated as the result of the same morpho-accentual process. If both the perfective and imperfective consecutives are subjected to the TAF constraint $\neg$ NO-FLOP-PROM, both shifts can be understood as a forced breach of Faithfulness for morphological reasons. Furthermore, as with the case of Limburg Dutch, the Anti-Faithfulness constraint is dominated, presumably by a constraint akin to Prince's 1990 Weight-to-Stress Principle, to account for the triggering and blocking of the shift in words with heavy penultimate syllables. Thus, while there are further details of the analysis to be worked out here, this case appears to represent a bi-directional shift of the type predicted by the TAF theory of accent shift.

\subsubsection{Case Study: Accent Shift in Tokyo Japanese}

As pointed out in the introduction, certain suffixes in Tokyo Japanese trigger a shift of the lexical accent of the base to which they are attached. Furthermore, this shift is to a default position for these affixed structures, showing that the morphologically triggered shift is grammar dependent. In this case study, I develop an analysis of this affixcontrolled process as an effect of the Anti-Faithfulness constraint $\neg$ NO-FLOP-PROM, similar to the analysis of the dragging tone mutation in Limburg Dutch and other grammar dependent affix-controlled processes.

The suffixes of interest here pattern in certain respects like compounds as they attach to an uninflected stem. These suffixes are mentioned in passing in McCawley 1968: 166-67 as oddities for his rules of noun-noun compounds. Later, Poser 1984 takes these cases head on, incorporating them into a complete typology of morpho-accentual rules as 'dependent affixes', so-called because they only trigger a process when they attach to an accented stem. Some examples of two representative suffixes are given below (from Poser 1984, with some additions). 
(114) Accent-shifting Suffix -mono 'thing'
a. /káki + mono/ $\rightarrow$ kakí-mono 'scroll'
yómi + mono/ $\rightarrow$ yomí-mono $\quad$ 'reading matter'
b. /nori + mono/ $\rightarrow$ nori-mono 'vehicle' /wasure + mono/ $\rightarrow$ wasure-mono $\quad$ 'forgotten item'

(115) Accent-shifting Suffix - ya 'keeper, seller of/house of X'
a.
$\begin{array}{lll}/ \text { koná }+ \text { ya/ } & \rightarrow & \text { koná-ya } \\ \text { /nomí }+ \text { ya/ } & \rightarrow & \text { nomí-ya }\end{array}$
$/$ kúzu + ya/ $\quad \rightarrow \quad$ kuzú-ya
'flour seller'
/sóba + ya/
$\rightarrow \quad$ sobá-ya
'bar, saloon'
'junk man'
'noodle house'
b. /kabu + ya/ $\rightarrow$ kabu-ya $\quad$ 'stockbroker'
/toma + ya/ $\rightarrow$ toma-ya 'mat seller'
/tindon + ya/ $\rightarrow$ tindon-ya $\quad$ 'traditional Japanese band'
/unsoo + ya/ $\rightarrow$ unsoo-ya 'forwarding agency'

The salient observation here is that these suffixes cause a shift of the lexical base accent to the stem-final syllable, as in (114a) and (115a). On the other hand, when these suffixes attach to an unaccented stem, the stem is left unaccented, creating unaccented words, as in $(114 b)$ and $(115 b)$.

A variation on this pattern is exemplified with the agentive suffix -te, which, like the two suffixes above, triggers a shift of a lexical accent. However, the shift of this accent is to the suffix itself, as shown in (116a) below.

(116) Agentive Suffix -te (Poser 1984: 50)
a. /káki + te/
$\rightarrow \quad$ kaki-té
/yómi + te/
$\rightarrow$ yomi-té
'writer'
'reader'
b. /katari + te/
$\rightarrow \quad$ katari-te
'narrator'
/kiki + te/
$\rightarrow \quad$ kiki-te
'hearer'

McCawley's description of the particle nagara 'though, in spite of', also fits this pattern, as it induces a shift of the accent of the base to the first syllable of the particle, as in: /ínoti + nagara/ $\rightarrow$ inoti-nágara 'in spite of life', cf. /miyako + nagara/ $\rightarrow$ miyako-nagara 'in spite of the city'. I will argue below that this class of suffixes is different in an important way from -mono and $-y a$ because they involve a banishment of the lexical accent from the stem, which invites an analysis in terms of dominance effects discussed in \$5.2.

Exploiting the full power of autosegmental representations, Poser 1984 treats the accent-shifting suffixes above as triggering an insertion of a link over a designated syllable. The fact that the shift in accent depends on the presence of an existing accent therefore 
follows from the assumption that no accents are inserted. Accented stems will undergo a shift, docking their accent to the inserted autosegmental link, but unaccented stems will be left unchanged. In what follows, I develop an alternative to this account which is more restrictive. This analysis also accounts for the fact that the shift is only found in words with accented stems, but more interestingly, it predicts that the shift will be towards the language particular default pattern for accent. Thus, in the case of the accent-shifting suffixes above, I will argue that it is no accident that these suffixes trigger a shift to the stem-final syllable or the first vowel of the suffix; these are the default positions for accent in compounds, and since these affixed words are compound-like, these are the predicted patterns of Anti-Faithfulness.

To be more concrete, I posit a structure for the affixed structures which is akin to the structures given in $\$ 3.3$ for noun-noun compounds. In particular, I propose that the words containing accent-shifting suffixes have a recursive PrWd, as shown below.

\section{(117) Proposed Structures for Words with Affix-Shifting Suffixes}
a. [[ kakí ]PrWd [mono] $\left.]_{P r W d}\right]_{P r W d}$
b. [[ kaki ]PrWd [ té ] PrWd]PrWd

These words are not structurally identical to compounds as they are not dominated by the higher level category used in the analysis of compounds, namely P-Comp. This difference is important, as these structures pattern differently than compounds in one important way: they may be unaccented. The absence of a superordinate P-Comp predicts this difference because the obligatory accent found in noun-noun compounds is due to a requirement that the PrWd head of the P-Comp must have an accent; these structures do not have an analogous head, and so they do not trigger an insertion of accent in words with unaccented morphemes.

But words with affix-shifting suffixes have some important similarities with compounds, and the related structures for these words provides a means of explaining these similarities. To begin first with a morphological fact, many of the suffixes above attach to the uninflected, or conjunctive, form of the verb, which is also observed in compounds. If we assume that these suffixes have the same basic morphological collocation as compounds, presumably attaching to morphological words, then the fact that these affixed words and compounds attach to the same verbal form can be tied to their similar morphological frame. A second important point is that the accentual defaults in words with accent-shifting suffixes are paralleled in compounds (as described in $\S 3.3$, following Kubozono 1995). Thus, in compounds with short second members, accent falls on the final syllable of the first member, as observed above in words with -mono and $-y a$. When the second member of a compound is long, however, accent is assigned to this member, with default initial accent on $\mathrm{N}_{2}$. Both of the affixed words above which have accent on the suffix, i.e., words with -te and nagara, have accent on the initial syllable of these morphemes (though -te is sub-minimal for the purposes of accent assignment in compounds, a point which I return to below). It is clear therefore that some of the assumptions made in the analysis of noun-noun compounds will apply in words with accent-shifting suffixes, which will become apparent in the analysis of particular affixes given below.

The phonological operation observed with suffixes like -mono is a shift of the base accent. Words with unaccented stems, however, are unaffected by these suffixes, showing that the accent shift is not an insertion of accent itself, but a mutation of an existing accent. Since one of the Prosodic Faithfulness constraints developed in \$1.2.2.1, NO-FLOPPROM, specifically bans this type of phonological activity, it is natural to model this accentual process as a forced violation of this type of Faithfulness. Thus, the negation of 
NO-FLOP-PROM, using the formula for Anti-Faithfulness constraints given in chapter 4, yields exactly the right tool for the job, as shown below.

(118) $\neg$ NO-FLOP-PROM

$\neg\left[\forall \mathrm{x} \forall \mathrm{y} \forall \mathrm{z}, \mathrm{x} \in\right.$ prominence, $\mathrm{y} \in$ sponsor, $\mathrm{z} \in$ link, if $\mathrm{x}$ and $\mathrm{y}$ are associated by $\mathrm{z}$ in $\mathrm{S}_{1}$, then $\exists x^{\prime} \exists y^{\prime} \exists z^{\prime}$ s.t. $(\mathrm{x}, \mathrm{y}, \mathrm{z}) R\left(\mathrm{x}^{\prime}, \mathrm{y}^{\prime}, \mathrm{z}^{\prime}\right)$ and $\mathrm{x}^{\prime}$ and $\mathrm{y}^{\prime}$ are associated by $\mathrm{z}$ ' in $\mathrm{S}_{2}$.]

To paraphrase this constraint, $\neg$ NO-FLOP-PROM entails that it must not be the case that corresponding prominences have corresponding sponsors; or alternatively, that corresponding prominences have corresponding links. The inclusion of autosegmental links is crucial in the definition, as it allows $\neg$ NO-FLOP-PROM to be satisfied by just the deletion and insertion of a link. This assumption will allow the non-overt satisfaction of $\neg$ NO-FLOP-PROM, which is necessary in Japanese, as shown by input-output mappings like /koná + ya/ $\rightarrow$ koná-ya. Thus, the analysis of the apparent lack of shift in these cases is handled in a similar way as non-overt Anti-Faithfulness in pre-accentuation.

As with all Anti-Faithfulness effects, they derive from Transderivational AntiFaithfulness, and hence, the important constraint rankings will involve OO-AntiFaithfulness, as shown below.

(119) Schematic Rankings

\section{a. Obligatory Accent Shift: $\neg$ OO-NO-FLOP-PROM >> OO-NO-FLOP-PROM \\ b. No Accent Shift: OO-NO-FLOP-PROM >> $\neg$ OO-NO-FLOP-PROM}

When the TAF constraint $\neg$ OO-NO-FLOP-PROM is ranked above its corresponding Faithfulness constraint, the result will be an obligatory accent shift. When the reverse ranking holds, no shift is predicted. In the now familiar way, these two rankings can be incorporated in the same constraint system using multiple correspondence relations, as shown below in (120a). The accent-shifting suffixes observed above will subcategorize for $\mathrm{OO}_{\mathrm{Dom}}$-correspondence, as shown in (121), which predicts that words with these

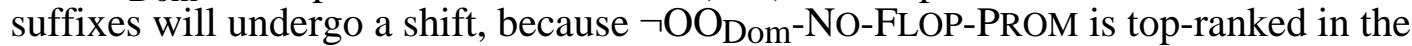
constraint hierarchy. A second important set of rankings, carried over from the analysis of compound accent given in chapter 3, is given in (120b). This ranking gives the PrWd-final default for accent in compounds with accented initial members and initial accent on finallyaccented members - an accentual default which is important in determining the landing site for shifted accents, as I will demonstrate below. ${ }^{18}$

(120) Accent Shift in Tokyo Japanese
a. $\neg \mathrm{OO}_{\text {Dom }}$-NO-FLOP-PM $>$ OO-NO-FLOP-PM $\gg>\mathrm{OO}_{\mathrm{Rec}^{-N O}-\mathrm{FLOP}-\mathrm{PM}}$
b. NONFInALITYComp $>$ Align-R(PM, PrWd) $\gg$ Align-L(PM, PrWd)

(121) Lexical Entries for Accenting-Shifting Suffixes

$\begin{array}{lll}- \text { mono } & \mathrm{N} & {\left[[\text { Noun }] \text { OODom } \_\right]} \\ -y a & \mathrm{~N} & {\left[\left[\text { Verb]oODom } \_\right]\right.}\end{array}$

\footnotetext{
${ }^{18}$ The NONFINALITY constraint here is somewhat different than the one used in $\$ 3.3$ as it is relativized to any prosodic compound, not just P-Comps, which will be important in deriving the avoidance of final accent in these words when the suffix is accented.
} 
While non-accent-shifting suffixes are not listed above, most Japanese suffixes do not pattern with -mono and -ya, showing that this morpho-accentual process is lexically idiosyncratic. Accordingly, the non-accent-shifting suffixes subcategorize for $\mathrm{OO}_{\mathrm{Rec}^{-}}$ correspondence and therefore do not trigger a shift.

The assumptions developed thus far yield precisely the pattern of accent shift observed above for suffixes like -mono, as I will now demonstrate. Starting first with words with accented stems, the attachment of -mono in such contexts triggers a shift to the stem-final syllable. This is because words with -mono are evaluated by $\neg \mathrm{OO}_{\mathrm{Dom}}-\mathrm{NO}-$ FLOP-PROM, and this constraint is top-ranked in the constraint hierarchy. Thus, if the accent of the base does not shift, as in (122a) below, such an output incurs a fatal violation of the top-ranked TAF constraint. A second alternative, shown in (122b), is also ruled out by $\neg \mathrm{OO}_{\text {Dom }}$-NO-FLOP-PROM because this candidate does not mutate the base in the relevant way. Succinctly, the TAF constraint requires a shift of the lexical accent within the base in the output. Given that the base káku only stands in correspondence with the stem of the suffixed form (see $\$ 4.2$ ), the candidate in (122b) has only undergone deletion of accent, not accent shift, and so it does not mutate the base in the right way (a point which is also crucial in words with -te discussed below). The winner then is the form which shifts the base accent to the default PrWd-final position.

(122) Accent Shift with Accented Base

\begin{tabular}{|lc|c|c|c|}
\hline \multicolumn{2}{|c|}{ Base } & /káki + mono/ & $\neg O_{\text {Dom }}$-NO-FLOP-PM & OO-NO-FLOP-PM \\
\hline \hline a. $\quad$ káku & {$[[$ káki]-[mono] $]$} & $* !$ & \\
\hline b. $\quad$ káku & {$[[$ kaki]]-[móno]] } & $* !$ & $*$ \\
\hline c. $\rightarrow$ káku & {$[[$ kakí]-[mono]] } & & \\
\hline
\end{tabular}

Words with unaccented stems will not undergo a shift for the simple reason that the TAF constraint $\neg$ OO-NO-FLOP-PROM is not relevant here. In this case, there is no accent in the base to shift, and so the condition specified in the antecedent to the implication of the constraint in (118) is not met. Thus, the two structures shown below are equal on the TAF constraint, and so the decision falls on the shoulders of the Faithfulness constraint IO-DEPPROM. In other words, the insertion of an accent into a default position does not lead to

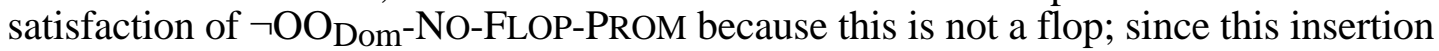
gives a gratuitous violation of Faithfulness, it is ruled out in favor of the unaccented word, as shown below.

(123) No Accent Shift with Unaccented Base

\begin{tabular}{|l|c|c|c|}
\hline \multicolumn{1}{|c|}{ Base } & $/$ nori + mono/ & $\neg$ OO $_{\text {Dom }}$-NO-FLOP-PM & IO-DEP-PROM \\
\hline \hline a. nori & {$[[$ norí]-[mono] $]$} & & $* !$ \\
\hline b. $\rightarrow$ nori & {$[[$ nori] $]-[$ mono] $]$} & & \\
\hline
\end{tabular}

This peculiar behavior of accent-shifting suffixes therefore receives a direct and natural account in TAF theory. The constraint $\neg \mathrm{OO}_{\text {Dom }}$-NO-FLOP-PM requires a mutation of an existing accent, but in unaccented words, there is no accent to be mutated, and so the TAF constraint is vacuously satisfied. 
Finally, the tableau below shows the role of the Alignment constraint in deriving default PrWd-final accent (with a hypothetical example). ${ }^{19}$ The first two options (124a-b) are ruled out in the same way they are above in (122); these structures lead to a fatal violation of the TAF constraint $\neg \mathrm{OO}_{\text {Dom }}$-NO-FLOP-PROM. Thus, the accent of the base must shift within the stem, but the TAF constraint says nothing about the landing site of the shifted accent (other than it must stay within the base). The decision in this case therefore goes to lower-ranking constraints in the grammar which are responsible for the accentual default pattern for these structures. As argued in detail in chapter 3, PrWd-final accent is the default position for compounds with accent on the first member. Since these structures are also compound-like in that they have two components wrapped by independent PrWds, this is the accentual default here too, as determined by the Alignment constraint ALIGN-R. So, contrasting the PrWd-penultimate and PrWd-final accents in (124c) and (124d) respectively, the latter is the winner because it fully satisfies ALIGN-R.

(124) Accent Shift with Long Accented Base

\begin{tabular}{|lc|c|c|c:c|}
\hline \multicolumn{2}{|c|}{ Base } & /títiti + mono/ & $\neg$ OODom-NO-FLOP-PM & OO-NO-FLOP-PM & ALIGN-R \\
\hline \hline a. $\quad$ títiti & [[títiti]-[mono]] & $* !$ & & $*$ \\
\hline b. $\quad$ títiti & [[tititi]-[móno]] & $* !$ & & $*$ \\
\hline c. $\quad$ títiti & [[titíti]-[mono]] & & $*$ & $* !$ \\
\hline d. $\rightarrow$ títiti & [[titití]-[mono]] & & $*$ & \\
\hline
\end{tabular}

This result shows that the morphologically triggered accent shifts are grammar dependent because the independently attested constraints on the distribution of accent predict the ultimate resting spot of a perturbed accent. As I show directly below, the shifted accent which lands on the first vowel of the suffix is also grammar dependent, but in a different way.

Suffixes like -te trigger a shift, but not to the final syllable of the stem which sponsors the accent. Instead, these suffixes trigger a shift to the first syllable of the suffix itself, as shown by the behavior of nagara above. Given the paucity of suffixes showing this pattern, it is not clear whether this is a linguistically significant pattern or just a quirk of these two suffixes. However, it is striking that these suffixes have the second accentual default pattern found in compounds, namely initial accent in the second member. To develop the parallel with the suffixes -mono and -ya above, I therefore treat this pattern as significant.

Accented, accent-shifting suffixes like -te are different than the unaccented ones, however, because they trigger a shift that is non-base-mutating by $\neg \mathrm{OO}_{\mathrm{Dom}}-\mathrm{NO}-\mathrm{FLOP}-$ PROM: the shift goes outside the stem itself (this is crucial in the analysis of -mono). Clearly, this TAF constraint is not at work in words with -te. However, its cousin, $\neg \mathrm{OO}-$ MAX-PROM, can be at play here, explaining the shift as a shunning of the base accent from the stem, i.e., a dominance effect in the framework developed in $\$ 5.2$ for such processes. Furthermore, the preservation of a lexical accent can be attributed to other factors,

\footnotetext{
${ }^{19}$ Ikuyo Kaneko (personal communication) points out that stems which would show a long distance shift of the type predicted here may in fact not be attested. For example, in words with trisyllabic stems and initial accent, the result is an unaccented word, as shown by /kísetu + mono/ $\rightarrow$ kisetu-mono 'seasonal thing'. If further investigation shows that this pattern is indeed a systematic gap, then a locality restriction on the position of the lexical accent, presumably foot-based, may need to be posited (see \$5.4.4 for a similar locality restriction on shifts in Aguaruna). This finding would not refute the claim that these shifts are grammar dependent, however, as shifts in disyllabic stems are rightward, to a stem-final syllable.
} 
presumably the IO-Faithfulness constraint MAX-PROMRoot. In other words, the behavior of -te involves a peculiar set of factors, involving both Anti-Faithfulness and RootFaithfulness, as depicted below.

(125) Accent Shift as Dominance Effect with Root-Faithfulness

\begin{tabular}{|c|c|c|c|c|}
\hline Base & /káki + te/ & $\neg \mathrm{OO}-\mathrm{MAX}-\mathrm{PM}$ & IO-MAX-PMRoot & NONFINALITY \\
\hline a. káku & [[káki]-[te]] & $* !$ & & \\
\hline b. káku & [[kakí]-[te]] & $* !$ & & \\
\hline c. káku & {$[[\mathrm{kaki}]-[\mathrm{te}]]$} & & $* !$ & \\
\hline d. $\rightarrow$ káku & [[kaki]-[té]] & & & * \\
\hline
\end{tabular}

The fully faithful form is ruled out because it violates the constraint requiring a deletion of base prosody, namely $\neg$ OO-MAX-PROM. The base accent is not simply shifted to the preferred base-final position because this does not satisfy the TAF constraint either (125b). Furthermore, the accent of the base cannot be deleted because this would violate an operative constraint here, namely IO-MAX-PROMRoot, as shown in (125c). The end result then is accent on the suffix itself (125d). This result is certainly marked in compounds because of the role of NONFINALITY in these cases, but as shown explicitly above, there are no other options here. A final important point here is that this effect is only possible under Stem-to-Stem correspondence (argued for in chapter 4, section 2), because the accent shift observed in (125d) can only be viewed as a deletion under this conception of OOcorrespondence.

In the case of the accented, accent-shifting particle nagara, the analysis is similar to that of -te, but the size of the morpheme permits satisfaction of NONFINALITY and, accordingly, gives leftmost accent in the second member. This pattern is of course the same pattern observed in compounds where the second member receives an accent, as in /minami + amerika/ $\rightarrow$ minami-ámerika 'South America'. Since these affixed structures are, by hypothesis, also compound-like, the predicted default here is initial accent on the second PrWd, as shown below.

(126) Default Initial Accent in Second Member

\begin{tabular}{|c|c|c|c|c|c|}
\hline Base & /ínoti + nagara/ & $\neg$ ОО-МАХ-РМ & NONFINAL & ALIGN-R & ALIGN-L \\
\hline a. ínoti & [[ínoti]-[nagara] ] & $* !$ & & & \\
\hline b. ínoti & [[inoti]-[nagará] ] & & $* !$ & * & $* !$ \\
\hline c. $\rightarrow$ ínoti & [[inoti]-[nágara] ] & & & $*$ & \\
\hline
\end{tabular}

The appeal to NONFINALITY to get the PrWd-initial default here involves a minor inconsistency with the analysis of compounds, as this constraint was relativized to the PComp in $\$ 3.3$, and these suffixed structures have no P-Comp (necessarily, because they may be unaccented). I assume therefore that the relevant prosodic category referred to by NONFINALITY is the highest prosodic category above the innermost PrWd, which generalizes to both compounds and these pseudo-compounds.

The treatment of this peculiar pattern of accent shift as a dominance effect is different from the usual type of dominance discussed in section $§ 5.2$, e.g., in words with the dominant unaccented suffix $-k k o$, as this type crucially preserves the accent of the root. 
I take this fact to be evidence for two co-existing TAF constraints: one is ranked above IOMAX-PROMRoot, while the other is not, as shown below.

(127) Dominance Effects in Japanese

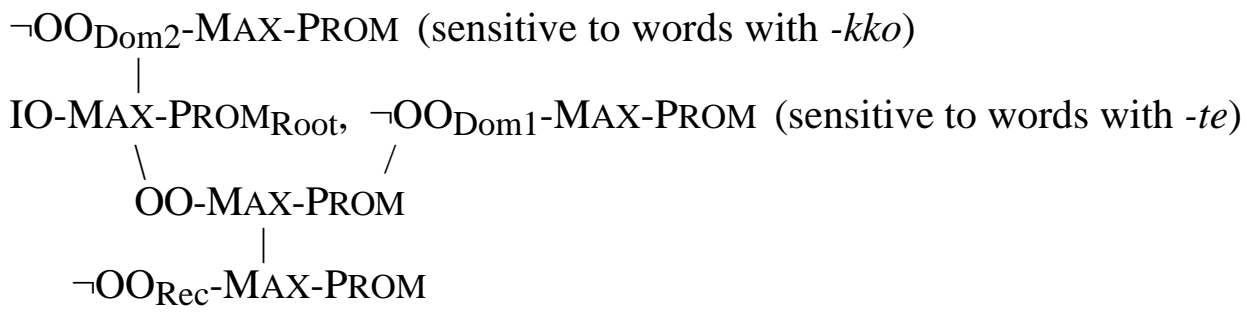

Thus, the top-ranked TAF constraint yields unaccented words because it dominates the Root Faithfulness constraint. On the other hand, this ranking does not hold with

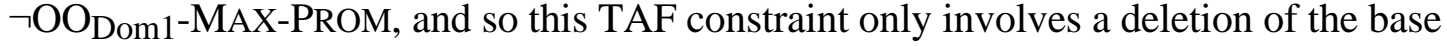
accent from the base itself, an expected consequence given the theory of Stem-to-Stem correspondence employed here. In sum, the range of affixed structures in Japanese represent different kinds of dominance effects which are negotiated via a fundamental tenet of the theory, constraint ranking.

To summarize the larger set of results, I have given an analysis of accent-shifting suffixes in Japanese which is consistent with all other types of affix-controlled accent. Suffixes like -mono, which induce an flop of the accent, and suffixes like -te, which cause a deletion, are both modelled in TAF theory as effects of an appropriate Anti-Faithfulness constraint. Thus, suffixes like -mono involved the Anti-Faithfulness constraint, $\neg \mathrm{OO}_{\mathrm{Dom}^{-}}$ NO-FLOP-PROM, which specifically requires a shift of accent in the derived forms. Suffixes like -te, on the other hand, trigger a dominance effect, which is due to high-

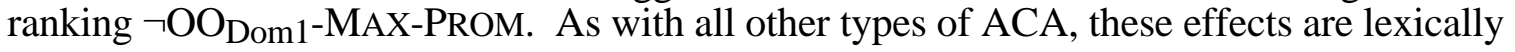
idiosyncratic and morphologically triggered: they correlate with the attachment of special suffixes. Furthermore, both of these effects are base-mutating, which as I have shown above, is an absolutely crucial factor in the analysis of these affixed words. Finally, both types of effects are found to be grammar dependent in the sense that independently necessary constraints dictate the outcome of the affix-controlled process. This property stems from the treatment of these affixed structures as compound-like, an assumption which appears to be unavoidable given the observed parallel with compounding morphology. With this assumed structure, the two accentual default patterns observed in these words followed from the assumptions made in the analysis of compounds. When the first member of the compound is accented, as in words with -mono, the shifted accent is to the PrWd-final syllable. However, when the accent is on the second member, i.e., on the suffix itself in words with te, the default pattern is for PrWd-initial accent, which again is consistent with the observed data. The conclusion is thus that the properties of accentshifting suffixes are explained in the same way as other affix-controlled phenomena.

\subsubsection{Extended Case Study: Accent in Aguaruna}

As illustrated in the introduction, the Jivaroan language Aguaruna exhibits a pattern of accent shift that is triggered by special suffixes. This morpho-phonological pattern was shown to display all of the properties of affix-controlled accentual processes, including grammar dependence. In this section, I construct an analysis of accent shift in Aguaruna that explains these properties as an effect of the TAF constraint $\neg$ NO-FLOP-PROM. This case study therefore provides further support for the claim that morphologically triggered 
accent shifts are grammar dependent, and offers further empirical support for the overall approach taken here.

This section is organized as follows. I start with a description of the distribution of accent in underived words in \$5.4.4.1 and give an analysis of the inventory of these accentual patterns in terms of constraint interaction between Prosodic Faithfulness and various prosodic well-formedness constraints. The interaction between accent placement and a rhythmic pattern of vowel deletion is then studied in $\$ 5.4 .4 .2$, as this pattern bears directly on the analysis of derived words. Finally, §5.4.4.3 examines morphologically triggered accent shifts and proposes an analysis within TAF theory.

\subsubsection{Distribution of Accent in Underived Words}

Aguaruna is a 'pitch accent' language in the sense that accent correlates with a rise in both $\mathrm{f} 0$ and intensity ${ }^{20}$ and that the distribution of accent is lexically governed. As illustrated in the following list, the accented vowel may be the first, second, or third vowel from the beginning of the word. It is also clear from these examples that accent can appear on either the first or second component of a syllable, e.g., waš ímau, cf. арии́риu, which shows that Aguaruna is a mora-accenting language. Furthermore, the constraints on the distribution of accent (discussed below) typically only make reference to vowel slots. I assume therefore that the mora is the accent-bearing unit in Aguaruna, and that consonants are not dominated by moras. Accented moras are represented here with a ' $M$ ' in the lefthand column.

(128) Frequencies for Accent Patterns (Payne 1990, from vocabulary in Larson 1963)

$\begin{array}{llll}\text { Mm } & \text { núka } & \text { 'leaf' } & 224 \\ \text { mMm } & \text { kawáu } & \text { 'parrot' } & 297 \\ \text { Mmm } & \text { káš ai } & \text { 'paca' } & 157 \\ \text { mmMm } & \text { wampukái } & \text { 'tadpole' } & 82 \\ \text { mMmm } & \text { maákai } & \text { 'mud' } & 123 \\ \text { Mmmm } & \text { ámuntai } & \text { 'buzzard' } & 45 \\ \text { mmMmm } & \text { apuúpuu } & \text { 'dolphin' } & 34 \\ \text { mMmmm } & \text { waš íimau } & \text { 'ocelot' } & 24 \\ \text { Mmmmm } & \text { áaNki as(a) } & \text { 'palm spear' } & 14 \\ \text { mMmmm } & \text { agálkiam(pa) } & \text { 'catfish' } & 5\end{array}$

The assertion made above that accent is phonemic in the language, and therefore marked in the lexicon, is in fact a contentious issue. While Pike \& Larson 1964 assume that accent is phonemic, Payne 1990 argues that accent is assigned on the peninitial mora by rule, and that a set of minor rules account for first and third mora accent. For concreteness, I follow Pike \& Larson in assuming that accent is marked lexically, but the analysis of accent shift presented below is fully compatible with the hypothesis that accent is assigned 'by rule' (in input-output mappings). If it turns out that Payne is correct, therefore, this assumption does not affect the overall argument.

\footnotetext{
${ }^{20}$ While pitch and intensity peaks do not coincide perfectly, the distribution of intensity peaks is predictable from pitch peaks (see Payne 1990: 166).
} 
The distribution of accent in bare substantives is subject to two over-arching constraints. First, as alluded to above, the accented mora is always within the first three moras of an underived word. Thus, while one finds words like apuúpuu, forms with accent beyond the third mora, e.g., *ариири́u, are systematically ruled out. A second important restriction is that the accented mora is never the (underlyingly) final mora of the word. There are no words such as nuká or kawaí which have accent of the last mora of a word. This last restriction is not always apparent from the surface form, however, as vowel deletion may create words with final mora accent, as in $/ \tilde{s}$ uúta/ $\rightarrow \breve{s}$ uút 'large cockroach'.

Consistent with the assumptions for Tokyo Japanese, I assume that pitch accent languages such as Aguaruna have a lexical prominence as the representation of accent, which at the surface is aligned with a high tonal target. This assumption is not critical in the analysis in any way, however, because the principles of Faithfulness and Faithfulness reversals can be applied with equal force to other types of prosodic structure. The constraints governing the realization of lexical accent in Aguaruna are therefore the same constraints employed in the analysis of stress-accent languages like Cupeño and Russian, namely the Prosodic Faithfulness constraints. The distributional gaps discussed above may be accounted for by ranking the Prosodic Faithfulness constraints in relation to other prosodic well-formedness constraints, which are given below.

(129) Constraints Governing the Inventory of Underived Words

\section{a. Constraint Encapsulation: PROS-FAITH = DEP-PROM, MAX-PROM, NO-FLOP- PROM}

b. ACCENTWINDOW: An accentual prominence must occur on one of the first three moras of a surface form.

c. NONFINALITY $(\mu)$ : An accentual prominence must not appear on the final mora of a word. (cf. Prince \& Smolensky 1993)

I assume for concreteness that the limitation of accent to the first third moras is due to the constraint ACCENTWINDOW (though this constraint is obviously not a serious proposal; see Green \& Kenstowicz 1995 for more substantive ideas). Specifically, when

ACCENTWINDOW is ranked above the IO-Prosodic Faithfulness constraints, lexical prominences which are outside of the accentual window will lead to an unfaithful mapping, as shown in (130b). Another important constraint ranking involves the domination of IOPROS-FAITH by the constraint NONFINALITY $(\mu)$, which is defined above. With NONFINALITY $(\mu)$ ranked above the Prosodic Faithfulness constraints, if a lexical prominence occurs in the final mora of a word in the input, the IO-mapping will also not be a faithful one (130a). The presence of a positional contrast in accent, however, shows that the Alignment constraints are dominated by IO-PROS-FAITH. Thus, as depicted in (130c), a medial prominence in the word has a corresponding medial prominence in the output, as shifting the prominence to an edge position violates Prosodic Faithfulness. 
(130) Illustration of Results for Inventory of Underived Words ( $\underline{V}=$ lexical accent position)

\begin{tabular}{|c|c|c|c|c|c|c|}
\hline Input & & Output & NONFINAL & WINDOW & P-FAITH & ALIGN-R \\
\hline \multirow[t]{2}{*}{ a. /CVCVCV́ / } & $\rightarrow$ & CVCV́ CE & & & $*$ & * \\
\hline & & *CVCVCV́ & $* !$ & & & \\
\hline \multirow{2}{*}{$\begin{array}{l}\text { b. /CVVCVV́ } \\
\text { CV/ }\end{array}$} & $\rightarrow$ & CVVCV́ $\underline{\mathrm{VCV}}$ & & & $*$ & $*$ \\
\hline & & $\begin{array}{l}{ }^{*} \mathrm{CVVCV} \underline{\underline{v}} \\
\mathrm{CV}\end{array}$ & & $* !$ & & $*$ \\
\hline \multirow[t]{2}{*}{ c. /CVCV́ CV/ } & $\rightarrow$ & CVCÉ CV & & & & $*$ \\
\hline & & $*_{\mathrm{CVCVCV}}$ & & & $* !$ & \\
\hline
\end{tabular}

A final point concerns the effects of NONFINALITY( $\mu)$ on forms like $\check{s}$ uút $(a)$, which have a deleted final vowel. I argue in the next subsection that these forms actually have covert structure word-finally, essentially a mora which does not dominate a vowel, i.e., [š uút $\Delta$ ]; thus, these forms do in fact conform to the generalization that accented moras are never word-final.

To summarize the above results, the following partial ordering of constraints accounts for the inventory of accentual patterns observed in underived words.

(131) Ranking for Underived Words

$\operatorname{NONFInALITY}(\mu)$, ACCENTWINDOW $>$ IO-PROSFAITH $>$ Alignment

This set of rankings accounts for the fact that the position of accent is generally unpredictable, as the IO-Prosodic Faithfulness constraints outrank the Alignment constraints which require accent to appear at a designated edge (the ordering of the two Alignment constraints with respect to each other is taken up directly below). This contrast in the position of accent is restricted, however, by two high-ranking constraints. Thus, because NONFINALITY $(\mu)$ dominates IO-PROSFAITH, accent may not appear on the final mora of a word. Furthermore, the ranking ACCENTWINDOW $>$ IO-PROSFAITH limits the range of the accentual contrast to the first three moras of a word.

\subsubsection{Vowel Deletion and Accent Shift}

Aguaruna has a phonological pattern of accent shift triggered by the application of vowel deletion. Since the edge orientation of the shift bears directly on the treatment of the morphological shifts, I examine and analyze this pattern first. The data and description of vowel deletion in Aguaruna is presented directly below, followed by the proposed analysis. 
Let us start with Payne's description of vowel deletion. ${ }^{21}$

(132) Description of Syncope (Payne 1990)

a. Syncope has a rhythmic basis: "Basically the elision rule looks at the third vowel from the beginning (or left) of the word, and elides that and every alternate vowel bounded [=surrounded] by consonants. At the end (or right) boundary of the word, Syncope stops short of eliding a penultimate vowel. Instead the final vowel is elided, if that vowel is immediately preceded by a consonant." (p. 164)

b. Synchronically, syncope makes no reference to accent placement. Historically, however, it may have developed from a vowel reduction process. Indeed, some vowels to be syncopated are retained as devoiced vowels in related dialects. (see p. $179,163)$

c. When the accented vowel is deleted, accent is shifted one mora to the right if there are three or more following vowels (underlyingly); otherwise accent is shifted one mora to the left. (see p. 179-80)

Like many other languages, for example Southeastern Tepehuan (Willett 1991, 1982), syncope in Aguaruna has a rhythmic basis, as it deletes vowels in alternating syllables. This pattern is illustrated by the following examples (vowels to be deleted are underlined in the underlying representation). Forms like ič inkaNminak show the preference for

dropping a final vowel over the penultimate vowel, even when the latter is predicted by the binary pairing of vowels. The two shifts in accent observed when the accented syllable is deleted are exemplified in (133b). Thus, while the pattern of leftward shift, as in $u N u ́ s ̌$ num, is very common, it is clear that the 'default' pattern is to shift the accent to the right because the environment for leftward shift is more specific, as I demonstrate below. This move is somewhat different from Payne's statement above, but this account is largely based on frequency (and not on the phonological composition of the string), as words with third mora accent and more than two following moras are rather rare.

\footnotetext{
${ }^{21}$ David Payne points out (personal communication) that the vowels in much previous work have been inconsistently transcribed, sometimes resulting in the treatment of a word-final long vowel as short (presumably to predict it from syncope). The reader interested in probing further into the details of the system is thus referred to Payne 1990 for the authoritative statement of this process, where this inconsistency is ironed out.
} 
(133) Exemplification of Syncope in Aguaruna

a. Deletion of an Unaccented Vowel

$$
\begin{aligned}
& \text { lič inaka/ ič inak 'clay pot' } \\
& \text { lič inaka }+ \text { nal } \\
& \text { lič inakka }+\mathrm{N} \underline{\mathrm{u}}+\mathrm{mi}+\text { nal } / \text { ič inka-N-mi-n 'your clay pot (Acc)' } \\
& \text { lič inakka }+\mathrm{N} \underline{\mathrm{u}}+\mathrm{mi}+\mathrm{na}+\mathrm{k \underline {i }} \text { ič inka-N-mi-na-k 'only your clay pot (Acc)' } \\
& \text { I } \\
& \text { b. Deletion of an Accented Vowel } \\
& \begin{array}{lll}
\text { /uhaNí }+ \text { kitij } & \text { uháN-kit } & \text { 'palm species (Compound)' } \\
\text { /uhaN } \underline{1}+\text { kiti }+ \text { nal/ } & \text { uhaN-kíti-n } & \text { 'palm species (Comp, Acc)' }
\end{array} \\
& \text { /uNuš } \underline{1}+\text { numil/ uNúš -num 'tree species (Compound)' } \\
& \text { /uNuš } \underline{1}+\text { numi + nal } \quad \text { uNuš -númi-n } \quad \text { 'tree species (Comp, Acc)' } \\
& \text { /uNuš } \underline{1}+\text { numi }+ \text { š akam/ uNuš -núm-š akam 'tree species (Also)' }
\end{aligned}
$$

To summarize, syncope in Aguaruna follows an alternating pattern, with a preference for deleting final vowels. Further, when the accented vowel is deleted, accent shifts to the left if there are less than three following vowels underlyingly; otherwise accent shifts to the right.

The rhythmic pattern of vowel deletion suggests a role for metrical structure in the analysis, as argued in detail in McCarthy 1998 for a similar body of facts in Bedouin dialects of Arabic. 22 Thus, if we assume that Aguaruna words are parsed left-to-right into iambs, it is possible to motivate vowel deletion as an effect of principles of rhythmic organization, i.e., the Iambic/ Trochaic Law of Hayes 1995 and Grouping Harmony of Prince 1990. Sketching the analysis informally below, in a sequence of CV syllables, the third vowel in the underlying representation in (134b) ends up in the weak position of the final iamb. The loss of this vowel can hence be explained as a need for a durational contrast in right-headed feet: the outright deletion of the vowel in the weak position satisfies this constraint, rather than the more usual strategy of lengthening the strong member of the iamb.

(134) Metrical Analysis of Syncope
a. /ič inakâl

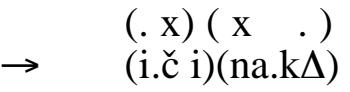
b. /ič inakka + nal
$(. x)(. x)$

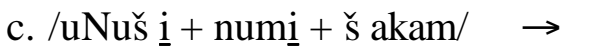
$(. \mathrm{x})(. \mathrm{x})(\mathrm{x}$.
$(\mathrm{uNu})(\check{\mathrm{s}} \Delta \cdot-$ num) $)(\check{\mathrm{s}}$ akam $)$

\footnotetext{
${ }^{22}$ See also Kager 1997 for a different approach to rhythmic vowel deletion in terms of the minimal violation of the constraints governing the prosodic organization of syllables.
} 
The fact that syncope only affects vowels which are surrounded by consonants also has a cogent explanation on this account. VV sequences will always either support their own foot or occupy the right member of a branching foot in standard foot typologies, and so they will be systematically ignored by the process. Furthermore, the fact that, in some final feet, the weak member is not deleted may be captured as a foot-type reversal to satisfy $\operatorname{NONFINALITY}(\mu)$, as argued in Prince \& Smolensky 1993 for Latin. Thus, in just those contexts where the head of the iambic foot would appear in the final position of the word, the canonical iamb switches to a left-headed trochee, as shown in (134c), which obviates the need for a durational contrast because the foot is a trochee, not an iamb. The metrical account is of course not the full story, since the complex patterns described above show some language particular intricacies which demand an explicit treatment. For example, the initial vowel is never lost, despite its position in the initial iamb, and so something else needs to be said here. Furthermore, the final vowel in the input-output mapping in (134ab) is not predicted to drop out in the metrical analysis just described — a problem that will also receive our attention below.

In addition to the theory-internal motivation for this analysis, the assumed structures help in describing two facts. First, as noted in $\$ 5.4 .4 .1$, accent is not allowed in the final mora of a word, but words which have lost the word-final vowel yield apparent exceptions to this generalization, e.g. /š uúta/ $\rightarrow \breve{s}$ uút. However, if forms such as these have a final $\mathrm{C} \Delta$ syllable, then these forms are consistent with final mora extrametricality. If final syllables have a mora, but are vowelless, then the accented mora in [š uú.t $\Delta$ ] is not final. Second, syncopated forms aside, only nasal consonants are allowed in the coda position (Payne 1990: 166). If the consonants which appear before the deleted vowels are re-syllabified with the preceding syllable, as in /ič inaka/ $\rightarrow$ [i.č i.nak], then syncope creates exceptions to an otherwise exceptionless constraint on coda consonants. Of course, if these underlyingly pre-vocalic consonants are syllabified as onsets at the surface, as assumed in (134), then this problem does not arise. McCarthy makes this same argument with different facts from Arabic.

Moving now to state these assumptions more formally, the constraint which is responsible for the durational contrast in iambs is given below.

(135) RHYM-SYNC

Avoid full vowels in the weak position of an iambic foot.

With this constraint top-ranked in the hierarchy, specifically outranking the anti-deletion constraint, MAX-V, and the constraint banning vowelless syllables, *EMPTY-NUC, rhythmic vowel deletion is the predicted outcome, as shown below.

(136) Rhythmic Vowel Deletion

\begin{tabular}{|c|c|c|c|}
\hline /CVCVCVCV ... & RHYM-SYNC & MAX-V & *EMPTY-NUC \\
\hline \hline $\begin{array}{c}\mathrm{x})(. \mathrm{x}) \\
(\mathrm{CVCV})(\mathrm{CVCV}) \ldots\end{array}$ & $* !$ & & \\
\hline $\begin{array}{c}(\mathrm{x})(\mathrm{x}) \\
(\mathrm{CVCV})(\mathrm{C} \Delta \mathrm{CV}) \ldots\end{array}$ & & $*$ & $*$ \\
\hline
\end{tabular}

The loser here has a full vowel in the weak syllable, in violation of RHYM-SYNC, leaving the candidate with an elided vowel in the third syllable as the winner. 
The first vowel of the word is never deleted, however, showing that initial syllables are special in some way. Following Beckman 1997 [1998], I treat this fact as evidence for a high-ranking Positional Faithfulness constraint which gives preferential treatment to initial syllables. Thus, with a position-sensitive MAX-V constraint ranked above RHYM-SYNC, deletion of the first vowel is not predicted, as shown below.

(137) No Vowel Deletion in the Initial Syllable

\begin{tabular}{|c|c|c|c|}
\hline & /CVCVCVCV ... I & $\sigma_{1}$-MAX-V & RHYM-SYNC \\
\hline & 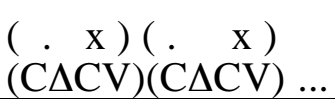 & $* !$ & \\
\hline$\rightarrow$ & 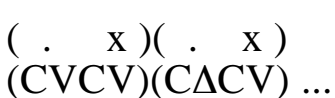 & & $*$ \\
\hline
\end{tabular}

The metrical analysis employed thus far does not account for the preference for dropping the final vowel of the word when it can, e.g., /ič inakal $\rightarrow[($ ič i $)(n a k \Delta)]$, since the fourth vowel here does not fall into a strong position. Furthermore, one cannot appeal to a foot-type reversal word-finally to account for the deletion of the final vowel in the weak member of a trochee because the final vowel in cases like /ič inaka + na/ $\rightarrow$ [(ič i) (n $\Delta \mathrm{ka})-\mathrm{n} \Delta]$ is not footed (or alternatively, it is not in the weak position if it supports a degenerate foot). It appears, however, that a different constraint is at work which handles these facts by favoring words that end in consonants. This constraint, called FINAL-C in McCarthy \& Prince 1993b, receives independent motivation from some additional distributional facts in the language. David Payne notes (personal communication) that the only vowel-final words in the language are those with just two vowels in the word or words which end in a VV sequence. Thus, if FINAL-C is ranked above MAX-V, the generalization that words tend to end in consonants can be treated with the same machinery used for describing the preference for apocope in the vowel deletion. This tack of course requires an analysis of the two classes of $\mathrm{V}$-final words, but these cases also lend themselves to a cogent analysis. First, the avoidance of apocope in $\mathrm{CV}(\mathrm{C}) \mathrm{V}$ words is straightforwardly handed as a two-vowel minimal word requirement, which is generally obeyed in the language. Furthermore, the lack of deletion of a VV in words that end with this sequence can be treated as an effect of stress: word-final VV will invariably be parsed as the head of an iamb, and because this is the preferred head of a light-heavy iamb and a possible monosyllabic iamb, these vowels will be protected from deletion as well.

Putting all of the pieces together, both RHYM-SYNC and the phonotactic constraint FINAL-C are necessary in Aguaruna vowel deletion, as illustrated in the following tableau. The winner here satisfies both constraints by deleting the third and fifth vowel, which contrasts with the two other candidates, which violate one or the other of the two highranking constraints. 
(138) Rhythmic and Non-Rhythmic Vowel Deletion

\begin{tabular}{|c|c|c|c|c|c|}
\hline & /ič inaka + na/ & FINAL-C & RHYM-SYNC & MAX-V & *EMPTY-NUC \\
\hline a. & 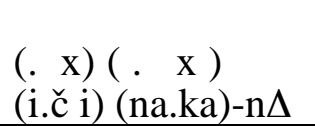 & & $* !$ & $*$ & * \\
\hline b. & 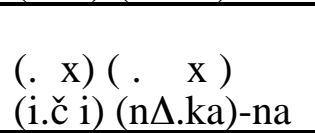 & $* !$ & & $*$ & $*$ \\
\hline c. & 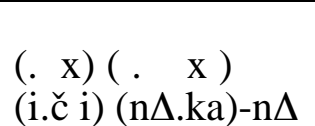 & & & $* *$ & $* *$ \\
\hline
\end{tabular}

The ranking arguments made thus far are summarized in the partial ordering given below.

(139) Ranking for Rhythmic Vowel Deletion

$$
\text { FINAL-C, } \sigma_{1} \text {-MAX-V }>\text { RHYM-SYNC }>\text { MAX-V, *EMPTY-NUC }
$$

Relating this grammar to Payne's description given in (132), the third vowel from the beginning of the word deletes when it is the weak member of an iamb, which by RHYMSYNC, must drop out. Only vowels which are surrounded by consonants undergo syncope because a VV sequence is always parsed as the strong syllable of an iamb, and hence, it is not be targeted by RHYM-SYNC. Furthermore, syncope only affects post-pen-initial vowels because when a single vowel appears in the first syllable, it is protected from RHYM-SYNC by the Positional Faithfulness constraint $\sigma_{1}$-MAX-V. Lastly, the independently motivated constraint FINAL-C accounts for the observed preference for deleting final vowels.

Moving now to the question of what happens when an accented vowel is deleted, let us review the following facts.

(140) Vowel Deletion with Accent Shift
a. /uNuš $\underline{1}+$ numi + nal/
uNuš -númi-n
Shift to Right
b. /uNuš $\underline{1}+$ numi//
uNúš -num
Shift to Left

When the accented vowel is lost, the accent typically shifts to the right, unless there are less than three underlying moras to the right of the accented vowel, in which case accent shifts to the left.

This type of observation (i.e., accent shifts triggered by deletion of the sponsoring element), is commonly attributed to HV's Faithfulness Condition, which, roughly speaking, entails that heads of feet be preserved throughout a derivation within the metrical constituent structure enclosing them. An initial problem with this approach to accent shift in Aguaruna is that lexical accents do not reliably fall in the head positions of the independently motivated syncope feet, and so a second metrical plane is required to account for the observed facts. Furthermore, the derivational implementation of this idea entails a unitary direction for the shift, but the facts of Aguaruna show that the accent of a deleted vowel shifts in two different directions. A final problem is that an analysis in terms of the Faithfulness Condition requires intermediate stages in the mapping from lexical to surface forms that are otherwise unmotivated, which I take to be fatal for this approach. 
The alternative I propose is that the edge orientation is described directly via the Alignment constraints, with an additional requirement that the prominence may not appear in the final foot. This result is achieved through the following constraint ranking, which builds on the constraint system given above for the basic accentual patterns by ranking the Alignment constraints with respect to each other, and by adding another NONFINALITY constraint relativized to the stress foot.

(141) Constraint Ranking for Accent Shift with Vowel Deletion

$$
\operatorname{NonFinAlity}(\mathrm{Ft})>\text { Align }(\mathrm{PM}, \mathrm{R}, \operatorname{PrWd}, \mathrm{R})>\text { Align (PM, L, PrWd, L) }
$$

While prominence typically does not shift because of high-ranking Prosodic Faithfulness, when the deletion of the sponsor of a prominence precludes a faithful mapping, a shift must take place. Because ALIGN-R dominates ALIGN-L, a shift to the right is preferred over a shift to the left, as illustrated below.

(142) Default Rightward Shift

\begin{tabular}{|c|c|c|}
\hline /uhaNí $+\mathrm{kiti}+\mathrm{na} /$ & ALIGN-R & ALIGN-L \\
\hline \hline (uhá) $(\mathrm{N} \Delta-\mathrm{ki})(\mathrm{ti}-\mathrm{n} \Delta)$ & $* * * * !$ & $*$ \\
\hline$\rightarrow($ uha $)(\mathrm{N} \Delta-\mathrm{kí})(\mathrm{ti}-\mathrm{n} \Delta)$ & $* *$ & $* * *$ \\
\hline
\end{tabular}

However, when a shift to the right would entail positing a prominence in the final foot of the word (i.e., when the deleted vowel is followed by just two underlying moras), the accent instead shifts to the left because NONFINALITY $(\mathrm{Ft})$ is ranked above ALIGN-R.

(143) Accent Shift with Final Foot Extrametricality

\begin{tabular}{|c|c|c|}
\hline /uhaNí + kiti $/$ & NONFINALITY(Ft) & ALIGN-R \\
\hline \hline (uha)(N $\Delta$-kí)t $\Delta$ & $* !$ & $*$ \\
\hline$\rightarrow$ (uhá)(N $\Delta$-ki)t $\Delta$ & & $* * *$ \\
\hline
\end{tabular}

The foot-based analysis here extends to all of the cases of leftward shift described by Payne. The only accented vowels eligible for deletion are the vowels dominated by the third mora because syncope starts with the third vowel from the beginning of the word, and lexical accent never goes beyond this third mora (this is the accentual window effect). Focusing on the vowels following the accented vowel (i.e., those following the third vowel), words with two subsequent underlying vowels or less may drop these vowels, as

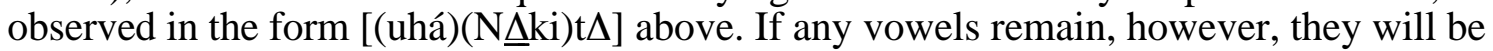
in the final foot and therefore ineligible for receiving the accent by NONFINALITY(Ft). If, on the other hand, the following vowels are protected from syncope because they appear in a VV cluster, then the final two vowels are likewise ineligible for accent because they are parsed as the strong member of the iamb, as in: /CVCVCÉ $-\mathrm{CVV} / \rightarrow[(\mathrm{CVCV})(\mathrm{C} \underline{\Delta}-\mathrm{CVV})]$.

The proposed analysis thus accounts for the 'default rightward shift, otherwise leftward shift under duress' through the constraint interaction shown in (144). However, the analysis does not yet account for the observed boundedness of accent shift, i.e., the fact that the widowed accent only shifts one mora to the right or the left. Since the morphological shifts examined below are also bounded in this sense, it is necessary to have 
an analysis of this fact as well. ${ }^{23}$ As currently stated, NO-FLOP-PROM does not require bounded accent shift, since any shift is a violation of this constraint, whether the accent shifts are bounded to the moraic sponsor or not. We therefore require a constraint which ensures that the shift will be local, i.e., to an adjacent mora rather than a non-adjacent one. I express this intuition with the following constraint, though other analyses are possible (see Myers 1997a and Bickmore 1996 for some leading ideas).

\section{(144) LOC-FLOP-PROM}

$\forall \mathrm{x} \forall \mathrm{y} \forall \mathrm{z}, \mathrm{x} \in$ prominence, $\mathrm{y} \in$ sponsor, $\mathrm{z} \in$ link, if $\mathrm{x}$ and $\mathrm{y}$ are associated by $\mathrm{z}$ in $\mathrm{S}_{1}$, and $\exists \mathrm{x}^{\prime} \exists \mathrm{y}^{\prime} \exists \mathrm{z}$ ' s.t. $(\mathrm{x}, \mathrm{y}, \mathrm{z}) R\left(\mathrm{x}^{\prime}, \mathrm{y}^{\prime}, \mathrm{z}^{\prime}\right)$ and $\mathrm{x}^{\prime}$ and $\mathrm{y}^{\prime}$ are not associated by $\mathrm{z}^{\prime}$ in $\mathrm{S}_{2}$, then $\exists y$ ' $\exists z$ ' s.t. $x$ ' and y' are associated by z' and y' and y' are adjacent in $S_{2}$.

Satisfaction of LOC-FLOP-PROM is only relevant in structures where the link between a prominence and its sponsor is severed in the IO-map, as stated in the second clause of the antecedent of the implication. The logic of this constraint entails that if there is a shift of a lexical prominence, then the result must be to a sponsor which is adjacent to the correspondent of the sponsor in the input. Applying this reasoning, if a lexical accent could be shifted more than one mora to the right (because the subsequent moras do not appear in the final foot), the ranking of LOC-FLOP-PROM above ALIGN-R predicts a shift exactly one mora to the right. Thus, when a candidate attempting to improve on edge alignment shifts the accent more than one mora, it incurs a violation LOC-FLOP-PROM, and is thus ruled out in favor of the candidate with a bounded shift, as depicted below.

(145) Bounded Accent Shift

\begin{tabular}{|c|c|c|}
\hline /CVCVCÉ $+\mathrm{CVVCVCV} /$ & LOC-FLOP-PROM & ALIGN-R \\
\hline$(\mathrm{CVCV})(\mathrm{C} \underline{\underline{\Delta}-\mathrm{CVV}})(\mathrm{CVC} \Delta)$ & $* !$ & $* *$ \\
\hline$\rightarrow \quad(\mathrm{CVCV})(\mathrm{C} \underline{\Delta}-\mathrm{CV} \mathrm{V})(\mathrm{CVC} \Delta)$ & & $* * *$ \\
\hline
\end{tabular}

The same result holds with the leftward shifts because LOC-FLOP-PROM also dominates ALIGN-L. In the mapping from /uhaNí-kiti// to [(uhá)(N $\Delta \mathrm{ki}) \mathrm{t} \Delta$ ], the competing candidate which defaults to initial accent is ruled out because it also incurs a violation of this topranked constraint.

To summarize, the rankings needed for bounded accent shifts are given below. I have also carried over the rankings from the previous subsection to present the complete constraint system.

\footnotetext{
${ }^{23}$ Furthermore, we require a phonological analysis of this fact as it cannot simply be assumed that the accent shifts observed here are immobile phonological accents whose $\mathrm{H}$ targets are somehow delayed in the phonetic implementation (as cogently argued for Chichewa in Myers \& Kim 1998). The phonological shifts interact with other phonological constraints, i.e., the Alignment constraints and the constraint responsible for leftward shift when the accent would appear in the final foot, which shows that the shifts are phonological in nature.
} 
(146) Summary of Rankings

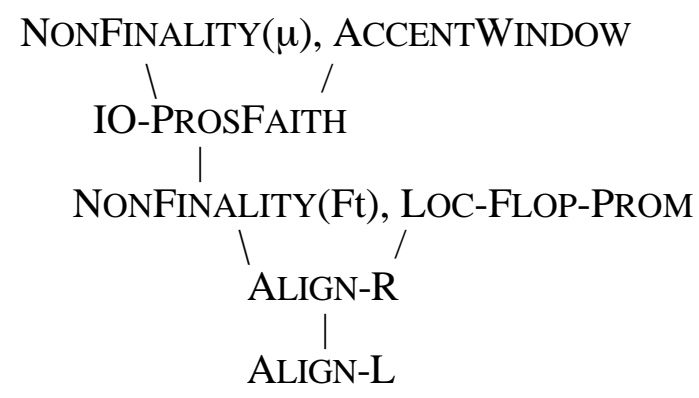

With LOC-FLOP-PROM ranked above the Alignment constraints, accent shifts will always be bounded to the mora which is the counterpart of the lexical sponsor for accent. Also, ALIGN-R dominates ALIGN-L, describing the fact that a shifted accent typically goes the right. If, however, the shifted accent would end up in the final foot of the word, then it shifts the left because of high-ranking NONFINALITY $(\mathrm{Ft})$. This constraint hierarchy will be directly relevant to the analysis of bounded rightward shift in the morphological environments examined in the next subsection.

\subsubsection{Accent Shift in Derived Words}

We may now proceed to the central facts of interest in this case study, namely the morphological pattern of accent shift. As illustrated in the introduction to this section, certain suffixes trigger a shift in the accent of the base. Since this affix-controlled accentual process has all of the properties of ACA, it may be explained in a general theory of Transderivational Anti-Faithfulness. I start first with the analysis of the basic distinction between accent-shifting and accent-neutral suffixes, and then move to extend the analysis to the behavior of a more complicated set of accent-shifting suffixes and the treatment of the locality conditions on accent shift.

Larson 1956 and Payne 1990 distinguish a wide range of suffixes, each with a distinct morpho-accentual behavior. For the present purposes, however, we only need to contrast the behavior of two classes, the accent-neutral and accent-shifting suffixes. The accent-neutral suffixes are like the stress-neutral suffixes in English in that they leave the accentuation of the base as it is in the underived form. This type of accentual behavior is exemplified below for the predicate-forming suffix $-\dot{t}$, which Payne glosses as is an $\mathrm{X}$ (object not present)'. The derived form is compared with the simplex nominative base, which forms the base for many suffixes.

(147) Accent-neutral - $\dot{t}$

\begin{tabular}{|c|c|c|}
\hline núka & núka-ì & 'leaf' \\
\hline č aNkín & č aNkína-i & 'basket' \\
\hline š uNkał́ m & š uNkaí ma-ì & 'bird (arrendajo)' \\
\hline kampaának & kampaánka-i & 'kind of palm' \\
\hline áaNki as & áaNki asa-i & 'palm spear' \\
\hline taátaanč & taátaanč i-i & 'water spider' \\
\hline
\end{tabular}

Other suffixes exhibiting accent-neutral behavior include: $-a$ 'is an X (object present)', the negation suffix $-\check{c} u u$, and $-\not c u$ 'maybe $\mathrm{X}$ '. 
Accent-shifting suffixes, on the other hand, cause the accent of the base to shift one mora to the right. This class of suffixes is actually a heterogeneous class, involving distinctions depending on the lexical prosody and syllable make-up of the base. However, since all accent-shifting suffixes have the basic properties of the suffixes discussed directly below, it is clear that our point of departure should be with these. In the closing remarks, I will discuss one of the variations on the basic pattern as it relates to the analysis and sketch an account which is consistent with the analysis given here.

(148) Accent-shifting Suffixes

$\begin{array}{ll}\text {-na } & \text { Accusative } \\ \text {-hai } ~ & \text { Comitative }(/ \mathrm{i} \sim / \text { is a nasalized vowel }) \\ \text {-nuu } & \text { Possessive }\end{array}$

The behavior of these suffixes is exemplified below, again opposing the simplex nominative with the derived forms showing the shift.

(149) Derived Words with Accent Shift

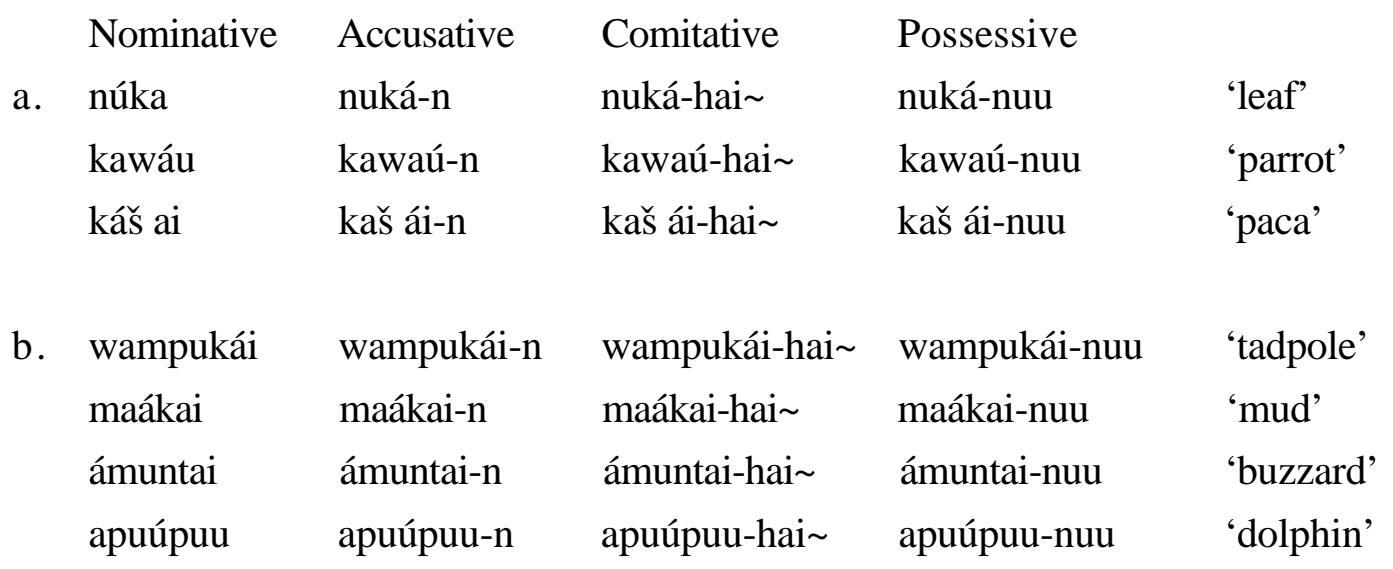

As shown by the contrast between the forms in (149a) and (149b), the accent of the base only shifts in the derived form if the base is three moras or less. This fact is significant, as it is observed in one form or another in all the accent-shifting suffixes. Payne suggests that this is a locality effect on the distance of the accent from the accent-shifting suffix, which would further diagnose this pattern as an affix-controlled process. But because locality effects of this kind are linked in an important way to the constraint requiring the shift, I need to set it aside for the moment while I develop the analysis of the basic distinction between accent-neutral and accent-shifting suffixes.

Before presenting the analysis in TAF theory, it is necessary to consider an alternative in which the shift is due to an inherent phonological property of the affix itself. After all, English has similar patterns of stress shift induced by suffixation, e.g., eléctric/electrícity. Since the rightward shift here also correlates with suffixation, perhaps the shift is simply a matter of the phonological composition of the suffixed structures. This parallel is clearly inappropriate, however, because the shifted accent does not conform to any generalizations counting from the left or right edge of the word. Suffixation triggers a shift of the lexically determined accent, which may occur on any of the first three moras; since the accent shift is bounded to the lexical accent, the position of the shifted accent in derived forms is not predictable in terms of mora count. 
A different idea is to say that the accent-shifting suffixes contribute an accent, or alternatively, that they simply add a link which is somehow merged with the base accent if it is close enough (as in the approach to accent-shifting suffixes in Poser 1984). Such an analysis is certainly possible here, and indeed, this is essentially the idea mentioned in passing in Payne 1990: 181. As with the case of Japanese, however, I develop an alternative here which is consistent with the more restrictive TAF theory of accent shifts. Thus, accent shift must be rightward and bounded because this is the independently motivated pattern of accent shift in the language. I do not see how the purely phonological account of these shifts would explain this fact, and as grammar dependence is a natural consequence of the theoretical model developed here, the TAF account has a clear advantage over the phonological analysis. Furthermore, a purely phonological analysis of the retraction of tonal accent in Limburg Dutch is highly undesirable (see argumentation in $\S 5.4 .2$ ), and I propose to develop an analysis of accent shifts in Aguaruna which is in line with this clear case of morphological shift.

In the now familiar way, the idiosyncratic distinction between whether or not an affix conditions an accentual process is derived through subcategorization of OOcorrespondence relations in the lexicon. Thus, assuming the lexical entries in (151) below, the accent-neutral suffixes are sensitive to the TAF constraint $\neg \mathrm{OO}_{\mathrm{Rec}}$-NOFLOP-PROM, which, because of its low rank in the hierarchy in (150), does not condition a shift in the base-derivative mappings. The suffixes in (151b), in contrast, trigger OO $_{\text {Dom }^{-}}$ correspondence, and since the Anti-Faithfulness constraint defined upon this relation is high-ranking, these suffixes are accent-shifting.

(150) Rankings for Accent-neutral/Accent-shifting Distinction

$$
\neg \mathrm{OO}_{\text {Dom }} \text {-NO-FLOP-PM }>\text { OO-NO-FLOP-PM }>\neg \mathrm{OO}_{\mathrm{Rec}^{-N O}-\mathrm{FLOP}-\mathrm{PM}}
$$

(151) Lexical Entries for Accent-neutral and Accent-shifting Suffixes

\begin{tabular}{|c|c|c|c|}
\hline a. & Pred $_{X(\text { pres.) }}$ & {$[[$ Stem $]$ OORec __ $]$} & \multirow[t]{4}{*}{ [Accent-Neutral] } \\
\hline$-a$ & Pred $_{X \text { (not pres.) }}$ & {$[[$ Stem $]$ OORec __ $]$} & \\
\hline$-\check{c} u u$ & $\mathrm{~N}_{\mathrm{Neg}}$ & 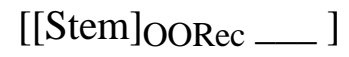 & \\
\hline$-\not \subset u$ & NPutative & {$\left[[\text { Stem }]_{\text {OORec }} \_\right]$} & \\
\hline \multirow[t]{3}{*}{ b. } & $\mathrm{N}_{\mathrm{Acc}}$ & {$[$ StemoODom _ _ ] } & \multirow[t]{3}{*}{ [Accent-Shifting] } \\
\hline & $\mathrm{N}_{\text {Com }}$ & [ StemoODom__ ] & \\
\hline & $\mathrm{N}_{\text {Poss }}$ & {$[$ StemoODom__ $]$} & \\
\hline
\end{tabular}

Applying these assumptions to a particular example, the accent-shifting suffix -na (which loses the final vowel in syncope) conditions a shift of the base accent because forms with this suffix are evaluated by the TAF constraint, $\neg \mathrm{OO}_{\text {Dom-NOFLOP-PROM, which is }}$ ranked above the OO-Prosodic Faithfulness constraint banning the shift. (152) Morphological Accent Shift

\begin{tabular}{|c|c|c|c|}
\hline Base & /núka + na $/$ & $\neg \mathrm{OO}_{\text {Dom-NO-FLOP-PROM }}$ & OO-NO-FLOP-PROM \\
\hline núka & núka-n & $* !$ & \\
\hline b. $\rightarrow$ núka & nuká-n & & * \\
\hline
\end{tabular}


The predicate-forming suffix $-\dot{i}$, on the other hand, does not trigger the shift, as shown below. This suffix subcategorizes for $\mathrm{OO}_{\mathrm{Rec}}$-correspondence; since the TAF constraint operating on this type of correspondence is so low-ranking, it cannot have an effect.

(153) No Morphological Accent Shift

\begin{tabular}{|c|c|c|c|c|}
\hline Base & $/$ núka $+\dot{i} /$ & $\neg O_{\text {Rec }}$ NO-FLOP & OO-NO-FLOP & $\neg$ OO-NO-FLOP \\
\hline \hline a. $\rightarrow$ núka & núka-i & & & $*$ \\
\hline b. núka & nuká-i & & $* !$ & \\
\hline
\end{tabular}

Therefore, as with dominant and pre- and post-accenting affixes, the presence or absence of a shift is due to the rank of the TAF constraint associated with a given suffix.

Returning to the behavior of the accusative suffix - $n a$, which is accent-shifting, this suffix triggers a flop of the base accent, which is bounded and rightward, i.e., the same basic pattern for phonologically triggered accent shifts. This fact shows that the morphological accent shifts exhibit grammar dependence and the analysis of this fact stems from the same basic principles applied in the analysis of grammar dependent ACA generally. Thus, an independently needed Alignment constraint, specifically ALIGN (PROM, R, PrWd, R), determines the edge orientation of the mutated accent. This result is illustrated in the tableau below, in which the competition between the two patterns of AntiFaithfulness is determined by the Alignment constraint.

(154) Grammar Dependence Part 1: Rightward Accent Shift

\begin{tabular}{|c|c|c|c|c|}
\hline Base & /kawáu + na/ & $\neg \mathrm{OO}_{\text {Dom-NO-FLOP }}$ & OO-NO-FLOP & ALIGN-R \\
\hline a. kawáu & kawáu-n & $* !$ & & \\
\hline b. kawáu & káwau-n & & $*$ & $* * !$ \\
\hline c. $\rightarrow$ kawáu & kawaú-n & & $*$ & \\
\hline
\end{tabular}

Another type of grammar dependence observed in the morphological shifts is that the mutated accent always shifts one mora to the right, but not all the way to the end of the word. This fact is also explained with the already existing constraint hierarchy, because the LOC-FLOP-PROM dominates ALIGN-R, and so the shifted accent will not migrate too far from its lexical sponsor. As illustrated below, the TAF constraint $\neg \mathrm{OO}_{\mathrm{Dom}-\mathrm{NO}-\mathrm{FLOP}}$ PROM requires a phonologically overt difference between the base and its derivative, but the rest of the grammar, specifically the ranking of IO-Prosodic Faithfulness relative to Alignment, ensures that the shift is bounded to the lexically accented mora.

(155) Grammar Dependence Part 2: Bounded Accent Shift

\begin{tabular}{|c|c|c|c|c|}
\hline Base & /káš ai + na/ & $\neg \mathrm{OO}_{\text {Dom }}-\mathrm{NO}-\mathrm{FLOP}$ & LOC-FLOP-PROM & ALIGN-R \\
\hline kášs ai & káš ai-n & $* !$ & & \\
\hline b. káš ai & kaš aí-n & & $* * !$ & \\
\hline c. $\rightarrow$ káš ai & kaš ái-n & & $*$ & * \\
\hline
\end{tabular}

The boundedness of the morphological shift is thus explained with the same constraint interaction used for the boundedness of phonological shifts, namely as the domination of ALIGN-R by LOC-FLOP-PROM. 
There is an apparent problem with the analysis concerning the grammar dependent nature of the shifts. Shifts due to syncope are to the right by default, but to the left if such a shift would yield an accent in the final foot of the word (see discussion above). Morphological shifts appear to be exclusively to the right, however, which is in conflict with the default-to-opposite nature of the phonological accent shifts. It turns out that this apparent inconsistency is really due to a combination of independently needed constraints which account for the absence of leftward shift. Recall that the morphological shift is only triggered in words with three moras or less (the crucial data is in (149)). This restriction, combined with the general avoidance of final mora accent, has the effect of precluding shifts from the third mora of a word; in order to have third mora accent, it is necessary to have a fourth mora, but bases with four moras or more generally do not shift. Returning to the main point, the only phonological context which would yield a leftward shift under the conditions stated in the analysis of syncope is a word with third mora accent and two moras beyond the third mora; only such a context would produce a shift from the first foot to the final foot, which is ruled out by NONFINALITY $(\mathrm{Ft})$. Furthermore, in words with a morphological shift, the shift of the base accent is always in a non-final foot, necessarily because of the size requirement on the shift, as shown below with some nominativepossessive pairs repeated from above.

(156) Foot Structures for Words with Accent Shift

$\begin{array}{ll}\text { (núka) } & \text { (nuká)-(nuu) } \\ \text { (kawáu) } & \text { (kawaú)-(nuu) } \\ \text { (káš ai) } & \text { (kaš ái)-(nuu) }\end{array}$

These shifts therefore do not incur a violation of NONFINALITY $(\mathrm{Ft})$, and so the shift is toward the default edge in the language, namely the right edge. Note also that words with a monosyllabic suffix, e.g., the accusative suffix -na, will also not trigger a leftward shift because all the words with the requisite size to undergo the shift, such as the three mora base, /kawáu + na/ $\rightarrow$ [(kawaú)-n $\Delta]$, will always be parsed by a single prosodic foot. Therefore, a shift into the final foot is unavoidable because there is only one; since any shift will violate NONFINALITY $(\mathrm{Ft})$, the default edge orientation kicks in again to give the observed shift to the right.

As for the size requirement on accent shift, I am only prepared to put forth a speculative analysis which makes use of the prosodic foot. To start, Payne makes the interesting suggestion that this restriction is a kind of locality effect. The idea here is that smaller nouns will bring the accent of the base 'close enough' to the suffix in order to trigger the shift; but in longer forms, the base accent and the suffix are too far apart, so there is no shift. If this is the correct way to analyze this pattern, then Aguaruna is indeed a strong case for the theory of accent shifts proposed here, as affix-controlled processes are generally subject to locality requirements. But as far as I can tell, the implementation of this idea will have some difficulties with contrasts like the following.
a. kawáu
kawaú-nuu
Base is $\leq \mu \mu \mu$ : Accent shifts
káš ai
kaš ái-nuu
b. wampukái
wampukái-nuu
Base is $\geq \mu \mu \mu$ : No shifts
maákai
maákai-nuu 
The lexical accent in the base forms here are identical in terms of their proximity to the suffix, and yet only the smaller nouns have a shift.

A different idea, in the spirit of Payne's original insight, is that the suffixes specifically mutate the accent in the main stress foot of the word, and that the suffix must be close enough to this primary accent. Thus, employing the foot structures argued for above in the analysis of syncope, we can characterize the initial foot as the main stress foot (realized as a pitch accent in this language), and if the suffix is close enough to the accent of the main stress foot, it can trigger a shift. To be more concrete, if the TAF constraint is conjoined with the Stem: PrWd Anchoring constraint in the domain of the prosodic foot (see $\$ 4.3$ for the details of constraint formulation), then the accent of the base will only shift if it is in the stem-final foot. Thus, this approach accounts for the cases in (157b) because the accent of the base is either not in the initial foot, as in the case of [(wampu)(kái)-nuu], or the accent of the first foot is not close enough, as in [(maá)(kai)nuu], the latter effect being crucial to the analysis of locality here.

Furthermore, the foot-based approach leads to an attractive approach to a different class of accent-shifting suffix, which is identical in all respects to the suffixes discussed here, except that in four mora words with third mora accent, the lexical accent is actually shifted. For example, the first person possessive suffix $-N(u)$, behaves this way; it shifts an accent in all of the three mora nouns shown above for other accent-shifting suffixes, but in addition, it shifts an accent in cases like: /wampukái $+\mathrm{Nu} / \rightarrow$ wampukaí- $N$ 'my tadpole', with stems with four moras and third mora lexical accent. A loosening of the requirement that the shifted accent be in the first foot yields a shift in precisely this context, as shown by: [(wampu)(kaí)-N]. The important point though is that a shift of initial or peninitial accents are still banned because the foot containing these accents would not be adjacent. As the details involved in implementing this idea in the current framework are too cumbersome to present in detail here, I leave the job of working out this problem formally for future research. To sum up, a first pass at an analysis of the size restriction is encouraging, as it fits in nicely with the model of affix-controlled accentual processes laid out in $\S 4.3$ to account for locality conditions on the application of the process.

To summarize the results established above, the proposed analysis achieves one of the central goals of this chapter, namely to account for the properties of accent shifts with the same basic toolbox used in the treatment of other affix-controlled accentual processes. Thus, the use of subcategorized correspondence relations sensitive to different TAF constraints is fundamental in distinguishing affixes by their morpho-accentual behavior. Furthermore, the explanation of this behavior via the TAF constraint $\neg$ OO-NOFLOP-PROM accounts for three additional properties of accent shift in Aguaruna, namely that it distinguishes a derivative from its base (i.e., it is morphological) and that the shift is basemutating and subject to locality conditions. Finally, the independently necessary constraints LOC-FLOP-PROM and ALIGN (PROM, R, PrWd, R) are crucial in the analysis of the boundedness and edge orientation of the shift, effectively characterizing the process as grammar dependent on par with the other types of ACA analyzed in this chapter. 


\section{BIBLIOGRAPHY}

Abe, Yasuaki. 1981. A Formal Theory of Pitch Accent in Japanese. University of Massachusetts Occasional Papers in Linguistics 7: 1-16.

Abe, Yasuaki. 1985. A Theory of Categorial Morphology and Agglutination in Japanese. Doctoral dissertation, University of Massachusetts, Amherst.

Adisasmito-Smith, Niken. 1998. Faithfulness Constraints and Consonant Patterns in Indonesian. In Matthew Pearson (ed.), UCLA Occasional Papers in Linguistics 21, Austronesian Formal Linguistics Association III-IV: 1-18.

Akinaga, Kazue (ed.), 1981. Meikai nihongo akusento jiten. [Clear Japanese Accent Dictionary]. Saneido, Japan.

Alderete, John. 1995a. Winnebago Accent and Dorsey's Law. In Beckman et al. (eds.), $21-51$.

Alderete, John. 1995b. Faithfulness to Prosodic Heads. Ms., University of Massachusetts, Amherst. [ROA-253-0498, http://ruccs.rutgers.edu/roa.html]

Alderete, John. 1996. Prosodic Faithfulness in Cupeño. To appear in Benedicto, Elena (ed.), University of Massachusetts Occasional Papers in Linguistics, Papers on Indigenous Languages. [ROA-131-0496]

Alderete, John. 1997a. Head Dependence in Stress Epenthesis Interaction. To appear in Hermans \& van Oostendorp (eds.).

Alderete, John. 1997b. Dissimilation as Local Conjunction. North East Linguistic Society 27: 17-31. [ROA-175-0297]

Alderete, John. 1997c. Multiple Correspondence and Base Optimization. Poster presented at Maryland Mayfest/Hopkins Optimality Workshop.

Alderete, John, Jill Beckman, Laura Benua, Amalia Gnanadesikan, John McCarthy, \& Suzanne Urbanczyk. 1998. Reduplication with Fixed Segmentism. To appear in Linguistic Inquiry. [ROA-226-1097]

Allen, Margaret. 1978. Morphological Investigations. Doctoral dissertation, University of Connecticut, Storrs.

Anderson, Stephen. 1992. A-Morphous Morphology. Cambridge: Cambridge University Press.

Anderson, Stephen \& Wayles Browne. 1973. On Keeping Exchange Rules in Czech. Papers in Linguistics VI: 445-82. 
Antilla, Arto \& Adams Bodomo. 1996. Stress and Tone in Dagaare. Ms., Stanford University and Norwegian University of Science and Technology.

Aoyagi, Seizô. 1969. A Demarcative Pitch of Some Prefix-Stem Sequences in Japanese. Onsei no Kenkyuu 14: 241-47.

Archangeli, Diana \& Douglas Pulleyblank. 1984. Extratonality and Japanese Accent. Ms., MIT and McGill University.

Archangeli, Diana \& Douglas Pulleyblank. 1987. Minimal and Maximal Rules: Effects of Tier Scansion. North East Linguistic Society 17: 16-35.

Aronoff, Mark. 1976. Word Formation in Generative Grammar. Cambridge, MA: MIT Press.

Austin, Peter. 1981. A Grammar of Diyari, South Australia. Cambridge: Cambridge University Press.

Bach, Emmon. 1968. Two Proposals Concerning the Simplicity Metric in Phonology. Glossia 2: 128-149.

Baerman, Matthew. The Evolution of Prosodic Constraints in Macedonian. Lingua 104: $57-78$.

Bailey, T. M. 1995. Nonmetrical Constraints on Stress. Doctoral dissertation, University of Minnesota. [http://cogsci1.psych.ox.ac.uk/PAPERS/toddabstract1.html]

Baković , Eric. 1996. Foot Harmony and Quantitative Adjustments. Ms., Rutgers University. [ROA-168-1296]

Baković , Eric. 1998. Unbounded Stress and Factorial Typology. To appear in Ron Artstein \& Madeline Holler (eds.), Rutgers Working Papers in Linguistics I. [ROA244-0298]

Barker, Chris. 1989. Extrametricality, the Cycle, and Turkish Word Stress. In Junko Itô \& Jeff Runner (eds.), Phonology at Santa Cruz 1: 1-34.

Bascom, Burt. 1959. Tonomechanics of Northern Tepehuan. Phonetica 4: 71-88.

Bascom, Burt. 1965. Proto-Tepiman. Doctoral dissertation, University of Washington.

Bat-El, Outi. 1993. Parasitic Metrification in the Modern Hebrew Stress System. The Linguistic Review 10: 189-210.

Bates, Dawn \& Barry Carlson. 1989. Prosodic Structure in Spokane Morphology. Working Papers of the Linguistics Circle of the University of Victoria 8: 75-95.

Beckman, Jill. 1995. Shona Height Harmony. In Beckman et al. (eds.), 53-75.

Beckman, Jill. 1997. Positional Faithfulness, Positional Neutralization and Shona Height Harmony. Phonology 14: 1-46. 
Beckman, Jill. 1997 [1998]. Positional Faithfulness. Doctoral dissertation, University of Massachusetts, Amherst. [Amherst, MA: Graduate Linguistic Student Association, ROA-234-1297]

Beckman, Jill, Laura Walsh Dickey, \& Suzanne Urbanczyk (eds.). 1995. University of Massachusetts Occasional Papers in Linguistics 18, Papers in Optimality Theory.

Beckman, Mary. 1986. Stress and Non-Stress Accent. Netherlands Phonetic Archives 7. Dordrecht: Foris.

Beckman, Mary E. \& Jan Edwards. 1994. Articulatory Evidence for Differentiating Stress Categories. In Keating (ed.), 7-33.

Beckman, Mary \& Janet Pierrehumbert. 1986. Intonational Structure in English and Japanese. Phonology 3: 255-310.

Bennett, Diana. 1981. Pitch Accent in Japanese: A Metrical Analysis. Masters thesis, University of Texas at Austin.

Benua, Laura. 1995. Identity Effects in Morphological Truncation. In Beckman et al., 77-136. [ROA-74-0000]

Benua, Laura, 1997 [1998]. Transderivational Identity: Phonological Relations between Words. Doctoral dissertation, University of Massachusetts, Amherst. [Amherst, MA: Graduate Linguistic Student Association, ROA-259-0498]

Bickmore, Lee. 1996. Bantu Tone Spreading and Displacement as Alignment and Minimal Misalignment. Ms., University of Albany. [ROA-161-1196]

Bickmore, Lee. 1997. Constraint High Tone Spans in Ekegusii Verbal Tonology. Ms., University of Albany. [ROA-77-0000]

Black, Andrew. 1993. Constraint-Ranked Derivation: A Serial Approach to Optimization. Doctoral dissertation, University of California, Santa Cruz.

Black, Deirdre. 1996. The Morphological and Phonological Structures of Spokane Lexemes. Doctoral dissertation, University of Victoria.

Blake, Susan J. 1998. The OCP and Root-Affix Faithfulness in St'át'imcets (Lillooet Salish). Ms., University of British Columbia.

Blevins, Juliette. 1993. A Tonal Analysis of Lithuanian Nominal Accent. Language 69: 237-73.

Blevins, Juliette. 1997. Rules in Optimality Theory. In Roca (ed.), 227-60.

Bloch, Bernard. 1946a. Studies in Colloquial Japanese I: Inflection. Journal of the American Oriental Society 66: 97-109. [Reprinted in Roy Andrew Miller (ed.), 1970, Bernard Bloch on Japanese, New Haven: Yale University Press.] 
Bloch, Bernard. 1946b. Studies in Colloquial Japanese III: Derivation of Inflected Words. Journal of the American Oriental Society 66: 304-15. [Reprinted in Roy Andrew Miller (ed.), 1970, Bernard Bloch on Japanese, New Haven: Yale University Press.]

Bolozky, Shmuel. 1982. Remarks on Rhythmic Stress in Modern Hebrew. Journal of Linguistics 18: 275-89.

Booij, Geert \& Jaap van Marle (eds.). 1995. Yearbook of Morphology 1994. Dordrecht: Kluwer.

Booij, Geert \& Jeroen van de Weijer (eds.). 1998. Phonology in Progress - Progress in Phonology: HILP Papers III. The Hague: Holland Academic Press.

Borowsky, Toni. 1986. Topics in the Lexical Phonology of English. Doctoral dissertation, University of Massachusetts, Amherst. [Amherst, MA: Graduate Linguistic Student Association]

Borowsky, Toni. 1993. On the Word Level. In Hargus \& Kaisse (eds.), 199-234.

Bright, William \& Jane Hill. 1967. The Linguistic History of the Cupeño. In Dell Hymes and W. Bittle (eds.), Studies in SouthWestern Ethnolinguistics, 351-71, The Hague: Mouton \& Co.

Broselow, Ellen \& John McCarthy. 1982. A Theory of Internal Reduplication. The Linguistic Review 3: 25-88.

Brown, Dunstan, Greville Corbett, Norman Fraser, Andrew Hippisley, and Alan Timberlake. 1996. Russian Noun Stress and Network Morphology. Linguistics 34: 53-107.

Browne, Wayles. 1978. Aspectual Morphology and VYGLJADET'. In Ernest A. Scatton, Richard D. Steele, \& Charles E. Gribble (eds.), Studies in Honor of Horace G. Lunt on the Occasion of His Sixtieth Birthday, Part 1, 46-53. [Folia Slavica 2.1-3: 46-53]

Buckley, Eugene. 1995. Uniformity in Extended Paradigms. To appear in Hermans \& van Oostendorp (eds.).

Burzio, Luigi. 1994. Principles of English Stress. Cambridge: Cambridge University Press.

Burzio, Luigi. 1996. Surface Constraints versus Underlying Representations. In Durand \& Laks (eds.), 123-42.

Burzio, Luigi. 1998. Multiple Correspondence. Lingua 104: 79-109.

Carlson, Barry. 1976. The N Shift in Spokane Salish. International Journal of American Linguistics 42: 133-39.

Carlson, Barry. 1989. Reduplication and Stress in Spokane. International Journal of American Linguistics 55: 204-13. 
Chafe, Wallace. 1977. Accent and Related Phenomena in the Five Nations Iroquois Languages. In Hyman (ed.), 209-30.

Chew, John J. 1964. Accent in Japanese Compounds. In Joseph K. Yamagiwa (ed.), Papers of the CIC Far Eastern Language Institute 1963, Ann Arbor: Committee of Institutional Cooperation.

Chew, John J. 1973. A Transformational Analysis of Modern Colloquial Japanese. Mouton: The Hague.

Chung, Sandra. Transderivational Relationships in Chamorro Phonology. Language 59: 35-66.

Clements, G. N. 1981. Akan Vowel Harmony: A Nonlinear Analysis. In G.N. Clements (ed.), Harvard Studies in Phonology, Vol. II, Part 1, 108-188, Bloomington: Indiana University Linguistics Circle.

Clements, G.N. \& K.C. Ford. 1979. Kikuyu Tone Shift and its Synchronic Consequences. Linguistic Inquiry 10: 179-210.

Clements, G.N. \& John Goldsmith. 1984. Autosegmental Studies in Bantu Tone: Introduction. In G.N. Clements \& John Goldsmith (eds.), Autosegmental Studies in Bantu Tone, 1-17, Dordrecht: Foris.

Cohn, Abigail. 1989. Stress in Indonesian and Bracketing Paradoxes. Natural Language and Linguistic Theory 7: 167-216.

Cohn, Abigail \& John McCarthy. 1994. Alignment and Parallelism in Indonesian Phonology. Ms., Cornell University and University of Massachusetts, Amherst. [ROA-25-0894]

Cook, Eung-Do. 1972. Stress and Related Rules in Tahltan. International Journal of American Linguistics 38: 231-33.

Corbett, Greville. 1982. Gender in Russian: An Account of Gender Specification and its Relationship to Declension. Russian Linguistics 6: 197-232.

Crosswhite, Catherine. 1997. Segmental vs. Prosodic Correspondence in Chamorro. Ms., University of California, Los Angeles.

Crowhurst, Megan. 1991. Demorification in Tübatulabal: Evidence from Initial Reduplication and Stress. North East Linguistic Society 21: 49-64.

Crowhurst, Megan. 1994. Foot Extrametricality and Template Mapping in Cupeño. Natural Language and Linguistic Theory 12: 177-202.

Crowhurst, Megan. 1996. An Optimal Alternative to Conflation. Phonology 13: 409-24.

Crowhurst, Megan \& Mark Hewitt. 1997. Boolean Operations and Constrain Interactions in Optimality Theory. Ms., University of North Carolina at Chapel Hill \& Brandeis University. [ROA-229-1197]

Culter, Anne. 1986. Forbear as a Homophone: Lexical Prosody Does Not Constraint Lexical Access. Language and Speech 29: 201-220. 
Cutler, Anne \& Chuck Clifton. 1984. The Use of Prosodic Information in Word Recognition. In Herman Boumaa \& Don G. Bouwhuis (eds.), Attention and Performance X. London: Lawrence Erlbaum Associates.

Czaykowska-Higgins, Ewa. 1993. Cyclicity and Stress in Moses-Columbia Salish (Nxa'amxcin). Natural Language and Linguistic Inquiry 11: 197-278.

Dixon, Robert M. W. 1977. A Grammar of Yidiñ. Cambridge: Cambridge University Press.

Duanmu, San. 1995. Alignment and the Cycle are Different. To appear in Hermans \& van Oostendorp (eds.). [ROA-93b-0000]

Durand, Jacques \& Bernard Laks (eds.). 1996. Current Trends in Phonology: Models and Methods, Vol. 1. European Studies Research Institute (ESRI), University of Salford, Salford, Manchester, UK

Elordieta, Gorka. 1997. Accent, Tone, and Intonation in Lekeitio Basque. In Fernando Martínez-Gil and Alfonso Morales-Front (eds.), Issues in the Phonology and Morphology of the Major Iberian Languages, 3-78, Washington D. C.: Georgetown University Press.

Fabb, Nigel. 1988. English Suffixation is Constrained only by Selectional Restrictions. Natural Language and Linguistic Theory 6: 527-39.

Féry, Caroline. 1996. German Foot and Word Stress in OT. To appear in Journal of Comparative Germanic Linguistics. [ROA-301-0399]

Fitzgerald, Colleen. 1997. O'odham Rhythms. Doctoral dissertation, University of Arizona, Tuscon. [ROA-190-0597]

Flemming, Edward. 1995. Auditory Representations in Phonology. Doctoral dissertation, University of California, Los Angeles.

Franks, Steven. 1989. The Monosyllabic Head Effect. Natural Language and Linguistic Theory 7: 551-63.

Franks, Steven. 1991. Diacritic Extrametricality versus Diacritic Accent: A Reply to Hammond. Phonology 8: 145-62.

Fraser, Norman \& Greville Corbett. 1995. Gender, Animacy, and Declensional Class Assignment: A Unified Account for Russian. In Booij \& van Marle (eds.), 123-150.

Frisch, Stephan, Michael Broe, \& Janet Pierrehumbert. 1996. The Role of Similarity in Phonotactic Constraints. Ms., Northwestern University.

Fromkin, Victoria A. (ed.). 1978. Tone: A Linguistic Survey. New York: Academic Press.

Fry, Dennis B. 1955. Duration and Intensity as Physical Correlates of Linguistic Stress. Journal of the Acoustical Society of America 27: 765-68. 
Fry, Dennis B. 1958. Experiments in the Perception of Stress. Language and Speech 1: 126-52.

Fudge, Erik. 1984. English Word-Stress. London: George, Allen \& Unwin.

Fukazawa, Haruka. 1998. Multiple Input-Output Faithfulness Relations in Japanese. Ms., University of Maryland, College Park.

Gafos, Diamandis. 1996a. The Articulatory Basis of Locality in Phonology. Doctoral dissertation, Johns Hopkins University.

Gafos, Diamandis. 1996b. The Two Faces of Anchoring. Ms., University of Massachusetts, Amherst.

Garde, Paul. 1968. L’Accent. Paris: Presses Universitaires de France.

Garde, Paul. 1973. Principles of the Synchronic Description of Stress. In Erik C. Fudge (ed.), Phonology: Selected Readings, 309-19, Harmondsworth: Penguin.

Garde, Paul. 1976. Histoire de l'Accentuation Slave. Paris: Institute d'Etudes Slaves.

Gessner, Suzanne. 1999. Tone Assimilation in Navaho. Ms., University of British Columbia.

Geytenbeek, Brian \& Helen Geytenbeek. 1971. Gidabal Grammar and Dictionary. Australian Aboriginal Studies No. 43. Canberra: A.C.T.

Gnanadesikan, Amalia. 1997. Phonology with Ternary Scales. Doctoral dissertation, University of Massachusetts, Amherst. [ROA-195-0597]

Goldsmith, John. 1976. Autosegmental Phonology. Doctoral dissertation, MIT.

Goldsmith, John. 1984. Tone and Accent in Tonga. In Clements \& Goldsmith (eds.), 19-51.

Goldsmith, John. 1987. Tone and Accent, and Getting the Two Together. Berkeley Linguistics Society 13: 88-104.

Goldsmith, John. 1990. Autosegmental \& Metrical Phonology. Oxford: Basil Blackwell.

Goldsmith, John (ed.). 1993. The Last Phonological Rule: Reflections on Constraints and Derivations. Chicago: University of Chicago Press.

Goldsmith, John (ed.). 1995. Handbook of Phonological Theory. Cambridge, MA: Basil Blackwell.

Goyvaerts, Didier L. \& Geoffrey K. Pullum (eds.). 1974. Essays on the Sound Pattern of English. Ghent: Scientific Publishers.

Green, Anthony. 1997. The Prosodic Structure of Irish, Scots Gaelic, and Manx. Doctoral dissertation, Cornell University. [ROA-196-0597] 
Green, Thomas \& Michael Kenstowicz. 1995. The Lapse Constraint. Ms., MIT. [ROA101-0000]

Greenberg, Joseph. 1966. Some Universals of Grammar with Particular Reference to the Order of Meaningful Elements. In J. H. Greenberg (ed.), Universals of Language (2nd edition), Cambridge, MA: MIT Press.

Gregersen, Edgar A. 1972. Consonant Polarity in Nilotic. In Erhard Voeltz (ed.), Third Annual Conference on African Linguistics, Bloomington: Indiana University.

Gribble, Charles E. 1981. Russian Root List, With a Sketch of Word Formation. Columbus: Slavica Publishers, Inc.

Halle, Morris. 1973a. The Accentuation of Russian Words. Language 49: 312-48.

Halle, Morris. 1973b. Prolegomena to a Theory of Word Formation. Linguistic Inquiry 4: 3-16.

Halle, Morris. 1990. Respecting Metrical Structure. Natural Language and Linguistic Theory 8: 149-79.

Halle, Morris. 1996. On Stress and Accent in Indo-European. Language 73: 275-313.

Halle, Morris \& Michael Kenstowicz. 1991. The Free Element Condition and Cyclic versus Noncyclic Stress. Linguistic Inquiry 22: 457-501.

Halle, Morris \& Samuel Jay Keyser. 1971. English Stress: Its Form, Its Growth, and Its Role in Verse. New York: Harper \& Row.

Halle, Morris \& Paul Kiparsky. 1981. Review of Histoire de l'Accentuation Slave, by Paul Garde. Language 57: 150-81.

Halle, Morris \& K.P. Mohanan. 1985. Segmental Phonology and Modern English. Linguistic Inquiry 16: 57-116.

Halle, Morris \& Jean-Roger Vergnaud. 1987a. An Essay on Stress. Cambridge, MA: MIT Press.

Halle, Morris \& Jean-Roger Vergnaud. 1987b. Stress and the Cycle. Linguistic Inquiry 18: $45-84$.

Halle, Morris \& Jean-Roger Vergnaud. 1991. A Reexamination of the Stress Erasure Convention in Spanish Stress. Linguistic Inquiry 23: 141-59

Hammond, Michael. 1984. Constraining Metrical Theory: A Modular Theory of Rhythm and Destressing. Doctoral dissertation, University of California, Los Angeles.

Hammond, Michael. 1986. The Obligatory-Branching Parameter in Metrical Theory. Natural Language and Linguistic Theory 4: 185-228.

Hammond, Michael. 1989a. Cyclic Secondary Stress and Accent in English. West Coast Conference on Formal Linguistics 8: 139-53. 
Hammond, Michael. 1989b. Lexical Stresses in Macedonian and Polish. Phonology 7: 19-38.

Haraguchi, Shosuke. 1977. The Tone Pattern of Japanese: An Autosegmental Theory of Tonology. Tokyo: Kaitakusha Publishers.

Haraguchi, Shosuke. 1991. A Theory of Stress and Accent. Dordrecht: Foris.

Hargus, Sharon \& Ellen Kaisse (eds.). 1993. Phonetics and Phonology 4: Studies in Lexical Phonology. San Diego: Academic Press.

Harris, James. 1983. Syllable Structure and Stress in Spanish: A Nonlinear Analysis. Cambridge, MA: MIT Press.

Harris, James. 1991. The Stress Erasure Convention and Cliticization in Spanish. Linguistic Inquiry 20: 339-63.

Hawkins, John A. \& Gary Gilligan. 1988. Prefixing and Suffixing Universals in Relation to Basic Word Order. Lingua 74: 219-257.

Hayes, Bruce. 1980. A Metrical Theory of Stress Rules. Doctoral dissertation, MIT.

Hayes, Bruce. 1982. Extrametricality and English Stress. Linguistic Inquiry 13: 227-50.

Hayes, Bruce. 1984. The Phonology of Rhythm in English. Linguistic Inquiry 15: 3374.

Hayes, Bruce. 1985. Iambic and Trochaic Rhythm in Stress Rules. Berkeley Linguistic Society 11: 429-46.

Hayes, Bruce. 1987. A Revised Parametric Metrical Theory. North East Linguistic Society 17: 274-89.

Hayes, Bruce. 1995. Metrical Stress Theory: Principles and Case Studies. Chicago: University of Chicago Press.

Hayes, Bruce. 1997. Anticorrespondence in Yidiñ. To appear in Hermans \& van Oostendorp (eds.).

Hermans, Ben. 1991. The Composite Nature of Accent: with Case Studies of Limburgian and Serbo-Croatian Pitch Accent. Doctoral dissertation, Free University of Amsterdam.

Hermans, Ben \& Marc van Oostendorp (eds.). Forthcoming. The Derivational Residue. Amsterdam: John Benjamins.

Hewitt, Mark. 1992. Vertical Maximization and Metrical Theory. Doctoral dissertation, Brandeis University.

Hewitt, Mark \& Megan Crowhurst. 1996. Conjunctive Constraints and Templates in Optimality Theory. North East Linguistic Society 26: 101-116.

Higurashi, Yoshiko. 1983. The Accent of Extended Word Structure in Tokyo Standard Japanese. Tokyo: EDUCA Inc. 
Hill, Jane. 1967. A Grammar of the Cupeño Language. Doctoral dissertation, University of California, Los Angeles.

Hill, Jane. 1970. A Peeking Rule in Cupeño. Linguistic Inquiry 1: 534-39.

Hill, Jane \& Kenneth Hill. 1968. Stress in the Cupan (Uto-Aztecan) Languages. International Journal of American Linguistics 34: 233-41.

Hill, Jane \& Rosinda Nolasquez. 1973. Mulu'wetam: The First People. Banning: Malki Museum Press.

Hirayama, Teruo. 1960. Zenkoku akusento jiten. [All Japan Accent Dictionary]. Tokyo: Tokyodo

Hock, Hans H. 1973. Exceptions and Synchronic Analogy in Sanskrit. Studies in the Linguistic Sciences 3: 81-101.

Holten, David. 1995. Assimilation and Dissimilation of Sundanese Liquids. In Beckman et al. (eds.), 167-80.

Hooper, Joan. 1976. An Introduction to Natural Generative Phonology. New York: Academic Press.

Hualde, José Ignacio. 1991. Basque Phonology. London: Routledge.

Hualde, José Ignacio. 1998. A Gap Filled: Post-Post-Initial Accent in Azkoitia Basque. Linguistics 36: 99-117.

Hualde, José Ignacio \& Xabier Bilbao. 1992. A Phonological Study of the Basque Dialect of Getxo. Anuario del Seminario del Filologia Vasca Julio de Urquijo 26: 1-118.

Hualde, José Ignacio \& Xabier Bilbao. 1993. The Prosodic System of the Basque Dialect of Getxo: A Metrical Analysis. Linguistics 31: 59-85.

van der Hulst, Harry. 1994. Separating Primary and Secondary Stress. Ms., University of Leiden.

van der Hulst, Harry. 1995. Word Accent. To appear in Harry van der Hulst (ed.), Word Prosodic Systems in the Languages of Europe, Berlin: Mouton de Gruyter.

van der Hulst, Harry \& Norval Smith (eds.). 1982. The Structure of Phonological Representations. Dordrecht: Foris.

van der Hulst, Harry \& Norval Smith. 1988. The Variety of Pitch Accent Systems: Introduction. In Harry van der Hulst \& Norval Smith (eds.), Autosegmental Studies in Pitch Accent, ix-xxiv, Dordrect: Foris.

Hung, Henrietta. 1994. The Rhythmic and Prosodic Organization of Edge Constituents. Doctoral dissertation, Brandeis University.

Hyman, Larry. 1977. On the Nature of Linguistic Stress. In Hyman (ed.), 37-82.

Hyman, Larry. 1978. Tone and/or Accent. In Napoli (ed.), 1-20. 
Hyman, Larry (ed.). 1977. Studies in Stress and Accent. Southern California Occasional Papers in Linguistics 4. Los Angeles: Department of Linguistics, University of Southern California.

Idsardi, William. 1991. Stress in Interior Salish. Chicago Linguistics Society 27: 24660 .

Idsardi, William. 1992. The Computation of Prosody. Doctoral dissertation, MIT.

Inkelas, Sharon. 1989. Prosodic Constituency in the Lexicon. Doctoral dissertation, Stanford University.

Inkelas, Sharon. 1994. Exceptional Stress-Attracting Suffixes in Turkish: Representation vs. the Grammar. To appear in Kager et al. (eds.).

Inkelas, Sharon. 1996. Dominant Affixes and the Phonology-Morphology Interface. In Ursula Kleinhenz (ed.), Studia Grammatica 41, Interfaces in Phonology: 128-154.

Inkelas, Sharon \& Draga Zec. 1988. Serbo-Croatian Pitch Accent. Language 64: 227-48.

Inkelas, Sharon \& Draga Zec (eds.). 1990. The Phonology-Syntax Connection. Chicago: The University of Chicago Press.

Inkelas, Sharon, Orhan Orgun, \& Cheryl Zoll. 1995. Exceptions and Static Phonological Patterns: Cophonologies versus Prespecification. Ms., University of California, Berkeley.

Itô, Junko \& Armin Mester. 1986. The Phonology of Voicing in Japanese: Theoretical Consequences for Morphological Assessibility. Linguistic Inquiry 17: 49-73

Itô, Junko \& Armin Mester. 1992. Weak Layering and Word Binarity. Linguistic Research Center Technical Report (LRC-92-09), University of California, Santa Cruz.

Itô, Junko \& Armin Mester. 1995a. The Core-Periphery Structure of the Lexicon and Constraints on Re-Ranking. In Beckman et al. (eds.), 181-209.

Itô, Junko \& Armin Mester. 1995b. Japanese Phonology: Constraint Domains and Structure Preserving. In Goldsmith (ed.), 817-38.

Itô, Junko \& Armin Mester. 1997. Correspondence and Compositionality: The GA-gyo Variation in Japanese Phonology. In Roca (ed.), 419-62. [ROA-145-0096]

Itô, Junko \& Armin Mester. 1998. The Phonological Lexicon. To appaer in Natsuko Tsujimura (ed.), Handbook of Japanese Linguistics. Oxford: Blackwell.

Itô, Junko, Yoshihisa Kitagawa, \& Armin Mester. 1996. Prosodic Faithfulness and Correspondence: Evidence from a Japanese Argot. Journal of East Asian Linguistics 5: 217-94. [ROA-146-0996]

Jahr, Ernst Hå kon, Toril Swan, Oddleif Leirbukt, \& Ove Lorentz (eds.). 1996. Nordlyd. Troms $\phi$ University Working Papers on Language and Linguistics 24, Papers from the Second Workshop on Comparative Germanic Phonology. Troms $\emptyset$ : University of Troms $\varnothing$. 
Jakobson, Roman. 1963. Opyt fonologicheskogo podxoda K istoricheskim voprosam slavjanskoj akcentologii. American contributions to the 5th International Congress of Slavists, 153-78.

Jakobson, Roman. 1965. Information and Redundancy in the Common Slavic Prosodic Pattern. Symbolae linguisticae in honorem Georgii Kurylowicz, 145-51. Wroclaw: Polska Akademija Nauk.

Jones, Danial \& Dennis Ward. 1969. The Phonetics of Russian. Cambridge: Cambridge University Press.

Kager, René. 1989. A Metrical Theory of Stress and Destressing in English and Dutch. Dordrecht: Foris.

Kager, René. 1992. Alternatives to the Iambic-Trochaic Law. Natural Language and Linguistic Theory 11: 381-432.

Kager, René. 1995. Surface Opacity of Metrical Structure in Optimality Theory. To appear in Hermans \& van Oostendorp (eds.).

Kager, René. 1996. Stem Stress and Peak Correspondence in Dutch. Ms., Utrecht University.

Kager, René. 1997. Rhythmic Vowel Deletion in Optimality Theory. In Roca (ed.), 46399.

Kager, René, Harry van der Hulst, \& Wim Zonneveld (eds.). 1998. The ProsodyMorphology Interface. Cambridge: Cambridge University Press.

Kageyama, Taro. 1982. Word Formation in Japanese. Lingua 57: 215-58.

Kaisse, Ellen. 1985. Some Theoretical Consequences of Stress Rules in Turkish. Chicago Linguistic Society 21: 199-209.

Katayama, Motoko. 1995. Loanword Accent and Minimal Re-Ranking in Japanese. In Walker et al., 1-12.

Katayama, Motoko. 1998. Optimality Theory and Japanese Loanword Phonology. Doctoral dissertation, University of California, Santa Cruz.

Keating, Patricia A. (ed.). 1994. Phonological Structure and Phonetic Form. Papers in Laboratory Phonology III. Cambridge: Cambridge University Press.

Kennedy, Chris. 1994. Morphological Alignment and Head Projection. In Merchant et al., 47-64. [ROA-139-0996]

Kenstowicz, Michael. 1994. Sonority Driven Stress. Ms., MIT. [ROA-33-1094]

Kenstowicz, Michael. 1995a. Cyclic vs. Non-Cyclic Constraint Evaluation. Phonology 12: 397-436. [ROA-31-0000]

Kenstowicz, Michael. 1995b. Quality-Sensitive Stress. Ms., MIT. 
Kenstowicz, Michael. 1996. Base-Identity and Uniform Exponence: Alternatives to Cyclicity. In Durand \& Laks (eds.), 363-93.

Kenstowicz, Michael. 1997. Uniform Exponence: Exemplification and Extension. Ms., MIT. [ROA-103-0000]

Kenstowicz, Michael \& Charles Kisseberth. 1977. Topics in Phonological Theory. New York: Academic Press.

Kim, Michael Jinhwa. 1996. The Tonal System of Accentual Languages. Doctoral dissertation, University of Chicago.

King, Alan R. 1994. The Basque Language. A Practical Introduction. Reno: University of Nevada Press.

Kiparsky, Paul. 1973. The Inflectional Accent in Indo-European. Language 49: 794-849.

Kiparsky, Paul. 1979. Metrical Structure Assignment is Cyclic. Linguistic Inquiry 10: 421-41.

Kiparsky, Paul. 1982a. From Cyclic to Lexical Phonology. In van der Hulst \& Smith (eds.), Part I, 131-75.

Kiparsky, Paul. 1982b. Lexical Morphology and Phonology. In I.-S. Yang (ed.), Linguistics in the Morning Calm, 3-91. Seoul: Hanshin Publishing Co.

Kiparsky, Paul. 1982c. The Lexical Phonology of Vedic Accent. Ms., MIT.

Kiparsky, Paul. 1984a. A Compositional Approach to Vedic Word Accent. In S. D. Joshi (ed.), Amrtadhara: Prof. R. N. Dandeka Felicitation Volume, 201-10. Jawahar Nagar Delhi: Ajanta Publications.

Kiparsky, Paul. 1984b. On the Lexical Phonology of Icelandic. In C. Elert, I. Johansson, \& E. Strangert (eds.), Nordic Prosody III, 135-164. Umea, Sweden: University of Umea.

Kiparsky, Paul. 1993. Blocking in Non-Derived Environments. In Hargus \& Kaisse (eds.), 277-313.

Kiparsky, Paul \& Morris Halle. 1977. Towards a Reconstruction of the Indo-European Accent. In Hyman (ed.), 209-38.

Kirchner, Robert. 1993. Round and Back Vowel Harmony and Disharmony: An Optimality Theoretic Account. Ms., University of California, Los Angeles.

Kirchner, Robert. 1996. Synchronic Chain Shifts in Optimality Theory. Linguistic Inquiry 27: 341-350.

Kisseberth, Charles. 1970. On the Functional Unity of Phonological Rules. Linguistic Inquiry 1: 291-306.

Krause, Scott. 1979. Topics in Chukchee Phonology and Morphology. Doctoral dissertation, University of Illinois. 
Kubozono, Haruo. 1988 [1993]. The Organization of Japanese Prosody. Doctoral dissertation, University of Edinburgh. [Tokyo: Kurosio Publishers]

Kubozono, Haruo. 1995. Constraint Interaction in Japanese Phonology: Evidence from Compound Accent. In Walker et al. (eds.), 21-38.

Kubozono, Haruo. 1997. Lexical Markedness and Variation: A Nonderivational Account of Japanese Compound Accent. West Coast Conference on Formal Linguistics 15: 273-87.

Kurata, Kiyoshi. 1984. Accent in Japanese Compounds. Ms., University of Massachusetts, Amherst.

Lakoff, George. 1993. Cognitive Phonology. In Goldsmith (ed.), 117-45.

Langdon, Margaret. 1970. A Grammar of Diegueño. The Mesa Grande Dialect. Berkeley: University of California Press.

Larson, Mildred. 1956. Aguaruna Noun Suffixation: A Tentative Statement. Información de Campo 10c. Yarinacocha, Pucallpa, Pera: Instituto Lingüístico de Verano.

Larson, Mildred. 1963. Vocabulario Aguaruna de Amazonas. Serie Lingüística Peruana 3. Yarinacocha, Pucallpa, Pera: Instituto Lingüístico de Verano.

Lehiste, Ilse \& Pavle Ivić . 1986. Word and Sentence Prosody in Serbocroatian. Cambridge, MA: MIT Press.

Liberman, Mark \& Alan Prince. 1977. On Stress and Linguistic Rhythm. Linguistic Inquiry 8: 249-336.

Lightner, Theodore M. 1972. Problems in the Theory and Phonology, Volume I: Russian and Turkish Phonology. Edmonton: Linguistic Research, Inc.

Lin, Yen-Hwei. 1998. Cyclic and Noncyclic Affixation in Piro. In Booij \& van de Weijer (eds.), 167-88.

Lubowicz, Anna. 1998. Derived Environment Effects in OT. Ms., University of Massachusetts, Amherst. [ROA-237-0198]

Martin, Samuel. 1952. Morphophonemics of Standard Colloquial Japanese. Language dissertation, no. 47. Baltimore: Linguistic Society of America.

Martin, Samuel. 1975. A Reference Grammar of Japanese. New Haven: Yale University Press.

Mascaró, Joan. 1976. Catalan Phonology and the Phonological Cycle. Doctoral dissertation, MIT.

Masuda, Koh (ed.). 1974. Kenkyusha's New Japanese-English Dictionary. Tokyo: Kenkyusha.

McCarthy, John. 1979a. Formal Problems in Semitic Phonology and Morphology. Doctoral dissertation, MIT. 
McCarthy, John. 1979b. On Stress and Syllabification. Linguistic Inquiry 10: 443-65.

McCarthy, John. 1984. Theoretical Consequences of Montañes Vowel Harmony. Linguistic Inquiry 15: 291-318.

McCarthy, John. 1995. Extensions of Faithfulness: Rotuman Revisited. To appear in Natural Language and Linguistic Theory. [ROA-110-0000]

McCarthy, John. 1997. Faithfulness and Prosodic Circumscription. To appear in Joost Dekkers, Frank van der Leeuw, \& Jeroen van de Weijer (eds.), The Pointing Finger: Conceptual Studies in Optimality Theory. The Hague: Holland Academic Press. [ROA-201-0697]

McCarthy, John. 1998. Serialism, OT and the Duke of York Gambit. Paper presented at Tübingen Syllable Conference.

McCarthy, John \& Alan Prince. 1986. Prosodic Morphology. Ms., University of Massachusetts, Amherst \& Brandeis University.

McCarthy, John \& Alan Prince. 1990. Foot and Word in Prosodic Morphology: The Arabic Broken Plural. Natural Language and Linguistic Theory 8: 209-83.

McCarthy, John \& Alan Prince. 1993a. Generalized Alignment. In Booij \& van Marle (eds.), 79-153.

McCarthy, John \& Alan Prince. 1993b. Prosodic Morphology I: Constraint Interaction and Satisfaction. Rutgers Center for Cognitive Science (RuCCs) Technical Report 3, University of Massachusetts, Amherst and Rutgers University. [Forthcoming MIT Press]

McCarthy, John \& Alan Prince. 1994a. The Emergence of the Unmarked: Optimality in Prosodic Morphology. North East Linguistics Society 24: 333-79.

McCarthy, John \& Alan Prince. 1994b. Two Lectures on Prosodic Morphology (Utrecht, 1994). Ms., University of Massachusetts, Amherst and Rutgers University. [ROA59-0000]

McCarthy, John \& Alan Prince. 1995. Faithfulness and Reduplicative Identity. In Beckman et al. (eds.), 249-384. [ROA-60-0000]

McCarthy, John \& Alan Prince. 1997. Faithfulness and Identity in Prosodic Morphology. To appear in Kager et al. (eds.). [ROA-216-0997]

McCawley, James D. 1968. The Phonological Component of a Grammar of Japanese. The Hague: Mouton.

McCawley, James D. 1978. What is a Tone Language? In Fromkin (ed.), 113-32.

Melvold, Janis. 1986. Cyclicity and Russian Stress. North East Linguistics Society 17: 467-81.

Melvold, Janis. 1990. Structure and Stress in the Phonology of Russian. Doctoral dissertation, MIT. 
Merchant, Jason, Jaye Padgett, \& Rachel Walker (eds.). 1994. Phonology at Santa Cruz 3. University of California, Santa Cruz.

Mester, Armin. 1994. The Quantitative Trochee in Latin. Natural Language and Linguistic Theory 12: 1-62.

Michelson, Karen. 1988. A Comparative Study of Lake-Iroquoian Accent. Dordrecht: Reidel.

Miner, Kenneth L. 1979. Dorsey's Law in Winnebago-Chiwere and Winnebago Accent. International Journal of American Linguistics 45: 25-33.

Mohanan, K.P. 1985. The Theory of Lexical Phonology. Dordrecht: Reidel.

Moreton, Elliott. 1996. Non-Computable Functions in Optimality Theory. Ms., University of Massachusetts, Amherst.

Munro, Pamela. 1977. Towards a Reconstruction of Uto-Aztecan Stress. In Hyman (ed.), 209-38.

Munro, Pamela. 1990. Stress and Vowel Length in Cupan Absolute Nouns. International Journal of American Linguistics 56: 217-50.

Myers, Scott. 1987a. Tone and the Structure of Words in Shona. Doctoral dissertation, University of Massachusetts, Amherst.

Myers, Scott. 1987b. Vowel Shortening in English. Natural Language and Linguistic Inquiry 3: 485-518.

Myers, Scott. 1991. Structure Preservation and the Strong Domain Hypothesis. Linguistic Inquiry 1991: 379-85.

Myers, Scott. 1997a. OCP Effects in Optimality Theory. Natural Language and Linguistic Theory 15: 847-92.

Myers, Scott. 1997b. Expressing Phonetic Naturalness in Phonology. In Roca (ed.), $125-52$.

Myers, Scott \& Sung-a Kim. 1998. The Timing of F0 Peaks in Chichewa: An Alternative to Nonlocal Tone Spread. Ms., University of Texas at Austin.

de Napoli, Joanna (ed.). 1978. Elements of Tone, Stress, and Intonation. Washington, D.C.: Georgetown University Press.

Nater, Hank F. 1989. Some Comments on the Phonology of Tahltan. International Journal of American Linguistics 55: 25-42.

Nelson, Nicole. 1998. Mixed Anchoring in French Hypocoristic Formation. To appear in Ron Artstein \& Madeline Holler (eds.), Rutgers Working Papers in Linguistics I.

Nespor, Marina \& Irene Vogel. 1986. Prosodic Phonology. Dordrecht: Foris. 
Ní Chiosáin, Máine \& Jaye Padgett. 1997. Markedness, Segment Realization, and Locality of Spreading. Linguistic Research Center Technical Report (LRC-97-01), University of California, Santa Cruz.

NHK. 1985. Nihongo hatsuon akusento ziten. [Japanese Pronounciation Accent Dictionary]. Tokyo: NHK.

Odden, David. 1994. Adjacency Parameters in Phonology. Language 70: 289-330.

Odden, David. 1995. Tone: African Languages. In Goldsmith (ed.), 444-75.

Odden, David. 1996. Patterns of Reduplication in Kikerewe. Ohio State University Working Papers in Linguistics 48, Papers in Phonology, 111-48.

Okoth-Okombo, Duncan. 1982. Dholuo Morphophonemics in a Generative Framework. Berlin: Dietrich Reimer Verlag.

Okuda, Kunio. 1971. Accentual Systems in Japanese Dialects. Doctoral dissertation, UCLA.

Orgun, Orhan. 1994. Monotonic Cyclicity and Optimality Theory. North East Linguistic Society 24: 461-74.

Orgun, Orhan. 1995. Correspondence and Identity Constraints in Two-Level Optimality Theory. Ms., University of California, Berkeley. [ROA-62-0000.]

Orgun, Orhan. 1997. Sign-Based Morphology and Phonology with Special Attention to Optimality Theory. Doctoral dissertation, University of California, Berkeley. [ROA171-0197]

Otsu, Yukio. 1980. Some Aspects of Rendaku in Japanese and Related Problems. In Yukio Otsu \& Ann Farmer (eds.), MIT Working Papers in Linguistics 2: Theoretical Issues in Japanese Linguistics, 207-27.

Parker, Steve. 1994. Coda Epenthesis in Huariapano. International Journal of American Linguistics 60: 95-119.

Parker, Steve. 1997. An OT Account of Laryngealization in Cuzco Quechua. In Stephan A. Marlett (ed.), Working Papers of the Summer Institute of Linguistics, University of North Dakota 41, Fargo, North Dakota.

Parker, Steve. 1998. Disjoint Metrical Tiers and Positional Markedness in Huariapano. Ms., University of Massachusetts, Amherst.

Pater, Joseph. 1994. Against the Underlying Representation of an 'Exceptional' English Stress Pattern. Ms., McGill University.

Pater, Joseph. 1995. On the Non-Uniformity of Weight-to-Stress and Stress Preservation Effects in English. Ms., McGill University.

Pater, Joe. 1996. Austronesian Nasal Substitution and other NC̣ Effects. To appear in Kager et al. (eds.). 
Payne, David. 1978. Aguaruna Pitch Accent. Información de Campo 373e. Yarinacocha, Pucallpa, Peru: Instituto Lingüístico de Verano.

Payne, David L. 1990. Accent in Aguaruna. In Doris L. Payne (ed.), Amazonian Linguistics: Studies in Lowland South American Languages, Austin: University of Texas Press.

Peperkamp, Sharon. 1997. Prosodic Words. Doctoral dissertation, University of Amsterdam.

Pesetsky, David. 1979. Russian Morphology and Lexical Theory. Ms., MIT.

Pierrehumbert, Janet \& Mary Beckman. 1988. Japanese Tone Structure. Cambridge, MA: MIT Press.

Pike, Kenneth. 1948. Tone languages. Ann Arbor: University of Michigan Press.

Pike, Kenneth \& Mildred Larson. 1964. Hyperphonemes and Non-Systematic Features of Aguaruna Phonemes. In A. H. Marckwardt (ed.), Studies in Languages and Linguistics in Honor of Charles G. Fries, 55-67, Ann Arbor: University of Michigan Press.

Pike, Kenneth \& Graham Scott. 1963. Pitch Accent and Non-Accented Phrases in Fore (New Guinea). Zeitschrift für Phonetik, Sprachwissenschaft und Kommunikations forschung 16: 179-89.

Poser, William. 1982. Phonological Representation and Action-At-A-Distance. In van der Hulst \& Smith (eds.), Part II, 121-58.

Poser, William. 1984. The Phonetics and Phonology of Tone and Intonation in Japanese. Doctoral dissertation, MIT.

Poser, William. 1986. Diyari Stress, Metrical Structure Assignment, and the Nature of Metrical Representations. West Coast Conference on Formal Linguistics 5: 178-91.

Poser, William. 1989. The Metrical Foot in Diyari. Phonology 6: 117-148.

Poser, William. 1990a. Evidence for Foot Structure in Japanese. Language 66: 78-105.

Poser, William. 1990b. Word-Internal Phrase Boundary in Japanese. In Inkelas \& Zec (eds.), 279-87.

Prince, Alan. 1983. Relating to the Grid. Linguistic Inquiry 14: 19-100.

Prince, Alan. 1985. Improving Tree Theory. Berkeley Linguistic Society 11: 471-90.

Prince, Alan. 1990. Quantitative Consequences of Rhythmic Organization. Parasession on the Syllable in Phonetics and Phonology, Chicago Linguistic Society 26: 355-98.

Prince, Alan. 1993. Minimal Violation. Paper presented at Rutgers Optimality Workshop I, Rutgers University.

Prince, Alan. 1997. Stringency and Anti-Paninian Hierarchies. LSA Linguistic Institute Lecture, Cornell University. 
Prince, Alan \& Paul Smolensky. 1991. Linguistics 247: Notes on Connectionism and Harmony Theory in Linguistics. Technical Report CU-CS-533-91, Department of Computer Science, University of Colorado, Boulder.

Prince, Alan \& Paul Smolensky. 1993. Optimality Theory: Constraint Interaction in Generative Grammar. Ms., Rutgers University \& University of Colorado at Boulder. [Forthcoming MIT Press]

Pulleyblank, Douglas. 1986. Tone in Lexical Phonology. Dordrecht: Reidel.

Pullum, Geoffrey. 1976. The Duke of York Gambit. Journal of Linguistics 12: 83-102.

Quirk, Randolph \& Sidney Greenbaum. 1973. A Concise Grammar of Contemporary English. San Diego: Harcourt Brace Jovanovich.

Radhakrishnan, R. 1981. The Nancowry Word: Phonology, Affixal Morphology and Roots of a Nicobarese Language. Carbondale, Illinois and Edmonton, Alberta: Linguistic Research.

Rehg, Kenneth L. 1993. Proto-Micronesian Prosody. In Jerold A. Edmondson \& Kenneth J. Gregerson (eds.), Tonality in Austronesian Languages, 25-46, Honolulu: University of Hawaii Press.

Revithiadou, Anthi. 1997. Feet Dependent on Heads: Morphological and Prosodic Dominance. In Walker et al., 79-101.

Revithiadou, Anthi. 1998. Marked Feet in the Pool. In Booij \& van de Weijer (eds.), 297-319.

Riad, Thomas. 1992. Structures in Germanic Prosody. A Diachronic Study with Special Reference to the Nordic Languages. Doctoral dissertation, Stockholm University.

Robbins, Scarlett. 1991. Lexicalized Metrical Foot Structure in Maidu. In Armin Mester \& Scarlett Robbins (eds.), Phonology at Santa Cruz 2: 95-116.

Roca, Iggy. 1992. On the Sources of Word Prosody. Phonology 9: 267-87.

Roca, Iggy (ed.). 1997. Derivations and Constraints in Phonology. Oxford: Clarendon Press.

Ross, John R. 1972. A Reanalysis of English Word Stress. In Michael K. Brame (ed.), Contributions to Generative Phonology, 229-323, Austin: University of Texas Press.

Rubach, Jerzy. 1984. Cyclic and Lexical Phonology: The Structure of Polish. Dordrecht: Foris Publications.

Samek-Lodovici, Vieri. 1993. A Unified Analysis of Cross-Linguistic Morphological Gemination. In Peter Ackema \& Maaike Schoorlemmer (eds.), CONSOLE I, 265-83, The Hague: Holland Academic Graphics. [ROA-149-1096]

Samek-Lodivici, Vieri. 1998. Opposite Constraints: Left and Right Focus-Alignment in Kanakuru. Lingua 104: 111-130. 
Selkirk, Elisabeth. 1980. The Role of Prosodic Categories in English Word Stress. Linguistic Inquiry 11: 563-605.

Selkirk, Elisabeth. 1982. The Syntax of Words. Cambridge: MIT Press.

Selkirk, Elisabeth. 1984. Phonology and Syntax: The Relation between Sound and Structure. Cambridge: MIT Press.

Selkirk, Elisabeth. 1986. On Derived Domains in Sentence Phonology. Phonology 3: 371-405.

Selkirk, Elisabeth. 1988. Dependency, Place and the Notion 'Tier'. Ms., University of Massachusetts, Amherst.

Selkirk, Elisabeth. 1993. Labial Relations. Ms., University of Massachusetts, Amherst.

Selkirk, Elisabeth. 1995a. Surface Restrictions in the Distribution of Lexical Contrasts: The Role of Root Faithfulness. Paper prepared for Linguistics 751, University of Massachusetts, Amherst.

Selkirk, Elisabeth. 1995b. Language-Particular Violation of a Universal Constraint: The OCP from the Perspective of Optimality Theory. Ms., Marrakech.

Selkirk, Elisabeth. 1995 [1996]. The Prosodic Structure of Function Words. In Beckman et al. (eds.), 439-69. [Also published in James Morgan \& Katherine Demuth (eds.), Bootstrapping from Speech to Grammar in Early Acquisition, Hillsdale, NJ: Lawrence Erlbaum].

Sezer, Engin. 1983. On Non-Final Stress in Turkish. Journal of Turkish Studies 5: 6169.

Shaw, Patricia. 1976. Dakota Phonology and Morphology. Doctoral dissertation, University of Toronto.

Shaw, Patricia. 1985. Modularism and Substantive Constraints in Dakota Lexical Phonology. Phonology Yearbook 2: 173-202.

Shaw, Patricia. 1992. Templatic Evidence for the Syllable Nucleus. North East Linguistic Society 92: 643-77.

Shibatani, Masayoshi. 1972. The Non-Cyclic Nature of Japanese Accentuation. Language 48: 584-95.

Shibatani, Masayoshi. 1990. The Languages of Japan. Cambridge: Cambridge University Press.

Siegel, Dorothy. 1974. Topics in English Morphology. Doctoral dissertation, MIT.

Sietsema, Brian. 1989. Metrical Dependencies in Tone Assignment. Doctoral dissertation, MIT.

Smith, Jennifer. 1996. Noun Faithfulness: Evidence from Accent in Japanese Dialects. To appear in Japanese/Korean Linguistics 7, Stanford: CSLI. 
Smith, Jennifer. 1997. Noun Faithfulness: On the Privileged Behavior of Nouns in Phonology. Ms., University of Massachusetts, Amherst. [ROA-242-0198]

Spencer, Andrew. 1991. Morphological Theory: An Introduction to Word Structure in Generative Grammar. Oxford: Blackwell Publishers.

Spencer, Andrew. 1998. Morphophological Operations. In Spencer \& Zwicky (eds.), $123-43$.

Spencer, Andrew \& Arnold M. Zwicky (eds.). 1998. The Handbook of Morphology. Oxford: Blackwell Publishers.

Sproat, Richard. 1985. On Deriving the Lexicon. Doctoral dissertation, MIT.

Stang, Christian. 1957. Slavonic Accentuation. Oslo: Universitetsforlaget.

Stankiewicz, Edward. 1962. The Singular-Plural Opposition in the Slavic Languages. International Journal of Slavic Linguistics and Poetics V: 1-15.

Stankiewicz, Edward. 1993. The Accentual Patterns of the Slavic Languages. Stanford, CA: Stanford University Press.

Steriade, Donca. 1988. Greek Accent: A Case of Preserving Structure. Linguistic Inquiry 19: 271-314.

Steriade, Donca. 1998. Lexical Conservatism and its Analysis. Ms., University of California, Los Angeles.

Suzuki, Keiichiro. 1998. A Typological Investigation of Dissimilation. Doctoral dissertation, University of Arizona, Tuscon. [ROA-281-0998]

Tesar, Bruce. 1998. An Iterative Strategy for Language Learning. Lingua 104: 131-45. [ROA-177-0297]

Tesar, Bruce \& Paul Smolensky. 1998. Learnability in Optimality Theory. Linguistic Inquiry 29: 229-68.

Townsend, Charles E. 1975. Russian Word-Formation. Columbus: Slavica Publishers, Inc.

Trubetzkoy, N. 1939. Grundzüge der Phonologie. Güttingen: Vandenhoeck and Ruprecht.

Truckenbrodt, Hubert. 1995. Phonological Phrases: Their Relation to Syntax, Focus, and Prominence. Doctoral dissertation, MIT.

Tsay, Jane S. 1991. Constraining Lexical Accent. North East Linguistics Society 21: 351-65.

Tsuchida, Ayako. 1997. Phonetics and Phonology of Japanese Vowel Devoicing. Doctoral dissertation, Cornell University.

Urbanczyk, Suzanne. 1996. Reduplication and Prosodic Morphology in Lushootseed. Doctoral dissertation, University of Massachusetts, Amherst. 
Vanderslice, Ralph \& Peter Ladefoged. 1972. Binary Suprasegmental Features and Transformational Word-Accentuation Rules. Language 48: 819-38.

Voegelin, C. F. 1935. Tübatulabal Grammar. University of California Publications in American Archaelogy and Ethnology 34: 55-190.

Walker, Rachel, Ove Lorentz, \& Haruo Kubozono (eds.). 1995. Phonology at Santa Cruz 4: Papers on Stress, Accent, and Alignment. University of California, Santa Cruz.

Walker, Rachel, Motoko Katayama, \& Daniel Karvonen (eds.). 1997. Phonology at Santa Cruz 5. University of California, Santa Cruz.

Walker, Rachel. 1996. Prominence-Driven Stress. Ms., University of California, Santa Cruz.

Walker, Rachel. 1998. Nazalization, Neutral Segments, and Opacity Effects. Doctoral dissertation, University of California, Santa Cruz.

Willett, Elizabeth. 1982. Reduplication and Accent in Southeastern Tepehuan. International Journal of American Linguistics 48: 168-84.

Willett, Thomas L. 1991. A Reference Grammar of Southeastern Tepehuan. Arlington: Summer Institute of Linguistics.

Williams, Edwin. 1981. On the Notions 'Lexically Related' and 'Head of a Word'. Linguistic Inquiry 12: 245-274.

Woo, Nancy. 1970. Tone in Northern Tepehuan. International Journal of American Linguistics 36: 18-30.

Woodbury, Anthony. 1987. Meaningful Phonological Processes: A Consideration of Central Alaskan Yupik Eskimo Prosody. Language 63: 685-740.

Yip, Moira. 1992. Reduplication with Fixed Melodic Material. North East Linguistic Society 22: 459-76.

Yip, Moira. 1995. Repetition and its Avoidance: The Case of Javanese. Ms., University of California, Irvine. [ROA-83-0000]

Yip, Moira. 1996. Feet, Tonal Reduction and Speech Rate at the Word and Phrase Level in Chinese. Paper presented at Utrecht Conference on Phasal Phonology. [ROA-1591196]

Zaliznjak, A. A. 1977. Grammatič eskij slovar' russkogo jazyka. Moscow: Russkij jazyk.

Zec, Draga. 1994. Footed Tones and Tonal Feet: Rhythmic Constituency in a Pitch Accent Language. Ms., Cornell University.

Zoll, Cheryl. 1996a. Parsing Below the Segment in a Constraint-Based Framework. Doctoral dissertation, University of California, Berkeley. [ROA-143-0996] 
Zoll, Cheryl. 1996b. Coincidence, Licensing and Suprasegmentals: License-Driven Tone Mapping. Invited talk at University of Massachusetts, Amherst.

Zoll, Cheryl. 1997. Conflicting Directionality. Phonology 14: 263-86. [ROA-151-1096]

Zubizarreta, Maria Luisa. 1982. A Metrical Acount of the Tone Pattern of Japanese: The Interaction of Harmony and Accent. In van der Hulst \& Smith (eds.), Part II, 159212. 